Florida International University

FIU Digital Commons

3-26-2019

\title{
Study of Charge Carrier Transport in Graphene and Graphite as Two Dimensional and Quasi-Two Dimensional Materials and Their Interfaces
}

Nalat Sornkhampan

Florida International University, nsorn001@fiu.edu

Follow this and additional works at: https://digitalcommons.fiu.edu/etd

Part of the Other Electrical and Computer Engineering Commons

\section{Recommended Citation}

Sornkhampan, Nalat, "Study of Charge Carrier Transport in Graphene and Graphite as Two Dimensional and Quasi-Two Dimensional Materials and Their Interfaces" (2019). FIU Electronic Theses and Dissertations. 4019.

https://digitalcommons.fiu.edu/etd/4019

This work is brought to you for free and open access by the University Graduate School at FIU Digital Commons. It has been accepted for inclusion in FIU Electronic Theses and Dissertations by an authorized administrator of FIU Digital Commons. For more information, please contact dcc@fiu.edu. 


\section{FLORIDA INTERNATIONAL UNIVERSITY}

Miami, Florida

\section{STUDY OF CHARGE CARRIER TRANSPORT IN GRAPHENE AND GRAPHITE AS TWO DIMENSIONAL AND QUASI-TWO DIMENSIONAL MATERIALS AND THEIR INTERFACES}

A dissertation submitted in partial fulfillment of the requirements for the degree of DOCTOR OF PHILOSOPHY

in ELECTRICAL AND COMPUTER ENGINEERING

by

Nalat Sornkhampan 
To: Dean John L. Volakis

College of Engineering and Computing

This dissertation, written by Nalat Sornkhampan, and entitled Study of Charge Carrier Transport in Graphene and Graphite as Two Dimensional and Quasi-Two Dimensional Materials and Their Interfaces, having been approved in respect to style and intellectual content, is referred to you for judgment.

We have read this dissertation and recommend that it be approved.

$\begin{array}{r}\hline \text { John L. Volakis } \\ \hline \text { Pete E. C. Markowitz } \\ \hline \text { Sakhrat Khizroev } \\ \hline \text { Yuriy A. Vlasov } \\ \hline \text { Jean H. Andrian, Co-Major Professor } \\ \hline \text { Grover L. Larkins, Co-Major Professor }\end{array}$

Date of Defense: March 26, 2019

The dissertation of Nalat Sornkhampan is approved.

Dean John L. Volakis
College of Engineering and Computing

College of Engineering and Computing

Andrés G. Gil

Vice President for Research and Economic Development and Dean of the University Graduate School

Florida International University, 2019 


\section{DEDICATION}

To the United States Air Force and the United States of America. 


\section{ACKNOWLEDGMENTS}

First and foremost, I would like to express my deepest gratitude to my major professor, Dr. Grover Larkins, for an opportunity to be a PhD candidate under his care. This dissertation would never have been completed without his support and guidance. His lecture was a perfect combination of academic and real-life lessons. Currently, I am working on finishing my $\mathrm{PhD}$ degree, the academic degree that I never imagined I could achieve. I would like to give my most grateful thanks to Dr. Larkins for helping me to make it happen. I will never forget the invaluable experience at the FIU Cryogenics \& Pulse Laser Deposition Laboratory.

Importantly, I must thank Dr. Yuriy Vlasov, Dr. Sakhrat Khizroev, Dr. Jean Andrian, Dr. Pete Markowitz, and the Dean of the College of Engineering and Computing, Dr. John Volakis, for agreeing to be members of my dissertation committee. It is an honor to have my doctoral dissertation under their supervision. I would like to thank Dr. Steven Prawer and his group at the University of Melbourne for the Hall effect and Raman spectra measurements, Dr. Deng of Dr. Paul Chu's group at the University of Houston for the susceptibility measurements, and Dr. Alex Gurevich of Old Dominion University and Dr. Harold Weinstock of Air Force Office of Scientific Research for the academic and financial supports.

I would also like to express my sincerest gratitude to my laboratory colleagues, Julian Gil Pinzon, Steward Schwarz, and Amber Woods, for their assiduity and dependability. Julian, Steward, and Amber have contributed a substantial amount of work to my research and dissertation. They have also been my precious friends since we were undergraduate students. 
I could not have come this far without the love and support from my family. Thanks to my wife, Salinee Kingbaisomboon, mother, Anongtip Chaisook, and father, Uthai Sornkhampan, for their immeasurable love and endless support.

Lastly, I am thankful for everyone who has directly or indirectly involved with my research and dissertation that I acknowledged or did not. I would gladly like to share this joy of success with everyone. 


\begin{abstract}
OF THE DISSERTATION
STUDY OF CHARGE CARRIER TRANSPORT IN GRAPHENE AND GRAPHITE AS

TWO DIMENSIONAL AND QUASI-TWO DIMENSIONAL MATERIALS AND
\end{abstract}

THEIR INTERFACES

by

Nalat Sornkhampan

Florida International University, 2019

Miami, Florida

Professor Grover L. Larkins, Co-Major Professor

Professor Jean H. Andrian, Co-Major Professor

Evidence of superconductivity in phosphorous-doped graphite and graphene has been observed at temperatures in the vicinity of $260 \mathrm{~K}$. This evidence includes transport current, magnetic susceptibility, Hall and Nernst measurements. All of these measurements indicate a transition of a type II superconductor without a phase of type I until below the limits of the measurement capabilities.

Vortex states are inferred from periodically repeated steps in the $R$ vs. $T$ characteristics of Highly Oriented Pyrolytic Graphite and exfoliated doped multilayer graphene. The presence of vortices has been confirmed with thermal gradient driven Nernst measurements. Magnetic susceptibility measurements have shown results qualitatively similar to those expected (and experimentally observed by others) for ultra-thin films 
(thickness « the London penetration depth). The magnetic susceptibility is negative for field-cooled and zero-field-cooled measurements. The susceptibility for field-cooled and zero-field-cooled measurements begin to diverge at approximately $260 \mathrm{~K}$. Hall effect measurements show a sign reversal in the Hall voltage as the temperature is reduced from $300 \mathrm{~K}$ to $78 \mathrm{~K}$. The Nernst effect confirms a Berezinskii-Kosterlitz-Thouless (BKT) vortex transition at $T \sim 40 \mathrm{~K}$ and several pinned vortices' melting temperatures which correlate with the resistive measurements.

Finally, in completeness, we have observed a charge BKT transition at $T \sim 4 \mathrm{~K}$ in both susceptibility and resistive measurements, and a vortex BKT transition in both the resistive, Nernst, and susceptibility measurements at $T \sim 40 \mathrm{~K}$. 


\section{TABLE OF CONTENTS}

\section{Part I}

Introduction

\section{CHAPTER}

PAGE

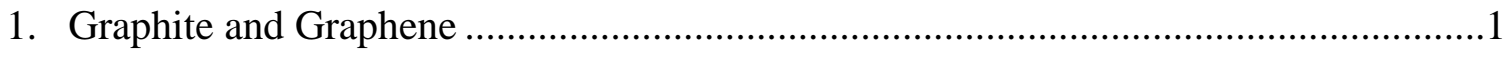

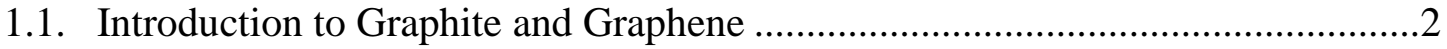

1.2. Highly Ordered Pyrolytic Graphite.............................................................

1.3. Graphene and Graphite Thin Film ..........................................................

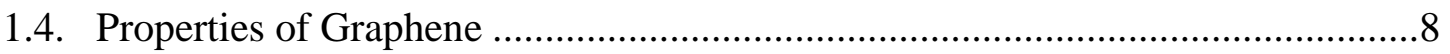

1.4.1. Physical and Mechanical Properties .....................................................

1.4.1.1. Lattice Structure .....................................................................8

1.4.1.2. Stress, Strain, and Melting Point........................................ 9

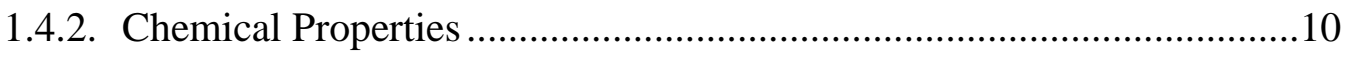

1.4.2.1. Covalent Functionalization ................................................10

1.4.2.2. Noncovalent Functionalization ...........................................12

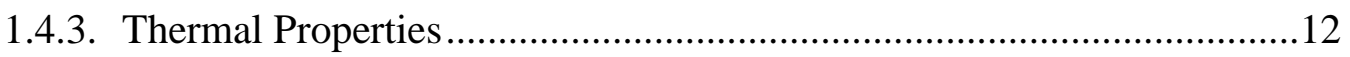

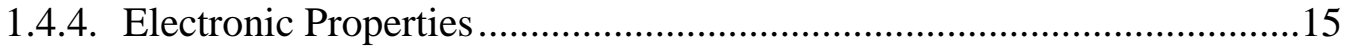

1.4.4.1. Electronic Band Structure ..................................................15

1.4.4.2. Quantum Hall Effect .........................................................17

1.4.4.3. Fractional Quantum Hall Effect..........................................21

1.4.5. Magnetic Properties .....................................................................25

1.4.5.1. Atomic Vacancies ............................................................25

1.4.5.2. Molecular Absorption .........................................................26

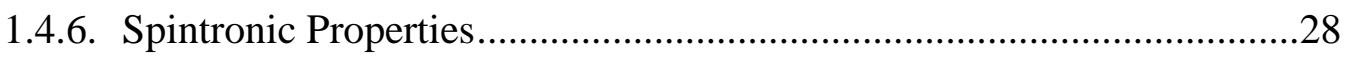

1.4.6.1. Introduction to Spintronics .............................................28

1.4.6.2. Spintronic Properties of Graphene..........................................30

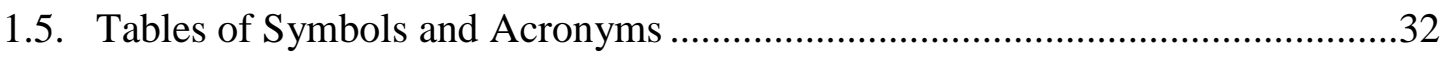

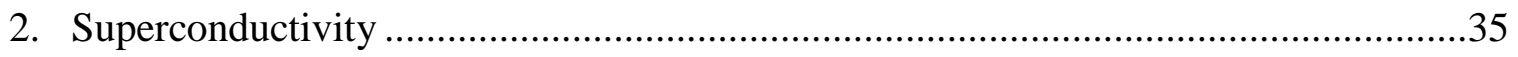

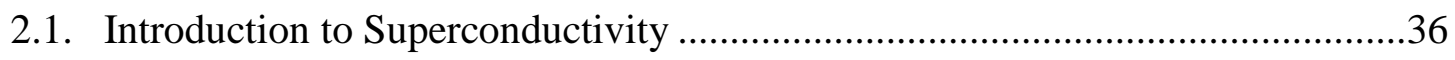

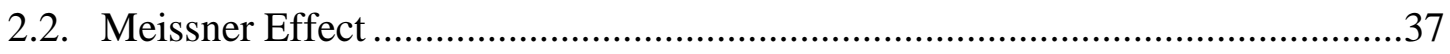

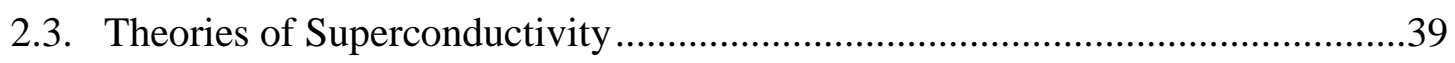

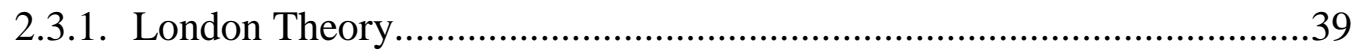

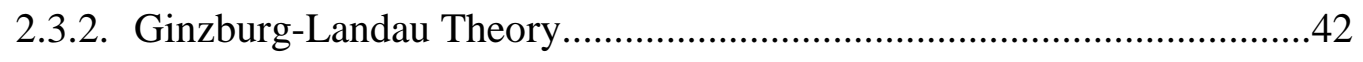

2.3.3. Bardeen-Cooper-Schrieffer Theory .................................................45

2.3.3.1. Cooper Pairs ...................................................................46

2.3.3.2. Microscopic Theory of Superconductivity ...........................48

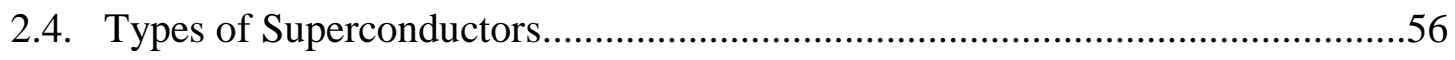


2.4.1. Type I Superconductors ....................................................................56

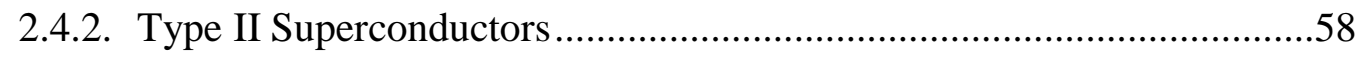

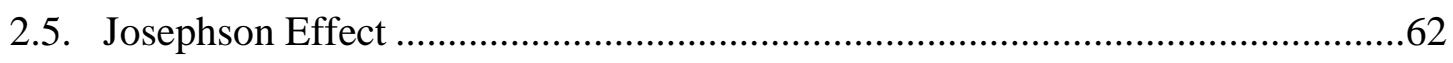

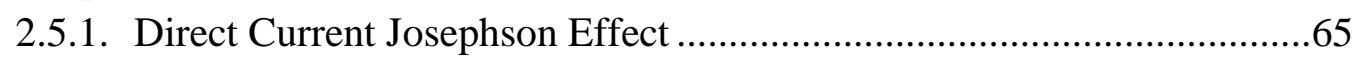

2.5.2. Alternating Current Josephson Effect ....................................................66

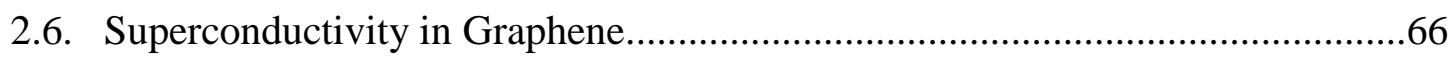

2.7. Berezinskii-Kosterlitz-Thouless Transition ......................................................69

2.7.1. Topological Defects and Phase Transitions ……………........................70

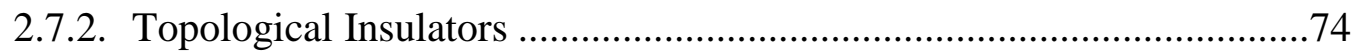

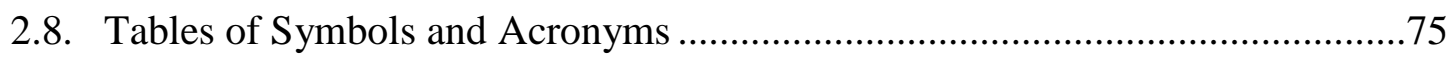

3. Electric Charge Carriers and Thermoelectric Effects ...............................................

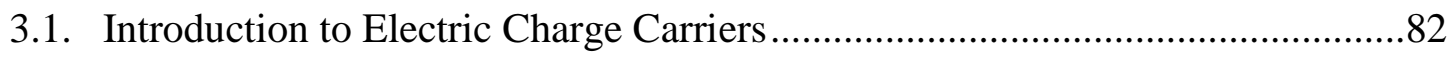

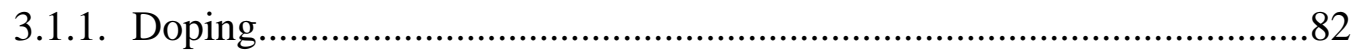

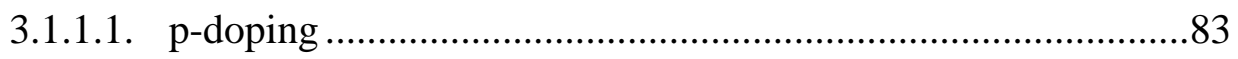

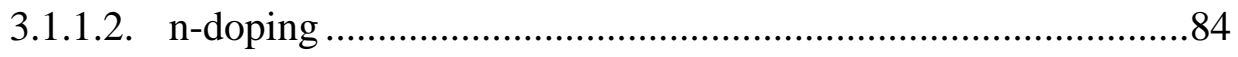

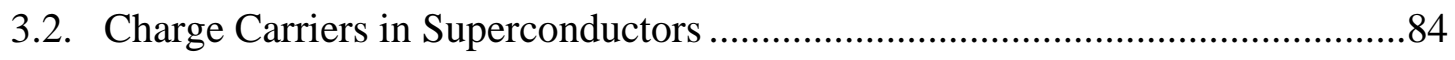

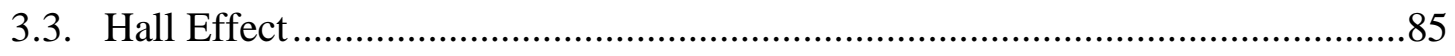

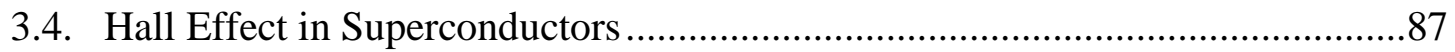

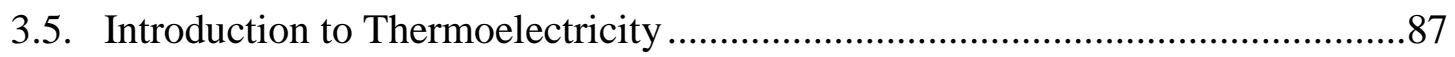

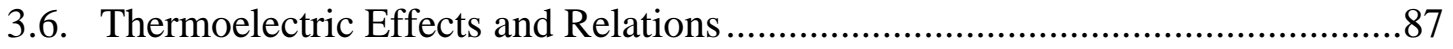

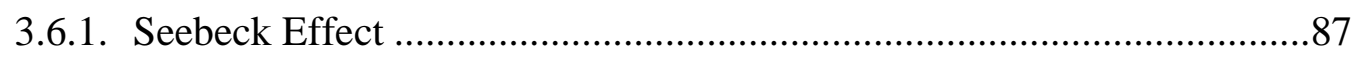

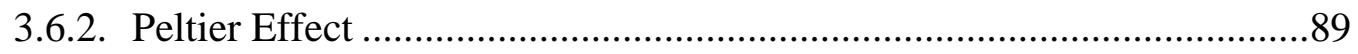

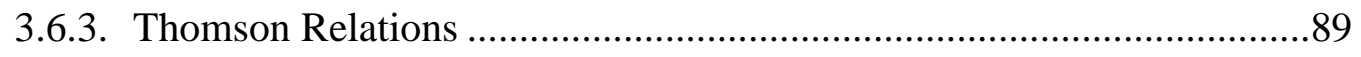

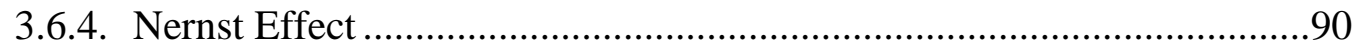

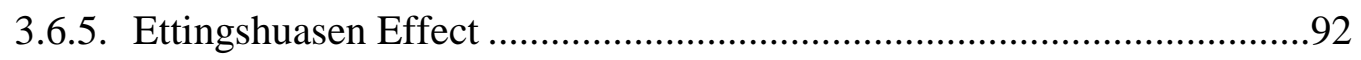

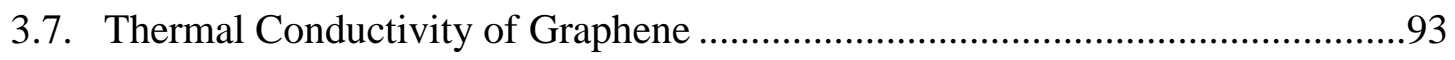

3.8. Tables of Symbols and Acronyms ..................................................................95 


\section{Part II}

\section{Experimentation}

CHAPTER

PAGE

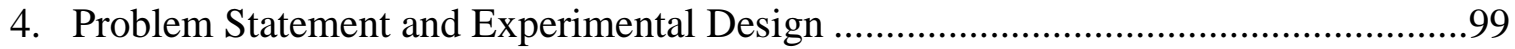

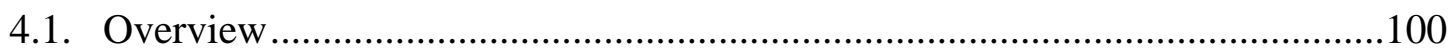

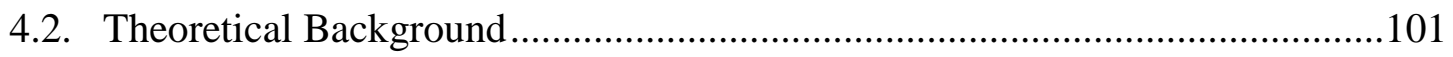

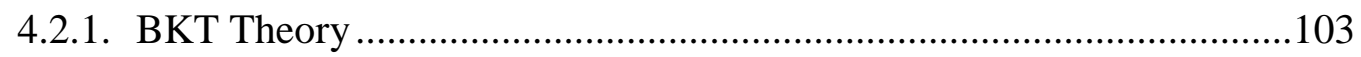

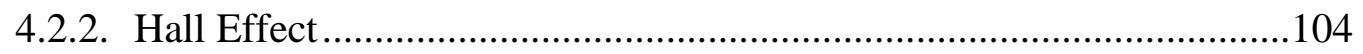

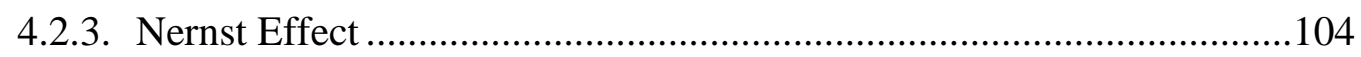

4.2.4. Pancake Vortices and Flux Pinning ..................................................105

4.2.5. Magnetic Characteristics in Thin-Film Superconductors ....................109

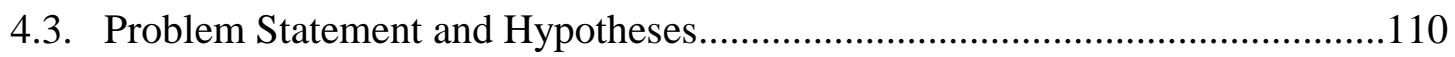

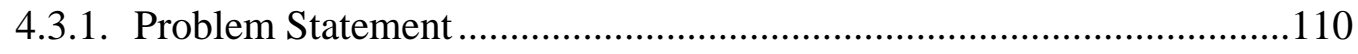

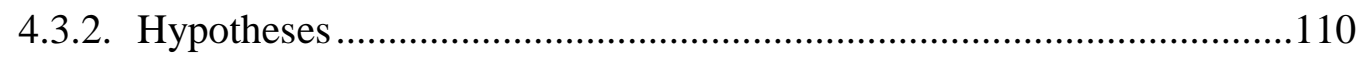

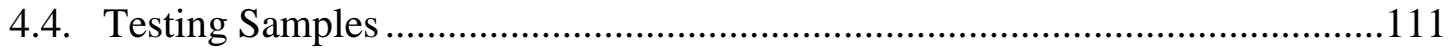

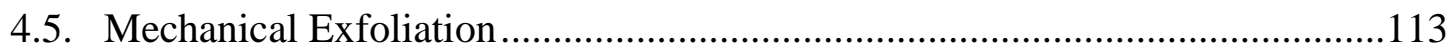

4.6. Experimental Design and Methodology .....................................................115

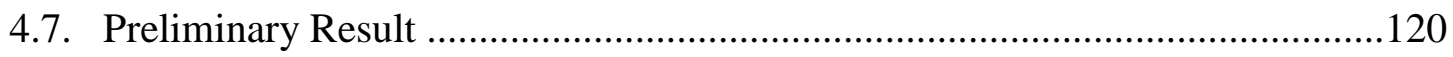

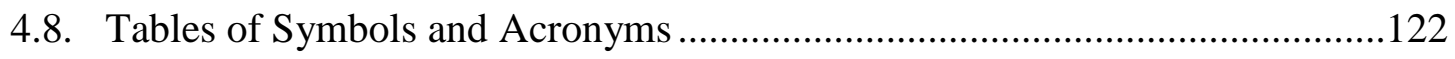

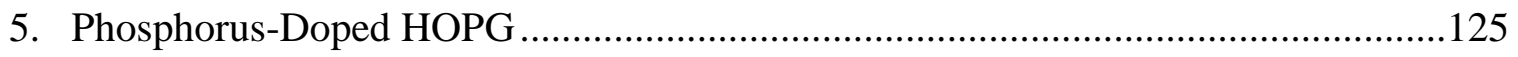

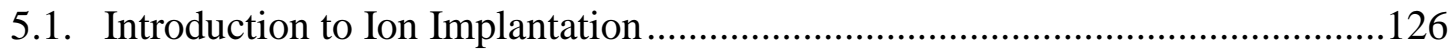

5.2. Introduction to Chemical Vapor Deposition..................................................130

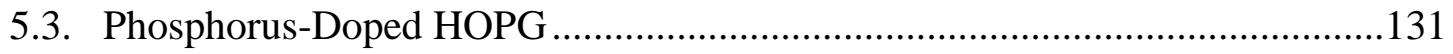

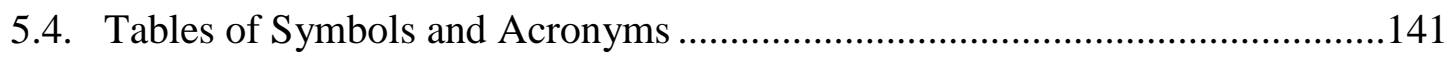

6. Results, Discussion, Conclusions, and Future Work ...........................................142

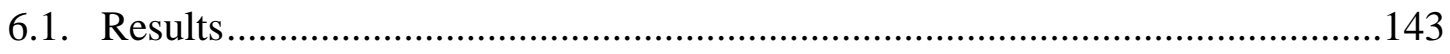

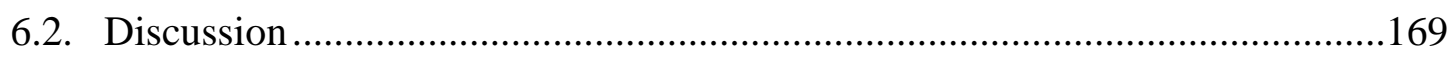

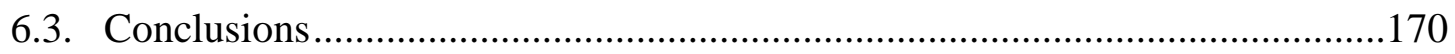

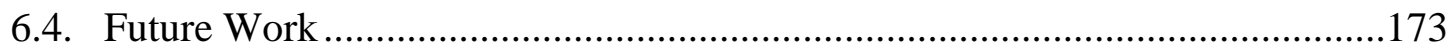

6.5. Tables of Symbols and Acronyms .......................................................... 175

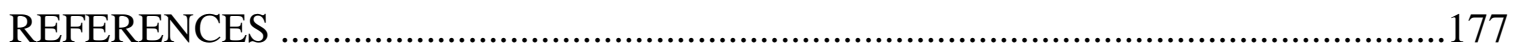

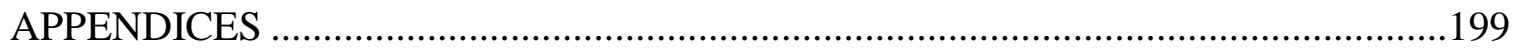

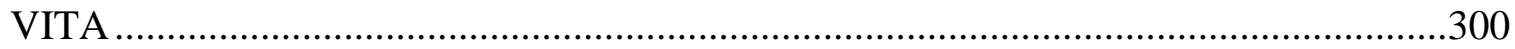




\section{LIST OF TABLES}

TABLE

PAGE

1.1: HOPG grades of quality. Image courtesy of MikroMasch [37].

1.2: Thermal conductivities of materials/mediums at room temperature.

1.3: Spin-dependent properties of graphene, metals, and semiconductor measured by spin valve measurements. Reprinted with permission from Fabian Jaroslav [64].....31

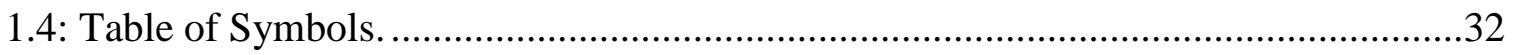

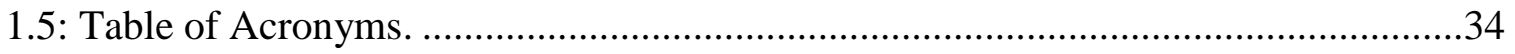

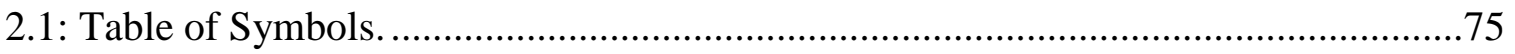

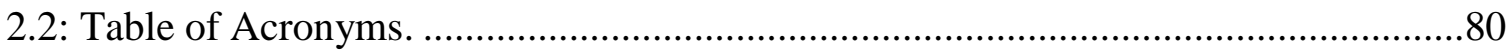

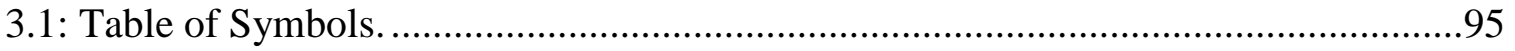

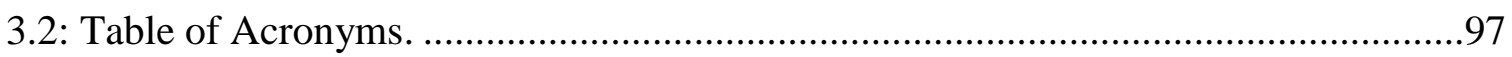

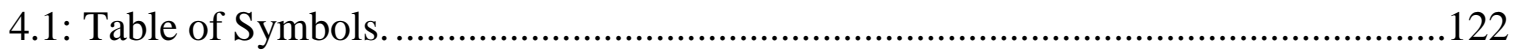

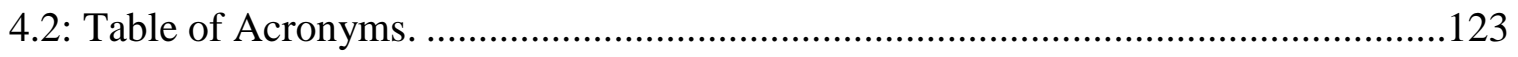

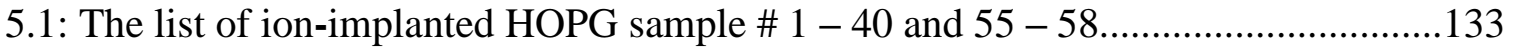

5.2: The ratio of the gas mixture to grow phosphorus doped-while-grown graphene on

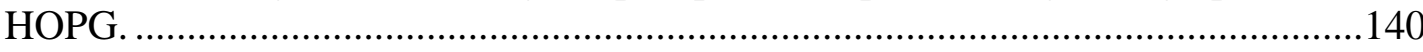

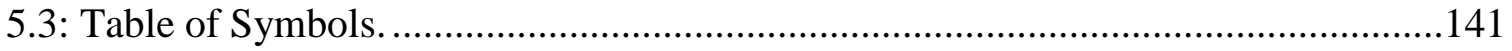

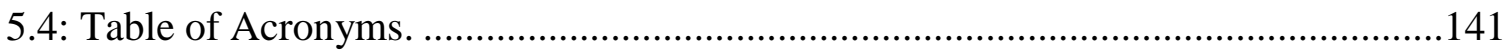

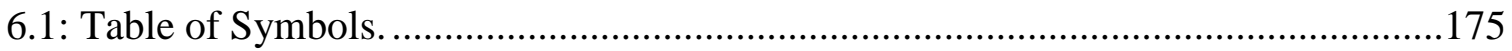

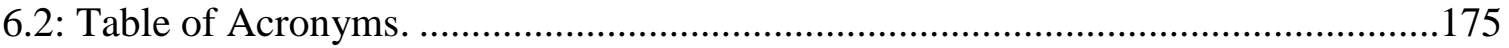




\section{LIST OF FIGURES}

\section{Chapter 1}

FIGURE

PAGE

1.1: Graphite. Reprinted with permission from Chowdhury Al-Amin [11] .....................2

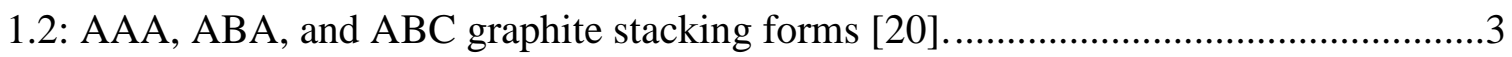

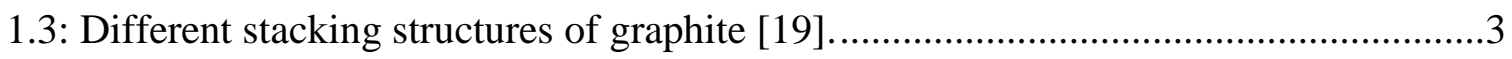

1.4: (a) $s, p_{x}$, and $p_{y}$ orbitals. (b) $s p^{2}$-hybridization..............................................

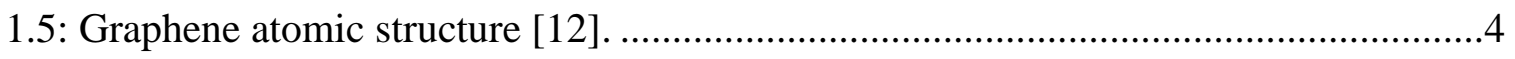

1.6: A model of changes of carbon structure during graphitization [262].......................6

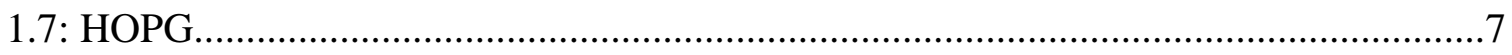

1.8: Nonequivalent carbon atoms $\mathrm{A}$ and $\mathrm{B}$ in a honeycomb lattice with armchair and zigzag edges. Reprinted with permission from S. -R. Eric Yang [40]...................... 8

1.9: (a) Graphene lattice with unit cell vectors in a real space. (b) Graphene reciprocal lattice with reciprocal lattice vectors and high symmetry points in a reciprocal space. Reprinted with permission from Vitor M. Pereira, National University of

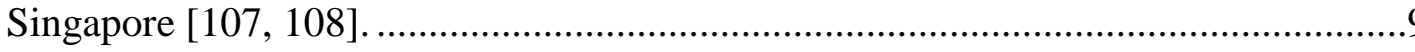

1.10: Schematic representation of the atomic structure of graphane and fluorographene. Image courtesy of GLOSSARY of NANO technology and related TERMS [52].

1.11: Chemical structure of graphene oxide. Image courtesy of The Green Optimistic [100].

1.12: Optothermal Raman measurement technique. Reprinted with permission from A.

A. Balandin [74].

1.13: Sublattices of nonequivalent carbon atoms A and B in graphene.

1.14: The Coordinates in the $k$-space of the Dirac points $\mathrm{K}$ and $\mathrm{K}^{\prime}$ [79]. .16 
1.16: A heterostructure used for Hall effect measurements. Reprinted with permission from Klaus von Klitzing, the 1985 Nobel Laureate in Physics [84].

1.17: A graph of typical measurement of the IQHE. The data was obtained from 2-D electron gas in a GaAs/GaAlAs heterojunction at $30 \mathrm{mK}$. Reprinted with permission from D. R. Leadley, University of Warwick (1997).

1.18: A schematic of a Hall bar where $V_{L}=I / \sigma_{x x}$ is the longitudinal voltage and $V_{H}=$ $I / \sigma_{x y}$ is the Hall voltage [106].

1.19: A graph of the integer quantum Hall effect in graphene. Reprinted with permission from Sir Sergeeyich Novoselov, the 2010 Nobel Laureate in Physics [82].

1.20: The results of the original experiment where the FQHE was discovered for filling factor $v=1 / 3$. Reprinted with permission from Horst Ludwig Störmer, the 1998 Nobel Laureate in Physics [91].

1.21: A graph of the FQHE where $\mathrm{N}$ is LL quantum number and $v$ indicates values of filling factors. Reprinted with permission from Daniel Chee Tsui, the 1998 Nobel Laureate in Physics [92].

1.22: Quantized Hall conductivity of graphene in FQHE for different filling factors [86].

1.23: (a) hydrogen chemisorption defect represented by $\Delta$. (b) the vacancy defect. Reprinted with permission from Prof. Yazyev Oleg, Ecole Polytechnique Federale de Lausanne [70].

1.24: (a) A hydrogen atom. (b) Graphene honeycomb lattice structure. (c) An absorbed hydrogen atom induces a magnetic moment on graphene. (d) The magnetic moments are added when hydrogen atoms are absorbed in the same sublattice. Reprinted with permission from Ivan Brihuega, Associate Professor, Universidad Autónoma de Madrid [73].

1.25: A spin torque acting on $\mu_{s}$ created by $B$.

1.26: (a) Random spin; electrons spin randomly in a material. (b) Spin alignment; electronic spins are aligned by an external magnetic field. (c) Unmagnetized; 
spins are located orderly in an unmagnetized solid state material. (d) Magnetized; spins are located orderly and aligned in a magnetized solid state material [43].

\section{Chapter 2}

FIGURE

PAGE

2.1: A graph of the electrical resistance of mercury, the resistance abruptly drops to

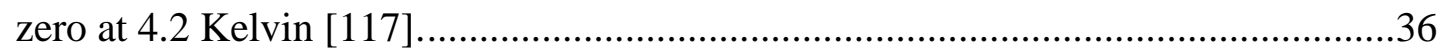

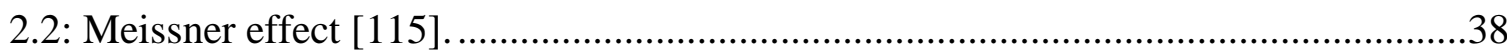

2.3: A permanent magnet is levitated by the repulsive force produced by the induced supercurrents in a superconductor where $\mathrm{N}$ and $\mathrm{S}$ represent magnetic north and south poles respectively. Reprinted with permission from Rod Nave, Georgia State University [116].

2.4: A permanent magnet is levitated over liquid nitrogen-cooled Yttrium Barium Copper Oxide (YBCO).

2.5: (a) Lattices of a conductor are attracted by an electron creating a phonon (red region). (b) Another electron is drawn by the phonon forming a Cooper pair [127]

2.6: A Feynman diagram showing the e-e interaction of a Cooper pair.

2.7: A diagram of the e-e interaction.

2.8: Pair states accessible to delocalization of a Cooper pair.

2.9: The characteristic of the temperature-dependent energy gap.

2.10: The relationship between $H_{c}$ and $T_{c}$ of type I superconductors.

2.11: The relationship of $M$ and $H$ of type I superconductors.

2.12: The phase diagram of type II superconductors.

2.13: The relationship of the $M$ and $H$ of type I superconductors [112] 
2.14: Magnetic flux lines penetrating a type II superconductor. The currents in the superconducting material generate a magnetic field which, together with the applied field, result in bundles of quantized flux [153].

2.15: The vortices of type II superconductors appear in the Shubnikov phase where the flux within each vortex is generated by a supercurrent [154].

2.16: Vortices in $\mathrm{NbSe}_{2}$ defined by scanning tunneling microscopy [152].

2.17: Two superconductors separated by a thin weak link.

2.18: A voltage is applied to a Josephson junction.

2.19: The process to obtain layers of Li-intercalated graphene [148].

2.20: $R v s . T$ of a thin-film graphene sample peeled off from P-implanted and then argon-implanted bulk HOPG sample \# 023. Curves 1, 2, 3, and 4 are four identical sequential runs with the same probe position $[56,57]$

2.21: $R$ vs. $T$ measurement of P-doped HOPG in the presence of magnetic fields from 0 to $1 \mathrm{~T}$. (These tests were performed at the University of Maryland by Dr. Paul Bach) [55]

2.22: $R$ vs. $T$ of thin film exfoliated from P-doped HOPG. Curve 1 was measured without an applied magnetic field. Curve 2 was measured under an applied magnetic field with the field strength of 0.035 tesla $[56,57]$

2.23: An illustration of the topological phase transition of the vortices. Image courtesy of J. Jarnestad / The Royal Swedish Academy of Sciences.

2.24: $R$ (in the log scale) $v s . T$ at different magnetic fields listed in the legend in panel [163].

2.25: Evolution of the temperature dependence of the sheet resistance $R(T)$ with thickness for a $B i$ film deposited onto $G e$. Reprinted with permission from David Haviland [166].

2.26: Sheet $R v s . T$ of amorphous $B i$ flims for a series of films with thicknesses, from top to bottom, of $11.15,11.25,11.37,11.38,11.43,11.48,11.55,11.65,11.75$, $11.85,11.95,12.03,12.17,12.27,12.4,12.55,12.65,12.85$, and $13.35 \AA$. 
Reprinted with permission from Allen M. Goldman, Regents Professor,

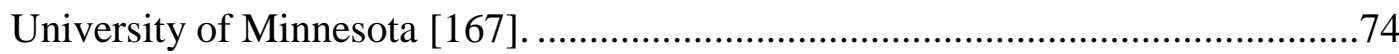

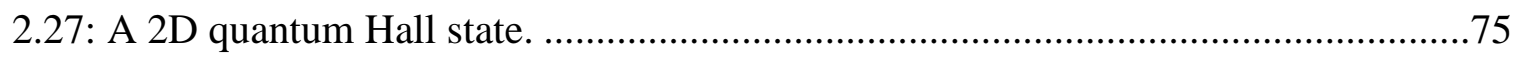

\section{Chapter 3}

FIGURE

PAGE

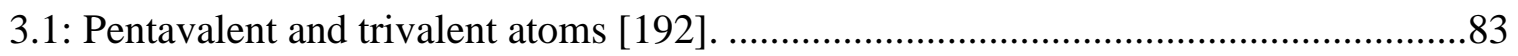

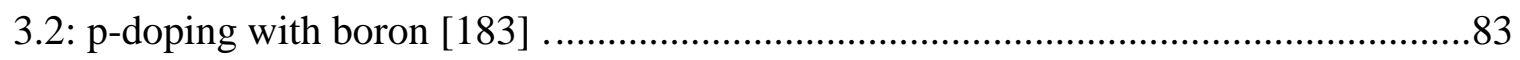

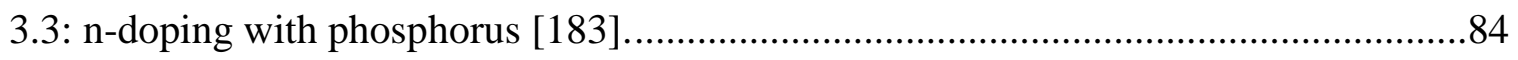

3.4: The Hall effect of negative charge carriers (electrons) [190]....................................86

3.5: The Hall effect of positive charge carriers [190] ...................................................86

3.6: A demonstration of the thermocouple Seebeck effect..............................................8

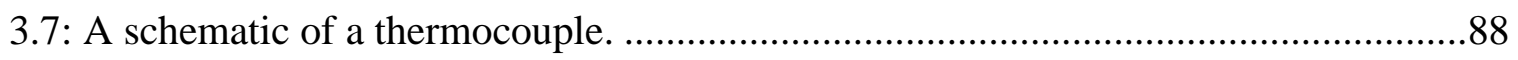

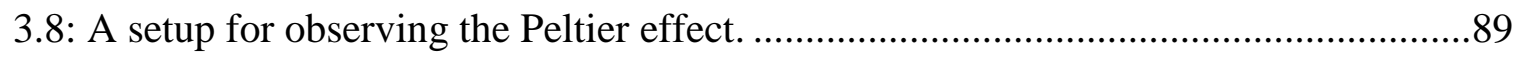

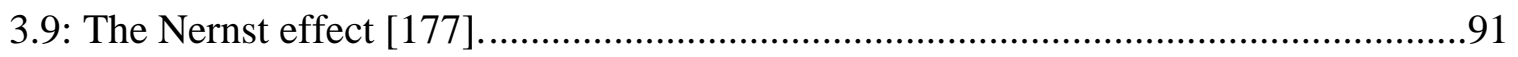

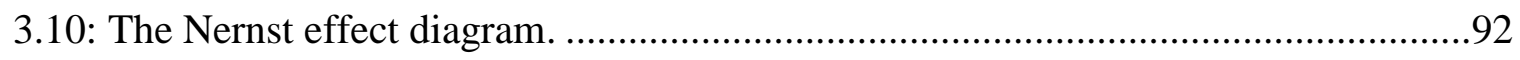

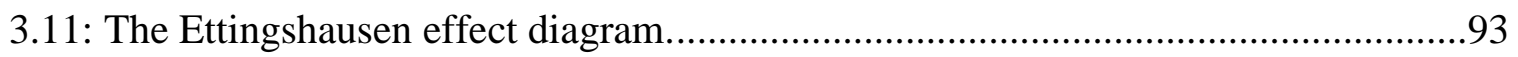

Chapter 4

FIGURE

PAGE

4.1: A flux line in a strongly layered superconductor is made up of pancake vortices in the superconducting layers interconnected by Josephson lines [234]..

4.2: The sample preparation devices. A vacuum device with a blue vacuum tube (left). A chuck with a through hole (right) [57]. .114

4.3: A HOPG sample secured to the chuck via vacuum seal [57]. .114 
4.4: (a) A roll of Kapton ${ }^{\circledR}$ film tape. (b) Mechanical exfoliation. (c) A graphene testing sample with a sheet of Kapton ${ }^{\circledR}$ film tape adhering underneath.

4.5: Top view of the simulated fixture. Image courtesy of Julian Gil Pinzon. .................116

4.6: Side view of the simulated fixture. Image courtesy of Julian Gil Pinzon. ...............117

4.7: Photograph of the Nernst fixture. The aluminum fixture is bolted to the cold head on the left, the thermometers, magnets, and heater are labeled. Note that the magnets have an approximately $1 \mathrm{~mm}$ space between themselves and the aluminum fixture. Image courtesy of Dr. Grover L. Larkins.

4.8: A graph of the room temperature Nernst effects of the undoped sample \# 401, layer \# 3 under 2,900 G magnetic field.

\section{Chapter 5}

FIGURE

PAGE

5.1: (a) Phosphorous implantations with different energies: $50 \mathrm{keV}, 100 \mathrm{keV}$ and 150 $\mathrm{keV}$ (dose constant $10^{15} / \mathrm{cm}^{2}$ ). (b) Phosphorous implantations with different doses: $10^{12} / \mathrm{cm}^{2}, 10^{14} / \mathrm{cm}^{2}$, and $10^{16} / \mathrm{cm}^{2}$ (energy constant at $200 \mathrm{keV}$ ). The shape of dose $10^{16} / \mathrm{cm}^{2}$ is different because it is above the amorphization limit, and different stopping parameters are applied for the amorphized region. Reprinted with permission from Sami Franssila [245].

5.2: Al contamination profiles resulting from a B implantation. For comparison, with SRIM simulated profiles are shown [254].

5.3: SRIM simulation results of implanted ion concentration for $2 \times 10^{21} \mathrm{Ti} / \mathrm{m}^{2}, 5 \times$ $10^{21} \mathrm{He} / \mathrm{m}^{2}$, and $5 \times 10^{21} \mathrm{D} / \mathrm{m}^{2}$ and displacement damage for $5 \times 10^{21} \mathrm{D} / \mathrm{m}^{2}$ as a function of depth in W (Pure Tungsten) [255].

5.4: Ion beam implanter. Reprinted with permission from the Plansee Group [249]......129

5.5: SRIM simulated distribution. (1) Phosphorus ions implanted in graphite at $E_{p}=10$ $\mathrm{keV}$. (2) damage in graphite lattice caused by the implant. (3) The probable region of effect [55].

5.6: A 5 by 5 array of HOPG substrates prepared for ion implantation. 
5.7: The assigned numbers of HOPG substrates

5.8: The specifications of implantation of the samples \# $201-225$.

5.9: This represents the simulated normalized distributions of implanted atoms and lattice damage caused by implanted atoms versus depth in HOPG. Curve 1: The calculated distribution of the implanted phosphorus. Curve 2: The computed damage caused by the implanted phosphorus. Curve 3: The calculated distribution of the implanted argon. Curve 4: The computed damage caused by the implanted argon [56].

5.10: A schematic of a PECVD system. Reprinted with permission from Jeon Geon Han [247].....

5.11: The CVD process includes the decomposition of methane to use the carbon needed in order to grow on the substrate surface. Reprinted with permission from ACS Material, LLC [259].

5.12: The most stable relaxed structures of phosphorus-doped graphene. The yellow and green balls represent the carbon and phosphorus atoms, respectively. Reprinted with permission from Jianmin Yuan, National University of Defense Technology [261].

5.13: An array of HOPG samples loaded on the PECVD machine's sample holder. Two sheets of copper were placed at the upper left corner of the array to indicate the position of the first sample in the series [57].

\section{Chapter 6}

FIGURE

PAGE

6.1: Measured $R v s . T$ of four thin films exfoliated from phosphorus implanted $\left(E_{p}=\right.$ $10 \mathrm{keV}$, dose $2 \times 10^{8} \mathrm{~cm}^{-2}$ ) HOPG samples. (a) HOPG-008, layer 3; (b) HOPG019, layer 3; (c) HOPG-019, layer 6; (d) HOPG-021, layer 7. The layer numbers indicate the number of multilayers peeled from the host sample, i.e. layer 7 would be the $7^{\text {th }}$ layer exfoliated from that sample [56]

6.2: ZFC and FC magnetic susceptibility plots along with the associated cooling and warming $R$ vs. $T$ plots for a film exfoliated from a doped-while-grown PECVD sample. The susceptibility measurements are courtesy of Dr. Deng of Dr. Paul Chu's group at the University of Houston. Note the onset of the charge carrier 
BKT transition at $T \sim 5 \mathrm{~K}$ and the onset of the vortex BKT transition at $T \sim 80 \mathrm{~K}$.

6.3: Raman spectra for a film on Kapton tape exfoliated from a phosphorous dopedwhile-grown CVD graphene sample. The peak ratios give a thickness of approximately 5 monolayers. Measurement courtesy of Laurens Henry Willems Van Beveren of Dr. Steven Prawer's group at the University of Melbourne [57]...146

6.4: Hall effect at $T=300 \mathrm{~K}$ and $T=80 \mathrm{~K}$ for the same film on Kapton tape exfoliated from a phosphorous doped-while-grown CVD graphene sample as is shown in the Raman spectrum in Figure 6.3. Measurement courtesy of Laurens Henry Willems Van Beveren of Dr. Steven Prawer's group at the University of Melbourne [57]...146

6.5: PECVD phosphorous doped-while-grown sample. Thermally driven Nernst voltage at 40 Gauss applied field. This is the difference of the Nernst signal with the magnetic field-oriented North Up through the sample subtracted from the South Up oriented field Nernst signal. This reduces any measurement errors due to thermoelectric or another non-field dependent phenomenon. Note the tilted negative peak at $T \sim 48 \mathrm{~K}$. This is characteristic of a vortex Nernst signal.

6.6: PECVD phosphorous doped-while-grown sample. Differential Nernst signal with magnetic field-oriented North Up through the sample subtracted from the Nernst signal with magnetic field-oriented South Up through the sample at an applied field of 40 Gauss. Note there are peaks at $T \sim 158 \mathrm{~K}$ and $T \sim 240 \mathrm{~K}$ that exhibit the tilted peak characteristic of a Nernst vortex signature. However, the peak at $T$ $\sim 240 \mathrm{~K}$ may be due to instrumentation.

6.7: Ion-implanted phosphorous followed by argon ion implant to create pinning. Magnetic field is 1500 Gauss and the plot is of the Nernst signal for the North Up oriented field subtracted from the South Up oriented Nernst signal. There are tilted peaks characteristic of Nernst vortex signals apparent at temperatures of $42 \mathrm{~K}, 70$ $\mathrm{K}$, and $238 \mathrm{~K}$.

6.8: Same data as in Figure 6.7 expanded to show the details of the $T \sim 70 \mathrm{~K}$ peak as well as some of the smaller peaks.

6.9: Further magnification of the data in Figure 6.7. Note that the peaks at $T \sim 130 \mathrm{~K}$ and $T \sim 238 \mathrm{~K}$ are clearly the tilted peak of a vortex signature in the Nernst effect. There are also small peaks in the vicinity of $T \sim 180 \mathrm{~K}$, but they are very small indicating few vortices are undergoing depinning at this temperature. 
6.10: $-V_{\text {Nernst }} / \Delta T$ and $\Delta T$ versus $T$ for sample 213 in a 1,500 Gauss magnetic field oriented normal to the sample's surface. There is 1 Watt of input power on the Nernst heater. This is the first run with the sample in a magnetic field. The cooling curve is, effectively, one enormous peak. The warming curve hints of the BKT transition seen in the later, lower power and lower gradient plots. Note the hysteresis.

6.11: $-V_{\text {Nernst }} / \Delta T$ curves from $20-180 \mathrm{~K}$ for warming direction only. Peak at approximately $36 \mathrm{~K}$ remains constant with respect to applied magnetic field. The peak amplitude is a strong function of the applied magnetic field. This peak is at the approximate BKT transition.

6.12: $-V_{\text {Nernst }} / \Delta T$ curves for cooling and warming direction cycle for the same sample as in Figure 6.11 from $20-70 \mathrm{~K}$. Peaks at approximately $36 \mathrm{~K}$ remain constant with respect to applied magnetic field. Each peak amplitude is a strong function of the applied magnetic field. These peaks are at the approximate BKT transition. Note the clear hysteresis between the cooling and the returning warming plots. .157

6.13: $-V_{\text {Nernst }} / \Delta T$ curves for cooling and warming direction cycle for the same sample as in Figure 6.11 taken with an applied field of 120 Gauss normal to the sample surface. Notice the hysteresis and the large step in the cooling curve that extends from virtually the start of the plot to about $224 \mathrm{~K}$.

6.14: $-V_{\text {Nernst }} / \Delta T$ curves for cooling and warming direction cycle for the same sample as in Figure 6.11 taken with an applied field of 290 Gauss normal to the sample surface. Notice the hysteresis and the large step in the cooling curve that extends from virtually the start of the plot to about $216 \mathrm{~K}$. There is structure in the cooling plot down to $178 \mathrm{~K}$. There is notable structure in the warming plot from a broad peak at about $210 \mathrm{~K}$ through to a broad peak at $260 \mathrm{~K}$.

6.15: $-V_{\text {Nernst }} / \Delta T$ curves for cooling and warming direction cycle for the same sample as in Figure 6.11 taken with an applied field of 641 Gauss normal to the sample surface. Notice the hysteresis and the large step in the cooling curve that extends from $265 \mathrm{~K}$ to about $216 \mathrm{~K}$. There is structure in the cooling plot down to 150 $\mathrm{K}$. There is notable structure in the warming plot from a broad peak at about 148 $\mathrm{K}$ through to a broad peak at $260 \mathrm{~K}$.

6.16: $-V_{\text {Nernst }} / \Delta T$ curves for cooling and warming direction cycle for the same sample as in Figure 6.11 taken with an applied field of 1,113 Gauss normal to the sample surface. Notice the hysteresis and the large step in the cooling curve that extends 
from $265 \mathrm{~K}$ to about $144 \mathrm{~K}$. There is structure in the cooling plot down to 144 $\mathrm{K}$. There is notable structure in the warming plot from a broad peak at about 164 $\mathrm{K}$ through to $260 \mathrm{~K}$

6.17: $-V_{\text {Nernst }} / \Delta T$ curves in the range of $140-300 \mathrm{~K}$ for cooling and warming direction cycle for the same sample as in Figure 6.11. This data was taken with 120 Gauss of magnetic field applied normal to the sample surface. Note the step at approximately $224 \mathrm{~K}$ in the cooling direction and the associated structure ranging from $260 \mathrm{~K}$ to $224 \mathrm{~K}$. There is hysteresis between the cooling and the returning warming plots.

6.18: $-V_{\text {Nernst }} / \Delta T$ curves in the range of $140-300 \mathrm{~K}$ for cooling and warming direction cycle for the same sample as in Figure 6.11. This data was taken with 290 Gauss of magnetic field applied normal to the sample surface. Note the step at approximately $216 \mathrm{~K}$ in the cooling direction and the structure from approximately $260 \mathrm{~K}$ to $180 \mathrm{~K}$. Note the structure from $200 \mathrm{~K}$ to $260 \mathrm{~K}$ in the warming plot. Cooling to warming hysteresis is present.

6.19: $-V_{\text {Nernst }} / \Delta T$ curves in the range of $140-300 \mathrm{~K}$ for cooling and warming direction cycle for the same sample as in Figure 6.11. This data was taken with 641 Gauss of magnetic field applied normal to the sample surface. Note the features that span from approximately $260 \mathrm{~K}$ to $160 \mathrm{~K}$ in the cooling direction and the structure between about $220 \mathrm{~K}$ and $265 \mathrm{~K}$ in the warming plot. Hysteresis is visible

6.20: $-V_{\text {Nernst }} / \Delta T$ curves in the range of $140-300 \mathrm{~K}$ for cooling and warming direction cycle for the same sample as in Figure 6.11. This data was taken with 1,113 Gauss of magnetic field applied normal to the sample surface. Note the step at approximately $144 \mathrm{~K}$ in the cooling direction and the structure from approximately $265 \mathrm{~K}$ to $144 \mathrm{~K}$. The structure in the returning warming plot begins with a broad inflection point at $160 \mathrm{~K}$ and ends near $265 \mathrm{~K}$. Hysteresis is visible.

6.21: $-V_{\text {Nernst }} / \Delta T$ curves in the range of $140-300 \mathrm{~K}$ for same sample as in figure 6.11. This is a collection of the cooling direction plots from Figures 6.17 through 6.20. Note how the steps and features have tended to shift lower in temperature as the applied field is increased.

6.22: $-V_{\text {Nernst }} / \Delta T$ curves in the range of $140-300 \mathrm{~K}$ for same sample as in Figure 6.11. This is a collection of the warming direction plots from Figures 6.17 through 6.20. Note how the steps and features have tended to shift lower in 
temperature as the applied field is increased. Notice how the broad peak in the 290 Gauss curve at $228 \mathrm{~K}$ has evolved as the applied field is stepped up. ............168

6.23: $\Delta T$ as a function of temperature both cooling and warming for the data presented in Figures 6.11 through 6.22..........................................................................169

6.24: Specific sketch of future phosphorus-doped graphene sample. Measurement unit is in millimeter. Image courtesy of Julian Gil Pinzon...........................................174 


\section{Part I}

\section{Introduction}

\section{Chapter 1}

\section{Graphite and Graphene}




\subsection{Introduction to Graphite and Graphene}

Graphite is a carbon polymorph found in metamorphic rocks. It is the most stable form of carbon under standard conditions for temperature and pressure (approximately 273 Kelvin and 1 Bar) [1 - 4]. Graphite consists of layers of carbon atoms uniquely bonded together. Each carbon atom is covalently bonded to three other atoms in the same plane creating hexagonal rings of carbon atoms (honeycomb lattices) through the plane. Each atomic layer in graphite is also weakly bonded to its adjacent layers with the interlayer spacing of $3.35 \AA[1-6]$. The properties of graphite vary depending on the type of graphitic stacking sequences $[8,9]$. Graphitic stacking refers to the way the carbon-bonded atomic layers are stacked, and there are four different types of stacking sequences that can occur in graphite: AAA stacking (simple hexagonal structure), ABA stacking (can be Bernal or orthorhombic structure), ABC stacking (rhombohedral structure), and turbostratic stacking (disordered structure).

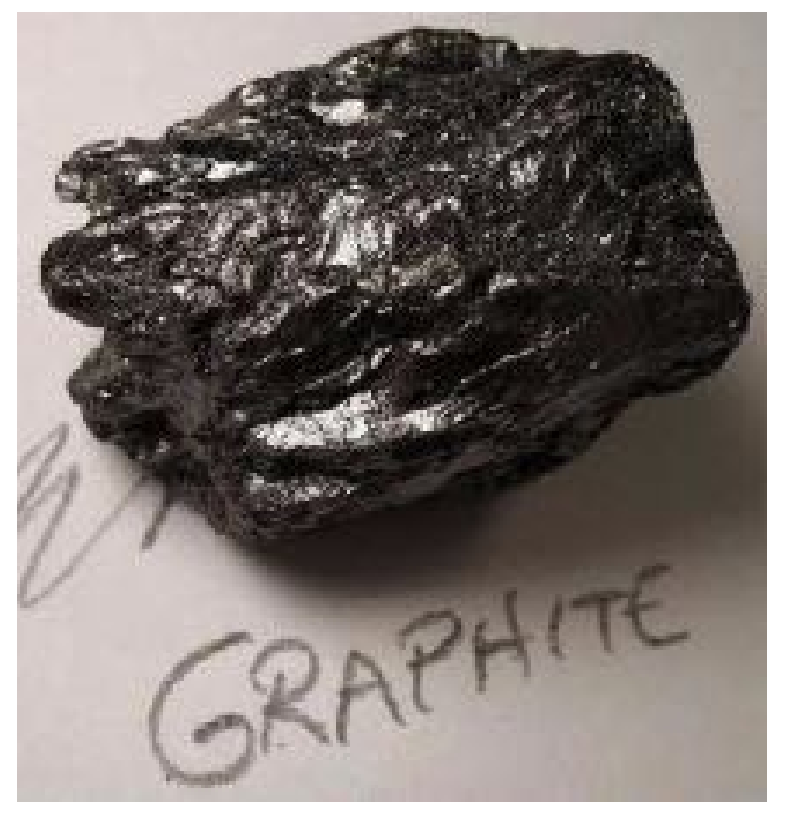

Figure 1.1: Graphite. Reprinted with permission from Chowdhury Al-Amin [11]. 

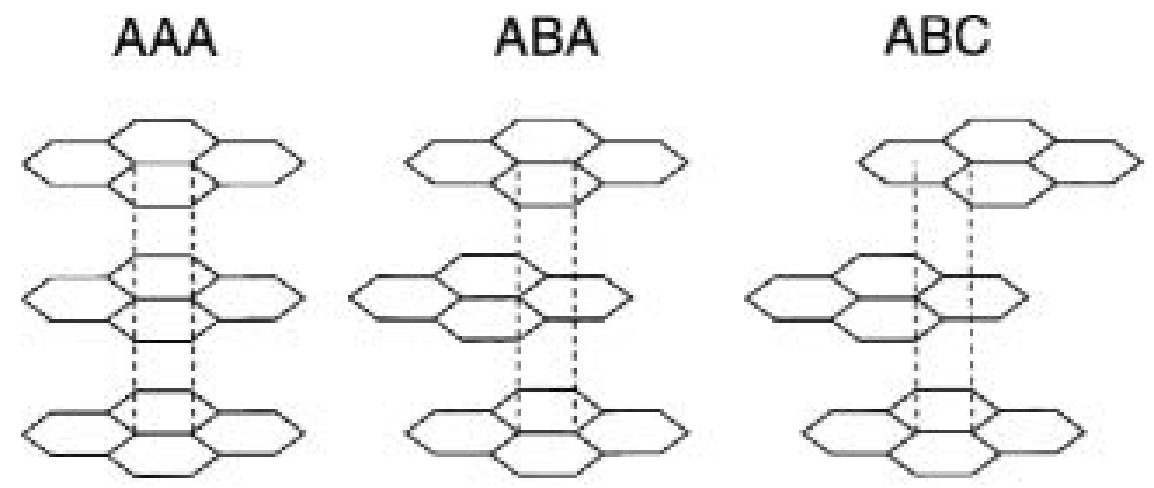

Figure 1.2: AAA, ABA, and $\mathrm{ABC}$ graphite stacking forms [20].

In nature, the composition of graphite is usually turbostratic. However, after graphitization (detail is in section 1.2), the composition happens to be approximately $85 \%$ Bernal $\mathrm{ABA}$ and $15 \% \mathrm{ABC}$, where $\mathrm{AAA}$ has only been observed in graphite intercalation compounds [19-26]. The most common stacking observed in graphite is the Bernal ABA, however orthorhombic ABA can be obtained by the shifting process in layer "B" of the Bernal ABA [19-21]. The force that bonds the carbon atomic layers in graphite together is a weak Van der Waals force [9, 27 - 29]. A monoatomic layer of graphite is known as "graphene" [5-7].

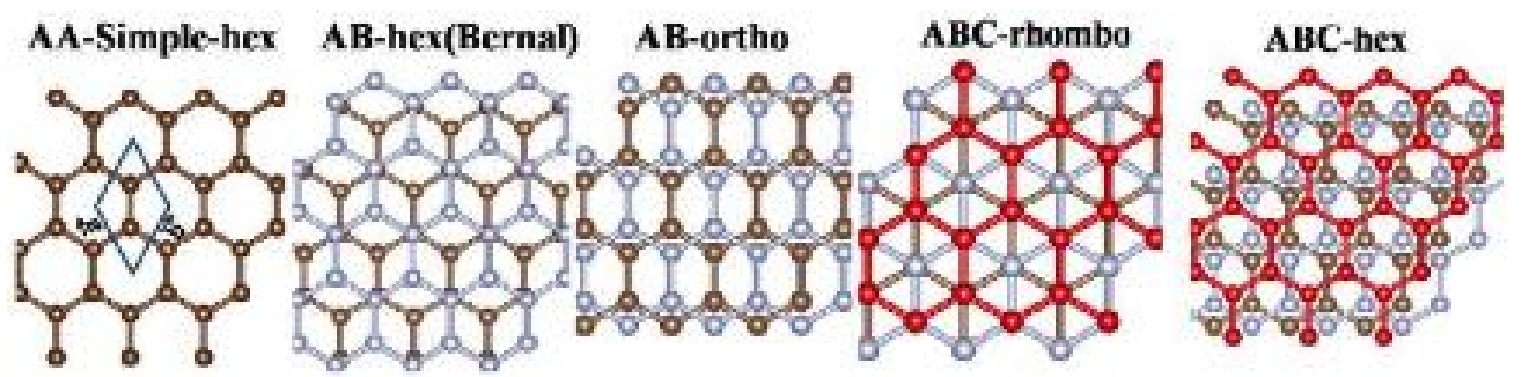

Figure 1.3: Different stacking structures of graphite [19].

Graphene was successfully exfoliated for the first time at the University of Manchester in 2004 by A. K. Geim and K. S. Novoserov, the 2010 Nobel Laureates in physics for the "groundbreaking experiments regarding the two-dimensional material 
graphene" $[95,96]$. Since graphene is a monoatomic layer material, it is claimed by A. K. Geim and K. S. Novoserov to be the 2-dimensional (2D) allotrope of carbon. The carbon atoms in an atomic layer of graphite (graphene) are hexagonally bonded together in 2D space which creates honeycomb lattice structures. In detail, these honeycomb lattices are formed by the $s p^{2}$ hybridization of the $2 s, 2 p_{x}$, and $2 p_{y}$ orbitals with the lattice constant, $a_{0}$, (the distance between two bonded carbon atoms) of $1.42 \AA$ and the angle between two bonds of $120^{\circ}[9-12,30]$.
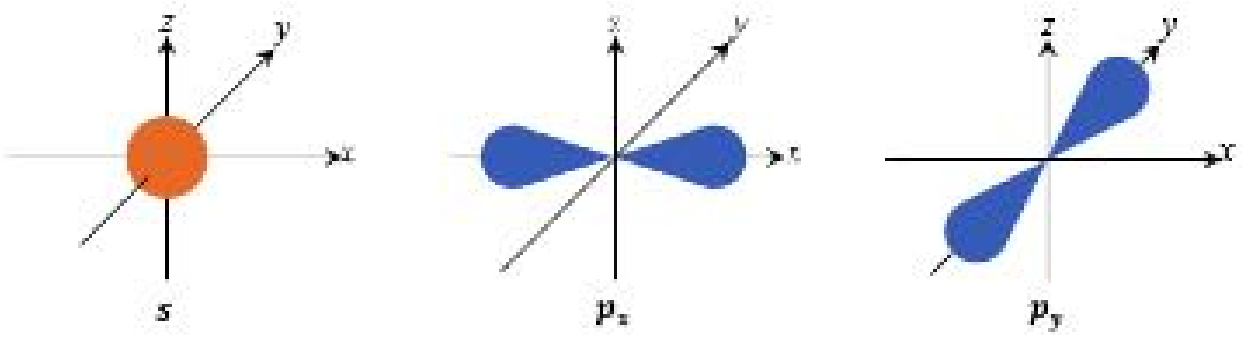

(a)

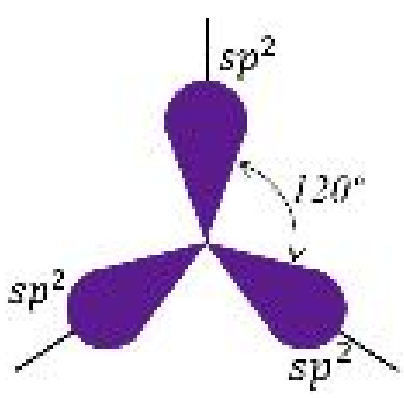

(b)

Figure 1.4: (a) $s, p_{x}$, and $p_{y}$ orbitals. (b) $s p^{2}$-hybridization.

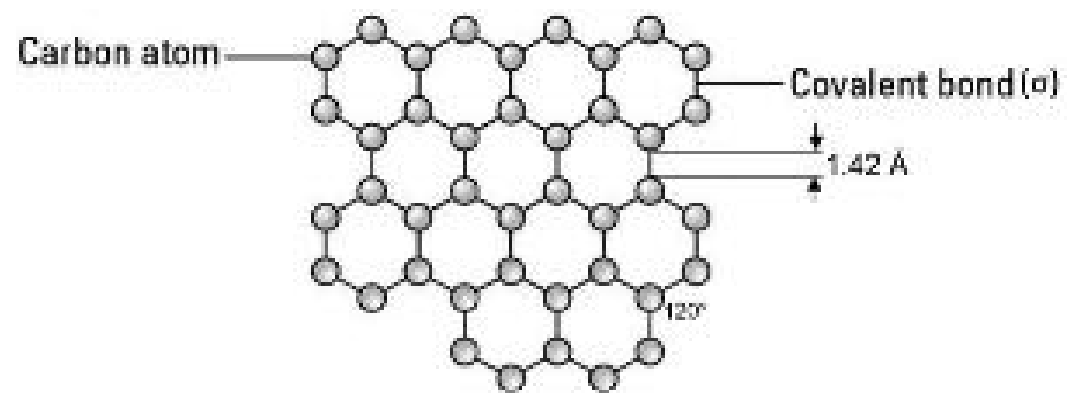

Figure 1.5: Graphene atomic structure [12]. 


\subsection{Highly Ordered Pyrolytic Graphite}

Pyrolytic graphite is a graphite material with a high degree of crystallographic orientation, obtained by graphitization (heat treatment) or by Chemical Vapor Deposition (CVD) at above $2,200{ }^{\circ} \mathrm{C}(2,473.15 \mathrm{~K})$. Graphitization or $\mathrm{CVD}$ at above $3,000{ }^{\circ} \mathrm{C}(3,273.15$ K) results in Highly Ordered Pyrolytic Graphite (HOPG) [109]. HOPG is a high-purity form of pyrolytic graphite which has impurity level less than 10 parts per million (ppm).

Graphitization is the formation of graphite by exposing turbostratic carbon to elevated temperatures over a long period of time. At elevated temperatures, carbon tends to migrate to the grain boundaries. The interlayer spacing of graphite and crystallite size also changes with temperature. When the graphitization temperature is between $1,500{ }^{\circ} \mathrm{C}$ and $2,000{ }^{\circ} \mathrm{C}(1,773.15 \mathrm{~K}$ and $2,273.15 \mathrm{~K})$, the interlayer spacing of turbostratic carbon decreases sharply from approximately $3.5 \AA$ to $3.4 \AA$. Eventually, the interlayer spacing approaches the graphitic interlayer spacing of $3.35 \AA$ at a temperature above $3,000{ }^{\circ} \mathrm{C}$ $(3,273.15 \mathrm{~K})$. Thus, any amorphous carbon materials can be graphitized. [31 - 36] 


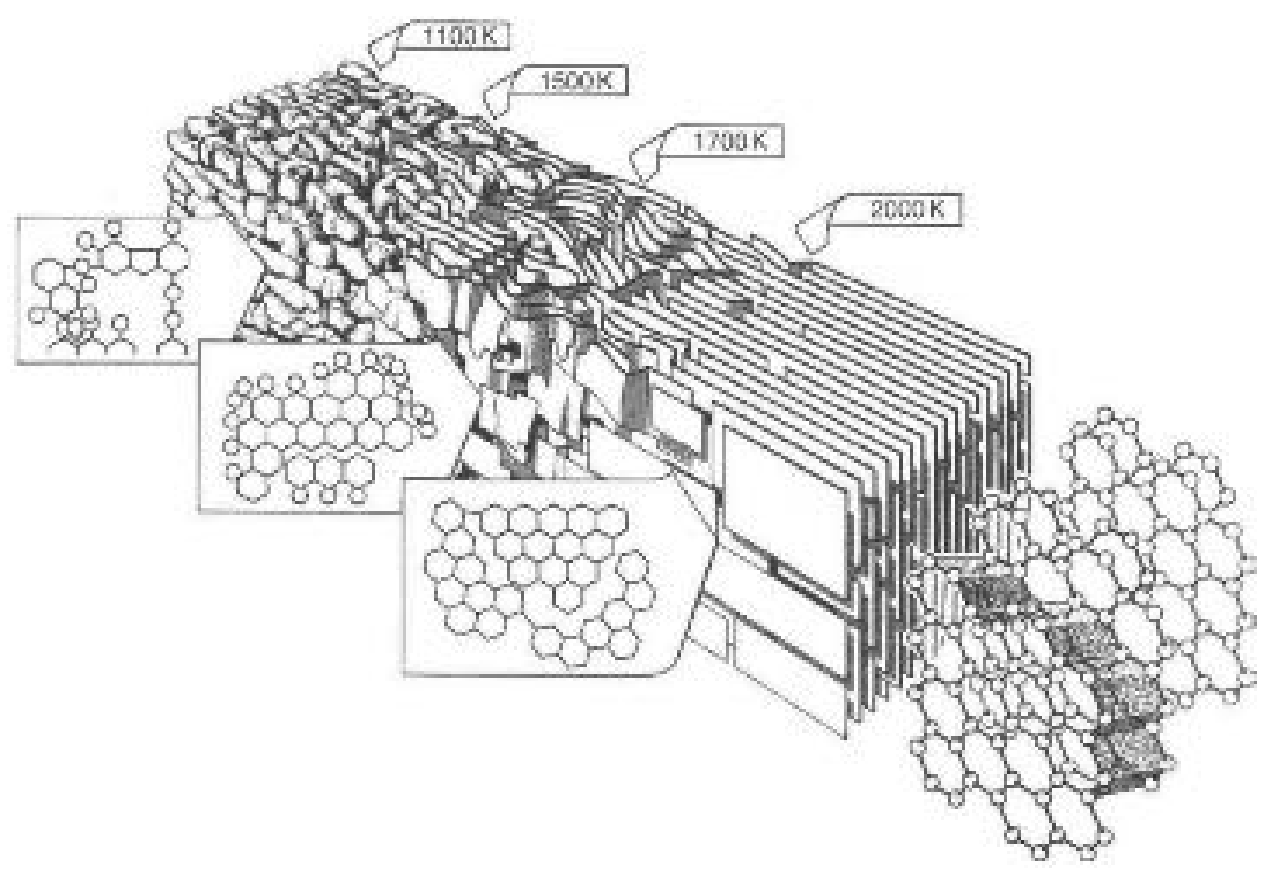

Figure 1.6: A model of changes of carbon structure during graphitization [262].

In this dissertation, HOPG materials are used as experimental substrates and to exfoliate undoped and doped samples. The experimental HOPG materials with dimensions of $10 \mathrm{~mm} \times 10 \mathrm{~mm} \times 1.2 \mathrm{~mm}$ each were ordered from NT-MDT Spectrum Instruments Company and MikroMasch Inc. From Micromasch's product specifications, HOPG has been categorized into three different grades of quality classified by the mosaicity, a measure of the arrangement of crystal lattices in a molecular plane, of materials. HOPG materials that have lesser angles of mosaic spread will be considered of a higher quality grade. Thus, the HOPG labeled as ZYA possesses the highest grade of quality, ZYB possesses the second highest grade of quality, and ZYH possesses the lowest grade of quality as shown in the Table 1.1 . 
Table 1.1: HOPG grades of quality. Image courtesy of MikroMasch [37].

\begin{tabular}{|c||c|c||}
\hline \multirow{2}{*}{ Grade } & \multicolumn{2}{|c|}{ Mosaic Spread } \\
\cline { 2 - 3 } & Value & Accuracy \\
\hline \hline ZYA & $0.4^{\circ}$ & $\pm 0.1^{\circ}$ \\
\hline \hline ZYB & $0.8^{\circ}$ & $\pm 0.2^{\circ}$ \\
\hline \hline ZYH & $3.5^{\circ}$ & $\pm 1.5^{\circ}$ \\
\hline
\end{tabular}

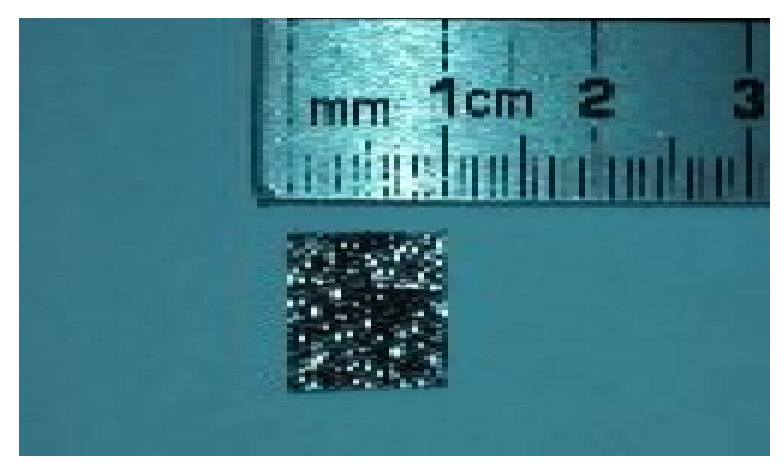

Figure 1.7: HOPG

\subsection{Graphene and Graphite Thin Film}

Many reports and experiments have concluded that properties of graphene can be diverse depending on the number of layers in the graphene material. Therefore, the graphene community has distinguished intrinsic (undoped) graphene into 3 categories by the number of layers, which are single-layer graphene (SLG, 1 layer), bilayer graphene (BLG, 2 layers), and few-layer graphene (FLG, 3 to 9 layers) due to the properties they exhibit. A structure consisting of 10 or more layers of graphene is considered a graphite thin film since it essentially exhibits properties of graphite [9, 13-18]. 


\subsection{Properties of Graphene}

\subsubsection{Physical and Mechanical Properties}

\subsubsection{Lattice Structure}

The carbon atoms in graphene are covalently bonded into a honeycomb lattice with armchair and zigzag edges as shown in Figure 1.8. However, the honeycomb lattice is not a Bravais lattice (the lattice that can perfectly fill 2D or 3D space without opening or overlapping by the repetition of its own units) because it is formed by nonequivalent carbon atoms $\mathrm{A}$ and $\mathrm{B}[9,38,39]$.

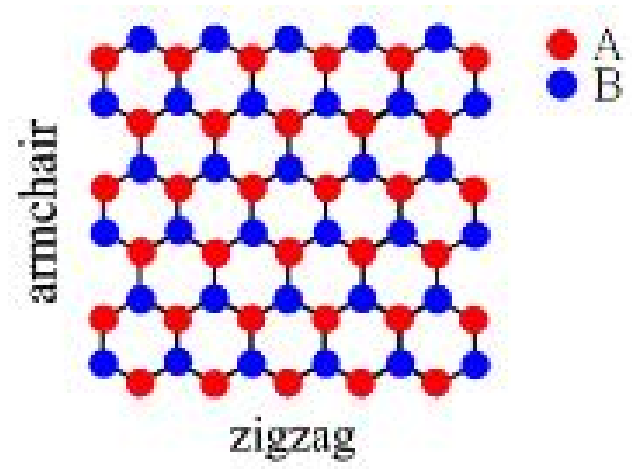

Figure 1.8: Nonequivalent carbon atoms $A$ and $B$ in a honeycomb lattice with armchair and zigzag edges. Reprinted with permission from S. -R. Eric Yang [40].

In section $1.1, a_{0}$ denotes the distance between two bonded carbon atoms with the length of $1.42 \AA$. Thus, a lattice spacing constant of graphene, $a$, will be equal to $\sqrt{3} a_{0}$ or $\sim 2.46 \AA$.

In real space, the unit cell vectors $\vec{a}_{1}$ and $\vec{a}_{2}$ can be written as:

$$
\vec{a}_{1}=a(\sqrt{3} / 2,1 / 2) \quad, \quad \vec{a}_{2}=a(\sqrt{3} / 2,-1 / 2)
$$

and the nearest-neighbor vectors $\vec{\delta}_{1}, \vec{\delta}_{2}$, and $\vec{\delta}_{3}$ will be:

$$
\vec{\delta}_{1}=a(1 / 2 \sqrt{3}, 1 / 2), \vec{\delta}_{2}=a(1 / 2 \sqrt{3},-1 / 2), \vec{\delta}_{3}=a(-1 / 2 \sqrt{3}, 0)
$$

In the reciprocal, the lattice vectors $\vec{b}_{1}$ and $\vec{b}_{2}$ can expressed as: 


$$
\vec{b}_{1}=(1 / a)(2 \pi / \sqrt{3}, 2 \pi) \quad, \quad \vec{b}_{2}=(1 / a)(2 \pi / \sqrt{3},-2 \pi)
$$

where $\Gamma, \mathrm{M}, \mathrm{K}$, and $\mathrm{K}$ ' represent high symmetry points in a reciprocal space [9].

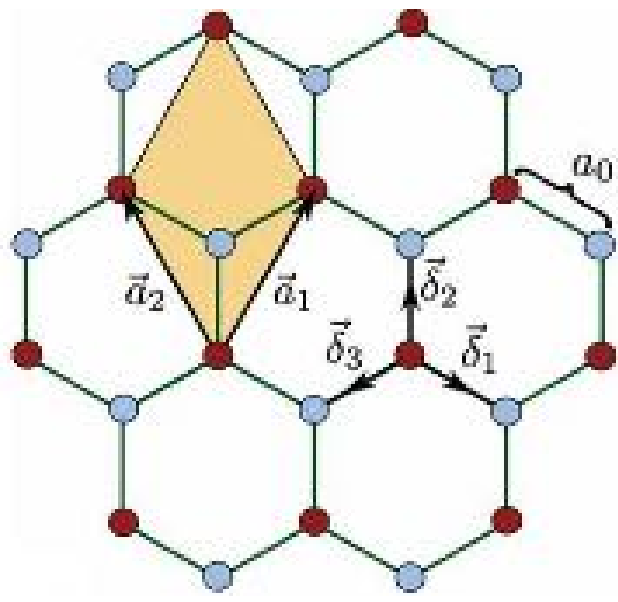

(a)

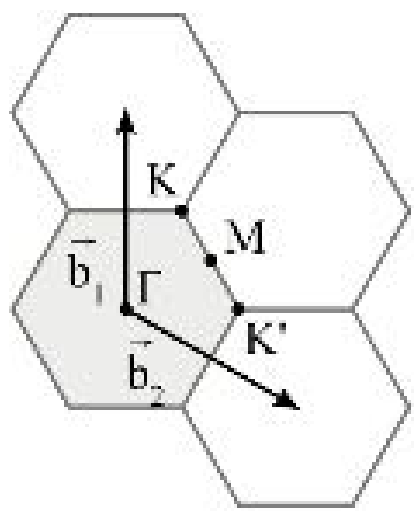

(b)

Figure 1.9: (a) Graphene lattice with unit cell vectors in a real space.

(b) Graphene reciprocal lattice with reciprocal lattice vectors and high symmetry points in a reciprocal space. Reprinted with permission from Vitor M. Pereira, National University of Singapore [107, 108].

\subsubsection{Stress, Strain, and Melting Point}

Graphene was confirmed to be the hardest material, since its values of Young's modulus (elastic modulus), $E$, third-order elastic stiffness, $D$, and intrinsic strength, $\sigma_{\text {int }}$, reach 1.0 terapascals $(\mathrm{TPa}),-2.0 \mathrm{TPa}$, and 130 gigapascals (GPa) respectively. $[9,41,44$, 45]. Other mechanical measurements of graphene also show that it has a tensile stress of $42 \mathrm{~N} / \mathrm{m}$, and a fracture strain of $25 \%$ which means graphene can expand nearly $25 \%$ before failing $[9,41]$.

In chemistry, graphene is a quasi-2D crystalline membrane which will become a $3 \mathrm{D}$ liquid phase when it is melted. This was described in the theory of $2 \mathrm{D}$ melting of Kosterlitz, Thouless, Halperin, Nelson, and Young also known as the KTHNY theory [46, 47]. With the intensive studies of the melting point of graphene, researchers and scientists 
have concluded that an unambiguously defined bulk melting temperature, $T_{m}$, of graphene is $4510 \mathrm{~K}[47-49]$.

\subsubsection{Chemical Properties}

Chemical reactions in the lattice structure of graphite have been tested and adapted in many purposes. However, moving from the $3 \mathrm{D}$ material of graphite to the $2 \mathrm{D}$ material of graphene, new avenues for chemistry emerge. Graphene is typically a 2D hexagonal array of carbon with $s p^{2}$ bonding, and this $s p^{2}$ bonding is a double bond of carbon atoms which is called a "carbon-carbon bond (C-C)". With this bonding structure, graphene can be covalently or noncovalently functionalized with other chemicals to improve or change its properties [9].

\subsubsection{Covalent Functionalization}

Covalent Functionalization of graphene is the breaking of $s p^{2}$ bonds of graphene using chemical reactions. For example, when graphene is hydrogenated, hydrogen atoms will covalently bond with carbon atoms of graphene and the bonding structure of the material will convert to $s p^{3}$. Since the chemical structure of graphene has been changed due to the hydrogenation, the hydrogenated graphene will be called "graphane" $[9,50]$. The same chemical bonding can also occur when graphene is fluorinated. Carbon and fluorine atoms are covalently bonded through the exposure of graphene to atomic fluorine formed by decomposing xenon difluoride $\left(\mathrm{XeF}_{2}\right)$ at $70{ }^{\circ} \mathrm{C}$. This covalent bonding forms another stoichiometric derivative of graphene called "fluorographene" $[9,51]$. 


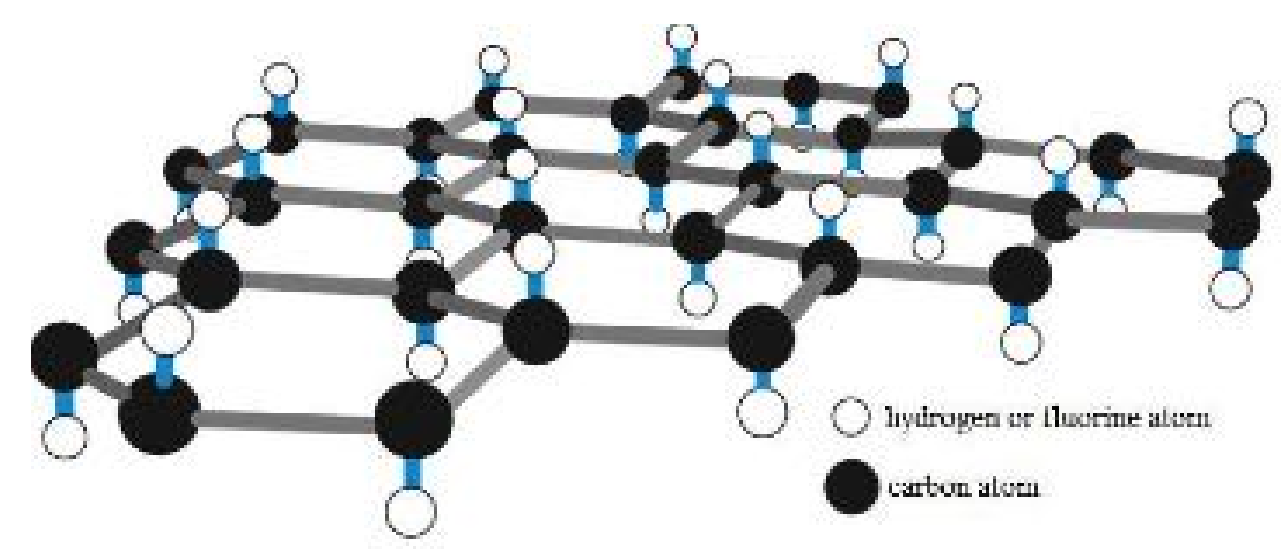

Figure 1.10: Schematic representation of the atomic structure of graphane and fluorographene. Image courtesy of GLOSSARY of NANO technology and related TERMS [52].

Oxidation is another way of covalent functionalization of materials in chemistry.

The oxidization of graphite can form a compound of carbon, oxygen, and hydrogen in various ratios, and the chemical composition of fully oxidized graphite has been estimated to be $\mathrm{C}_{4} \mathrm{O}(\mathrm{OH})$ [53]. The oxidized graphite is called "graphite oxide", and when it is exfoliated into a single layer it will be called "graphene oxide (GO)". Due to the dispersibility of graphite oxide, it is easy to exfoliate GO from graphite oxide using sonication in water $[9,54]$.

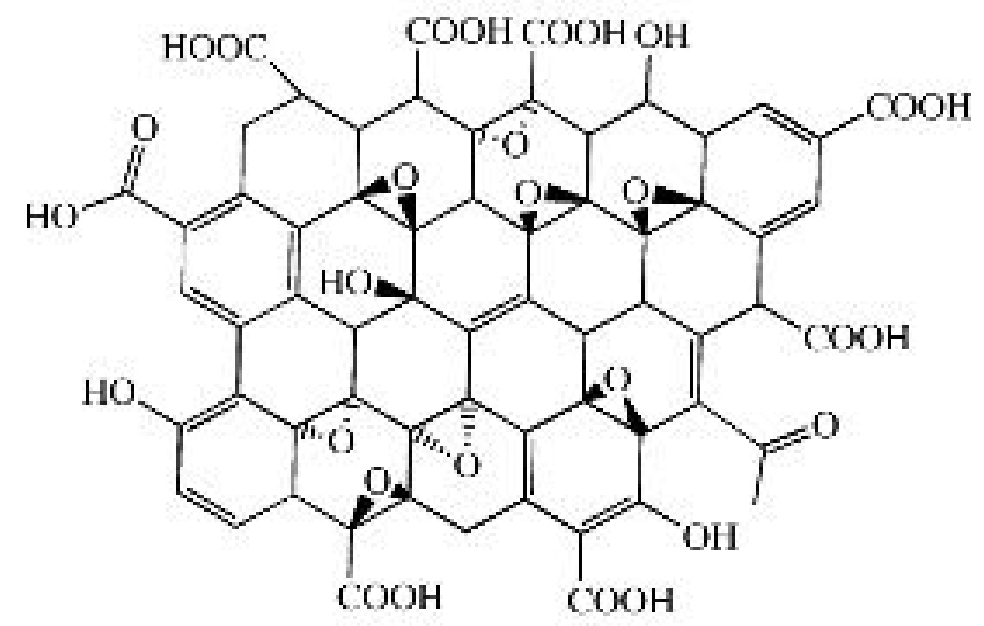

Figure 1.11: Chemical structure of graphene oxide. Image courtesy of The Green Optimistic [100]. 


\subsubsection{Noncovalent Functionalization}

Noncovalent Functionalization of graphene changes the properties of graphene by

depositing or doping a graphite substrate with other chemicals without breaking its $s p^{2}$ hybridization, then mechanically exfoliating the functionalized layers of the graphite substrate which can be chemically deposited or doped graphene. For example, negative type dopant such as boron and positive type dopant such as phosphorus can be deposited into HOPG substrates to shift the superconducting transition in 3D materials of graphite and the 2D materials of graphene [55 - 57].

\subsubsection{Thermal Properties}

The measurement of thermal conductivity of graphene using the optothermal Raman technique was developed by Balandin et al. [74, 77]. The $\mathrm{G}$ mode (elongation of the $\mathrm{C}-\mathrm{C}$ bond) shift decreases linearly from $\sim 1,584 \mathrm{~cm}^{-1}$ at $-200{ }^{\circ} \mathrm{C}$ to $\sim 1,578 \mathrm{~cm}^{-1}$ at $100{ }^{\circ} \mathrm{C}$. The frequency shift of $\mathrm{G}$ mode as a function of temperature enables a Raman spectrometer to function as an optical thermometer which results in the measured thermal conductivity, $K$, of mechanically exfoliated graphene to be $\sim 3,000 \mathrm{~W} / \mathrm{m} \cdot \mathrm{K}$ near room temperature ( 298 K). Cai et al. [75]. also used Raman technique on CVD grown graphene. The result shows a value of $K$ is approximately equal to $2,500 \mathrm{~W} / \mathrm{m} \cdot \mathrm{K}$ near room temperature and $1,400 \mathrm{~W} / \mathrm{m} \cdot \mathrm{K}$ at $500 \mathrm{~K}[9,74,75]$. 


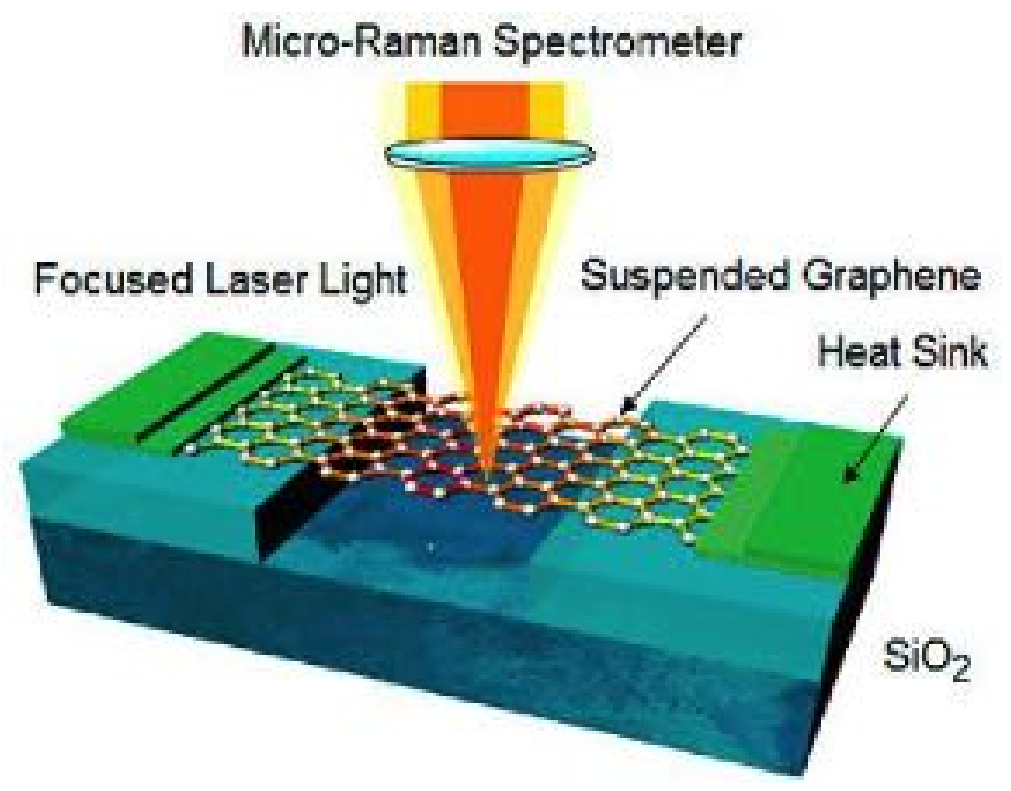

Figure 1.12: Optothermal Raman measurement technique. Reprinted with permission from A. A. Balandin et al [74].

Compared to other thermal conductors, graphene has higher thermal conductivity. Several studies have found that graphene has a high potential for heat conduction based on the size of the sample in the micrometer scale which contradicts Fourier's law (the law of thermal conduction). In many experiments [74-77], the researchers found that the larger the segment of graphene, the more heat it could transfer. Theoretically, graphene could absorb heat with its thermal conductivity increases logarithmically. This could be because of the stable atomic bonding pattern of graphene as well as being a 2D material [78]. 
Table 1.2: Thermal conductivities of materials/mediums at room temperature.

\begin{tabular}{|c|c|}
\hline Material/Medium & Thermal conductivity, $K,(\mathrm{~W} / \mathrm{m} \cdot \mathrm{K})$ \\
\hline Aluminum & 205 \\
\hline Beryllium & 218 \\
\hline Brass & 109 \\
\hline Bronze & 110 \\
\hline Copper & 401 \\
\hline Diamond & 1,000 \\
\hline Gold & 310 \\
\hline Graphene [9] & $\begin{array}{c}\sim 2,500 \text { (CVD grown) } \\
\sim 3,000 \text { (mechanically cleaved) }\end{array}$ \\
\hline Graphite & 168 \\
\hline Indium & 86 \\
\hline Iron & 80 \\
\hline Lithium & 301 \\
\hline Magnesium & 156 \\
\hline Mercury & 8.3 \\
\hline Oxygen & 0.024 \\
\hline Silver & 429 \\
\hline Stainless Steel & 16 \\
\hline Titanium & 22 \\
\hline Vacuum & 0 \\
\hline Water & 0.58 \\
\hline Zinc & 116 \\
\hline
\end{tabular}




\subsubsection{Electronic Properties}

Graphene exhibits unique characteristics for electron transport and other electronic properties. In this section, the electronic properties of graphene such as the electronic band structure, quantum Hall effect, and fractional quantum Hall effect will be briefly explained.

\subsubsection{Electronic Band Structure}

Section 1.4.1.1. shows that the lattice structure of graphene is a hexagonal lattice formed by the $s p^{2}$ hybridization of nonequivalent carbon atoms in a 2D plane and each carbon atom is covalently bonded to the other three nearest neighbor atoms. Because of the honeycomb lattice formed by nonequivalent carbon atoms, A and B, it has been described as consisting of two triangular sublattices A and B. This sublattice description was first used in 1947 by P. L. Wallace to calculate the energy bands of the band structure of monolayer graphite $[9,58]$. The energy bands of monolayer graphite can be derived as:

$$
E_{ \pm}\left(k_{x}, k_{y}\right)= \pm t_{0} \sqrt{1+4 \cos ^{2} \frac{a_{0}}{2} k_{x}+4 \cos \frac{a_{0}}{2} k_{x} \cos \sqrt{3} \frac{a_{0}}{2} k_{y}}
$$

where "+" applies to the upper band, "-" the lower band, $t_{0} \approx 2.7 \mathrm{eV}$ is the nearest neighbor hopping energy, $a_{0} \approx 2.46 \AA$ is a lattice spacing constant of graphene, and $k_{x, y}$ is the $2 \mathrm{D} k$-space $[9,59]$.

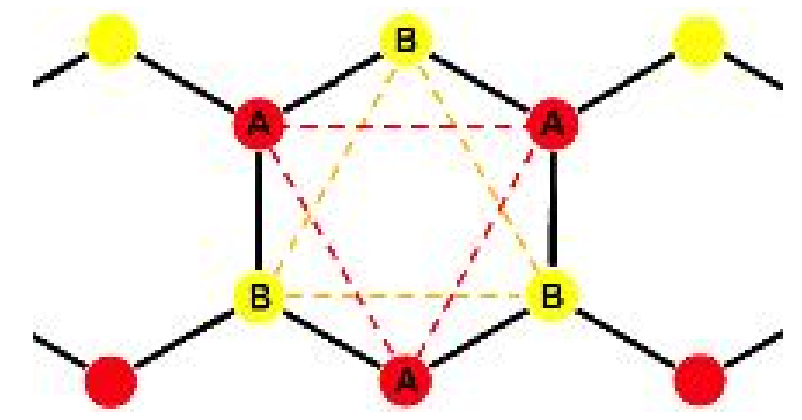

Figure 1.13: Sublattices of nonequivalent carbon atoms $A$ and $B$ in graphene. 
The band structure of graphene exhibits valence and conduction bands intersecting at high symmetry points $\mathrm{K}$ and $\mathrm{K}^{\prime}$ in the reciprocal space or $k$-space $\left(k_{x}, k_{y}\right)$. At the $\mathrm{K}$ and $\mathrm{K}^{\prime}$ points, the electronic dispersion corresponds to massless relativistic particles (Dirac fermions) which means the charge carriers satisfy an equation of motion that has the same form as a relativistic wave equation (Dirac equation). Thus, $\mathrm{K}$ and $\mathrm{K}$ ' are referred to as the charge neutrality points or "Dirac points" $[9,41,79]$. The coordinates in $k$-space of the Dirac points $\mathrm{K}$ and $\mathrm{K}$ ' can be expressed as:

$$
\mathrm{K}=\left(1 / a_{0}\right)(2 \pi / \sqrt{3}, 2 \pi / 3) \text { and } \mathrm{K}^{\prime}=\left(1 / a_{0}\right)(2 \pi / \sqrt{3},-2 \pi / 3)
$$

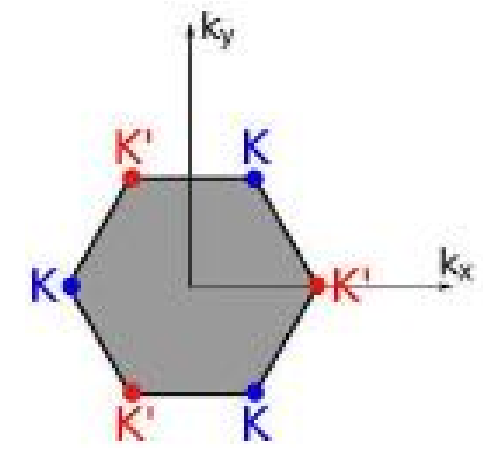

Figure 1.14: The Coordinates in the $k$-space of the Dirac points $K$ and $K^{\prime}$ [79].

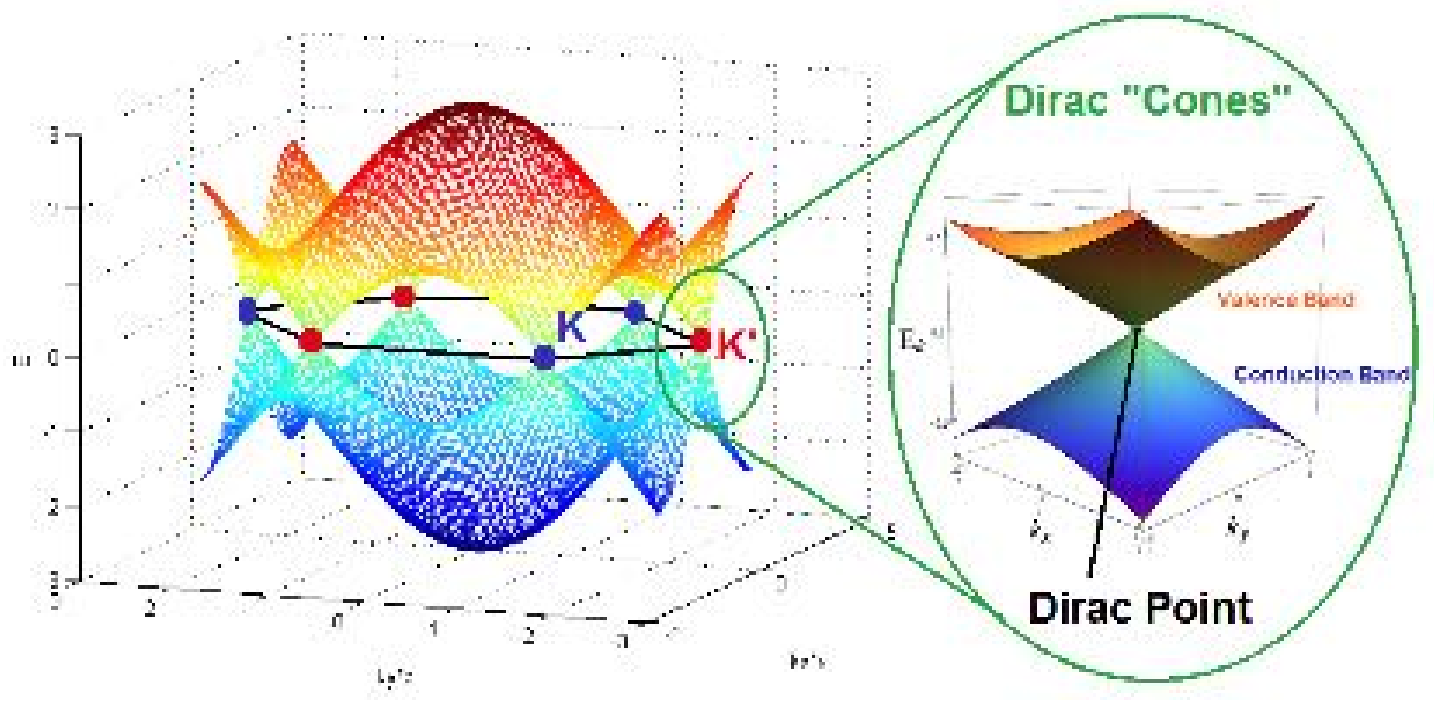

Figure 1.15: Band Structure of graphene [79]. 


\subsubsection{Quantum Hall Effect}

The Quantum Hall Effect (QHE) or Integer Quantum Hall Effect (IQHE) is a quantum-mechanical version of the Hall effect. It can be observed in a thin layer or 2D electron systems near absolute zero temperature $(0 \mathrm{~K})$ in a strong magnetic flux density. In 1980, von Klitzing found that the simple one-electron picture for the Hall effect of an ideal 2D system in a strong magnetic flux density, $B$, leads to the correct value for the quantized Hall resistance at integer filling factors of the degenerate energy levels, the discrete set of energy levels of a particle that become equally spaced with the gap between each level proportional to the magnetic flux density, known as Landau Levels (LLs) [84]. The discovery of the QHE led von Klitzing to be awarded the Nobel prize in physics 1985 and to a new metrological standard.

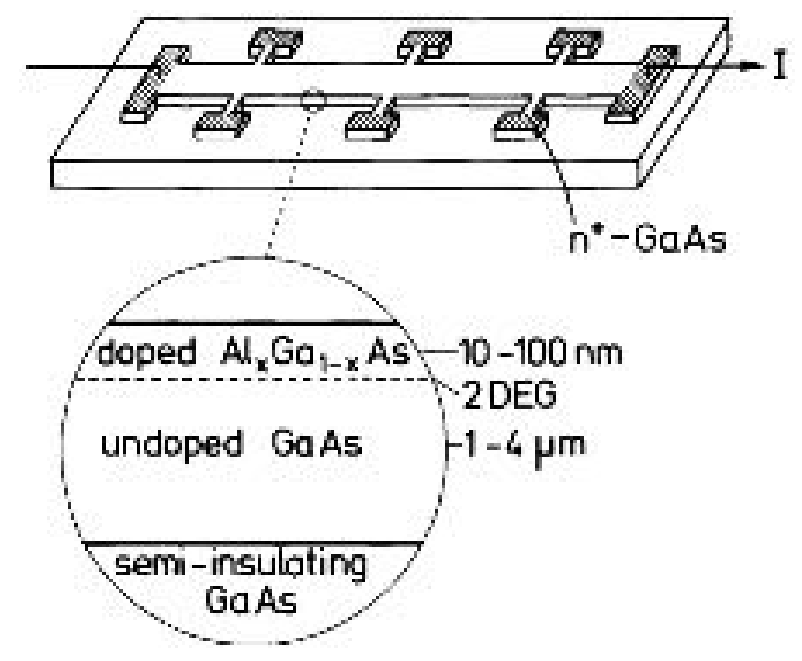

Figure 1.16: A heterostructure used for the Hall effect measurements. Reprinted with permission from Klaus von Klitzing, the 1985 Nobel Laureate in Physics [84].

The resistance quantum was designated as the von Klitzing constant which is:

$$
R_{K}=\frac{h}{e^{2}} \approx 25.813 \mathrm{k} \Omega
$$


where $h \approx 6.626 \times 10^{-34} \mathrm{~m}^{2} \mathrm{~kg} / \mathrm{s}$ is the Planck's constant and $e \approx 1.602 \times 10^{-19} \mathrm{C}$ is the elementary electric charge.

In the quantum state, the Hall resistivity $\rho_{x y}$ and the longitudinal resistivity $\rho_{x x}$ exhibit interesting behavior as shown in Figure 1.16.

When a strong magnetic flux density is applied at low temperature, $\rho_{x y}$ exhibits quantized plateaus as:

$$
\rho_{x y}=\frac{R_{K}}{p}
$$

where $p$ is an integer.

When $\rho_{x y}$ is on a plateau, the longitudinal resistivity vanishes: $\rho_{x x}=0$. This vanishing of $\rho_{x x}$ results in a contrary conversion of a resistivity to a conductivity. Hence, the Hall conductivity and longitudinal conductivity can be expressed as:

$$
\sigma_{x y}=\frac{-\rho_{x y}}{\rho_{x x}^{2}+\rho_{x y}^{2}} \quad \text { and } \quad \sigma_{x x}=\frac{\rho_{x x}}{\rho_{x x}^{2}+\rho_{x y}^{2}}
$$

respectively $[9,41,80-81]$. 


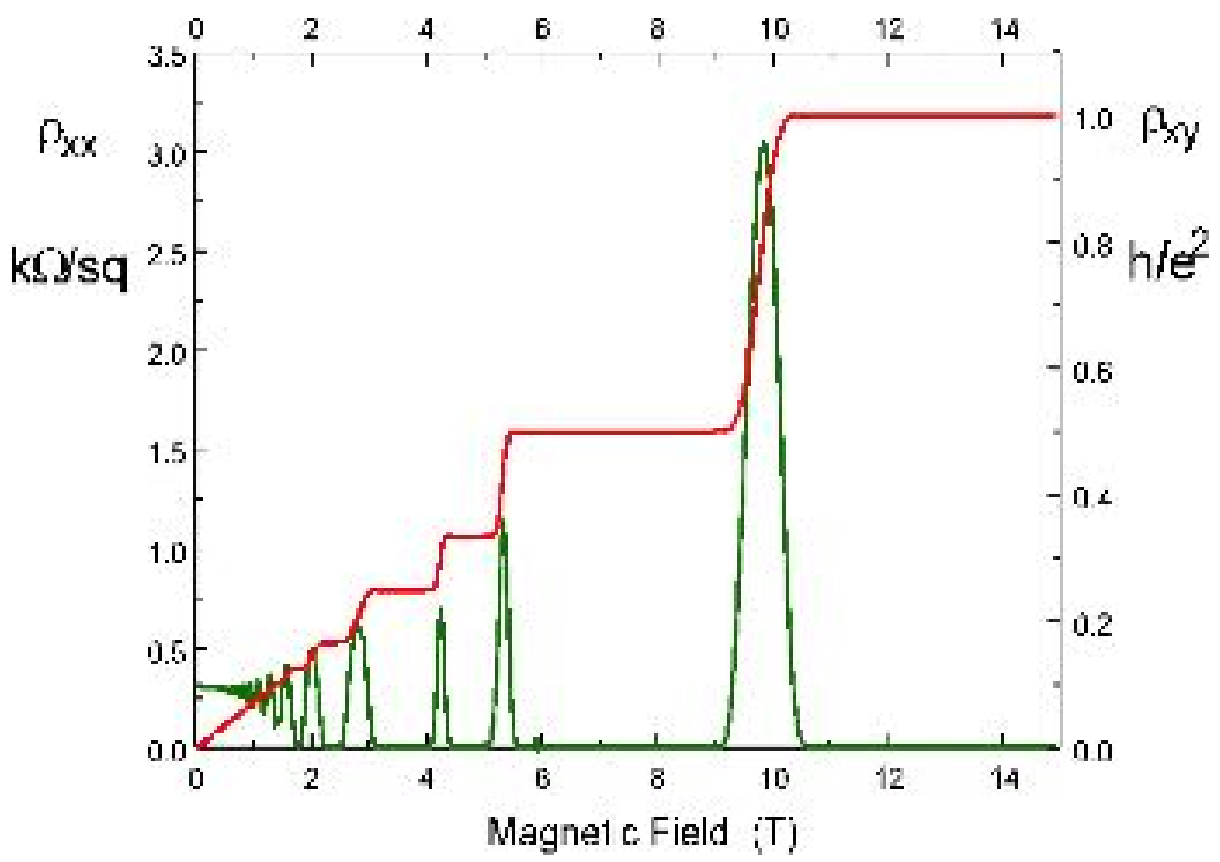

Figure 1.17: A graph of typical measurement of the IQHE.

The data was obtained from 2-D electron gas in a GaAs/GaAlAs heterojunction at $30 \mathrm{mK}$. Reprinted with permission from D. R. Leadley, University of Warwick (1997).

In graphene, the observation of QHE shows an anomalous 2D electron system which leads to a new type of IQHE known as Unconventional Quantum Hall Effect (UQHE) or Quantum Anomalous Hall Effect (QAHE). UQHE exhibits an anomalous behavior with half-integer filling factors. The Hall conductivity of the UQHE in graphene is given by:

$$
\sigma_{x y}= \pm\left(i+\frac{1}{2}\right) \frac{4 e^{2}}{h}
$$

where $i$ is a nonnegative integer [85-87]. 


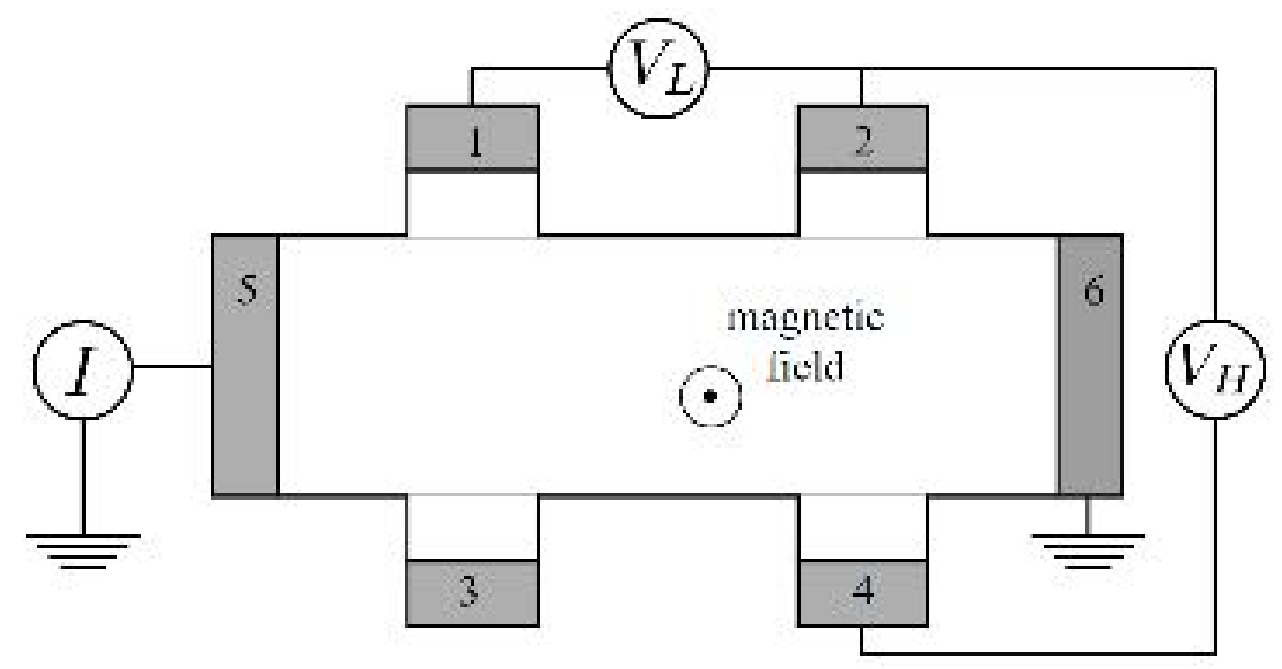

Figure 1.18: A schematic of a Hall bar where $V_{L}=I / \sigma_{x x}$ is the longitudinal voltage and $V_{H}=I / \sigma_{x y}$ is the Hall voltage [106].

The work of Novoselov et al. [83]. also shows that the QHE in graphene can survive at high temperatures. The $\sigma_{x y}$ exhibits a set of quantized plateaus situated symmetrically due to the massless Dirac like nature of the charge carriers. The energy of Dirac fermions in quantized fields can be derived as:

$$
E_{N}=\sqrt{2 e B \hbar v_{F}^{2}|p|}
$$

where $B$ is a magnetic flux density, $\hbar \approx 6.582 \times 10^{-16} \mathrm{eV} \cdot \mathrm{s}$ is the reduced Planck's constant, $v_{F} \approx 10^{6} \mathrm{~m} / \mathrm{s}$ is the Fermi velocity, and $p$ is an integer.

Novoselov et al. have also made assumptions about their experiment that the factors which may help the QHE in graphene to survive at room temperature can be attributed to the large cyclotron gap, $\hbar \omega_{c}$, characteristic to Dirac fermions in graphene, the mobility of Dirac fermions, $\mu$, does not chance from liquid-helium to room temperature $[9,41,82,83]$. 


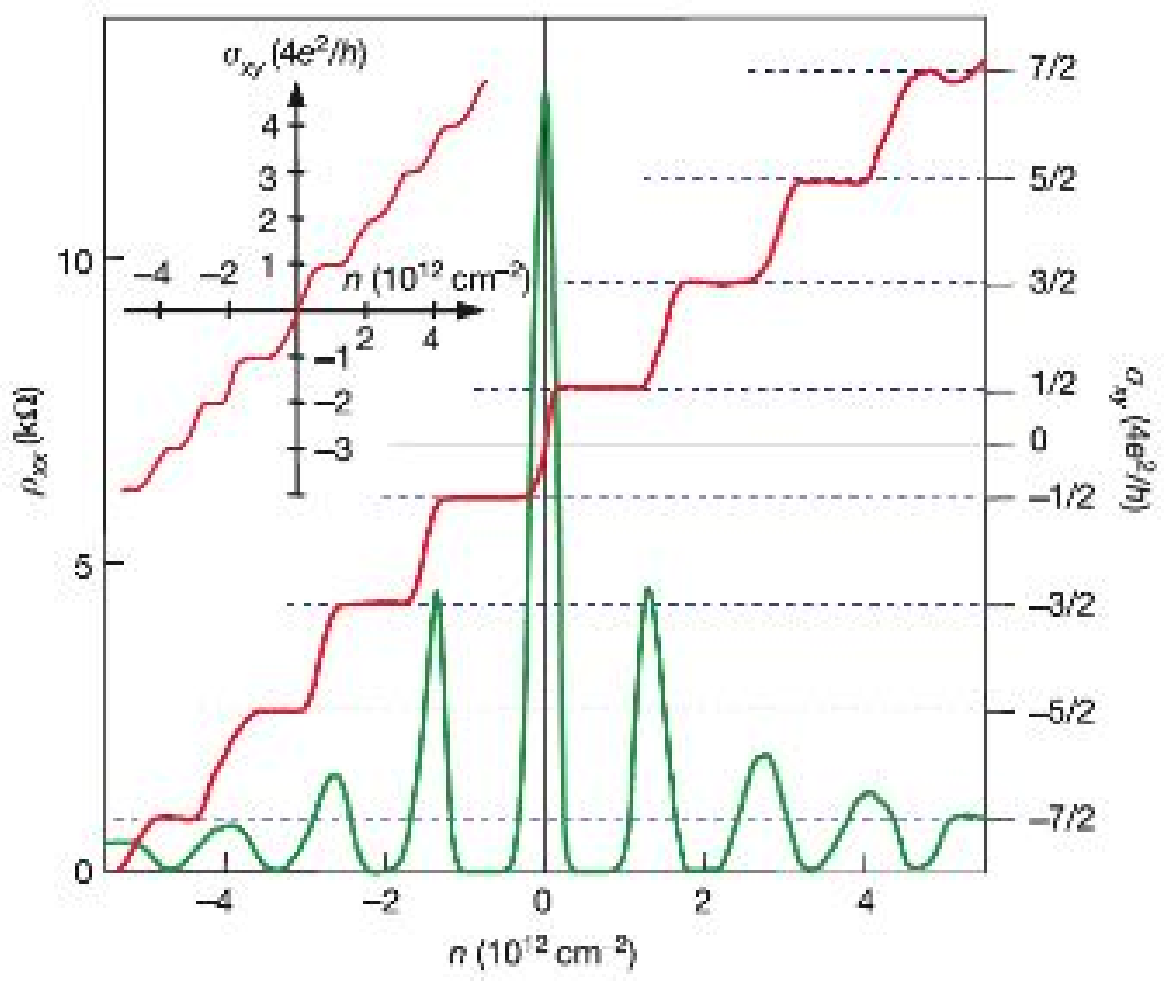

Figure 1.19: A graph of the integer quantum Hall effect in graphene. Reprinted with permission from Sir Konstantin Sergeevich Novoselov, the 2010 Nobel Laureate in Physics [82].

\subsubsection{Fractional Quantum Hall Effect}

In a strong magnetic flux density, $B$, and at a temperature near absolute zero, electron gas in 2D system condenses into a quantum liquid state, and the Hall plateaus of this quantum liquid are corresponding to a fractional value of the filling factor, $v$, of the Hall resistivity as:

$$
\rho_{x y}=R_{H}=\frac{R_{K}}{v}
$$

where $v$ is a fractional filling factor with the rational number of $p / q$.

The fraction $p / q$ will be defined as $p \neq q$ where $p$ is an integer and $q$ is an odd integer except for two factors $5 / 2$ and $7 / 2$. The discovery of a new form of quantum fluid with fractionally charged excitations led R. B. Laughlin, H. L. Störmer, and D. C. Tsui to 
be awarded the 1998 Nobel prize in physics. Thus, the wavefunction of the ground state to describe the behavior of the quantum liquid for the original $1 / 3$ effect was proposed by $R$. B. Laughlin as:

$$
\Psi_{q}\left(z_{1}, z_{2}, \ldots, z_{N}\right)=\prod_{j<k>}^{N}\left(z_{j}-z_{k}\right)^{q} \exp \left[-\frac{1}{4 \ell_{B}^{2}} \sum_{j}^{N}\left|z_{j}\right|^{2}\right]
$$

where $q$ is an odd integer, $z_{j}=x_{j}+i y_{j}$ is the complex coordinate of the $j^{\text {th }}$ particle, $\ell_{B}=$ $\sqrt{\hbar / m \omega_{c}}$ is the magnetic length, with $\omega_{c}=e B / m$ is the cyclotron frequency, $e \approx$ $1.602 \times 10^{-19} \mathrm{C}$ is the elementary electric charge, $B$ is a magnetic flux density, and $m \approx$ $9.109 \times 10^{-31} \mathrm{~kg}$ is the mass of an electron [88 - 90]. This counterintuitive physical phenomenal of the fractional Hall filling factors has been called a "Fractional Quantum Hall Effect (FQHE)". 


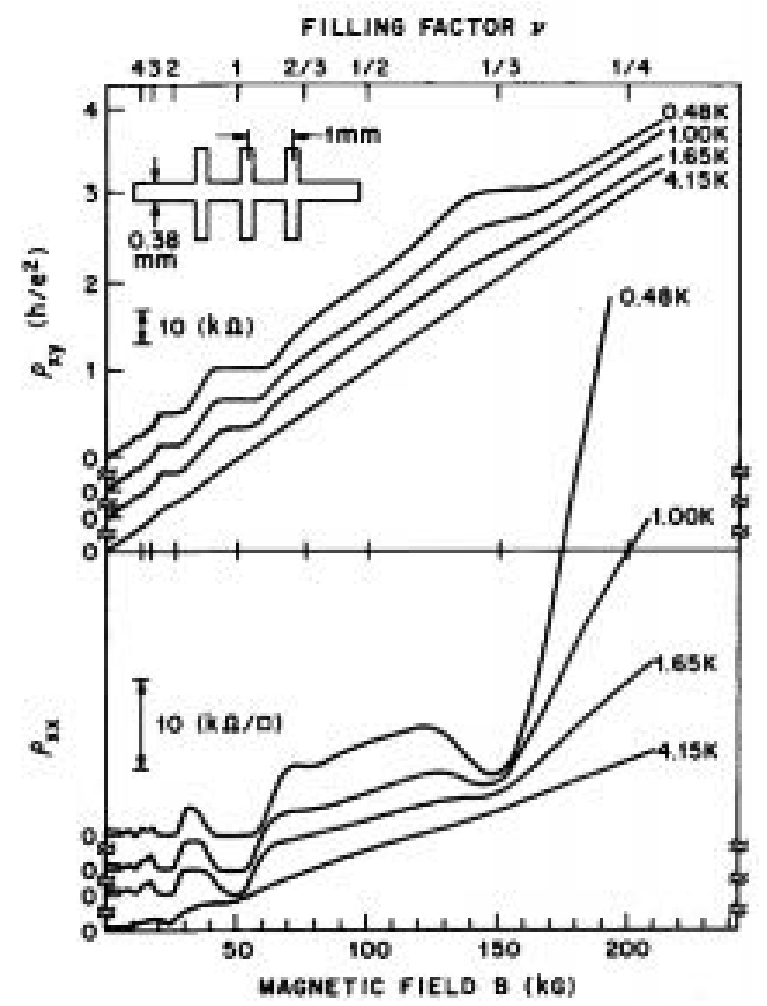

Figure 1.20: The results of the original experiment where the FQHE was discovered for filling factor $v=1 / 3$. Reprinted with permission from Horst Ludwig Störmer, the 1998 Nobel Laureate in Physics [91].

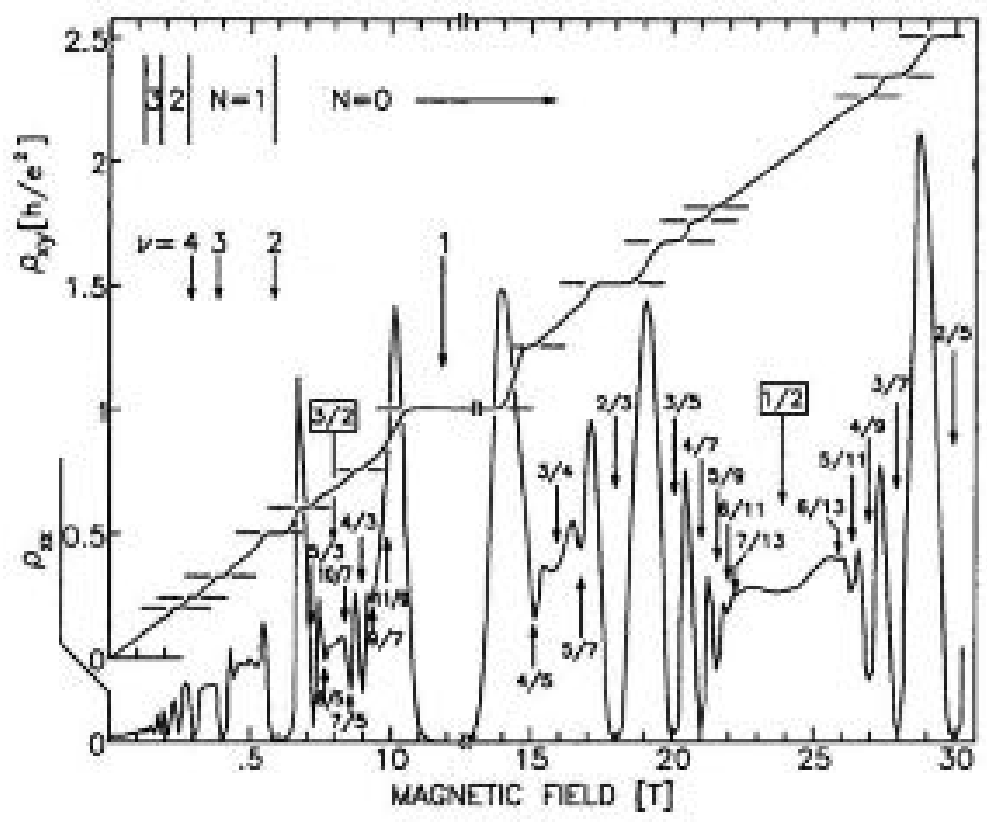

Figure 1.21: A graph of the FQHE where $\mathrm{N}$ is LL quantum number and $v$ indicates values of filling factors. Reprinted with permission from Daniel Chee Tsui, the 1998 Nobel Laureate in Physics [92]. 
The FQHE depends upon the combined effects of the magnetic flux density and Coulomb interaction between electrons which require lower temperature, stronger magnetic flux density, and higher mobility compared to IQHE. In graphene, the variation of $\sigma_{x y}$ with respect to different values of filling factors, $v$, has been recorded as shown in the graph of Figure 1.20.

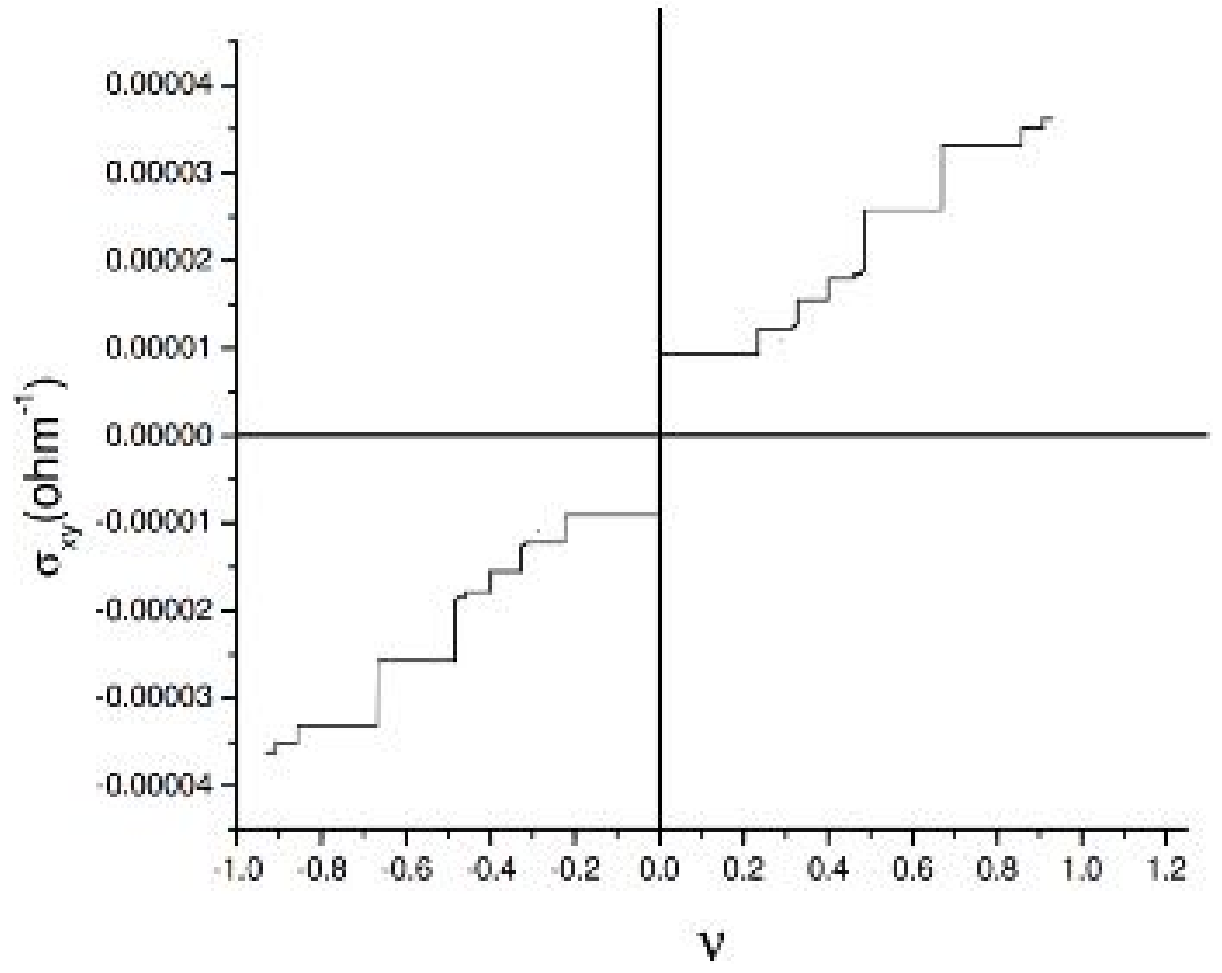

\section{Figure 1.22: Quantized Hall conductivity of graphene in FQHE for different filling factors [86].}

In 2010, Dean et al. also observed features of the FQHE in graphene in which Hall plateaus appear at the filling factor $v= \pm 4\left(n+\frac{1}{2}\right)$ where $n=0,1,2, \ldots$ is the integer index of the LLs [93].

For $n=0$, the fractional filling factors, $v=\frac{1}{3}, \frac{2}{3}$, and $\frac{4}{3}$.

For $\mathrm{n}=1$, the fractional filling factors, $v=\frac{7}{3}, \frac{8}{3}, \frac{10}{3}, \frac{11}{3}$, and $\frac{13}{3}$. 


\subsubsection{Magnetic Properties}

Graphene is a nonmetallic material containing no magnetic atoms. Magnetism in graphene comes from the local states caused by defects or molecular adsorption.

\subsubsection{Atomic Vacancies}

Vacancies of atoms are the most common type of intrinsic defect in a crystal lattice structure. In graphene, vacancies of carbon atoms alter the molecular symmetry of the honeycomb structure and induce magnetism [65-67]. The evidence of magnetism induced by point defects in graphene was reported in the works of Ugeda et al. that by removing some carbon atoms, magnetic moments will form near these atomic vacancies which induce magnetic properties in graphene $[97,98]$.

Besides the vacancies of atoms, defects in graphene can also be created by external doping. By doping boron (B) and nitrogen (N) atoms into graphene, the atoms around the border regions of the hexagonally bonded honeycomb structure of graphene and boron nitride $(\mathrm{BN})$ are localized and they are responsible for magnetism [68, 69].

Yazyev and Helm observed magnetism induced by the presence of quasi-localized defect states in the case of both defect types (vacancy and doping) [70]. The hydrogen chemisorption defect gives rise to the strong Stoner ferromagnetism [101 - 103] with a magnetic moment of $1 \mu_{B}$ per defect, and the vacancy defect gives $1.12 \mu_{B}$ to $1.53 \mu_{B}$ per

defect depending on the defect concentration where $\mu_{B}=\frac{e \hbar}{2 m} \approx 5.788 \times 10^{-5} \mathrm{eV} / \mathrm{T}$ is Bohr magneton; the natural unit for expressing the magnetic moment of an electron (an electron has intrinsic magnetic moment approximately 1 Bohr magneton) [94]. 


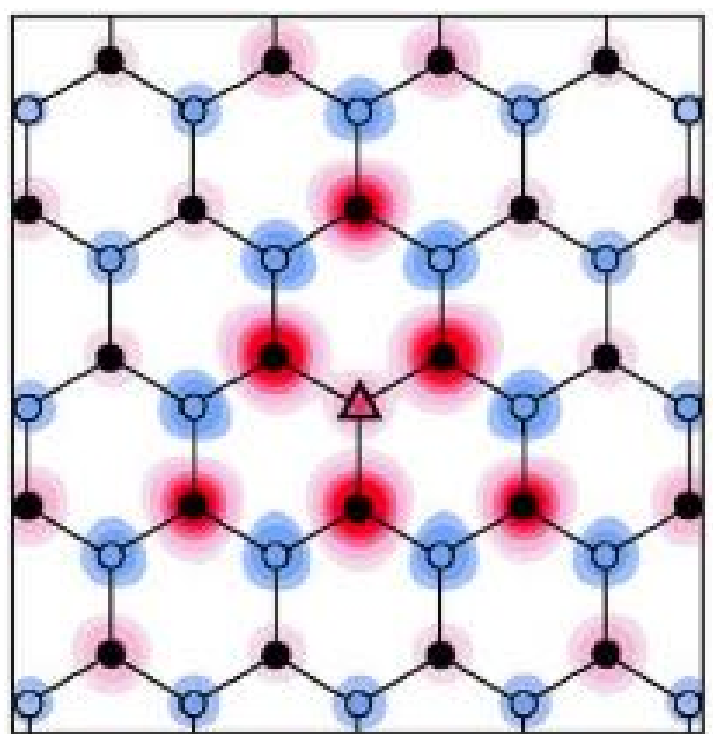

(a)

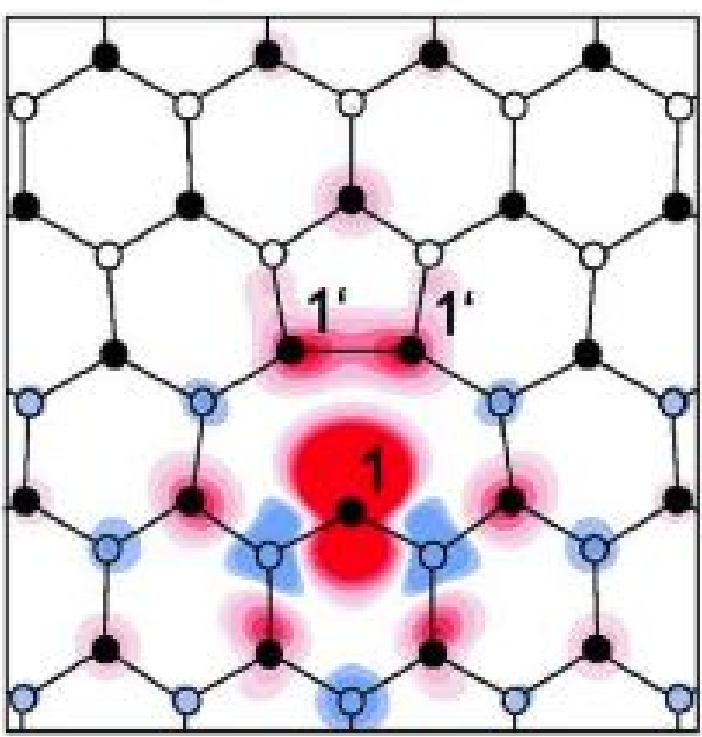

(b)

Figure 1.23: (a) hydrogen chemisorption defect represented by $\Delta$. (b) the vacancy defect. Reprinted with permission from Prof. Yazyev Oleg, Ecole Polytechnique Federale de Lausanne [70].

\subsubsection{Molecular Absorption}

The remarkable results of magnetism in graphene are present when hydrogen atoms are absorbed onto the surface of graphene [72]. The absorption leads to magnetic moments on neighboring carbon atoms, and spin-polarized states are mainly localized around the adsorptive hydrogen $[104,105]$.

Theoretically, if a hydrogen atom is absorbed onto the surface of graphene, it can induce a magnetic moment to the surface due to its magnetic properties [99]. However, when hydrogen atoms are absorbed by nonequivalent carbon atoms in the different sublattices, the magnetic moments induced by hydrogen atoms of sublattices A and B will repeal each other which results the hydrogen absorbed graphene to lose its magnetic moment. On the other hand, if hydrogen atoms are absorbed by the carbon atoms in the 
same sublattice, the magnetic moments of absorbed hydrogen atoms will be added [6972].

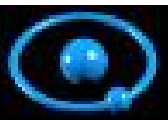

(a)

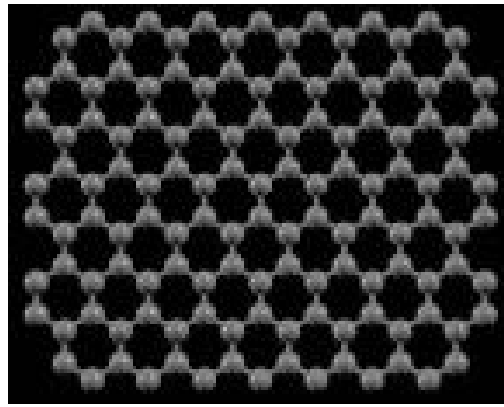

(b)

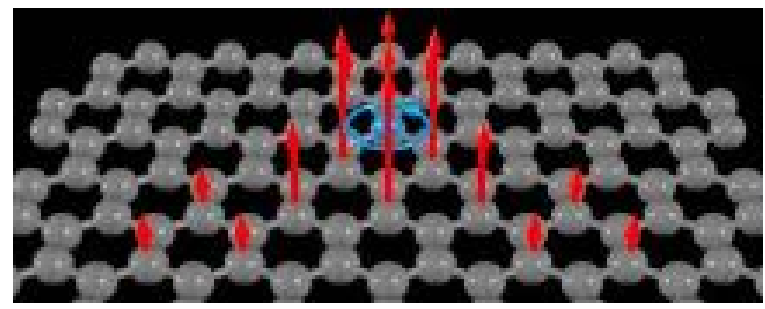

(c)

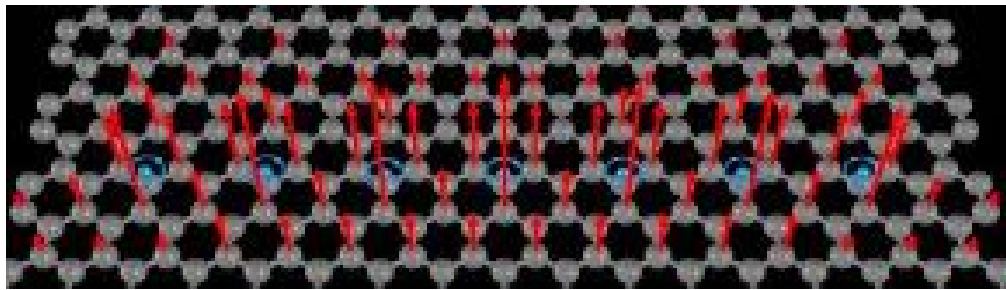

(d)

Figure 1.24: (a) A hydrogen atom.

(b) Graphene honeycomb lattice structure.

(c) An absorbed hydrogen atom induces a magnetic moment on graphene.

(d) The magnetic moments are added when hydrogen atoms are absorbed in the same sublattice. Reprinted with permission from Ivan Brihuega, Associate Professor, Universidad Autónoma de Madrid [73]. 


\subsubsection{Spintronic Properties}

\subsubsection{Introduction to Spintronics}

Spintronics or spin-electronics is advanced electronics that aims to exploit the spin of electrons. The spin of electrons is an intrinsic property of electrons which creates a quantum phenomenon known as a spin angular momentum of electrons, $S$, given by:

$$
S=\sqrt{s(s+1)} \hbar
$$

where $s=\frac{1}{2}$ is a spin quantum number and $\hbar=\frac{h}{2 \pi} \approx 6.582 \times 10^{-16} \mathrm{eV} \cdot \mathrm{s}$ is the reduced Planck's constant. And the spin magnetic dipole moment, $\mu_{s}$, can be expressed as:

$$
\mu_{s}=g \mu_{B} S
$$

where $g=-2$ is a dimensionless magnetic moment quantity of electron known as "Landé $g$-factor" and $\mu_{B}=\frac{e \hbar}{2 m} \approx 5.788 \times 10^{-5} \mathrm{eV} / \mathrm{T}$ is Bohr magneton where $e \approx 1.602 \times 10^{-19}$ $\mathrm{C}$ is the elementary electric charge and $m \approx 9.109 \times 10^{-31} \mathrm{~kg}$ is the mass of an electron [42].

The relationship between $\mu_{s}$ and the magnetic flux density generated by the relative motion of the electron and the nucleus, $B$, can be defined by the change of energy equation:

$$
\Delta E=-\mu_{s} \cdot B
$$

A change of energy in the spin of electron occurs because $B$ creates a spin torque that rotates $\mu_{s}$ as shown in Figure 1.23. 


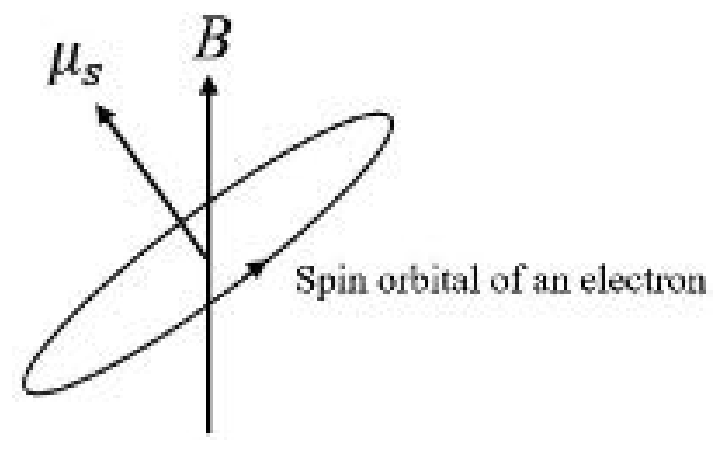

Figure 1.25: A spin torque acting on $\mu_{s}$ created by $B$.

The spin torque can be calculated as:

$$
\tau=\mu_{s} \times B
$$

While $B$ is proportional to the angular momentum of a particle, $L$, and $\mu_{s}$ is proportional to $S$, the change of energy equation can be rewritten as:

$$
\Delta E \propto S \cdot L
$$

where the relationship between $S$ and $L$ is call the "spin-orbit interaction" [61, 62].

Because $\mu_{s}$ can be affected by $B$, spintronics engineers attempt to manipulate the spins of electrons in materials by applying an external magnetic field to spintronic devices. When electrons move through a nonmagnetic medium, they will exhibit random spins. However, the spins can be aligned up or down according to the direction of the applied magnetic field. In solid state materials, the spins might be aligned in an orderly fashion on a crystal lattice forming a nonmagnetic material. Or the spins may be on a lattice and be aligned as a magnetic material $[42,43]$. 


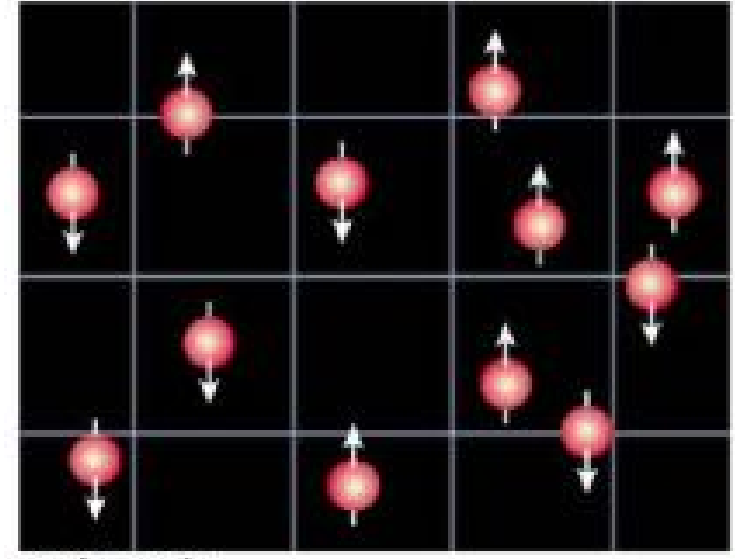

random spin

(a)

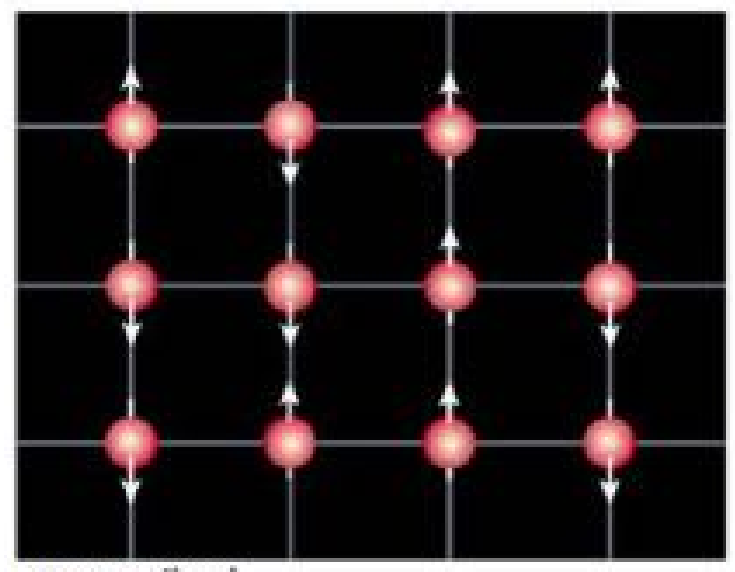

unmagnetized

(c)

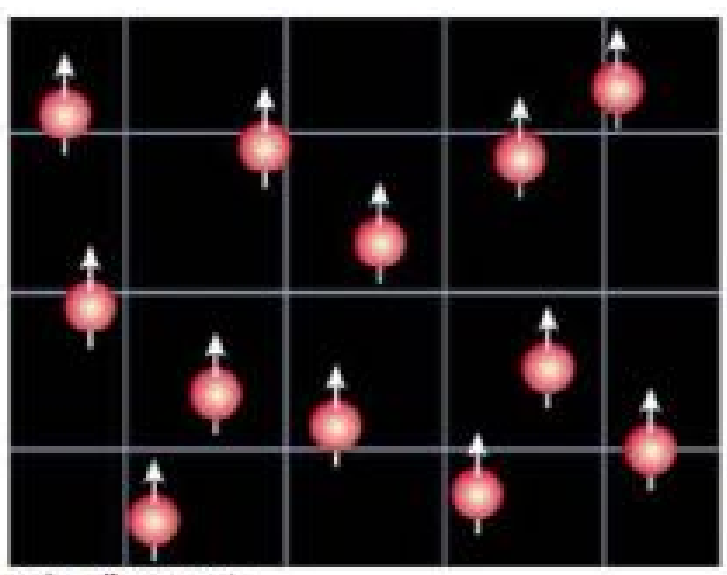

spin alignment

(b)

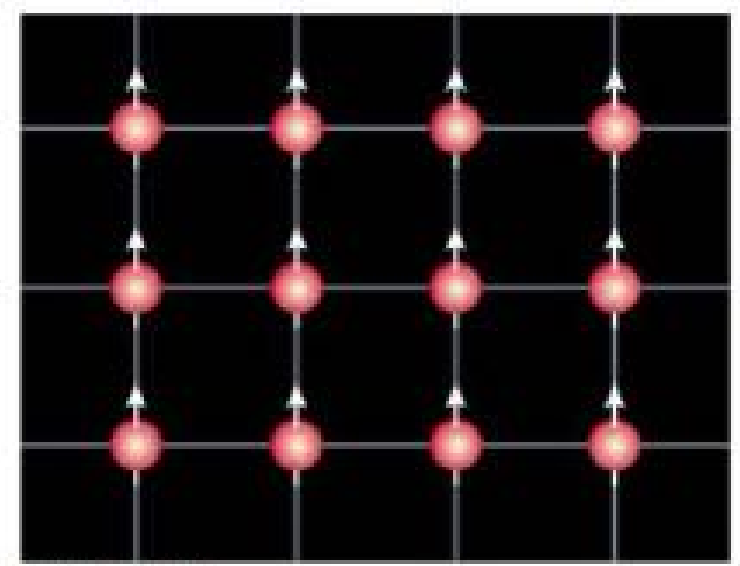

magnetized

(d)

Figure 1.26: (a) Random spin; electrons spin randomly in a material.

(b) Spin alignment; electronic spins are aligned by an external magnetic field.

(c) Unmagnetized; spins are located orderly in an unmagnetized solid state material. (d) Magnetized; spins are located orderly and aligned in a magnetized solid-state material [43].

\subsubsection{Spintronic Properties of Graphene}

Since the exfoliation of graphene was achieved, there have been many reports and experiments in the field of graphene spintronics. Many investigations conclude that graphene has the potential to be a promising material for spintronics due to its remarkable electronic spin properties such low intrinsic spin-orbit interaction which causes graphene 
to have a longer period of electron spin lifetimes compared to other materials $[9,63,64]$ as shown in Table 1.3.

Table 1.3: Spin-dependent properties of graphene, metals, and semiconductor measured by spin valve measurements. Reprinted with permission from Fabian Jaroslav [64].

\begin{tabular}{|c|c|c|c|c|}
\hline \multicolumn{2}{|l|}{ Spin Channel } & \multirow{2}{*}{$\begin{array}{l}\text { Spin lifetime } \\
\sim 42 \mathrm{ps} \text { at } 4.2 \mathrm{~K} \\
\sim 11 \text { ps at } 300 \mathrm{~K}\end{array}$} & \multirow{2}{*}{$\begin{array}{l}\begin{array}{l}\text { Spin } \\
\text { lengths }\end{array} \\
\sim 1 \mu \mathrm{m} \text { at } 4.2 \mathrm{~K} \\
\sim 0.4 \mu \mathrm{m} \text { at } 300 \mathrm{~K}\end{array}$} & \multirow{2}{*}{$\begin{array}{l}\text { Spin signals } \\
\sim 1 \mathrm{~m} \Omega \text { at } 4.2 \mathrm{~K} \\
\sim 0.5 \mathrm{~m} \Omega \text { at } 300 \mathrm{~K}\end{array}$} \\
\hline Metals & $\mathrm{Cu}$ & & & \\
\hline & $\mathrm{Al}$ & $\begin{array}{l}\sim 100 \mathrm{ps} \text { at } 4.2 \mathrm{~K} \\
\sim 45 \mathrm{ps} \text { at } 300 \mathrm{~K}\end{array}$ & $\begin{array}{l}\sim 0.6 \mu \mathrm{m} \text { at } 4.2 \mathrm{~K} \\
\sim 0.4 \mu \mathrm{m} \text { at } 300 \mathrm{~K}\end{array}$ & $\begin{array}{l}\sim 12 \mathrm{~m} \Omega \text { at } 4.2 \mathrm{~K} \\
\sim 0.5 \mathrm{~m} \Omega \text { at } 300 \mathrm{~K}\end{array}$ \\
\hline & $\mathrm{Ag}$ & $\begin{array}{l}\sim 20 \mathrm{ps} \text { at } 5 \mathrm{~K} \\
\sim 10 \mathrm{ps} \text { at } 300 \mathrm{~K}\end{array}$ & $\begin{array}{l}\sim 1 \mu \mathrm{m} \text { at } 5 \mathrm{~K} \\
\sim 0.3 \mu \mathrm{m} \text { at } 300 \mathrm{~K}\end{array}$ & $\begin{array}{l}\sim 9 \mathrm{~m} \Omega \text { at } 5 \mathrm{~K} \\
\sim 2 \mathrm{~m} \Omega \text { at } 300 \mathrm{~K}\end{array}$ \\
\hline \multirow[t]{3}{*}{ Semiconductor } & $\begin{array}{l}\text { Highly } \\
\text { doped } \\
\text { Si }\end{array}$ & $\begin{array}{l}-10 \text { ns at } 8 \mathrm{~K} \\
-1.3 \mathrm{~ns} \text { at } 300 \mathrm{~K}\end{array}$ & $\begin{array}{l}-2 \mu \mathrm{m} \text { at } 8 \mathrm{~K} \\
-0.5 \mu \mathrm{m} \text { at } 300 \mathrm{~K}\end{array}$ & $\begin{array}{l}-30 \mathrm{~m} \Omega \text { at } 8 \mathrm{~K} \\
-1 \mathrm{~m} \Omega \text { at } 300 \mathrm{~K}\end{array}$ \\
\hline & $\mathrm{GaAs}$ & $\begin{array}{l}24 \text { ns at } 10 \mathrm{~K} \\
4 \mathrm{~ns} \text { at } 70 \mathrm{~K}\end{array}$ & $6 \mu \mathrm{m}$ at $50 \mathrm{~K}$ & $\sim 30 \mathrm{~m} \Omega$ at $50 \mathrm{~K}$ \\
\hline & $\begin{array}{l}\text { Highly } \\
\text { doped } \\
\mathrm{Ge}\end{array}$ & $\begin{array}{l}- \text { I ns at } 4 \mathrm{~K} \\
-300 \text { ps at } 100 \mathrm{~K}\end{array}$ & $-0.6 \mu \mathrm{m}$ at $4 \mathrm{~K}$ & $\begin{array}{l}0.1-1 \Omega \text { at } 4 \mathrm{~K} \\
0.02-0.1 \Omega \text { at } 200 \mathrm{~K}\end{array}$ \\
\hline \multicolumn{2}{|l|}{ Graphene } & $\begin{array}{l}0.5-2 \text { ns at } 300 \\
K \\
1-6 \text { ns at } 4 K\end{array}$ & $\begin{array}{l}3-10 \mu \mathrm{m} \text { at } 300 \mathrm{~K} \\
(-100 \mu \mathrm{m} \text { fit from } \\
\text { local MR data) }\end{array}$ & $\begin{array}{l}130 \Omega \text { at } 300 \mathrm{~K} \\
(1 \mathrm{M} \Omega \text { for local MR at } \\
1.4 \mathrm{~K} \text { ) }\end{array}$ \\
\hline
\end{tabular}

There are also reports of spin injection and transport in graphene with ferromagnetic electrodes in the non-local geometry. Depending on the interface between graphene and the electrodes, the interfacial contacts can be categorized into three classes: pinhole contacts, transparent contacts, and tunneling contacts. The interface between graphene and ferromagnetic electrodes can also be used to measure the local magnetoresistance, the difference in the resistance between the magnetization alignments of two electrodes, which is a sign of spin transport [64]. 


\subsection{Tables of Symbols and Acronyms}

Table 1.4: Table of Symbols.

\begin{tabular}{|c|c|c|}
\hline Symbols & Description & Value \\
\hline$a_{0}$ & $\begin{array}{l}\text { The distance between two covalently bonded } \\
\text { carbon atoms in graphene. }\end{array}$ & $1.42 \AA$ \\
\hline$a$ & The lattice spacing constant of graphene. & $\sqrt{3} a_{0}$ \\
\hline$\vec{a}_{1}$ & A unit cell vector of graphene. & $a(\sqrt{3} / 2,1 / 2)$ \\
\hline$\vec{a}_{2}$ & A unit cell vector of graphene. & $a(\sqrt{3} / 2,-1 / 2)$ \\
\hline $\overrightarrow{\delta_{1}}$ & A nearest-neighbor vector of graphene. & $a(1 / 2 \sqrt{3}, 1 / 2)$ \\
\hline $\overrightarrow{\delta_{2}}$ & A nearest-neighbor vector of graphene. & $a(1 / 2 \sqrt{3},-1 / 2)$ \\
\hline$\vec{\delta}_{3}$ & A nearest-neighbor vector of graphene. & $a(-1 / 2 \sqrt{3}, 0)$ \\
\hline$\vec{b}_{1}$ & A reciprocal lattice vector of graphene & $(1 / a)(2 \pi / \sqrt{3}, 2 \pi)$ \\
\hline$\vec{b}_{2}$ & A reciprocal lattice vector of graphene & $(1 / a)(2 \pi / \sqrt{3},-2 \pi)$ \\
\hline$\Gamma$ & $\begin{array}{l}\text { A high symmetry point in the reciprocal space } \\
\text { of graphene. }\end{array}$ & - \\
\hline M & $\begin{array}{l}\text { A high symmetry point in the reciprocal space } \\
\text { of graphene. }\end{array}$ & - \\
\hline $\mathrm{K}$ & $\begin{array}{l}\text { A high symmetry point in the reciprocal space } \\
\text { of graphene. }\end{array}$ & $\left(\frac{1}{a_{0}}\right)\left(\frac{2 \pi}{\sqrt{3}}, \frac{2 \pi}{3}\right)$ \\
\hline $\mathrm{K}^{\prime}$ & $\begin{array}{l}\text { A high symmetry point in the reciprocal space } \\
\text { of graphene. }\end{array}$ & $\left(\frac{1}{a_{0}}\right)\left(\frac{2 \pi}{\sqrt{3}}, \frac{-2 \pi}{3}\right)$ \\
\hline$E$ & Young's modulus. & - \\
\hline$D$ & Third-order elastic stiffness. & - \\
\hline$\sigma_{\text {int }}$ & Intrinsic strength. & - \\
\hline$T_{m}$ & Melting temperature. & - \\
\hline $\mathrm{XeF}_{2}$ & Xenon difluoride. & - \\
\hline $\mathrm{C}_{4} \mathrm{O}(\mathrm{OH})$ & $\begin{array}{l}\text { The chemical composition of fully oxidized } \\
\text { graphite. }\end{array}$ & - \\
\hline$K$ & Thermal conductivity. & - \\
\hline$R_{K}$ & von Klitzing constant. & $25.813 \mathrm{k} \Omega$ \\
\hline
\end{tabular}




\begin{tabular}{|c|c|c|}
\hline$h$ & The Planck's constant. & $\approx 6.626 \times 10^{-34} \mathrm{~m}^{2} \mathrm{~kg} / \mathrm{s}$ \\
\hline$e$ & The elementary electric charge. & $\approx 1.602 \times 10^{-19} \mathrm{C}$ \\
\hline$\rho_{x y}$ & Hall resistivity. & - \\
\hline$\rho_{x x}$ & Longitudinal resistivity. & - \\
\hline$\sigma_{x y}$ & Hall conductivity. & - \\
\hline$\sigma_{x x}$ & Longitudinal Conductivity. & - \\
\hline$p$ & An integer. & $\ldots,-2,-1,0,1,2, \ldots$ \\
\hline$i$ & A nonnegative integer. & $0,1,2,3, \ldots$ \\
\hline$E_{N}$ & $\begin{array}{l}\text { The energy of Dirac fermions in quantized } \\
\text { fields. }\end{array}$ & - \\
\hline$B$ & Magnetic flux density. & - \\
\hline$\hbar$ & The reduced Planck's constant. & $\approx 6.582 \times 10^{-16} \mathrm{eV} \cdot \mathrm{s}$ \\
\hline$v_{F}$ & The Fermi velocity. & $\approx 10^{6} \mathrm{~m} / \mathrm{s}$ \\
\hline$\hbar \omega_{c}$ & Cyclotron gap. & - \\
\hline$\mu$ & The mobility of Dirac fermions. & - \\
\hline$R_{H}$ & Hall resistivity. & - \\
\hline$v$ & A fractional filling factor (a rational number). & - \\
\hline$q$ & An odd integer. & $\ldots,-5,-3,-1,1,3,5, \ldots$ \\
\hline$\Psi_{q}$ & The quantum liquid wavefunction. & - \\
\hline$\ell_{B}$ & Magnetic length. & - \\
\hline$\omega_{c}$ & Cyclotron frequency. & - \\
\hline$m$ & The mass of an electron. & $\approx 9.109 \times 10^{-31} \mathrm{~kg}$ \\
\hline$n$ & Index of Landau Levels. & $0,1,2, \ldots$ \\
\hline$\mu_{B}$ & Bohr magneton. & $\approx 5.788 \times 10^{-5} \mathrm{eV} / \mathrm{T}$ \\
\hline$S$ & A spin angular momentum of electrons. & - \\
\hline$S$ & A spin quantum number. & $1 / 2$ \\
\hline$\mu_{s}$ & Spin magnetic dipole moment. & - \\
\hline$g$ & $\begin{array}{l}\text { A dimensionless magnetic moment quantity } \\
\text { of electron known as "Landé } g \text {-factor". }\end{array}$ & -2 \\
\hline
\end{tabular}




\begin{tabular}{|c|l|c|}
\hline$\Delta E$ & A change of energy in the spin of electron & - \\
\hline$\tau$ & Spin torque. & - \\
\hline$L$ & The angular momentum of a particle. & - \\
\hline
\end{tabular}

Table 1.5: Table of Acronyms.

\begin{tabular}{|c|c|}
\hline Acronym & Description \\
\hline 2D & 2-dimensional \\
\hline 3D & 3-dimensional \\
\hline B & Boron \\
\hline BLG & Bilayer graphene \\
\hline BN & Boron nitride \\
\hline C-C & Carbon-carbon \\
\hline CVD & Few-layer graphene \\
\hline FLG & Fractional Quantum Hall Effect \\
\hline FQHE & Graphene oxide \\
\hline GO & Highly Ordered Pyrolytic Graphite \\
\hline HOPG & Integer Quantum Hall Effect \\
\hline IQHE & Kosterlitz, Thouless, Halperin, Nelson, and Young \\
\hline KTHNY & Landau Levels \\
\hline LLs & Qneonventional Quantum Hall Effect \\
\hline N & Quantum Hall Effect \\
\hline QHE & Single-layer graphene \\
\hline SLG & UQHE \\
\hline
\end{tabular}


Chapter 2

Superconductivity 


\subsection{Introduction to Superconductivity}

Superconductivity is a phenomenon of the disappearance of electrical resistance and magnetic field in a material when the material reaches its critical temperature, $T_{c}$, and critical magnetic field, $H_{c}$. When a material exhibits its superconducting state, it is called a "superconductor".

Superconductivity was discovered in 1911 by a Dutch physicist H. K. Onnes, the 1913 Nobel Laureate in Physics, while he was working on the cryogenics of mercury. When mercury was cooled by liquid helium, at $4.2 \mathrm{~K}$, its electrical resistance abruptly drops from $0.002 \Omega$ to a millionth part as shown in Figure 2.1 [117].

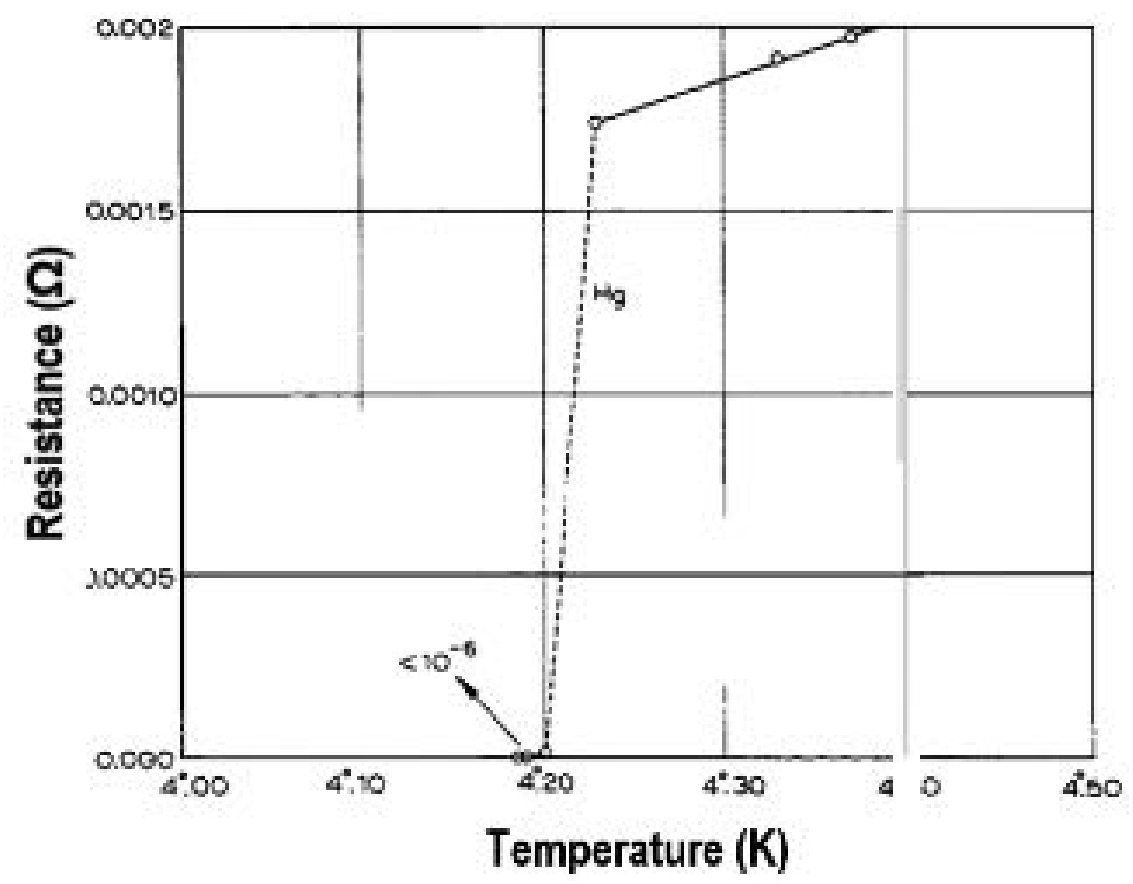

Figure 2.1: A graph of the electrical resistance of mercury, the resistance abruptly drops to zero at 4.2 Kelvin [117].

Since the first superconductor was discovered, many elements have been tested for superconductivity and a number of effects have been discovered and theory proposed. For example, if a superconducting material is placed on a magnet which has a magnetic field 
lower than $H_{c}$ of the material, a repulsive force will levitate to a height where the force of repulsion balances its weight. This phenomenon of levitation is a proof of near-perfect diamagnetism in superconductors which is called the "Meissner Effect" [112].

\subsection{Meissner Effect}

The Meissner effect or Meissner-Ochsenfeld effect is a quantum phenomenon where all magnetic fields penetrating through a material are repelled when the material reaches its superconducting state. The effect was named after the German physicists W. Meissner and R. Ochsenfeld who discovered this phenomenon in 1933.

When the temperature, $T$, of a material is lowered to the critical temperature, $T_{c}$, the material will reach its superconducting state. In that state, the interior magnetic field in the material will decrease, on the other hand, the exterior magnetic field will increase. If the interior field is completely repelled, the exterior field will increase to the maximum due to the conservation law of magnetic flux. The Meissner effect shows that a superconductor will not allow magnetic fields to easily penetrate through it. That is because the

microscopic magnetic dipoles induced in a superconductor will oppose the applied magnetic field, $H$, with the magnetic flux density, $B$. Therefore, beside a perfect conductor, a superconductor is also said to be a "perfect diamagnetic material" $[110,111]$.

Since a superconductor is also a perfect diamagnetic material, if a superconductor is placed in a magnetic field, the magnetic field will be repelled by the diamagnetic property of the superconductor causing a repulsive force that will trap the superconductor into the air. This phenomenon is known as the "Meissner levitation". 

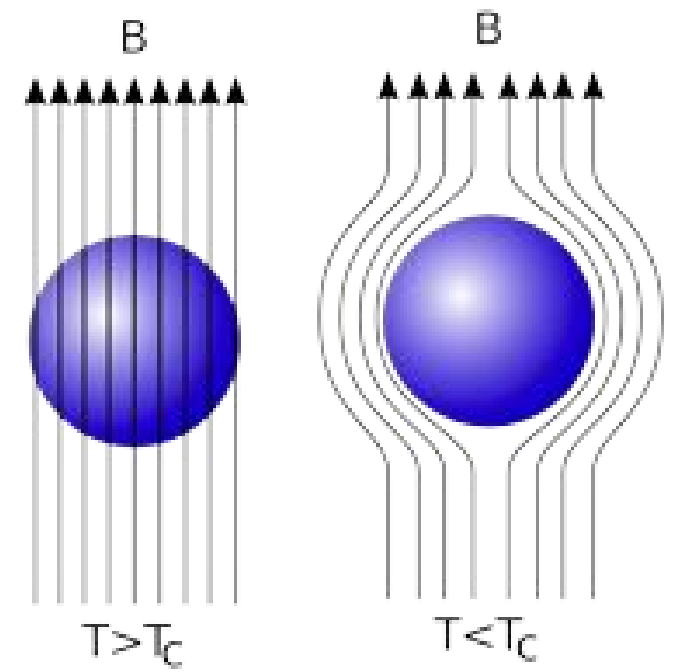

Figure 2.2: The Meissner effect [115].

If a small magnet is placed on a superconductor, the magnet will be lifted by the repulsive force produced by the induced supercurrents in the superconductor. The induced supercurrents will act as mirror images of the magnetic poles of the magnet and repel the magnetic field away from the superconductor [116].

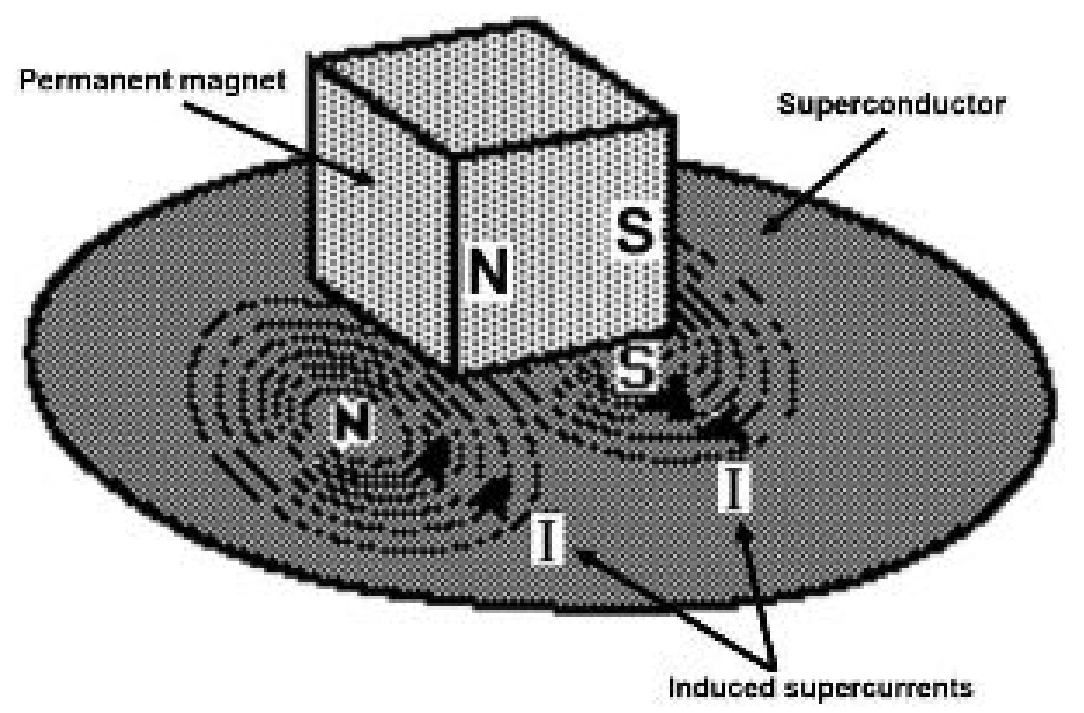

Figure 2.3: A permanent magnet is levitated by the repulsive force produced by the induced supercurrents in a superconductor where $\mathbf{N}$ and $\mathbf{S}$ represent magnetic north and south poles respectively. Reprinted with permission from Rod Nave, Georgia State University [116]. 


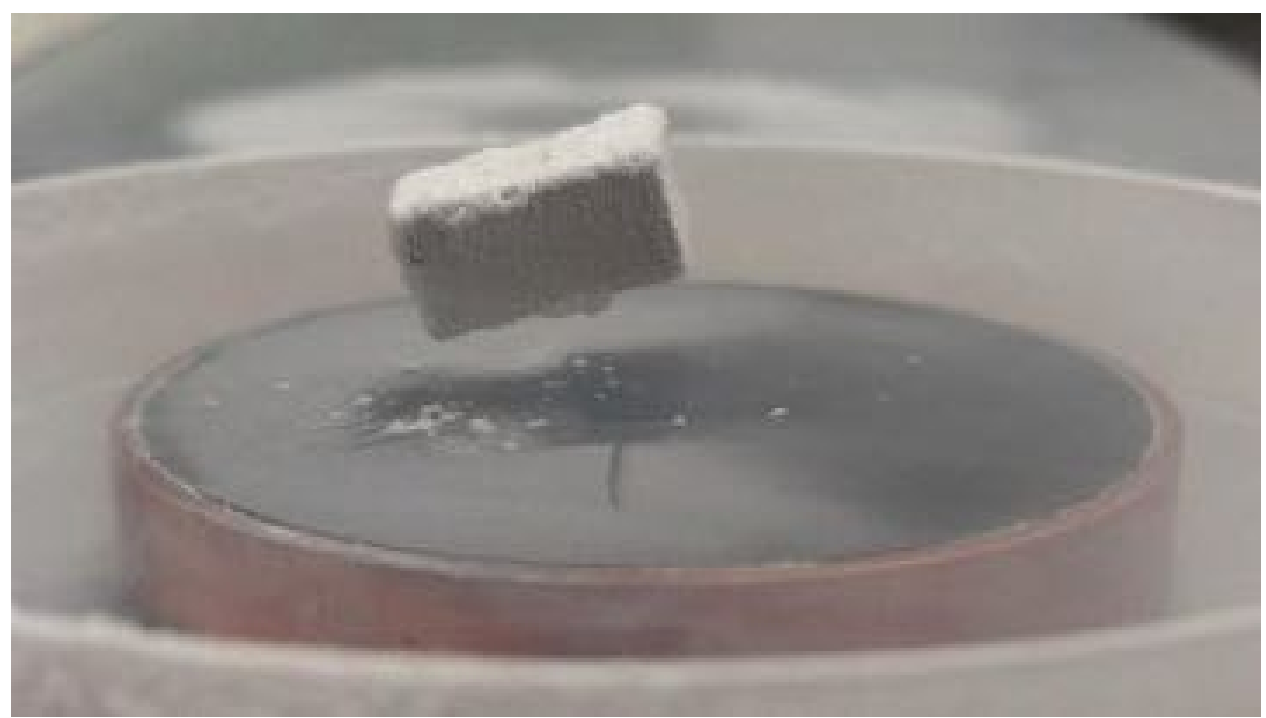

Figure 2.4: A permanent magnet is levitated over liquid nitrogen-cooled Yttrium Barium Copper Oxide (YBCO).

\subsection{Theories of Superconductivity}

\subsubsection{London Theory}

In 1935, "the electromagnetic equations of the supraconductor" were proposed by F. London and H. London to describe the electromagnetic behavior of superconductors where Ohm's law of normal current density, $j_{n}$, is replaced by supercurrent density, $j_{s}$ [113]. In the standard Drude "free electron gas" model [134], normal electrical conductivity is defined as:

$$
\sigma_{n}=\frac{e^{2} n_{n} \tau}{m}
$$

where $e$ is the elementary electric charge, $n_{n}$ is the number of free electrons per unit volume of a normal conductor, $\tau$ is the mean free time between electron collision which is also known as relaxation time, and $m$ is the mass of an electron. The Drude model results a linear relationship between normal current density, $j_{n}$, and an electric field, $E$, as: 


$$
j_{n}=\sigma_{n} E=\left(\frac{e^{2} n_{n} \tau}{m}\right) E=-n_{n} v_{n} e
$$

where $v_{n}$ is the velocity of electrons in a normal conductor [118, 119].

In a superconductor, $\mathrm{F}$. and $\mathrm{H}$. London assumed $\tau$ to be infinite, since the electrical resistance in a superconductor is proved to be zero (no collision). Then, when a normal conductor reaches its superconducting state, the normal current density is replaced by the supercurrent density which can be defined as:

$$
j_{s}=-n_{s} v_{s} e
$$

By applying Newton's classical equation of motion to the electrons, the electric force in a superconductor will be:

$$
F_{s}=m \frac{d v_{s}}{d t}=-e E
$$

where $v_{s}$ is the velocity of the electrons in the superconductor.

This classical equation of motion was used to derive the first London equation:

$$
\frac{d v_{s}}{d t}=-\frac{e E}{m}
$$

The total derivative and the partial derivative of the velocity of electrons in a superconductor can be interchangeably employed as:

$$
\frac{d v_{s}}{d t}=\frac{\partial v_{s}}{\partial t}+\left(v_{s} \cdot \nabla\right) v_{s}
$$

where $\nabla=\hat{\imath} \frac{\partial}{\partial x}+\hat{\jmath} \frac{\partial}{\partial y}+\hat{k} \frac{\partial}{\partial z}$ is the differential operation and $\left(v_{s} \cdot \nabla\right) v_{s}$ is the appearance of a non-linear term which will vanish if the superconducting system is symmetry [112]. Thus, the "first London equation" is obtained:

$$
\frac{\partial j_{s}}{\partial t}=-n_{s} e \frac{\partial v_{s}}{\partial t}=\frac{e^{2} n_{s} E}{m}
$$


where $n_{s}$ is the number of free electrons per unit volume of a superconductor [110 - 112].

The second London equation is derived by taking the curl of the first London equation together with taking Maxwell's equation into account as:

$$
\nabla \times\left(\frac{\partial j_{s}}{\partial t}\right)=\nabla \times\left(\frac{e^{2} n_{s} E}{m}\right)=\frac{e^{2} n_{s}(\nabla \times E)}{m}
$$

From Maxwell's equation $(\nabla \times E)=-\left(\frac{\partial B}{\partial t}\right)$, the equation can be derived as:

$$
\frac{e^{2} n_{s}(\nabla \times E)}{m}=-\frac{e^{2} n_{s}}{m}\left(\frac{\partial B}{\partial t}\right)=-\frac{1}{\mu_{0} \lambda_{L}^{2}}\left(\frac{\partial B}{\partial t}\right)
$$

where $B$ is a magnetic flux density, $\mu_{0}=4 \pi \times 10^{-7}$ Henries per meter $(\mathrm{H} / \mathrm{m})$ is the magnetic permeability in free space, and $\lambda_{L}=\sqrt{m c^{2} / 4 \pi n_{S} e^{2}}$ is the London penetration depth. Therefore, the "second London equation" can be expressed as:

$$
\nabla \times j_{s}=-\frac{1}{\mu_{0} \lambda_{L}^{2}} B=-\frac{c}{4 \pi \lambda_{L}^{2}} B
$$

where $c \approx 3 \times 10^{8} \mathrm{~m} / \mathrm{s}$ is the speed of light measured in a vacuum.

In a superconductor, $E$ does not change quickly, the fourth Maxwell's equation will be:

$$
\nabla \times B=\frac{4 \pi}{c} j_{s}
$$

By using the vector relation:

$$
\nabla \times(\nabla \times B)=\nabla(\nabla \cdot B)-\nabla^{2} B=\nabla \times\left(\frac{4 \pi}{c} j_{s}\right)
$$

where $(\nabla \cdot B)=0$ because of the nonexistence of the magnetic monopole by the definition of the second Maxwell's equation $[111,112]$. Therefore:

$$
\nabla \times \frac{4 \pi}{c} j_{s}=-\nabla^{2} B=-\frac{4 \pi n_{s} e^{2}}{m c^{2}} B
$$




$$
-\nabla \times \frac{4 \pi}{c} j_{s}=\nabla^{2} B=\frac{1}{\lambda_{L}^{2}} B
$$

The equation that determines the spatial variation of $B$ is:

$$
\nabla^{2} B-\frac{B}{\lambda_{L}^{2}}=0
$$

and the equation that determines the spatial variation of $j_{s}$ is:

$$
\nabla^{2} j_{s}-\frac{j_{s}}{\lambda_{L}^{2}}=0
$$

\subsubsection{Ginzburg-Landau Theory}

In 1950, V. L. Ginzburg and L. D. Landau proposed a theory which introduced a pseudo-wave function order parameter, $\psi$, and other parameters of superconductors. Thus, the theory was named after them as Ginzburg-Landau (GL) theory. According to the GL theory, the numbers of electrons, $n_{s}$, in a superconductor can be described as:

$$
n_{s}=|\psi|^{2}
$$

and the free energy density of a material in a superconducting state can be written in a Taylor series as:

$$
f_{s}=f_{n}+\alpha|\psi|^{2}+\frac{1}{2} \beta|\psi|^{4}+\cdots
$$

where $f_{n}$ is the free energy density of a material in a normal state, $\alpha$ is the coefficient of superconducting pair density which is proportional to $|\psi|^{2}$, and $\beta$ is the coefficient of pair interaction term which is proportional to $|\psi|^{4}[120]$.

This form of free energy density can be fully expanded as a function of magnetic flux density when $B \neq 0$ as:

$$
f_{s}(B)=f_{n}(B)+\alpha|\psi|^{2}+\frac{1}{2} \beta|\psi|^{4}+\frac{1}{2 m}|(-i \hbar \nabla-e A) \psi|^{2}+\frac{B^{2}}{2 \mu_{0}}
$$


where $A$ is the magnetic vector potential of $B$ which can be defined as $B=\nabla \times A$.

In a superconducting state, $B$ can be considered as zero which makes the surface kinetic energy density, $\frac{1}{2 m}|(-i \hbar \nabla-e A) \psi|^{2}$, and the magnetic energy, $\frac{B^{2}}{2 \mu_{0}}$, negligible $[112,121]$. Therefore, the total free energy density can be simply described as:

$$
f=f_{s}-f_{n}=\alpha|\psi|^{2}+\frac{1}{2} \beta|\psi|^{4}
$$

The equation can be minimized by setting the derivative of $f$ with respect to $|\psi|$ to be equal to zero as:

$$
\frac{\partial f}{\partial|\psi|}=\left(2 \alpha|\psi|+\frac{4}{2} \beta|\psi|^{3}\right)=2|\psi|\left(\alpha+\beta|\psi|^{2}\right)=0
$$

Thus, $|\psi|$ can be determined as $|\psi|=0$ when $T>T_{c}$ (normal state) and $|\psi|=$ $\sqrt{-\frac{\alpha}{\beta}}$ when $T<T_{c}$ (superconducting state) [121].

In a superconducting state of a material when $T<T_{c}$ where $|\psi| \neq 0$, it indicates that $\beta>0$ and $\alpha<0$.

To find the minimum free energy density, the value of $|\psi|=\sqrt{-\frac{\alpha}{\beta}}$ is substituted into the free energy equation as:

$$
f_{\text {min }}=\left(f_{s}-f_{n}\right)_{\text {min }}=\alpha\left(\sqrt{-\frac{\alpha}{\beta}}\right)^{2}+\frac{1}{2} \beta\left(\sqrt{-\frac{\alpha}{\beta}}\right)^{4}=-\frac{\alpha^{2}}{2 \beta}
$$

The free energy density of a superconductor, $f_{s}$, can also be determined by subtracting the magnetic field energy of a superconductor, $\frac{B_{c}^{2}}{2 \mu_{0}}$, from $f_{n}$ as:

$$
f_{s}=f_{n}-\frac{B_{c}^{2}}{2 \mu_{0}}
$$


where $B_{c}$ is the critical magnetic flux density of the material and $\mu_{0}$ is the magnetic permeability in free space. This leads to:

$$
\begin{gathered}
\frac{B_{c}^{2}}{2 \mu_{0}}=\frac{\alpha^{2}}{2 \beta} \\
B_{c}=\sqrt{\frac{\mu_{0} \alpha^{2}}{\beta}}
\end{gathered}
$$

From the equation $n_{s}=|\psi|^{2}$, the London penetration depth can be rewritten as:

$$
\lambda_{L}=\sqrt{\frac{m c^{2}}{4 \pi e^{2}|\psi|^{2}}}=\sqrt{\frac{m \beta}{\mu_{0} e^{2} \alpha^{2}}}
$$

If the surface kinetic energy density and the magnetic field energy are taken into account, the derivative of the full form of free energy density equation:

$$
f_{s}(B)=f_{n}(B)+\alpha|\psi|^{2}+\frac{1}{2} \beta|\psi|^{4}+\frac{1}{2 m}|(-i \hbar \nabla-e A) \psi|^{2}+\frac{B^{2}}{2 \mu_{0}}
$$

can be minimized as:

$$
\frac{\partial f_{s}(B)}{\partial|\psi|}=\alpha|\psi|+\beta|\psi|^{3}+\frac{1}{2 m}(-i \hbar \nabla-2 e A)^{2} \psi=0
$$

where $\frac{1}{2 m}(-i \hbar \nabla-2 e A)^{2} \psi$ is the minimized surface kinetic energy density.

When $B=0$, hence $A=0$, the minimized surface kinetic energy density equation can be written as $\frac{-\hbar^{2} \psi}{2 m}$.

The surface kinetic energy density of a superconductor can develop over a certain length in space, $x$, therefore the changes of the surface kinetic energy density of a superconductor can be calculated by taking the second derivative of $\psi$ of the minimized surface kinetic energy density term with respect to a certain length in space, then the total free energy density at $B=0$ can be minimized as: 


$$
\frac{\partial f_{s}(0)}{\partial|\psi|}=\alpha|\psi|+\beta|\psi|^{3}-\frac{\hbar^{2}}{2 m} \cdot \frac{d^{2} \psi}{d x^{2}}=0
$$

If the function of free energy density which corresponds to $x$ is introduced as $f(x)=\frac{\psi}{\psi_{\infty}}$, then the minimized free energy density equation can be rewritten as:

$$
\alpha \psi_{\infty} f(x)+\beta \psi_{\infty}^{3} f^{3}(x)-\frac{\psi_{\infty} \hbar^{2}}{2 m} \cdot \frac{d^{2} f(x)}{d x^{2}}=0
$$

where $\psi_{\infty}$ is the pseudo-wave function in a superconductor which approaches an infinite value.

In a superconducting state, where $\alpha<0$, the pseudo-wave function will be:

$$
\psi_{\infty}=\sqrt{n_{s}}=|\psi|=\sqrt{-\frac{\alpha}{\beta}}
$$

where $-\frac{\alpha}{\beta}>0$, and the minimized free energy density equation will be:

$$
f(x)-f^{3}(x)-\frac{\hbar^{2}}{2 m \alpha} \cdot \frac{d^{2} f(x)}{d x^{2}}=0
$$

where $-\frac{\hbar^{2}}{2 m \alpha}=\frac{\hbar^{2}}{2 m|\alpha|}=\xi_{G L}^{2}$ which $\xi_{G L}$ is the GL coherence length [122]. The ratio between the London penetration depth and the GL coherence length is called the GL parameter, $\kappa$, where $\kappa$ is given as:

$$
\kappa=\frac{\lambda_{L}}{\xi_{G L}}
$$

\subsubsection{Bardeen-Cooper-Schrieffer Theory}

The Bardeen-Cooper-Schrieffer theory is commonly known as BCS theory. It was proposed by J. Bardeen, L. N. Cooper, and J. R. Schrieffer who jointly received the Nobel Prize in Physics 1972 for their theory of superconductivity [123 - 125]. 


\subsubsection{Cooper Pairs}

In 1956, L. N. Cooper proposed that two electrons in a metal bound together by a small attraction at low temperatures [128]. While an electron is passing through the lattices of a conductor, the electron is attracting the lattices because the electron possesses a negative charge which will attract the positive charges from the lattices described by the Coulomb's law of charges [126]. When the lattices are attracted by the electron, they are distorted from their origins. This distortion of the lattices or "virtual" phonon creates a region of positive charge which can attract electrons. This interaction of the electron and the virtual phonon is called an "electron-phonon interaction", and the phonon created by the distortion of the lattices may be referred as a virtual phonon because it is localized and does not propagate through the lattice like a wave. Due to the Pauli exclusion principle which describes that two or more identical Fermions cannot occupy the same quantum state, therefore only one electron which is drawn by the phonon will have a weak bond to the first electron that attracts the lattices, and the two electrons will have different spin states. A pair of the two electrons formed by the weak bond between them is called a “Cooper pair” [127]. 


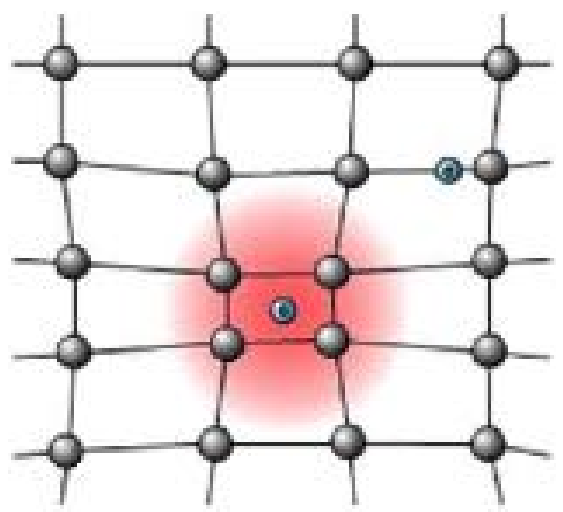

(a)

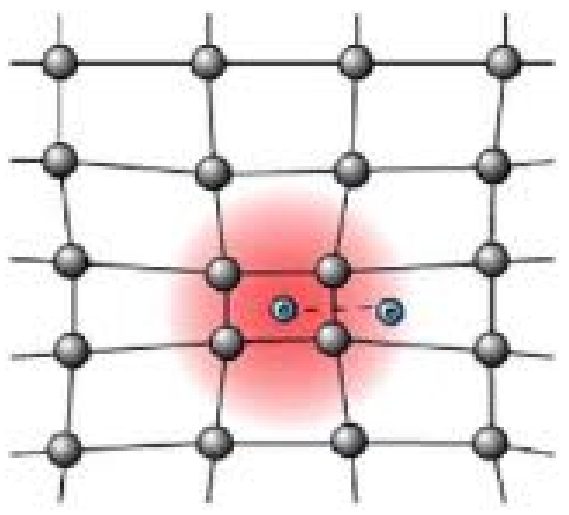

(b)

Figure 2.5: (a) Lattices of a conductor are attracted by an electron creating a phonon (red region). (b) Another electron is drawn by the phonon forming a Cooper pair [127].

In general, electrons are not attracted to each other. The weak bond between two electrons in a Cooper pair is a phonon which constitutes a coupling between two electrons known as electron-electron (e-e) interaction depicted by a Feynman diagram as shown in Figure 2.6.

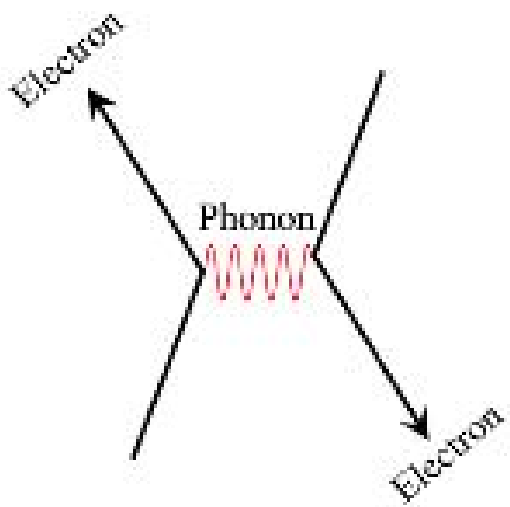

Figure 2.6: A Feynman diagram showing the e-e interaction of a Cooper pair.

Consider an example where the energy of the phonon bond of the two electrons in a Cooper pair is approximately $10^{-3} \mathrm{eV}$, therefore thermodynamic energy can easily break the bond of a Cooper pair when the temperature of the conductor is too high $\left(T>T_{C}\right)$. According to the formula of energy $E=k_{B} T$ where $k_{B} \approx 8.62 \times 10^{-5} \mathrm{eV} / \mathrm{K}$ is Boltzmann's constant, if the thermodynamic energy in the conductor should not be greater 
than $10^{-3} \mathrm{eV}$ in order to keep the bonding of Cooper pairs, then the temperature of the conductor must be equal to or below $11.6 \mathrm{~K}$. Thus, Cooper pairs can be formed at any temperature, but they can only continue to exist when the thermodynamic energy is below the pair breaking value. This occurs when the thermal energy is below $11.6 \mathrm{~K}$ in this work $[127-129]$.

\subsubsection{Microscopic Theory of Superconductivity}

In quantum mechanics, electrons are treated as wave-particles and the planewave product function of the two-electron system was described by L. N. Cooper as:

$$
\psi\left(\vec{k}_{1}, \vec{k}_{2} ; \vec{r}_{1}, \vec{r}_{2}\right)=\frac{1}{\Omega} e^{i\left(\vec{k}_{1} \cdot \vec{r}_{1}+\vec{k}_{2} \cdot \vec{r}_{2}\right)}
$$

where $\vec{k}_{1}$ and $\vec{k}_{2}$ are the states that electrons occupy in the wavevector space $(k-$ space) which $\vec{k}_{1}$ is assumed to have a spin up and $\vec{k}_{2}$ is assumed to have a spin down, $\vec{r}_{1}$ and $\vec{r}_{2}$ are the coordinate of electron one and electron two, and $\Omega$ is a box of volume $[110-112$, 130].

This planewave function can be written in the center-of-mass and relative-motion coordinates as:

$$
\psi(\vec{K}, \vec{k} ; \vec{R}, \vec{r})=\frac{1}{\Omega} e^{i(\vec{K} \cdot \vec{R}+\vec{k} \cdot \vec{r})}
$$

where $\vec{R}=\frac{1}{2}\left(\vec{r}_{1}+\vec{r}_{2}\right)$ is the location of the center of mass, $\vec{r}=\vec{r}_{1}-\vec{r}_{2}$ is the relative electron-position coordinate, $\vec{K}=\left(\vec{k}_{1}+\vec{k}_{2}\right)$ is the momentum of the center of mass, $\vec{k}=$ $\frac{1}{2}\left(\vec{k}_{1}-\vec{k}_{2}\right)$ is the difference of the momenta $[111,130]$.

And the Hamiltonian of this two-electron system is:

$$
\mathcal{H}=n_{k} \varepsilon_{k}+V_{k k^{\prime}}
$$


where $\varepsilon_{k}=\frac{\hbar^{2}}{2 m} \vec{k}^{2}=\frac{\hbar^{2}}{2 m}\left(k_{x}^{2}+k_{y}^{2}+k_{z}^{2}\right)=\frac{\hbar^{2} k^{2}}{2 m}$ is the free electron energy in $k$-space, $V_{k k^{\prime}}$ is the matrix elements of the electron interaction potential, and $n_{k}$ is the number of electrons in $k$-space in the case of a two-electron system it can be consider as $n_{k}=2$.

Without interactions of electrons, the term $V_{k k^{\prime}}$ is negligible and the Hamiltonian of each electron is:

$$
\mathcal{H}_{0}=\varepsilon_{k}=\frac{\hbar^{2}}{2 m} \vec{k}^{2}=-\frac{\hbar^{2}}{2 m} \nabla^{2}
$$

and the Eigenvalue equation is given by:

$$
\mathcal{H}_{0} \psi_{k}=\varepsilon_{k} \psi_{k}
$$

where $\psi_{k}$ represents the planewave function in $k$-space.

When electrons interact with each other in a two-electron system, the Hamiltonian becomes:

$$
\mathcal{H}_{0}\left(\vec{r}_{1}, \vec{r}_{2}\right)=-\frac{\hbar^{2}}{2 m} \nabla_{1}^{2}-\frac{\hbar^{2}}{2 m} \nabla_{2}^{2}+V_{k k^{\prime}}
$$

where the matrix elements of the electron interaction potential, $V$, is:

$$
V_{k k^{\prime}}=\left\langle\vec{k}_{\uparrow}^{\prime},-\vec{k}_{\downarrow}^{\prime}\left|V\left(\vec{r}_{1}-\vec{r}_{2}\right)\right| \vec{k}_{\uparrow},-\vec{k}_{\downarrow}\right\rangle
$$

which represents the scattering by $V\left(\vec{r}_{1}-\vec{r}_{2}\right)$ of the pair state occupying $\left|\vec{k}_{\uparrow},-\vec{k}_{\downarrow}\right\rangle$ into the other pair state $\left|\vec{k}_{\uparrow}^{\prime},-\vec{k}_{\downarrow}^{\prime}\right\rangle$ where $\vec{k}_{\uparrow}$ and represents $\vec{k}_{1}$ before emitting a phonon and $-\vec{k}_{\downarrow}$ represents $\vec{k}_{2}$ before absorbing a phonon.

In the e-e interaction, the electron of the wavevector $\vec{k}_{\uparrow}$ emits a phonon, $\gamma$, and the electron of the wavevector $-\vec{k}_{\downarrow}$ absorbs it which results new wavevectors $\vec{k}_{\uparrow}^{\prime}=\vec{k}_{\uparrow}-\gamma$ and $-\vec{k}_{\downarrow}^{\prime}=-\vec{k}_{\downarrow}+\gamma$ as shown in Figure 2.7. 


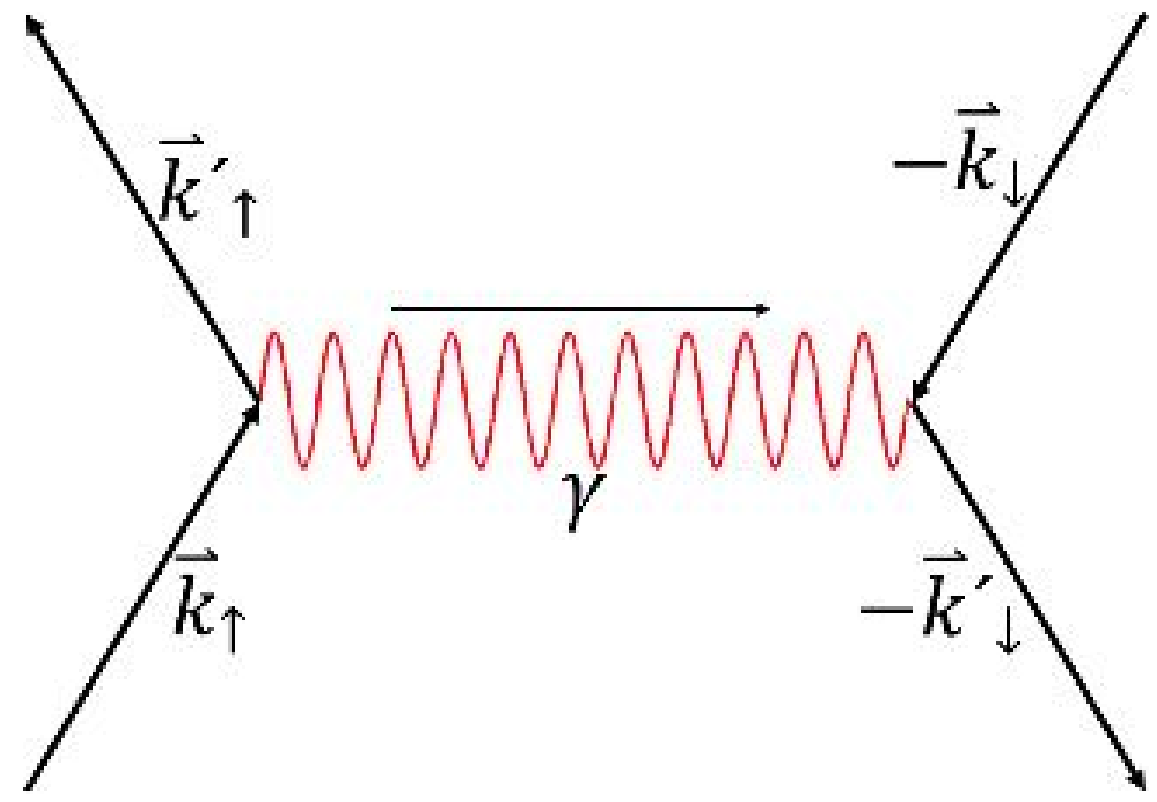

Figure 2.7: A diagram of the e-e interaction.

The simplification of the BCS theory is done by approximating:

$$
V_{k k^{\prime}}=\left\{\begin{array}{cl}
-V & \text { if } \varepsilon_{F}<\varepsilon_{k} \text { and } \varepsilon_{k^{\prime}}<\left(\varepsilon_{F}+\hbar \omega_{D}\right) \\
0 & \text { otherwise }
\end{array}\right.
$$

where $\varepsilon_{F}$ is the Fermi energy, $\hbar \omega_{D}$ is the energy of the highest energy phonon, known as the Debye energy, and $\varepsilon_{k}=\frac{\hbar^{2} k^{2}}{2 m}$ and $\varepsilon_{k^{\prime}}=\frac{\hbar^{2} k^{\prime 2}}{2 m}$ are the free electron energies in $k$-space of the states $k$ and $k^{\prime}$ respectively [110-112].

At energies below the Fermi level, all the states are occupied. Above the cutoff energy, a Cooper pair cannot be formed. Therefore, the range from $\varepsilon_{F}$ to $\left(\varepsilon_{F}+\hbar \omega_{D}\right)$ is the energy range of states available for the electron delocalization of a Cooper pair [112].

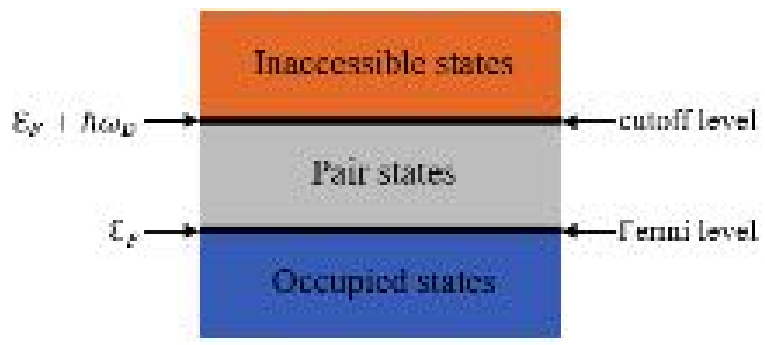

Figure 2.8: Pair states accessible to delocalization of a Cooper pair. 
The total summation of $V_{k k^{\prime}}$ with the coefficient $h_{k^{\prime}}$ is equal to:

$$
\sum_{\vec{k}_{\uparrow}^{\prime}} V_{k k^{\prime}} h_{k^{\prime}}=\left(E_{\text {Eigen }}-2 \varepsilon_{k}\right) h_{k}
$$

where $E_{\text {Eigen }}$ is the energy Eigenvalue of the electrons in the e-e interaction, and $h_{k}$ and $h_{k^{\prime}}$ are the probabilities that the states $k$ and $k^{\prime}$ are occupied respectively $[110-112,131]$.

Since $V_{k k^{\prime}}=-V$ where $\varepsilon_{F}<\varepsilon_{k}$ and $\varepsilon_{k^{\prime}}<\left(\varepsilon_{F}+\hbar \omega_{D}\right)$, then:

$$
-V \sum_{\vec{k}_{\uparrow}^{\prime}} h_{k^{\prime}}=\left(E_{\text {Eigen }}-2 \varepsilon_{k}\right) h_{k}
$$

The coefficient $h_{k}$ can be calculate as:

$$
\begin{gathered}
h_{k}=\left(-V \sum_{\vec{k}_{\uparrow}^{\prime}} h_{k^{\prime}}\right) /\left(E_{\text {Eigen }}-2 \varepsilon_{k}\right) \\
h_{k}=-V \sum_{\vec{k}_{\uparrow}^{\prime}} \frac{h_{k^{\prime}}}{\left(E_{\text {Eigen }}-2 \varepsilon_{k}\right)}
\end{gathered}
$$

The total wavefunction must be antisymmetric with respect to the exchange state of the two electrons. If the spin part is antisymmetric, then the spatial part must be symmetric [132]. Therefore, the probabilities that states $k$ and $k^{\prime}$ are occupied must be, by definition, equal, hence:

$$
h_{k} \equiv h_{k^{\prime}}
$$

The relation can then be derived to be:

$$
\begin{gathered}
-\frac{1}{V}=\sum_{\vec{k}_{\uparrow}^{\prime}} \frac{1}{\left(E_{\text {Eigen }}-2 \varepsilon_{k}\right)} \\
\frac{1}{V}=\sum_{\vec{k}_{\uparrow}^{\prime}} \frac{1}{\left(2 \varepsilon_{k}-E_{\text {Eigen }}\right)}
\end{gathered}
$$


The summation over $\vec{k}_{\uparrow}^{\prime}$ can be substituted by the integral over total energy with respect to the kinetic energy of a free electron, $\varepsilon$, when $V \neq 0$ :

$$
\frac{1}{V}=N(0) \int_{0}^{\hbar \omega_{D}} \frac{d \varepsilon}{2 \varepsilon-E_{\text {Eigen }}}
$$

where $N(0)$ is the density of states at the Fermi surface [131].

Since the interaction potential is not equal to zero, $V \neq 0$, only within the range of the Fermi level, $\varepsilon_{F}$, to the cutoff level, $\varepsilon_{F}+\hbar \omega_{D}$, the range of the integration can be:

$$
\frac{1}{V}=N\left(\varepsilon_{F}\right) \int_{\varepsilon_{F}}^{\varepsilon_{F}+\hbar \omega_{D}} \frac{d \varepsilon}{2 \varepsilon-E_{\text {Eigen }}}
$$

which results:

$$
\frac{1}{V}=\frac{1}{2} N\left(\varepsilon_{F}\right) \ln \left(\frac{2 \varepsilon_{F}-E_{\text {Eigen }}+2 \hbar \omega_{D}}{2 \varepsilon_{F}-E_{\text {Eigen }}}\right)
$$

where $N\left(\varepsilon_{F}\right)$ is the density of states above the Fermi level that has an almost constant value.

If the interaction between the electrons is weak, it will result a weak electron interaction potential and the electron coupling limit will be $V N\left(\varepsilon_{F}\right) \ll 1$. Thus, the energy Eigenvalue of the electrons in the e-e interaction can approximately be:

$$
E_{\text {Eigen }}=2 \varepsilon_{F}-2 \hbar \omega_{D} e^{-2 / V N\left(\varepsilon_{F}\right)}
$$

and the binding energy of the Cooper pair can be calculated as:

$$
\begin{gathered}
E_{\text {Eigen }}-2 \varepsilon_{F}=-2 \hbar \omega_{D} e^{-2 / V N\left(\varepsilon_{F}\right)} \\
2 \varepsilon_{F}-E_{\text {Eigen }}=2 \hbar \omega_{D} e^{-2 / V N\left(\varepsilon_{F}\right)}
\end{gathered}
$$


where $2 \hbar \omega_{D} e^{-2 / V N\left(\varepsilon_{F}\right)}=\varepsilon_{b}$ is the binding energy which can be used to approximate the average distance between the two electrons in a Cooper pair, $\delta$, as:

$$
\delta=\frac{\hbar v_{F}}{\varepsilon_{b}}
$$

where $v_{F}$ is the Fermi velocity [112].

At the absolute zero, $T=0$, the excitation energy of electrons is:

$$
E_{k}=\sqrt{\Delta_{k}^{2}+\left(\varepsilon_{k}-\varepsilon_{F}\right)^{2}}
$$

where $\Delta_{k}$ is the superconducting energy gap in the state $k$ which can be obtained by:

$$
\Delta_{k}=-\sum_{\vec{k}^{\prime} \uparrow} V_{k k^{\prime}} v_{k^{\prime}} u_{k^{\prime}}=V \sum_{\vec{k}^{\prime} \uparrow} v_{k^{\prime}} u_{k^{\prime}}
$$

where $v_{k^{\prime}}$ and $u_{k^{\prime}}$ are the probabilities of occupancy and vacancy in the state $k^{\prime}$ and the probabilities of occupancy and vacancy in the state $k$ are denoted by $v_{k}$ and $u_{k}$ respectively.

The relationship between the probabilities of occupancies and vacancies in states $k$ and $k^{\prime}$ can be defined as:

$$
\begin{gathered}
v_{k}^{2}+u_{k}^{2}=1 \\
\text { and } \\
v_{k^{\prime}}^{2}+u_{k^{\prime}}^{2}=1
\end{gathered}
$$

and the value of $\Delta_{k}$ can be assumed that:

$$
\Delta_{k}= \begin{cases}\Delta & \text { if }\left|\varepsilon_{k}-\varepsilon_{F}\right|<\hbar \omega_{D} \\ 0 & \text { otherwise }\end{cases}
$$

where the superconducting energy gap, $\Delta$, can be obtained from the following: 


$$
\begin{gathered}
\frac{2}{V}=N(0) \int_{-\hbar \omega_{D}}^{\hbar \omega_{D}} \frac{d\left(\varepsilon_{k}-\varepsilon_{F}\right)}{\sqrt{\Delta^{2}+\left(\varepsilon_{k}-\varepsilon_{F}\right)^{2}}} \\
\Delta=\frac{\hbar \omega_{D}}{\sinh \left(\frac{1}{V N(0)}\right)}=2 \hbar \omega_{D} e^{-1 / V N(0)}
\end{gathered}
$$

The superconducting energy gap approaches zero when $T \rightarrow T_{c}$, therefore the temperature-dependent superconducting energy gap, $\Delta(T)$, can be derived from:

$$
\begin{gathered}
\frac{2}{V}=N(0) \int_{-\hbar \omega_{D}}^{\hbar \omega_{D}} \frac{d\left(\varepsilon_{k}-\varepsilon_{F}\right)}{\sqrt{\Delta^{2}(T)+\left(\varepsilon_{k}-\varepsilon_{F}\right)^{2}}} \tanh \left(\frac{\sqrt{\left(\varepsilon_{k}-\varepsilon_{F}\right)^{2}+\Delta^{2}(T)}}{2 k_{B} T}\right) \\
\Delta(T) \approx 3.2 k_{B} T_{c}\left(1-\frac{T}{T_{c}}\right)^{1 / 2}
\end{gathered}
$$

when $T$ is approaching $T_{c}$ [111].

When $T=T_{c}$, the $\Delta(T)$ will be equal to zero which is:

$$
\begin{gathered}
\frac{1}{V}=N(0) \int_{0}^{\hbar \omega_{D}} \frac{d\left(\varepsilon_{k}-\varepsilon_{F}\right)}{\left(\varepsilon_{k}-\varepsilon_{F}\right)} \tanh \left(\frac{\left(\varepsilon_{k}-\varepsilon_{F}\right)}{2 k_{B} T_{c}}\right) \\
\frac{1}{V N(0)}=\ln \left(\frac{1.14 \hbar \omega_{D}}{k_{B} T_{c}}\right) \\
k_{B} T_{C}=1.14 \hbar \omega_{D} e^{-1 / V N(0)}
\end{gathered}
$$

The normalized energy gap, $\Delta(0)$, can be determined by:

$$
\begin{gathered}
\frac{\Delta}{k_{B} T_{C}}=\frac{\left(2 \hbar \omega_{D} e^{-1 / V N(0)}\right)}{\left(1.14 \hbar \omega_{D} e^{-1 / V N(0)}\right)}=\frac{2}{1.14} \approx 1.754 \\
\Delta=1.754 k_{B} T_{c}=\Delta(0)
\end{gathered}
$$

where $k_{B} \approx 8.62 \times 10^{-5} \mathrm{eV} / \mathrm{K}$ is the Boltzmann's constant. 
According to the BCS theory, the $\Delta$ is specific to a superconductor and it will remain zero in normal states above the critical temperature of the superconductor. The graph in Figure 2.9 shows the relationship between the temperature dependence of reduced energy gap, $\frac{\Delta(T)}{\Delta(0)}$, and the reduced temperature, $\frac{T}{T_{c}}$, in the BCS theory, and it is a valid approximation in most cases of superconductors $[110,168,169]$.

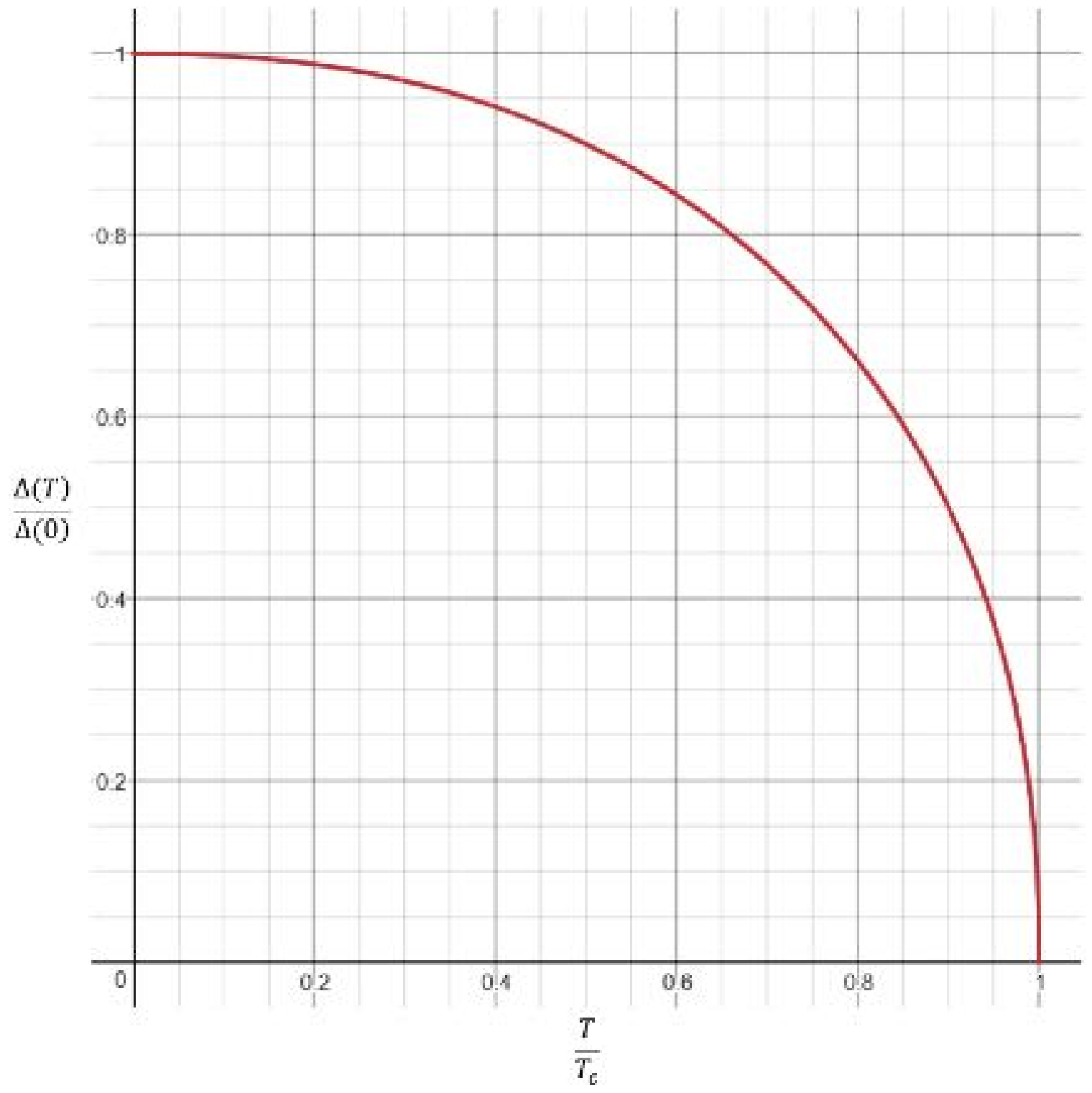

Figure 2.9: The characteristic of the temperature-dependent energy gap. 


\subsection{Types of Superconductors}

Superconductors can be classified into two types depending on their behavior in an external magnetic field. The difference between type I and type II superconductors are described in the sections 2.4.1. and 2.4.2 respectively.

\subsubsection{Type I Superconductors}

In type I superconductors, the superconductivity abruptly vanishes when the applied magnetic field, $H$, is greater than the critical magnetic field, $H_{c}$. In Figure 2.9, the graph of the relationship between $H_{c}$ and $T_{c}$ shows the superconductivity of type I superconductors is in the region under $H_{c}$ and $T_{c}$ only.

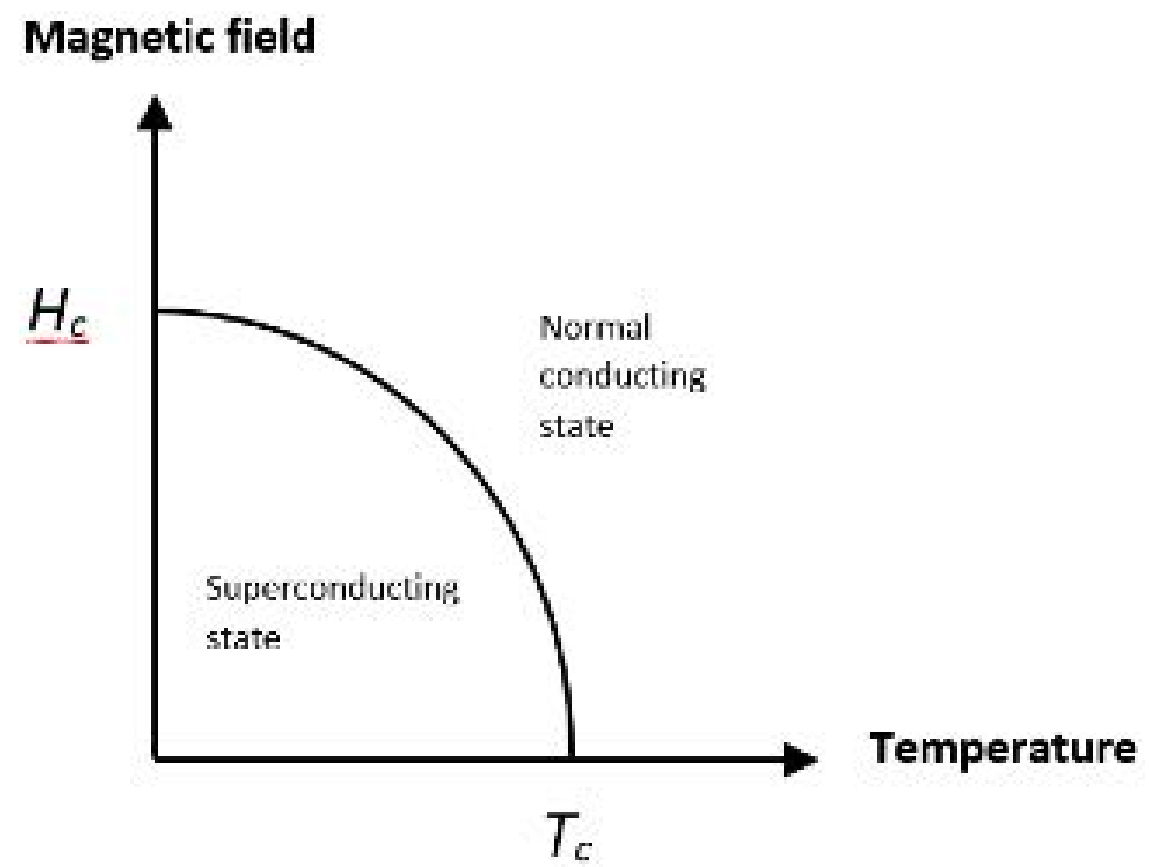

Figure 2.10: The relationship between $H_{c}$ and $T_{c}$ of type I superconductors.

From section 2.2, when a material is in its superconducting state, it is also a perfect diamagnetic material which completely repels the magnetic field penetrating through it [111]. In general, the relationship between magnetic flux density, $B$, and the applied magnetic field, $H$, is defined by: 


$$
B=\mu_{0}(H+M)
$$

for a magnetizing magnetic field, and:

$$
B=\mu_{0} H
$$

for a demagnetizing magnetic field $[112,135]$ where $\mu_{0}$ is the magnetic permeability in free space, and $M$ is a magnetic polarization which is commonly known as a magnetization.

Because of the diamagnetic characteristic, a superconductor prevents a weak magnetic field from penetrating it. This implies that $B$ is equal to zero inside the bulk of a superconducting material which leads to the relation:

$$
M=-H
$$

From the relationship between $M$ and $H$ in the magnetic susceptibility equation $M=\chi H$, therefore $\chi=-1$, where $\chi$ is a magnetic susceptibility. The graph of the relationship of the $M$ and $H$ of type I superconductors is shown in Figure 2.11 where the slope represents $\chi=-1$.

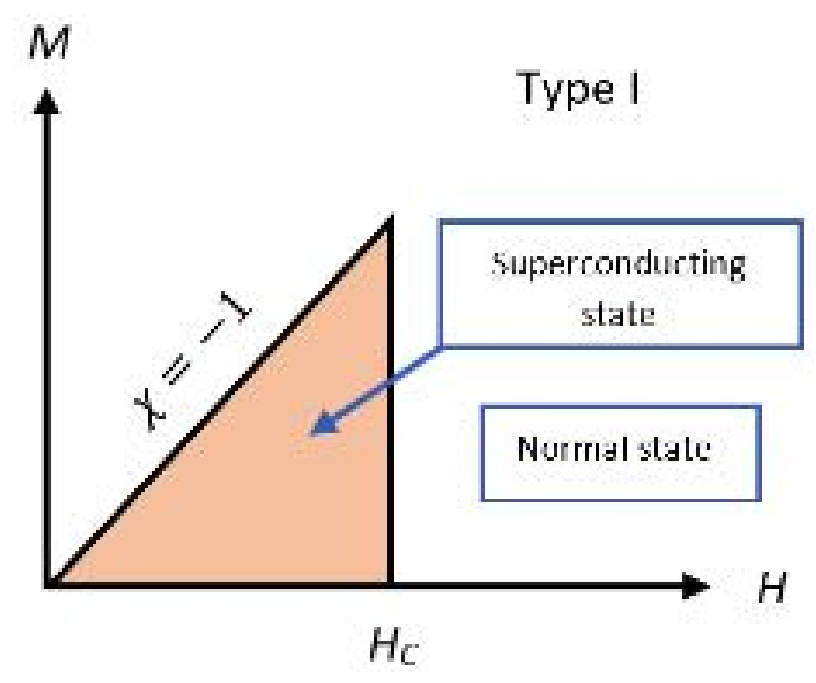

Figure 2.11: The relationship of the $M$ and $H$ of type I superconductors. 
The GL parameter, $\kappa$, can be used to determine the type of a superconductor where $\kappa=\lambda_{L} / \xi_{G L}$ which $\lambda_{L}$ is the London penetration depth and $\xi_{G L}$ is the GL coherence length. If $\kappa<\frac{1}{\sqrt{2}}$, the superconductor is a type I superconductor [110 - 114].

\subsubsection{Type II Superconductors}

Superconductors that have different magnetic behavior from type I superconductors are categorized as type II superconductors. Type II superconductors will not abruptly lose their superconductivity when the applied magnetic field is higher than the critical field, but they will gradually lose their superconductivity when the magnetic field increases until they completely lose their superconductivity when they reach their normal conducting states. The state that type II superconductors gradually lose their superconductivity is called the "mixed state" between the normal conducting and superconducting states. This is also called the "semi-superconducting state".

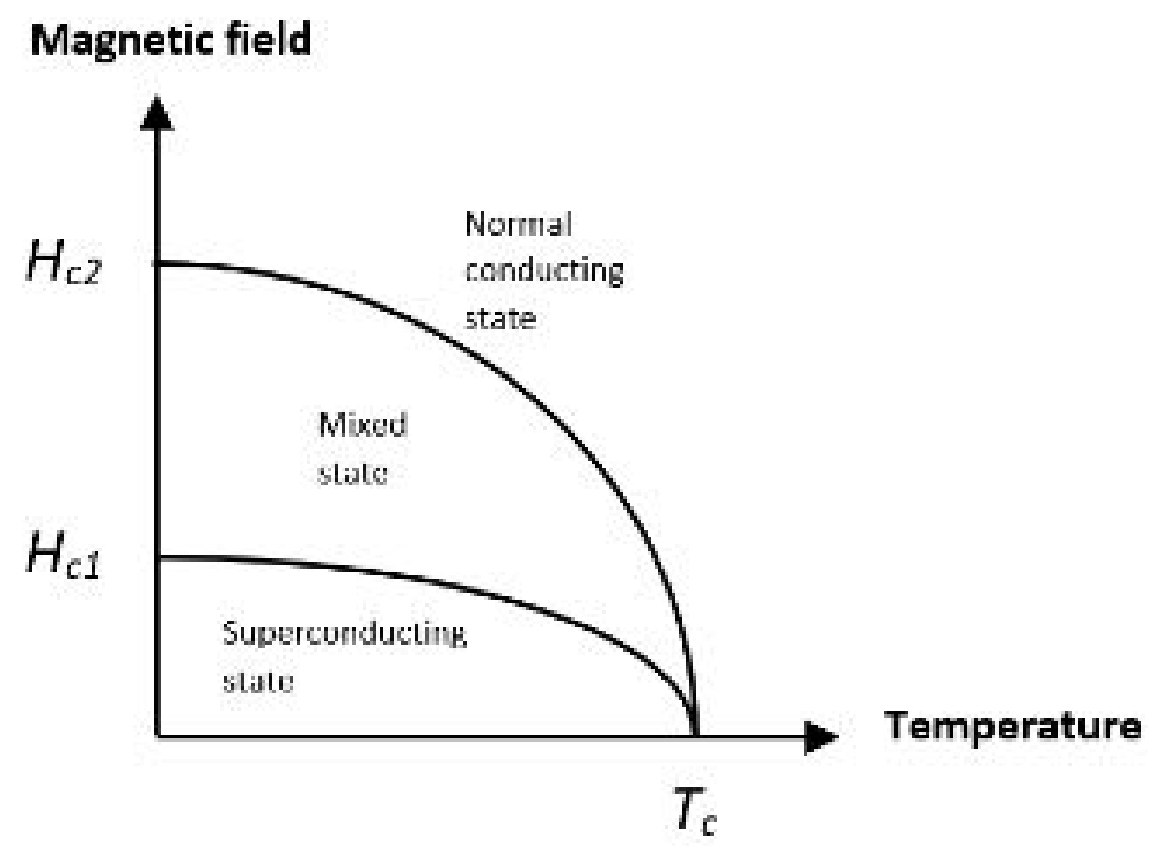

Figure 2.12. The phase diagram of type II superconductors. 
As shown in Figure 2.12, type II superconductors start to lose their superconductivity at the lower critical magnetic field, $H_{c 1}$, and completely lose their superconductivity at the upper critical magnetic field, $H_{c 2}$.

When the applied magnetic field of a type II superconductor is less than its lower critical magnetic field, $H<H_{c 1}$, the type II superconductor will have the same characteristic of a type I superconductor. When the applied magnetic field, $H$, of a type II superconductor is less than the lower critical magnetic field, $H_{c 1}$, the material will be perfectly diamagnetic that yields $\chi=-1$ which gives $M=-H$ and $B=0$.

When $H_{c 1}<H<H_{c 2}$, the material will be in the semi-superconducting state and remains diamagnetic $(\chi<0)$ but is no longer perfectly so $|\chi|<1$. When $H_{c 2}<H$, the material becomes normal [112] as shown in Figure 2.13.

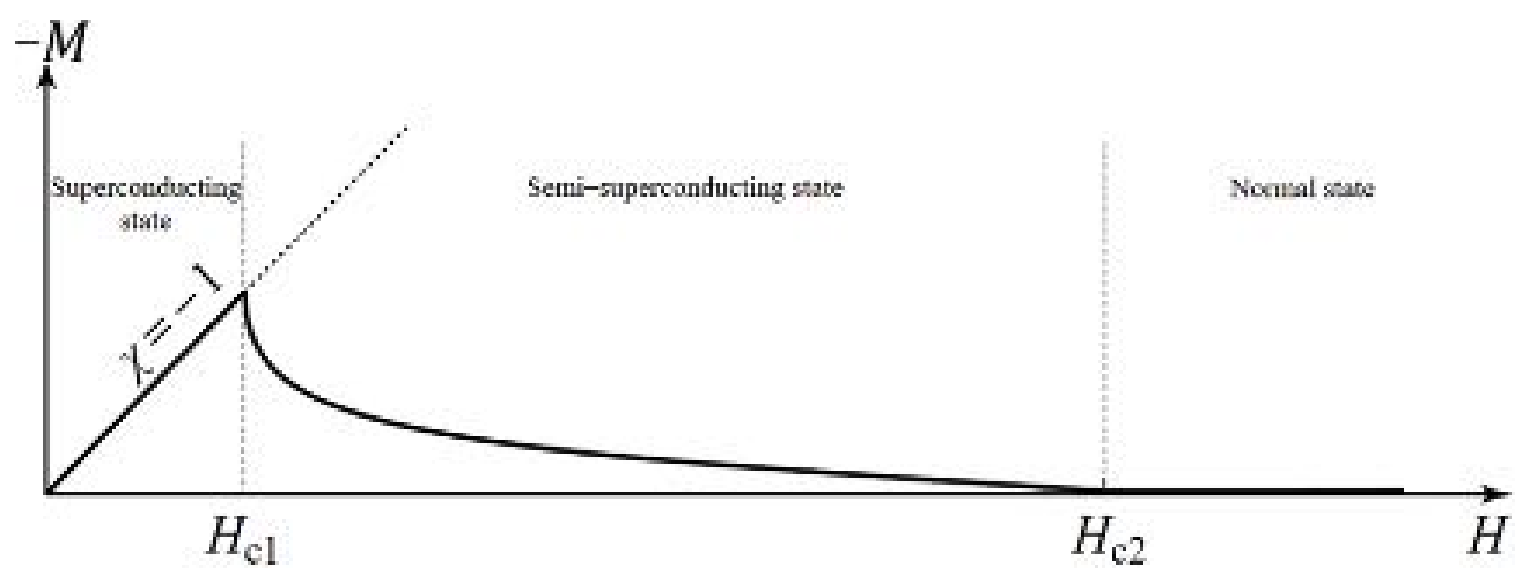

Figure 2.13: The relationship of the $M$ and $H$ of type I superconductors [112].

The mixed state or semi-superconducting state of type II superconductors also known as "Shubnikov phase" which was named after L. V. Shubnikov a Soviet physicist who experimentally discovered the phenomenon of type II superconductivity [149 - 151]. Unfortunately, Shubnikov was accused of an attempt to organize an "anti-Soviet strike", 
arrested, and executed by the KGB when he was only 36 years of age. This was considered a great loss to Physics.

A. A. Abrikosov, one of the 2003 Nobel Laureates in Physics, stated that in the Shubnikov phase, magnetic fields do not fully penetrate through a superconductor. However, they penetrate the superconductor in narrow columns and each column holds a quantum of magnetic flux with a supercurrent surrounding it. Each column where magnetic fields penetrate through has a supercurrent that flows around it like a whirlpool. The columns of magnetic fields in type II superconductors, which supercurrents flow around, are called "Abrikosov vortices". [152].

By applying the description of the numbers of electrons in a superconductor, $n_{s}=|\psi|^{2}$, from the GL theory into the second London equation, $\nabla x j_{s}$, the supercurrent density, $j_{s}$, will be:

$$
\begin{gathered}
\nabla \times j_{s}=-\frac{1}{\mu_{0} \lambda_{L}^{2}} B=-\frac{n_{s} e^{2}}{m c} B \\
\nabla \times j_{s}=-\frac{|\psi|^{2} e^{2}}{m c} B=-\frac{|\psi|^{2} e^{2}}{m c} \nabla \times A \\
j_{s}=-\frac{|\psi|^{2} e^{2}}{m c} A
\end{gathered}
$$

When the phase of wave function, $\theta$, is taken into account, the pseudo-wave function order parameter will be:

$$
\psi=|\psi| e^{i \theta}
$$

and the supercurrent density will be:

$$
j_{s}=\frac{\hbar e}{m}|\psi|^{2}\left(\nabla \theta-\frac{2 e}{\hbar c} A\right)
$$


In the polar coordinate system, the wave function can be converted to be as follows:

$$
|\psi| e^{i \theta}=r e^{i \varphi}
$$

where $r$ is the amplitude of wave function on the radial coordinate in the polar coordinate system and $\varphi$ is the phase of wave function on the angular coordinate in the polar coordinate system.

In the vicinity of $\psi=0, \theta=\varphi$, and $\nabla \theta$ has only a $\varphi$-component which is equal to:

$$
\frac{1}{r} \cdot \frac{\partial \theta}{\partial \varphi}=\frac{1}{r}
$$

Hence, it is much larger than the second term in the supercurrent density equation, and the current forms a vortex [152].

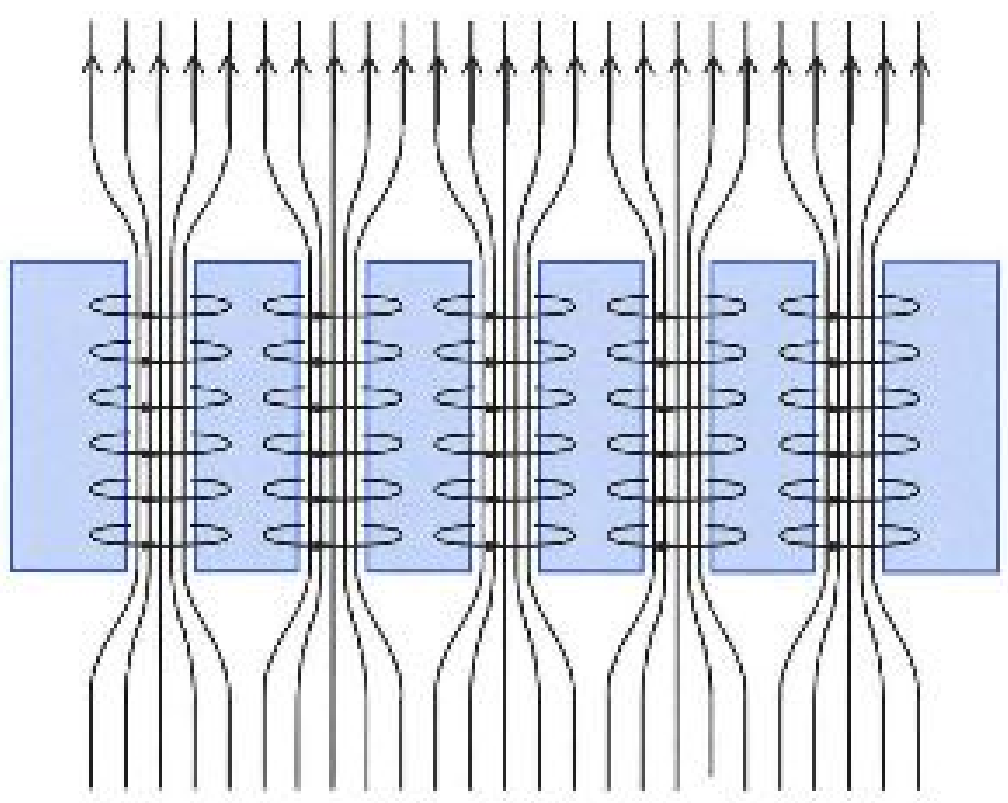

Figure 2.14: Magnetic flux lines penetrating a type II superconductor. The currents in the superconducting material generate a magnetic field which, together with the applied field, result in bundles of quantized flux [153]. 

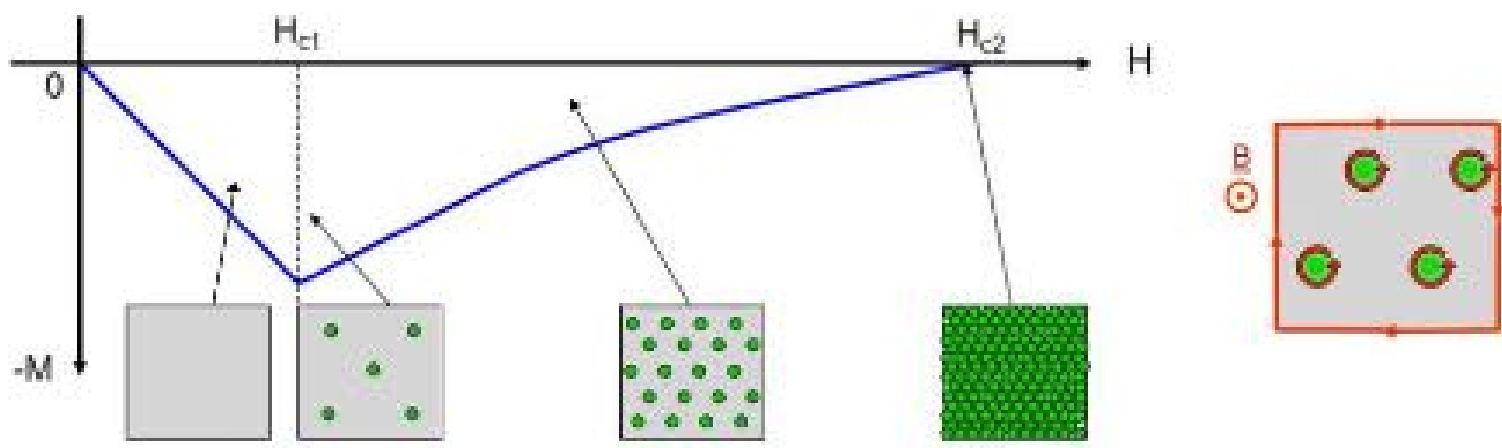

Figure 2.15: The vortices of type II superconductors appear in the Shubnikov phase where the flux within each vortex is generated by a supercurrent [154].

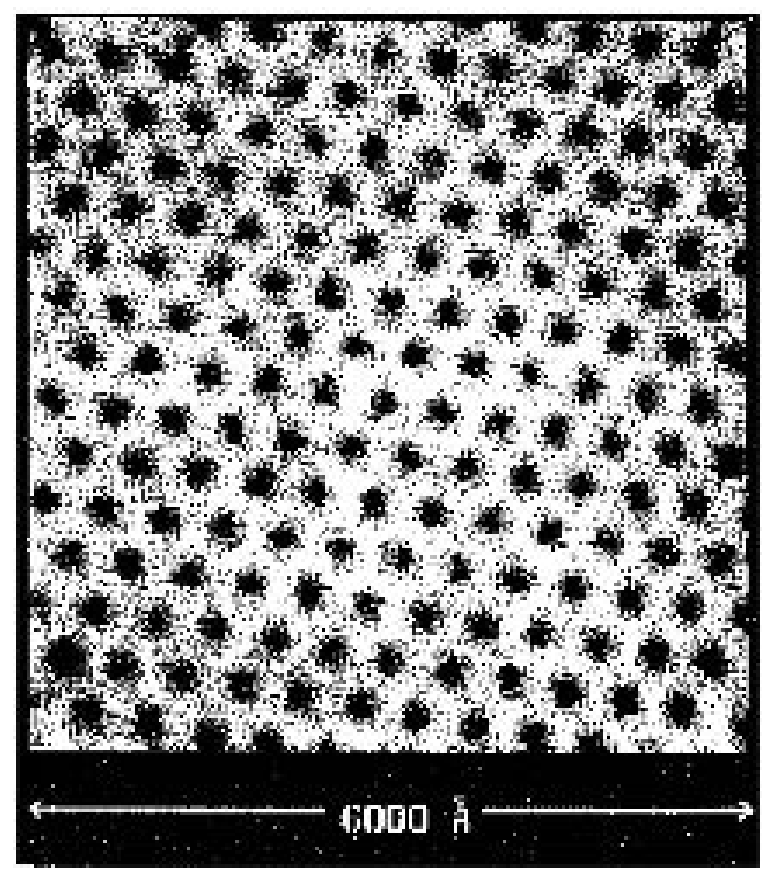

Figure 2.16: Vortices in $\mathrm{NbSe}_{2}$ defined by scanning tunneling microscopy [152].

The GL parameter, $\kappa$, of a type II superconductor is greater than $\frac{1}{\sqrt{2}}$ and, as a result, the surface kinetic energy density of the superconductor is negative [152].

\subsection{Josephson Effect}

The Josephson effect was proposed by B. D. Josephson, one of the 1973 Nobel Laureates in Physics. The Josephson effect states that the supercurrent can flow through two superconductors separated by a thin weak link which is usually measured in nanometer 
scale without any applied voltage. When two superconductors are separated by a weak link, this creates region of lowered order parameter in the weak link and a junction between the superconductors called the "Josephson Junction" is formed. Tunneling of the electron-pairs through the weak link called "Josephson Tunneling" [136].

The weak link between the two superconductors may be an insulator which forms a superconductor-insulator-superconductor (S-I-S) junction, a normal conductor which makes a superconductor-normal conductor-superconductor (S-N-S) junction, or a constricted superconductor which forms a superconductor-constriction-superconductor (Sc-S) junction.

The wavefunctions of superconductor 1 and superconductor 2 can be written as:

$$
\Psi_{1}=\sqrt{n_{1}} e^{i \theta_{1}} \text { and } \Psi_{2}=\sqrt{n_{2}} e^{i \theta_{2}}
$$

where $n_{1}, n_{2}$ are the densities of Cooper pairs and $\theta_{1}, \theta_{2}$ are the phases in superconductor 1 and superconductor 2 , respectively.

\begin{tabular}{|c|c|c|}
\hline Superconductor 1 & Weak link & Superconductor 2 \\
\hline$\Psi_{1}$ & $\Psi_{2}$
\end{tabular}

Figure 2.17: Two superconductors separated by a thin weak link.

The dynamic time evolution of the two wavefunctions of the superconductors on each side of a coupled Josephson junction can be determined by the following Schrödinger equations:

$$
\begin{aligned}
& i \hbar \frac{\partial \Psi_{1}}{\partial t}=U_{1} \Psi_{1}+K \Psi_{2} \\
& i \hbar \frac{\partial \Psi_{2}}{\partial t}=U_{2} \Psi_{2}+K \Psi_{1}
\end{aligned}
$$


where $U_{1}, U_{2}$ are energies of the wavefunctions in superconductor 1 and superconductor 2 and $K$ is a coupling constant.

Substituting $\Psi_{1}$ and $\Psi_{2}$ with $\sqrt{n_{1}} e^{i \theta_{1}}$ and $\sqrt{n_{2}} e^{i \theta_{2}}$ into the Schrödinger equations of the dynamic time evolution of the wavefunctions gives:

$$
\begin{gathered}
\hbar \frac{\partial n_{1}}{\partial t}=2 K \sqrt{n_{1} n_{2}} \sin \emptyset \\
\hbar \frac{\partial n_{2}}{\partial t}=-2 K \sqrt{n_{1} n_{2}} \sin \emptyset \\
\hbar \frac{\partial \theta_{1}}{\partial t}=-K \sqrt{\frac{n_{2}}{n_{1}}} \cos \emptyset+\frac{e}{2 \hbar} V_{\text {source }} \\
\hbar \frac{\partial \theta_{2}}{\partial t}=-K \sqrt{\frac{n_{1}}{n_{2}}} \cos \emptyset-\frac{e}{2 \hbar} V_{\text {source }}
\end{gathered}
$$

where $\varnothing$ is the phase difference which equals to $\theta_{2}-\theta_{1}$, and $V_{\text {source }}$ is the applied voltage that can affect the energy levels of the wavefunctions as:

$$
V_{\text {source }}=\frac{U_{2}-U_{1}}{2 e}
$$

this equation yields:

$$
(2 e) V_{\text {source }}=U_{2}-U_{1}=\hbar \frac{\partial \emptyset}{\partial t}
$$

The time derivative of the density of Cooper pairs describes a charge transport, thus the supercurrent density, $j_{s}$, can be determined by:

$$
\frac{\partial n_{1}}{\partial t}=-\frac{\partial n_{2}}{\partial t}=j_{S}
$$

Therefore, the supercurrent density equation can be derived as:

$$
j_{s}=j_{c} \sin \emptyset
$$


where $j_{C}=\frac{2 K \sqrt{n_{1} n_{2}}}{\hbar}$ is the critical current density [110 - 112].

\subsubsection{Direct Current Josephson Effect}

When an applied direct current (DC), $I_{D C}$, is smaller than the critical current, $I_{c}$, it can pass through the Josephson junction without any voltage drop across the junction as in the equation:

$$
I_{D C}=I_{S}=I_{C} \sin \emptyset
$$

where $I_{S}$ and $I_{C}$ are the supercurrent and critical current which are equal to the supercurrent density, $j_{s}$, and critical current density, $j_{c}$, divided by the cross-sectional area of the superconductors.

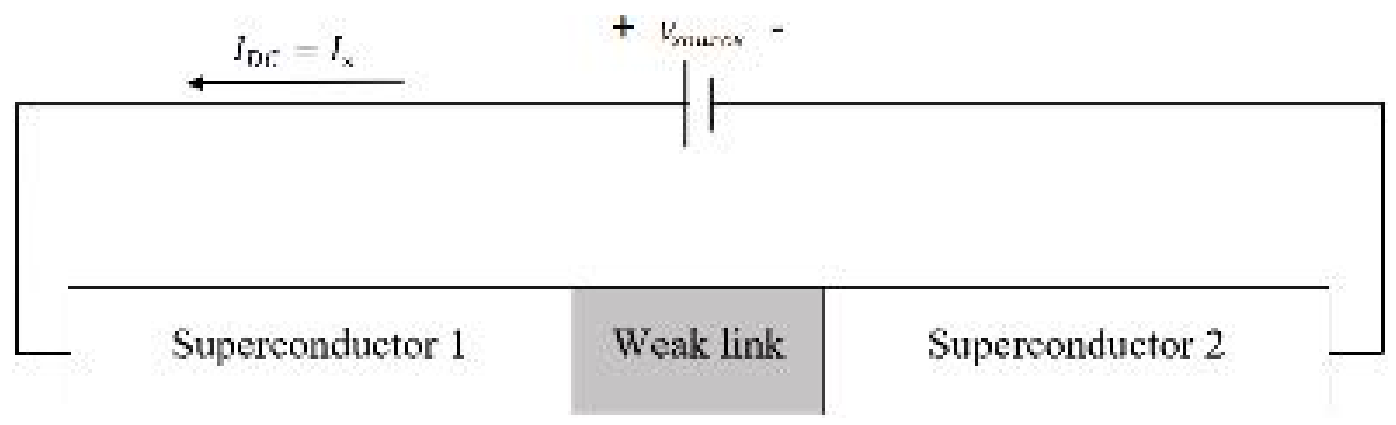

Figure 2.18: A voltage is applied to a Josephson junction.

The phase difference can be calculated as:

$$
\emptyset=\sin ^{-1}\left(\frac{I_{s}}{I_{c}}\right)
$$

When an applied DC current is greater than $I_{c}$, a voltage drop across the Josephson junction appears $[111,112]$. 


\subsubsection{Alternating Current Josephson Effect}

When there is a voltage drop across the junction, the constant DC current will start to oscillate due to the potential differences in the two superconductors and the phase difference will be defined according to the time evolution as:

$$
\varnothing(t)=\varnothing(0)+\frac{(2 e) V_{\text {source }}}{\hbar} t
$$

and the alternating current $(\mathrm{AC})$ will be:

$$
I_{A C}(t)=I_{C} \sin \left(\varnothing(0)+\omega_{J} t\right)
$$

This equation shows that:

$$
\omega_{J}=\frac{(2 e) V_{\text {source }}}{\hbar}
$$

which yields:

$$
f_{J}=\frac{(2 e) V_{\text {source }}}{2 \pi \hbar}
$$

where $\omega_{J}$ is the Josephson angular velocity and $f_{J}$ is the Josephson frequency $[111,112]$.

\subsection{Superconductivity in Graphene}

Since the exfoliation of single-layer graphene (SLG) was achieved in 2004, superconductivity in intrinsic graphene has not been observed. The superconductivity in graphene is experimentally induced by placing graphene on a superconductor or modifying properties of graphene by adding other chemicals into the atomic structure of graphene [137 - 139]. For example, placing graphene on a superconductor was done by growing SLG on a 30-nanometer rhenium (Re) thin-film via chemical vapor deposition (CVD). The result of the experiment shows that the critical temperature, $T_{c}$, on the graphene layer is approximately equal to $2.1 \mathrm{~K}$ [139]. 
Intercalating graphene with alkali metal ions such as lithium (Li) or alkaline earth metal ions such as calcium $(\mathrm{Ca})$ shows the superconductivity in the intercalated graphene which can be explained by the electron-phonon coupling enhancement that arises from the presence of an intercalant-derived band as well as graphitic $\pi$-bands $[144,145]$ at the Fermi level [140 - 143]. In the intercalated graphene where a bulk of graphite is inserted with Li or $\mathrm{Ca}$ ions then exfoliated as graphene shows that the $T_{c}$ was recorded at $11.5 \mathrm{~K}$ for $\mathrm{Ca}$ intercalated bilayer (BLG) graphene and at $7.4 \mathrm{~K}$ in Li-intercalated few-layer (FLG) graphene [146, 147].

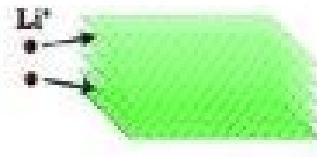

Graphite

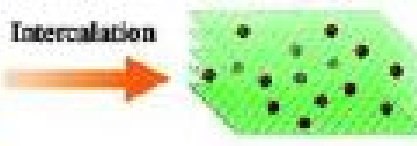

$u_{x} C$,
Expansien

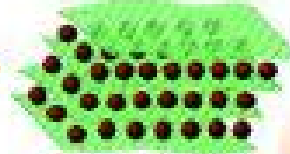

Expanded Graphite

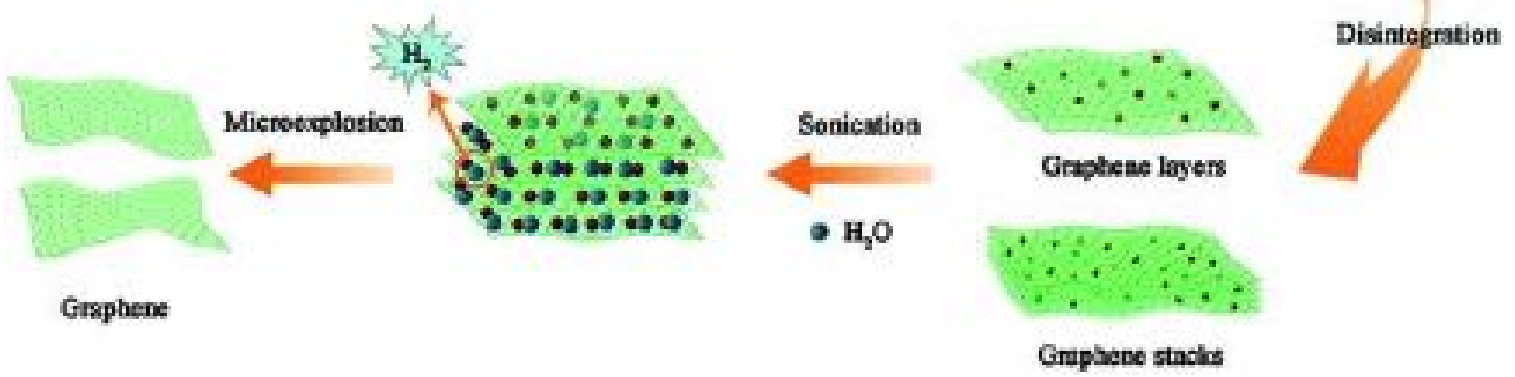

Figure 2.19: The process to obtain layers of Li-intercalated graphene [148].

Implanting graphene with phosphorous $(\mathrm{P})$ exhibited deviations from the expected rise in resistance $(R)$ as the temperature $(T)$ is reduced to some point above $100 \mathrm{~K}$. The relatively large drop in resistance at lower temperatures was also considered a possible indication of superconductivity in the sample. Figure 2.20 shows that phosphorusimplanted and then argon-implanted graphene led to the discovery of a strong connection between resistance and temperature [159-161]. 


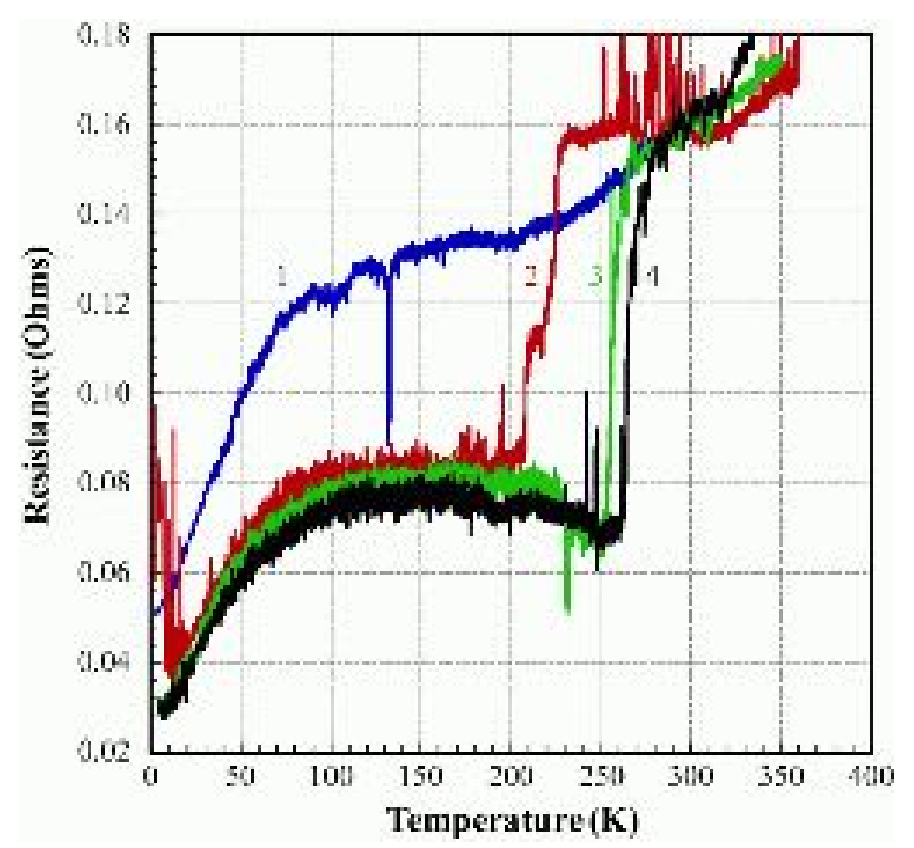

Figure 2.20: $R v s . T$ of a thin-film graphene sample peeled off from phosphorusimplanted and then argon-implanted bulk HOPG sample \# 023. Curves 1, 2, 3, and 4 are four identical sequential runs with the same probe position [56, 57].

The apparent superconductivity in phosphorus-doped graphene and HOPG can be quenched by a magnetic field (as shown in Figure 2.21 and 2.22 below).

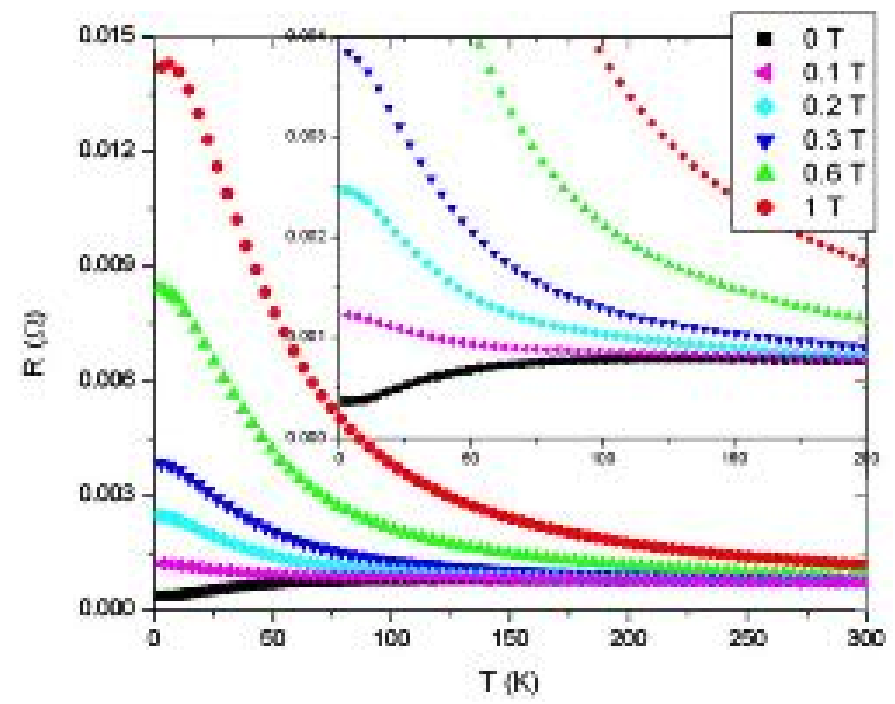

Figure 2.21: $R$ vs. $T$ measurement of phosphorus-doped HOPG samples \# 005 in the presence of magnetic field from 0 to $1 \mathrm{~T}$. (These tests were performed at the University of Maryland by Dr. Paul Bach) [55]. 


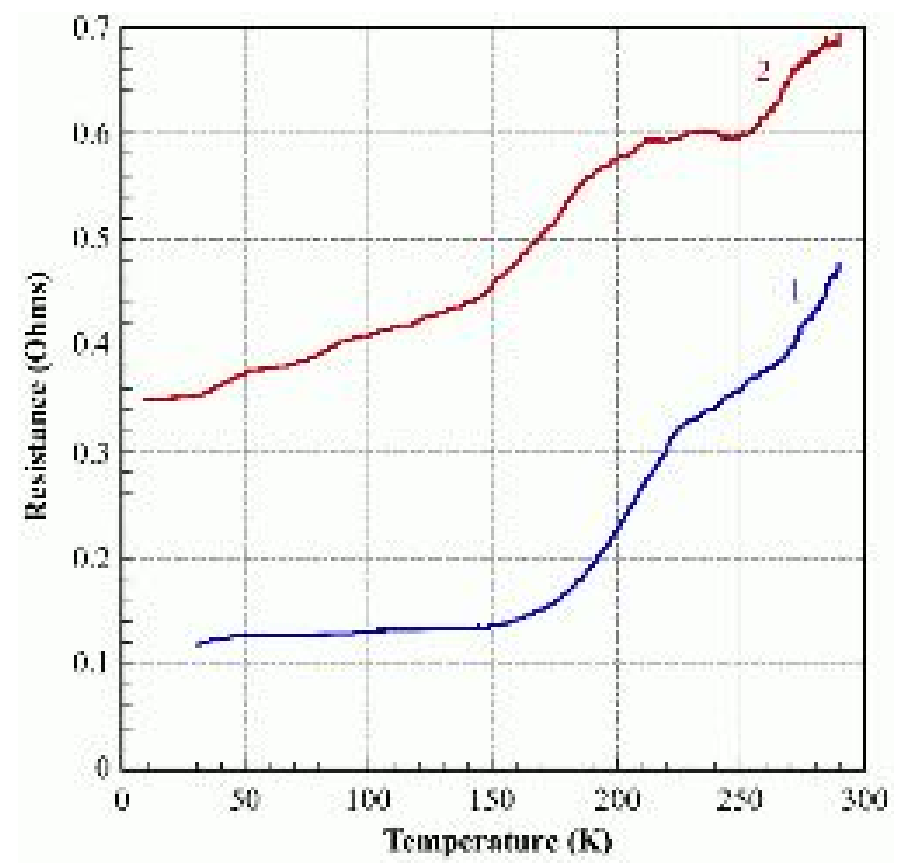

Figure 2.22: $R$ vs. $T$ of thin film exfoliated from phosphorus-doped HOPG.

Curve 1 was measured without an applied magnetic field.

Curve 2 was measured under an applied magnetic field with the field strength of 0.035 tesla $[56,57]$.

The doped-while-grown graphene was prepared in a CVD system using plasmaenhanced CVD on HOPG substrates. The HOPG was being used as a seed crystal, as it was easier to remove the doped-while-grown graphene layers from the HOPG via a mechanical exfoliation than to remove graphene layers from a copper foil sheet. [55 - 57].

\subsection{Berezinskii-Kosterlitz-Thouless Transition}

In 2016, the Nobel Prize in Physics was awarded to D. J. Thouless, F. D. M. Haldane, and J. M. Kosterlitz for their theoretical discoveries of topological phase transitions and topological phases of matter $[155,156]$. The Berezinskii-KosterlitzThouless (BKT) transition is an unconventional phase transition in 2-dimensional (2D) materials occurring through topological defects in materials such as point-defects or 
vortices (or antivortices). In 1972, the work of D. J. Thouless, and J. M. Kosterlitz [157] shows that at:

$$
k_{B} T_{c} \approx \frac{1}{2} q^{2}
$$

a phase transition to a conducting state of material begins to occur where $k_{B}$ is the Boltzmann's constant, $T_{c}$ is the critical temperature corresponding to a phase transition into a superconducting state, and $q$ is the charge of particles.

The critical temperature at which a single dislocation (a vortex) is likely to occur is at which the free energy, $F_{v}$, change sign. For the $x y$-model (2D model), it gives

$$
k_{B} T_{c}=\frac{\pi \hbar^{2} \rho}{2 m_{e f f}}=\pi J
$$

where $J$ is the spin-spin coupling constant which $\hbar$ is the reduced Planck's constant, $\rho$ is the density of particles or superfluid per unit area, and $m_{e f f}$ is the effective atomic mass of particles which is not necessarily the same as the atomic mass for a particle moving on a substrate $[157,158]$.

\subsubsection{Topological Defects and Phase Transitions}

In the BKT transition, free energy, $F_{v}$, associates with each vortex core as a vortex

core is a region where the pseudo-wave function order parameter, $\psi=\sqrt{\rho} e^{i \theta}$, of the thin film superconductor or superfluid (fluid helium that reaches its property of zero viscosity) surface vanishes which causes a finite free energy to exist. Each vortex corresponds to a hole in the 2D material (thin film or superfluid surface) and the free energy of a single vortex is 


$$
F_{v}=E_{v}-T S_{v}=\pi J \ln \left(\frac{L}{a}\right)-T k_{B} \ln \left(\frac{L^{2}}{a^{2}}\right)
$$

where $E_{v}=\pi J \ln \left(\frac{L}{a}\right)$ is the vortex energy, $S_{v}=k_{B} \ln \left(\frac{L^{2}}{a^{2}}\right)$ is the entropy, $L$ is the finite system size, and $a$ is the material lattice spacing. Thus, a vortex can be considered as a topological defect [156 - 158].

At the BKT characteristic transition temperature, $T_{B K T}=\frac{\pi J}{2 k_{B}}$, the vortex energy, $E_{v}$, balances the entropy, $S_{v}$, which is considered as a topological phase transition of the vortices. At $T>T_{B K T}$, the vortices are free to move, and the material will be electrical conducting, while at $T<T_{B K T}$, the vortices will bound in pairs of zero vorticity and the material will be electrical insulating.

The interaction energy between vortices at under $T_{B K T}$ can be calculate as

$$
E_{\text {pair }}=2 \pi J \ln \left(\frac{d}{a}\right)
$$

where $d$ is the distance between two vortices of a vortex pair $[156,157]$.

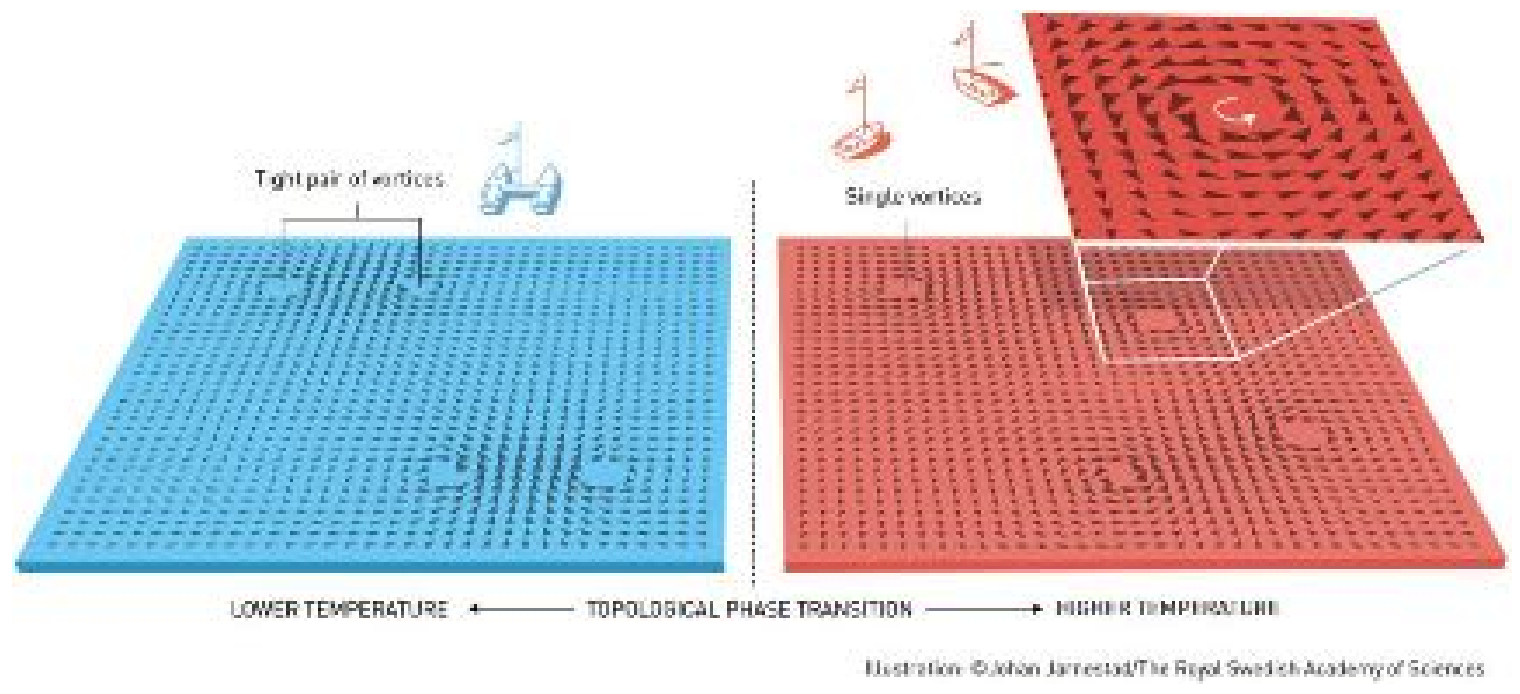

Figure 2.23: An illustration of the topological phase transition of the vortices. Image courtesy of J. Jarnestad / The Royal Swedish Academy of Sciences. 
The work of Mironov et al. [163] shows that the phase transition of a material depends on $T$ and $B$. The $T_{B K T}$ of a niobium titanium nitride $(N b T i N)$ thin film (10nm) starts to emerge at $T \approx 0.2 \mathrm{~K}$ for $B=0.12 T$. However, in a higher $B$, the phase transition will emerge at a higher $T$ as shown in In Figure 2.24.

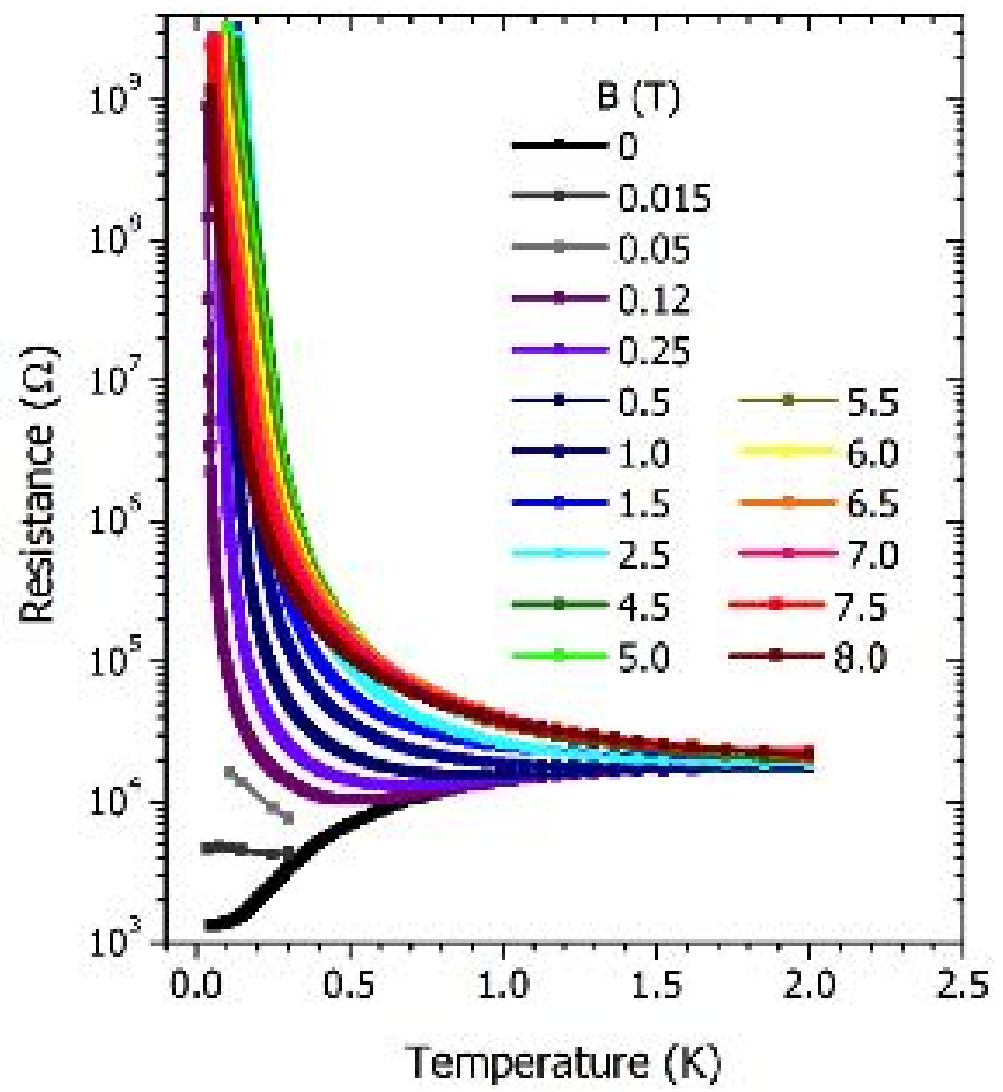

Figure 2.24: $R$ (in the $\log$ scale) $v s . T$ at different magnetic fields listed in the legend in panel [163].

The works of A. M. Goldman et al. show that the thickness of material also has an impact on its phase transition [165 - 167]. 


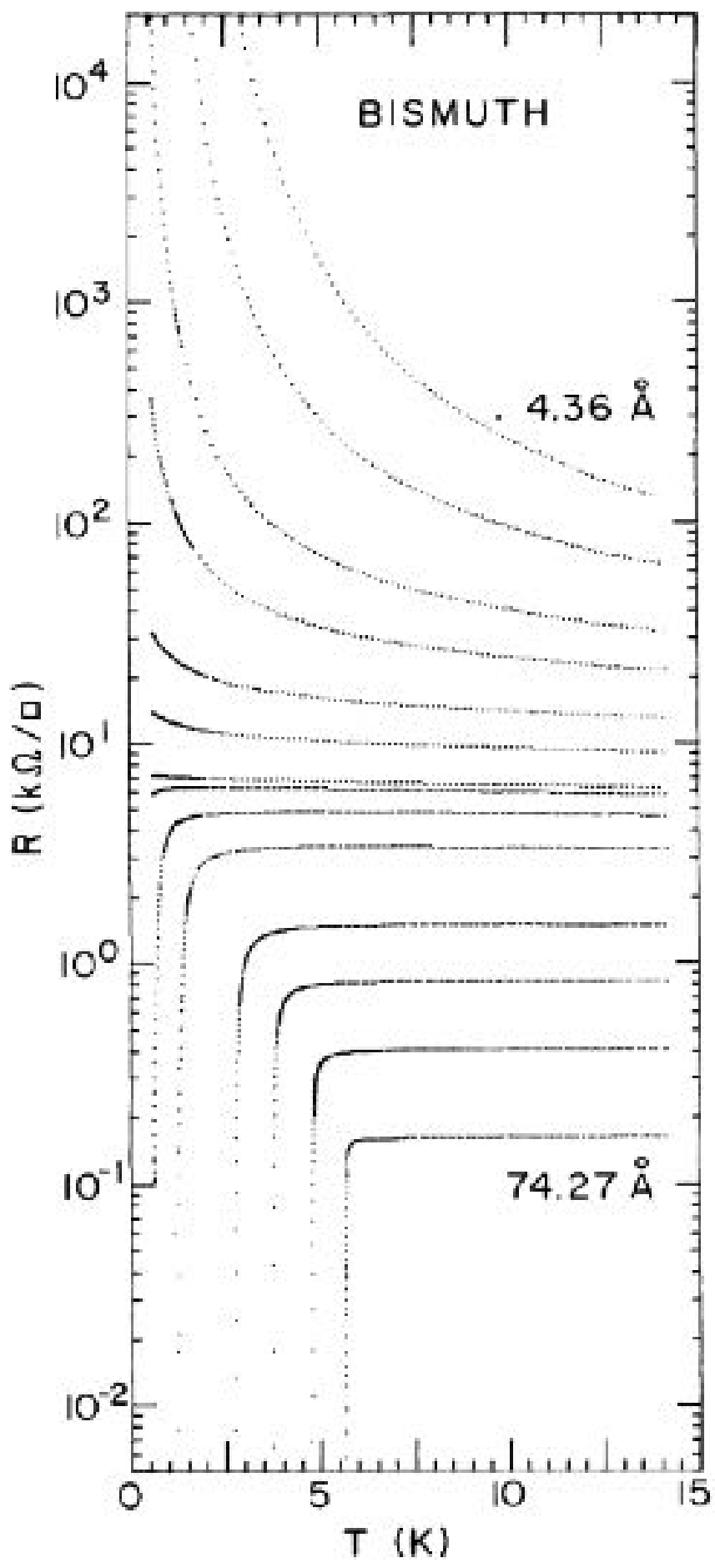

Figure 2.25: Evolution of the temperature dependence of the sheet resistance $R(T)$ with thickness for a $B i$ film deposited onto $G e$. Reprinted with permission from David Haviland [166]. 


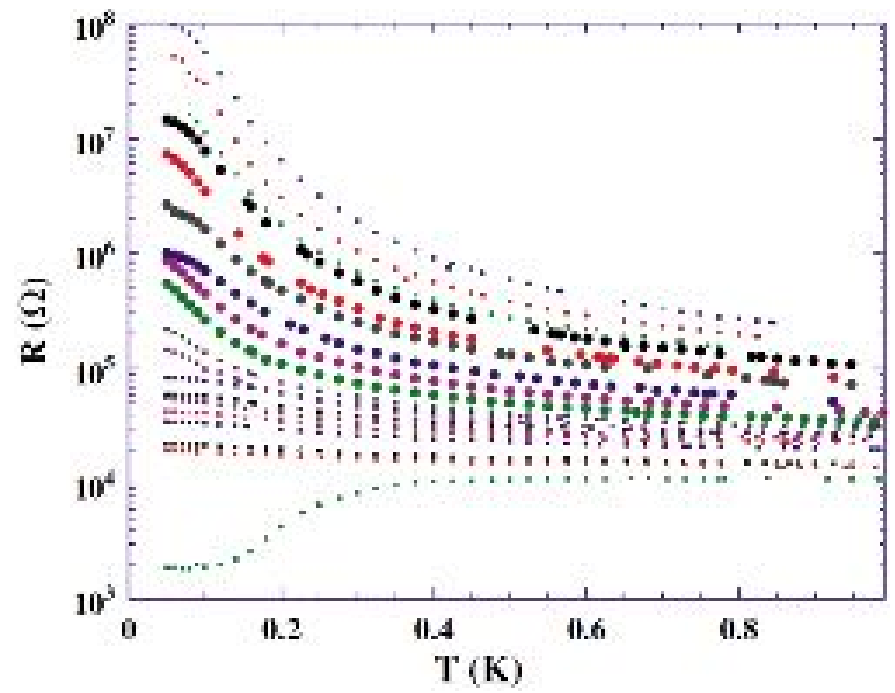

Figure 2.26: Sheet $R$ vs. $T$ of amorphous $B i$ flims for a series of films with thicknesses, from top to bottom, of 11.15, 11.25, 11.37, 11.38, 11.43, 11.48, 11.55, $11.65,11.75,11.85,11.95,12.03,12.17,12.27,12.4,12.55,12.65,12.85$, and $13.35 \AA$. Reprinted with permission from Allen M. Goldman , Regents Professor, University of Minnesota [167].

Thus, the phase transition of a thin material under the influence of an applied magnetic field can be analyzed as at below $T_{B K T}$, the material is in an insulating phase where the vortices are bound together, but at above $T_{B K T}$, the material is in conducting state where the vortices are free to move [164].

\subsubsection{Topological Insulators}

A topological insulator is a material which will not conduct current through the bulk of the material but will carry current along the surface. In a 2D material, if a certain magnetic flux density penetrates through it, charges would localize in closed orbital circles (trapped in loops). While charges are trapped in loops, the material cannot conduct electricity. However, around the edges of the material, charges can travel in open orbits without any losses of energy. While on the edges of a topological insulator conducts 
electricity, but in the middle of the material is electrically insulated is called a "quantum Hall state" of a 2D material. [159-162].

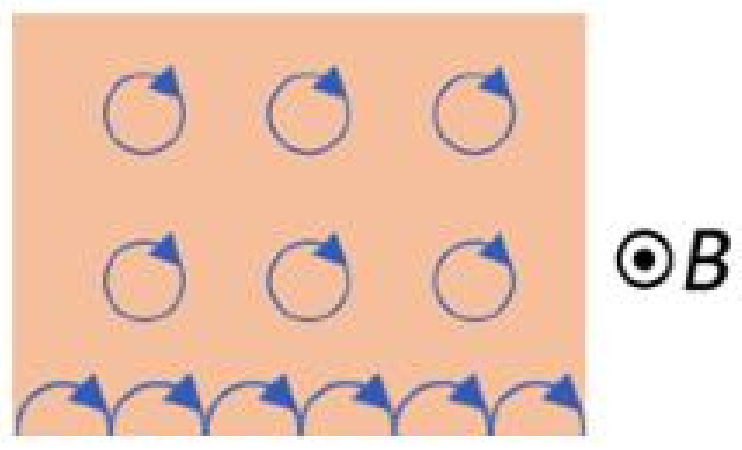

Figure 2.27: A 2D quantum Hall state.

\subsection{Tables of Symbols and Acronyms}

Table 2.1: Table of Symbols

\begin{tabular}{|c|c|c|}
\hline Symbols & Description & Value \\
\hline$T$ & Temperature. & - \\
\hline$T_{c}$ & Critical temperature. & - \\
\hline$H$ & An applied magnetic field. & - \\
\hline$H_{c}$ & Critical magnetic field. & - \\
\hline $\mathrm{N}$ & Magnetic north pole. & - \\
\hline $\mathrm{S}$ & Magnetic south pole. & - \\
\hline$j_{n}$ & Normal current density. & - \\
\hline$j_{S}$ & Supercurrent density. & - \\
\hline$\sigma_{n}$ & Normal electrical conductivity. & - \\
\hline$n_{n}$ & $\begin{array}{l}\text { The number of free electrons per unit volume of } \\
\text { a normal conductor. }\end{array}$ & - \\
\hline$\tau$ & $\begin{array}{l}\text { The mean free time between electron collision } \\
\text { which is also known as relaxation time. }\end{array}$ & - \\
\hline$e$ & The elementary electric charge. & $\approx 1.602 \times 10^{-19} \mathrm{C}$ \\
\hline$m$ & The mass of an electron. & $\approx 9.109 \times 10^{-31} \mathrm{~kg}$ \\
\hline$E$ & An electric field. & - \\
\hline
\end{tabular}




\begin{tabular}{|c|c|c|}
\hline$v_{n}$ & The velocity of electrons in a normal conductor. & - \\
\hline$n_{s}$ & $\begin{array}{l}\text { The number of free electrons per unit volume of } \\
\text { a superconductor. }\end{array}$ & - \\
\hline$v_{S}$ & The velocity of electrons in a superconductor. & - \\
\hline$t$ & Time. & - \\
\hline$\nabla$ & The differential operation. & $\hat{\imath} \frac{\partial}{\partial x}+\hat{\jmath} \frac{\partial}{\partial y}+\hat{k} \frac{\partial}{\partial z}$ \\
\hline$B$ & Magnetic flux density. & - \\
\hline$B_{c}$ & Critical magnetic flux density. & - \\
\hline$\mu_{0}$ & The magnetic permeability in free space. & $4 \pi \times 10^{-7} \mathrm{H} / \mathrm{m}$ \\
\hline$\lambda_{L}$ & London penetration depth. & - \\
\hline$c$ & The speed of light measured in a vacuum. & $\approx 3 \times 10^{8} \mathrm{~m} / \mathrm{s}$ \\
\hline$\psi$ & A pseudo-wave function order parameter. & - \\
\hline$f_{S}$ & $\begin{array}{l}\text { The free energy density of a material in a } \\
\text { superconducting state. }\end{array}$ & - \\
\hline$f_{n}$ & $\begin{array}{l}\text { The free energy density of a material in a normal } \\
\text { state. }\end{array}$ & - \\
\hline$f$ & Total free energy density. & - \\
\hline$f_{\min }$ & Minimum free energy density. & - \\
\hline$\alpha$ & $\begin{array}{l}\text { The coefficient of superconducting pair density } \\
\text { which is proportional to }|\psi|^{2} \text {. }\end{array}$ & - \\
\hline$\beta$ & $\begin{array}{l}\text { The coefficient of pair interaction term which is } \\
\text { proportional to }|\psi|^{4} \text {. }\end{array}$ & - \\
\hline$A$ & Magnetic vector potential of $B$. & - \\
\hline$h$ & The Planck's constant. & $\begin{array}{c}\approx 6.626 \times 10^{-34} \\
\mathrm{~m}^{2} \mathrm{~kg} / \mathrm{s}\end{array}$ \\
\hline$\hbar$ & The reduced Planck's constant. & $\approx 6.582 \times 10^{-16} \mathrm{eV} \cdot \mathrm{s}$ \\
\hline$x$ & A certain length in space. & - \\
\hline$\psi_{\infty}$ & $\begin{array}{l}\text { Pseudo-wave function in a superconductor which } \\
\text { approaches an infinite value. }\end{array}$ & - \\
\hline$\xi_{G L}$ & The GL coherence length. & - \\
\hline$\kappa$ & The GL parameter. & - \\
\hline
\end{tabular}




\begin{tabular}{|c|c|c|}
\hline$k_{B}$ & The Boltzmann's constant. & $\approx 8.62 \times 10^{-5} \mathrm{eV} / \mathrm{K}$ \\
\hline $\overrightarrow{k_{1}}$ & $\begin{array}{l}\text { The states that spin-up electrons occupy in the } \\
\text { wavevector space. }\end{array}$ & - \\
\hline$\vec{k}_{2}$ & $\begin{array}{l}\text { The states that spin-down electrons occupy in the } \\
\text { wavevector space. }\end{array}$ & - \\
\hline$\vec{r}_{1}$ & The coordinate of electron one. & - \\
\hline$\vec{r}_{2}$ & The coordinate of electron two. & - \\
\hline$\Omega$ & A box of volume. & - \\
\hline$\vec{K}$ & The momentum of the center of mass. & - \\
\hline$\vec{k}$ & The difference of the momenta. & - \\
\hline$\vec{R}$ & The location of the center of mass. & - \\
\hline$\vec{r}$ & The relative electron position coordinate. & - \\
\hline $\mathcal{H}$ & The Hamiltonian. & - \\
\hline$n_{k}$ & The number of electrons in $k$-space. & - \\
\hline V & The electron interaction potential. & - \\
\hline$V_{k k^{\prime}}$ & $\begin{array}{l}\text { The matrix elements of the electron interaction } \\
\text { potential. }\end{array}$ & - \\
\hline $\mathcal{H}_{0}$ & The Hamiltonian of each electron. & - \\
\hline$\vec{k}_{\uparrow}$ & $\begin{array}{l}\text { A wave vector which represents } \vec{k}_{1} \text { before } \\
\text { emitting a phonon. }\end{array}$ & - \\
\hline$-\vec{k}_{\downarrow}$ & $\begin{array}{l}\text { A wave vector which represents } \vec{k}_{2} \text { before } \\
\text { absorbing a phonon. }\end{array}$ & - \\
\hline$\vec{k}_{\uparrow}^{\prime}$ & $\begin{array}{l}\text { A new wave vector resulted from the phonon } \\
\text { emission of } \vec{k}_{1} \text {. }\end{array}$ & - \\
\hline$-\vec{k}_{\downarrow}^{\prime}$ & $\begin{array}{l}\text { A new wave vector resulted from the phonon } \\
\text { absorption of } \vec{k}_{2} \text {. }\end{array}$ & - \\
\hline$\varepsilon_{F}$ & The Fermi energy. & - \\
\hline$\hbar \omega_{D}$ & The energy of a phonon known as Debye energy. & - \\
\hline$\varepsilon_{k}$ & $\begin{array}{l}\text { The free electron energy in } k \text {-space of the states } \\
k \text {. }\end{array}$ & - \\
\hline$\varepsilon_{k^{\prime}}$ & $\begin{array}{l}\text { The free electron energy in } k \text {-space of the states } \\
k^{\prime} \text {. }\end{array}$ & - \\
\hline
\end{tabular}




\begin{tabular}{|c|c|c|}
\hline$E_{\text {Eigen }}$ & $\begin{array}{l}\text { Energy Eigenvalue of the electrons in the e-e } \\
\text { interaction. }\end{array}$ & - \\
\hline$h_{k}$ & The probability that the state $k$ is occupied. & - \\
\hline$h_{k^{\prime}}$ & The probability that the state $k^{\prime}$ is occupied. & - \\
\hline$N(0)$ & The density of states at the Fermi surface. & - \\
\hline$N\left(\varepsilon_{F}\right)$ & The density of states above the Fermi level. & - \\
\hline$\varepsilon_{b}$ & The binding energy. & - \\
\hline$\delta$ & $\begin{array}{l}\text { The average distance between the two electrons } \\
\text { in a Cooper pair. }\end{array}$ & - \\
\hline$v_{F}$ & The Fermi velocity. & - \\
\hline$\Delta_{k}$ & The superconducting energy gap in the state $k$. & - \\
\hline$v_{k}$ & The probability of occupancy in the state $k$. & - \\
\hline$v_{k^{\prime}}$ & The probability of occupancy in the state $k^{\prime}$. & - \\
\hline$u_{k}$ & The probability of vacancy in the state $k$. & - \\
\hline$u_{k^{\prime}}$ & The probability of vacancy in the state $k^{\prime}$. & - \\
\hline$\Delta$ & The superconducting energy gap & - \\
\hline$\Delta(T)$ & $\begin{array}{l}\text { The temperature-dependent superconducting } \\
\text { energy gap. }\end{array}$ & - \\
\hline$\chi$ & Magnetic susceptibility. & - \\
\hline$H_{c 1}$ & Lower critical magnetic field. & - \\
\hline$H_{c 2}$ & Upper critical magnetic field. & - \\
\hline$\Psi_{1}$ & The wavefunction of the superconductor 1. & - \\
\hline$\Psi_{2}$ & The wavefunction of the superconductor 2 . & - \\
\hline$n_{1}$ & $\begin{array}{l}\text { The density of Cooper pairs in the } \\
\text { superconductor } 1 .\end{array}$ & - \\
\hline$n_{2}$ & $\begin{array}{l}\text { The density of Cooper pairs in the } \\
\text { superconductor } 2 \text {. }\end{array}$ & - \\
\hline$\theta$ & $\begin{array}{l}\text { The phase of wave function (in the Cartesian } \\
\text { coordinate system). }\end{array}$ & - \\
\hline$r$ & $\begin{array}{l}\text { The amplitude of wave function on the radial } \\
\text { coordinate in the polar coordinate system. }\end{array}$ & - \\
\hline$\varphi$ & $\begin{array}{l}\text { The phase of wave function on the angular } \\
\text { coordinate in the polar coordinate system. }\end{array}$ & - \\
\hline
\end{tabular}




\begin{tabular}{|c|l|c|}
\hline $\mathrm{NbSe}_{2}$ & Niobium diselenide. & - \\
\hline$\theta_{1}$ & The phase in superconductor 1. & - \\
\hline$\theta_{2}$ & The phase in superconductor 2. & - \\
\hline$U_{1}$ & $\begin{array}{l}\text { The energy of the wavefunctions in the } \\
\text { superconductor 1. }\end{array}$ & - \\
\hline$U_{2}$ & $\begin{array}{l}\text { The energy of the wavefunctions in the } \\
\text { superconductor 2. }\end{array}$ & - \\
\hline$K$ & A coupling constant. & - \\
\hline$\varnothing$ & Phase difference. & - \\
\hline$V_{s o u r c e}$ & Applied voltage. & - \\
\hline$j_{c}$ & Critical current density. & - \\
\hline$I_{D C}$ & Applied direct current. & - \\
\hline$I_{S}$ & Supercurrent. & - \\
\hline$I_{C}$ & Critical current. & - \\
\hline$\omega_{J}$ & The Josephson angular velocity. & - \\
\hline$f_{J}$ & The Josephson frequency. & - \\
\hline$T_{B K T}$ & The BKT characteristic transition temperature. & - \\
\hline$J$ & The spin-spin coupling constant. & - \\
\hline$\rho$ & The density of particles per unit area. & - \\
\hline$m_{e f f}$ & Effective atomic mass. & - \\
\hline$F_{v}$ & The free energy of a single vortex. & - \\
\hline$E_{v}$ & Vortex energy. & - \\
\hline$S_{v}$ & Entropy. & - \\
\hline$E_{p a i r}$ & The interaction energy between vortices. & - \\
\hline$d$ & $\begin{array}{l}\text { The distance between two vortices of the vortex } \\
\text { pair. }\end{array}$ & - \\
\hline GbTiN & Niobium titanium nitride. & - \\
\hline$B i$ & Bismuth. & - \\
\hline${ }_{2}$ & Germanium. & - \\
\hline
\end{tabular}


Table 2.2: Table of Acronyms.

\begin{tabular}{|c|c|}
\hline Acronym & Description \\
\hline $2 \mathrm{D}$ & 2-dimensional \\
\hline $\mathrm{AC}$ & Alternating current \\
\hline BCS & Bardeen-Cooper-Schrieffer \\
\hline BKT & Berezinskii-Kosterlitz-Thouless \\
\hline BLG & Bilayer graphene \\
\hline $\mathrm{Ca}$ & Calcium \\
\hline CVD & Chemical Vapor Deposition \\
\hline DC & Direct current \\
\hline e-e & Electron-electron \\
\hline FLG & Few-layer graphene \\
\hline GL & Ginzburg-Landau \\
\hline HOPG & Highly Ordered Pyrolytic Graphite \\
\hline $\mathrm{Li}$ & Lithium \\
\hline $\mathrm{P}$ & Phosphorous \\
\hline$R$ & Resistance \\
\hline$R$ vs. $T$ & Resistance versus Temperature \\
\hline $\operatorname{Re}$ & Rhenium \\
\hline $\mathrm{RF}$ & Radio frequency \\
\hline S-c-S & Superconductor-constriction-superconductor \\
\hline S-I-S & Superconductor-insulator-superconductor \\
\hline S-N-S & Superconductor-normal conductor-superconductor \\
\hline SLG & Single-layer graphene \\
\hline$T$ & Temperature \\
\hline YBCO & Yttrium Barium Copper Oxide \\
\hline
\end{tabular}




\section{Chapter 3}

Electric Charge Carriers and Thermoelectric Effects 


\subsection{Introduction to Electric Charge Carriers}

Electric charge is a physical property of a material that causes the material to experience a force when placed in an electromagnetic field. Basically, electric charge is classified into two types: positive and negative charges. Electric charge can move from one place to another in a material via mobile particles that can carry electric charge. These particles are "electric charge carriers".

In metals, the charges are usually negative. The negative charge carriers are free electrons that have the ability to move under the influence of an electric field. The free electrons in a metal are from the valence electrons from each metal atom. These electrons can move freely within the crystal structure.

On the other hand, the absence of valence electrons in atoms creates "holes". Holes are the charge carriers that carry positive charge. Holes or electron holes commonly refer to the vacancies of electrons in the valence band of atoms which leave a net positive charge at the hole's location. In general, the electric charge of a hole is equal to the electric charge of an electron with opposite polarity.

\subsubsection{Doping}

Doping is a process to induce more available charge carriers in the material for conduction by introducing impurities into the crystal structure of a material in order to modify its conductivity. This process is often utilized in semiconductors such as silicon. The most common dopants in silicon and other tetravalent semiconductors are trivalent dopants such as boron (B), aluminum (Al), gallium (Ga), and indium (In) which have an ability to accept electrons, and pentavalent dopants such as phosphorus (P), arsenic (As), antimony $(\mathrm{Sb})$, or bismuth $(\mathrm{Bi})$ which have an ability to donate electrons. A similar 
situation exists for compound III-V and II-VI semiconductors. We will restrict our discussion to the tetravalent materials as carbon, like silicon, is tetravalent.

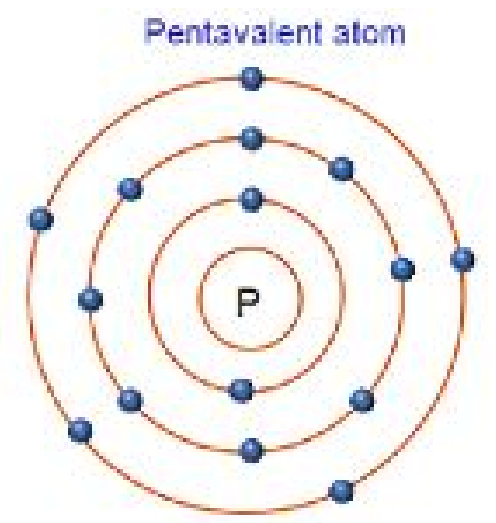

Phosphorus
Trivalent atom

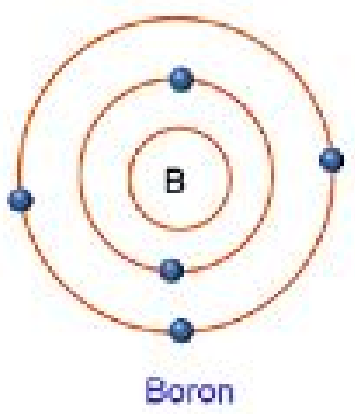

\section{Figure 3.1: Pentavalent and trivalent atoms [192].}

\subsubsection{1. p-doping}

p-doping or hole doping is the process of adding a trivalent dopant or an electron acceptor to a material. Trivalent dopants have three valence electrons in their outer shell, therefore they can catch an additional outer electron. When a trivalent dopant atom is added into an intrinsic semiconductor, it would leave a "hole" instead of the fourth electron. An intrinsic semiconductor such as Silicon $(\mathrm{Si})$ is a material that has number of excited electrons equal to number of holes [183].

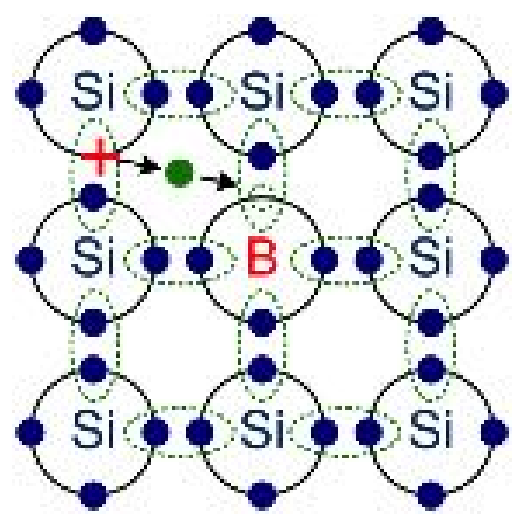

Figure 3.2: p-doping with boron [183]. 


\subsubsection{2. n-doping}

In contrast to $\mathrm{p}$-doping, $\mathrm{n}$-doping or electron doping is involves adding pentavalent impurities or electron-donor atoms to a material. Pentavalent dopants have five valence electrons in their outer shell and donate their free electron to the semiconductor [183].

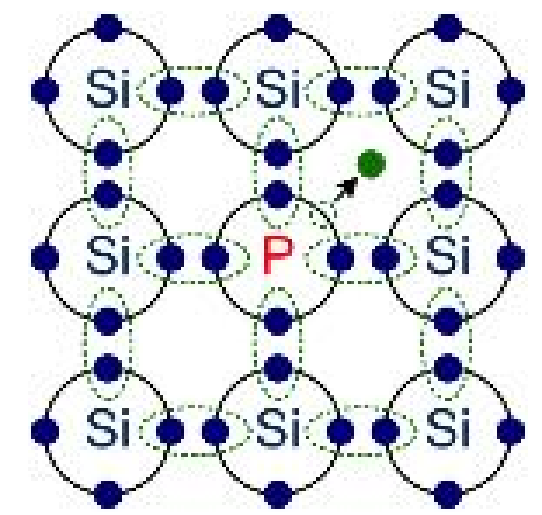

Figure 3.3: n-doping with phosphorus [183].

\subsection{Charge Carriers in Superconductors}

In conventional, Bardeen-Cooper-Schreiffer (BCS), superconductors, lattice vibrations (phonons) do not impede the motion of the electrons. Moreover, the lattice vibrations draw electrons into the regions of the phonons creating phonon mediated or linked pairs of electrons. These phonon paired electrons are known as Cooper pairs. Cooper pairs are the dominant charge carriers in conventional superconductors. In the base BCS theory superconductor a spherical pair wavefunction shell is presumed and, for many metallic superconductors this model is surprisingly accurate.

In unconventional superconductors and the newer high- $T_{c}$ superconductors, electron pairs have been shown to exist. However, the pairing does not obey the BCS theory and the pair wavefunction cannot be described by the simple spherical $s$-wave order parameter (attractive forces between pairs are isotropic in all spatial directions) in the base 
BCS theory $[184,185]$. The mechanism(s) of two negatively charged electrons bonding together and transport characteristics of charge carriers in unconventional superconductors are still being studied. Many experiments have used electron- or hole-doped materials to investigate the electron pairing and charge carriers in unconventional superconductors [186 - 188]. In the work of Di Bernardo et al., single-layer graphene (SLG) has revealed evidence of an unconventional superconductor while it was placed in proximity to an electron-doped oxide superconductor [189]. The results of their work has led to a larger study of charge carrier transport in electron-doped graphene and graphite with the hypothesis that electron-doped SLG, bilayer graphene (BLG), few-layer graphene (FLG), or graphite thin film can be an unconventional superconductor.

\subsection{Hall Effect}

The Hall effect is the demonstration of the forces acting on charge carriers in a conductor in a magnetic field. It was discovered by the American physicist Edwin Hall in 1879. The basics of this effect are shown in Figures 3.4 and 3.5. The current density, $J$, is in the $x$-direction and magnetic flux density, $B$, penetrates through the plane in the $y$ -

direction. The magnetic force, $F$, is upward for both cases ( $z$-direction). The charge carriers in each case are pushed toward the upper edge of the plane by the magnetic force:

$$
F_{z}=|q| v_{d} B
$$

where $|q|$ is the magnitude of the electric charge and $v_{d}$ is the drift velocity of the charge carriers. 


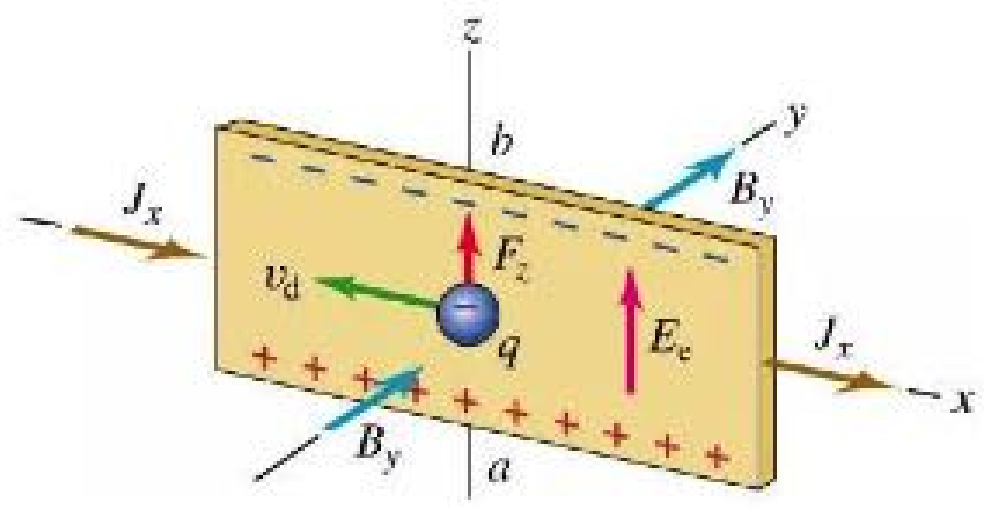

Figure 3.4: The Hall effect of negative charge carriers (electrons) [190].

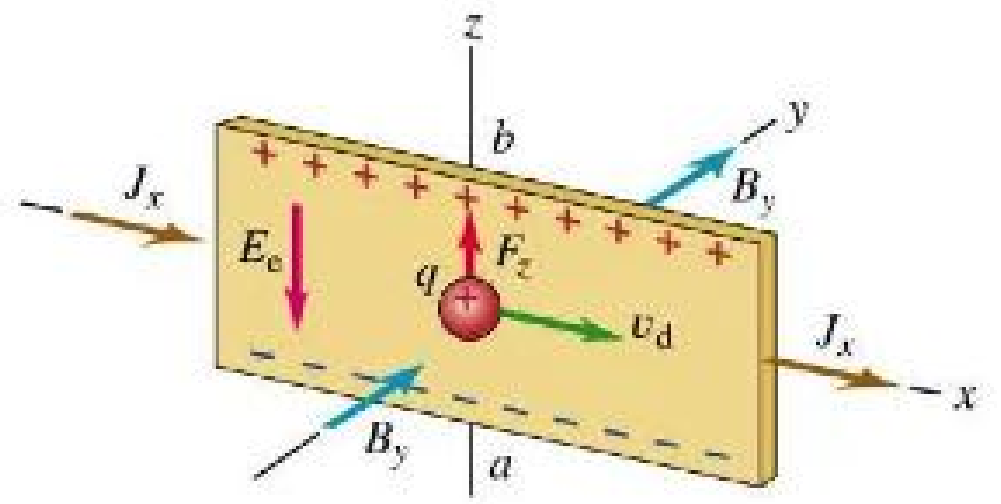

Figure 3.5: The Hall effect of positive charge carriers [190].

If charge carriers in the plane are negative (electrons), as in Figure 3.4, the negative charges will accumulate at the upper edge of the plane, leaving the lower edge with positive charges. The accumulation of charge carriers continues until the transverse electric field, $E_{e}$, with the magnitude $|q| E$ becomes equal to the magnetic force in the $z$-direction, $F_{z}$.

If the charge carriers are positive, as in Figure 3.5, then the accumulated charges at the upper edge will be positive, and the potential difference is opposite to the negative charge carrier situation [190]. 


\subsection{Hall Effect in Superconductors}

In most cases, if a conventional superconductor is in its superconducting state, the magnetic field is entirely expelled from the superconductor. Therefore, there will not be a Hall effect in the material. If the superconductor is in the mixed state, where both superconducting and Meissner phases are "mixed" with "normal", non-superconducting volumes, the Hall effect will be present in the "normal" non-superconducting cores of the vortices, and the Hall conductivity will show the same sign as in the normal state. However, in high- $T_{c}$ and some conventional superconductors, the Hall effect sign changes in the superconducting state. This effect is called the "Hall anomaly". The sign change is a result of vortex dynamics, and not a spurious Hall effect resulting from extrinsic effects such as atomic defects. It has become a challenge for the theorists to explain why sign changes appear in the combination of the different terms of the total Hall conductivity and there is an ongoing body of research into this [191].

\subsection{Introduction to Thermoelectricity}

Thermoelectricity is a process where electricity is generated by applying a temperature difference between two sides of a material. The thermoelectric effect can also be used to generate a temperature difference between two sides of a material by the application of an electrical current. As a result, thermoelectricity can be a bi-directional conversion of heat into electricity or electricity into heat [171].

\subsection{Thermoelectric Effects and Relations}

\subsubsection{Seebeck Effect}

The thermoelectric effect was first observed by T. J. Seebeck in 1821 and it was named after him as the "Seebeck effect" [174]. The Seebeck experiment showed that if two 
dissimilar metals are joined together then connected to a galvanometer to form a closed circuit as shown in Figure 3.6, heating the junction between the metals induces an electric current to flow in the circuit. The joined metals are called a "thermocouple" [170].

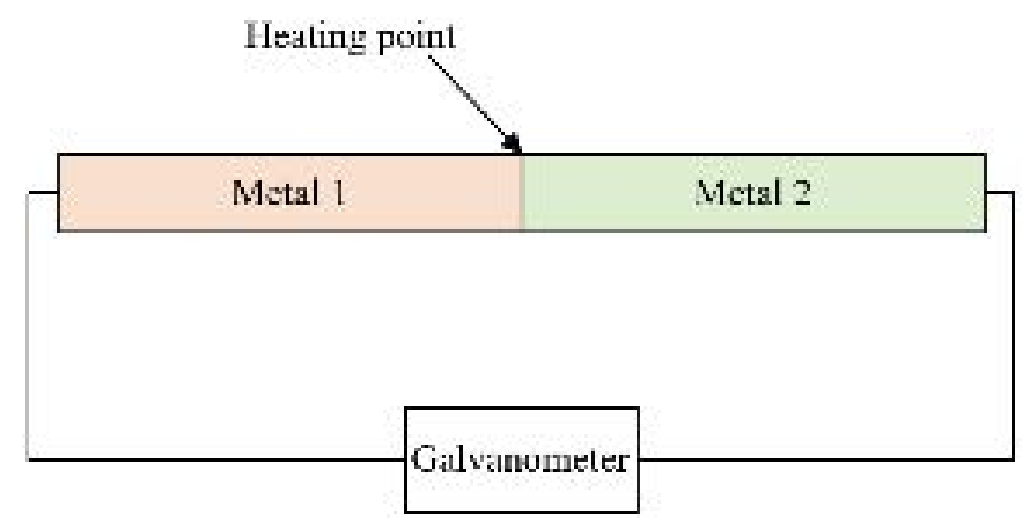

\section{Figure 3.6: A demonstration of the thermocouple Seebeck effect.}

In an open loop circuit, the magnitude of the thermoelectric voltage is found to be proportional to the temperature difference at the thermal junction. The Seebeck coefficient is then defined to be:

$$
S_{A B}=\frac{V_{t h}}{\Delta T}
$$

where $S_{A B}$ is the Seebeck coefficient of $A$ and $B$ where $A$ and $B$ are different types of metals, $V_{t h}$ is a thermoelectric voltage, and $\Delta T$ is a temperature difference [170, 172, 175].

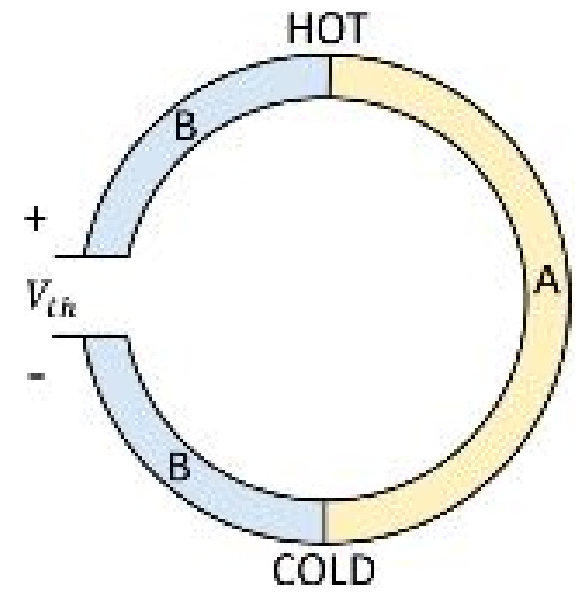

Figure 3.7: A schematic of a thermocouple. 
The Seebeck coefficient, $S_{A B}$, is also known as thermoelectric power which is an electromotive force with the SI unit of volts per kelvin (V/K) that drives an electric current from hot junction to cold junction through $A$ [170].

\subsubsection{Peltier Effect}

The Peltier effect was discovered in 1834 by J. C. A. Peltier. It was the second thermoelectric effect discovered. The Peltier effect is that when an electric current is flowing through a junction of two different metals $A$ and $B$ (thermocouple), heat will be increased (heating) or reduced (cooling) depending on the direction of the electric current flow [170].

The Peltier coefficient can be defined as:

$$
\Pi_{A B}=\Pi_{A}-\Pi_{B}=\frac{Q}{I}
$$

where $Q$ is a temperature changing rate at the junction, $I$ is an electric current, $\Pi_{A B}$ is the Peltier coefficient of the junction between metals $A$ and $B$, and $\Pi_{A}$ and $\Pi_{B}$ are the Peltier coefficients of metals $A$ and $B$ repectively.

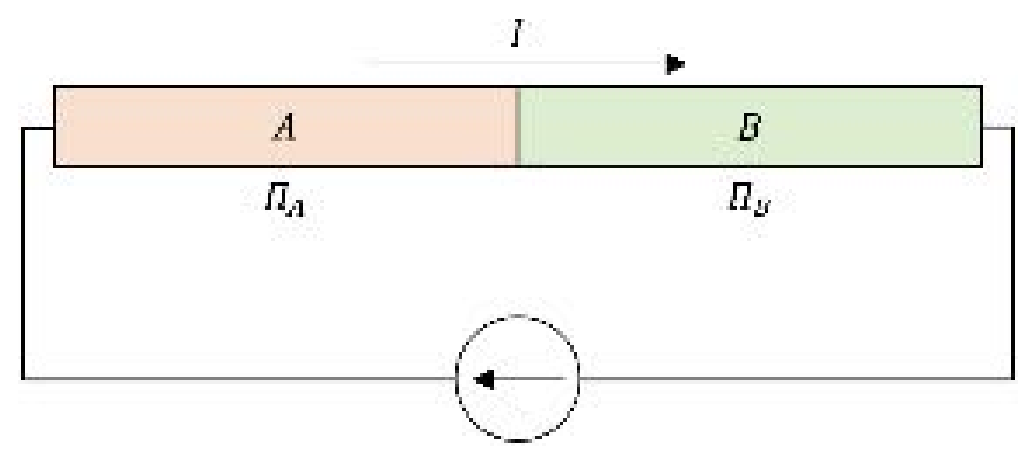

Figure 3.8: A setup for observing the Peltier effect.

\subsubsection{Thomson Relations}

W. Thomson, the $1^{\text {st }}$ Baron Kelvin of Largs (Lord Kelvin), determined the value of the absolute zero as to be approximately -273.15 degrees Celsius $\left({ }^{\circ} \mathrm{C}\right)$ or -459.67 
degrees Fahrenheit $\left({ }^{\circ} \mathrm{F}\right)$. The use of Thompson's titular name, Kelvin, as the unit of absolute temperature is in his honor.

Lord Kelvin expressed that the Seebeck and Peltier effects obey the theory of thermoelectric energy conversion. Thus, the Seeback and Peltier coefficients are relevant to each other as:

$$
\Pi_{A B}=T \cdot S_{A B}
$$

which is the first Thomson relation $[170,176]$.

Lord Kelvin also showed the connection between his coefficient (Thomson coefficient) and the Seebeck coefficient at the junction of two metals $A$ and $B$ in the second Thomson relation as:

$$
\tau_{A B}=\tau_{A}-\tau_{B}=T \frac{d S_{A B}}{d T}
$$

where $\tau_{A B}$ is the Thomson coefficient of the junction between metals $A$ and $B, \tau_{A}$ and $\tau_{B}$ are the Thomson coefficients of metals $A$ and $B$ repectively.

\subsubsection{Nernst Effect}

In 1886, W. H. Nernst, the Nobel Laureate in Chemistry (1920), and his colleague, A. von Ettingshausen, jointly discovered the thermoelectric phenomena which were named after them. The Nernst effect, also known as the first Nernst-Ettingshausen effect, is a thermoelectric phenomenon where a Nernst current density arises when a magnetic field, $H$, is applied perpendicularly to the thermal gradient (the difference of temperature in the direction of the heat flow) in a conductor. 


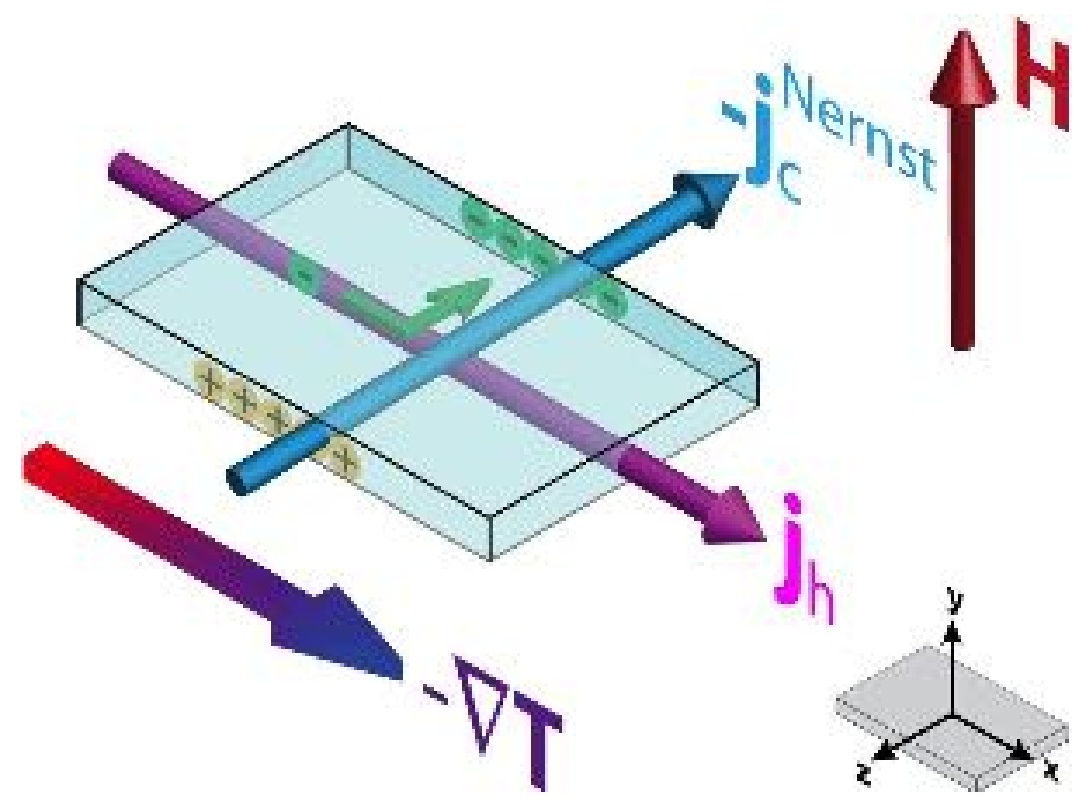

Figure 3.9: The Nernst effect [177].

As shown in Figure 3.9, the Nernst current density, $j_{c}^{\text {Nernst }}$, is driven in the transverse direction ( $z$-direction) of the heat flow or corresponding thermal current density (heat current), $j_{h}$, in the $x$-direction when $H$ is applied along the $y$-direction. The electric charges of $j_{c}^{\text {Nernst }}$ causes a transverse electric field, $E_{e}$, to occur in the conductor. Particularly, the Nernst effect depends on the heat flow rather than on a heat current $[170$, 177]

To simplify the calculation of the Nernst coefficient, a $x y$-plane diagram is presented in Figure 3.10. 


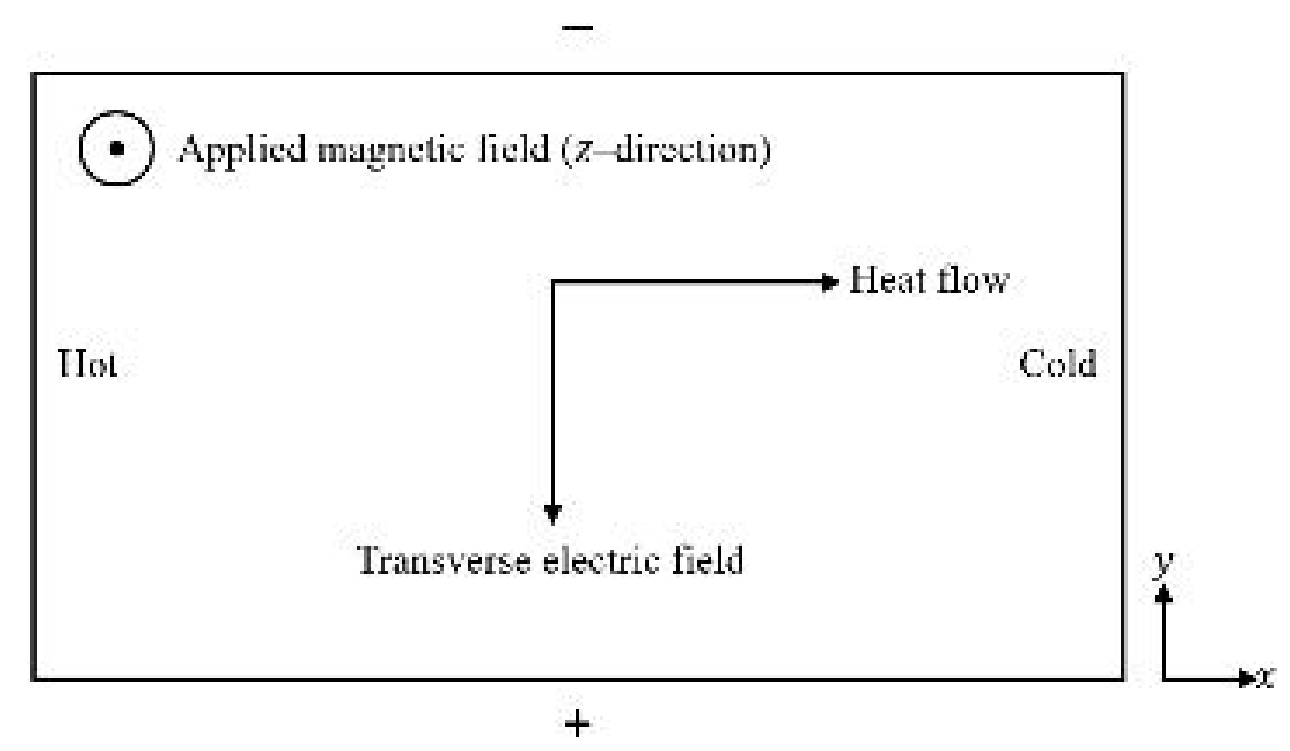

Figure 3.10: The Nernst effect diagram.

The Nernst coefficient, $N$, is defined as:

$$
|N|=\frac{E_{y}}{\nabla T_{x} H_{z}}=\frac{\left(\frac{d V}{d y}\right)}{H_{z}\left(\frac{d T}{d x}\right)}
$$

where $E_{y}=\frac{d V}{d y}$ is the electric field in the $y$-direction (transverse electric field), $V$ is a transverse voltage, $H_{z}$ is an applied magnetic field in the z-direction, and $\nabla T_{x}=\frac{d T}{d x}$ is the thermal gradient in the $x$-direction.

\subsubsection{Ettingshausen Effect}

The Ettingshausen effect or the second Nernst-Ettingshausen effect is a thermoelectric effect where a thermal gradient is a result from a transverse magnetic field and longitudinal flow of electric current [170] as shown in Figure 3.11. 


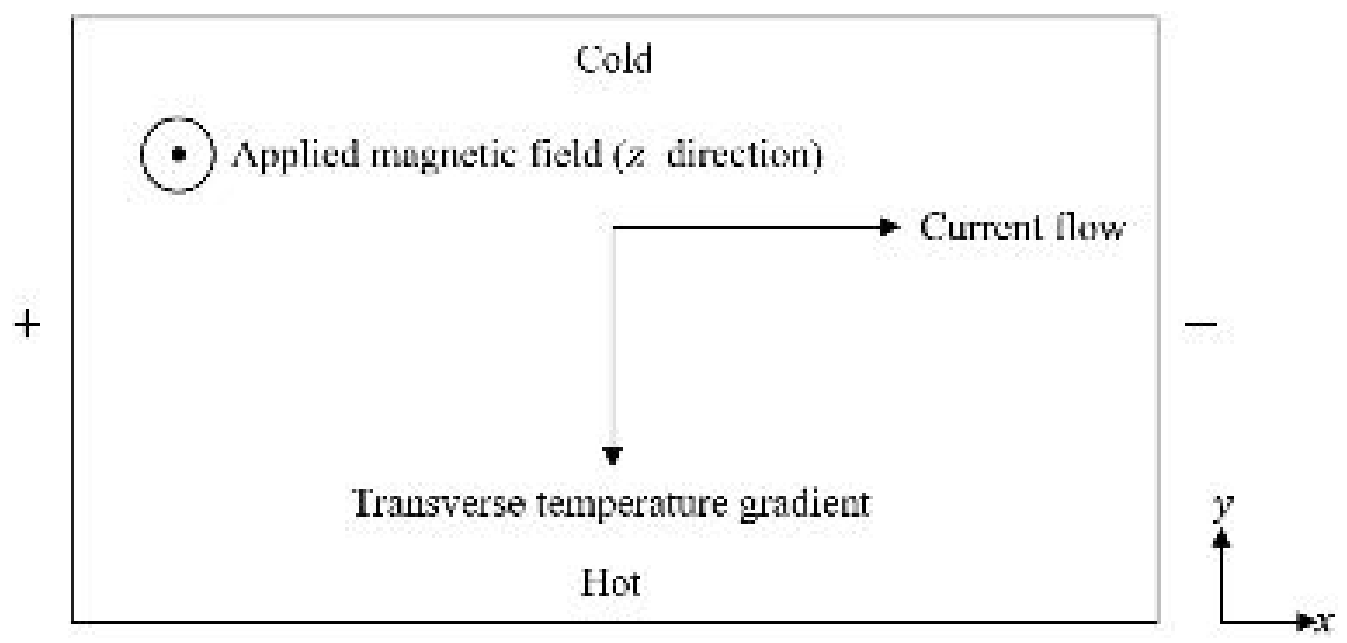

Figure 3.11: The Ettingshausen effect diagram.

The Ettingshausen coefficient, $P$, is defined as:

$$
|P|=\frac{\left(\frac{d T}{d y}\right)}{H_{z} I_{x}}
$$

where $I_{x}$ is the electric current flow in the $x$-direction.

\subsection{Thermal Conductivity of Graphene}

The thermal transport in graphene essentially depends on the quantized oscillations of the crystal lattice which propagate as phonons. Phonon propagation through the2dimensional (2D) materials shows differences as compared to 1-dimensional (1D) and 3dimensional (3D) materials. In 2D systems, the thermal conductivity, $K$, goes logarithmically as:

$$
K \approx \ln (n)
$$

where $n$ is the number of atoms [173].

The phonon dispersion relation of graphene comprises three acoustic branches and three optical branches. The branches are associated with out-of-plane, in-plane longitudinal, and in-plane transverse atomic motions. The six phonon dispersion branches 
of graphene are originated from the $\Gamma$-point of graphene reciprocal lattice in the reciprocal space. The three dispersion branches which correspond to the acoustic mode are a ZA (outof-plane), a TA (in-plane transverse), and an LA (in-plane longitudinal) branches, and the remaining three branches which correspond to the optical mode are a ZO (out-of-plane), a TO (in-plane transverse), and an LO (in-plane longitudinal) branches [178].

In graphene, the thermal conductivity is size-dependent since a weak scattering of low energy phonons by other phonons within the sheet is present. Thus, the phonon scattering on the sheet boundaries causes their relaxation. In order to make the thermal conductivity size-independent, the graphene sheet must be sufficiently large $(>10 \mu \mathrm{m})$. The phonon part of the thermal conductivity is:

$$
K_{p}=\sum_{j} C_{j}(\omega) v_{j}^{2}(\omega) \tau_{j}(\omega) d \omega
$$

where $\tau_{j}(\omega)$ is the phonon relaxation time, $j$ is the index of phonon polarization branches, $C_{j}(\omega)$ is the contribution to heat capacity from the $j$-th branch, and $v_{j}(\omega)$ is the phonon group velocity of the $j$-th branch [173].

The relevant phonon branches, which contribute in $K_{p}$, include one LA branch and two TA branches, and the Bose-Einstein distribution function of phonons will be:

$$
N_{0}\left(\omega_{j}\right)=1 /\left(\exp \left[\hbar \omega_{j} / k_{B} T\right]-1\right)
$$

where $\omega_{j}$ is the phonon frequency of the of the $j$-th branch.

Due to spatial confinement of acoustic phonons in nanostructures of graphene, the phonon energy spectra are quantized. The quantization of the phonon energy spectra results a decrease of the phonon group velocity. 
Where $s=T A, L A$ is the index denotes the TA and LA branches of phonon, the thermal conductivity of graphene is obtained as:

$$
K=\frac{1}{4 \pi T Z_{0}} \sum_{s=T A, L A} \int_{q_{\min }}^{q_{\max }}\left[E_{q}^{s} v_{s}(q)\right]^{2} \tau_{U, s}^{K}(q)\left(-\frac{\partial N_{0}}{\partial E_{q}^{s}}\right) q d q
$$

where $q$ is the wave vector, $Z_{0}=0.335 \mathrm{~nm}$ is the thickness of a graphene layer, $E_{q}^{s}=$ $\hbar \omega_{s}(q)$ is the phonon energy, $v_{s}(q)=d \omega_{s}(q) / d q$ is the phonon group velocity, $\tau_{U, S}^{K}(q)$ is the Umklapp relaxation time obtained by taking into account of different lifetimes for the LA and TA phonon branches [173].

The steady state Bose-Einstein function is given as follows:

$$
N_{0}\left(E_{q}\right)=\frac{1}{e^{\frac{E_{q}^{S}}{k_{B} T}}-1}
$$

The calculation of the thermal conductance of graphene in a pure ballistic region has obtained a value which was translated to a thermal conductivity, $K \approx 6.6 \mathrm{~kW} / \mathrm{mK}$. This result showed the higher $K$ is expected for the ballistic regime when no scattering is included [180, 181]. However, in experiments, there is scattering on defects, edges, or impurities, and thus the transport is partially diffusive. The measurement reports of thermal conductivity of graphene found that the near room-temperature thermal conductivity of partially suspended SLG was in the range of $\sim 3-5 \mathrm{~kW} / \mathrm{mK}$ depending on the size of graphene flake $[77,179,180]$.

\subsection{Tables of Symbols and Acronyms}

Table 3.1: Table of Symbols

\begin{tabular}{|c|l|c|}
\hline Symbols & Description & Value \\
\hline$T_{C}$ & Critical temperature. & - \\
\hline
\end{tabular}




\begin{tabular}{|c|l|c|}
\hline$J$ & Current density. & - \\
\hline$B$ & Magnetic flux density. & - \\
\hline$F$ & A magnetic force. & - \\
\hline$|q|$ & The magnitude of the electric charge. & - \\
\hline$v_{d}$ & The drift velocity of the charge carriers. & - \\
\hline$E_{e}$ & A transverse electric field. & - \\
\hline$E$ & An electric field. & - \\
\hline$S_{A B}$ & The Seebeck coefficient of metals $A$ and $B$. & - \\
\hline$V_{t h}$ & A thermoelectric voltage. & - \\
\hline$\Delta T$ & A temperature difference. & - \\
\hline$\Pi_{A B}$ & $\begin{array}{l}\text { The Peltier coefficient of the junction between } \\
\text { metals } A \text { and } B .\end{array}$ & - \\
\hline$\Pi_{A}$ & The Peltier coefficients of metals $A$. & - \\
\hline$\Pi_{B}$ & The Peltier coefficients of metals $B$. & - \\
\hline$Q$ & A temperature changing rate at the junction. & - \\
\hline$I$ & An electric current. & - \\
\hline$T$ & Temperature. & - \\
\hline$\tau_{A B}$ & $\begin{array}{l}\text { The Thomson coefficient of the junction between } \\
\text { metals } A \text { and } B .\end{array}$ & - \\
\hline$\tau_{A}$ & The Thomson coefficient of the metals $A$. & - \\
\hline$\tau_{B}$ & The Thomson coefficient of the metals $B$. & - \\
\hline$j_{C}^{N e r n s t}$ & The Nernst current density. & - \\
\hline$H$ & An applied magnetic field. & - \\
\hline$N$ & The Nernst coefficient. & - \\
\hline$V$ & A transverse voltage. & - \\
\hline$\nabla T$ & Thermal gradient. & - \\
\hline$P$ & The Ettingshausen coefficient. & - \\
\hline$K$ & Thermal conductivity. & - \\
\hline$n$ & Number of atoms. & - \\
\hline
\end{tabular}




\begin{tabular}{|c|l|c|}
\hline$\Gamma$ & $\begin{array}{l}\text { A high symmetry point in the reciprocal space of } \\
\text { graphene. }\end{array}$ & - \\
\hline$K_{p}$ & The phonon part of the thermal conductivity. & - \\
\hline$\tau_{j}(\omega)$ & The phonon relaxation time. & - \\
\hline$j$ & The index of phonon polarization branches. & - \\
\hline$C_{j}(\omega)$ & $\begin{array}{l}\text { The contribution to heat capacity from the } j \text {-th } \\
\text { branch. }\end{array}$ & - \\
\hline$v_{j}(\omega)$ & The phonon group velocity of the $j$-th branch. & - \\
\hline$N_{0}\left(\omega_{j}\right)$ & $\begin{array}{l}\text { The Bose-Einstein distribution function of } \\
\text { phonons. }\end{array}$ & - \\
\hline$\omega_{j}$ & The phonon frequency of the of the $j$-th branch. & - \\
\hline$q$ & The wave vector. & - \\
\hline$Z_{0}$ & The thickness of a graphene layer. & - \\
\hline$E_{q}^{S}$ & The phonon energy. & - \\
\hline$v_{S}(q)$ & The phonon group velocity. & - \\
\hline$\tau_{U, S}^{K}(q)$ & The Umklapp relaxation time. & - \\
\hline$N_{0}\left(E_{q}\right)$ & The steady state Bose-Einstein function. & - \\
\hline
\end{tabular}

Table 3.2: Table of Acronyms.

\begin{tabular}{|c|c|}
\hline Acronym & Description \\
\hline 1D & 1-dimensional \\
\hline 2D & 2-dimensional \\
\hline 3D & 3-dimensional \\
\hline Al & Aluminum \\
\hline As & Arsenic \\
\hline B & Boron \\
\hline BCS & Bardeen-Cooper-Schrieffer \\
\hline Bi & Bismuth \\
\hline BLG & Bilayer graphene \\
\hline
\end{tabular}




\begin{tabular}{|c|c|}
\hline FLG & Few-layer graphene \\
\hline Ga & Gallium \\
\hline In & Indium \\
\hline LA & In-plane longitudinal (acoustic mode) \\
\hline LO & In-plane longitudinal (optical mode) \\
\hline P & Phosphorous \\
\hline Sb & Silicon \\
\hline Si & Single-layer graphene \\
\hline SLG & In-plane transverse (acoustic mode) \\
\hline TA & In-plane transverse (optical mode) \\
\hline TO & Out-of-plane (acoustic mode) \\
\hline ZA & Out-of-plane (optical mode) \\
\hline ZO & \\
\hline
\end{tabular}




\section{Part II}

\section{Experimentation}

\section{Chapter 4}

\section{Problem Statement and Experimental Design}




\subsection{Overview}

Carbon has many allotropes exhibiting different chemical, mechanical, and electrical properties. Graphene, a single layer of hexagonally-bonded carbon atoms, has been widely studied [ $1-5]$ because of its unique thermodynamic stability, electronic band structure, orientation of atoms [219, 220], and other properties. In 2005, A. K. Geim and K. S. Novoselov successfully obtained the first single-layer graphene by mechanical exfoliation of HOPG. Their groundbreaking experiments on graphene led them to be awarded the 2010 Nobel Prize in Physics. Mechanically exfoliated graphene can be further separated into 2D (single layer) or quasi-2D (multilayer) conducting materials. These can then be used as test objects to study possible electron pairing and BKT transitions necessary for superconductivity.

The thermoelectric Nernst effect can be used to investigate charge carrier transport in mechanically exfoliated SLG, BLG and FLG as 2D and quasi-2D materials. In this dissertation, a Voltage versus Temperature $(V v s . T)$ Nernst effect measurement was used as the main method to test and identify the behavior of electric charge carriers in graphene under an applied thermal gradient and magnetic field. In conductors, the electric charge carriers are electrons. A thermal gradient in a conductor can cause electrons to diffuse from the hot end to the cold end of the material [170], A magnetic field perpendicular to the charge diffusion current exerts a transverse force on the moving charge carriers due to the Lorentz force. This Lorentz force pushes the charge carriers to one side of the conductor in a direction perpendicular to both the thermally diffusing charge flow and the applied magnetic field [233]. 
A similar effect also occurs in a mixed state superconductor when magnetic vortices are present and mobile. They, analogously to thermally diffusing charge carriers, move via diffusion from the hot end of the material to the cold end. The application of an external magnetic field perpendicular to the diffusing vortices also gives rise to a force perpendicular to both the diffusing vortices and the applied magnetic field. This pushes the vortices transverse to the thermal gradient and, as a result of the motion of the magnetic flux gives rise to an electric field via:

$$
\nabla \times \mathbf{E}=-\partial \mathbf{B} / \partial t
$$

where the equation is the differential form of Maxwell's third equation.

The purpose of this study is to examine the behavior of charge carrier transport in doped and undoped graphene in the temperature range between $\sim 290 \mathrm{~K}$ to $\geq 10 \mathrm{~K}$ via a thermoelectric Nernst effect measurement, and to explore any means to increase the critical temperature, $T_{c}$, of graphene to be close to the room temperature or even above it, if it is possible.

\subsection{Theoretical Background}

It has been hypothesized that the close coupling or strong scattering of electrons by both phonons and plasmons in graphene indicates a potential for superconductivity at considerable temperatures in doped graphene [201 - 206]. Typically, graphene is a nonmetallic material containing nonmagnetic atoms which does not react to an externally applied magnetic field. The magnetism in graphene comes from the local states caused by atomic vacancy defects or molecular adsorption as mentioned in Section 1.4.5. Vacancies

of carbon atoms break the molecular symmetry of the honeycomb structure causing 
magnetic flux pinning that induces magnetism in graphene [212 - 214], and chemical absorption leads to a forming of magnetic moments on neighboring carbon atoms causing spin-polarized states to mainly localize around the adsorbed atoms [210,211]. Bombarding graphene with argon (Ar) via ion implantation can cause atomic vacancy defects, and doping graphene with a chemical such as boron $(\mathrm{B})$, nitrogen $(\mathrm{N})$, or phosphorus $(\mathrm{P})$ can cause the atoms around the border regions of the honeycomb structure of graphene to localize. Then the graphene can be responsible for magnetism [55, 56, 208, 209].

The work of Larkins et al. shows that boron-doped HOPG samples show no sign of possible superconductivity at any temperature down to below $20 \mathrm{~K}$ [55]. On the other hand, phosphorus-implanted HOPG samples exhibit a deviation from the expected monotonic rise in resistance as temperature goes down at some point above $100 \mathrm{~K}$. Their following work [56] was able to observe a response consistent with the presence of magnetic field flux vortices in phosphorus-implanted and in phosphorus-doped multilayer graphene. The observation of possible superconductivity in phosphorus-implanted and in phosphorusdoped multilayer graphene has led to the thermal gradient-based Nernst effect measurements to study and understand more about the transports and behaviors of electrons in 2D and quasi-2D materials under an influence of a magnetic field at temperatures above and below the $T_{B K T}$. The phosphorus-implanted and phosphorus-doped-while-grown multilayer graphene has exhibited an evidence of superconductivity (or another unknown physical phenomenon) with a $T_{c}$ in the region of $260 \mathrm{~K}$ [56] which is considered to be in a region of high- $T_{c}$ superconductors. Thus, this dissertation was conducted to observe large Nernst signals below and above the hypothesized $T_{c}$ of the samples to confirm the 
existences of vortex transport and high- $T_{C}$ superconductivity in phosphorus implanted and phosphorus doped graphene.

\subsubsection{BKT Theory}

The motion of vortices is created by phase fluctuations above the BKT characteristic transition temperature, $T_{B K T}$, and the Nernst Effect can be attributed to this motion of vortices [200]. Many experiments have demonstrated that an enhanced Nernst magnetic vortex signal is observed in hole-doped (electron-accepter doped) cuprates when a motion of vortices is induced in a superconductor at temperatures significantly above the superconducting transition temperature, $T_{c}[194-198]$.

Susceptibility and transport measurements on a 2D superconductor should demonstrate a characteristic double BKT transition. At temperatures below $T_{B K T}$, there should be a magnetic vortex BKT transition at a higher temperature, and a charge BKT transition at a lower temperature [237]. This represents a characteristic signature of superconductivity in two dimensions and, along with diamagnetic susceptibility which tracks these BKT transitions, is strong evidence of superconductivity.

The charge BKT transition is the phenomenon where charge carriers becoming "frozen". As a result, the freezing of charge carriers causes conduction and magnetic screening to cease which makes the susceptibility and the conduction becoming effectively zero at and below this temperature. The magnetic vortex BKT transition is where magnetic vortices in a $2 \mathrm{D}$ material become frozen in a lattice and unable to move. In multilayer graphene, not only vortices in the same layer freeze into a lattice, but those in adjacent layers would freeze as well. The transition temperature will be higher for lattices with all vortices in the same layer and lower for vortices in adjacent layers. This is due to the 
distance between adjacent vortices in the same layer being smaller than the inter-vortex distance of adjacent vortices in adjacent layers. As a result, the repulsive force between the same layer vortices is stronger than between adjacent layer vortices. This leads to the expectation that the transition will be somewhat broadened as compared with the simple single layer model's transition. This effect should be visible in both the susceptibility (magnetization) and transport (resistance and Nernst) data.

\subsubsection{Hall Effect}

In Hall effect measurements on mixed-state multilayered superconductors with weak flux pinning, it has been widely observed that the presence of a vortex state with mobile vortices often leads to a sign reversal in the Hall voltage as the sample goes through the transition [237 - 241]. This sign reversal in the Hall voltage is also seen in thin-film materials with anomalous ferromagnetism. Anomalous ferromagnetism is only found in materials with $d$ shell and/or $f$ shell electrons and these materials also universally exhibit susceptibilities and magnetizations greater than zero [242, 243]. Magnetization and susceptibility measurements of the doped graphene demonstrate hysteresis in the fieldcooled (FC) and zero-field-cooled (ZFC) data. This is consistent with diamagnetism (negative susceptibility) and magnetic field storage (hysteresis with the susceptibility of ZFC films more negative than FC films). This is not consistent with ferromagnetism.

\subsubsection{Nernst Effect}

A conductor exhibits a transverse voltage when a magnetic field is applied on a perpendicular direction of the direction of thermal gradient [232]. In a conventional superconductor, a large Nernst magnetic vortex signal below $T_{c}$ is a sign of vortex

transport. But it was unexpected that large Nernst magnetic vortex signals were discovered 
above the $T_{C}$ in high- $T_{C}$ superconductors (hole-doped cuprates) [194-199]. A description of this phenomenon was raised by Pourret et al. that a large Nernst magnetic vortex signal in conventional superconductors and cuprates is generated by vortices as they are displaced by an applied thermal gradient which exerts a force on the vortices [207].

\subsubsection{Pancake Vortices and Flux Pinning}

Several works and experiments [221 - 228] have been done to characterize and model the behavior of magnetic vortices in layered superconductors lead to the conclusion that pancake vortices are the preferred vortex form in this extremely anisotropic material (the ratio of the in-plane resistivity to the normal direction resistivity in pure multilayer graphene is greater than $100,000: 1)[229,230]$. Normally, the thicknesses of hightemperature superconducting thin films are often less than 200 layers. When the superconducting layers are separated by relatively thick insulating or normal conducting layers, the formation of an Abrikosov vortex is only possible within the superconducting layers. These vortices are generally called "pancake vortices", due to their flat shape [234]. For multilayer graphene (graphene and graphite are diamagnetic materials with significant magnetic anisotropy [231]) with graphitic layers $<200$, interlayer distances $\sim 3.4-3.5 \AA$, and relatively long magnetic penetration depths, if the graphene was superconducting, A magnetic self-pinning attractive force or coupling force, $F\left(r_{j}, j, i\right)$, that tended to bring the two pancake vortices into alignment in layers $i$ and $j$ would be given as:

$$
F\left(r_{j}, j, i\right) \approx \hat{\rho}\left(\varphi_{j}\right)\left(\frac{\Phi_{0}}{2 \pi \Lambda}\right)^{2} \frac{\left(\sqrt{\rho_{j}^{2}+|i-j|^{2} s^{2}}\right)-|i-j| s}{\rho_{j}}
$$


where $\hat{\rho}\left(\varphi_{j}\right)=\hat{\imath} \cos \varphi_{j}+\hat{\jmath} \sin \varphi_{j}$ is the unit vector pointing from the $z$ axis (an axis that passes through the center of pancake in layer $i), r_{j}=\left(\rho_{j}, \varphi_{j}\right)$ is the coordinates of pancake vortices in layer $j, \Phi_{0}$ is magnetic flux quantum, $s$ is the interlayer spacing, and $\Lambda=2 \lambda_{\|}^{2} / s$ is the $2 \mathrm{D}$ thin-film screening length which $\lambda_{\|}$is the effective penetration depth parallel to the graphene planes [223].

Where the layers $i$ and $j$ are adjacent and the vortices are directly vertically aligned, the coupling force reduces to:

$$
F(z, 0,1) \approx \hat{z} \frac{\Phi_{0}^{2} s^{2}}{16 \pi^{2} \lambda_{\|}^{4}}
$$

where $z$ is the axis of the pancake vortices in layer $i$.

In general, pancake vortices in different layers attract to each other via an attractive force caused by the magnetic field associated with each pancake vortex. In multilayer superconducting materials, pancake vortices will be stacked together by a flux line of attractive force. This line of force is a Josephson interaction between adjacent layers of a layered superconductor, therefore it is called the "Josephson coupling or Josephson line" $[222,234,235]$. 


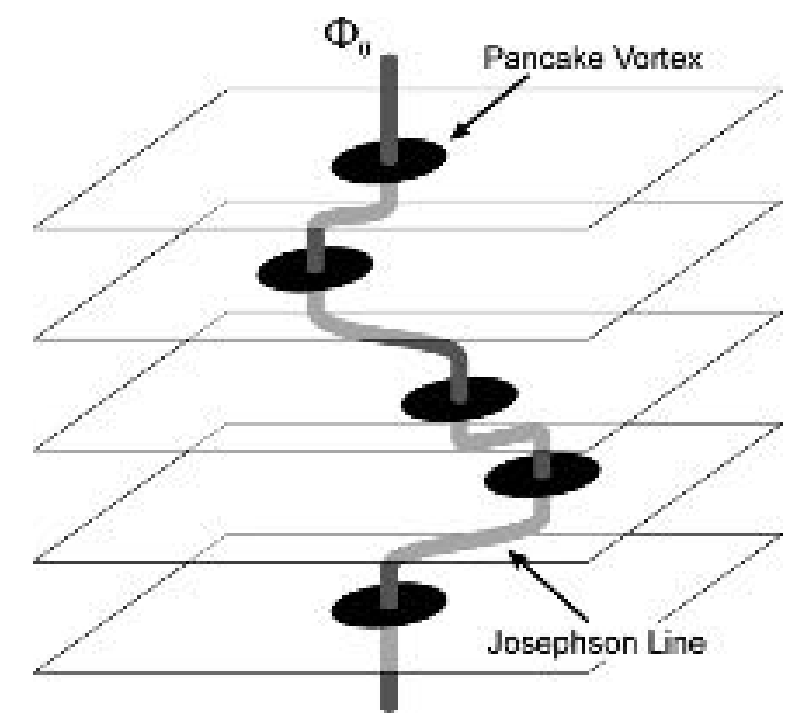

Figure 4.1: A flux line in a strongly layered superconductor is made up of pancake vortices in the superconducting layers interconnected by Josephson lines [234].

From $F(z, 0,1) \approx \hat{z} \frac{\Phi_{0}^{2} s^{2}}{16 \pi^{2} \lambda_{\|}^{4}}$, it is obvious that the coupling force between two pancake vortices in adjacent layers is proportional to the square of the interlayer spacing and inversely proportional to the fourth power of the magnetic field penetration depth within the layer. Since the interlayer spacing in graphene stacks is $\sim 3.4-3.5 \AA$, in the absence of external pinning, flux-flow resistance is a probable factor in the resistance of graphene to very low temperatures. The single pancake vortex pinning energy is given by:

$$
U_{0}=\left(\phi_{0} / 4 \pi\right)^{2} s / \lambda_{a b}^{2}
$$

where $\lambda_{a b}$ is the in-plane penetration depth which corresponds to $\lambda_{\|}$[224].

This pinning energy gives a self-pinning characteristic temperature for a single pancake vortex in $\mathrm{YBa}_{2} \mathrm{Cu}_{3} \mathrm{O}_{7-\mathrm{x}}$ of $1,200 \mathrm{~K}$ and vortex motion begins to be a problem at about $1 / 20$ of that temperature [224]. The expression $U_{0} / k_{B}=1,200 \mathrm{~K}$ is given for $\mathrm{YBa}_{2} \mathrm{Cu}_{3} \mathrm{O}_{7-\mathrm{X}}$ where the flux motion temperature regime is $\left(U_{0} / k_{B}\right) / 20=60 \mathrm{~K}$. 
Given that the measured normal state conduction anisotropy in multilayer graphene is significantly greater than conduction anisotropy of $\mathrm{YBa}_{2} \mathrm{Cu}_{3} \mathrm{O}_{7-\mathrm{x}}$, if the $\lambda_{a b}$ is the same, the expected temperature where a flux motion regime begins in graphene would be about $10 \mathrm{~K}$. This is expected to be an upper-value estimated temperature. Realistic estimates for the onset temperature for flux flow could be lower than $10 \mathrm{~K}$. Hence, even if the multilayer and highly anisotropic material is superconducting with Cooper pairs, the resistance would not be "zero" even at low temperatures and currents.

The strength of a pinning force of a pancake vortex to an atomic vacancy caused by ion-implantation is related to the size of the vacancy and $\lambda_{a b}$ that governs the physical size of the pancake vortex. At low temperatures, a pancake vortex could be pinned to a vacancy. However, the pinning force of a vortex would not be strong in this highly anisotropic material unless there were other pancake vortices pinned to similar vacancies in adjacent layers. In this case, the vacancies would provide condensation sites for the formation of columns of pancake vortices called "stacks" and the pinning forces of these stacks could be calculated as the summation of the vortices in the stack's mutual magnetic fields and Josephson interactions (Josephson lines) [224].

To deform or "melt" a stack requires a temperature that is proportional to the pinning energy from the inter-vortex pinning of the stack. Usually, numbers of pancake vortices in each stack are varied depending on the size of the vacancy and $\lambda_{a b}$. Thus, stacks with different numbers of vortices would melt at different temperatures. Once a stack is melted, all the vortices which were in the stack are now free to move and immediately contribute resistive losses in the material. This would lead to the expectation of upward steps in resistance at the melting temperatures of the various stacks. Thermal effects and 
angular misalignment of some of the vortices in the stacks (tilted stacks of pancake vortices) would give rise to smearing of the steps.

In other doping processes which do not cause a copious amount of atomic vacancies, pancake vortices would still form but would be less likely to stack vertically as there would be no columnar defect to stack along. For example, A doped-while-grown material would be less likely to have sharp steps as the pancake vortices would be less vertically aligned and more tilted away from the normal to the layers (greater spread in layer-to-layer offsets). If the vortices are separated by additional layers and not vertically stacked, the magnetic interaction will be lowered by the separation. However, the interaction potential will still be logarithmic and repulsive giving rise to a BKT transition.

\subsubsection{Magnetic Characteristics in Thin-Film Superconductors}

Magnetization measurements on thin-film superconductors where the penetration depth is many times greater than the film's thickness have shown that the magnetization is negative and has a "valley" or quasi-parabolic shape as a function of temperature and/or applied field. This is quite different from what can be seen form thick conventional superconductors where pancake vortices are not formed, and the applied magnetic field is expelled from the bulk of the superconductors $[56,57,236]$. In AC susceptibility measurements [55 - 57], the primary differences between thin films where the thickness is

considerably less than the magnetic penetration depth and materials with a unity ratio of thickness to penetration depth are

1. A smaller net signal as the magnetic screening is smaller;

2. A broader and depressed transition as a function of temperature due to the field penetration and vortices. 
For a thin film that has its thickness measured in nanometers, many films may be required to stack together to provide sufficient magnetic screening to obtain a stable and comprehensible signal. The magnetic screening fraction of a thin film usually behaves as:

$$
S F=1-e^{(-Z / \Lambda)}
$$

where $Z$ is the total sample thickness perpendicular to the applied magnetic field.

\subsection{Problem Statement and Hypotheses}

\subsubsection{Problem Statement}

A vortex in a superconductor can only exist in a mixed state superconductor. Vortex motion can only be observed for temperatures and magnetic fields below the upper critical zone. The term critical zone refers to the condition when the material is under the upper critical field $\left(H_{c 2}\right)$ and the critical temperature $\left(T_{c}\right)$ which are interdependent functions of each other. In the presence of thermal gradient, the resulting Nernst signal is a tilted peak, not a step, with a steep onset on the low temperature side and a longer tail on the high temperature side. As an extension of this problem, an array of pinned vortices with a common de-pinning temperature should be formed in the material. These vortices would give rise to a Nernst signal with a similar tilted peak characteristic shape. For a sample such as hypothesized phosphorous doped then argon damaged graphene, a series of Nernst tilted peak signals at temperatures correlating to the various depinning energies for vortices in the sample is highly anticipated to be observed.

\subsubsection{Hypotheses}

- The Nernst signal for a charge versus a vortex is differentiable insofar as a charge leads to a slope change with onset at the activation temperature and a vortex leads to a tilted peak at the depinning temperature. For a sample with multiple pinning 
energies, there should be multiple depinning temperatures observable in the Nernst signal.

- Vortices in different layers of superconducting phosphorous doped then argon damaged graphene are expected to be interconnected by Josephson lines through the columnar defects caused by the argon bombardment.

- $T \sim 40 \mathrm{~K}$ is expected to be the melting temperature of pinned vortices [110] which is also the same temperature where the thermoelectric Figure of Merit $(Z T)$ of the graphene-based topological insulators with nanopores [244] is found to reach its maximum of $Z T \sim 3$.

- Phosphorous-nano-doped graphene can become a superconductor when properly doped. The critical temperature for a flux-flow vortex state appears to be above $260 \mathrm{~K}$ in this material.

- The critical temperature in doped-while-grown graphene samples should be considerably higher than ion-implanted and ion-damaged graphene samples. This is because the phonon coherence lengths, Debye temperatures, and electron mean free paths are larger for samples with less damage to the lattice.

- Doping graphene using phosphorus or other electron-donor dopants could create a high- $T_{c}$ superconductor.

\subsection{Testing Samples}

As mentioned in Section 1.2, the host samples or substrates to create thin-film testing samples of this work was HOPG ZYH specimen with the dimensions of $10 \mathrm{~mm} \times 10 \mathrm{~mm} \times 1.2 \mathrm{~mm}$, a mosaic spread of $3.5^{\circ} \pm 1.5^{\circ}$, gran size of $30-40 \mathrm{~nm}$, and density of $2.255-2.265 \mathrm{gcm}^{-3}$ [26]. Bulks of HOPG were ordered from MikroMasch Inc. 
and NT-MDT Spectrum Instruments Company. After doping and peeling (mechanical exfoliation) the substrates, the testing samples were numbered and categorized into three types which were phosphorus and argon implanted samples (samples \# 023 and 201 - 225), phosphorus doped-while-grown samples (sample \# 151, 163 - 170), and undoped samples (sample \# 401 and 402).

For the ion-implanted samples \# 023 and 201 - 225, phosphorus was implanted into bulks of HOPG ZYH via ion implantation. Then, argon was used to deliberately damage the phosphorus-implanted HOPG to create vacancy columns for pinning of magnetic flux. The energy of the implanted phosphorus was $10 \mathrm{keV}$ with the implantation dose of $1.2 \times 10^{8} \mathrm{~cm}^{-2}$. All the ion-implanted samples were ion implanted by the CuttingEdge Ions, LLC on a mail-in basis. After the ion implantation was done, the HOPG substrates were mechanically exfoliated using silicon adhesive Kapton ${ }^{\circledR}$ film tape [216] to remove multilayer graphene sheets to be the testing samples.

From the previous work of Larkins et al. [56], the consecutive Resistance versus Temperature ( $R$ vs. $T$ ) measurements of a peeled thin-film of the sample \# 023 had shown large and abrupt drops of resistance at high temperatures for a superconductor (Figure 2.18). Therefore, the sample \# 023 was selected to be one of the samples used in this work.

For the doped-while-grown samples \# 151, 163, and 170, the samples were prepared in a proven Chemical Vapor Deposition (CVD) system using Plasma-Enhanced CVD (PECVD) [215] on HOPG substrates. The HOPG was used as a seed crystal as it was easier to remove the doped-while-grown layer from the HOPG via exfoliation than a monolayer from copper foil. The gas mixture to create the phosphorus doped-while-grown samples on HOPG substrates was methane at 20 Standard Cubic Centimeters per Minute 
(sccm), hydrogen at $10 \mathrm{sccm}$, argon at $14 \mathrm{sccm}$, and $0.1 \%$ phosphine in an argon carrier at

$5 \mathrm{sccm}$. After the PECVD process, the samples were obtained via exfoliation using Kapton ${ }^{\circledR}$ film tape.

For samples \# 401 and 402, the samples were exfoliated with the same method directly from undoped HOPG substrates.

\subsection{Mechanical Exfoliation}

In this dissertation, the mechanical exfoliation was the procedure to obtain the graphene testing samples from HOPG substrates. The polyimide silicon adhesive Kapton ${ }^{\circledR}$ film tape [216] was the main tool to perform the exfoliation. Due to the usage of the tape in a high vacuum, the uniform silicone adhesive layer of the tape would not allow air bubbles to be trapped in between the tape and the graphene sheets which were peeled off from HOPG substrates.

To mechanically exfoliate a testing sample, a chuck with a through hole and a vacuum device in Figure 4.2 were used to hold down the substrate (a bulk of HOPG) in place as shown in Figure 4.3. After the substrate was set up on the chuck, a layer of Kapton ${ }^{\circledR}$ film tape was laid on top of the substrate and carefully pressed down to remove all air bubbles between the tape and the substrate. Then the tape was gently removed off the substrate and a multilayer graphene sample was obtained as shown in Figure 4.4.

Since the Kapton ${ }^{\circledR}$ film tape has high electrical insulation and is lighter than other insulators [193], after the tape was used to produce testing samples, it was left under the testing samples to insulate the sample from the experimental fixture during the experiment. 


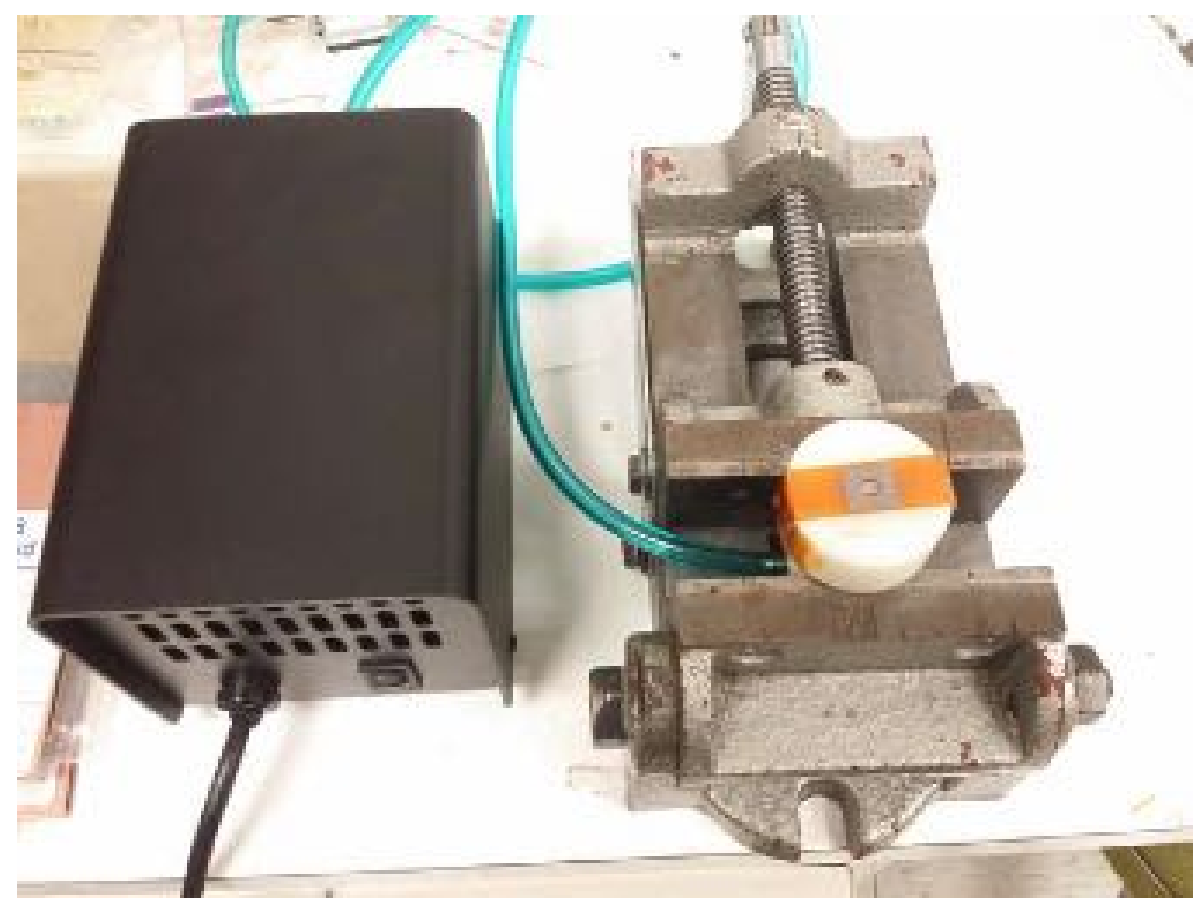

Figure 4.2: The sample preparation devices.

A vacuum device with a blue vacuum tube (left). A chuck with a through hole (right) [57].

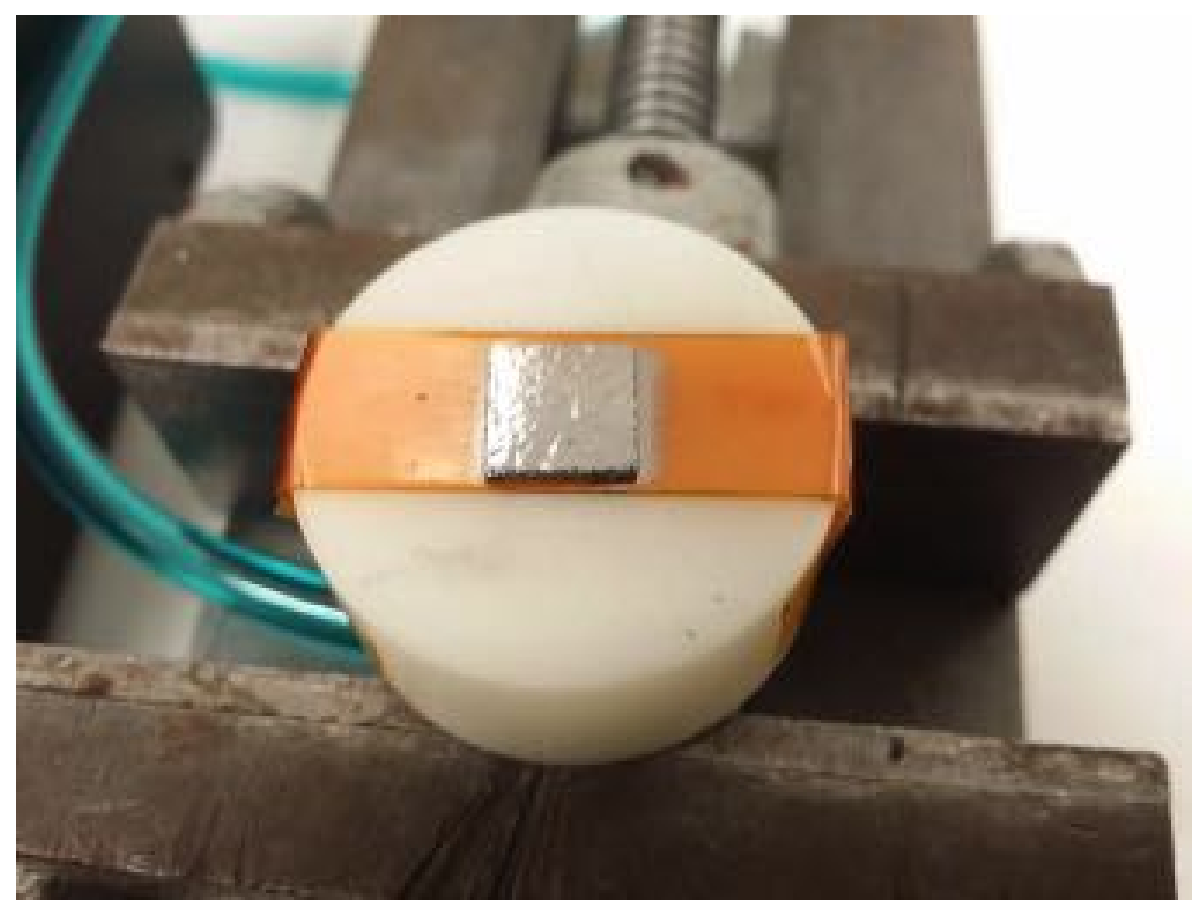

Figure 4.3: A HOPG sample secured to the chuck via vacuum seal [57]. 


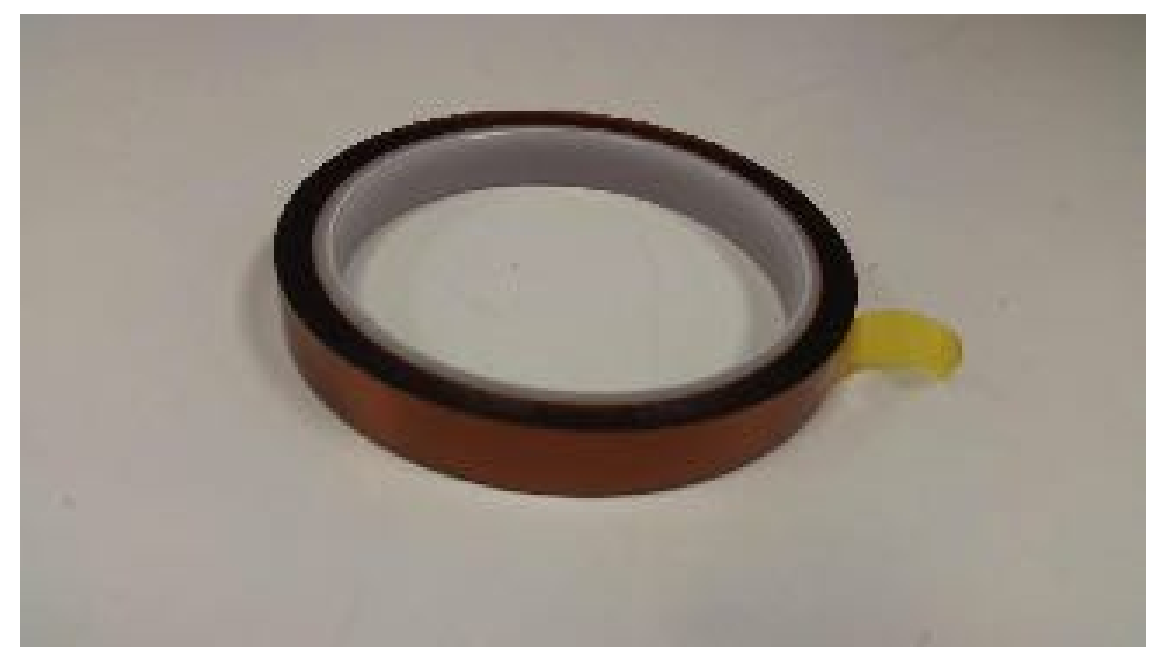

(a)

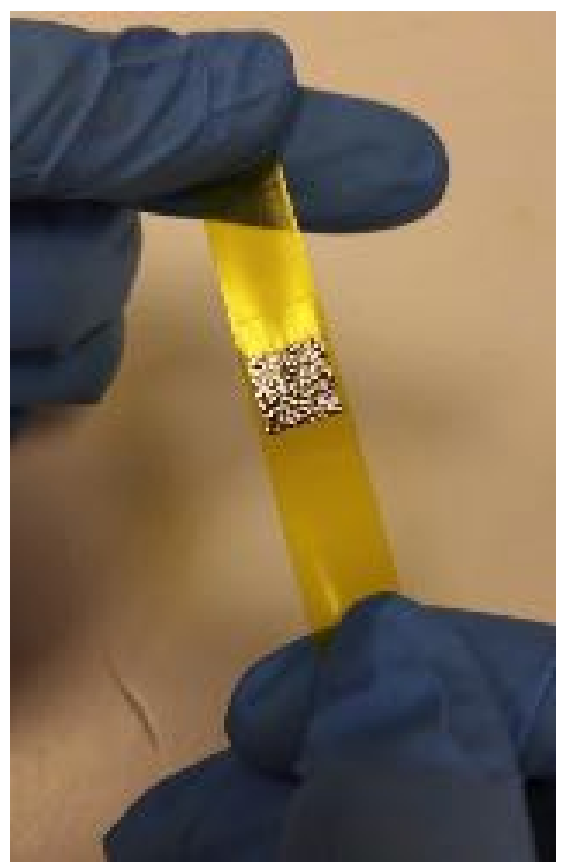

(b)

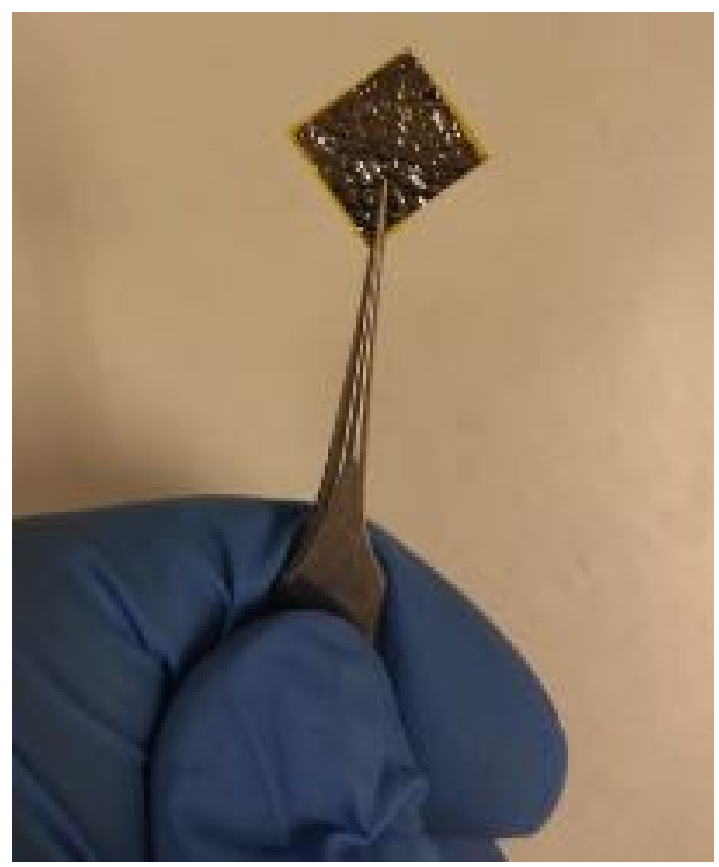

(c)

Figure 4.4: (a) A roll of Kapton ${ }^{\circledR}$ film tape. (b) Mechanical exfoliation. (c) A graphene testing sample with a sheet of Kapton ${ }^{\circledR}$ film tape adhering underneath.

\subsection{Experimental Design and Methodology}

After testing samples were prepared, the samples were mounted on a custom-made fixture in the cryogenic chamber of the closed-circle refrigeration system. The diagram in 
Figure 4.5 shows that the fixture was designed to have one mounting hole and four measurement pin holes. The mounting hole was designed to mount the fixture onto the cryogenic refrigerator's cold finger. Two measurement pin holes were used to install voltage measuring pins to measure the transverse thermoelectric voltage across the sample undergoing testing. The other two measurement pins holes were used to install pins to supply an electric current when a current for Resistance or Hall type measurements was desired.

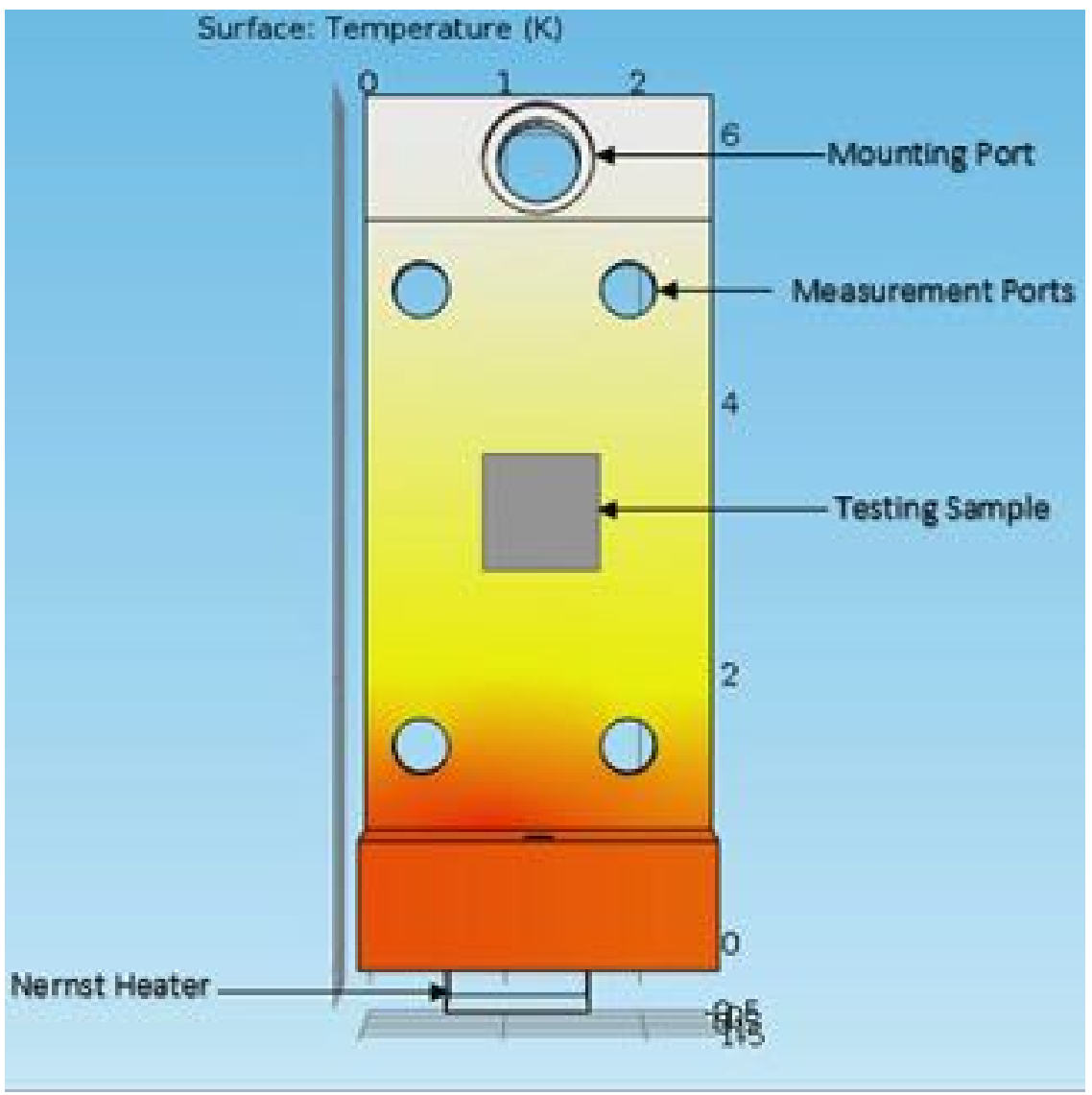

Figure 4.5: Top view of the simulated fixture. Image courtesy of Julian Gil Pinzon. 


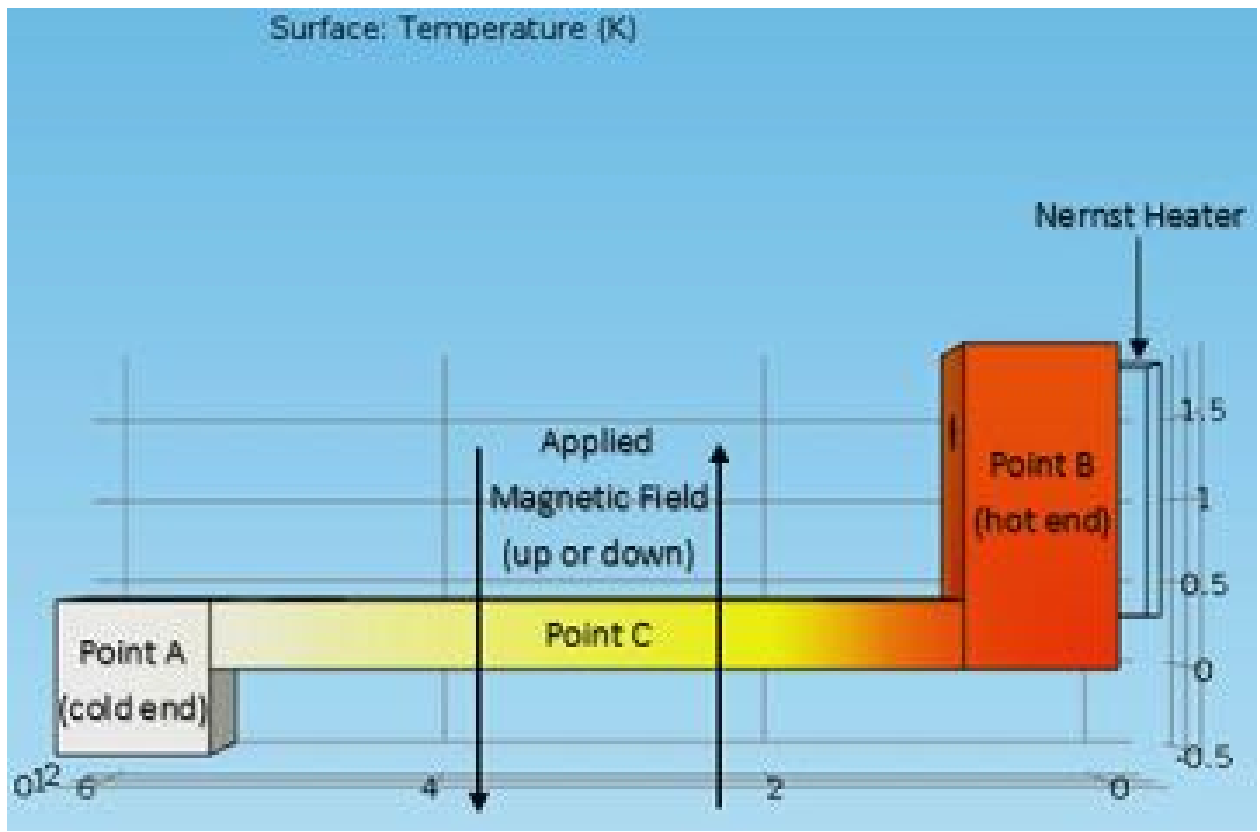

Figure 4.6: Side view of the simulated fixture. Image courtesy of Julian Gil Pinzon.

The experimental fixture was made from aluminum alloy 6061 [217] which is a paramagnetic material that can efficiently transfer heat and does not retain magnetization from the externally applied magnetic field. The cold end of the fixture was anchored to the cold head on the closed cycle refrigerator leaving the other end (hot end) free floating. The hot end (free-floating end) of the fixture had a resistive heater (Nernst Heater) attached to it. A $10 \Omega$-power film resistor model no. MP930 TO-220 with the tolerance of $\pm 1 \%$ [218] was used as the Nernst Heater to drive a thermal gradient from the hot end (Point B) to the cold end (Point A) as shown in Figure 4.6. Phosphor bronze contacts were used to make the contact to the sample and care was taken to place them at the same distance along the thermal gradient fixture to minimize differences in the temperature at the contacts. These contacts were placed on a line nominally normal to the thermal gradient (transverse to the anticipated thermally driven flux flow). 
During a thermoelectric Nernst effect measurement, a magnetic flux density, $B$, was applied perpendicularly to the thermal gradient, $\nabla T$, under the testing sample at Point $\mathrm{C}$ in Figure 4.6. Temperature sensors were installed at the points $\mathrm{A}, \mathrm{B}$, and $\mathrm{C}$ to observe temperatures at the cold end, hot end, and under the testing sample. These sensors were also used to estimate the thermal gradient and heat transport through the testing material for the data calculations.

To run an experiment, a graphene sample was placed on the fixture at point $\mathrm{C}$ in Figure 4.6 with the Kapton ${ }^{\circledR}$ film tape under the sample insulating the sample and the fixture. The Kapton ${ }^{\circledR}$ film tape was also used as an insulator preventing electrons to transport from the sample to the aluminum fixture. After a sample was placed on the experimental fixture and probed with voltage measuring pins, 1 - 3 watts of electric power was supplied to the Nernst Heater to create a thermal gradient through the experimental fixture. A magnetic field was applied perpendicularly to the sample where an adjustable magnetic field was generated by a DC copper solenoid, and a non-adjustable magnetic field was generated by neodymium rare earth disc magnets.

The DC copper solenoid was placed outside of the refrigerator's vacuum shroud to apply an adjustable magnetic field where the magnetic field could be adjusted from 0 to 300 Gauss. To apply a non-adjustable magnetic field, disc magnets were inserted in the gap between the Nernst fixture and the cold head without touching the fixture in order to minimize distortion of the thermal gradient.

The setup of a sample on the fixture, thermometers, Nernst Heater, and neodymium rare earth disc magnets are shown in Figure 4.7. 


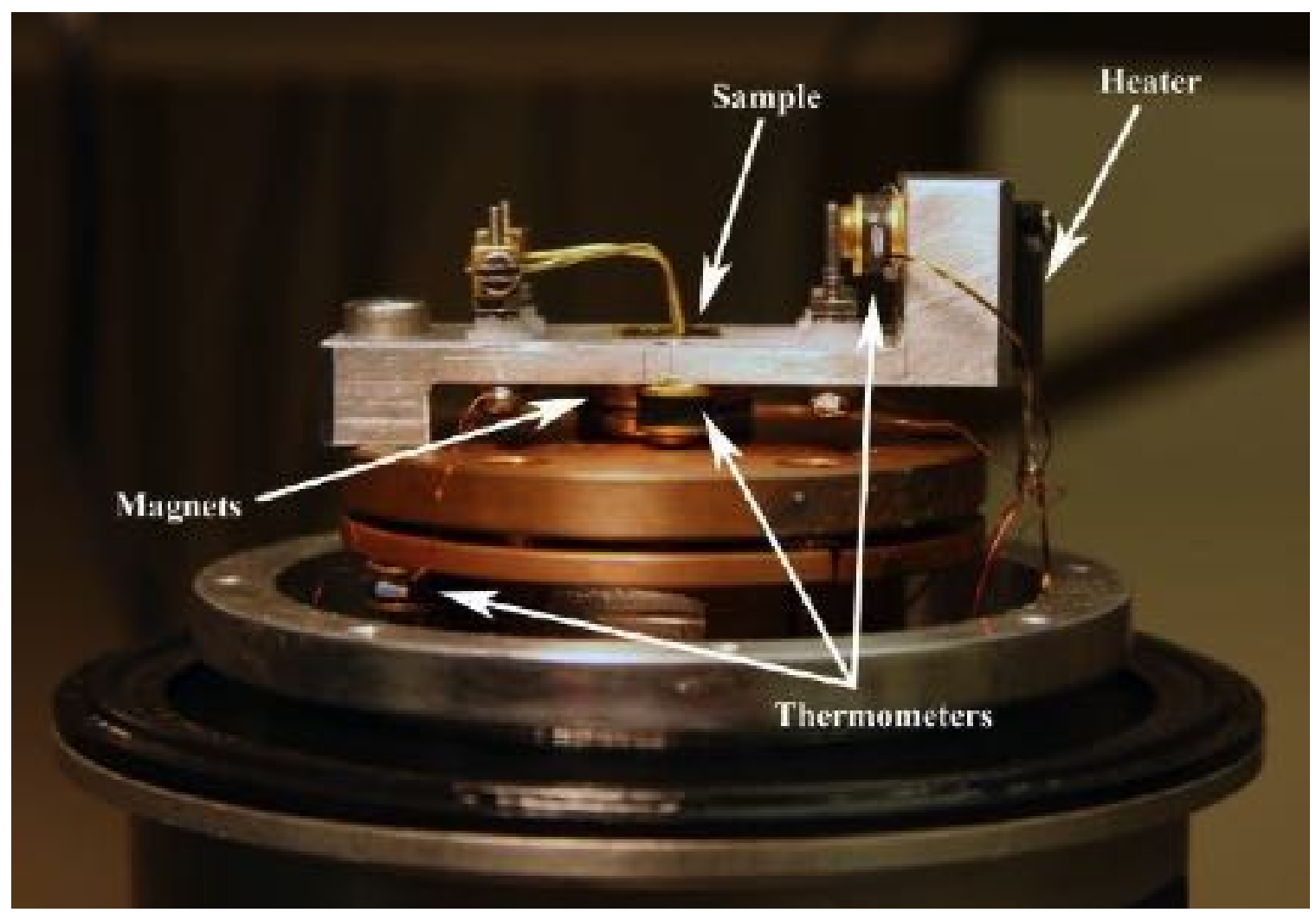

Figure 4.7: Photograph of the Nernst fixture. The aluminum fixture is bolted to the cold head on the left, the thermometers, magnets, and heater are labeled. Note that the magnets have an approximately $1 \mathrm{~mm}$ space between themselves and the aluminum fixture. Image courtesy of Dr. Grover L. Larkins.

After the experiment was set up in a high vacuum cryogenic system, the $V$ vs. $T$ characteristic of the sample was measured. The voltage was measured in microvolt-scale by a Keithley 2182A Nanovoltmeter. The temperatures at points A, B, and C in Figure 4.6 were measured by LakeShore 330 and LakeShore 335 Temperature Controllers from room temperature $(\sim 290 \mathrm{~K})$ down to $40 \mathrm{~K}$ at the position under the testing sample (point $\mathrm{C}$ in Figure 4.6). A LabVIEW program was used to control the compressor (F-70 Sumitomo) via a serial communication interface port and the data collecting instruments (LakeShore 330, LakeShore 335, and Keithley 2182A) via General Purpose Interface Bus (GPIB). The 
Measurement speed was approximately $5 \mathrm{~s}$ per final averaged data point recorded. In this experiment, an electrical current was not applied to the sample.

To change the orientation of the non-adjustable magnetic field required opening the refrigeration system at ambient temperature, flipping the magnets, re-evacuating the system, and re-running the experiment of the same sample. This was important as the field might not be completely symmetrically reversed, and there was a possibility of mechanical motion of the contacts; despite this, the results would be remarkably consistent and symmetric. In the Nernst experiment, a magnetic field was applied normal to the surface of the sample and a thermal gradient perpendicular to the field is applied. Magnetic vortices (as well as charge carriers) then thermally diffuse from the cold end to the hot end of the sample. While moving, they experienced a Lorentz force due to the magnetic field, and a voltage (electromotive force) was induced in the direction of the cross product of the diffusion velocity and the magnetic bias field. Placing the electrodes on both edges of the sample in the direction of the Lorentz force allowed the induced electromotive force to be detected as a DC voltage.

\subsection{Preliminary Result}

The designed system was examined by performing a few trial runs to capture the Nernst effect voltages in a sample at room temperature. Trial runs of the undoped sample \# 401 layer \# 3 with an applied magnetic field of 2,900 G (0.29 T) showed that the Nernst effect voltage across the testing sample was affected by the increasing temperature from the heat flow. Figure 4.8 displays the voltages across the sample (transverse voltages) of 4 trial runs at room temperature. The data of each run have been captured for 900 seconds (15 minutes). When the Nernst heater was on, heat flowed from B to A and increased to 
the maximum depending on the power supplied to the heater. The polarities of the transverse voltages were determined by the polarity of the magnetic field from the magnet underneath the testing sample. In this dissertation, when the magnetic field penetrated out of the plain of the testing sample, it was labeled as "North up" and when the magnetic field penetrated onto the plain of the testing sample, it was labeled as "North down" (or "South up" in later experiments in Chapter 6).

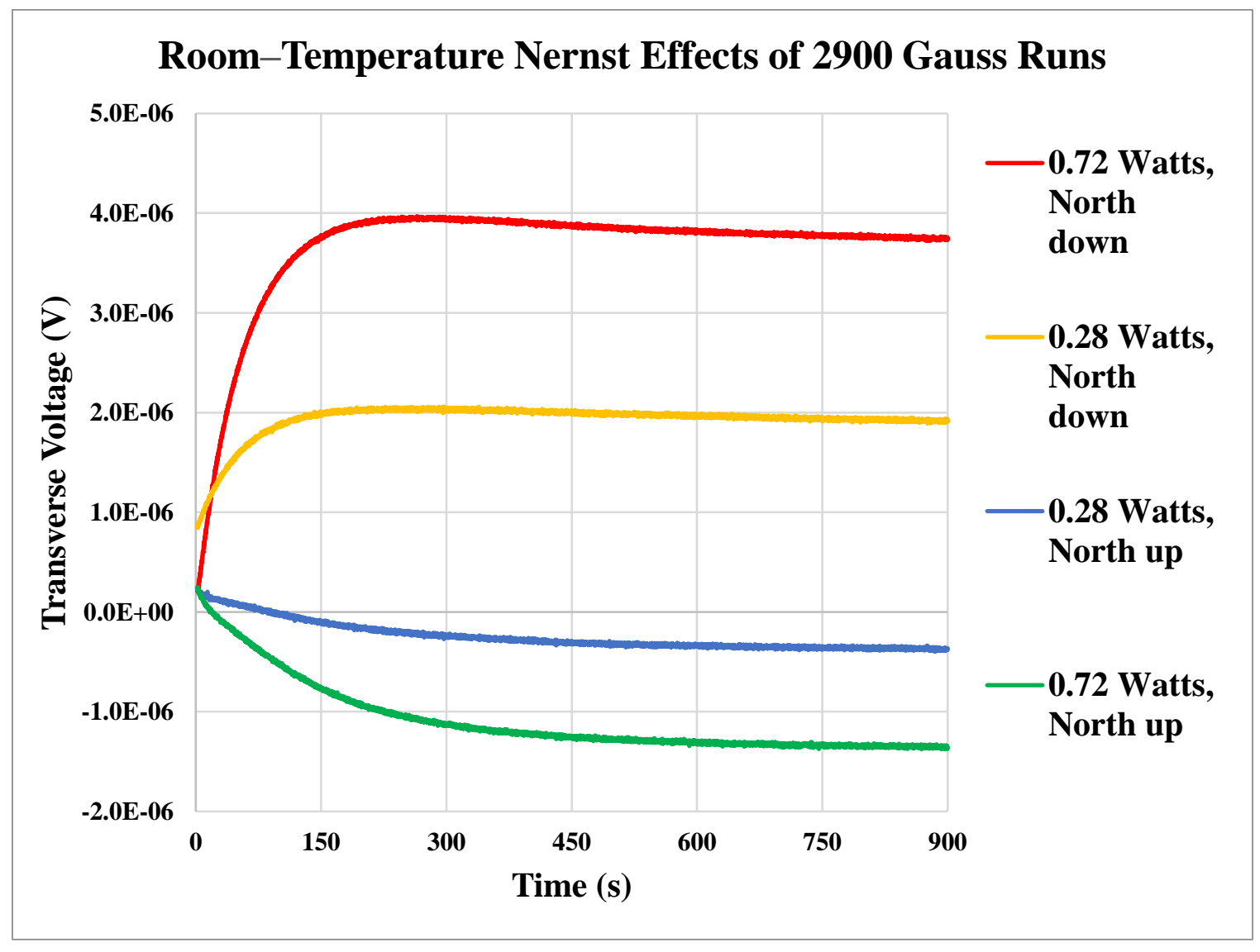

Figure 4.8: A graph of the room temperature Nernst effects of the undoped sample \# 401, layer \# 3 under 2,900 G magnetic field.

The graph of Voltage versus Time ( $V v s . t)$ in Figure 4.8 shows that the transverse voltage of each run was increasing (or decreasing in the opposite polarity of the magnetic 
flux density) when the temperature increased from the room-temperature to the maximum, and the voltage became stable when the temperature has stabilized (reached its maximum).

The graphs indicated that the voltage across the sample, $V$, was proportional to the thermal gradient, $\nabla T$, caused by the applied power of the Nernst Heater and the strength of the magnetic field, $H$. And the polarity of the transverse voltage was determined by the polarity of $H$ as in the thermoelectric Nernst effect equation:

$$
\frac{d V}{d y}=|N| H_{z} \cdot \nabla T_{x}
$$

where $N$ is the Nernst coefficient.

\subsection{Tables of Symbols and Acronyms}

Table 4.1: Table of Symbols.

\begin{tabular}{|c|l|c|}
\hline Symbols & \multicolumn{1}{|c|}{ Description } & Value \\
\hline$T_{B K T}$ & BKT characteristic transition temperature. & - \\
\hline$T_{c}$ & Critical temperature. & - \\
\hline$F$ & $\begin{array}{l}\text { A magnetic self-pinning attractive force or } \\
\text { coupling force. }\end{array}$ & $\hat{\imath} \cos \varphi_{j}+\hat{\jmath} \sin \varphi_{j}$ \\
\hline$\hat{\rho}\left(\varphi_{j}\right)$ & The unit vector pointing from the $z$ axis. & $\left(\rho_{j}, \varphi_{j}\right)$ \\
\hline$r_{j}$ & The coordinates of pancake vortices in layer $j$. & Wb \\
\hline & & or \\
$\Phi_{0}$ & Magnetic flux quantum. & $h c / 2 e \approx 2.07 \times 10^{-7}$ \\
& & $\mathrm{Gauss} \mathrm{cm}^{2}$ \\
\hline$S$ & The interlayer spacing. & - \\
\hline$\Lambda$ & The 2D thin-film screening length. & - \\
\hline$\lambda_{\|}$ & $\begin{array}{l}\text { The effective penetration depth parallel to the } \\
\text { graphene planes. }\end{array}$ & - \\
\hline$Z$ & The axis of the pancake vortices in layer $i$. & - \\
\hline
\end{tabular}




\begin{tabular}{|c|l|c|}
\hline$U_{0}$ & The single pancake vortex pinning energy. & - \\
\hline$\lambda_{a b}$ & The in-plane penetration depth. & - \\
\hline$k_{B}$ & The Boltzmann's constant. & - \\
\hline$S F$ & The magnetic screening fraction of a thin film. & - \\
\hline$Z$ & $\begin{array}{l}\text { The total sample thickness perpendicular to the } \\
\text { applied magnetic field. }\end{array}$ & - \\
\hline$Z T$ & Thermoelectric Figure of Merit. & - \\
\hline$\nabla T$ & Thermal gradient. & - \\
\hline$\nabla T_{x}$ & The thermal gradient on the $x$ axis. & - \\
\hline$H$ & Magnetic field. & - \\
\hline$H_{Z}$ & The magnetic field on the $z$ axis. & - \\
\hline$N$ & The Nernst coefficient. & - \\
\hline
\end{tabular}

Table 4.2: Table of Acronyms.

\begin{tabular}{|c|c|}
\hline Acronym & Description \\
\hline $2 \mathrm{D}$ & 2-dimensional \\
\hline Ar & Argon \\
\hline AC & Alternating Current \\
\hline B & Berezinskii-Kosterlitz-Thouless \\
\hline BKT & Bilayer graphene \\
\hline BLG & Chemical Vapor Deposition \\
\hline CVD & Few-layer graphene \\
\hline FLG & Highly Ordered Pyrolytic Graphite \\
\hline HOPG & Nitrogen \\
\hline N & Phosphorous \\
\hline P & Plasma-Enhanced Chemical Vapor Deposition \\
\hline PECVD & Resistance \\
\hline$R$ &
\end{tabular}




\begin{tabular}{|c|c|}
\hline$R$ vs. $T$ & Resistance versus Temperature \\
\hline SLG & Single-layer graphene \\
\hline$T$ & Temperature \\
\hline$V$ & Voltage \\
\hline$V v s . T$ & Voltage versus Temperature \\
\hline$V v s . t$ & Voltage versus Time \\
\hline
\end{tabular}


Chapter 5

Phosphorus-Doped HOPG 


\subsection{Introduction to Ion Implantation}

Ion implantation is a low-temperature process to modify electronic properties of a substrate which is usually used to implant electron-donor or electron-acceptor atoms into silicon to create a P-type or N-type semiconductor. In the ion implantation, ions of the dopant are accelerated to hit and penetrate the substrate. Then the accelerated ions are decelerated by collisional and stochastic processes and come to rest within femtoseconds at the top micrometer layers [245]. The concentration of the dopant in an ion-implanted substrate is commonly defined by the dose and energy.

Ions (charged atoms or molecules) are created via an enormous electric field stripping away an electron. The ions are filtered and accelerated toward a target substrate, where they are buried in the substrate. The depth of the implantation depends on the acceleration energy (voltage), and the dose is controlled by integrating the measured ion current. This integration process tends to minimize noise in the measurement of the ion current, resulting in several decimal places of accuracy in the dose [248].

At low doses (below $10^{14} / \mathrm{cm}^{2}$ ), the predominant damage type is point defects such as vacancies and interstitials, or clusters of point defects. At high doses, extended defects are created, and even amorphization can take place. High dose phosphorus and arsenic implants can amorphized a target such as silicon. The critical dose for amorphization is approximately $10^{14} / \mathrm{cm}^{2}[245]$. 


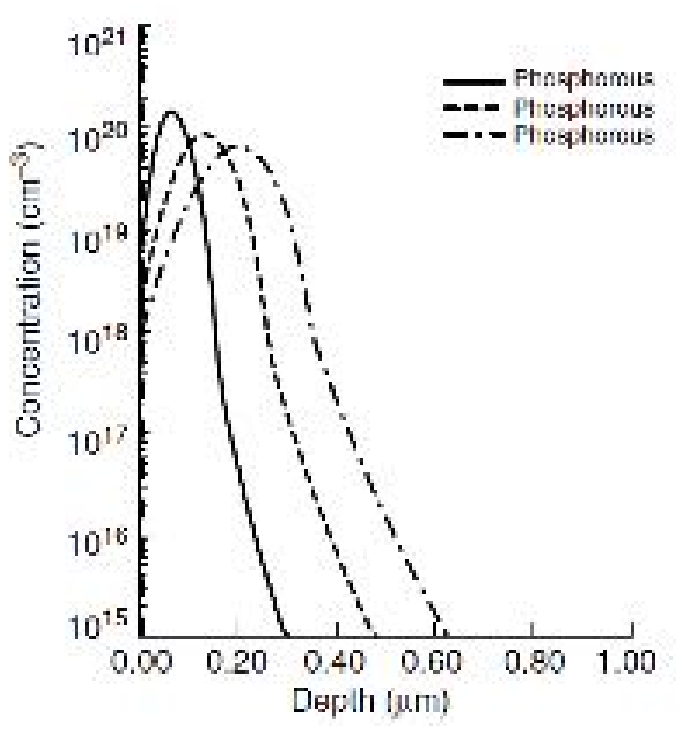

(a)

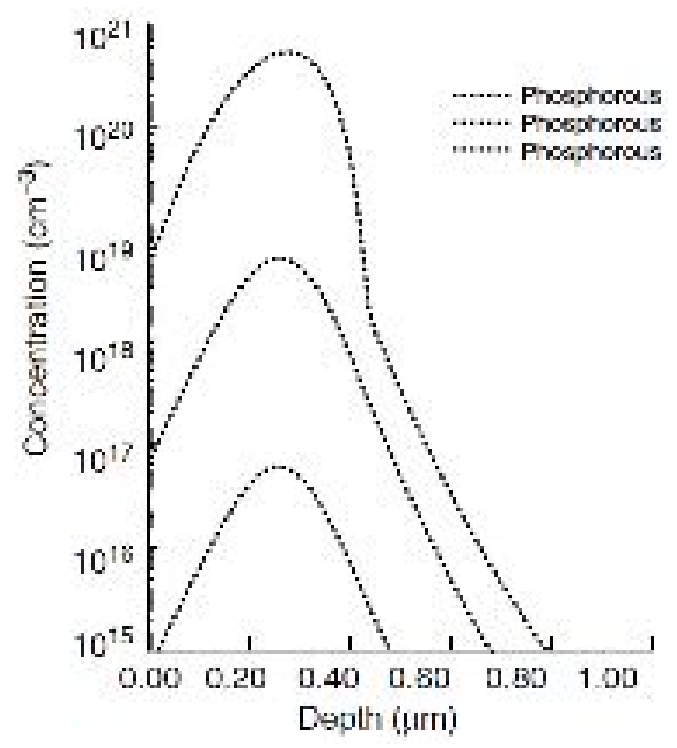

(b)

Figure 5.1: (a) Phosphorous implantations with different energies: $50 \mathrm{keV}, 100 \mathrm{keV}$ and $150 \mathrm{keV}$ (dose constant $10^{15} / \mathrm{cm}^{2}$ ). (b) Phosphorous implantations with different doses: $10^{12} / \mathrm{cm}^{2}, 10^{14} / \mathrm{cm}^{2}$, and $10^{16} / \mathrm{cm}^{2}$ (energy constant at $200 \mathrm{keV}$ ). The shape of dose $10^{16} / \mathrm{cm}^{2}$ is different because it is above the amorphization limit, and different

stopping parameters are applied for the amorphized region. Reprinted with permission from Sami Franssila [245].

Point defects created by ion implantation cannot be seen by physical analysis, but extended defects like dislocations can be seen by Transmission Electron Microscopy (TEM). Amorphization can be measured by TEM or by X-Ray Diffraction (XRD).

Implantation simulation such as the Monte Carlo (MC) simulation offers many advantages over semi-analytical implantation simulations. The Monte Carlo simulation can predict not only ranges and straggles, but it also enables physically based damage prediction, including amorphization. The MC simulations are, of course, more computationally intensive than the semi-analytic ones. The simulator SRIM (Simulation of Ranges of Ions in Matter) is a widely used MC simulator for implantation and other ionbeam processes [245]. 
SRIM is a group of programs which calculate the stopping and range of ions into matter using a quantum mechanical treatment of ion-atom collisions (assuming a moving atom as an "ion", and all target atoms as "atoms"). This calculation is made very efficient using statistical algorithms which allow the ion to make jumps between calculated collisions and then averaging the collision results over the intervening gap [253]. Examples of SRIM simulation are shown in Figure 5.2 and 5.3.

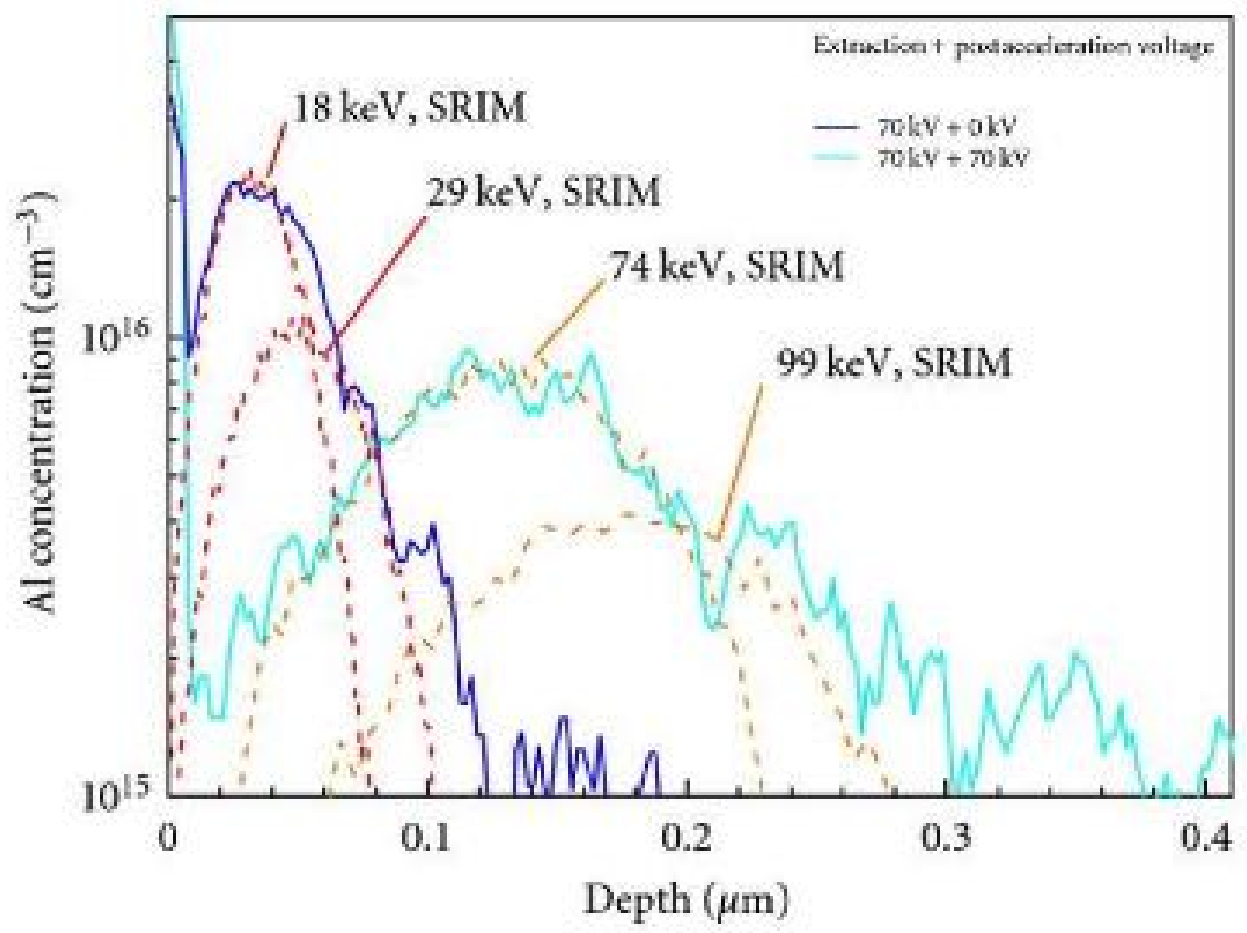

Figure 5.2: Al contamination profiles resulting from a $B$ implantation. For comparison, with SRIM simulated profiles are shown [254]. 


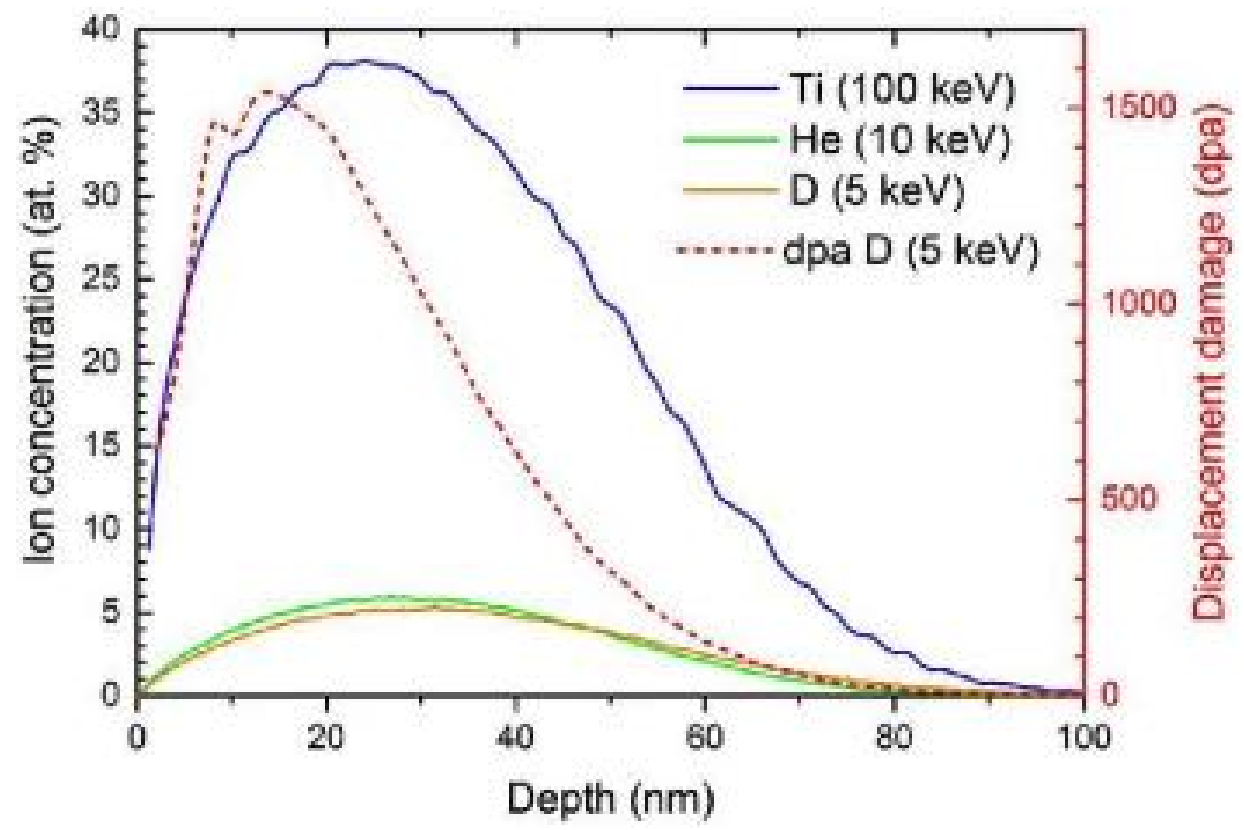

Figure 5.3: SRIM simulation results of implanted ion concentration for $2 \times 10^{21}$ $\mathrm{Ti} / \mathrm{m}^{2}, 5 \times 10^{21} \mathrm{He} / \mathrm{m}^{2}$, and $5 \times 10^{21} \mathrm{D} / \mathrm{m}^{2}$ and displacement damage for $5 \times 10^{21} \mathrm{D} / \mathrm{m}^{2}$ as a function of depth in W (Pure Tungsten) [255].

An ion-beam implanter is the machine used to perform the ion implantation process.

A diagram of an ion beam implanter is shown in Figure 5.4.

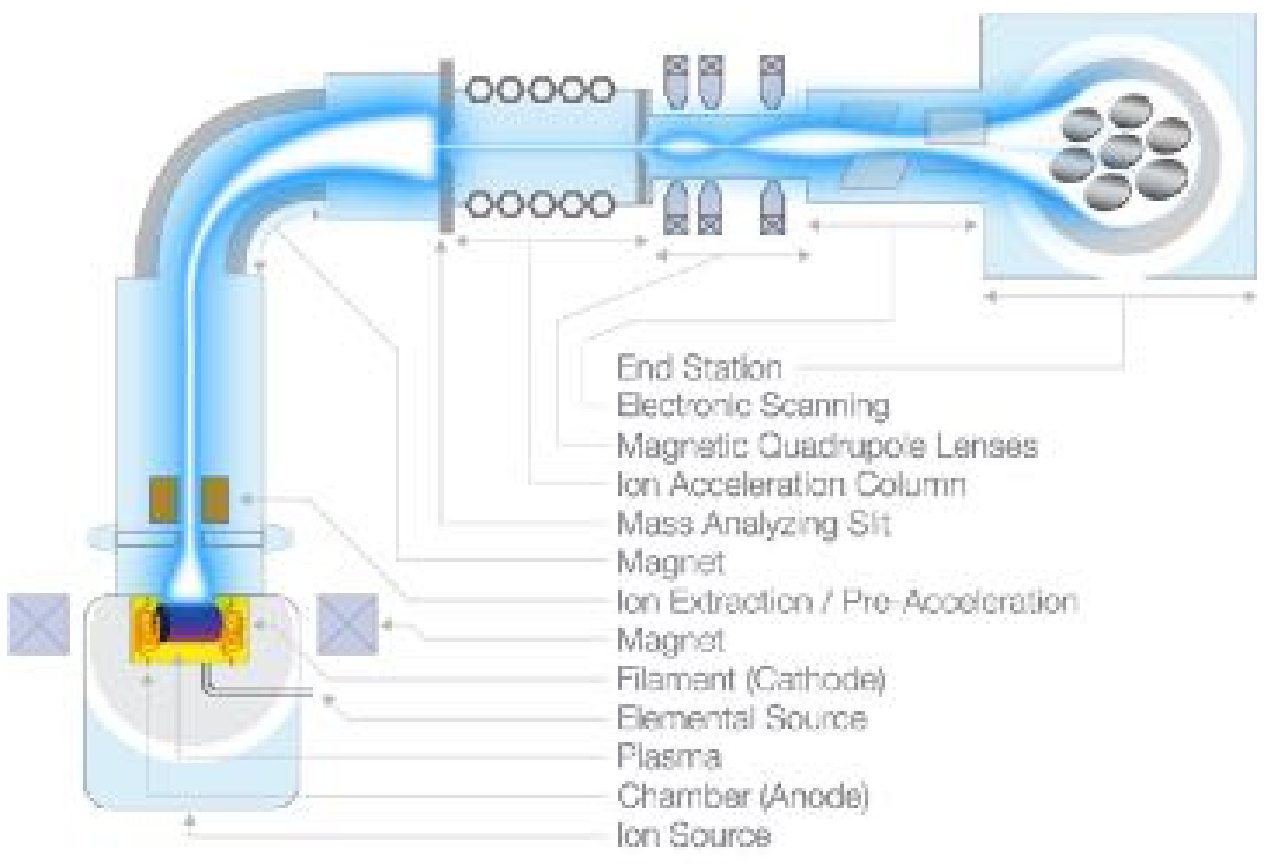

Figure 5.4: Ion-beam implanter. Reprinted with permission from the Plansee Group [249]. 
The ion source is a radio frequency (RF), and the multicusp ion source produces the desired beam species. The beam passes through a pre-acceleration section, known as the ion source extraction. The bias voltage gives the beam sufficient energy to allow selection of the desired species required for implantation by a 90-degrees mass analyzing magnet. The mass analyzing magnet is positioned along the beam path between the source and the acceleration column deflects ions through controlled arcuate paths to filter ions from the beam with the mass analyzing slit while allowing certain other ions to enter the ion acceleration column. In order to achieve higher ion energies and effectively penetrate a substrate, the ions must be accelerated in the ion accelerating column. Quadrupole lenses are installed at locations along the beam line for optimal beam focusing according to the ion optics requirements, and electronic scanning systems are used for correction of the lens aberration and for scanning the ion beam [250 - 252].

\subsection{Introduction to Chemical Vapor Deposition}

Chemical Vapor Deposition (CVD) is a process to produce high quality, purity, and performance solid materials. In a CVD reaction chamber, the depositing materials are delivered in a gas phase called "gas precursors" flow into the vicinity of the substrate, where they decompose and react to deposit a film on the substrate. Decomposition of depositing gases can normally be induced either by temperature or plasma. The temperature-induced CVD is called Rapid Thermal CVD (RTCVD), and the plasmainduced CVD is called Plasma-Enhanced CVD (PECVD). Typically, RTCVD processes in the range of 300 to $900{ }^{\circ} \mathrm{C}$, and PECVD processes at $300{ }^{\circ} \mathrm{C}$, but there is no fundamental lower limit to deposition temperature [245, 246]. 


\subsection{Phosphorus-Doped HOPG}

For testing samples where ion implantation doping was used to implant phosphorus followed by argon atoms (sample \# 1 - 40, 55 - 58, and 201 - 225), the implantation was performed by CuttingEdge Ions, LLC. According to the result from the works of Larkins et al., phosphorus-implanted graphite and graphene showed evidence of superconductivity. Thus, this research which is the continuation of [56], the phosphorus-implanted samples would absolutely be considered.

After phosphorus was implanted into the HOPG substrates, argon was used to deliberately damage the phosphorus-implanted HOPG to create vacancy columns for pinning of magnetic flux. The energy of the implanted phosphorus was $10 \mathrm{keV}$ with doses ranging from $6 \times 10^{7} \mathrm{~cm}^{-2}$ to $1.2 \times 10^{9} \mathrm{~cm}^{-2}$. In the samples where argon was implanted, an energy of $5 \mathrm{keV}$ and a dose of $1.2 \times 10^{8} \mathrm{~cm}^{-2}$ were used for the argon implantation. The implantation was performed at a room temperature while the substrates were placed at 7 degrees of tilt to avoid channeling.

Figure 5.5 is the SRIM simulation of phosphorus ions implanted in graphite. The simulation was used predict the implant ranges and straggles, determine the final atom position after implantation, and avoid implant saturation. 


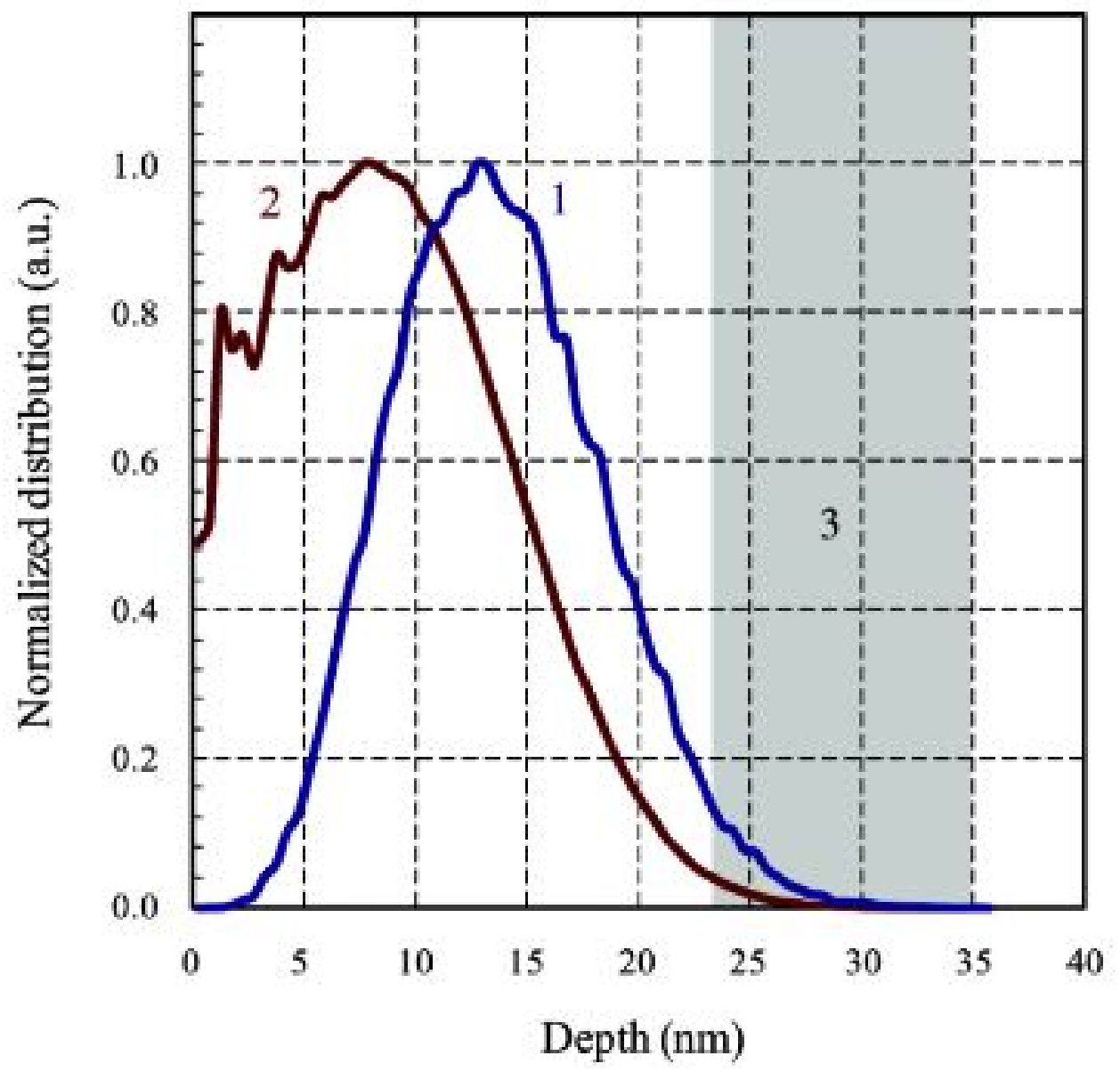

Figure 5.5: SRIM simulated distribution.

(1) Phosphorus ions implanted in graphite at $E_{p}=10 \mathrm{keV}$.

(2) Damage in graphite lattice caused by the implant.

(3) The probable region of effect [55]. 
Table 5.1: The list of ion-implanted HOPG samples \# 1 - 40 and 55 - 58 .

\begin{tabular}{|c|c|c|c|c|c|c|c|c|}
\hline $\begin{array}{l}\text { HOPG } \\
\text { sample } \\
\text { number }\end{array}$ & Type & $\begin{array}{c}\text { Substrate } \\
\text { manufacturer }\end{array}$ & $\begin{array}{c}\text { Implant } \\
\text { (ion implantation) }\end{array}$ & $\begin{array}{c}\text { Energy } \\
(\mathrm{KeV})\end{array}$ & $\begin{array}{c}\text { Current } \\
(\mathbf{n A} \mathbf{A})\end{array}$ & $\begin{array}{l}\text { Implantation } \\
\text { dose }\left(\mathrm{cm}^{-2}\right)\end{array}$ & $\begin{array}{c}\text { Dopant / }{ }^{\circ} \mathbf{C} \\
\text { (thermal diffusion) }\end{array}$ & Comments \\
\hline 001 & $\mathrm{ZYH}$ & MikroMasch & boron & 10 & - & $4.0 \times 10^{8}$ & $\mathrm{~B}\left(1200^{\circ} \mathrm{C}\right)$ & New B-diffusant B154 \\
\hline 002 & $\mathrm{ZYH}$ & MikroMasch & boron & 10 & - & $8.0 \times 10^{8}$ & $\mathrm{P}\left(1200^{\circ} \mathrm{C}\right)$ & New P-diffusant P509 \\
\hline 003 & $\mathrm{ZYH}$ & MikroMasch & boron & 10 & - & $2.0 \times 10^{9}$ & $\mathrm{~B}\left(1200^{\circ} \mathrm{C}\right)$ & New B-diffusant B154 \\
\hline 004 & $\mathrm{ZYH}$ & MikroMasch & boron & 10 & - & $4.0 \times 10^{9}$ & - & \\
\hline 005 & $\mathrm{ZYH}$ & MikroMasch & phosphorus & 10 & - & $1.2 \times 10^{8}$ & - & \\
\hline 006 & $\mathrm{ZYH}$ & MikroMasch & phosphorus & 10 & - & $3.0 \times 10^{8}$ & - & \\
\hline 007 & $\mathrm{ZYH}$ & MikroMasch & phosphorus & 10 & - & $5.9 \times 10^{8}$ & none $\left(900^{\circ} \mathrm{C}\right)$ & \\
\hline 008 & $\mathrm{ZYH}$ & MikroMasch & phosphorus & 10 & - & $1.2 \times 10^{9}$ & none $\left(900^{\circ} \mathrm{C}\right)$ & \\
\hline 009 & $\mathrm{ZYH}$ & MikroMasch & phosphorus & 10 & - & $1.2 \times 10^{8}$ & - & \\
\hline 010 & $\mathrm{ZYH}$ & MikroMasch & phosphorus & 10 & - & $6 \times 10^{7}$ & - & \\
\hline 011 & $\mathrm{ZYH}$ & NT-MDT & phosphorus & 10 & - & $1.2 \times 10^{8}$ & - & \\
\hline 012 & $\mathrm{ZYH}$ & NT-MDT & phosphorus & 10 & - & $6 \times 10^{7}$ & - & \\
\hline 013 & $\mathrm{ZYH}$ & NT-MDT & phosphorus & 5 & - & $1.2 \times 10^{8}$ & - & \\
\hline 014 & $\mathrm{ZYH}$ & NT-MDT & phosphorus & 5 & - & $6 \times 10^{7}$ & - & \\
\hline 015 & $\mathrm{ZYH}$ & NT-MDT & - & - & - & - & - & Scratch sample \\
\hline 016 & $\mathrm{ZYH}$ & NT-MDT & phosphorus & 10 & 1.04 & $1.2 \times 10^{8}$ & $\mathrm{~B}\left(900^{\circ} \mathrm{C}\right)$ & New B-diffusant B154 \\
\hline 017 & $\mathrm{ZYH}$ & NT-MDT & phosphorus & 10 & 1.04 & $1.2 \times 10^{8}$ & $\mathrm{P}\left(900^{\circ} \mathrm{C}\right)$ & New P-diffusant P509 \\
\hline 018 & $\mathrm{ZYH}$ & NT-MDT & phosphorus & 10 & 1.04 & $1.2 \times 10^{8}$ & - & \\
\hline 019 & $\mathrm{ZYH}$ & NT-MDT & phosphorus & 10 & 1.04 & $1.2 \times 10^{8}$ & - & \\
\hline 020 & $\mathrm{ZYH}$ & NT-MDT & phosphorus & 10 & 1.04 & $1.2 \times 10^{8}$ & - & \\
\hline 021 & $\mathrm{ZYH}$ & NT-MDT & phosphorus & 10 & 1.04 & $1.2 \times 10^{8}$ & - & \\
\hline 022 & $\mathrm{ZYH}$ & NT-MDT & phosphorus & 10 & 2 & $1.2 \times 10^{8}$ & - & \\
\hline 023 & $\mathrm{ZYH}$ & NT-MDT & phosphorus & 10 & 2 & $1.2 \times 10^{8}$ & - & \\
\hline 024 & ZYH & NT-MDT & phosphorus & 10 & 2 & $1.2 \times 10^{8}$ & - & \\
\hline 025 & $\mathrm{ZYH}$ & NT-MDT & phosphorus & 10 & 2 & $1.2 \times 10^{8}$ & - & \\
\hline 026 & $\mathrm{ZYH}$ & NT-MDT & sulphur & 10 & 2 & $6.0 \times 10^{7}$ & - & \\
\hline 027 & ZYH & NT-MDT & sulphur & 10 & 2 & $1.2 \times 10^{8}$ & - & \\
\hline 028 & ZYH & NT-MDT & sulphur & 10 & 4 & $3.0 \times 10^{8}$ & - & \\
\hline 029 & $\mathrm{ZYH}$ & NT-MDT & sulphur & 10 & 4 & $6.0 \times 10^{8}$ & $\mathrm{~B}\left(1200^{\circ} \mathrm{C}\right)$ & Old B-diffusant B154 \\
\hline 030 & $\mathrm{ZYH}$ & NT-MDT & phosphorus & 10 & 2 & $1.2 \times 10^{8}$ & - & \\
\hline 031 & ZYA & NT-MDT & phosphorus & 10 & 1.04 & $1.2 \times 10^{8}$ & $\mathrm{~B}\left(900^{\circ} \mathrm{C}\right)$ & Old B-diffusant B154 \\
\hline 032 & ZYA & NT-MDT & phosphorus & 10 & 1.04 & $1.2 \times 10^{8}$ & $\mathrm{P}\left(900^{\circ} \mathrm{C}\right)$ & Old P-diffusant \\
\hline 033 & ZYA & NT-MDT & - & - & - & - & $\mathrm{B}\left(1000^{\circ} \mathrm{C}\right)$ & Old B-diffusant B154 \\
\hline 034 & ZYA & NT-MDT & - & - & - & - & none $\left(1200^{\circ} \mathrm{C}\right)$ & \\
\hline 035 & $\mathrm{ZYH}$ & MikroMasch & phosphorus & 10 & 2 & $1.2 \times 10^{8}$ & - & \\
\hline 036 & $\mathrm{ZYH}$ & MikroMasch & phosphorus & 10 & 2 & $1.2 \times 10^{8}$ & - & \\
\hline 037 & $\mathrm{ZYH}$ & MikroMasch & phosphorus & 10 & 2 & $1.2 \times 10^{8}$ & - & \\
\hline 038 & $\mathrm{ZYH}$ & MikroMasch & phosphorus & 10 & 2 & $1.2 \times 10^{8}$ & - & \\
\hline 039 & $\mathrm{ZYH}$ & MikroMasch & phosphorus & 10 & 2 & $1.2 \times 10^{8}$ & - & \\
\hline 040 & $\mathrm{ZYH}$ & MikroMasch & - & - & - & - & - & Scratch sample \\
\hline 055 & ZYB & NT-MDT & $\mathrm{Ca}$ & 10 & & $2.5 \mathrm{E} 7$ & - & \\
\hline 056 & ZYB & NT-MDT & $\mathrm{Ca}$ & 10 & & $1.2 \mathrm{E} 8$ & - & \\
\hline 057 & ZYB & NT-MDT & $\mathrm{Ca}$ & 10 & & $6.0 \mathrm{E} 8$ & - & \\
\hline 058 & ZYB & NT-MDT & $\mathrm{Ca}$ & 10 & & $3.0 \mathrm{E} 9$ & - & \\
\hline
\end{tabular}


The samples \# 201 - 225 are the most recent phosphorus and argon implanted samples ordered from CuttingEdge Ions, LLC. To ion implant, 25 HOPG substrates were set up as a 5 by 5 array attached to a silicon carrier wafer via double-sided carbon tape as shown in Figure 5.6. The numbers of the substrates were assigned as in Figure 5.7. The specifications of the implantation are listed in Figure 5.8.

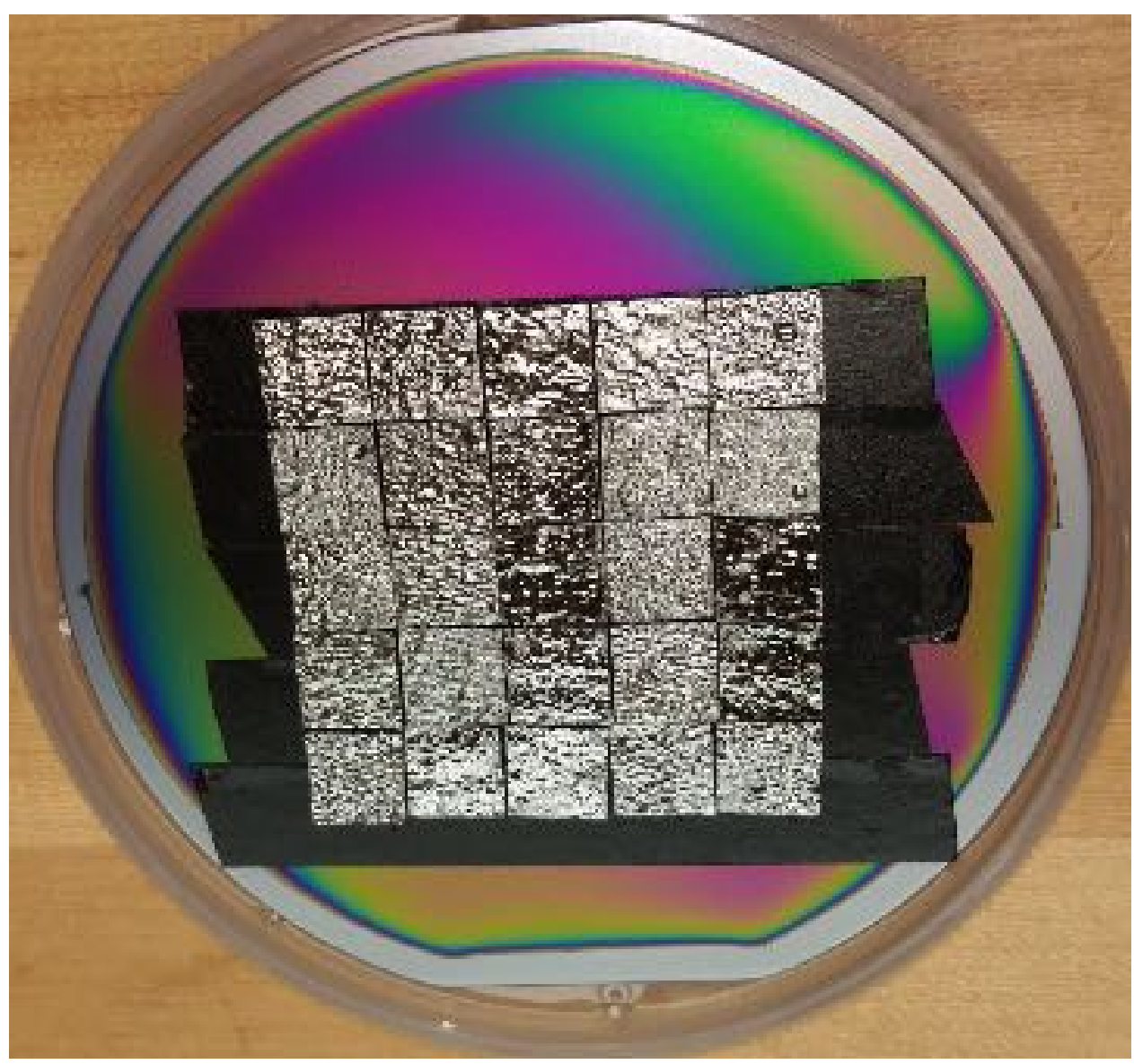

Figure 5.6: A 5 by 5 array of HOPG substrates prepared for ion implantation. 


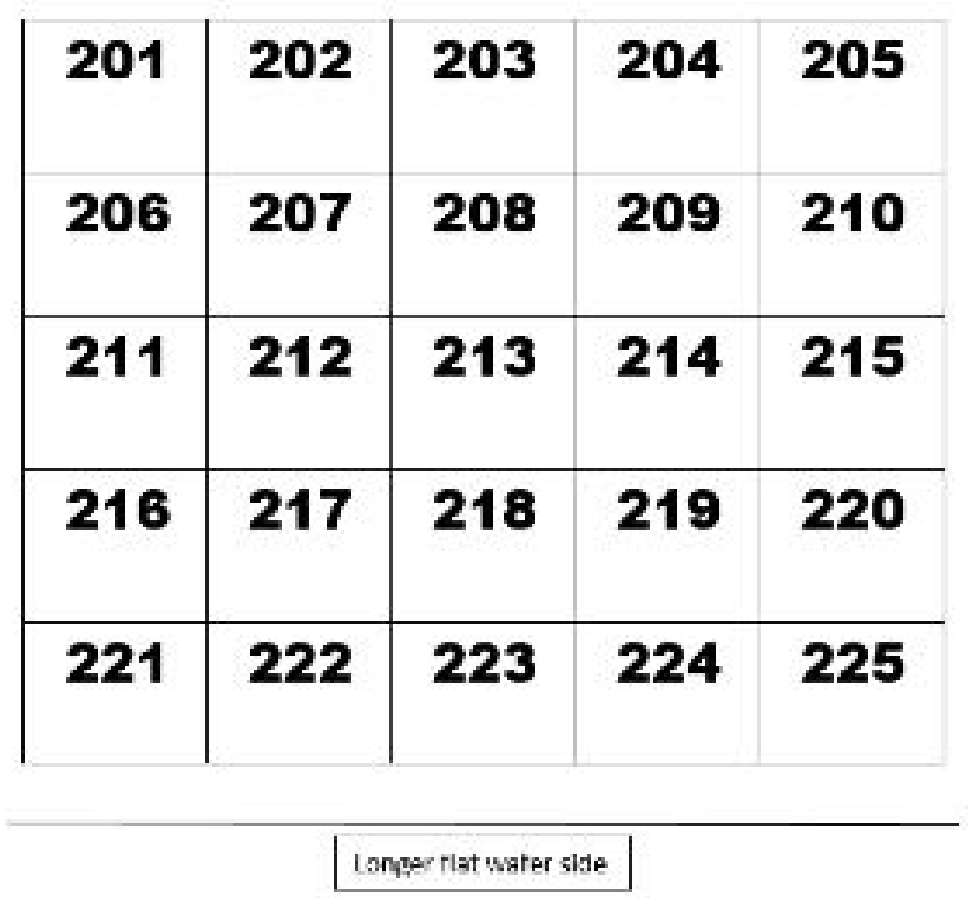

Figure 5.7: The assigned numbers of HOPG substrates.

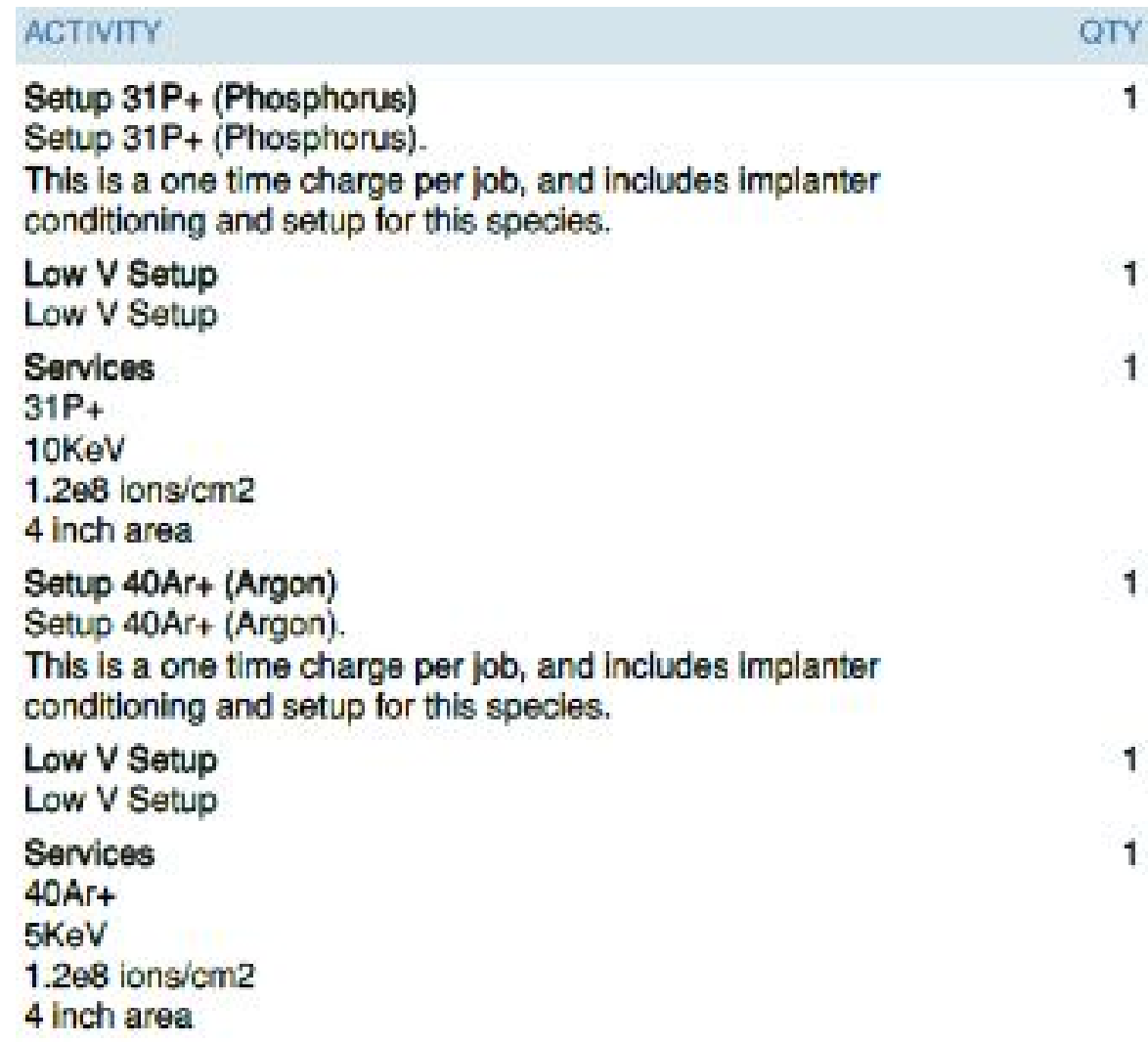

Figure 5.8: The specifications of implantation of the samples \# $201-225$. 
In Figure 5.9, the computed depth profile of the ion-implanted phosphorous in graphite and the computed damage profile are shown in curves 1 and 2 . The corresponding computed profiles for the ion-implanted argon in graphite are shown in curves 3 and 4 . Since there is no characterized implantation model for the stopping power of HOPG as a substrate we selected the closest substrate material in the library, graphite, for the simulation.

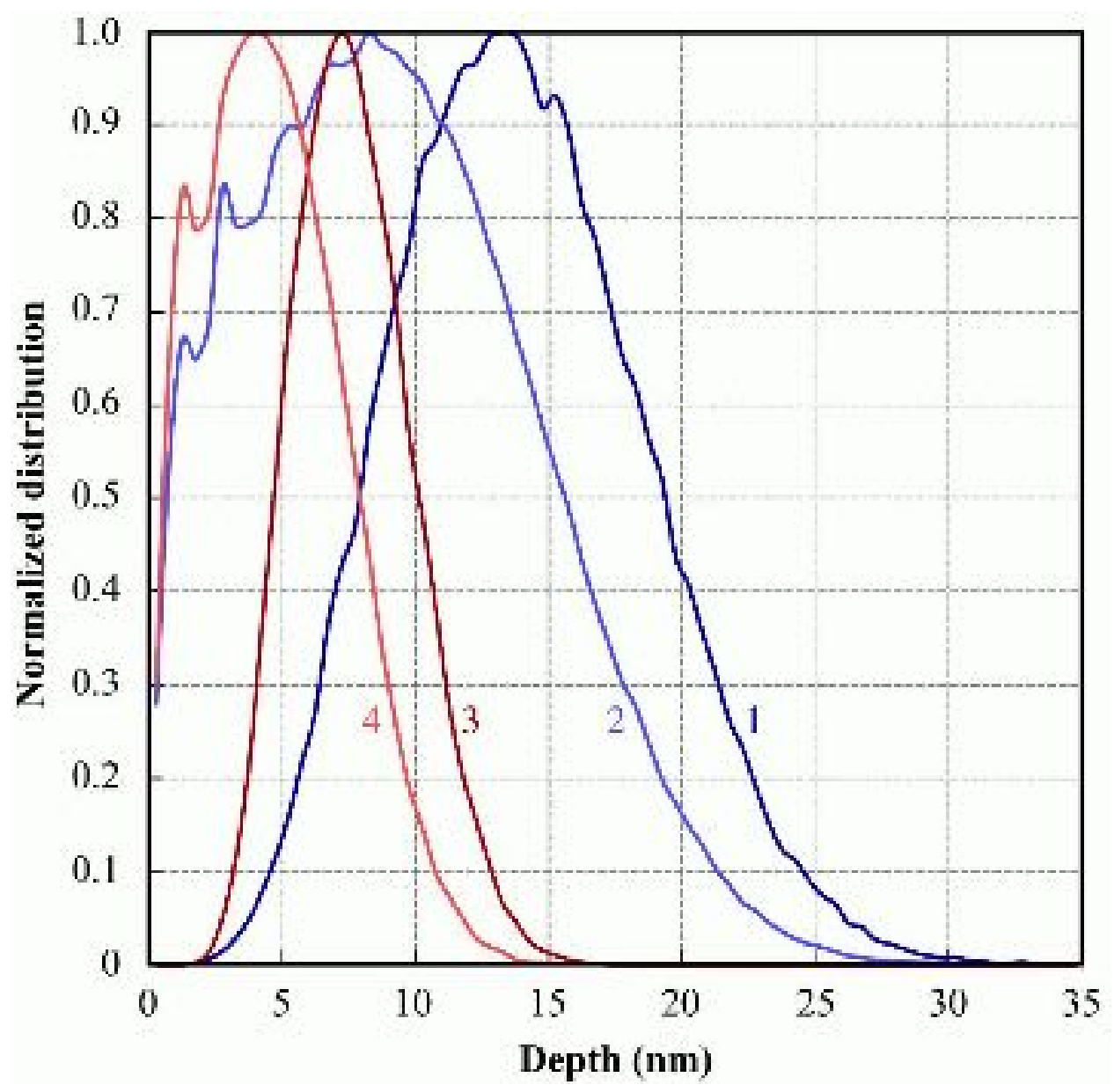

Figure 5.9: This represents the simulated normalized distributions of implanted atoms and lattice damage caused by implanted atoms versus depth in HOPG.

Curve 1: The calculated distribution of the implanted phosphorus.

Curve 2: The computed damage caused by the implanted phosphorus.

Curve 3: The calculated distribution of the implanted argon.

Curve 4 : The computed damage caused by the implanted argon [56]. 
For the phosphorus doped-while-grown samples (sample \# 151, 163, and 170), the method used to deposit phosphorus into the doped-while-grown samples on the substrates was PECVD. Deposition is achieved by introducing reactant gases between parallel top and bottom electrodes and an RF power supply. The capacitive coupling between the electrodes excites the reactant gases into a plasma, which induces a chemical reaction and results in the reaction product being deposited on the substrate. The substrate is typically heated to $600{ }^{\circ} \mathrm{C}$ or $800{ }^{\circ} \mathrm{C}$, depending on the specific requirements [215].

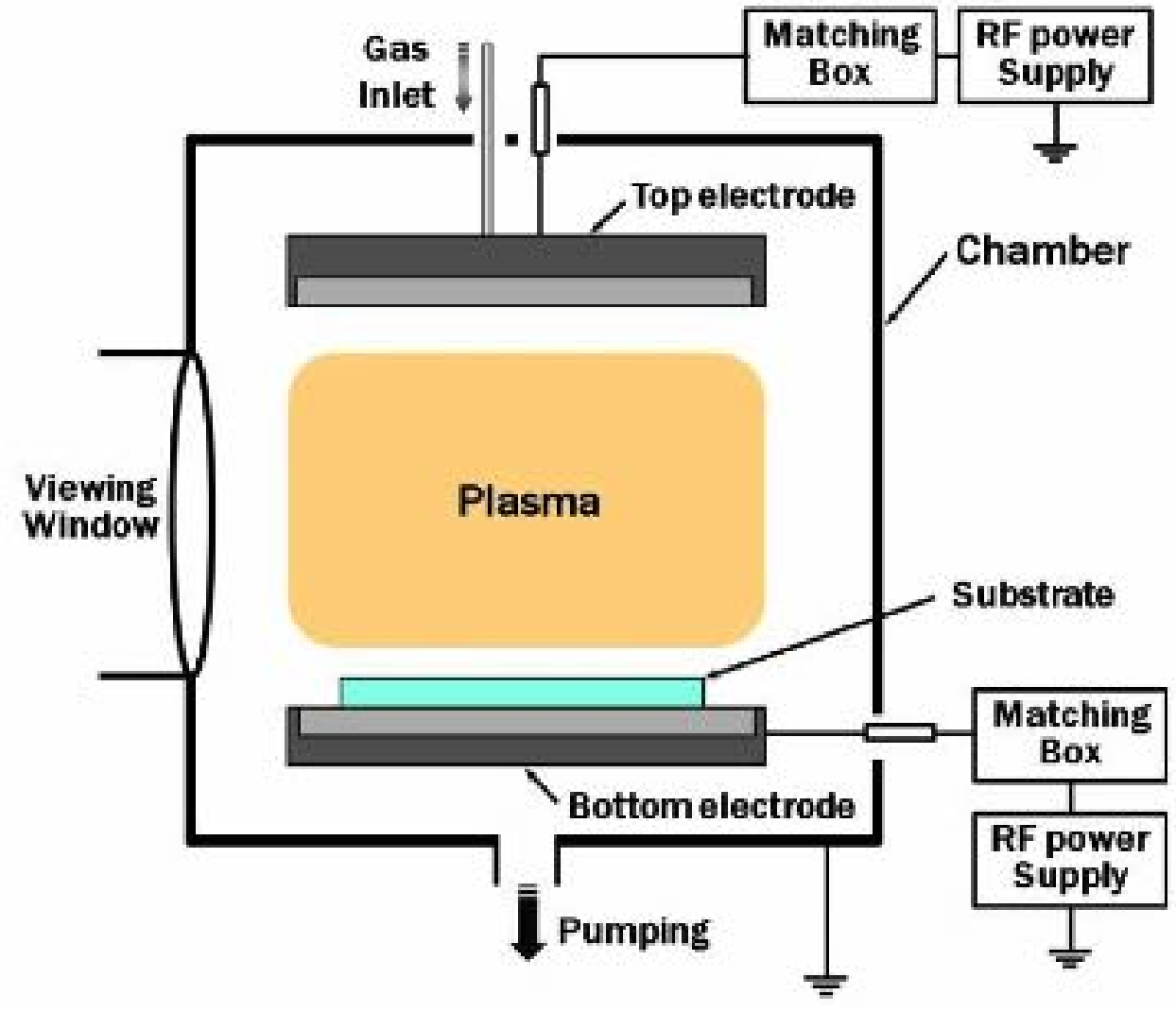

Figure 5.10: A schematic of a PECVD system. Reprinted with permission from Jeon Geon Han [247].

In this dissertation, the method of producing phosphorus doped-while-grown graphene on HOPG was modelled after the CVD growth of graphene on a sheet of a transition metal such as copper $(\mathrm{Cu})$ or Nickel $(\mathrm{Ni})$. The process of CVD grown graphene 
on $\mathrm{Cu}$ or $\mathrm{Ni}$ usually uses methane $\left(\mathrm{CH}_{4}\right)$ and molecular hydrogen $\left(\mathrm{H}_{2}\right)$ as gas sources. A hydrocarbon compound such as methane has highly stable saturated molecules, thus the dehydrogenation in the gas phase of $\mathrm{CH}_{x}$ to $\mathrm{CH}_{x-1}$ is highly endothermic. At an elevated temperature, hydrocarbon atoms are thermally decomposed, and surface absorption, desorption, or segregation of carbon occurs depending on carbon-metal solubility for graphene growth. Homogeneous layer/layers of graphene can be grown by simply annealing the metallic substrate due to graphitization of amorphous carbon previously deposited on the surface or coming from furnace walls desorption. By reducing the partial pressure of methane gas in the overall gas flow, the concentration of carbon precursors can be made to be low enough to favor the growth of graphene honeycomb structure over nucleation $[256-260]$.

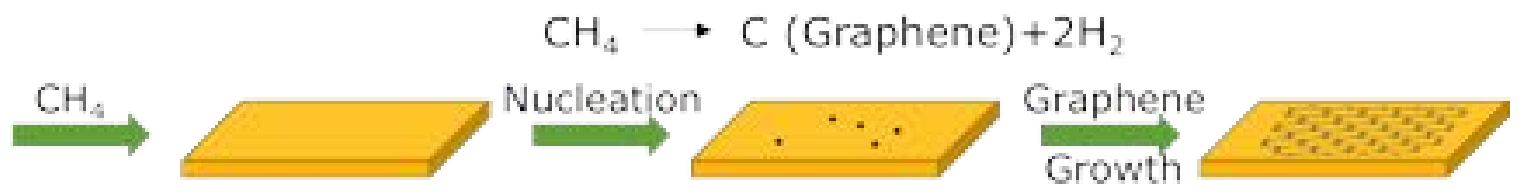

Figure 5.11: The CVD process includes the decomposition of methane to use the carbon needed in order to grow on the substrate surface. Reprinted with permission from ACS Material, LLC [259].

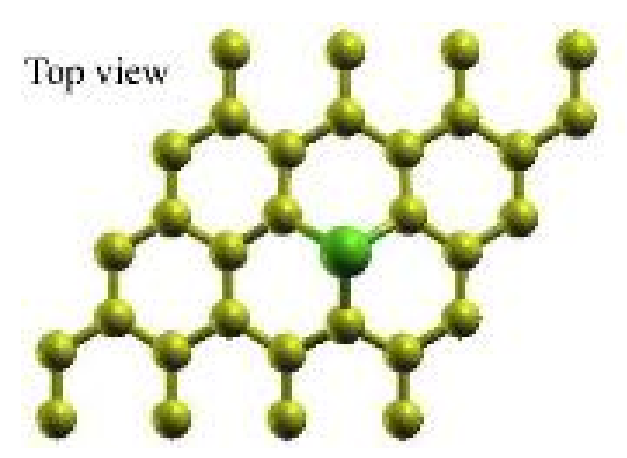

Side vicw

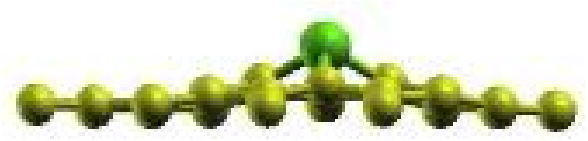

Figure 5.12: The most stable relaxed structures of phosphorus-doped graphene. The yellow and green balls represent the carbon and phosphorus atoms, respectively. Reprinted with permission from Jianmin Yuan, National University of Defense Technology [261]. 
To create the doped samples, $\mathrm{CH}_{4}$ was used as the main gas source to release carbon atoms by dehydrogenation. $\mathrm{H}_{2}$ cleaned and crystallized the substrates as diluent gas of the carbon precursor. Indication of the important role of hydrogen in determining the graphene growth kinetics and in limiting the graphene thickness comes from previous observations such as that when the fraction of $\mathrm{CH}_{4}$ with respect to $\mathrm{H}_{2}$ is increased, the graphene growth on $\mathrm{Cu}$ is no longer self-limiting [260]. $\mathrm{Ar}$ assists the annealing by providing a protective atmosphere. Annealing a substrate under an inert gas atmosphere reduces the presence of oxygen to avoid or minimize the chance of oxidation or contamination. $\mathrm{PH}_{3}$ is used to provide phosphorus dopant atoms via dehydrogenation to attain the doped-while-grown graphene.

The phosphorus doped-while-grown graphene samples were prepared via a PECVD on HOPG bulks under an argon atmosphere with a plasma power of $30 \mathrm{~W}$ for 15 minutes (900 seconds). Bulks of HOPG were used as bed substrates because removing the phosphorus doped-while-grown layers from the HOPG substrates was easier than removing them from $\mathrm{Cu}$ or $\mathrm{Ni}$ sheets. Due to the weak Van der Waals force of HOPG, a mechanical exfoliation technique to remove layers of graphene from the substrates was simply performed by using Kapton ${ }^{\circledR}$ adhesive tape. To grow phosphorus doped-whilegrown graphene on HOPG bulks required methane at $20 \mathrm{sccm}$ (Standard Cubic Centimeters per Minute), hydrogen at $10 \mathrm{sccm}$, argon at $14 \mathrm{sccm}$, and $0.1 \%$ phosphine in an argon carrier gas at $5 \mathrm{sccm}$ as the ratio of the gas mixture as shown in Table 5.2.

In order to redress the interlayer spacing of the HOPG and incinerate unwanted particles, the HOPG substrates were graphitized at the temperature of $900{ }^{\circ} \mathrm{C}$ in the reaction chamber under the vacuum pressure of $2 \times 10^{-6}$ Torr for 30 minutes before the deposition 
process. Then, the PECVD took place when the gas inlets allowed the gas mixture to flow into the chamber when the pressure and temperature reached the requirements.

Table 5.2: The ratio of the gas mixture to grow phosphorus doped-while-grown graphene on HOPG.

\begin{tabular}{|c|c|c|c|}
\hline \multicolumn{4}{|c|}{ Flow Rate (sccm) } \\
\hline $\begin{array}{c}\text { Methane } \\
\left(\boldsymbol{C H}_{\mathbf{4}}\right)\end{array}$ & $\begin{array}{c}\text { Hydrogen } \\
\left(\boldsymbol{H}_{\mathbf{2}}\right)\end{array}$ & $\begin{array}{c}\text { Argon } \\
(\boldsymbol{A r})\end{array}$ & $\begin{array}{c}\text { Phosphine* } \\
\left(\boldsymbol{P H}_{\mathbf{3}}\right)\end{array}$ \\
\hline 20 & 10 & 14 & 5 \\
\hline
\end{tabular}

* Phosphine was 1000 ppm (Parts per Million) in argon.

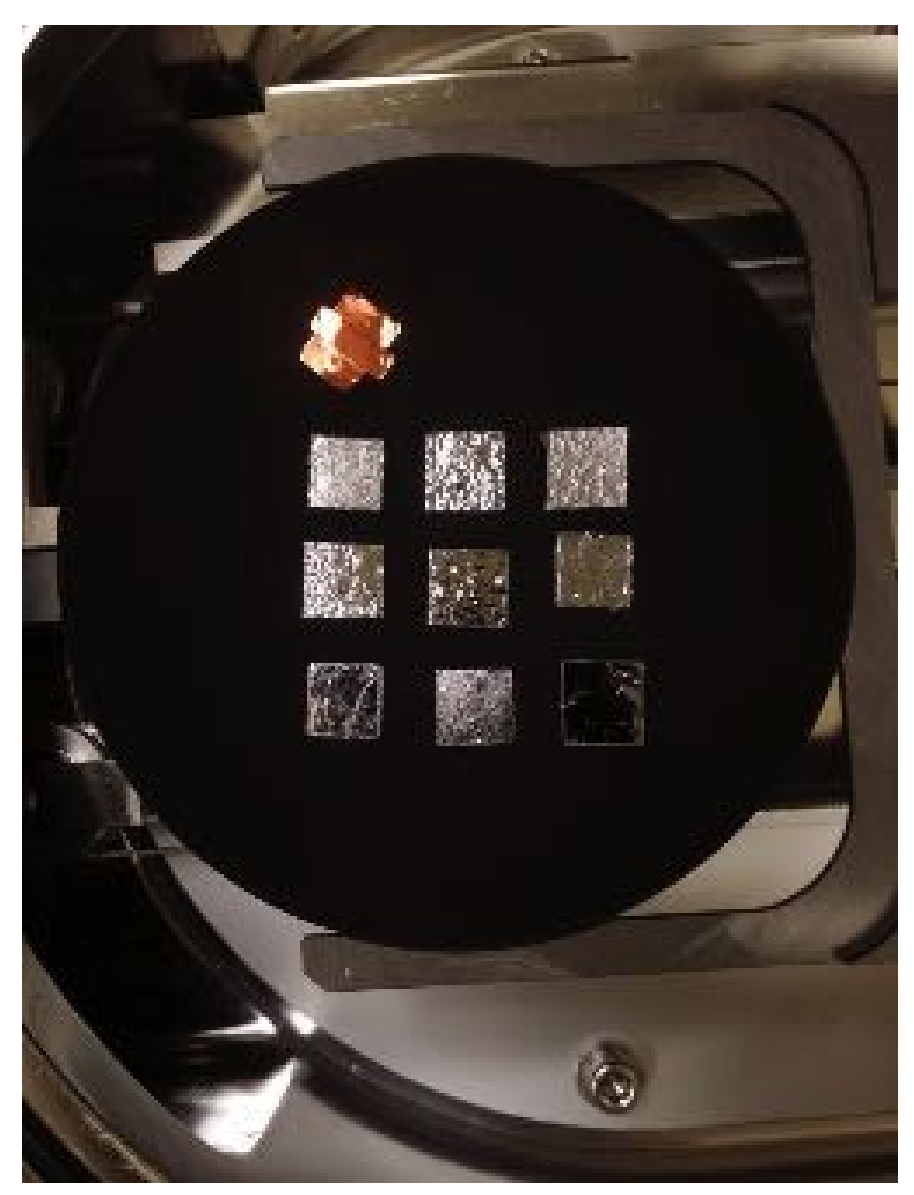

Figure 5.13: An array of HOPG samples loaded on the PECVD machine's sample holder. Two sheets of copper were placed at the upper left corner of the array to indicate the position of the first sample in the series [57]. 


\subsection{Tables of Symbols and Acronyms}

Table 5.3: Table of Symbols.

\begin{tabular}{|c|l|c|}
\hline Symbols & \multicolumn{1}{|c|}{ Description } & Value \\
\hline $\mathrm{CH}_{4}$ & The chemical formula of methane. & - \\
\hline $\mathrm{H}_{2}$ & The chemical formula of molecular hydrogen. & - \\
\hline $\mathrm{PH}_{3}$ & The chemical formula of phosphine. & - \\
\hline
\end{tabular}

Table 5.4: Table of Acronyms.

\begin{tabular}{|c|c|}
\hline Acronym & Description \\
\hline $2 \mathrm{D}$ & 2-dimensional \\
\hline $\mathrm{Ar}$ & Argon \\
\hline $\mathrm{Cu}$ & Copper \\
\hline CVD & Chemical Vapor Deposition \\
\hline HOPG & Highly Ordered Pyrolytic Graphite \\
\hline $\mathrm{MC}$ & Monte Carlo \\
\hline $\mathrm{Ni}$ & Nickel \\
\hline ppm & Parts per Million \\
\hline PECVD & Plasma-Enhanced Chemical Vapor Deposition \\
\hline RF & Radio Frequency \\
\hline RTCVD & Rapid Thermal Chemical Vapor Deposition \\
\hline $\mathrm{sccm}$ & Standard Cubic Centimeters per Minute \\
\hline SRIM & Simulation of Ranges of Ions in Matter \\
\hline TEM & Transmission Electron Microscopy \\
\hline XRD & X-Ray Diffraction \\
\hline
\end{tabular}




\section{Chapter 6}

Results, Discussion, Conclusions, and Future Work 


\subsection{Results}

The key to understanding the potential causes for the observed results is to examine number of $R v s . T$ characteristics, shown in Figure 6.1, of similarly exfoliated films taken from bulk HOPG implanted using phosphorous ions.
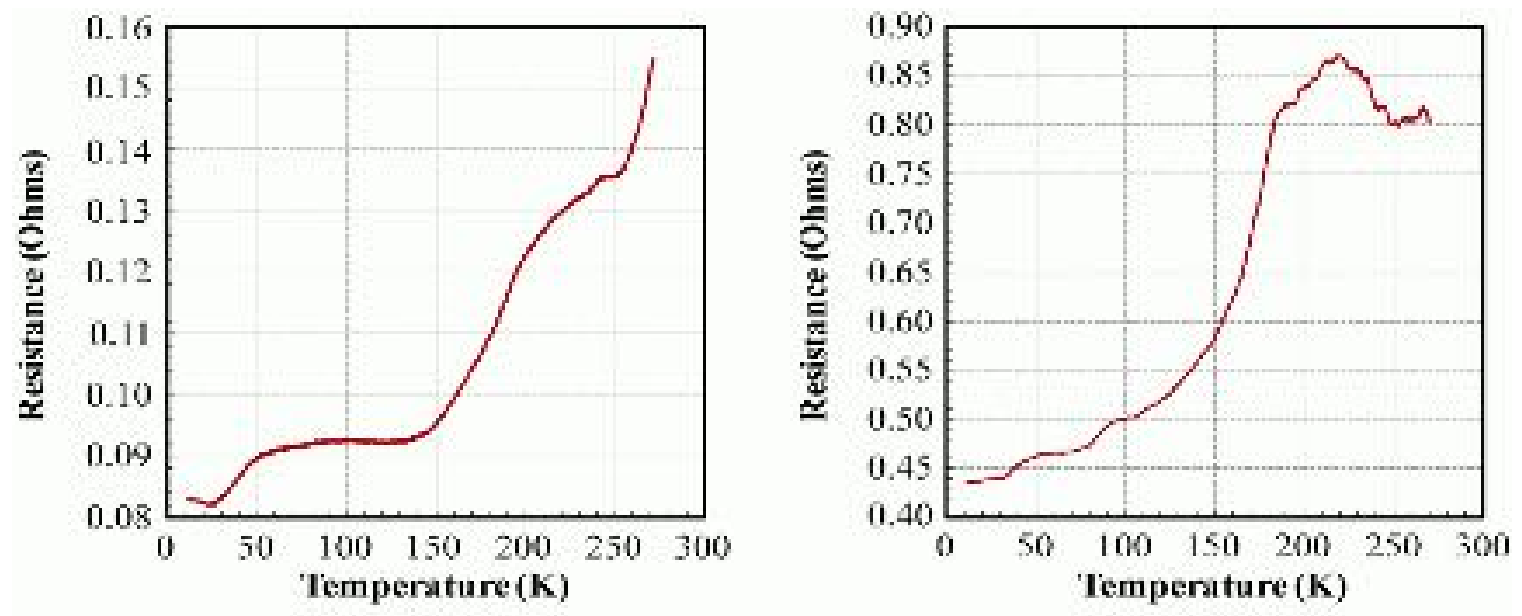

a

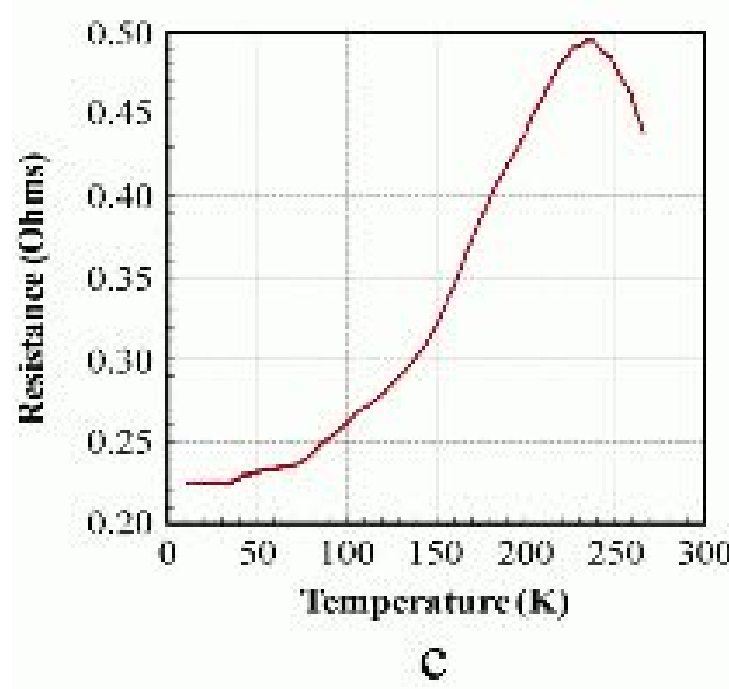

b

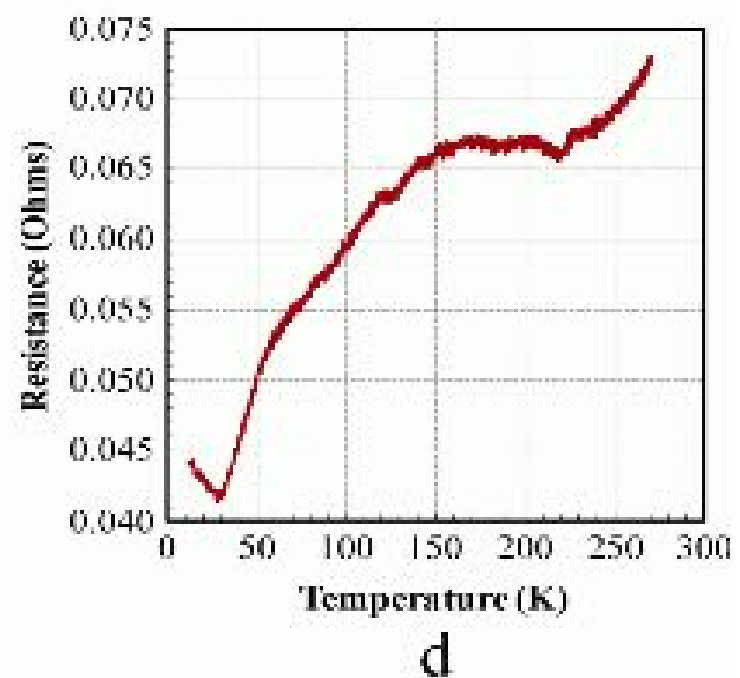

Figure 6.1: Measured $R$ vs. $T$ of four thin films exfoliated from phosphorus implanted $\left(E_{p}=10 \mathrm{keV}\right.$, dose $2 \times 10^{8} \mathrm{~cm}^{-2}$ ) HOPG samples. (a) HOPG-008, layer 3; (b) HOPG-019, layer 3; (c) HOPG-019, layer 6; (d) HOPG-021, layer 7. The layer numbers indicate the number of multilayers peeled from the host sample, i.e. layer 7 would be the $7^{\text {th }}$ layer exfoliated from that sample [56]. 
Comparing characteristics (a) through (d) in Figure 6.1, there is a step-in resistance at a temperature of approximately $50-60 \mathrm{~K}$ in all the samples. When the data is closely examined, there is a second resistance step at temperatures approximately between $100-$ $120 \mathrm{~K}, 150-180 \mathrm{~K}$, and $200-240 \mathrm{~K}$. These steps in the $R v s . T$ characteristics cause a suspicion that the features are due to magnetic vortex lattice melting and subsequent flux flow losses.

To see if additional lattice damage by neutral ion species could increase pinning, which could only occur if magnetic vortices were present, and reduce losses, a sample which had been previously implanted with phosphorous but had not yet been exfoliated was sent back for implantation with argon. This implantation was done at reduced energy $(5 \mathrm{keV})$ and the same dose as the phosphorous implantation $\left(2 \times 10^{8} \mathrm{~cm}^{-2}\right)$ to place the damage in front of the peak in the phosphorous.

The Superconducting QUantum Interference Device (SQUID) magnetometer measurements of a doped-while-grown exfoliated thin-film graphite is shown with the $R$ vs. $T$ plots for the same sample superposed in Figure 6.2. The plots consist of a Zero-FieldCooled (ZFC) magnetization run followed by a Field-Cooled (FC) magnetization run. The hysteresis loop in the ZFC to FC curves begins to open at a temperature of approximately $260 \mathrm{~K}$. The $R v s . T$ plots were done prior to the samples being tested the susceptibility versus temperature ( $S v s . T$ ). It is evident on the combined $R v s . T$ and $S v s . T$ plots that there is a charge carrier Berezinski-Kosterlitz-Thouless (BKT) transition at low temperatures, nominally below $T \sim 5 \mathrm{~K}$ in Figure 6.2. It is also clear that there is hysteresis in the susceptibility data up to temperatures in excess of $240 \mathrm{~K}$ which is qualitatively matched by the cooling versus warming of $R$ vs. $T$ data. The 5 Oersted (Oe) ZFC data 
exhibits a small but distinct downturn at a temperature of approximately $80-90 \mathrm{~K}$ which correlates with the inflection point in the $R v s . T$. This is the BKT transition for the magnetic vortices. Hence, for temperatures from approximately $80 \mathrm{~K}$ through $240 \mathrm{~K}$ the sample is in the flux flow regime.

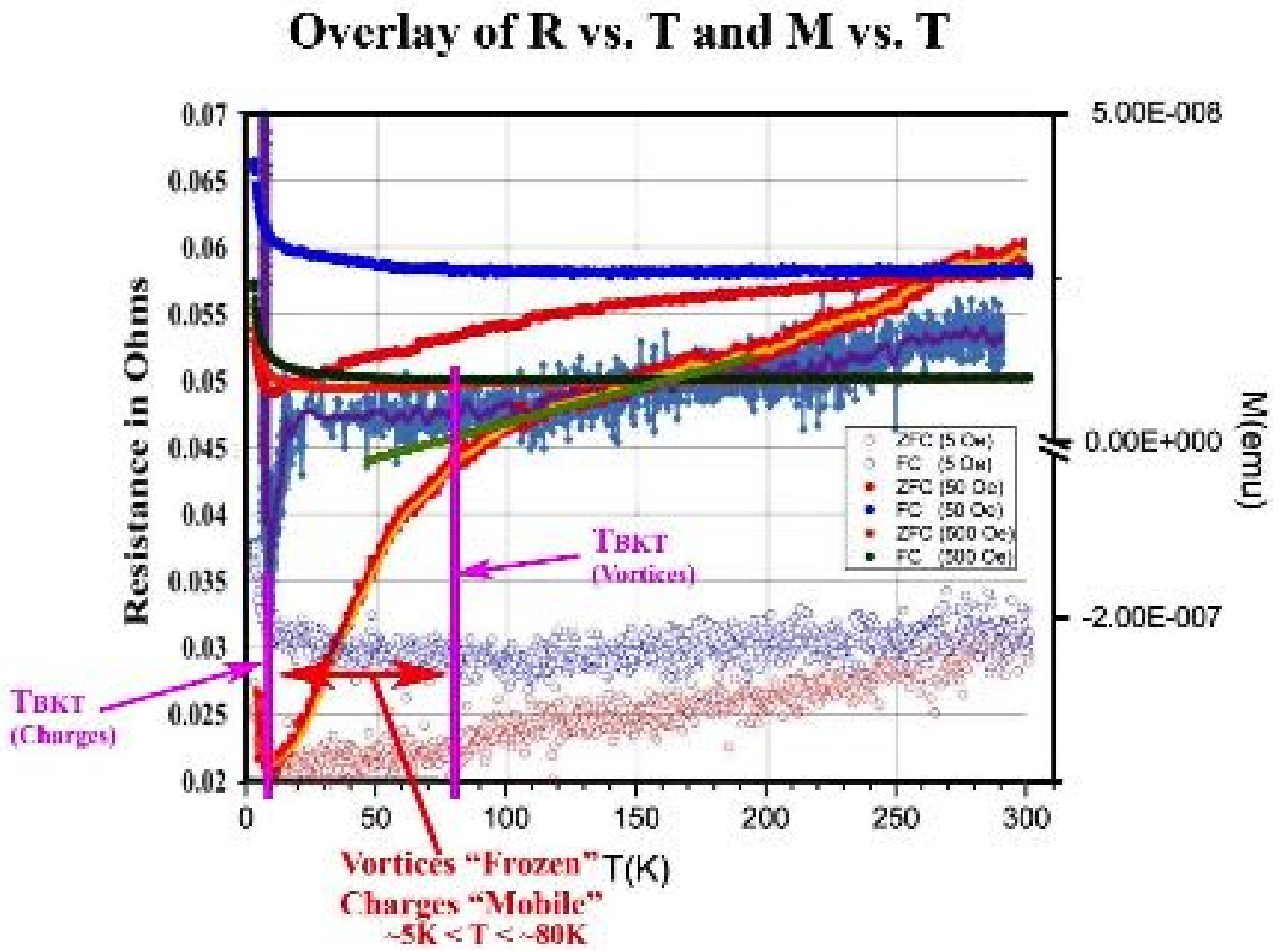

Figure 6.2: ZFC and FC magnetic susceptibility plots along with the associated cooling and warming $R$ vs. $T$ plots for a film exfoliated from a doped-while-grown PECVD sample. The susceptibility measurements are courtesy of Dr. Deng of Dr. Paul Chu's group at the University of Houston. Note the onset of the charge carrier BKT transition at $T \sim 5 \mathrm{~K}$ and the onset of the vortex BKT transition at $T \sim 80 \mathrm{~K}$.

A Raman spectrum of an exfoliated sample was done to estimate the film's thickness. Hall effect measurements have also been done on the same sample that was tested the Raman spectrum. 


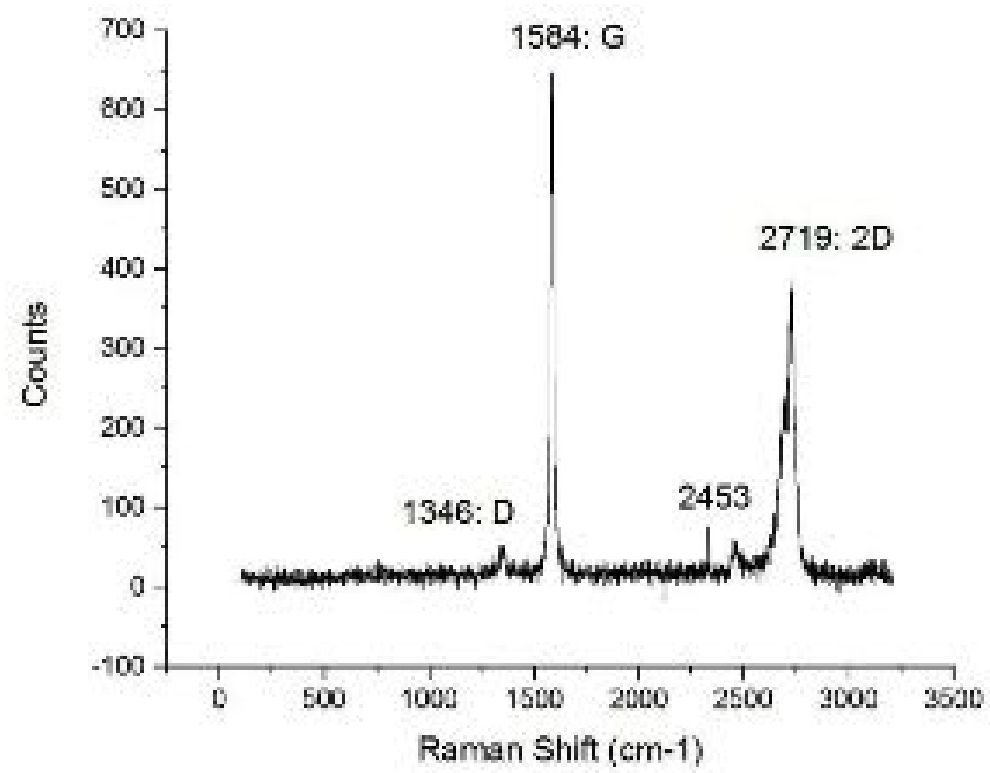

Figure 6.3: Raman spectra for a film on Kapton tape exfoliated from a phosphorous doped-while-grown CVD graphene sample. The peak ratios give a thickness of approximately 5 monolayers. Measurement courtesy of Laurens Henry Willems Van Beveren of Dr. Steven Prawer's group at the University of Melbourne [57].

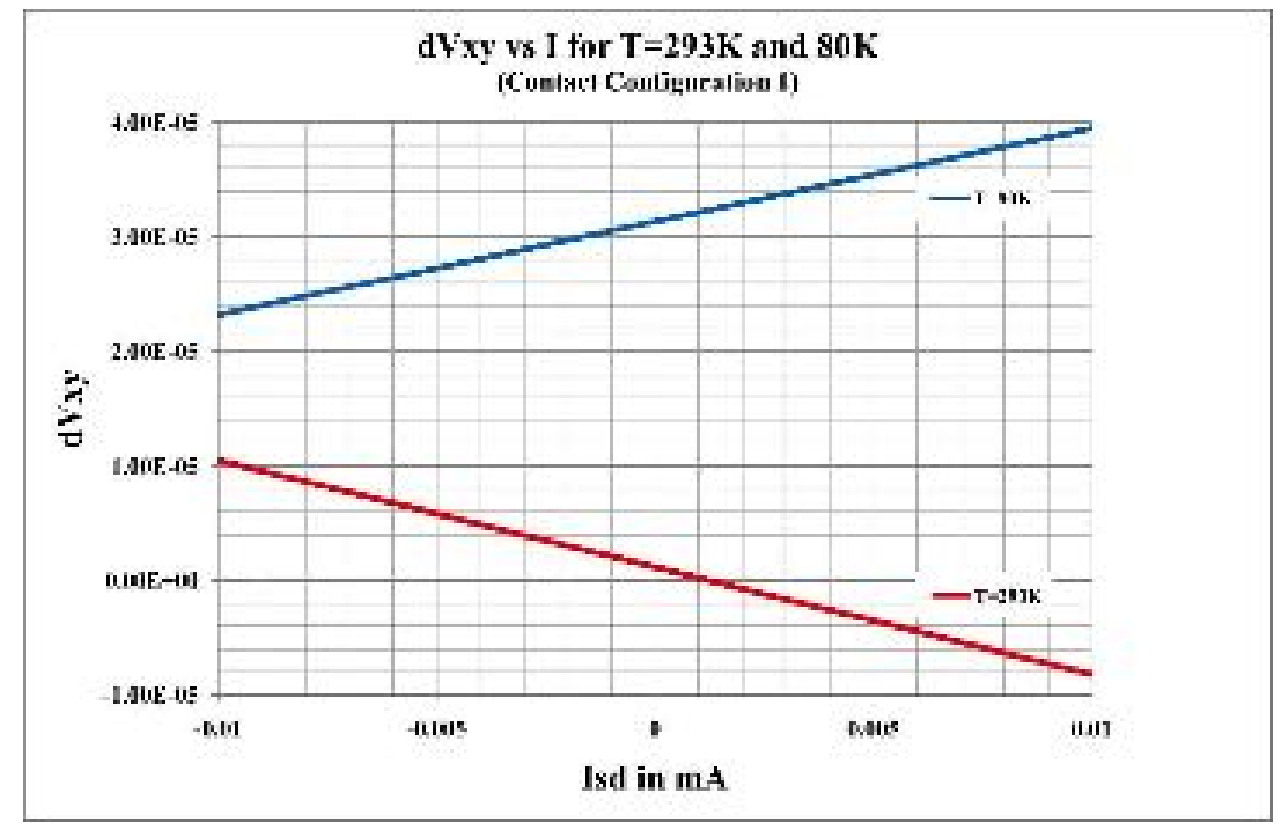

Figure 6.4: Hall effect at $T=300 \mathrm{~K}$ and $T=80 \mathrm{~K}$ for the same film on Kapton tape exfoliated from a phosphorous doped-while-grown CVD graphene sample as is shown in the Raman spectrum in Figure 6.3. Measurement courtesy of Laurens Henry Willems Van Beveren of Dr. Steven Prawer's group at the University of Melbourne [57]. 
The results from Dr. Prawer's group also included van der Pauw configuration Hall effect measurements at temperatures of $297 \mathrm{~K}$ and $80 \mathrm{~K}$ in Figure 6.4. Note that the change of sign in the Hall voltage is real and not due to instrumentation; neither the contacts nor the extraction calculations were altered between the two runs. The sign change is an indication of the possible presence of a vortex state superconductor and, although not conclusive in and of itself, a strong indicator that this material is a superconductor. This was one of the motivations for attempting to measure the Nernst effect in this material to conclusively determine if magnetic vortices were present.

The differential Nernst effect measurements $\left(V_{\text {Nernst }} / \Delta T\right.$ North subtracted from $V_{\text {Nernst }} / \Delta T$ South) for a small applied field are shown in Figure 6.4. The Nernst heater power was 1 Watt for the data in Figures 6.5 - 6.9. 


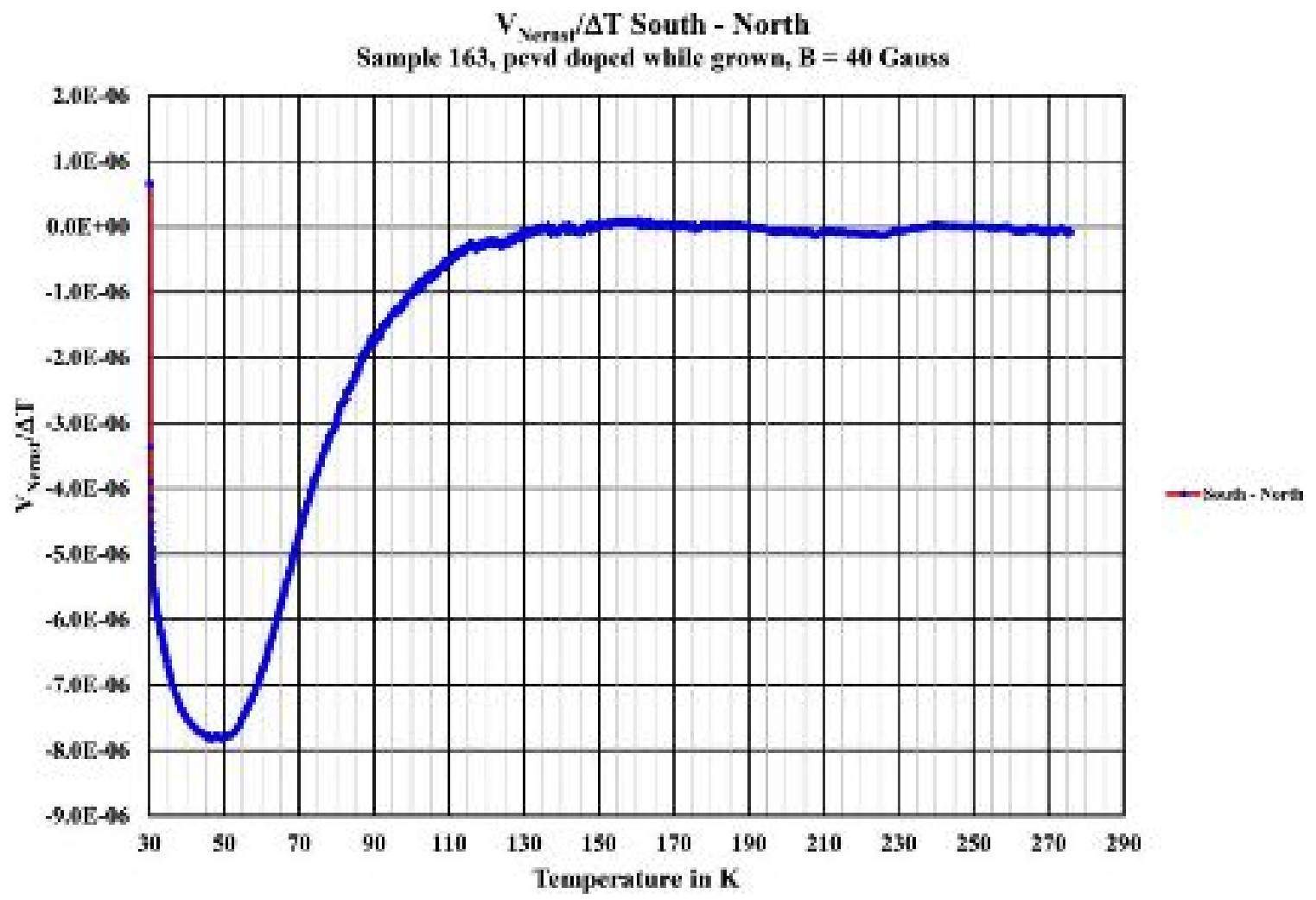

Figure 6.5: PECVD phosphorous doped-while-grown sample. Thermally driven Nernst voltage at 40 Gauss applied field. This is the difference of the Nernst signal with the magnetic field-oriented North Up through the sample subtracted from the South Up oriented field Nernst signal. This reduces any measurement errors due to thermoelectric or another non-field dependent phenomenon. Note the tilted negative peak at $T \sim 48 \mathrm{~K}$. This is characteristic of a vortex Nernst signal.

From the observation, there is a large peak (negative) in the South Up - North Up Nernst signals at approximately $50 \mathrm{~K}$ which extends up to a temperature of approximately $130 \mathrm{~K}$. They appear to be smaller peaks at temperatures above $100 \mathrm{~K}$ as well. Figure 6.6 displays this region more clearly. 


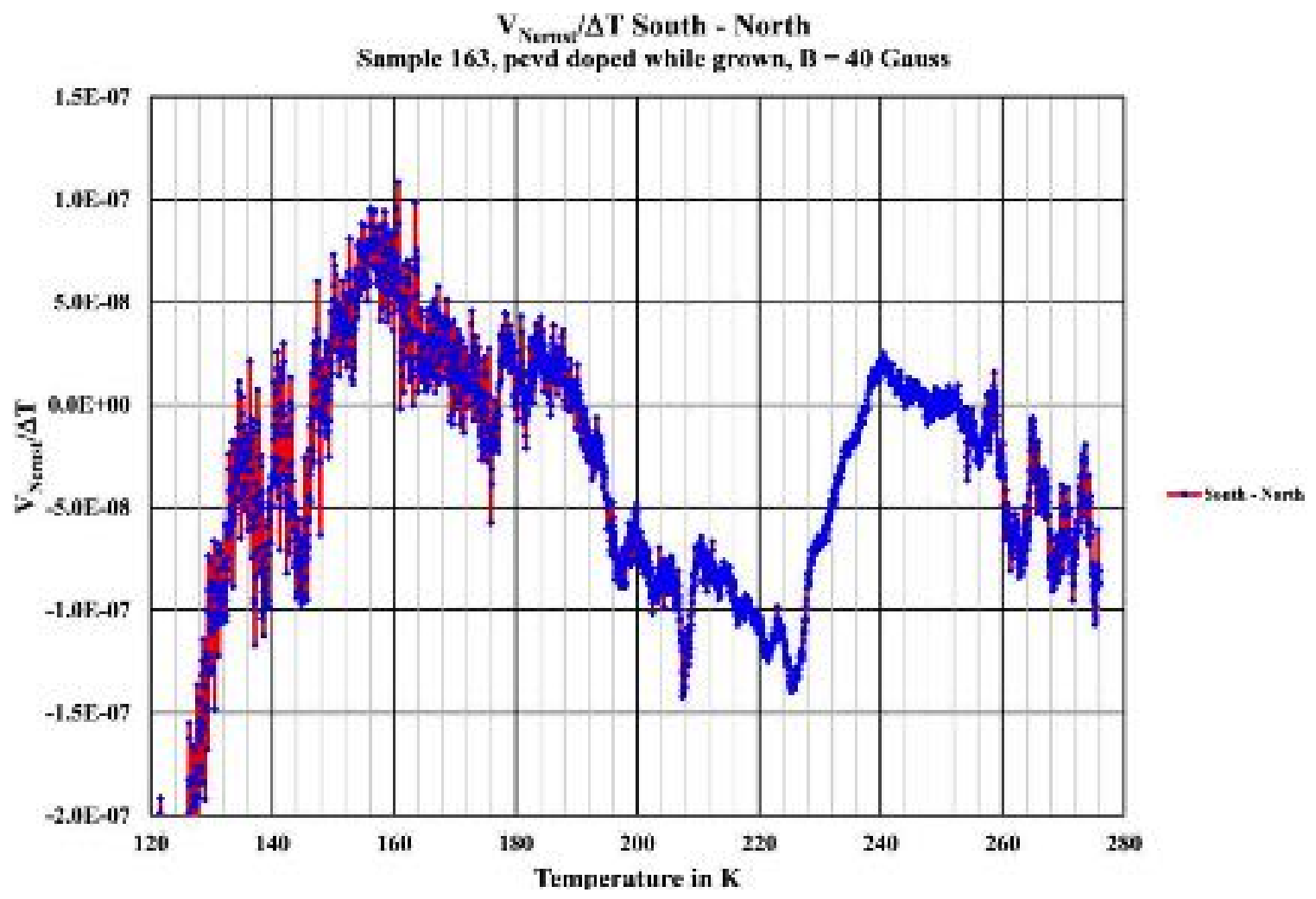

Figure 6.6: PECVD phosphorous doped-while-grown sample. Differential Nernst signal with magnetic field-oriented North Up through the sample subtracted from the Nernst signal with magnetic field-oriented South Up through the sample at an applied field of 40 Gauss. Note there are peaks at $T \sim 158 \mathrm{~K}$ and $T \sim 240 \mathrm{~K}$ that exhibit the tilted peak characteristic of a Nernst vortex signature. However, the peak at $T \sim 240 \mathrm{~K}$ may be due to instrumentation.

Figure 6.7 is the differential (North subtracted from South) $V_{\text {Nernst }} / \Delta T$ for a sample with deliberate argon ion implant damage to enhance pinning. It is also for a considerably larger magnetic field of 1,500 Gauss. The additional damage is expected to give rise to peaks in the Nernst signal at the depinning temperatures of the various pinning sites. Even at full scale, it is apparent that there are peaks in the Nernst signal at $T \sim 42 \mathrm{~K}, 70 \mathrm{~K}$, and $238 \mathrm{~K}$. 
Sample 23: $\mathrm{V}_{\text {Nernst }}($ South up $) / \Delta T-V_{\text {Nernst }}($ North up) $/ \Delta T$

1500 Gauss (Warming)

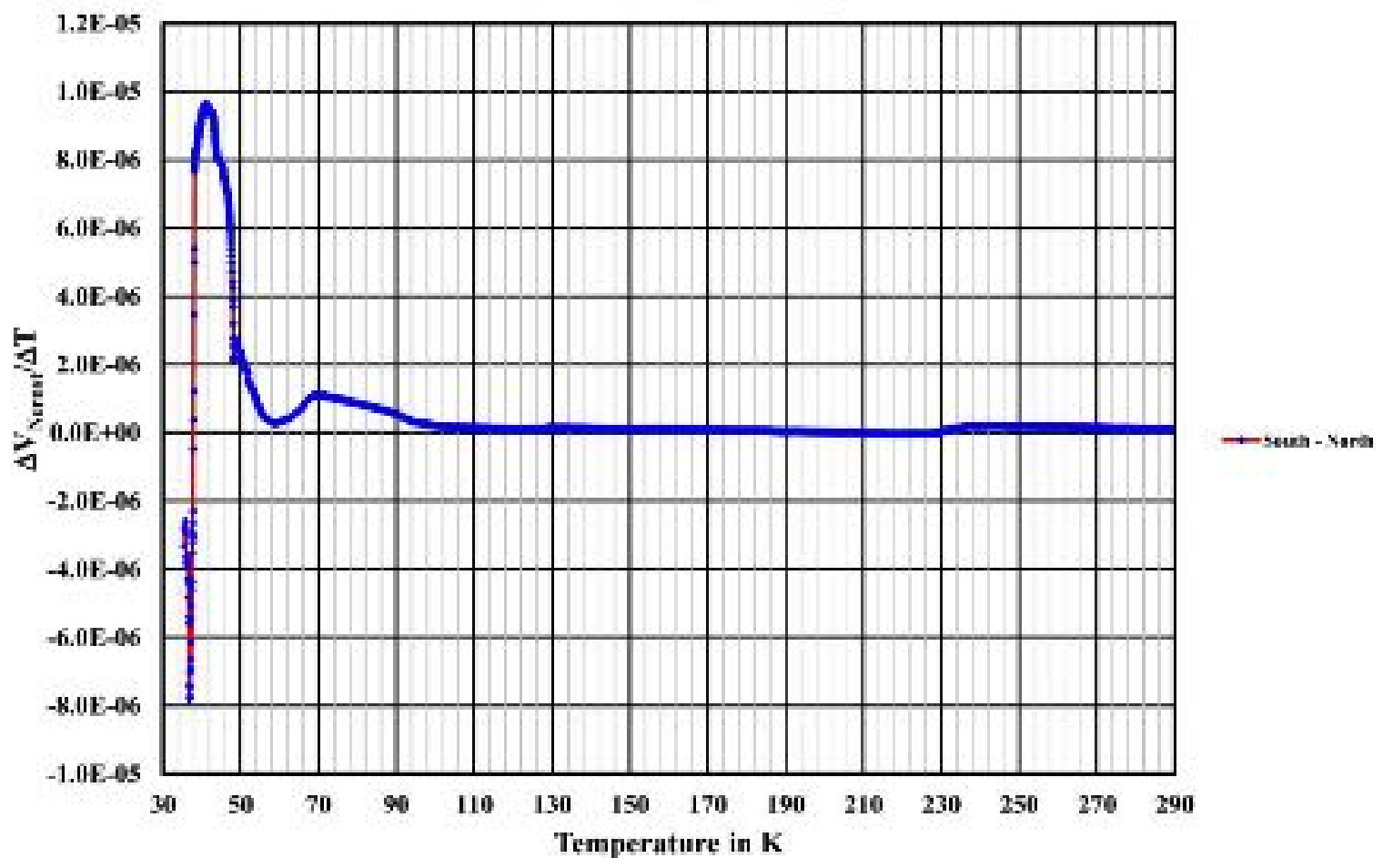

Figure 6.7: Ion-implanted phosphorous followed by argon ion implant to create pinning. Magnetic field is $\mathbf{1 5 0 0}$ Gauss and the plot is of the Nernst signal for the North Up oriented field subtracted from the South Up oriented Nernst signal. There are tilted peaks characteristic of Nernst vortex signals apparent at temperatures of $42 \mathrm{~K}, 70 \mathrm{~K}$, and $238 \mathrm{~K}$.

Figure 6.8 is an expansion of the plot in Figure 6.7 to obtain a better resolution at the peak at $T \sim 70 \mathrm{~K}$. the observation in Figure 6.8 shows that this peak is a classical Nernst tilted peak characteristic of a vortex signal. In addition, there is also a somewhat smaller but nonetheless distinguishable peak at a temperature of approximately $130 \mathrm{~K}$ which is not clear on the full-scale plot. The periodicity of these peaks suggested that an additional small peak should be present at a temperature of approximately $180 \mathrm{~K}$. The further magnification of the plot is shown in Figure 6.8. As can be seen on the plot, there are some small peaks near $180 \mathrm{~K}$. However, these small peaks are not nearly as prominent as those at $130 \mathrm{~K}$ and 
$238 \mathrm{~K}$. This suggests that there either are few pinning sites at this energy or that the existing vortices are preferentially pinned at other sites.

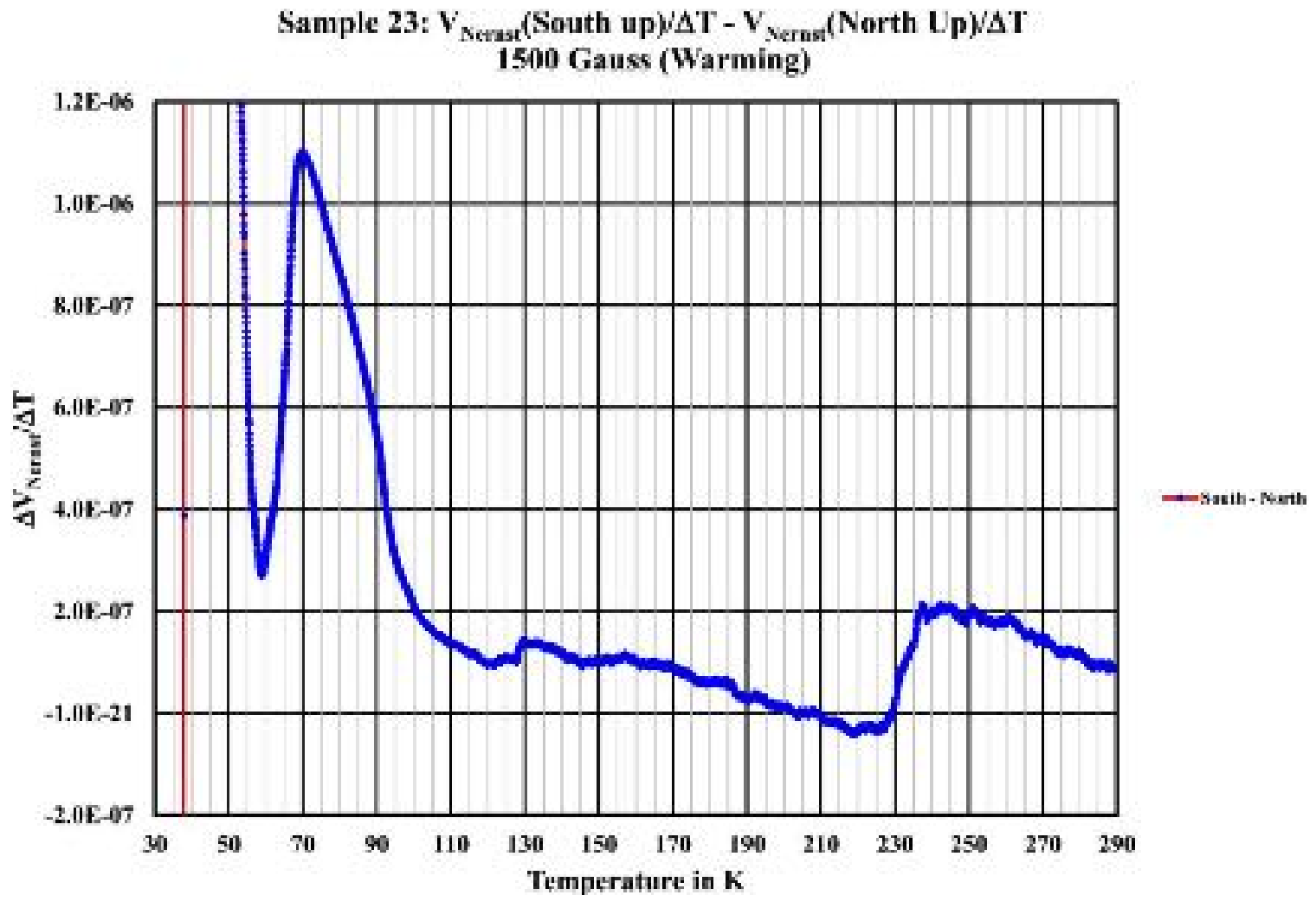

Figure 6.8: Same data as in Figure 6.7 expanded to show the details of the $T \sim 70 \mathrm{~K}$ peak as well as some of the smaller peaks. 


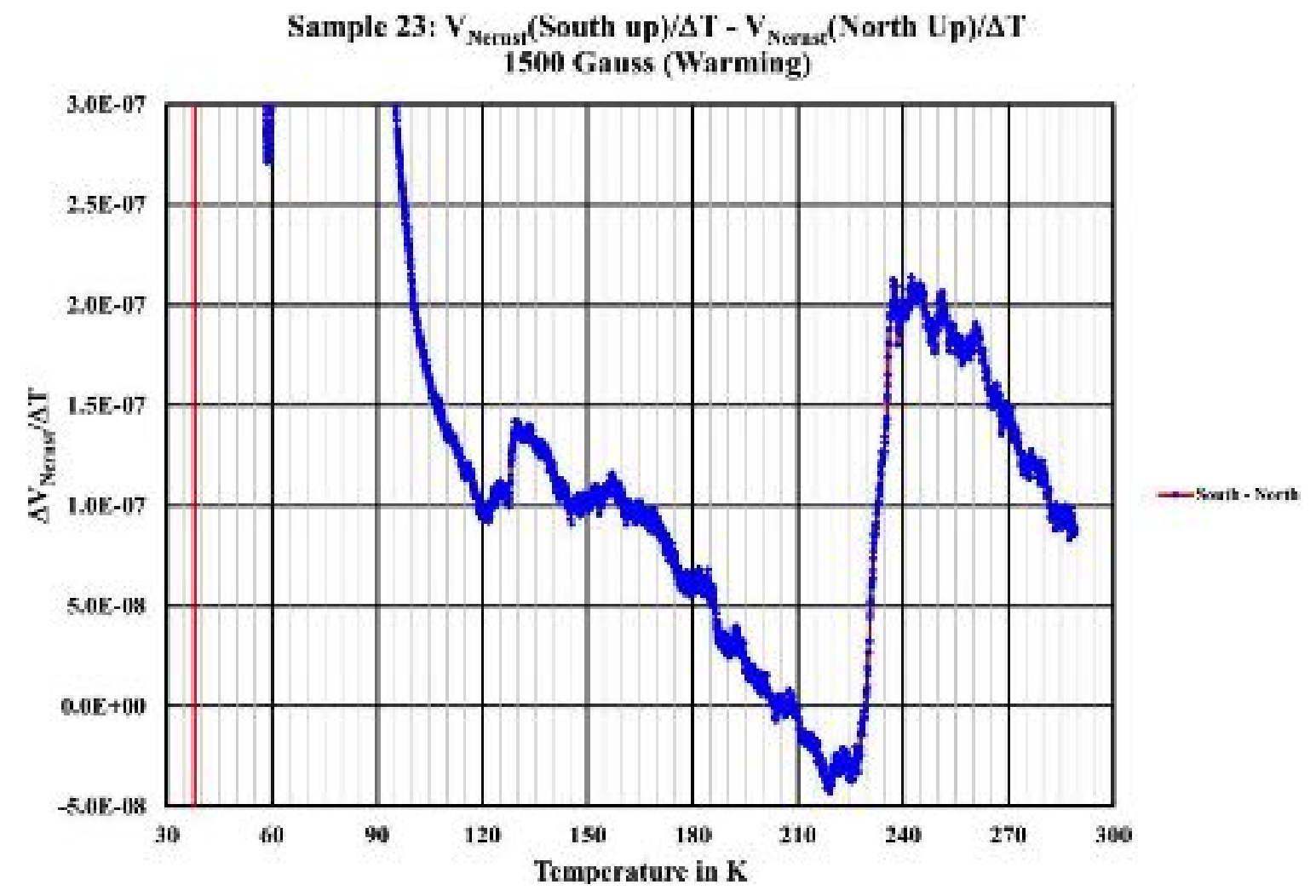

Figure 6.9: Further magnification of the data in Figure 6.7. Note that the peaks at $T$ $\sim 130 \mathrm{~K}$ and $T \sim 238 \mathrm{~K}$ are clearly the tilted peak of a vortex signature in the Nernst effect. There are also small peaks in the vicinity of $T \sim 180 \mathrm{~K}$, but they are very small indicating few vortices are undergoing depinning at this temperature.

For further examination on the Nernst effect in phosphorous and argon implanted samples, identical samples were created in July of 2018 with the same procedure which was ion implanted with phosphorous and then ion damaged with argon. The samples were also created by the same company using the same conditions as the prior samples which were done in 2011.

One of the made-in-2018 samples was selected to test, and the results were qualitatively identical to the samples that were seven years in storage. A few questions which were addressed by this experiment were;

1) were these results repeatable over a period of years? 
2) what did the cooling-warming cycle look like?

The first question was answered as "yes", despite a seven-year gap in time between runs the behavior is reproducible. The second question was more difficult to answer with the older samples as they were thin, fragile and well measured (hence, mechanically damaged). The results of the cooling cycle are particularly problematic as the vibration of the refrigerator adds serious mechanical noise to the cooling results due to contact motion. After an effort to ensure a measurable contact on a new implanted and damaged sample, the plot shown in Figure 6.10 was obtained. As an experimental note, a typical Nernst cooling cycle takes approximately 20,000 data points and a warming plot consists of from 380,000 to 400,000 data points. The simple $-V_{N e r n s t} / \Delta T$ plots are shown from Figures $6.10-6.22$. 
Sample 213: Run 1, 1500 Gauss, Cooling and Warming $-V_{\text {Nermo }} / \Delta T$ vs T, Large Thermal Gradient

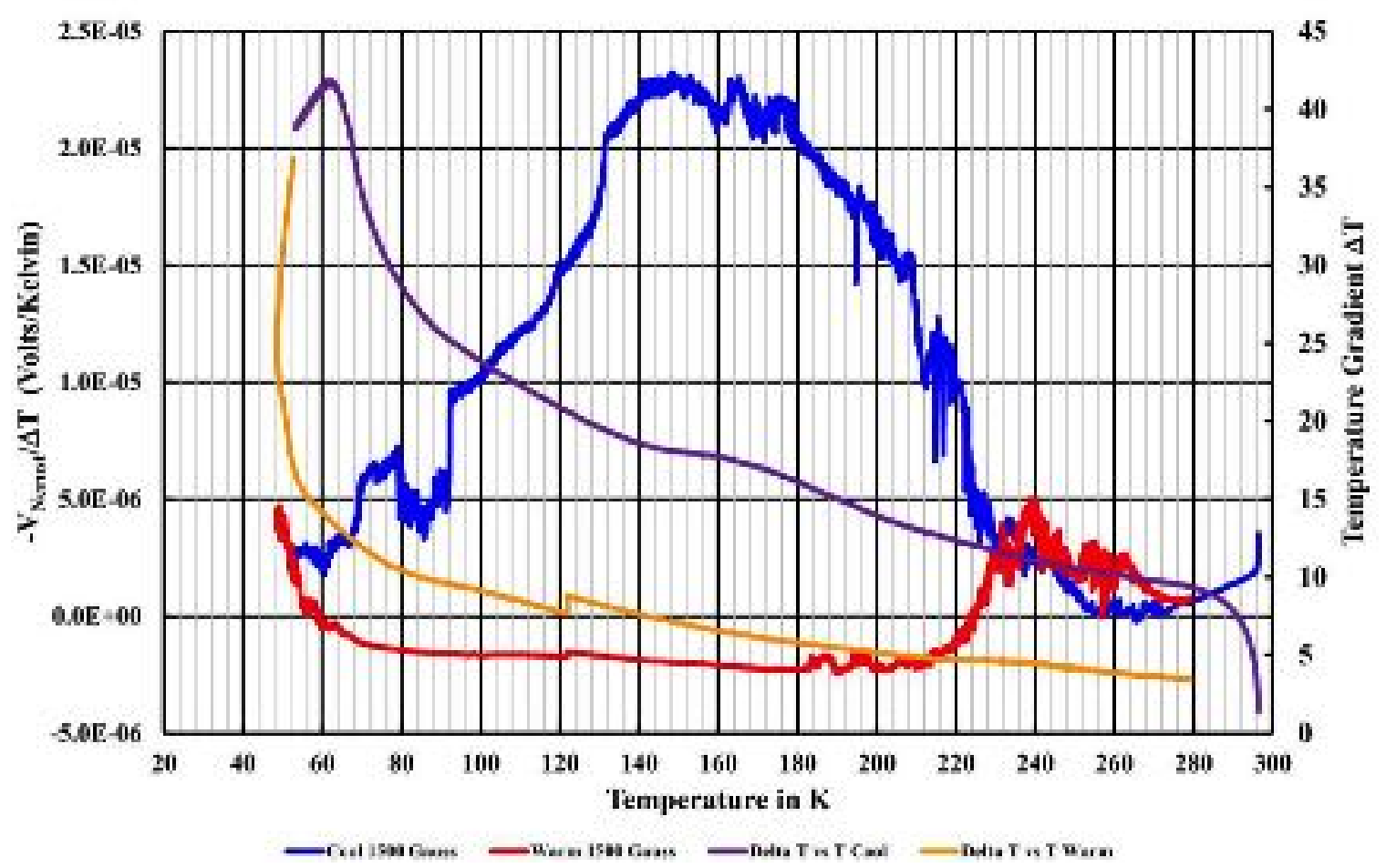

Figure 6.10: $-V_{N e r n s t} / \Delta T$ and $\Delta T$ versus $T$ for sample 213 in a 1,500 Gauss magnetic field oriented normal to the sample's surface. There is 1 Watt of input power on the Nernst heater. This is the first run with the sample in a magnetic field. The cooling curve is, effectively, one enormous peak. The warming curve hints of the BKT transition seen in the later, lower power and lower gradient plots. Note the hysteresis.

The cooling curve has a large Nernst voltage signal which begins at $T \sim 245 \mathrm{~K}$ and that there is a large, obvious, and magnetically induced hysteretic character to the Nernst signal between the cooling and warming data. This hysteresis is consistent with the eventual magnetic field exclusion at low temperature. The small step in the warming data at $T \sim 122 \mathrm{~K}$ does appear to be due to a small jump in the reading of one of the gradientmeasuring thermometers. The other peaks in the warming curve are many data points each and should be considered as real measurements. In the warming curve, the large Nernst features are at $T \sim 50 \mathrm{~K}$ and $220 \mathrm{~K}<T<280 \mathrm{~K}$. The low-temperature Nernst feature is 
the onset of the vortex BKT transition melting signal and the Nernst signal nominally at $T$ $\sim 236 \mathrm{~K}$ is the actual superconducting to normal transition.

Figure 6.10 also includes the thermal gradient information on the secondary vertical axis. This data is what is divided into the measured Nernst voltage to give the $V_{N e r n s t} / \Delta T$ plots. An examination of this thermal gradient data makes it clear that the $V_{\text {Nernst }} / \Delta T$ plots are real signals and not a thermally driven electric effect.

The same sample was tested further at various magnetic field intensities with a Nernst heater power of 0.2 Watts to extend the measurements to lower temperatures in order to examine the features at $T$ between 30 to $40 \mathrm{~K}$. The overall results of this experiment are shown in Figures 16-27.

In Figure 6.11, an obvious peak of each run can be seen at $T \sim 36 \mathrm{~K}$ which corresponds with the expected BKT transition. At below $36 \mathrm{~K}$, the antivortices have been expelled and a vortex lattice has been formed (hence, very few vortices can move), and the Nernst signal is small. As the sample transitions through $36 \mathrm{~K}$ during the warming run, the BKT lattice melts, only a few vortices are free to move, and the Nernst signal is large until they are balanced by antivortices. Once the balancing between vortices and antivortices occurs the Nernst signal becomes smaller. 
$-V_{\text {Nirns }} / \Delta T$ vs. T (North up)

Sample 213: Ran 18, 1113 Gauss, Run 17, 641 Gauss; Run 16, 290 Gauss \& Run 15, 120 Gauss

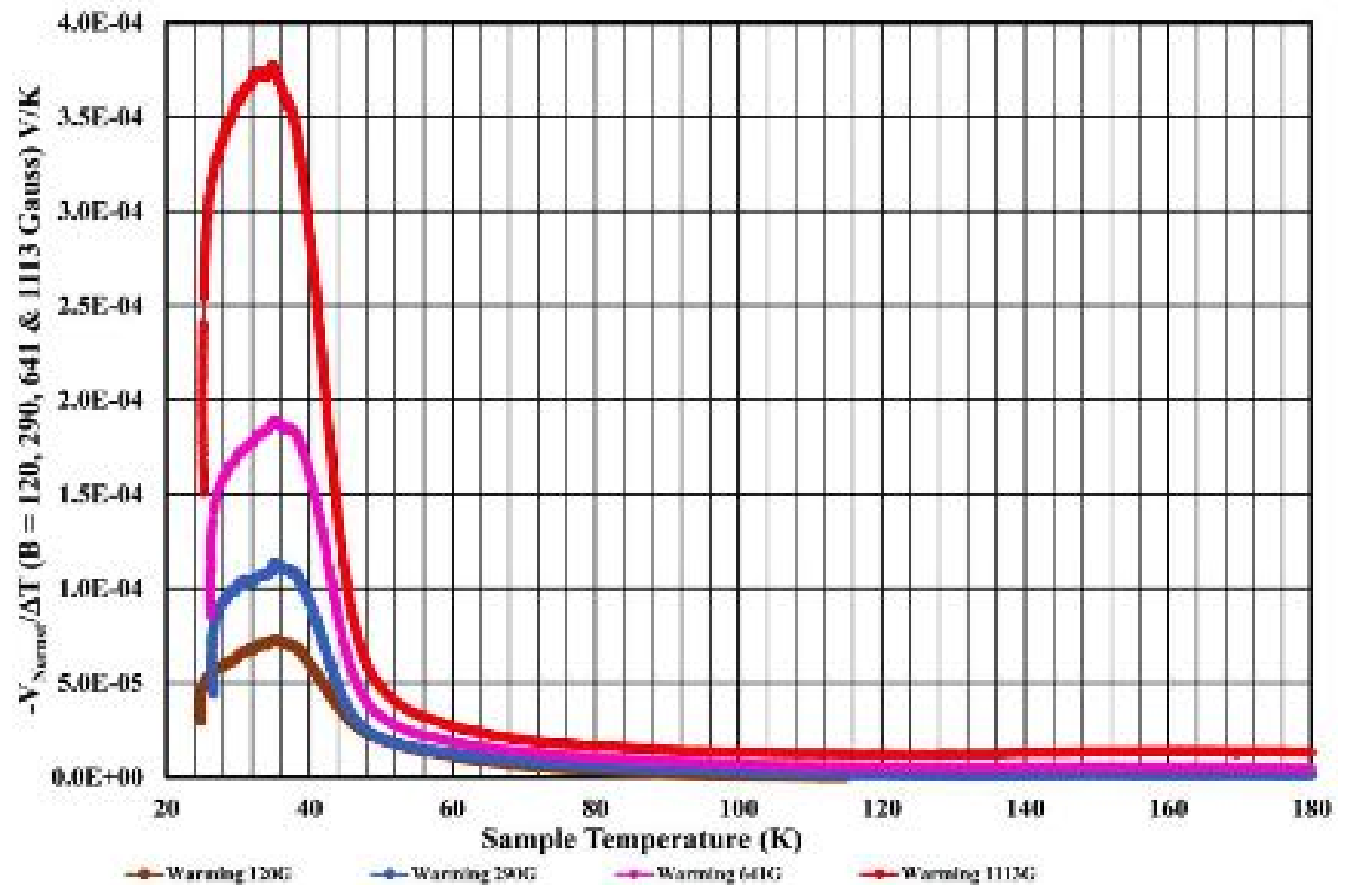

Figure 6.11: $-V_{\text {Nernst }} / \Delta T$ curves from $20-180 \mathrm{~K}$ for warming direction only. Peak at approximately $36 \mathrm{~K}$ remains constant with respect to applied magnetic field. The peak amplitude is a strong function of the applied magnetic field. This peak is at the approximate BKT transition.

In figure 6.12, the cooling plots of the same runs of the warming plots shown in figure 15 are added in order to observe a temperature hysteresis of each run. As seen in the figure, there is an obvious hysteresis between the cooling and warming plots of each run. This hysteresis indicates that magnetic flux occurred and trapped in the material which validates the presence of magnetic vortices. 
YkunsisT va. T (Nurth up)

Sample 213: 12un 18, 113 Gauss; Kun 17,611 Gauss; Ruv 16, 240 Gauss \& Ron 15, 120 Gauss

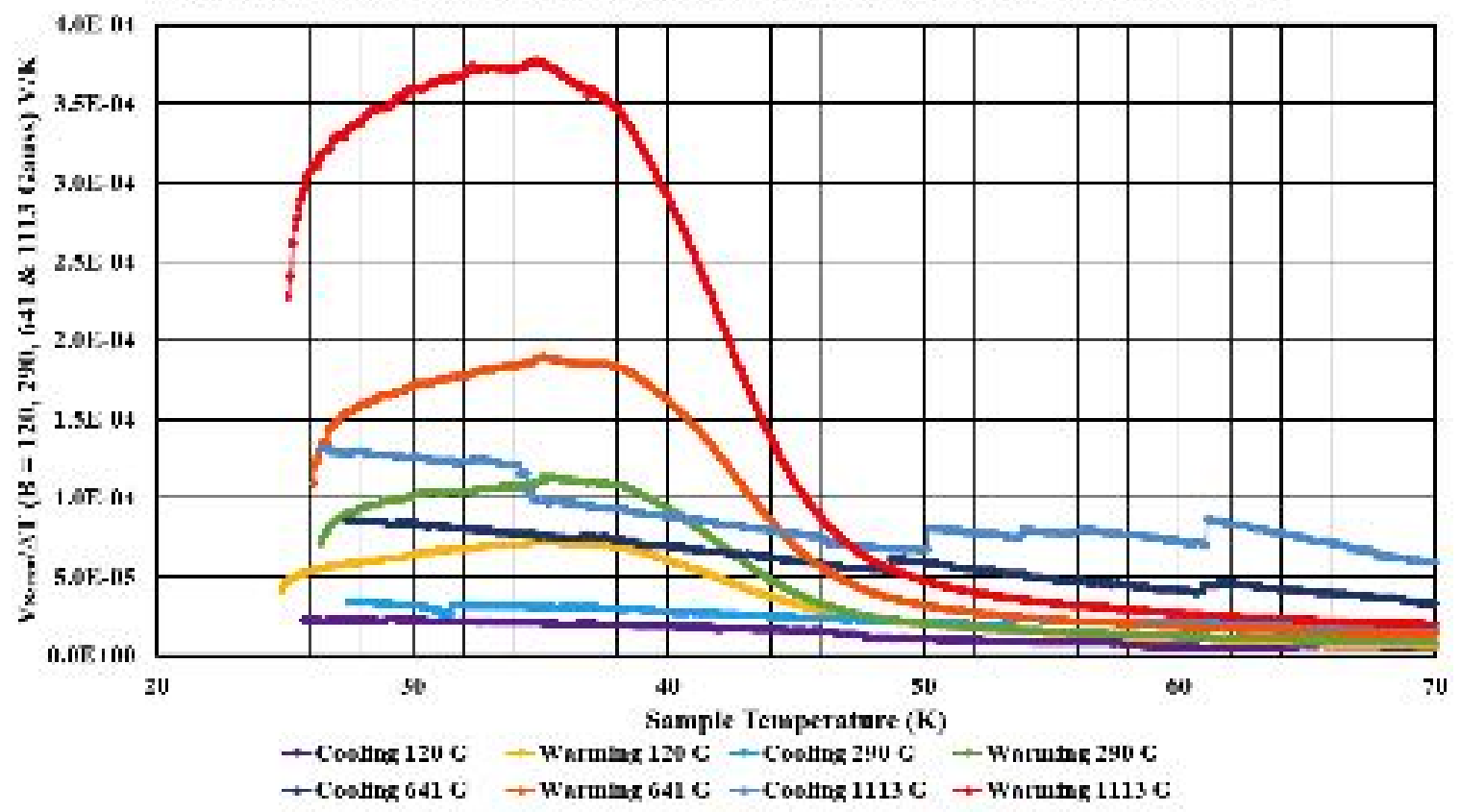

Figure 6.12: $-V_{\text {Nernst }} / \Delta T$ curves for cooling and warming direction cycle for the same sample as in Figure 6.11 from 20 - $70 \mathrm{~K}$. Peaks at approximately $36 \mathrm{~K}$ remain constant with respect to applied magnetic field. Each peak amplitude is a strong function of the applied magnetic field. These peaks are at the approximate BKT transition. Note the clear hysteresis between the cooling and the returning warming plots.

Figures 6.13 through 6.16 show the entirety of each cooling - warming Nernst measurement for a single applied magnetic field normal to the sample's surface on each plot. The vertical axis has been selected to allow better visibility of structure in the curves at the upper end of the temperature scale and the low-temperature BKT peaks have been allowed to go off scale as they are comprehensively presented in Figures 6.11 and 6.12.

Figure 6.13 is a plot of the cooling and warming $-V_{N e r n s t} / \Delta T$ curves with 120 Gauss of magnetic field applied normal to the sample's surface. The figure presents a large step in the cooling curve that extends from virtually the start of the plot to about $224 \mathrm{~K}$ and that there are additional features at about $180 \mathrm{~K}$ and $63 \mathrm{~K}$. The warming curve appears to 
be smooth with some structure from $216 \mathrm{~K}$ to $265 \mathrm{~K}$ and, perhaps, a broad peak in the 265

$\mathrm{K}$ region. The hysteresis shown is indicative of flux trapping.

$-\mathrm{V}_{\mathrm{v} \text { trmul }} / \Delta \mathrm{T}$ vs. T (North up)

Sumple 213: Run 15, 120 Gauss

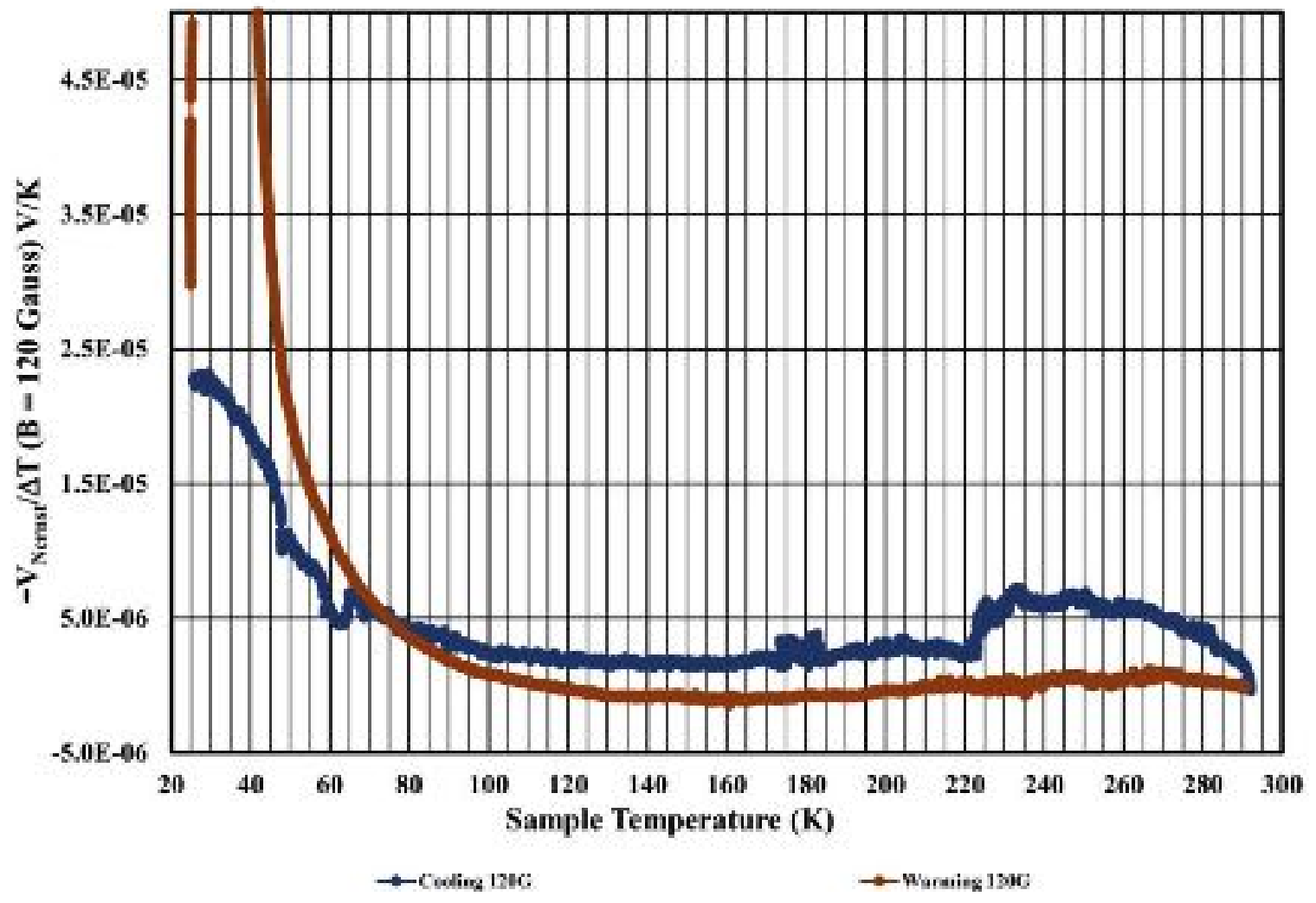

Figure 6.13: $-V_{\text {Nernst }} / \Delta T$ curves for cooling and warming direction cycle for the same sample as in Figure 6.11 taken with an applied field of 120 Gauss normal to the sample surface. Notice the hysteresis and the large step in the cooling curve that extends from virtually the start of the plot to about $224 \mathrm{~K}$.

A plot of the cooling and warming $-V_{\text {Nernst }} / \Delta T$ curves with 290 Gauss of magnetic field applied normal to the sample's surface is presented in Figure 6.14. There is a large step in the cooling curve that extends from virtually the start of the plot down to about $216 \mathrm{~K}$. There are additional features at about $180 \mathrm{~K}$ and $53 \mathrm{~K}$. The warming curve appears to be smooth with some structure from $210 \mathrm{~K}$ to $265 \mathrm{~K}$. The warming curve has a definite peak at $234 \mathrm{~K}$ and, perhaps, a broad peak in the $265 \mathrm{~K}$ region. The hysteresis 
shown is indicative of flux trapping. The fact that the cooling curve appears to drop to the warming curve in the notches at $53 \mathrm{~K}, 185 \mathrm{~K}, 195 \mathrm{~K}$ and again at $232 \mathrm{~K}$ tends to strongly suggest that there is unstable "catch and release" flux trapping occurring during cooling.

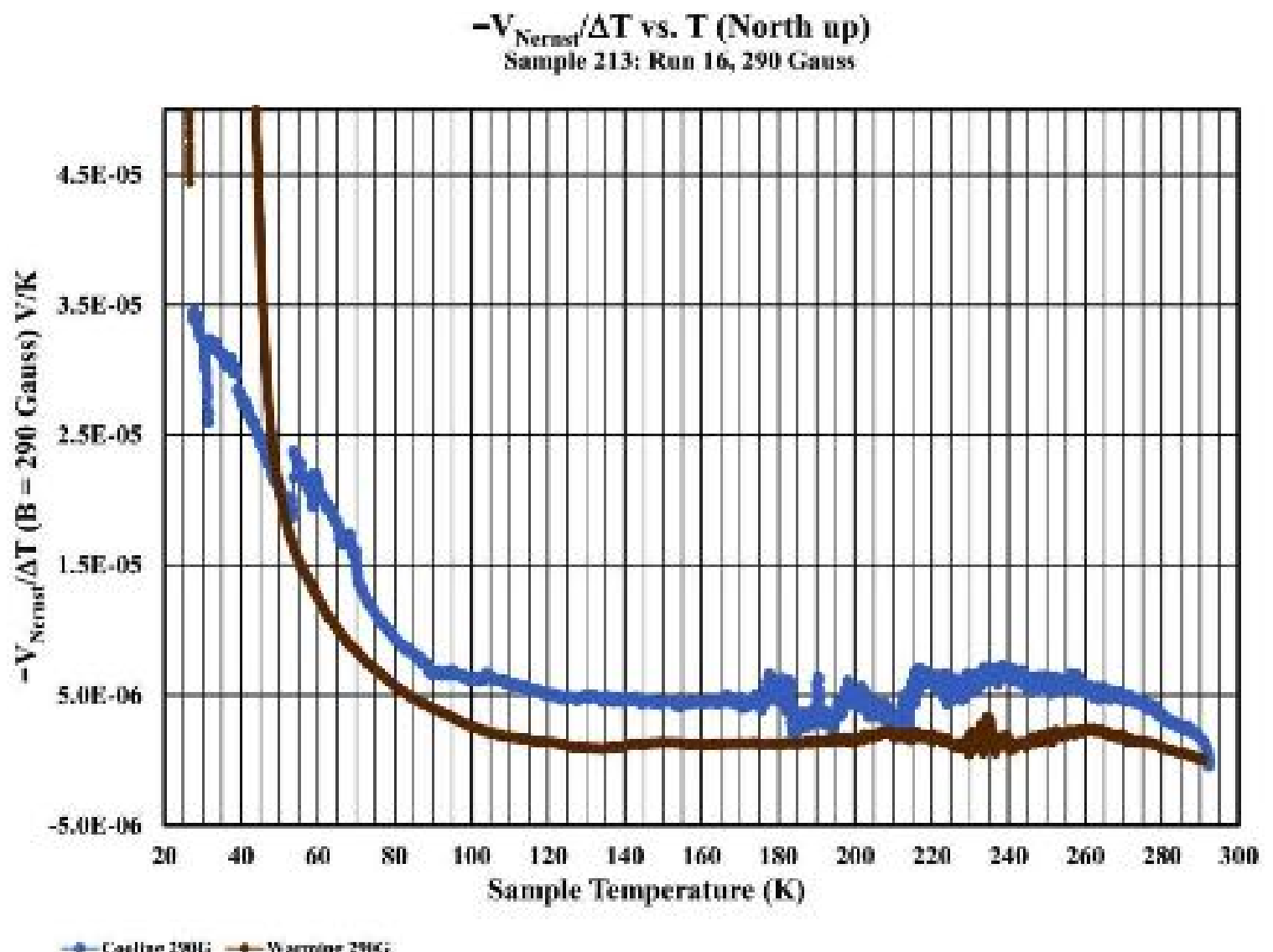

Figure 6.14: $-V_{N e r n s t} / \Delta T$ curves for cooling and warming direction cycle for the same sample as in Figure 6.11 taken with an applied field of 290 Gauss normal to the sample surface. Notice the hysteresis and the large step in the cooling curve that extends from virtually the start of the plot to about $216 \mathrm{~K}$. There is structure in the cooling plot down to $178 \mathrm{~K}$. There is notable structure in the warming plot from a broad peak at about $210 \mathrm{~K}$ through to a broad peak at $260 \mathrm{~K}$.

A plot of the cooling and warming $-V_{\text {Nernst }} / \Delta T$ curves with 641 Gauss of magnetic field applied normal to the sample's surface is presented in Figure 6.15. There is a large step in the cooling curve that extends from virtually the start of the plot down to about $216 \mathrm{~K}$. There are additional features at about $180 \mathrm{~K}$ and $60 \mathrm{~K}$. Figure 6.11 also shows 
a notch at $48 \mathrm{~K}$ in the cooling curve for this sample, this is off scale in Figure 6.15. This is the $53 \mathrm{~K}$ notch in the 290 Gauss cooling curve which has moved downwards with the applied field. In addition, the peak at $80 \mathrm{~K}$ has noticeably broadened from the 290 Gauss curve in Figure 6.14, and now extends from $180 \mathrm{~K}$ down to $155 \mathrm{~K}$.

The warming curve in Figure 6.15 appears to be smooth with structure from $210 \mathrm{~K}$ to $265 \mathrm{~K}$. The curve has definite peaks at $232 \mathrm{~K}$ and $255 \mathrm{~K}$. The fact that the cooling curve appears to drop to the warming curve in the notches at $155 \mathrm{~K}, 195 \mathrm{~K}$, and again at $225 \mathrm{~K}$ tends to strongly suggest that there is unstable "catch and release" flux trapping occurring during cooling. 


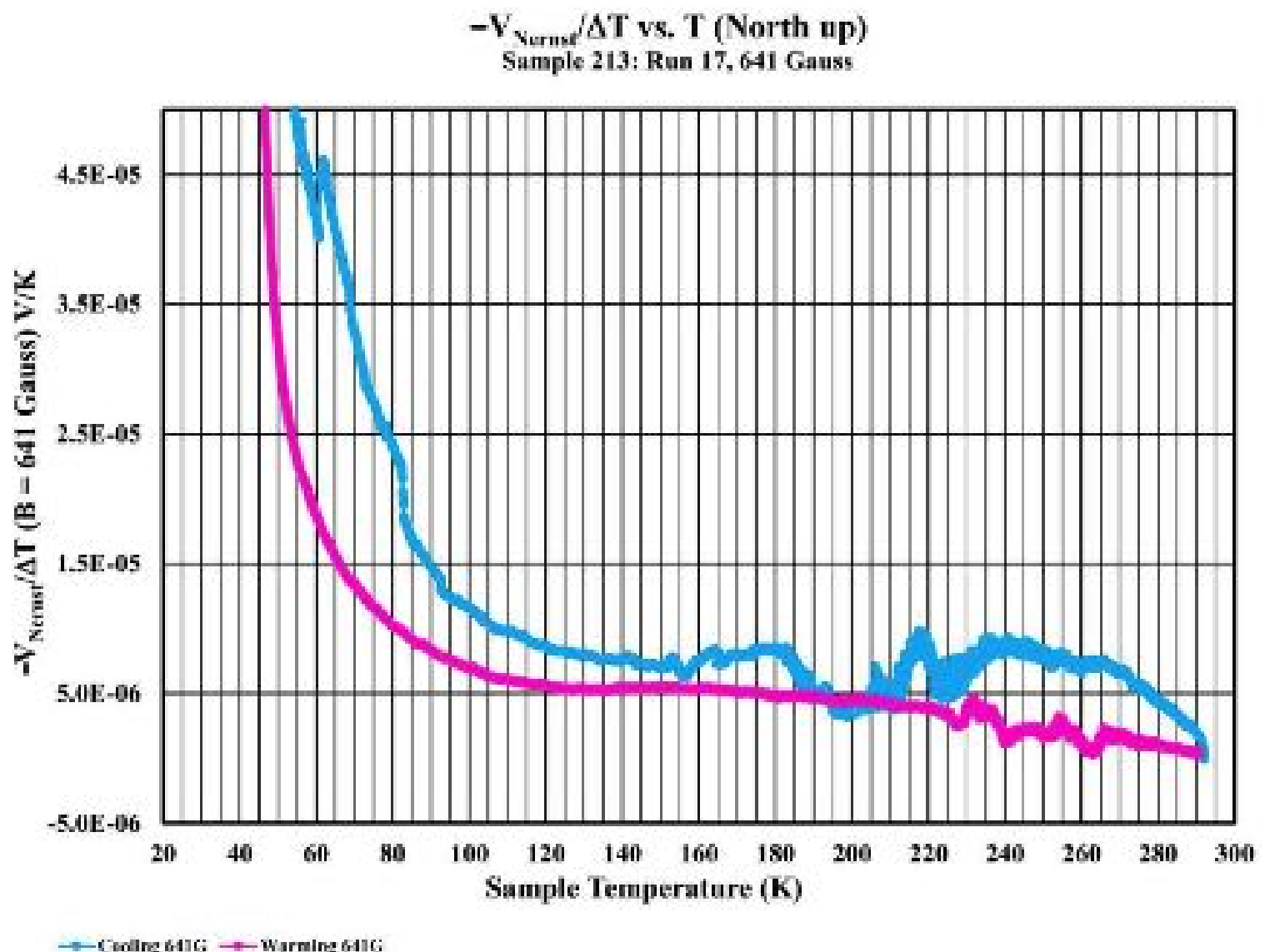

Figure 6.15: $-V_{\text {Nernst }} / \Delta T$ curves for cooling and warming direction cycle for the same sample as in Figure 6.11 taken with an applied field of 641 Gauss normal to the sample surface. Notice the hysteresis and the large step in the cooling curve that extends from $265 \mathrm{~K}$ to about $216 \mathrm{~K}$. There is structure in the cooling plot down to $150 \mathrm{~K}$. There is notable structure in the warming plot from a broad peak at about $148 \mathrm{~K}$ through to a broad peak at $260 \mathrm{~K}$.

Figure 6.16 shows the sample with 1,113 Gauss of magnetic field applied normal to the sample's surface. There are obvious changes in the cooling curve; the large step in the cooling curve that extended from $265 \mathrm{~K}$ to $216 \mathrm{~K}$ in the 641 Gauss data of figure 20 now is a single broad step from $265 \mathrm{~K}$ to about $144 \mathrm{~K}$. The warming data in Figure 6.16 has changed. There is a broad flat-topped tilted peak extending from $145 \mathrm{~K}$ to about 227 $\mathrm{K}$. There is a peak at $230 \mathrm{~K}$ and a notch at $240 \mathrm{~K}$. 
More interestingly, in Figure 6.16, the hysteresis between $144 \mathrm{~K}$ and $216 \mathrm{~K}$ has closed. This tends to suggest that, in this region and at this field, there is no mobile flux trapped. As the hysteresis opens again above $220 \mathrm{~K}$ this may be indicative of the fact that there are equal numbers of vortices and antivortices in this region at an applied field of 1,113 Gauss.

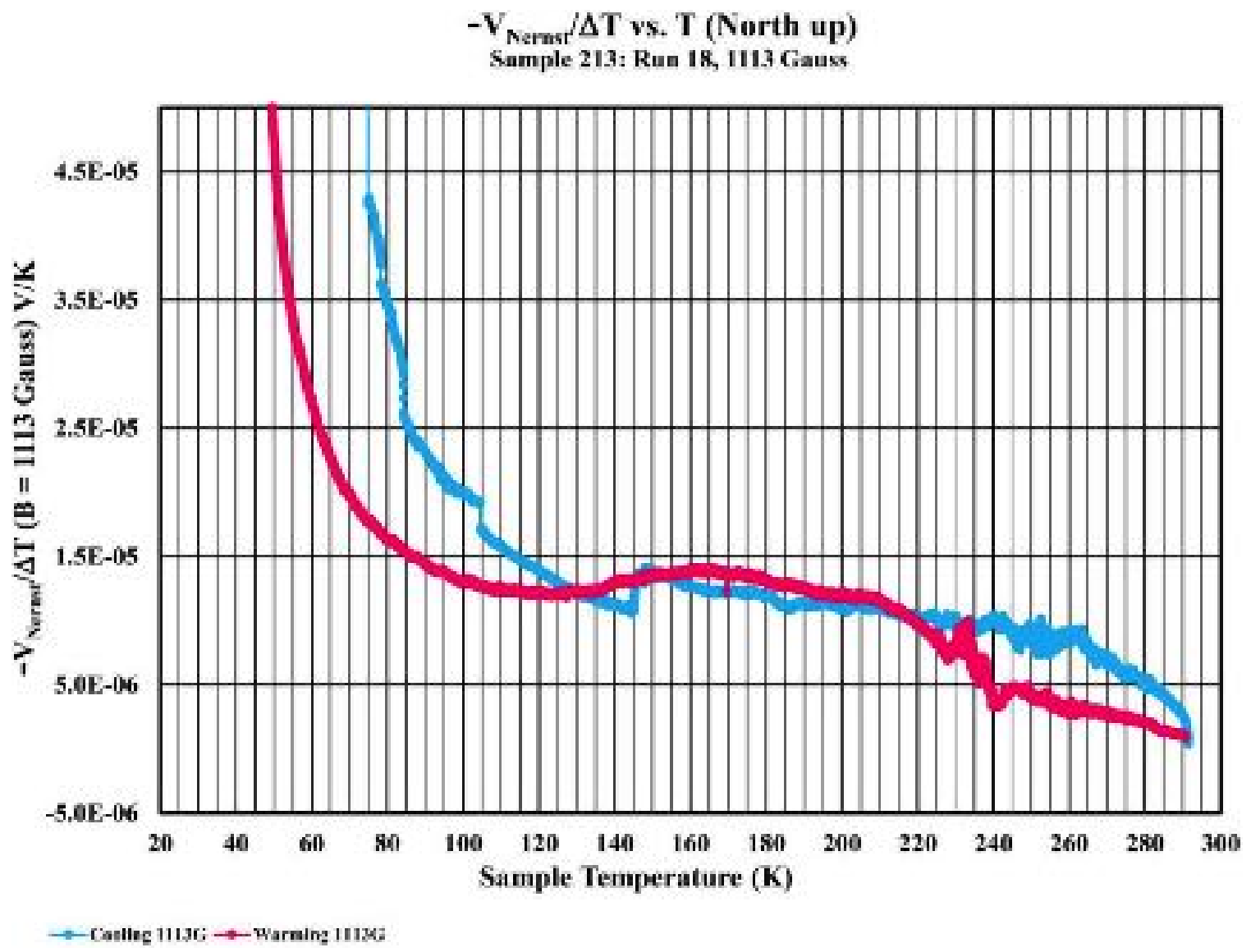

Figure 6.16: $-V_{N e r n s t} / \Delta T$ curves for cooling and warming direction cycle for the same sample as in Figure 6.11 taken with an applied field of 1,113 Gauss normal to the sample surface. Notice the hysteresis and the large step in the cooling curve that extends from $265 \mathrm{~K}$ to about $144 \mathrm{~K}$. There is structure in the cooling plot down to $144 \mathrm{~K}$. There is notable structure in the warming plot from a broad peak at about $164 \mathrm{~K}$ through to $260 \mathrm{~K}$.

Figures 6.17 through 6.20 are a closer look at the upper end of the temperature scales of Figures 6.13 through 6.16. The step in the cooling curve at $144 \mathrm{~K}$ in Figure 6.20 
was investigated and found to contain 50 data points. The progression of structures to lower temperatures with increases in the applied magnetic field is now more distinct. The upper end of the temperature range of the $V_{N e r n s t} / \Delta T$ plots clearly has structures in it through to temperatures in the range of $260-265 \mathrm{~K}$.

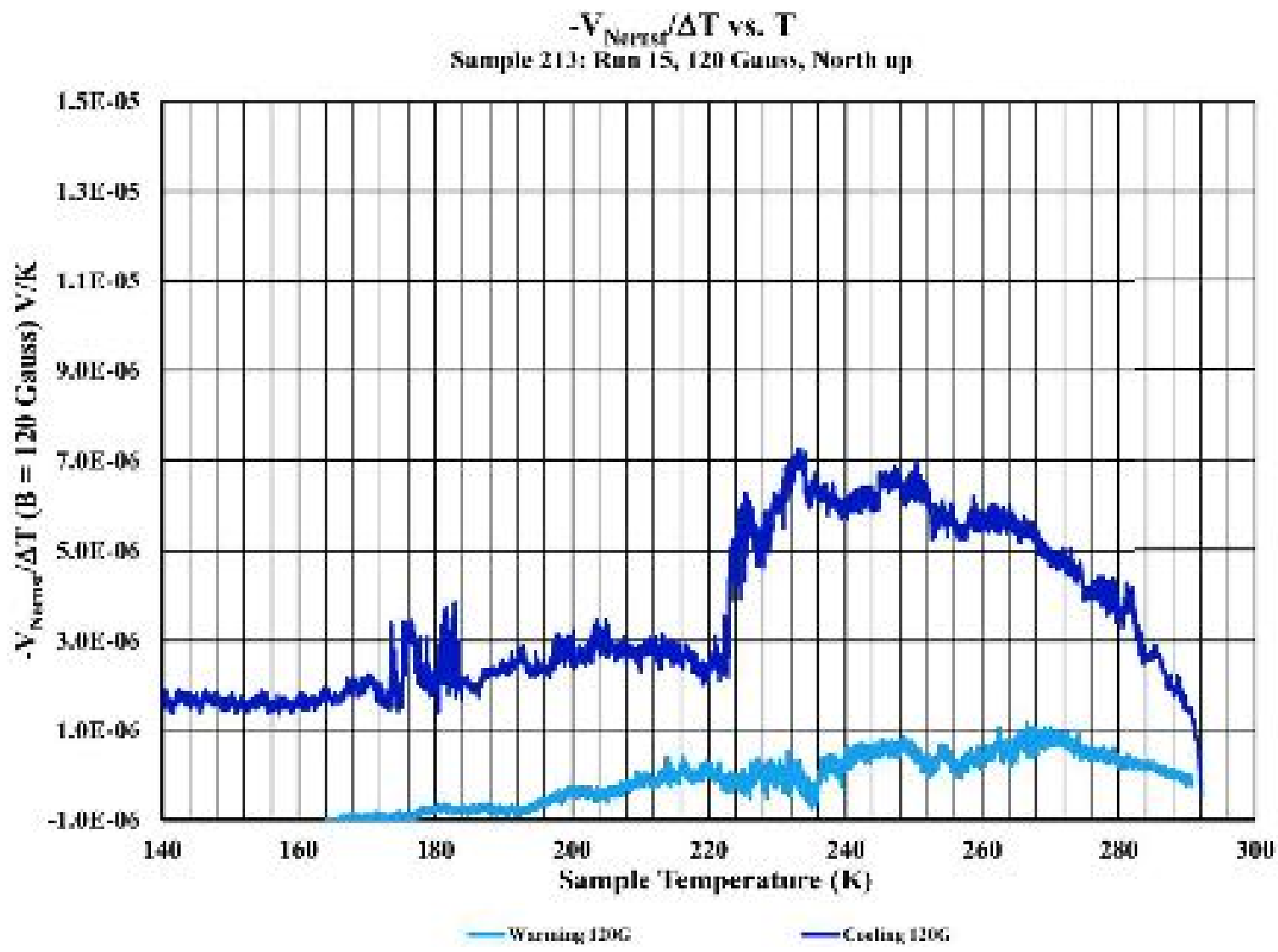

Figure 6.17: $-V_{N e r n s t} / \Delta T$ curves in the range of $140-300 \mathrm{~K}$ for cooling and warming direction cycle for the same sample as in Figure 6.11. This data was taken with 120 Gauss of magnetic field applied normal to the sample surface. Note the step at approximately $224 \mathrm{~K}$ in the cooling direction and the associated structure ranging from $260 \mathrm{~K}$ to $224 \mathrm{~K}$. There is hysteresis between the cooling and the returning warming plots. 
$-Y_{\text {Aerns }}$ ST YS. T

Sumple 213: Run 16, 291 Gisuss, North up

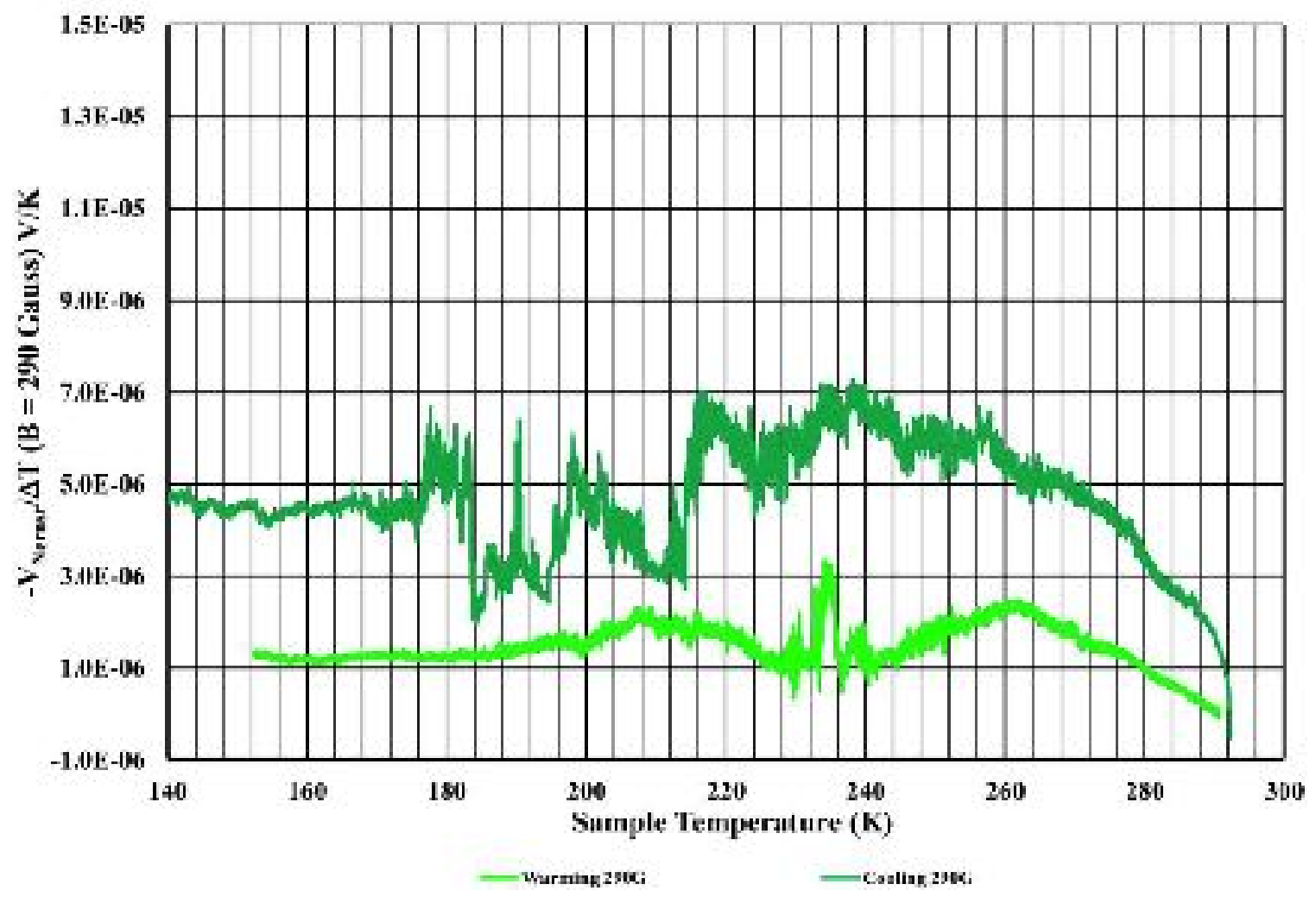

Figure 6.18: $-V_{N e r n s t} / \Delta T$ curves in the range of $140-300 \mathrm{~K}$ for cooling and warming direction cycle for the same sample as in Figure 6.11. This data was taken with 290 Gauss of magnetic field applied normal to the sample surface. Note the step at approximately $216 \mathrm{~K}$ in the cooling direction and the structure from approximately $260 \mathrm{~K}$ to $180 \mathrm{~K}$. Note the structure from $200 \mathrm{~K}$ to $260 \mathrm{~K}$ in the warming plot. Cooling to warming hysteresis is present. 


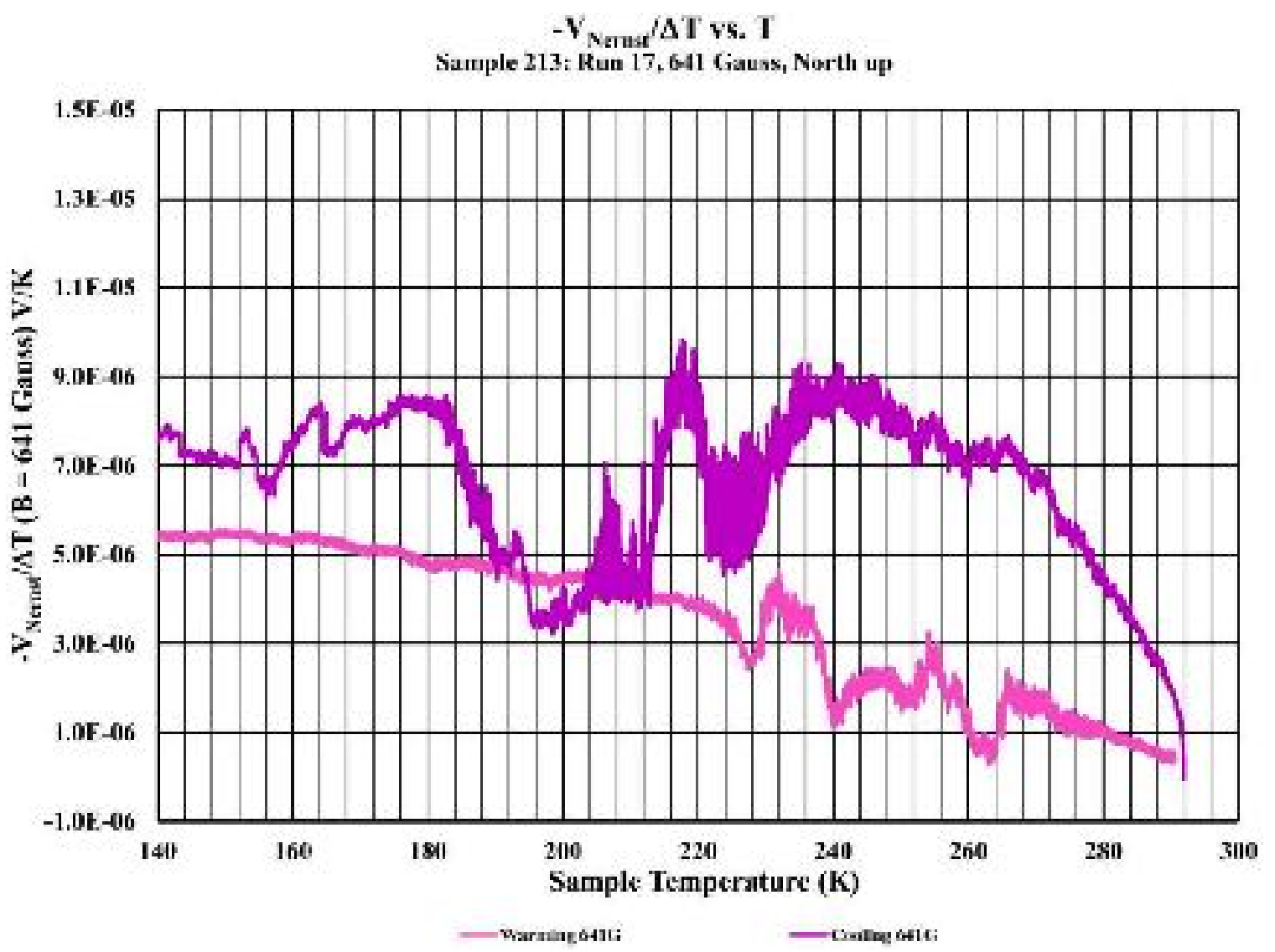

Figure 6.19: $-V_{N e r n s t} / \Delta T$ curves in the range of $140-300 \mathrm{~K}$ for cooling and warming direction cycle for the same sample as in Figure 6.11. This data was taken with 641 Gauss of magnetic field applied normal to the sample surface. Note the features that span from approximately $260 \mathrm{~K}$ to $160 \mathrm{~K}$ in the cooling direction and the structure between about $220 \mathrm{~K}$ and $265 \mathrm{~K}$ in the warming plot. Hysteresis is visible. 


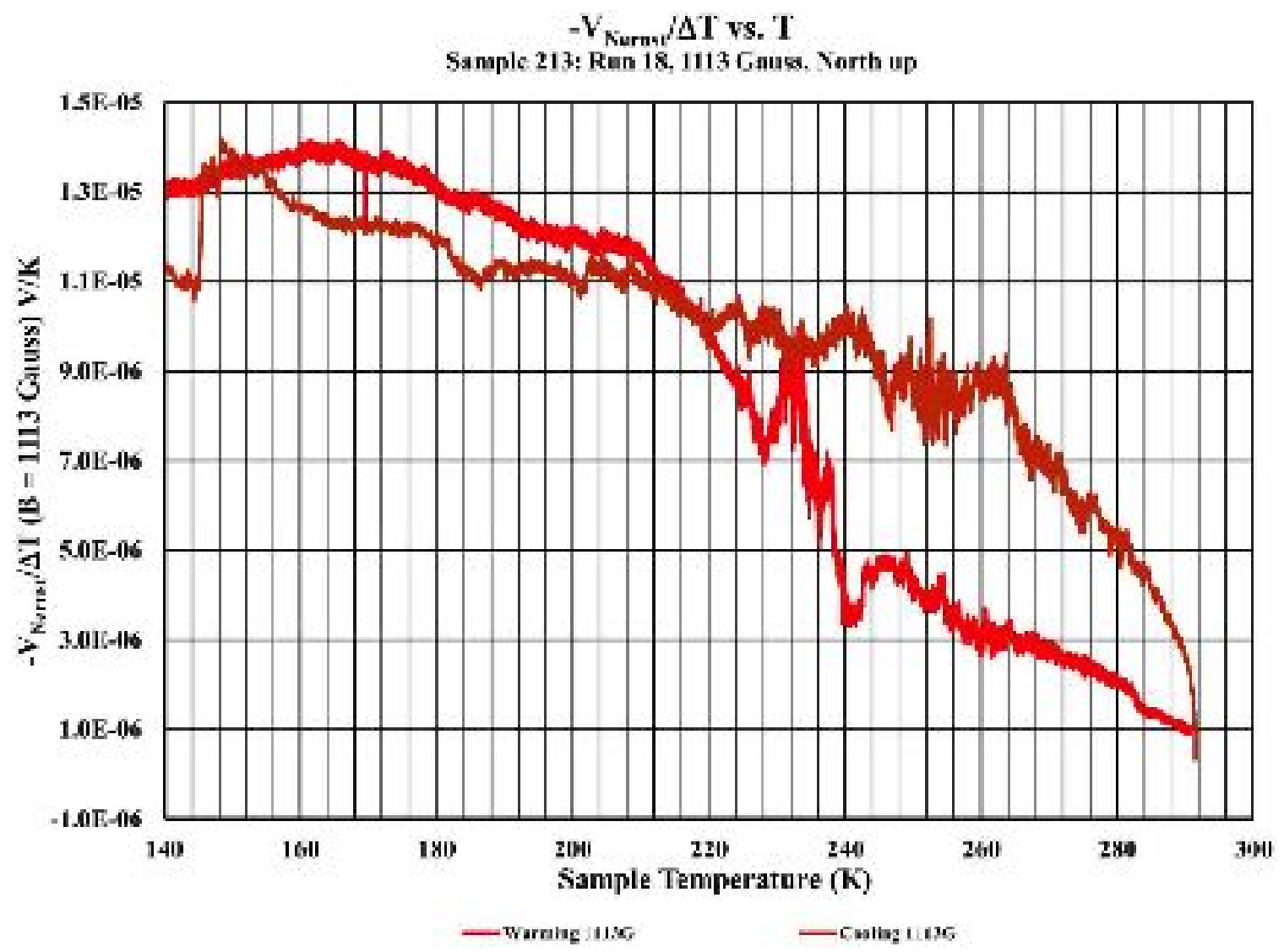

Figure 6.20: $-V_{\text {Nernst }} / \Delta T$ curves in the range of $140-300 \mathrm{~K}$ for cooling and warming direction cycle for the same sample as in Figure 6.11. This data was taken with 1,113 Gauss of magnetic field applied normal to the sample surface. Note the step at approximately $144 \mathrm{~K}$ in the cooling direction and the structure from approximately $265 \mathrm{~K}$ to $144 \mathrm{~K}$. The structure in the returning warming plot begins with a broad inflection point at $160 \mathrm{~K}$ and ends near $265 \mathrm{~K}$. Hysteresis is visible.

In the prior four figures (Figures $6.17-6.20$ ), the structure in the cooling data at approximately $180 \mathrm{~K}$ fills in and becomes part of the overall larger step in the final 1,113 Gauss plot. It does not appear to move. The structure in the warming data at about $240 \mathrm{~K}$ also appears to remain stationary. These stationary features are a signature of pinning. The fact that they are large enough to be visible on the Nernst data is suggestive of there being a considerable number of flux bundles or stacks pinned with this energy. 
Figure 6.21 is the aggregation of all the cooling direction plots shown individually in Figures 6.17 through 6.20 and highlights the evolution of the structure in the curves as the magnetic field is increased.

$-V_{\text {Vernst }} / \Delta T$ vs. T

Sample 213: Run 15, 120 Gaass; Run 16,290 Gauss; Run 17, 641 Gauss; \& Run 1K, 1113

Gunss; all North up

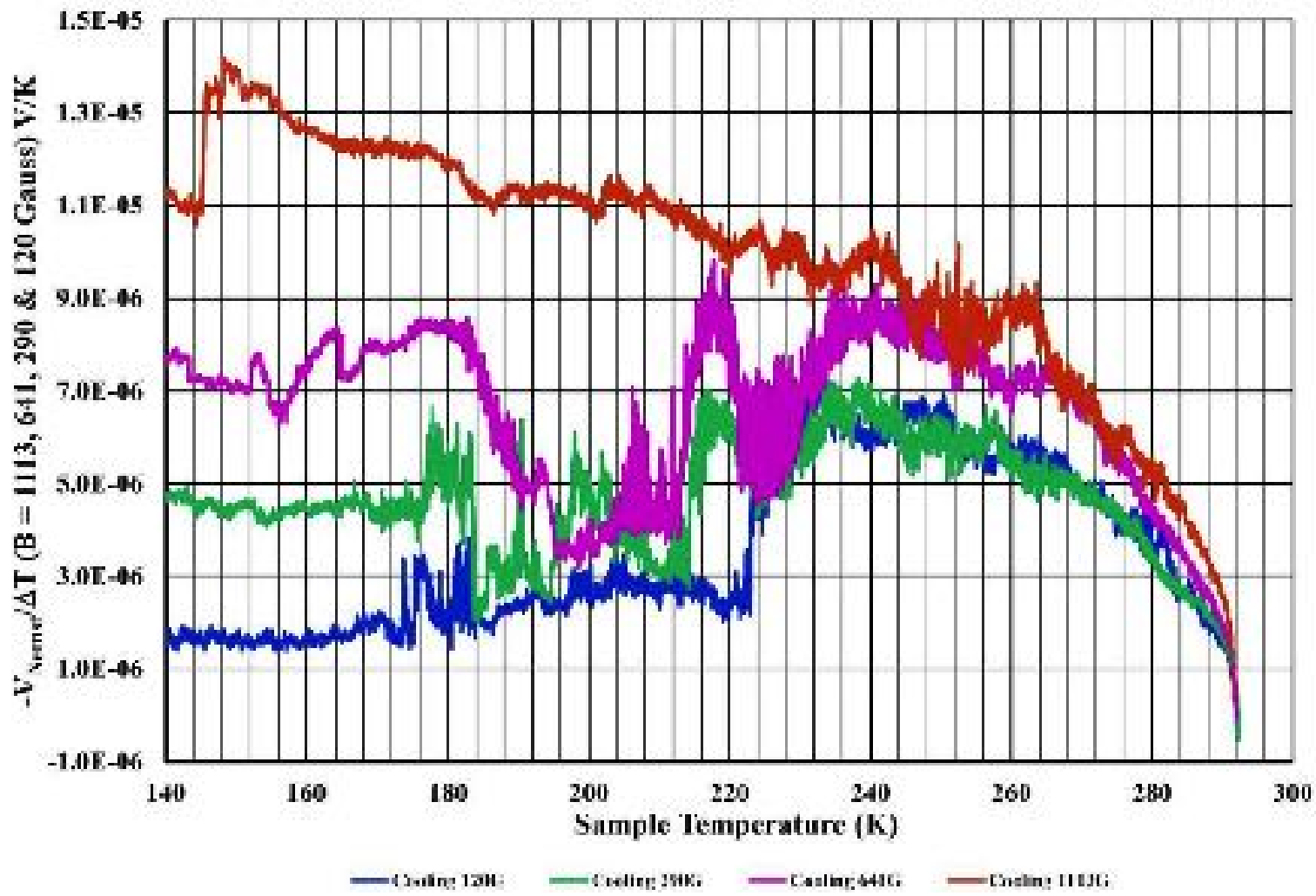

Figure 6.21: $-V_{\text {Nernst }} / \Delta T$ curves in the range of $140-300 \mathrm{~K}$ for same sample as in figure 6.11. This is a collection of the cooling direction plots from Figures 6.17 through 6.20. Note how the steps and features have tended to shift lower in temperature as the applied field is increased.

Figure 6.22 below is the aggregation of all the warming direction plots shown individually in Figures 6.17 through 6.20. This aggregate plot highlights the evolution of the structure in the curves as the magnetic field is increased. 


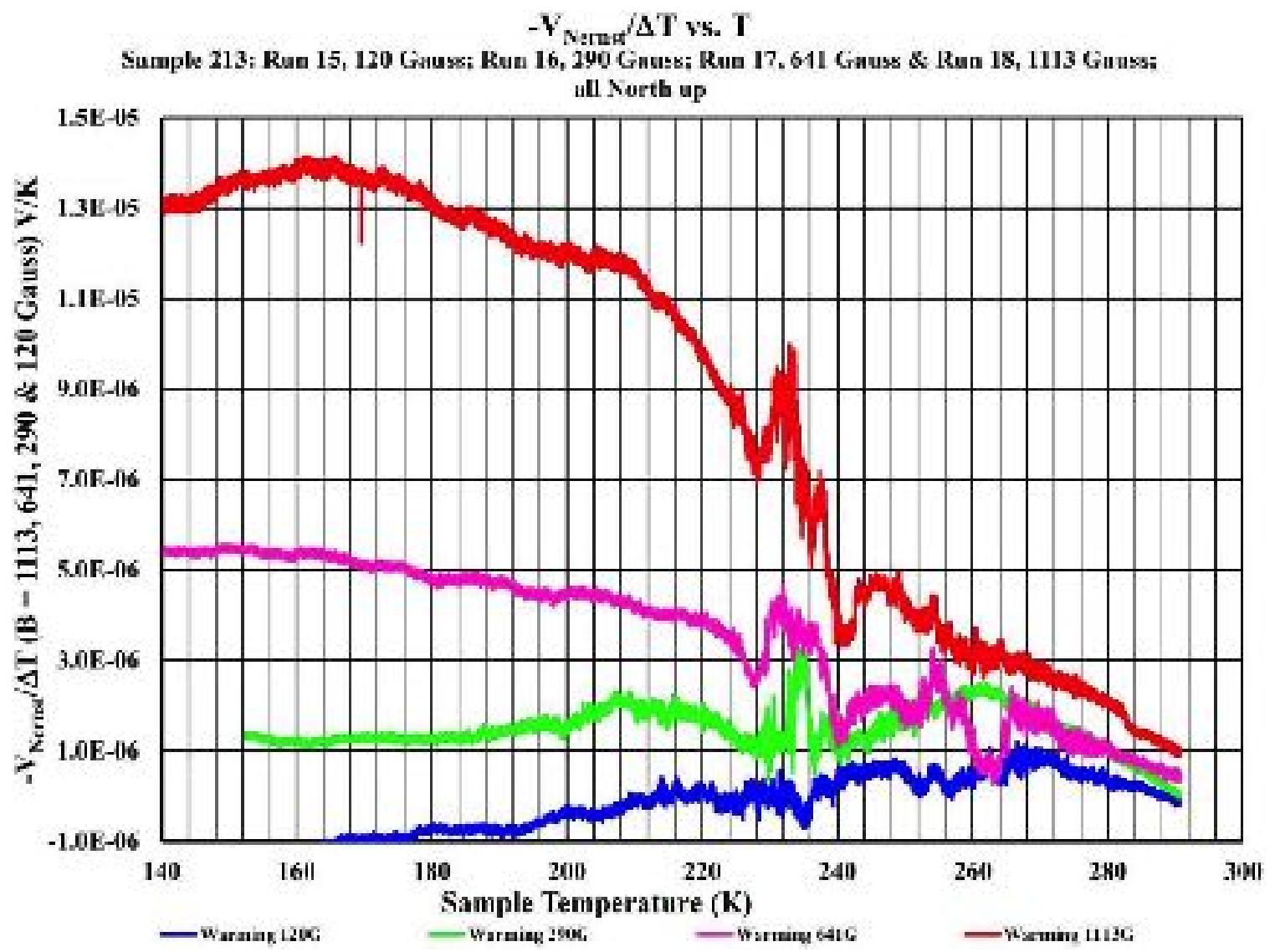

Figure 6.22: $-V_{\text {Nernst }} / \Delta T$ curves in the range of $140-300 \mathrm{~K}$ for same sample as in Figure 6.11. This is a collection of the warming direction plots from Figures 6.17 through 6.20. Note how the steps and features have tended to shift lower in temperature as the applied field is increased. Notice how the broad peak in the 290 Gauss curve at $228 \mathrm{~K}$ has evolved as the applied field is stepped up.

The consistency of the thermal gradient plots from run to run that are presented in aggregate in Figure 6.23 which eliminates any possibility that the observed Nernst signal in the data is the result of a thermally driven effect. 


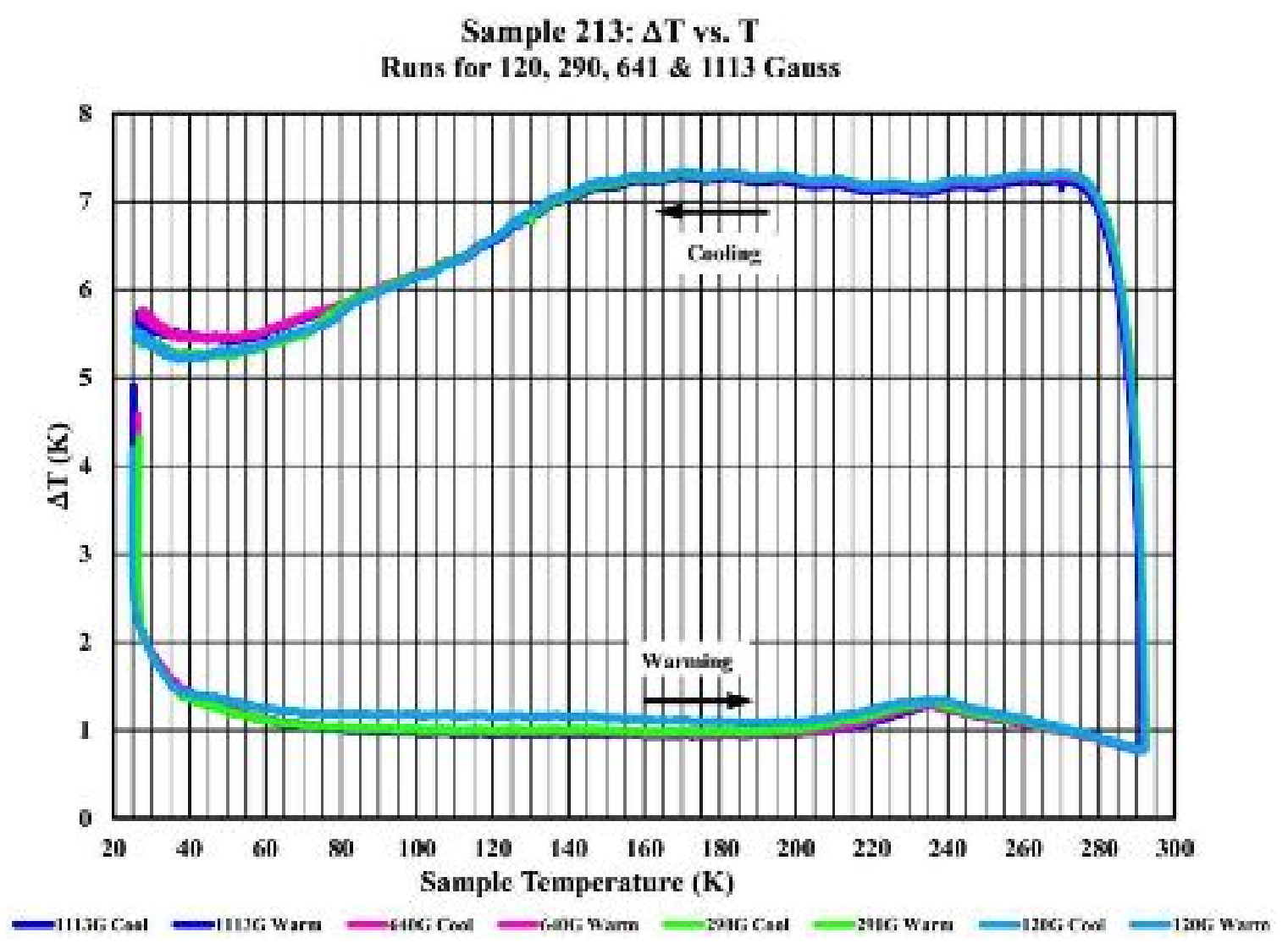

Figure 6.23: $\Delta T$ as a function of temperature both cooling and warming for the data presented in Figures 6.11 through 6.22.

\subsection{Discussion}

From the experiment, a response consistent with the presence of magnetic field flux vortices in phosphorous (electron donor) implanted HOPG and in phosphorous doped exfoliated multilayer graphene has been observed. The repeated nature of the observed steps in the $R v s . T$ characteristics of the material is consistent with the melting of stacks of pancake vortices of differing lengths at different temperatures. The lack of zero resistance at low temperatures is also consistent with pancake vortex behavior in the fluxflow regime. This allows the use of a single phenomenon, magnetic pancake vortices, to describe the features observed. The presence of magnetic vortices requires and is direct 
evidence of, superconductivity. The small Meissner effect may simply mean that the volume fraction of material involved is quite small or that the penetration depth is significantly larger than the sample thickness.

The extensive additional Nernst effect measurements have confirmed the presence of magnetic vortices, highlighted the magnetic vortex BKT transition and, in several instances, permitted the identification of pinning temperatures for stacks of these vortices well above the BKT transition.

In addition, once again, the temperature of $260-265 \mathrm{~K}$ has shown, this time magnetically, that there is evidence that the sample still retains superconductivity as high as $260-265 \mathrm{~K}$.

The material that was subjected to post doping argon implantation (damage) showed a discontinuous step in resistance at a temperature of about $265 \mathrm{~K}$. In [55], a summary of which is included in the background portion of this paper, that "the ultimate critical temperature in this system is in excess of $100 \mathrm{~K}$ and, may very likely be considerably higher if damage incurred during the doping can be further minimized". This conclusion has not been voided and, based on the material with phosphine in the PECVD growth gas mixture, may well be valid for a transition at a temperature of greater than 300 K.

\subsection{Conclusions}

As a result of high volume of our experimental work, we conclude that the lack of zero resistance at low temperature does not fundamentally exclude superconductivity as a mechanism for the material's observed electrical and magnetic behavior. The high degree of anisotropy in graphite and graphene, in fact, predicts that if the material is a 
superconductor there would be significant magnetic flux-flow losses to very low temperatures.

The magnetic response of phosphorous doped HOPG and graphene is likewise as would be expected for superconductors of similar physical characteristics. That is

1) that the superconducting region is thin in comparison to the London (magnetic) penetration depth;

2) that the high level of anisotropy in the material is favorable for the formation of pancake vortices. Additionally, it has been experiment demonstrated that the resistance vs. temperature curve can be quenched by the application of a magnetic field. This is likewise what would be anticipated from a superconductor.

The magnetization and susceptibility results for phosphorous-doped HOPG and graphene suggest

1) a transition temperature above $260 \mathrm{~K}$ for the magnetization based upon the ZFC to FC Hysteresis loop;

2) a transition temperature above $150 \mathrm{~K}$ based upon the $\mathrm{AC}$ susceptometer measurements. There is no trace of ferromagnetism in the results as both the magnetization and susceptibility are negative in the lower temperature region.

Finally, the Hall effect measurement shows a sign change in the Hall voltage when cooling the sample from room temperature to a temperature of $80 \mathrm{~K}$. This is observed in other, known and accepted, superconductors that are in the flux flow region of the mixed or vortex state. It is also seen in anomalous ferromagnetism. Since there are

1) no ferromagnetic atoms; 
2) no atoms with $d$ or $f$ electron shells in the sample it is highly unlikely that ferromagnetism is involved in these Hall measurements. The fact that the magnetization and magnetic susceptibility are both negative also tends to eliminate ferromagnetism as a candidate for this sign reversal in the Hall voltage.

The repeated steps in the resistance versus temperature characteristics of phosphorous doped HOPG and graphene are independent on how the material is doped. Adding damage causes these steps to become nearly discontinuous steps at elevated temperatures. This is consistent with the results that are expected for thin superconducting films without and with damage.

The fact that these steps in the Nernst voltage vs. temperature experiment are likewise observed in the differential Nernst experiment as tilted-peak vortex type peaks is confirmation that the steps represent the melting of pinned vortices at these temperatures. The simple Nernst experiment likewise has highlighted some of the same features as the differential measurement. There is a correlation for the observed temperatures, whether they are a resistive step, a differential Nernst peak or a Nernst step, peak or structure, throughout all the various measurements.

The results presented both confirm the model of Pearl or pancake vortices on a string as well as answering the fundamental question underpinning the entire hypothesis; the material is a superconductor. This is strongly reinforced by the hysteresis in the Nernst signal in a thermal cycling experiment where the only remaining explanation for the disappearance of the Nernst signal in the warming data is that the greater portion of the magnetic flux has been expelled from the sample at low temperature. 
This evidence of superconductivity in doped graphene/graphite with a de-pairing (critical) temperature in the region of $260 \mathrm{~K}$ is conclusive. This material is a superconductor. It screens magnetic fields. It exhibits drops in resistivity as vortices become pinned. The BKT transition for both charge carriers and magnetic vortices is present. Differential Nernst magnetic vortex peaks have demonstrated the depinning temperatures of vortices as well as the fundamental BKT transition of the vortex liquid to a vortex lattice.

In conclusion, the evidence forces us to conclude that phosphorous doped Highly Oriented Pyrolytic Graphite (and phosphorous doped graphene) is a superconductor with a transition temperature above $260 \mathrm{~K}$. In fact, some of our experimentally obtained data hints at a possibility that the transition temperature in the best samples may approach room temperature. More work can be done to confirm the limitations, observations and proposed mechanism of the observed effect; however, the effect is only consistent with the material being a mixed state superconductor.

In addition, the fact that this data is qualitatively reproducible with many, now hundreds, of samples and over a time span of eight years is a compelling reason to conclude that this is not a fleeting "Unidentified Superconducting Object".

\subsection{Future Work}

As a continuation of the research to investigate superconductivity and other phenomena of phosphorus-doped graphene and graphite as 2D and quasi-2D materials, some of the doped graphene samples will be etched in Hall-bar shape by a laser engraver as shown in Figure 6.24. 
The purpose of etching the samples is to measure Current-Voltage (I-V) characteristic curve of the samples while a nanoampere direct current (DC) is applied longitudinally across the etched sample, a uniformed magnetic field is applied into or out of the plane, and longitudinal and Hall voltages are measured as shown in Figure 1.18 in chapter 1. Furthermore, a quantum Hall effect (QHE), unconventional quantum Hall effect (UQHE), or quantum anomalous Hall effect (QAHE) in phosphorus-doped graphene is expected to be seen in this future experiment.

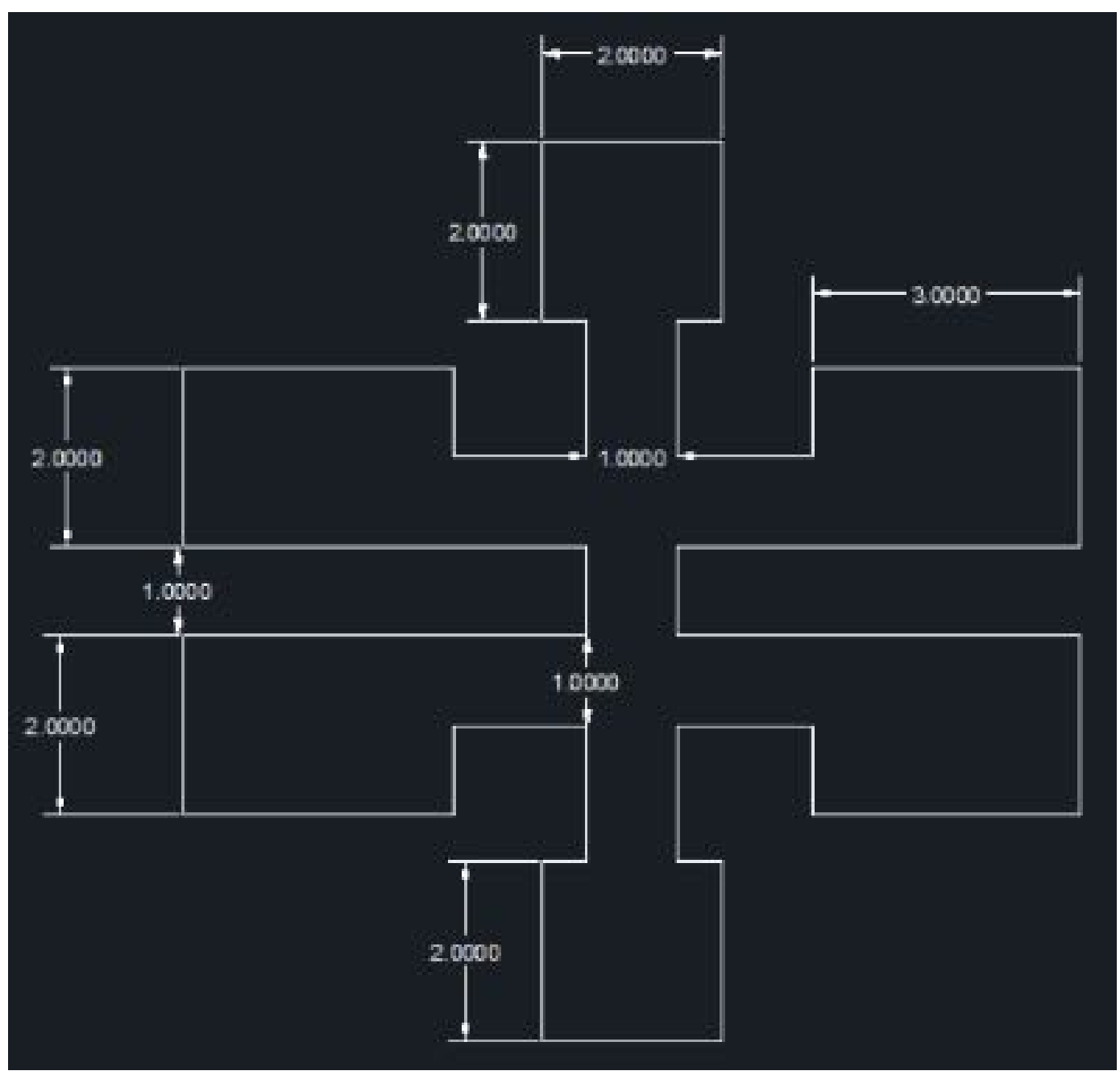

Figure 6.24: Specific sketch of etched phosphorus-doped graphene samples. Measurement unit is in millimeter. Image courtesy of Julian Gil Pinzon. 
6.5. Tables of Symbols and Acronyms

Table 6.1: Table of Symbols.

\begin{tabular}{|c|l|c|}
\hline Symbols & \multicolumn{1}{|c|}{ Description } & Value \\
\hline$E_{p}$ & Implantation Energy. & - \\
\hline$V_{\text {Nernst }}$ & Nernst Voltage. & - \\
\hline$\Delta T$ & Temperature Difference. & - \\
\hline$d$ & $\begin{array}{l}\text { A type of subshell (a subdivision of electron } \\
\text { shells separated by electron orbitals). }\end{array}$ & $\begin{array}{l}\text { A type of subshell (a subdivision of electron } \\
\text { shells separated by electron orbitals). }\end{array}$ \\
\hline$f$
\end{tabular}

Table 6.2: Table of Acronyms.

\begin{tabular}{|c|c|}
\hline Acronym & Description \\
\hline $2 \mathrm{D}$ & 2-dimensional \\
\hline $\mathrm{AC}$ & Alternating Current \\
\hline BKT & Berezinski-Kosterlitz-Thouless \\
\hline CVD & Chemical Vapor Deposition \\
\hline $\mathrm{DC}$ & Direct Current \\
\hline HOPG & Highly Ordered Pyrolytic Graphite \\
\hline $\mathrm{FC}$ & Field-Cooled \\
\hline $\mathrm{I}-\mathrm{V}$ & Current-Voltage \\
\hline $\mathrm{Oe}$ & Oersted \\
\hline PECVD & Plasma-Enhanced Chemical Vapor Deposition \\
\hline QAHE & Quantum Anomalous Hall Effect \\
\hline QHE & Quantum Hall Effect \\
\hline$R$ vs. $T$ & Resistance versus Temperature \\
\hline SQUID & Superconducting QUantum Interference Device \\
\hline$S$ vs. $T$ & Susceptibility versus Temperature \\
\hline
\end{tabular}




\begin{tabular}{|c|c|}
\hline UQHE & Unconventional Quantum Hall Effect \\
\hline ZFC & Zero-Field-Cooled \\
\hline
\end{tabular}




\section{REFERENCES}




\section{LIST OF REFERENCES}

[1] J. S. Kukesh and L. Pauling "The Problem of The Graphite Structure," American Mineralogist 35, p.125, 1950.

[2] P. Atkins "The Elements of Physical Chemistry," $3^{\text {rd }}$ ed. W.H. Freeman and Company, NY, 1993.

[3] S. Evans "Carbon and Its Allotropes," Chemistry 405, 2001.

[4] N. W. Winter and F. H. Ree "Stability of the Graphite and Diamond Phase of Finite Carbon Clusters," Detonation Symposium Snowmass, UCRL-JC-127880 preprint, 1998.

[5] D. W. Olson “Graphite,” U.S. Geological Survey Minerals Yearbook, 2003.

[6] D. Kopeliovich "Graphite," Materials Engineering, SubsTech Substances \& Technologies, Substech.com, online, 2013.

[7] University of Waterloo "Graphite," Earth Sciences Museum, Detailed Rocks and Minerals Articles, uwaterloo.ca, online, 2017.

[8] R. R. Haering "Band Structure of Rhombohedral Graphite," Canadian Journal of Physics 36, pp. $352-362,1958$.

[9] J. H. Warner, F. Schaffel, M. Rummeli, and A. Bachmatiuk "Graphene: Fundamentals and emergent applications," $1^{\text {st }}$ ed., Elsevier, print, 2013.

[10] B. Hannesson "Introduction to Graphene," 16 ECTS thesis of a B.Sc. degree in physics, School of Engineering and Natural Sciences, University of Iceland, 2016.

[11] C. G. Al-Amin “Advanced Graphene Microelectronic Devices," FIU Electronic Theses and Dissertations 2512, 2016.

[12] E. Boysen, N. C. Muir, D. Dudley, and C. Peterson "Graphene: Sheets of CarbonBased Nanoparticles,” Nanotechnology for Dummies, $2^{\text {nd }}$ ed. Dummies, 2011.

[13] A. C. Ferrari, J. C. Meyer, V. Scardaci, C. Casiraghi, M. Lazzeri, F. Mauri, S. Piscanec, D. Jiang, K.S. Novoselov, S. Roth, and A. K. Geim "Raman Spectrum of Graphene and Graphene Layers," Physical Review Letters, 97, 187401, 2006.

[14] K. S. Novoselov, A. K. Geim, S. V. Morozov, D. Jiang, Y. Zhang, S. V. Dubonos, I. V. Grigorieva, A. A. Firsov "Electric Field Effect in Atomically Thin Carbon Films," Science 306, p. 666, 2004.

[15] K. S. Novoselov, E. McCann, S. V. Morozov, V. I. Fal'ko, M. I. Katsnelson, U. Zeitler, D. Jiang, F. Schedin, and A. K. Geim "Unconventional quantum Hall effect 
and Berry's phase of $2 \pi$ in bilayer graphene," Nature Physics, vol. 2, issue 3, pp. 177 - 180, 2006.

[16] Y. Zhang, J. P. Small, W. V. Pontius, and P. Kim "Fabrication and electric-fielddependent transport measurements of mesoscopic graphite devices," Apply Physics Letters 86, 073104, 2005.

[17] C. Berger, Z. Song, T. Li, X. Li, A. Y. Ogbazghi, R. Feng, Z. Dai, A. N. Marchenkov, E. H. Conrad, P. N. First, and W. A. de Heer "Ultrathin Epitaxial Graphite: 2D Electron Gas Properties and a Route toward Graphene-based Nanoelectronics," Journal Physics Chemistry B 108, pp. 19912 - 19916, 2004.

[18] J. S. Bunch, Y. Yaish, M. Brink, K. Bolotin, and P. L. McEuen "Coulomb Oscillations and Hall Effect in Quasi-2D Graphite Quantum Dots," Nano Letters vol. 5, no. 2, pp. $287-290,2005$.

[19] P. Anees, M. C. Valsakumar, S. Chandra, and B. K. Panigrahi "Ab initio study on stacking sequences, free energy, dynamical stability and potential energy surfaces of graphite structures," IOP Publishing, Modelling and Simulation in Materials Science and Engineering 22, 035016, 2014.

[20] A. H. R. Palser "Interlayer interactions in graphite and carbon nanotubes," Physical and Theoretical Chemistry Laboratory, South Parks Road, Oxford, UK, 1999.

[21] G. Savini, Y. J. Dappe, S. Öberg, J. C. Charlier, M. I. Katsnelson, and A. Fasolino "Bending modes, elastic constants and mechanical stability of graphitic systems," Carbon 49, pp. $62-69,2011$.

[22] D. Tománek, S. G. Louie, H. J. Mamin, D. W. Abraham, R. E. Thomson, E. Ganz and J. Clarke "Theory and observation of highly asymmetric atomic structure in scanning-tunneling-microscopy images of graphite," Physical Review B 35, 7790, 1987.

[23] J. D. Bernal "The structure of graphite," Proceedings of The Royal Society A, The Royal Society Publishing, vol. 106, issue 740, pp. 749 - 7731924.

[24] H. Lipson and A. R. Stokes "The structure of graphite," Proceedings of The Royal Society A, The Royal Society Publishing, vol. 181, issue 984, pp. 101 - 105, 1942.

[25] L. Samuelson, Inder P. Batra, and C. Roetti "A comparison of electronic properties of various modifications of graphite," Solid State Communications, vol. 33, issue 7, pp. $817-820,1980$. 
[26] L. Samuelson and I. P. Batra "Electronic properties of various stages of lithium intercalated graphite," Journal of Physics C: Solid State Physics, vol. 13, no. 27, pp. $5105-5124,1980$.

[27] J. S. Rowlinson "Cohesion: a scientific history of intermolecular forces," Cambridge University Press, 2002.

[28] J. D. Van der Waals "The equation of state for gases and liquids," Nobel Lecture, The Nobel Prize in Physics, 1910.

[29] J. Israelachvili "Intermolecular and Surface Forces," London: Academic Press, 1991.

[30] G. Peschel "Carbon-Carbon bonds: Hybridization," Gina Peschel Handout, Freie Universität Berlin, 2011.

[31] AIST-NT Advanced Integrated Scanning Tools for Nano Technology "HOPG," AIST-NT SPM accessories web store, online, 2017.

[32] POCO GRAPHITE An Entegris Company "Properties and Characteristics of Graphite," POCOGRAPHIT, INC., Specialty Materials, For industrial applications, online, 2015.

[33] J. D. Brook and G. H. Taylor "The Formation of Some Graphitizing Carbons," Chemistry and Physics of Carbon 4, pp. 243 - 283, 1968.

[34] Graphite Concept Product "Graphitization," Graphite Concept Products Co. Ltd., online, 2017.

[35] Corrosionpedia, "Graphitization," Corrosionpedia Inc., online, 2017.

[36] H. Marsh, E. A. Heintz, and F. Rodriguez-Reinoso "Introduction to carbon technologies," Universidad de Alicante, Alicante, Spain, 1997.

[37] MikroMasch, "HOPG HIGHLY ORDERED PYROLYTIC GRAPHITE," Test Structure, MikroMasch, a trademark of Nanoworld AG operated by ISB Ltd., online, 2017.

[38] J. N. Fuchs and M. O. Goerbig "Introduction to the physical Properties of Graphene," Lecture Note, University of California, Santa Barbara, 2008.

[39] L. Pauling, "The Nature of Chemical Bonds," Cornell University Press, 1960.

[40] Y. H. Jeong and S. -R. Eric Yang "Graphene Nanosystems and Low-Dimensional Chern-Simons Topological Insulators," Cornell University Library, Condensed 
Matter, Mesoscale and Nanoscale Physics, arXiv:1511.00805v1 [cond-mat.meshall], 2015.

[41] W. Bao "Electrical and Mechanical Properties of Graphene," Doctoral Dissertation, University of California Riverside, 2012.

[42] C. G. Stefanita "Magnetism Basics and Applications," Springer, Condensed Matter Physics, Springer-Verlag Berlin Heidelberg, 2012.

[43] S. D. Sarma "Spintronics," American Scientist, vol. 89, pp. 516 - 523, 2001.

[44] C. Lee, X. D. Wei, J. W. Kysar, and J. Hone "Measurement of The Elastic Properties and Intrinsic Strength of Monolayer Graphene," Science 321, pp. 385 388, 2008.

[45] I. A. Ovid'ko "Mechanical Properties of Graphene," Reviews on advanced materials science 34, pp. 1 - 11, 2013.

[46] H. H. von Grunberg, P. Keim, G. Maret "Phase Transitions in Two-Dimensional Colloidal Systems," Universität Konstanz, Germany, 2007.

[47] J. H. Los, K. V. Zakharchenko, M. I. Katsnelson, and A. Fasolino "Melting Temperature of Graphene," Physical Review B 91, 045415, 2015.

[48] K. V. Zakharchenko, A. Fasolino, J. H. Los, and M. I. Katsnelson, "Melting of Graphene: From Two to One Dimension," Journal of Physics: Condensed Matter IOP science 23, 202202, 2011.

[49] E. Ganz, A. B. Ganz, L. M. Yang, and M. Dornfeld "The Initial Stages of Melting of Graphene Between $4000 \mathrm{~K}$ and $6000 \mathrm{~K}$," Physical Chemistry Chemical Physics 19, 3756, 2017.

[50] D. C. Elias, R. R. Nair, T. M. G. Mohiuddin, S. V. Morozov, P. Blake, M. P. Halsall, A. C. Ferrari, D. W. Boukhvalov, M. I. Katsnelson, A. K. Geim, K. S. Novoselov "Control of graphene's properties by reversible hydrogenation: evidence for graphene," Science 323, pp. 610-613, 2009.

[51] R. R. Nair, W. Ren, R. Jalil, I. Riaz, V. G. Kravets, L. Britnell, P. Blake, F. Schedin, A. S. Mayorov, S. Yuan, M. I. Katsnelson, H. M. Cheng, W. Strupinski, L. G. Bulusheva, A. V. Okotrub, I. V. Grigorieva, A. N. Grigorenko, K. S. Novoselov, A. K. Geim "Fluorographene: a two-dimensional counterpart of Teflon," Small 6, pp. $2877-2884,2010$. 
[52] Goldt Ilya V., Shlyakhtin Oleg A., and Ioffe Ilya N. "graphane (rus. графан) hydrogenated graphene," Glossary of Nanotechnology and Related Terms, Rusnano, online, 2011.

[53] F. A. de la Cruz and J. M. Cowley "Structure of Graphitic Oxide," Nature 196, pp. $468-469,1962$.

[54] M. Nasrollahzadeh, F. Babaei, P. Fakhric, and B. Jalehc "Synthesis, characterization, structural, optical properties and catalytic activity of reduced graphene oxide/copper nanocomposites," RSC Advances, issue 14, 2015.

[55] G. L. Larkins, and Y. A. Vlasov, "Indications of superconductivity in doped highly oriented pyrolytic graphite," Superconductor Science and Technology, vol. 24, no. 092001, 2011.

[56] G. L. Larkins, Y. A. Vlasov, and K. J. Holland, "Evidence of superconductivity in doped graphite and graphene," Superconductor Science and Technology, 29, 1, $015015,2016$.

[57] K. J. Holland, "Doping as a Possible Means to create Superconductivity in Graphene," FIU Electronic Theses and Dissertations 2550, 2016.

[58] P. L. Wallace "The Band Structure of Graphite," Physical Review 71, pp. 622 634, 1947.

[59] Q. Luo "The elementary electronic properties of graphene," Department of Physics and Astronomy, University of Tennessee, 2010.

[60] R. M. White "Quantum Theory of Magnetism," Springer, Springer Series in SolidState Sciences 32, Magnetic Properties of Materials $3^{\text {rd }}$ ed. 2007.

[61] AK Lectures "Spin-Orbit Interaction," Quantum-Mechanical Theory of Atoms, Modern Physics, aklectures.com, online, 2014.

[62] University of St. Andrew "Spin-orbit interaction (or "coupling")," PHY 4021 Lecture Note, 2011.

[63] N. Tombros, C. Jozsa, M. Popinciuc, H. T. Jonkman, and B. J. van Wees "Electronic Spin Transport and Spin Precession in Single Graphene Layers at Room Temperature," Physics of Nanodevices and Molecular Electronics, Zernike Institute for Advanced Materials, Nijenborgh 4, 9747 AG Groningen, The Netherlands, 2007. 
[64] W. Han, R. K. Kawakami, M. Gmitra, and J. Fabian "Graphene Spintronics," Nature Nanotechnology, Review Article, 2014.

[65] Z. Bao, J. Shia, M. Yang, S. Zhang, and M. Zhang "Magnetism Induced by D3Symmetry Tetra-Vacancy Defects in Graphene," Chemical Physics Letters, vol. 510, issues $4-6$, pp. $246-251,2011$.

[66] A. M. Valencia and M. J. Caldas "Vacancy in Graphene: Insight on Magnetic Properties from Theoretical Modeling," Physical Review B 96, 125431, 2017.

[67] M. Sepioni "Magnetic Properties of Graphene," Doctoral Thesis, University of Manchester, 2012.

[68] S. Okada and A. Oshiyama "Magnetic Ordering in Hexagonally Bonded Sheets with First-Row Elements," Physical Review Letters, vol. 87, 146803, 2001.

[69] E. Kan, Z. Li, and J. Yang "Magnetism in Graphene System," Nano, vol. 03, issue 06, pp. $433-442,2008$.

[70] O. V. Yazyev and L. Helm "Defect-Induced Magnetism in Graphene," Physical Review B 75, 125408, 2007.

[71] D. W. Boukhvalov, M. I. Katsnelson, and A. I. Lichtenstein "Hydrogen on graphene: Electronic structure, total energy, structural distortions and magnetism from first-principles calculations," Physical Review B 77, 035427, 2008.

[72] H. González-Herrero, J. M. Gómez-Rodríguez, P. Mallet, M. Moaied, J. J. Palacios, C. Salgado, M. M. Ugeda, J. Y. Veuillen, F. Yndurain, and I. Brihuega, "Atomicscale control of graphene magnetism by using hydrogen atoms," Science, vol. 352, issue 6284, pp. $437-441,2016$.

[73] I. Brihuega "Hydrogen makes graphene magnetic," YouTube, An illustrative video describing the work "Atomic-scale control of graphene magnetism using hydrogen atoms", Category: Science \& Technology, online, 2016.

[74] A. A. Balandin "Thermal Properties of Graphene and Nanostructure Carbon Materials," Review Article, Nature Materials 10, pp. 569 - 581, 2011.

[75] W. Cai, A. L. Moore, Y. Zhu, X. Li, S. Chen, L. Shi, and R. S. Ruoff "Thermal Transport in Suspended and Supported Monolayer Graphene Grown by Chemical Vapor Deposition," Nano Letters 10, pp. 1645 - 1651, 2010.

[76] The Engineering Tool Box "Thermal Conductivity of common Materials and Gases," Resources, Tools and Basic Information for Engineering and Design of Technical Applications!, The Engineering Tool Box.com, online, 2017. 
[77] A. A. Balandin, S. Ghosh, W. Bao, I. Calizo, D. Teweldebrhan, F. Miao and C. N. Lau "Extremely High Thermal Conductivity of Graphene: Experimental Study," Nano Letters 8, pp. $902-907,2008$.

[78] Graphene-info The Graphene Experts "Graphene thermal conductivity introduction and latest news," Graphene-Info, online, 2017.

[79] MuonRay "The Spacecraft That Requires no Fuel: Graphene Photoelectric Solar Sails," Awesome Inc., muonray.blogspot.com, online, 2016.

[80] D. R. Cooper, B. D’Anjou, N. Ghattamaneni, B. Harack, M. Hilke, A. Horth, N. Majlis, M. Massicotte, L. Vandsburger, E. Whiteway, and V.Yu "Experimental Review of Graphene," Review Article, International Scholarly Research Network, Condensed Matter Physics, vol. 2012, Article ID 501686, 2011.

[81] D. Tong “The Quantum Hall Effect,” TIFR Infosys Lectures, 2016.

[82] K. S. Novoselov, A. K. Geim, S. V. Morozov, D. Jiang, M. I. Katsnelson, I. V. Grigorieva, S. V. Dubonos, and A. A. Firsov "Two-dimensional gas of massless Dirac fermions in graphene," Nature, vol. 438, no. 7065, pp. 197 - 200, 2005.

[83] K. S. Novoselov, Z. Jiang, Y. Zhang, S. V. Morosov, H. L. Stormer, U. Zeitler, J. C. Maan, G. S. Boebinger, P. Kim, and A. K. Geim "Room-Temperature Quantum Hall Effect in Graphene," Science 315, 1379, 2007.

[84] K. von Klitzing "The Quantized Hall Effect," Nobel Lecture, The Nobel Prize in Physics, 1985.

[85] Z. Jiang, Y. Zhang, Y. W. Tan, H. L. Stormer, and P. Kim "Quantum Hall Effect in Graphene," Elsevier, Science Direct, Solid State Communications, Exploring graphene: Recent research advances, vol. 143, issue 1-2, pp. 14 - 19, 2007.

[86] S. Sahoo "Quantum Hall Effect in Graphene: Status and Prospects," Indian Journal of Pure \& Applied Physics, vol. 49, pp. 367 - 371, 2011.

[87] Z. Li "Unconventional Quantum Hall Effect in Graphene," Physics Essay, Department of Physics, University of Illinois at Urbana-Champaign, 2008.

[88] R. B. Laughlin "Anomalous Quantum Hall Effect: An Incompressible Quantum Fluid with Fractionally Charged Excitations," Physics Review Letters vol. 50, no. 18, pp. $1395-1398,1983$.

[89] R. B. Laughlin "Fractional Quantization," Nobel Lecture, The Nobel Prize in Physics, 1998. 
[90] B. L. Johnson "Understanding the Laughlin wave function for the fractional quantum Hall effect,” American Journal of Physics 70, pp. 401 - 405, 2002.

[91] H. L. Störmer "The Fractional Quantum Hall Effect," Nobel Lecture, The Nobel Prize in Physics, 1998.

[92] D. C. Tsui "Interplay of Disorder and Interaction in Two-Dimensional Electron Gas in Intense Magnetic Fields," Nobel Lecture, The Nobel Prize in Physics, 1998.

[93] C. R. Dean, A. F. Young, P. Cadden-Zimansky, L. Wang, H. Ren, K. Watanabe, T. Taniguchi, P. Kim, J. Hone, and K. L. Shepard "Multicomponent fractional quantum Hall effect in graphene," Nature Physics, 7, pp. 639 - 696, 2011.

[94] A. S. Mahajan and A. A. Rangwala "Electricity and Magnetism," 23 rd ed. McGrawHill, print, p. 315, 2007.

[95] A. K. Geim, "Random Walk to Graphene," Nobel Lecture, The Nobel Prize in Physics, 2010.

[96] K. S. Novoselov, "Graphene: Materials in The Flatland," Nobel Lecture, The Nobel Prize in Physics, 2010.

[97] M. M. Ugeda, I. Brihuega, F. Guinea, and J. M. Gómez-Rodríguez, “The missing atom as a source of carbon magnetism," Physical Review Letters, 104, 096804, 2010 .

[98] M. M. Ugeda, D. Fernández-Torre, I. Brihuega, P. Pou, A. J. Martínez-Galera, R. Pérez, and J. M. Gómez-Rodríguez, "Point defects on graphene on metals," Physical Review Letters, 107, 116803, 2011.

[99] E. Ishiguro and S. Koide, "Magnetic Properties of the Hydrogen Molecules," Physical Review, vol. 94, no. 2, pp. 350 - 357, 1954.

[100] B. Jerew "Graphene Oxide Offers New Hope for Water Decontamination," The Green Optimistic, greenoptimistic.com, online, 2013.

[101] E. C. Stoner, "Collective electron ferromagnetism in metals and alloys," J. Phys. Radium, 12 (3), pp. 372 - 388, 1951.

[102] P. Mohn, "Magnetism in the Solid State," Springer, Springer Series in Solid-State Sciences 134, print, 2003.

[103] K. H. J. Buschoe and F. R. De Boer, "Physics of Magnetism and Magnetic Materials," Kluwer Academic Publishers, print, 2004. 
[104] J. O. Sofo, G. Usaj, P. S. Cornaglia, A. M. Suarez, A. D. Hernandez-Nieves, and C. A. Balseiro, "Magnetic structure of hydrogen-induced defects on graphene," Physical Review B 85, 115405, 2012.

[105] W. Li, M. Zhao, Y. Xia, R. Zhang, and Y. Mu "Covalent-adsorption induced magnetism in graphene," Journal of Materials Chemistry, 19 (48), pp. 9274 -9282, 2009.

[106] Topology in Condensed Matter, "How to measure the Hall effect," topocondmat.org, online, 2014.

[107] V. M. Pereira, A. H. Castro Neto, and N. M. R. Peres, "Tight-binding approach to uniaxial strain in graphene," Physical Review B 80, 045401, 2009.

[108] J. Sivek, "File:Real and reciprocal space unit vectors of graphene lattice.svg," wikimedia commons, Category: Graphene, online, 2015.

[109] A. W. Moore, "Highly oriented pyrolytic graphite," Chemistry and physics of carbon, vol. 11, pp. $69-187,1973$.

[110] M. Tinkham "Introduction to Superconductivity," $2^{\text {nd }}$ ed. Dover, print, 1996.

[111] T. V. Duzer and C. W. Turner "Principle of Superconductive Device and Circuit," $2^{\text {nd }}$ ed. Prentice Hall PTR, print, 1999.

[112] P. Mangin and R. Kahn "Superconductivity An Introduction," Springer, print, 2017.

[113] F. London and H. London "The electromagnetic equations of the supraconductor," Proceedings of the Royal Society A: Mathematical, Physical and Engineering Sciences. 149, pp. $71-88,1935$.

[114] ENCYCLOÆDIA BRITANNICA "Superconductivity," Physics, britannica.com, Encyclopædia Britannica, Inc., online, 2017.

[115] MuonRay "Nature of Magnetism," Awesome Inc., muonray.blogspot.com, online, 2014.

[116] R. Nave "Magnetic Levitation and Levitation Currents," HyperPhysics, Condensed Matter, Georgia State University, online, 2016.

[117] H. K. Onnes "Investigations into the properties of substances at low temperatures, which have led, amongst other things, to the preparation of liquid helium," Nobel Lecture, The Nobel Prize in Physics, 1913. 
[118] K. McElroy "Notes on the Drude model," Introduction to Solid State Physics, Physics Department University of Colorado, 2017.

[119] A. Bid "Lecture1-Drude Model," Condensed Matter Physics, Indian Institute of Science, 2017.

[120] H. J. Fink and S. B. Haley "Surface Induced Anomalous Superconductivity," International Journal of Modern Physics B, vol. 17, Issue 11, 2171, 2003.

[121] G. Rangarajan "Mod-01 Lec-29 Ginsburg - Landau Theory, Flux Quantization," nptelhrd, YouTube, Indian Institute of Technology, Department of Physics, Condensed Matter Physics Lecture - 29, Category: Education, online, 2013.

[122] M. Suzuki and I. S. Suzuki "Lecture Note on Solid State Physics Ginzburg-Landau Theory for Superconductivity," Department of Physics, State University of New York at Binghamton, 2007.

[123] J. Bardeen "Electron-Phonon Interactions and Superconductivity," Nobel Lecture, The Nobel Prize in Physics, 1972.

[124] L. N. Cooper "Microscopic Quantum Interference Effects in The Theory of Superconductivity," Nobel Lecture, The Nobel Prize in Physics, 1972.

[125] J. R. Schrieffer "Macroscopic Quantum Phenomena from Pairing in Superconductors," Nobel Lecture, The Nobel Prize in Physics, 1972.

[126] H. D. Young and R. G. Freedman "University Physics," Pearson, $12^{\text {th }}$ ed., p. 716, print, 2007.

[127] 9. 4. D - Superconductors "How is Superconductivity Explained?," quarkology.com science made simple, Acuity Education, online, 2012.

[128] R. Nave "Model of Pair Attraction," HyperPhysics, Condensed Matter, Georgia State University, online, 2016.

[129] M. Lufaso "Chapter 9: Superconductivity," Solid State Chemistry, Lecture Note, University of North Florida, 2017.

[130] L. N. Cooper "Bound Electron Pairs in a Degenerate Fermi Gas," Physical Review, vol. 104 , no. $4,1956$.

[131] J. Bardeen, L. N. Cooper, and J. R. Schrieffer, "Theory of Superconductivity," Physical Review, vol. 108, no. 5, 1957.

[132] H. G. Katzgraber "Phase Transition," Proseminar in Theoretical Physics, Institut für theoretische Physik, ETH Zürich, 2007. 
[133] G. Rangarajan "Mod-01 Lec-30 Cooper Pairs," nptelhrd, YouTube, Indian Institute of Technology, Department of Physics, Condensed Matter Physics Lecture-30, Category: Education, online, 2013.

[134] A. F. J. Levi "Essential Classical Mechanics for Device Physics," IOPscience, print, 2016.

[135] C. G. Stefanita "Magnetism Basics and Applications," Springer, Condensed Matter Physics, Springer-Verlag Berlin Heidelberg, 2012.

[136] B. D. Josephson "Possible new effects in superconductive tunnelling," Physics Letters, vol. 1, issue 7, pp. $251-253,1962$.

[137] V. N. Kotov, B. Uchoa, V. M. Pereira, F. Guinea, and A. H. Castro Neto "Electronelectron interactions in graphene: current status and perspective," Reviews of Modern Physics 84, 1067 - 1125, 2012.

[138] A. Di Bernardo, O. Millo, M. Barbone, H. Alpern, Y. Kalcheim, U. Sassi, A. K. Ott, D. De Fazio, D. Yoon, M. Amado, A. C. Ferrari, J. Linder, and J. W. A. Robinson " $p$-wave triggered superconductivity in single-layer graphene on an electron-doped oxide superconductor," Nature Communications 8: 14024, 2017.

[139] C. Tonnoir, A. Kimouche, J. Coraux, L. Magaud, B. Delsol, B. Gilles, and C. Chapelier "Induced Superconductivity in Graphene Grown on Rhenium," Physical Review Letters 111, 246805, 2013.

[140] D. M. Guzman, H. M. Alyahyaei, and R. A. Jishi "Superconductivity in graphenelithium," 2D Materials, 1, 021005, 2014.

[141] R. Al-Jishi "Model for superconductivity in graphite intercalation compounds," Physical Review B 28, 112, 1983.

[142] R. A. Jishi, M. S. Dresselhaus, and A. Chaiken "Theory of the upper critical field in graphite intercalation compounds," Physical Review B 44, 10248, 1991.

[143] R. A. Jishi and M. S. Dresselhaus "Superconductivity in graphite intercalation compounds," Physical Review B 45, 12465, 1992.

[144] D. R. Cooper, B. D’Anjou, N. Ghattamaneni, B. Harack, M. Hilke, A. Horth, N. Majlis, M. Massicotte, L. Vandsburger, E. Whiteway, and V. Yu "Experimental Review of Graphene," International Scholarly Research Network, ISRN Condensed Matter Physics, vol. 2012, Article ID 501686, 2012.

[145] A. Streitwieser, C. H. Heathcock, and E. M. Kosower "Introduction to organic chemistry," $4^{\text {th }}$ ed., Macmillan, print, 1992. 
[146] I. I. Marzin and A. V. Balatsky "Superconductivity in Ca-intercalated bilayer graphene," Philosophical Magazine Letters, 90:10 pp. 731 - 738, 2010.

[147] A. P. Tiwari, S. Shin, E. Hwang, S. G. Jung, T. Park, and H. Lee "Superconductivity at $7.4 \mathrm{~K}$ in Few Layer Graphene by Li-intercalation," Journal of Physics: Condensed Matter, vol. 29, 445701, 2017.

[148] H. Huang, Y. Xia, X. Tao, J. Du, J. Fang, Y. Gan and W. Zhang "Highly efficient electrolytic exfoliation of graphite into graphene sheets based on $\mathrm{Li}$ ions intercalation-expansion-microexplosion mechanism," Journal of Materials Chemistry, issue 21, 2012.

[149] A. G. Shepelev and D. Larbalestier "The discovery of type II superconductors," CERN Courier, Produced for CERN by IOP Publishing, online, 2011.

[150] A. G. Shepelev "The Discovery of Type II Superconductors (Shubnikov Phase)," Superconductor, Sciyo, ch. 2, pp. 17 - 46, print, 2010.

[151] J. N. Rjabinin and L. W. Shubnikow "Magnetic Properties and Critical Currents of Supra-conducting Alloys," Nature, vol. 135, no. 3415, pp. 581 - 582, 1935.

[152] A. A. Abrikosov “Type II superconductors and The Vortex Lattice," Nobel Lecture, The Nobel Prize in Physics, 2003.

[153] Adwaele "Flux lines in a type two superconductor01," English Wikipedia, 2012.

[154] M. Wilson “The mixed state in Type II superconductors," SlidePlayer, Presentation transcript: 10, slideplayer.com, online, 2016.

[155] F. D. M. Haldane, “Topological Quantum Matter,” Nobel Lecture, The Nobel Prize in Physics, 2016.

[156] J. M. Kosterlitz, “Topological Defects and Phase Transitions,” Nobel Lecture, The Nobel Prize in Physics, 2016.

[157] J. M. Kosterlitz and D. J. Thouless, "Ordering, metastability and phase transitions in two-dimensional systems," Journal of Physics C: Solid State Physics, vol. 6, no. 7, pp. $1181-1203,1973$.

[158] M. R. Beasley, J. E. Mooij, and T. P. Orlando, "Possibility of Vortex-Antivortex Pair Dissociation in Two-Dimensional Superconductors," Physical Review Letters, vol. 42, no. 17, pp. $1165-1168,1979$.

[159] C. L. Kane and E. J. Mele, “A New Spin on the Insulating State,” Science, vol. 314, 5806, pp. $1692-1693,2006$. 
[160] B. I. Halperin, "Quantized Hall conductance, current-carrying edge states, and the existence of extended states in a two-dimensional disordered potential," Physical Review B, vol. 25, no. 4, pp. 2185 -2190, 1982.

[161] Q. Niu, D. J. Thouless, and Y. S. Wu, "Quantized Hall conductance as a topological invariant," Physical Review B, vol. 31, no. 6, pp. 3372 - 3377, 1985.

[162] F. Zhang, "What in the world is topological quantum matter?," TED-ED, YouTube, Category: Education, online, 2017.

[163] A. Y. Mironov, D. M. Silevitch, T. Proslier, S. V. Postolova, M. V. Burdastyh, A. K. Gutakovskii, T. F. Rosenbaum, V. M. Vinokur, and T. I. Baturina "Charge Berezinskii-Kosterlitz-Thouless transition in superconducting NbTiN films," Cornell University Library, Condensed Matter, Superconductivity, arXiv:1707.09679v1 [cond-mat.supr-con], 2017.

[164] D. Packard, "Introduction to the Berezinskii-Kosterlitz-Thouless Transition," University of Illinois at Urbana-Champaign, Loomis Laboratory of Physics, Physics 563: Phase Transitions and the Renormalization Group, Term essays, pp. $1-11,2013$.

[165] A. M. Goldman, "Superconductor-insulator transitions in the two-dimensional limit," Physica E, vol. 18, pp. 1-6, 2003.

[166] D. B. Haviland, Y. Liu, and A. M. Goldman, "Onset of Superconductivity in the Two-Dimensional Limit," Physical Review Letters, vol. 62, no. 18, pp. 2180 2183, 1989.

[167] L. M. Hernandez, A. Bhattacharya, K. A. Parendo, and A. M. Goldman, "Electrical Transport of Spin-Polarized Carriers in Disordered Ultrathin Films," Physical Review Letters, vol. 91, no. 12, 126801, 2003.

[168] P. Townsend and J. Sutton, "Investigation by Electron Tunneling of the Superconducting Energy Gaps in Nb, Ta, Sn, and Pb," Physical Review, vol. 128, no. 2 , pp. $591-595,1962$.

[169] X. Zhang, Y. S. Oh, Y. Liu, L. Yan, S. R. Saha, N. P. Butch, K. Kirshenbaum, K. H. Kim, J. Paglione, R. L. Greene, and I. Takeuchi, "Evidence of a universal and isotropic $2 \Delta / k_{B} T_{c}$ ratio in 122-type iron pnictide superconductors over a wide doping range," Physical Review B, vol. 82, 020515, 2010.

[170] H. J. Goldsmid, "Introduction to Thermoelectricity," Springer, Springer Series in Materials Science, Springer Berlin Heidelberg, vol. 121, print, 2010.

[171] D. L. Chandler, "Explained: Thermoelectricity," MIT News Office, online, 2010. 
[172] G. S. Nolas, J. Sharp, and H. J. Goldsmid, "Thermoelectrics: Basic Principles and New Materials Developments," Springer, Springer Series in Materials Science, Springer Science \& Business Media, vol. 45, print, 2013.

[173] S. E. Shafraniuk "Thermoelectricity and Heat Transport in Graphene and Other 2D Nanomaterials," Elsevier, Micro and Nano Technologies, $1^{\text {st }}$ ed., print, 2017.

[174] E. Velmre, "Thomas Johann Seebeck and his contribution to the modern science and technology," IEEE, Electronics Conference (BEC), $12^{\text {th }}$ Biennial Baltic, INSPEC Accession Number: 11649381, 2010.

[175] D. C. K. MacDonald, "Thermoelectricity: An Introduction to the Principles," Dover Books on Physics, Dover Publication, Inc., reprint, 2016.

[176] W. Thomson, "On a Mechanical Theory of Thermo-Electric Currents," Proceedings of the Royal Society of Edinburgh, pp. 91 - 98, 1851.

[177] S. Meyer, Y. T. Chen, S. Wimmer, M. Althammer, T. Wimmer, R. Schlitz, S. Geprägs, H. Huebl, D. Ködderitzsch, H. Ebert, G. E. W. Bauer, R. Gross, and S. T. B. Goennenwein, "Observation of the spin Nernst effect," Nature Materials, vol. 16, pp. $977-981,2017$.

[178] M. Pourfath, "Numerical Study of Quantum Transport in Carbon Nanotube Based Transistors," Ph.D. Dissertation, Vienna University of Technology, Vienna, Austria, 2007.

[179] S. Ghosh, I. Calizo, D. Teweldebrhan, E. P. Pokatilov, D. L. Nika, A. A. Balandin, W. Bao, F. Miao, and C. N. Lau, "Extremely high thermal conductivity of graphene: Prospects for thermal management applications in nanoelectronic circuits," Applied Physics Letters 92, 151911, 2008.

[180] D. L. Nika, S. Ghosh, E. P. Pokatilov and A. A. Balandin, "Thermal conductivity of graphene flakes: Comparison with bulk graphite," Cornell University Library, Condensed Matter, Materials Science, arXiv:0904.0607v1 [cond-mat.mtrl-sci], 2009.

[181] J. W. Jiang, J. S. Wang, and B. Li, "Thermal conductance of graphene and dimerite," Cornell University Library, Condensed Matter, Materials Science, arXiv:0902.1836v2 [cond-mat.mtrl-sci], 2009.

[182] Physics and Radio-Electronics "Majority \& minority carriers," Electronics devices and circuits, Semiconductor, Physics and Radio-Electronics, physics-and-radioelectronics.com, online, 2015. 
[183] P. Laube, "Fundamentals: Doping: $\mathrm{n}$ - and p-semiconductors," Semiconductor Technology from A to Z, halbleiter.org, online, 2018.

[184] M. Sigrist, "Introduction to Unconventional Superconductivity," AIP Conference Proceedings 789, 165, 2005.

[185] S. Haas and K. Maki, "Unconventional Superconductivity," Physics \& Astronomy Newsletters, Department of Physics \& Astronomy, University of Southern California, 2001.

[186] H. Eisaki, N. Kaneko, D. L. Feng, A. Damascelli, P. K. Mang, K. M. Shen, Z. X. Shen, and M. Greven, "Effect of chemical inhomogeneity in bismuth-based copper oxide superconductors," Physical Review B 69, 064512, 2004.

[187] P. A. Denis, "Chemical Reactivity of Electron-Doped and Hole-Doped Graphene," The Journal of Physical Chemistry C 117, 8, pp. 3895 - 3902, 2013.

[188] D. Manske, I. Eremin, and K. H. Bennemann, "Theory for electron- and hole-doped cuprate superconductors: d-wave symmetry order parameter," Europhysics Letters, vol. 53, no. 3, 2001.

[189] A. Di Bernardo, O. Millo, M. Barbone, H. Alpern, Y. Kalcheim, U. Sassi, A.K. Ott, D. De Fazio, D. Yoon, M. Amado, A.C. Ferrari, J. Linder, and J.W.A. Robinson, "p-wave triggered superconductivity in single-layer graphene on an electron-doped oxide superconductor," Nature Communications, vol. 8, no. 14024, 2017.

[190] H. D. Young and R. G. Freedman "University Physics," Pearson, $12^{\text {th }}$ ed., p. 716, print, 2007.

[191] V. Berseth, "Mixed State Hall Effect in a Twinned $\mathrm{YBa}_{2} \mathrm{Cu}_{3} \mathrm{O}_{7-\delta}$ Single Crystal," Doctoral Thesis, École polytechnique fédérale de Lausanne (Swiss Federal Institute of Technology in Lausanne), Switzerland, 1999.

[192] Physics and Radio-Electronics "Extrinsic semiconductor," Electronics devices and circuits, Semiconductor, Physics and Radio-Electronics, physics-and-radioelectronics.com, online, 2015.

[193] FAA insulation aging test results, DOT/FAA Tech Report AR-08/2, 2008.

[194] Z. A. Xu, N. P. Ong, Y. ang, T. Kakeshita, and S. Uchida, "Vortex-like excitations and the onset of superconducting phase fluctuation in underdoped $\mathrm{La}_{2-x} \mathrm{Sr}_{x} \mathrm{CuO}_{4}$," Nature 406 pp. 486 - 488, 2000. 
[195] Y. Wang, Z. A. Xu, T. Kakeshita, S. Uchida, S. Ono, Y. Ando, and N. P. Ong, "Onset of the vortexlike Nernst signal above $T_{c}$ in $\mathrm{La}_{2-} \mathrm{Sr}_{x} \mathrm{CuO}_{4}$ and $\mathrm{Bi}_{2} \mathrm{Sr}_{2-}$ ${ }_{y} \mathrm{La}_{y} \mathrm{CuO}_{6}$, , Physical Review B 64, 224519, 2001.

[196] Y. Wang, N. P. Ong, Z. A. Xu, T. Kakeshita, S. Uchida, D. A. Bonn, R. Liang, and W. N. Hardy, "High Field Phase Diagram of Cuprates Derived from the Nernst Effect," Physical Review Letters 88, 257003, 2002.

[197] Y. Wang, S. Ono, Y. Onose, G. Gu, Y. Ando, Y. Tokura, S. Uchida, and N. P. Ong, "Dependence of Upper Critical Field and Pairing Strength on Doping in Cuprates," Science, vol. 299, issue 5603, pp. 86 - 89, 2003.

[198] Y. Wang, L. Li, and N. P. Ong, "Nernst effect in high- $T_{c}$ superconductors," Physical Review B 73, 024510, 2006.

[199] R. Li and Z. S. She, "A quantitative vortex-fluid description of Nernst effect in Bibased cuprate high-temperature superconductors," New Journal of Physics, vol. 19, 113028, 2017.

[200] A. Sergeev, M. Yu. Reizer, and V. Mitin, "Heat current in the magnetic field: Nernst-Ettingshausen effect above the superconducting transition," Physical Review B 77, 064501, 2008.

[201] V. G. Kogan, "Interaction of vortices in thin superconducting films and the Berezinskii-Kosterlitz-Thouless transition,” Physical Review B 75, 064514, 2007.

[202] B. Uchoa and A. H. Castro Neto, "Superconducting States of Pure and Doped Graphene," Physical Review Letters 98, 146801, 2007.

[203] A. Bostwick, T. Ohta, J. L. McChesney, T. Seyller, K. Horn, E. Rotenberg, "Renormalization of graphene bands by many-body interactions," Elsevier, Science Direct, Solid State Communications, Exploring graphene: Recent research advances, vol. 143, issue $1-2$, pp. $63-71,2007$.

[204] E. Perfetto and J. González, "Superconductivity in multi-walled carbon nanotubes and doped graphite," Elsevier, Science Direct, Physica C: Superconductivity and its Applications, Proceedings of the 8th International Conference on Materials and Mechanisms of Superconductivity and High Temperature Superconductors (M2SHTSC VIII), vol. 460 - 462, pp. 1039 - 1040, 2007.

[205] S. Pathak, V. B. Shenoy, and G. Baskaran, "Possibility of High $T_{c}$ Superconductivity in doped Graphene," Cornell University Library, Condensed Matter, Superconductivity, arXiv:0809.0244v1 [cond-mat.supr-con], 2008. 
[206] S. Pathak, V. B. Shenoy, and G. Baskaran, "Possible high-temperature superconducting state with a $d+i d$ pairing symmetry in doped graphene," Physical Review B 81, 085431, 2010.

[207] A. Pourret, P. Spathis, H. Aubin, and K. Behnia, "Nernst effect as a probe of superconducting fluctuations in disordered thin films," New Journal of Physics, vol. 11, 055071, 2009.

[208] E. Kan, Z. Li, and J. Yang, "Magnetism in graphene systems," Nano, vol. 3, no. 6, pp. $433-442,2008$.

[209] S. Okada and A. Oshiyama, "Magnetic Ordering in Hexagonally Bonded Sheets with First-Row Elements," Physical Review Letters 87, 146803, 2001.

[210] J. O. Sofo, Gonzalo Usaj, P. S. Cornaglia, A. M. Suarez, A. D. Hernández-Nieves, and C. A. Balseiro, "Magnetic structure of hydrogen-induced defects on graphene," Physical Review B 85, 115405, 2012.

[211] W. Li, M. Zhao, Y. Xia, R. Zhang, and Y. Mu, "Covalent-adsorption induced magnetism in graphene," Journal of Materials Chemistry 19, 9274 - 9282, 2009.

[212] Z. Bao, J. Shia, M. Yang, S. Zhang, and M. Zhang, "Magnetism induced by D3symmetry tetra-vacancy defects in graphene," Chemical Physics Letters, vol. 510, issue $4-6$, pp. $246-251,2011$.

[213] A. M. Valencia and M. J. Caldas, "Single vacancy defect in graphene: Insights into its magnetic properties from theoretical modeling," Physical Review B 96, 125431, 2017.

[214] M. Sepioni, "Magnetic properties of graphene," Thesis, The University of Manchester, 2013.

[215] Plasma-Therm "PECVD," Deposition Technologies, PECVD, Plasma-Therm, plasma-therm.com, online, 2014.

[216] KAPTONTAPE.COM, "Kapton ${ }^{\circledR}$ Tapes, RoHS Compliant," kaptontape.com., online, 2018.

[217] ALCOA, “Alloy 6061," Understanding Extruded Aluminum Alloys, Alcoa Engineered Products, Alcoa Corporation, online, 2018.

[218] CADDOCK High Performance Film Resistors, "MP900 and MP9000 Series KoolPak $^{\circledR}$ Power Film Resistors TO-126, TO-220 and TO-247 Style," Caddock Electronics, Inc, online, 2017. 
[219] J. Hass, W. A. de Heer and E. H. Conrad, "The growth and morphology of epitaxial multilayer graphene,” Journal of Physics: Condensed Matter 20, 323202, 2008.

[220] J. Hass, F. Varchon, J. E. Millán-Otoya, M. Sprinkle, N. Sharma, W. A. de Heer, C. Berger, P. N. First, L. Magaud, and E. H. Conrad, "Why Multilayer Graphene on $4 H-\operatorname{SiC}(000 \overline{1})$ Behaves Like a Single Sheet of Graphene," Physical Review Letters 100, 125504, 2008.

[221] J. W. Guikema, H. Bluhm, D. A. Bonn, R. Liang, W. N. Hardy, and K. A. Moler, "Two-dimensional vortex behavior in highly underdoped $\mathrm{YBa}_{2} \mathrm{Cu}_{3} \mathrm{O}_{6+\mathrm{x}}$ observed by scanning Hall probe microscopy," Physical Review B 77, 104515, 2008.

[222] J. R. Clem, "Two-dimensional vortices in a stack of thin superconducting films: A model for high-temperature superconducting multilayers," Physical Review B 43, 7837, 1991.

[223] T. Pe, M. Benkraouda, and J. R. Clem, "Magnetic coupling of two-dimensional pancake vortex lattices in a finite stack of thin superconducting films with transport currents in the two outermost layers," Physical Review B 55, 6636, 1997.

[224] J. R. Clem, "Magnetic Flux Penetration into the High-Temperature Superconductors," in Physics and Materials Science of Vortex States, Flux Pinning and Dynamics, R. Kossowsky, Ed. Kluwer Academic Publishers, pp. 57 - 80, 1999.

[225] J. R. Clem, "Pancake Vortices," Journal of Superconductivity and Novel Magnetism, vol. 17, issue 5, pp. 613 - 629, 2004.

[226] S. J. Bending and M. J. W. Dodgson, "Vortex chains in anisotropic superconductors," Journal of Physics: Condensed Matter, vol. 17, no. 35, 2005.

[227] D. Milliken, T. Silver, and S. X. Dou, "Irradiation of HTS for Enhancement of Critical Current," Springer, Frontiers in Superconducting Materials, pp. 555 - 588, 2005.

[228] B. M. Kessler, Ç. Ö. Girit, A. Zettl, and V. Bouchiat, "Tunable Superconducting Phase Transition in Metal-Decorated Graphene Sheets," Physical Review Letters 104, 047001, 2010.

[229] I. L. Spain, A. R. Ubbelohde, and D. A. Young, "Electronic Properties of Well Oriented Graphite," Philosophical Transactions of The Royal Society A: Mathematical, Physical, and Engineering Sciences, vol. 262, no. 1128, pp. 345 386, 1967.

[230] I. L. Spain, "The Electronic Properties of Graphite," Chemistry and Physics of Carbon, vol. 8, pp. 1 - 150, 1973. 
[231] K. S. Krishnan, "Magnetic Anisotropy of Graphite," Nature 133, pp. 174 - 175, 1934.

[232] K. Behnia and H. Aubin, "Nernst effect in metals and superconductors: a review of concepts and experiments," Reports on Progress in Physics, vol. 79, no. 4, 2016.

[233] R. Nave "Hall Effect," HyperPhysics, Electricity and Magnetism, Georgia State University, online, 2016.

[234] A. Engel, "Vortex Dynamics and Superconducting Phase Diagrams in $\mathrm{Ta}_{\mathrm{x}} \mathrm{Ge}_{1-\mathrm{x}} / \mathrm{Ge}$ Multilayers with Coplanar Defects," A thesis submitted to the Victoria University of Wellington in fulfilment of the requirements for the degree of Doctor of Philosophy in Physics, Victoria University of Wellington, 2001.

[235] G. Blatter, M. V. Feigel'mann, V. B. Geshkenbein, A. I. Larkin, and V. M. Vinokur, "Vortices in high temperature superconductors," Reviews of Modern Physics 66, $1125,1994$.

[236] G. Berdiyorov, "Vortex Structure and Critical Parameters in Superconducting Thin Films with Arrays of Pinning Centers," Ph.D. Dissertation, University of Antwerpen, Antwerpen, Belguim, 2007.

[237] T. I. Baturina and V. M. Vinokur, "Superinsulator-superconductor duality in two dimensions," Annals of Physics, vol. 331, pp. 236 - 257, 2013.

[238] S. J. Hagen, C. J. Lobb, R. L. Greene, and M. Eddy, "Flux-flow Hall effect in superconducting $\mathrm{Tl}_{2} \mathrm{Ba}_{2} \mathrm{CaCu}_{2} \mathrm{O}_{8}$ films," Physical Review B 43, 6246(R), 1991.

[239] N. B. Kopnin, B. I. Ivlev, and V. A. Kalatsky, "The flux-flow hall effect in type II superconductors. An explanation of the sign reversal," Journal of Low Temperature Physics, vol. 90, issue 1-2, pp. 1 - 13, 1993.

[240] N. B. Kopnin and A. V. Lopatin, "Flux-flow Hall effect in clean type-II superconductors," Physical Review B 51, 15291, 1995.

[241] N. B. Kopnin, "Hall effect in moderately clean superconductors and the transverse force on a moving vortex," Physical Review B 54, 9475, 1996.

[242] S. D. Ha, R. Jaramillo, D. M. Silevitch, F. Schoofs, K. Kerman, J. D. Baniecki, and S. Ramanathan, "Hall effect measurements on epitaxial $\mathrm{SmNiO}_{3}$ thin films and implications for antiferromagnetism," Physical Review B 87, 125150, 2013.

[243] H. Chen, Q. Niu, and A. H. MacDonald, "Anomalous Hall Effect Arising from Noncollinear Antiferromagnetism,” Physical Review Letters 112, 017205, 2014. 
[244] P. H. Chang, M. S. Bahramy, N. Nagaosa, and B. K. Nikolic̀, "Giant thermoelectric effect in graphene-based topological insulators with nanopores," Nano Letters 14, 7, pp. 3779 - 3784, 2014.

[245] S. Franssila, "Introduction to Micro Fabrication," Wiley \& Sons, Ltd., $1^{\text {st }}$ ed., print, 2004.

[246] Q. Zhang, D. Sandoa, and V. Nagarajan, "Chemical route derived bismuth ferrite thin films and nanomaterials," Journal of Materials Chemistry C, issue 19, pp. 4092 $-4124,2016$.

[247] J. S. Lee, S. B. Jin, N. Vichiansan, J. G. Han, M. Mori, K. Leksakul, " $\mathrm{SiC}_{\mathrm{x}} \mathrm{H}_{\mathrm{y}}$-based hydrophobic thin films with good chemical and mechanical properties synthesized by PECVD at various substrate temperatures," Current Applied Physics, vol. 15, issue 11, pp. $1342-1347,2015$.

[248] A. Doolittle, "Lecture 5 Ion Implantation Reading Chapter 5," Lecture5-Ion Implantation.ppt, ECE 6450, Georgia Institute of Technology, online, 2018.

[249] PLANSEE, "Components and spare parts for ion implantation.," Component, Products, PLANSEE The Plansee Group, online, 2018.

[250] Case Technology, “The Ion Implanter," Ion Implantation, Case Technology, Inc., online, 1996.

[251] Z. Yu, "Introduction to Ion Beam Biotechnology," Springer, National Key Laboratory of Ion Beam Bioengineering, Institute of Plasma Physics, Chinese Academy of Sciences, Hefei, China, 2006.

[252] S. Pezzagna and J. Meijer, "High-Resolution Ion Implantation from keV to MeV," INTECHOPEN, ION IMPLANTATION Edited by Mark Goorsky, INTECHOPEN.COM, 2012.

[253] J. F. Ziegler, J. P. Biersack, and M. D. Ziegler, "SRIM The Stopping and Range of Ions in Matter," Elsevier, print, 2010.

[254] V. Haublein, H. Ryssel, and L. Frey, "Purity of Ion Beams: Analysis and Simulation of Mass Spectra and Mass Interferences in Ion Implantation," Advances in Materials Science and Engineering, vol. 2012, Article ID 610150, 2011.

[255] N. Catarino, M. A. Dias, J. G. Lopes, I. Jepu, and E. Alves, "Helium and deuterium irradiation effects in tungsten-based materials with titanium," Elsevier, Surface \& Coatings Technology, 2018. 
[256] G. Kalita and M. Tanemura, "Fundamentals of Chemical Vapor Deposited Graphene and Emerging Applications," IntechOpen, Graphene Materials Advanced Applications, 2017.

[257] B. Hu, H. Ago, C. M. Orofeo, Y. Ogawa, and M. Tsuji, "On the nucleation of graphene by chemical vapor deposition," Royal Society of Chemistry, New Journal of Chemistry, 36, pp. 73 - 77, 2012.

[258] J. Yu, J. Li, W. Zhang, and H. Chang, "Synthesis of high quality two-dimensional materials via chemical vapor deposition," Royal Society of Chemistry, Chemical Science, 6, pp. $6705-6716,2015$.

[259] ACS Material Advanced Chemicals Supplied, "Introduction to Graphene," ACS Material Blog-Introduction to Graphene, ACS Material, LLC, online, 2017.

[260] R. Muñoz and C. Gómez-Aleixandre, "Review of CVD Synthesis of Graphene," WILEY-VCH, CVD Chemical Vapor Deposition, vol. 19, issue 10-11-12, pp. 279 $-322,2013$.

[261] J. Dai and J. Yuan, "Modulating the electronic and magnetic structures of P-doped graphene by molecule doping," Journal of Physics Condensed Matter, 22, 225501, 2010.

[262] H. Marsh and F. R. -Reinoso, “Activated Carbon," Elsevier, Elsevier Science \& Technology Books, print, 2006. 
APPENDICES 


\section{Appendix 1 - Photographs of The Nernst Fixture}

Image courtesy of Amber Woods.
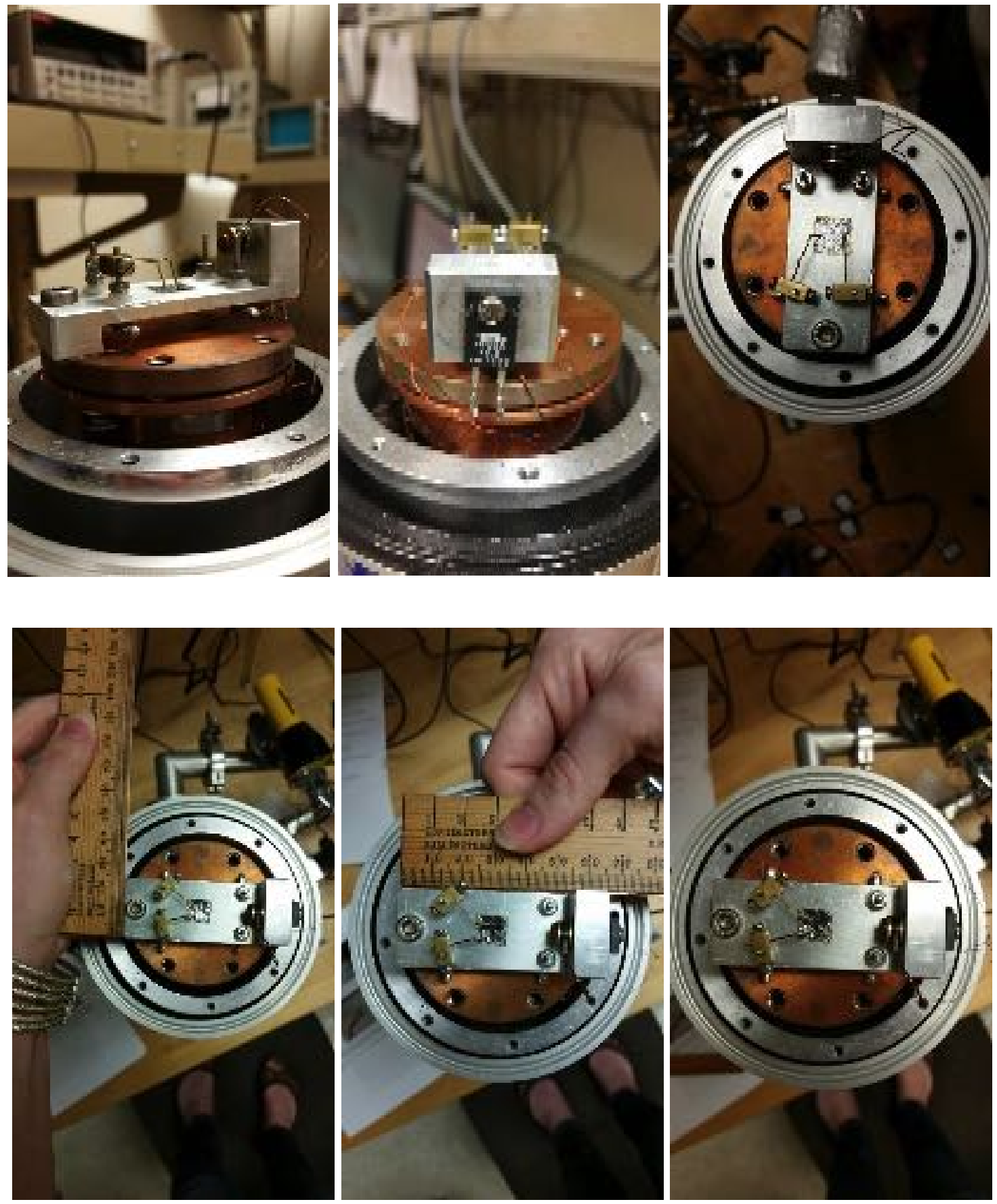


\author{
Appendix 2 - LabVIEW
}

\title{
NALAT's Cooling and Warming LabVIEW Program.vi
}

The program was developed by Nalat Sornkhampan.

\section{Front Panel}

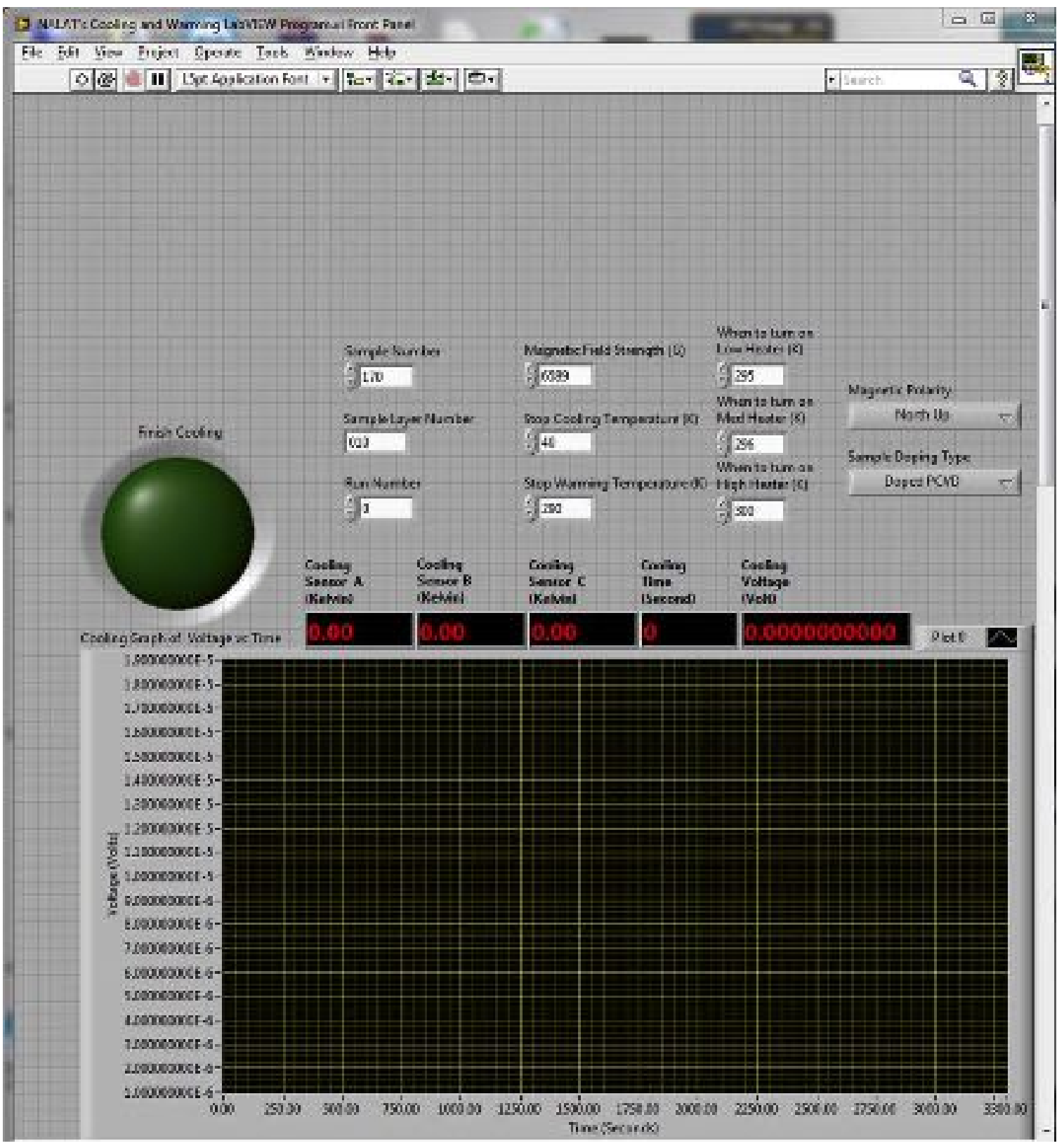









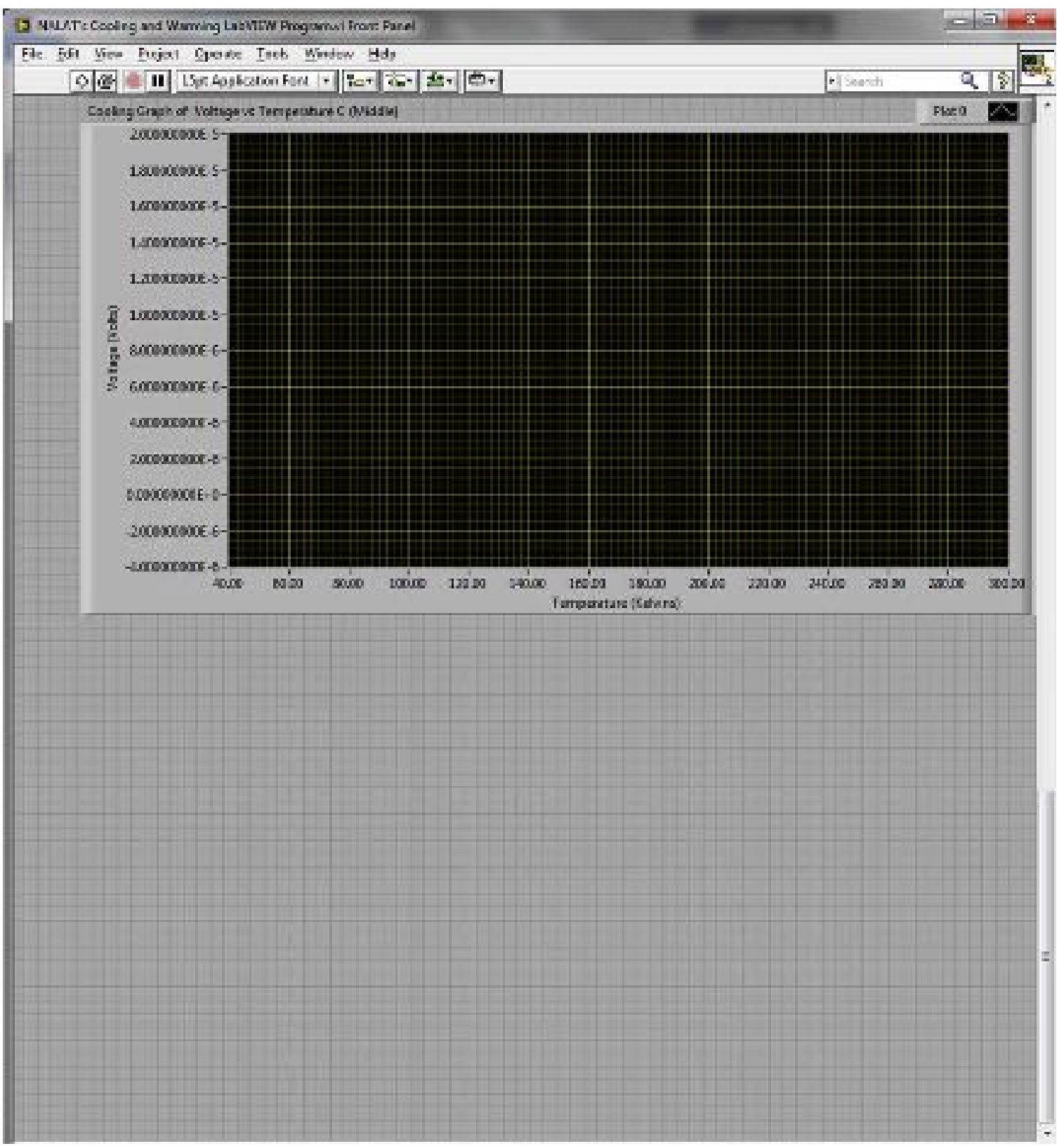




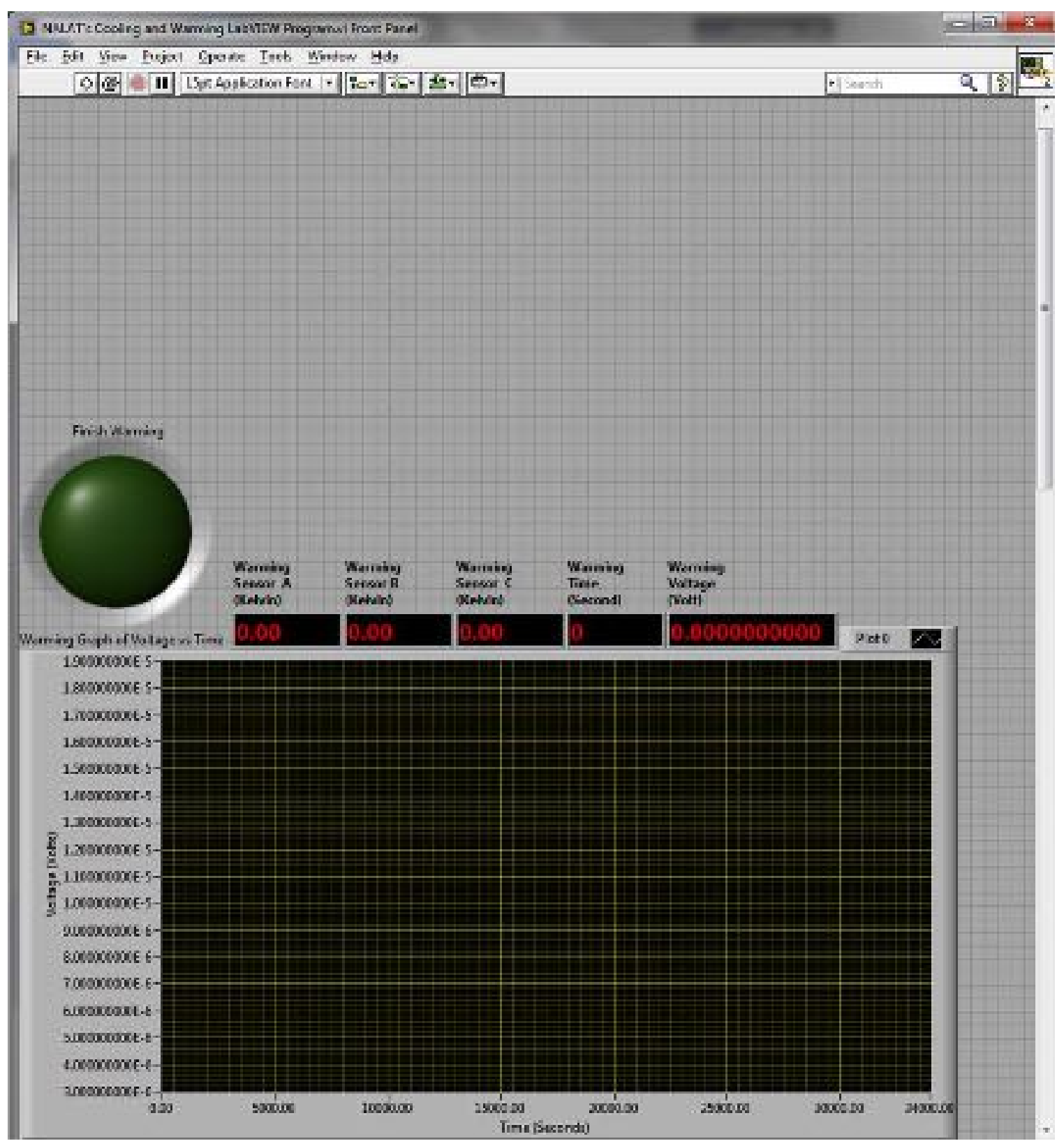




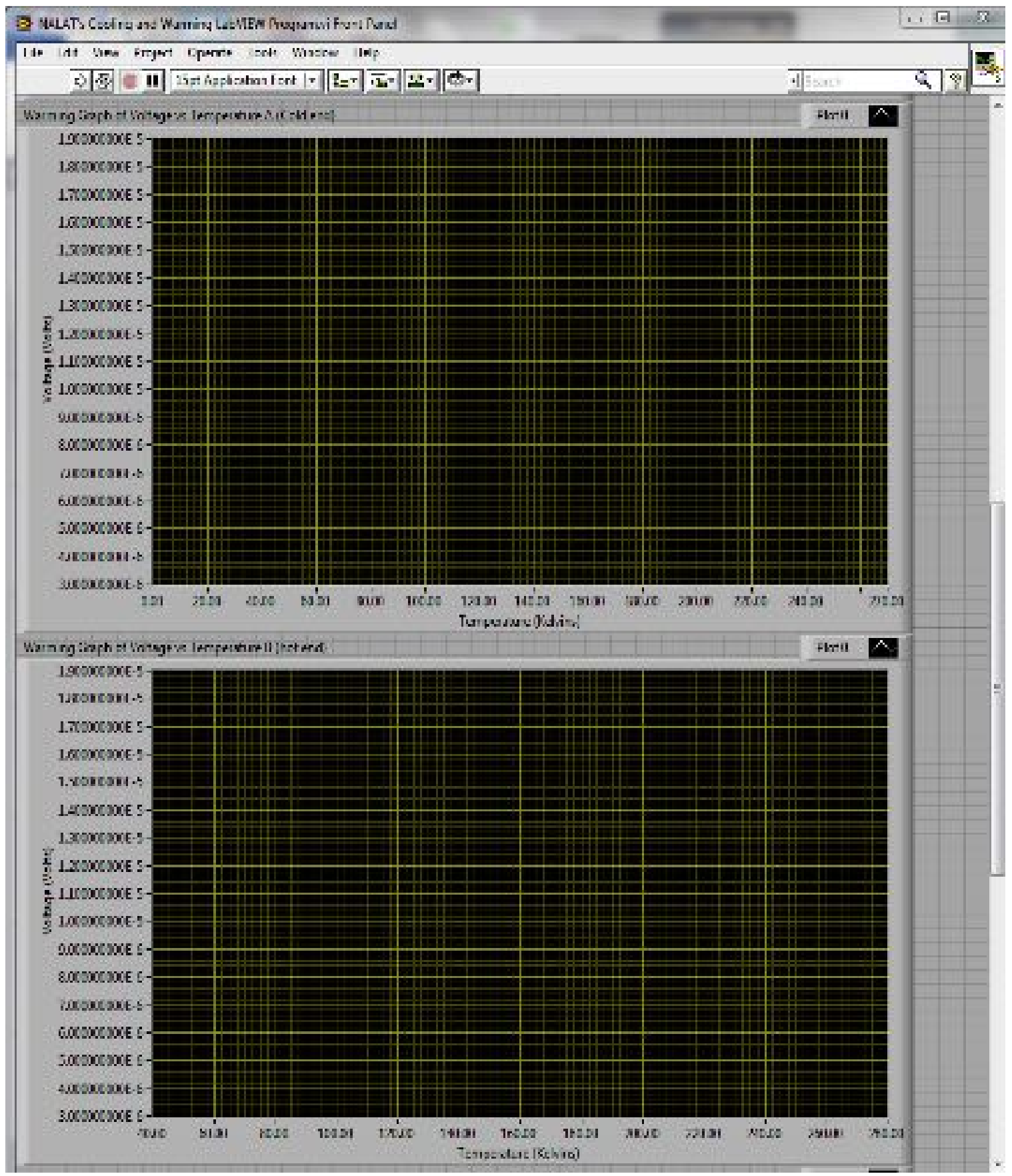




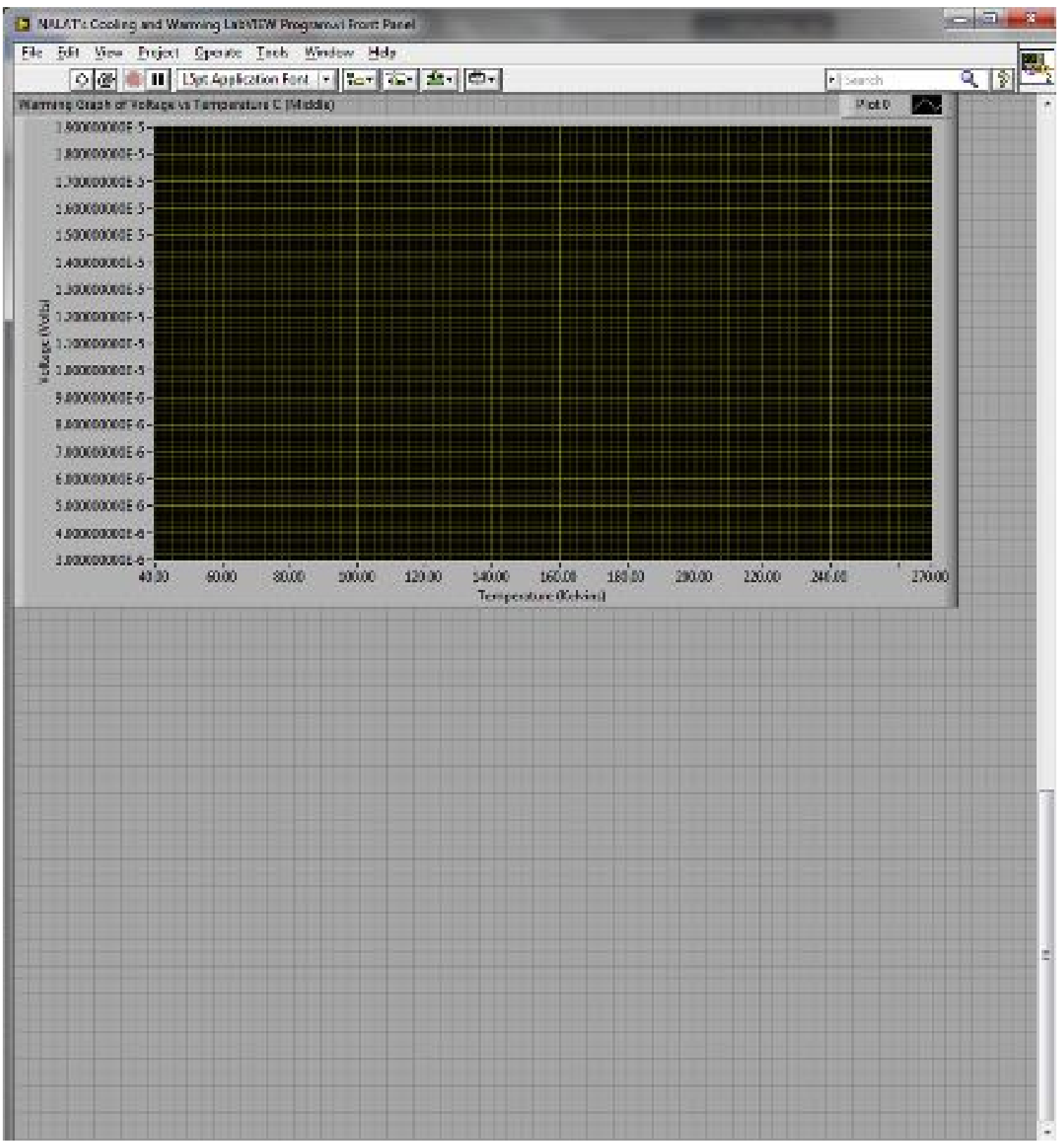




\section{Block Diagram}

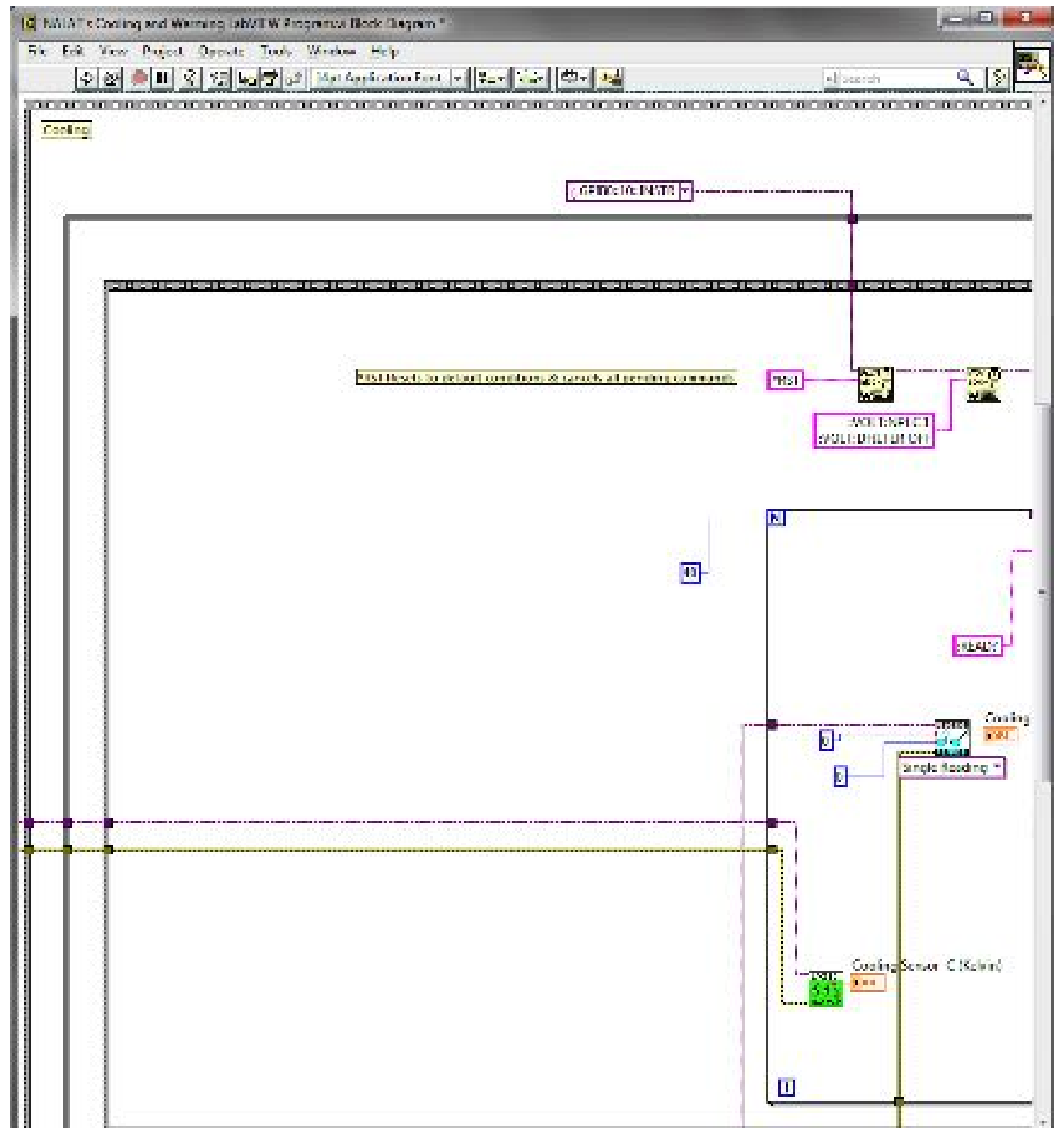




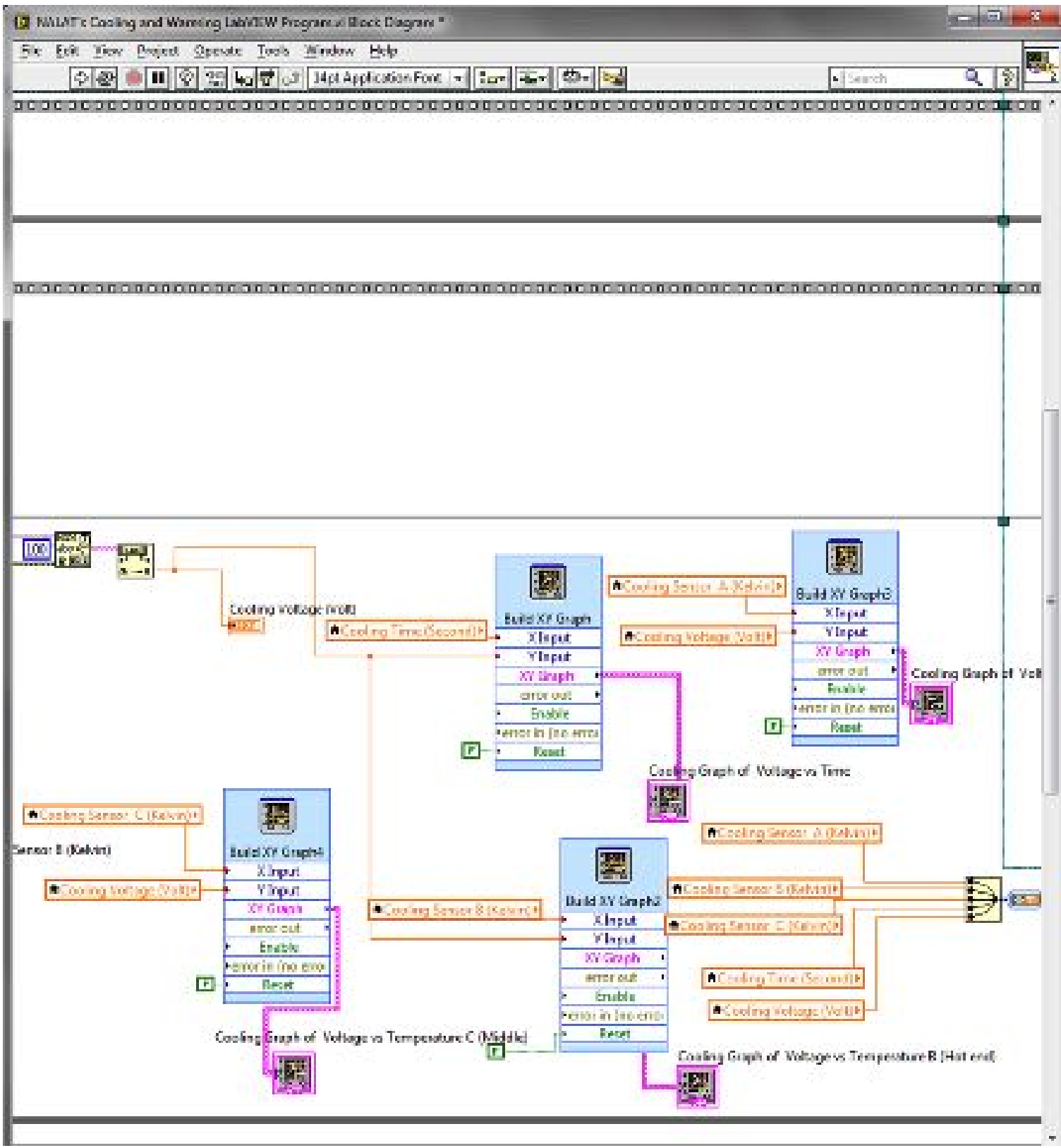




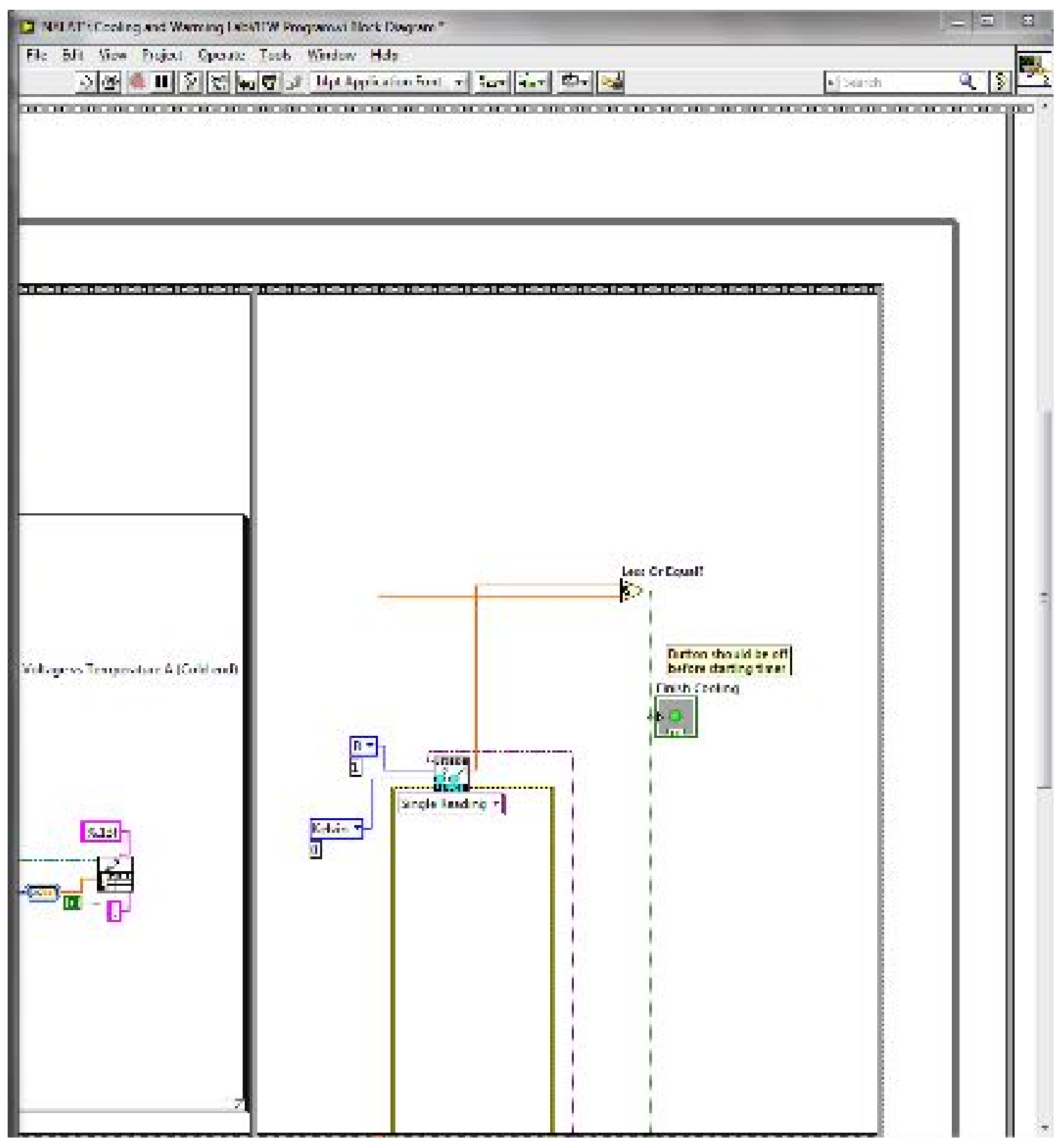




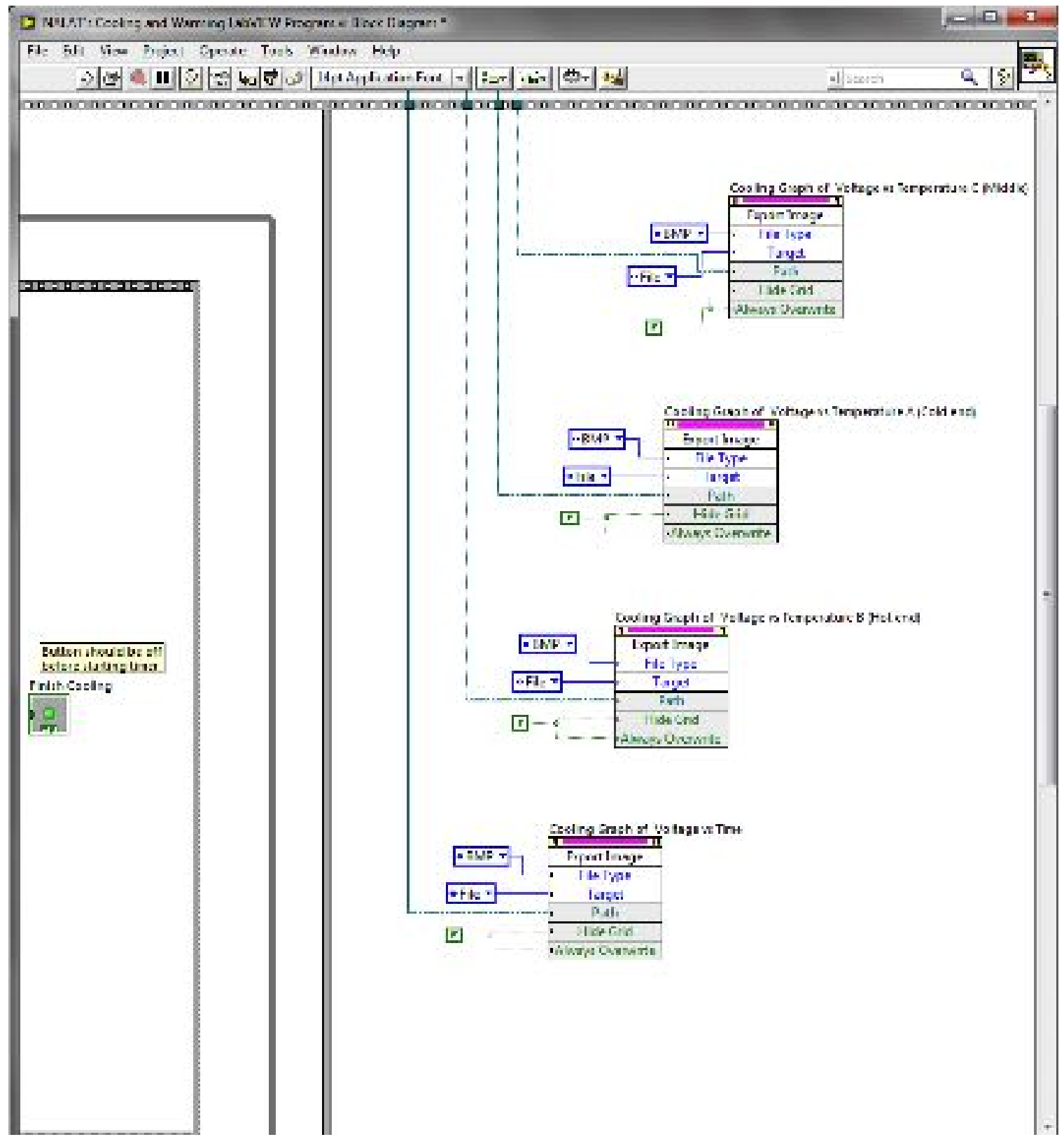




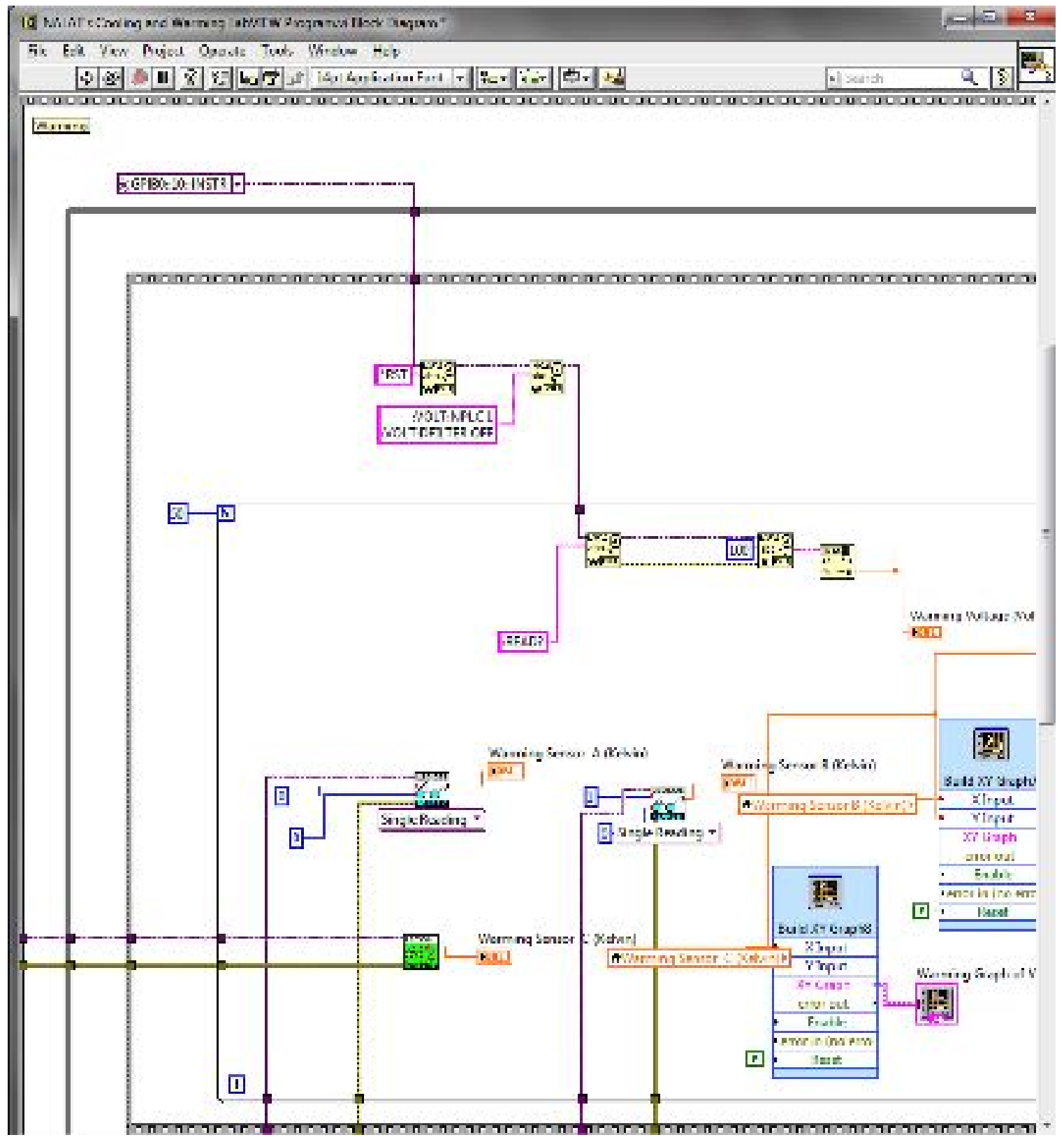




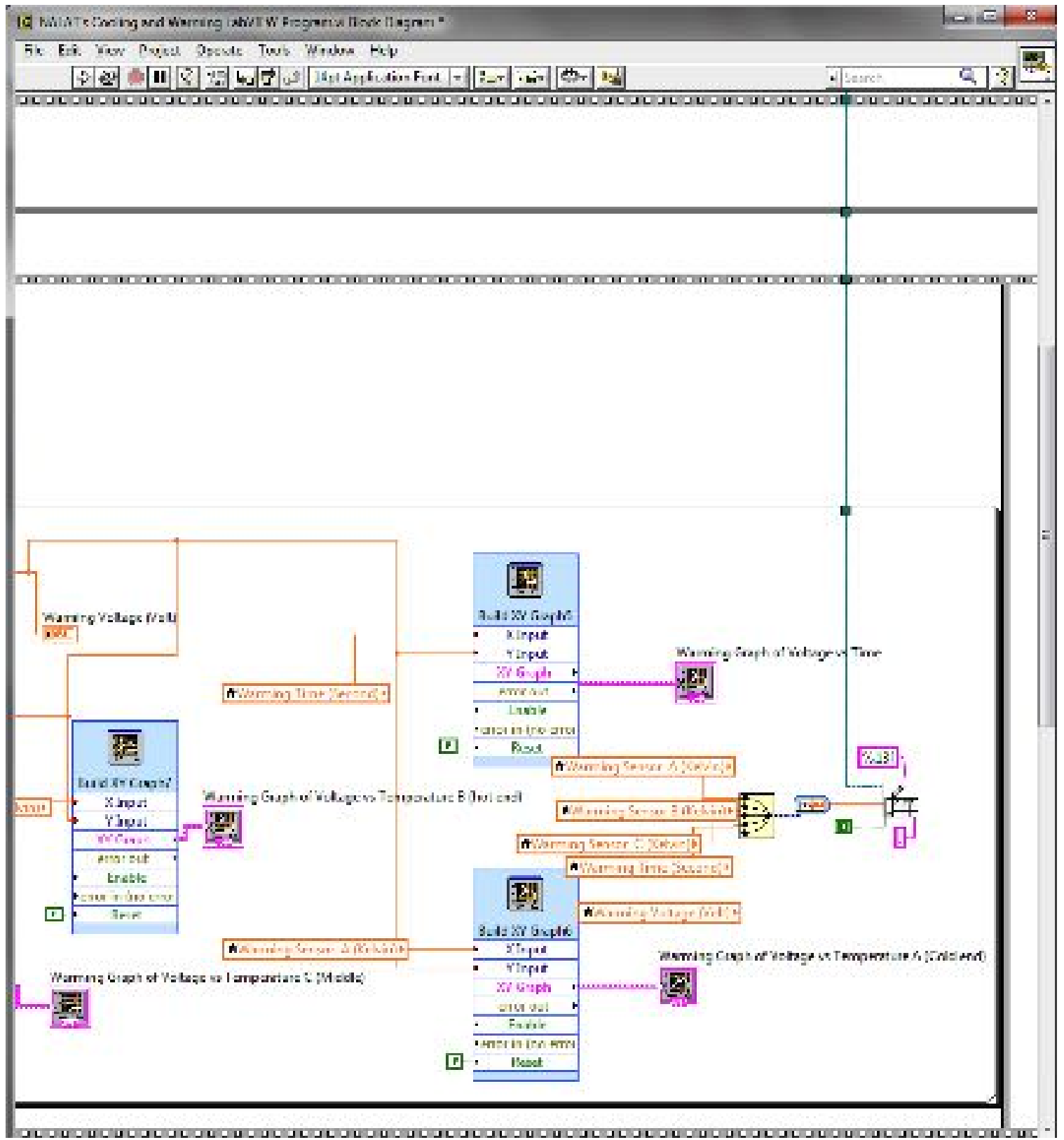




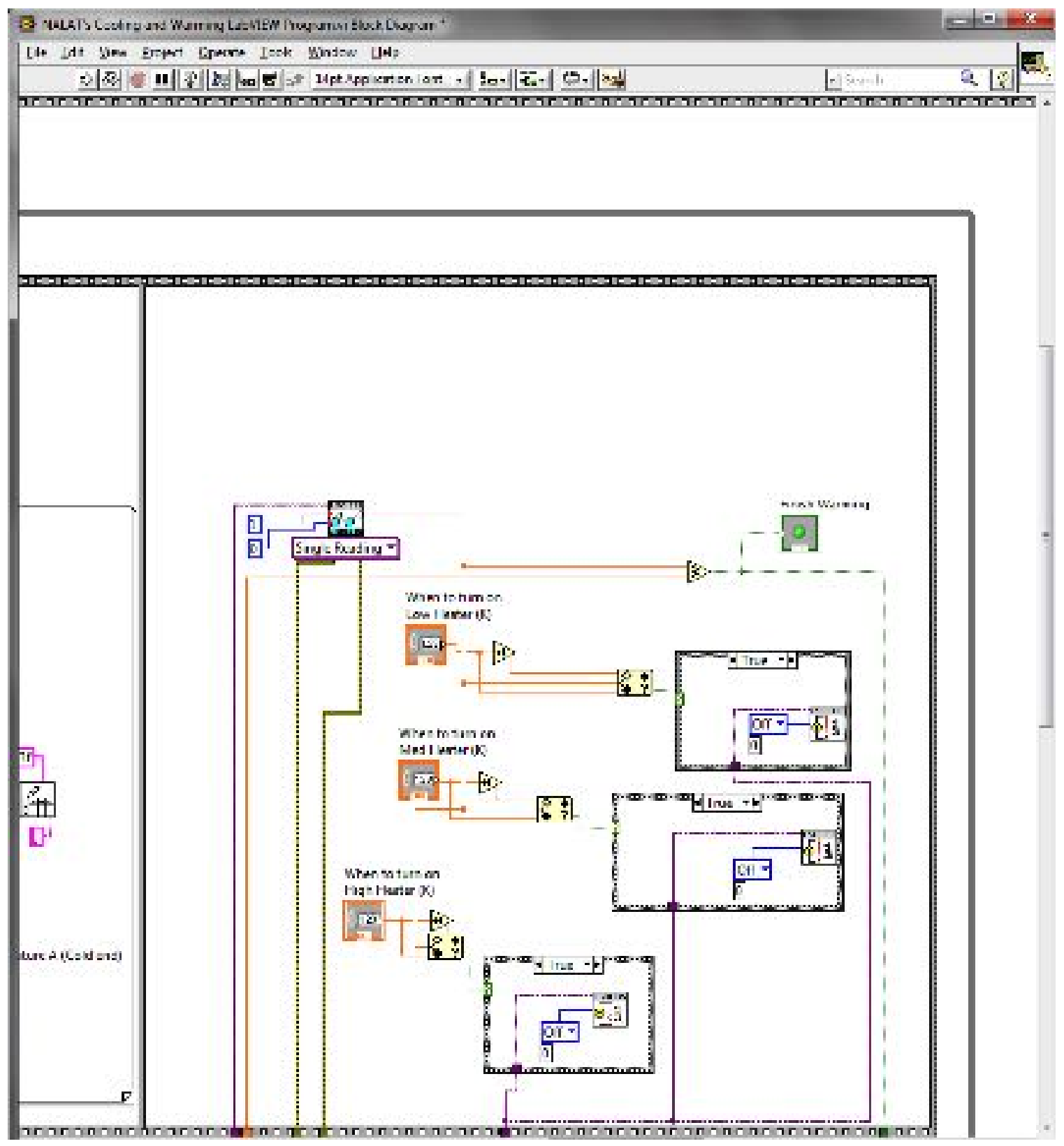




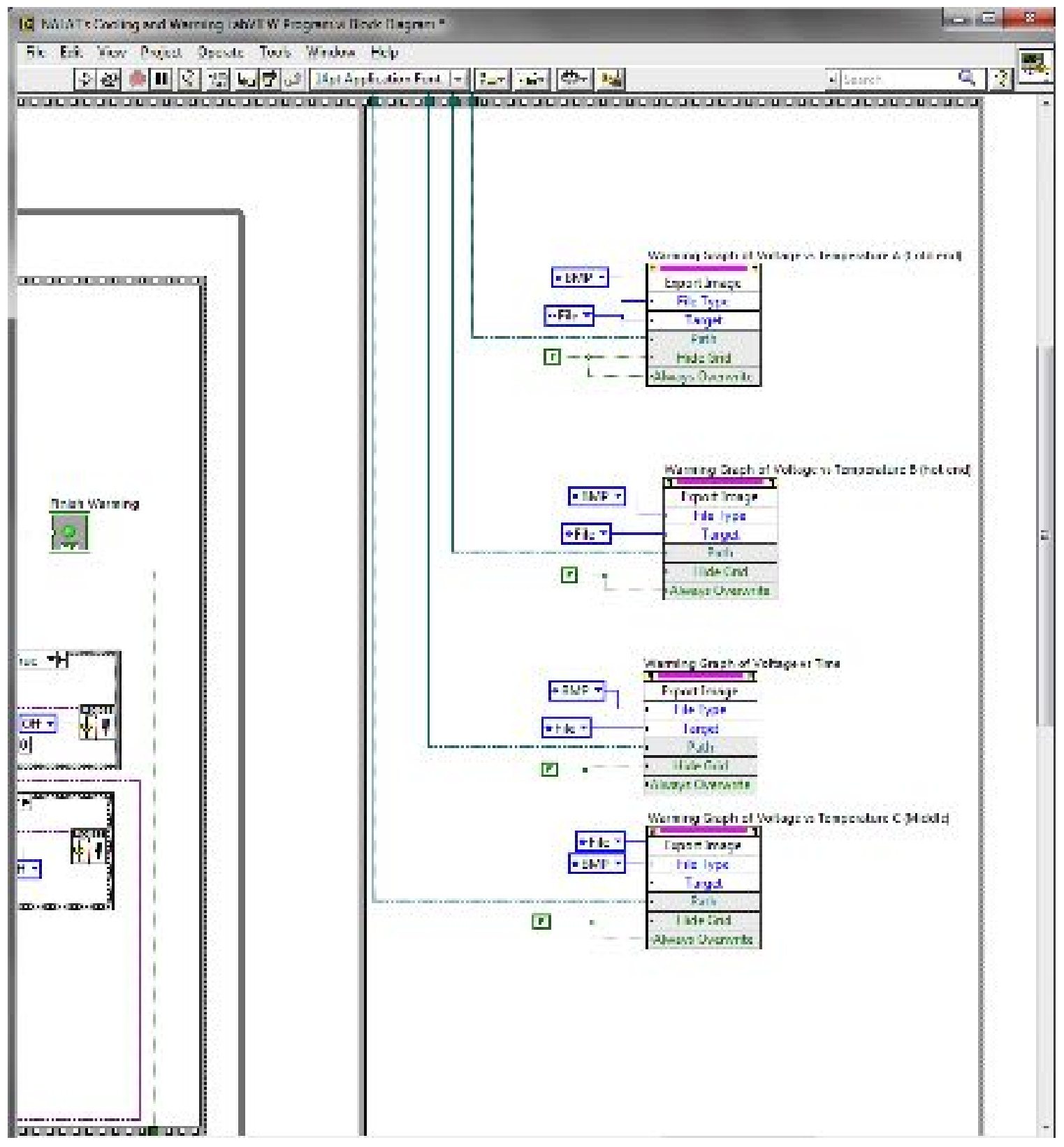


Appendix 3 - List of Tested Samples

\begin{tabular}{|c|c|c|}
\hline Sample \# & Layer \# & Doping Process \\
\hline 023 & 001 & Ion implantation \\
\hline 151 & 008 & Chemical Vapor Deposition \\
\hline 163 & 009 & Chemical Vapor Deposition \\
\hline 170 & 010 & Chemical Vapor Deposition \\
\hline 213 & 001 & Ion implantation \\
\hline 401 & 003 & Non-doping \\
\hline 402 & 005 & Non-doping \\
\hline
\end{tabular}




\section{Appendix 4 - Voltage versus Temperature Plots}

The data were collected by Nalat Sornkhampan.

I. Sample \# 023, layer \# 001
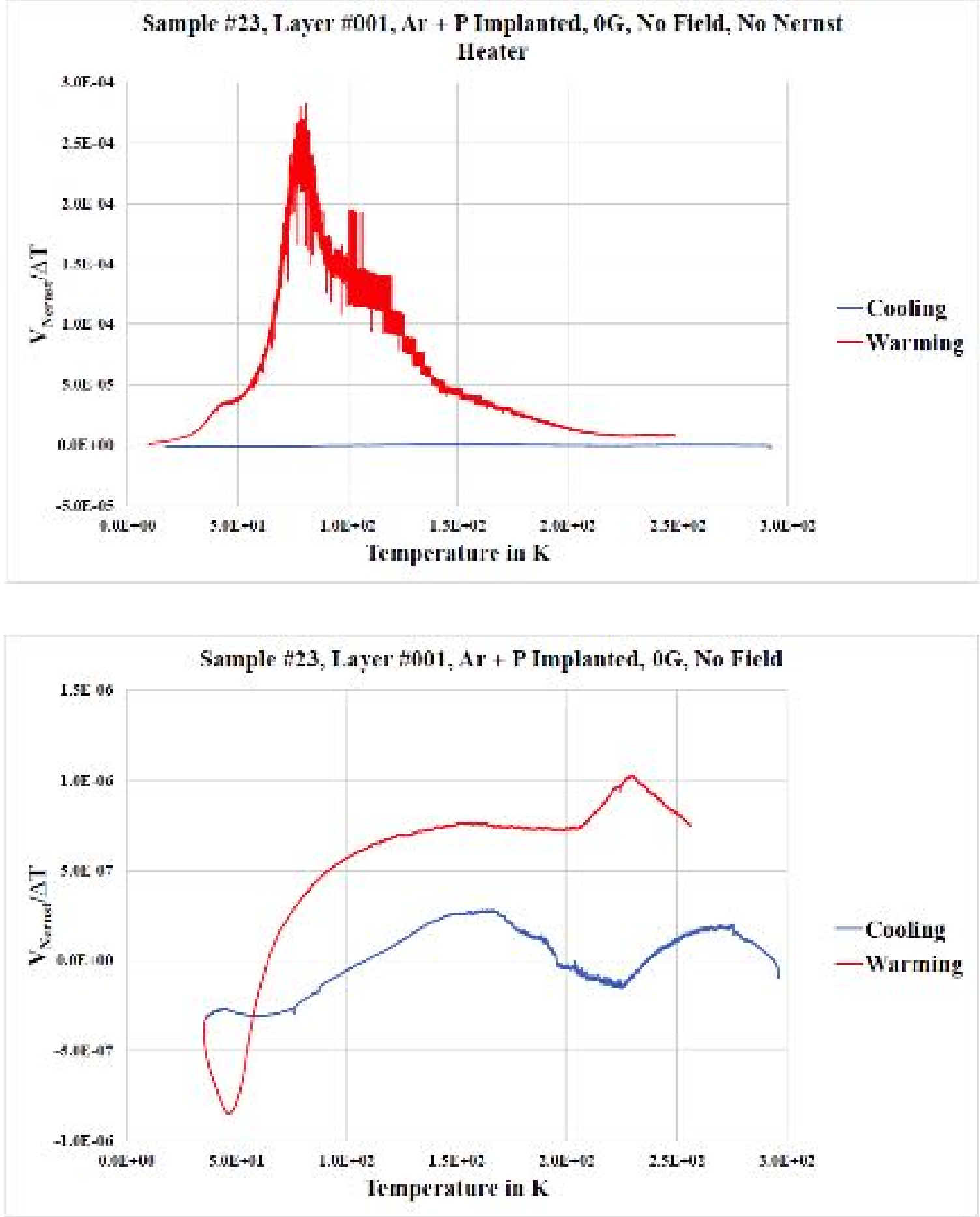

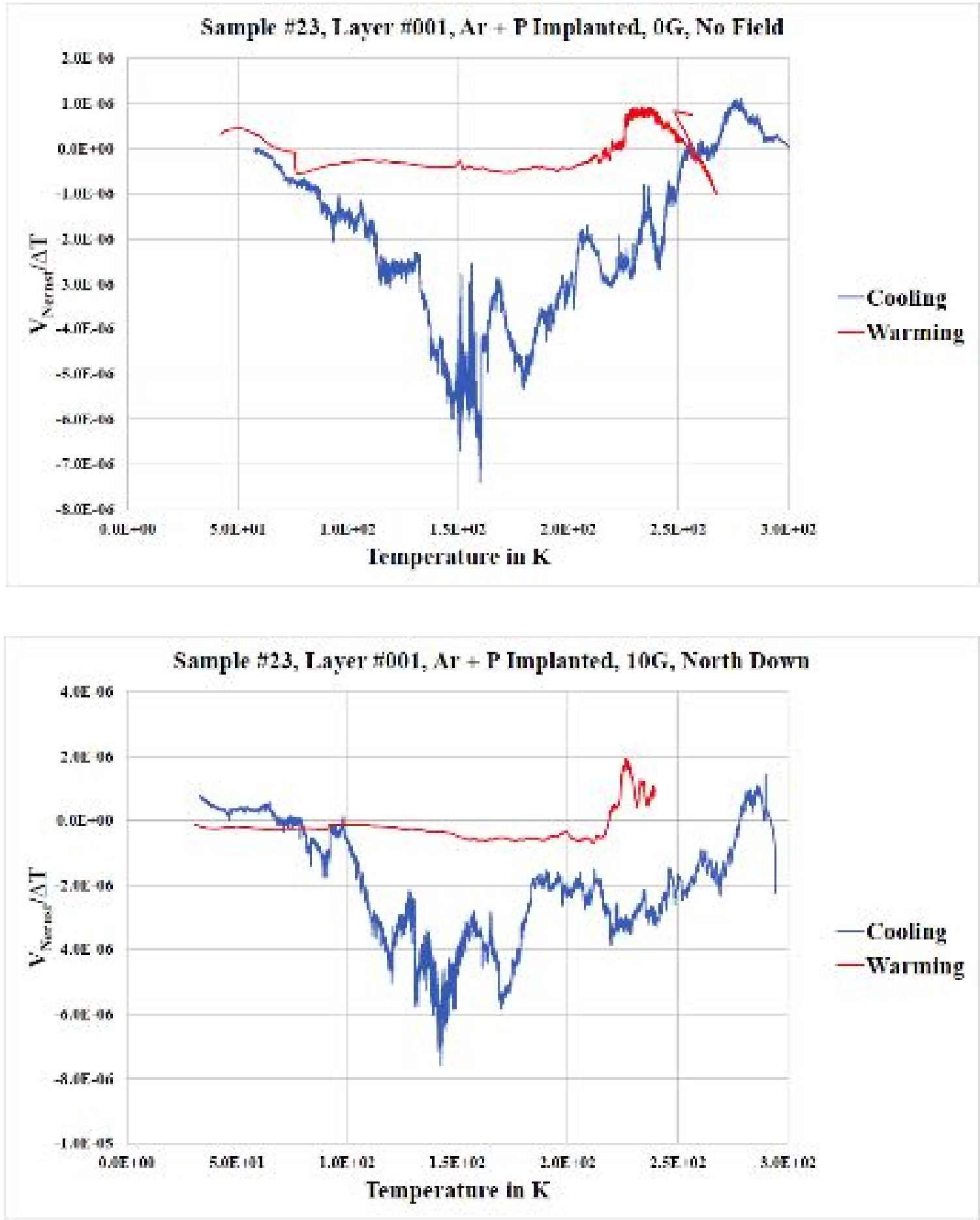

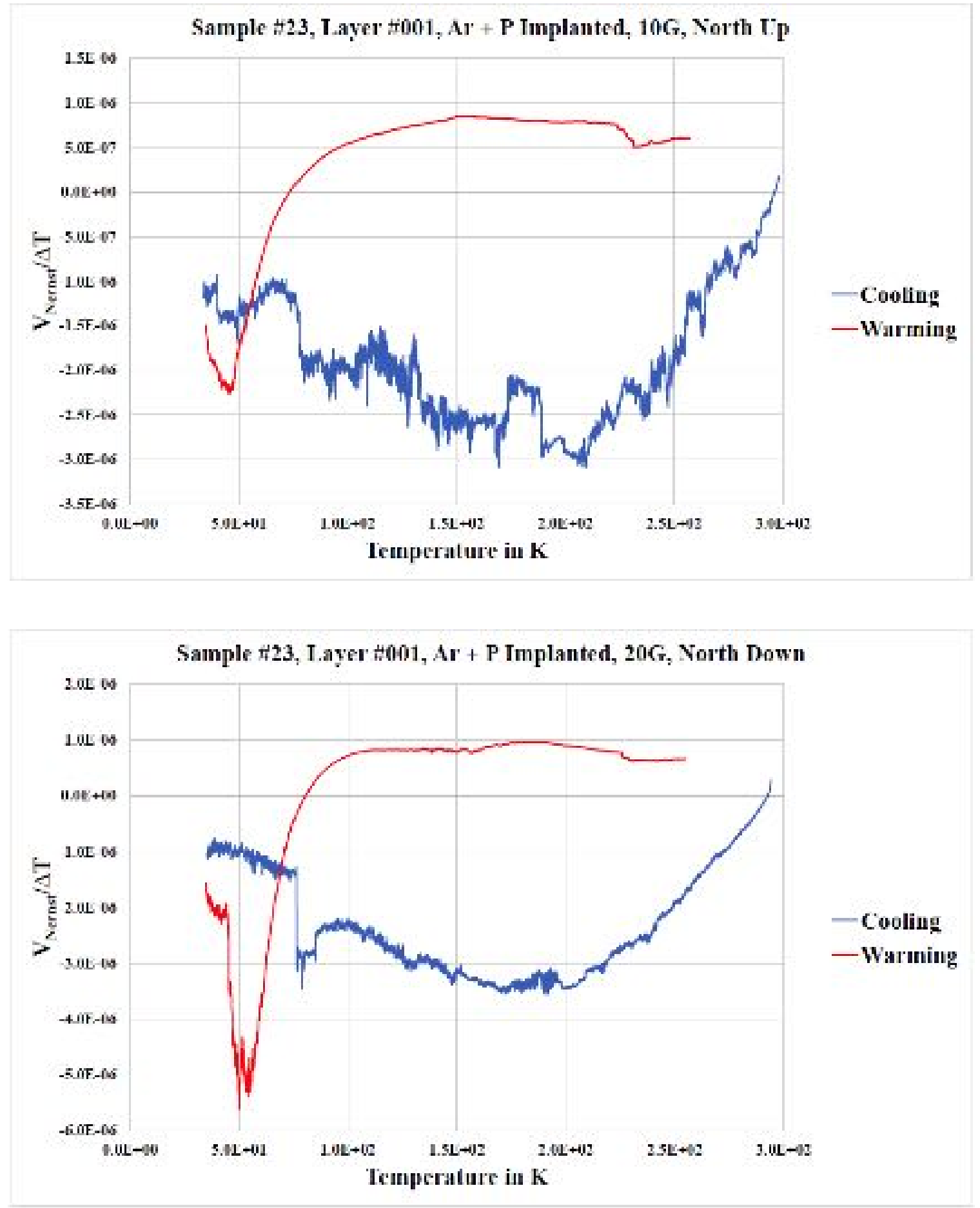

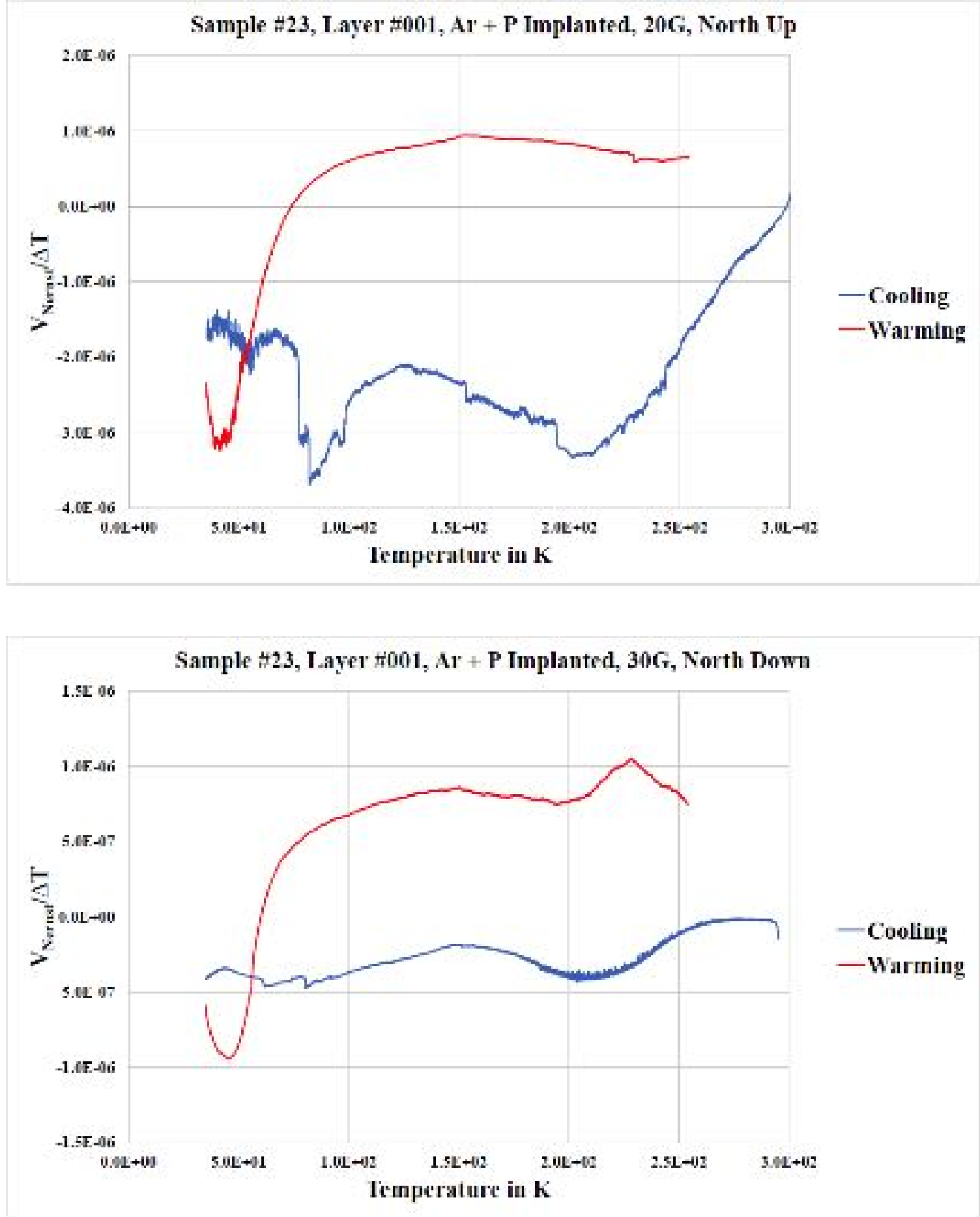

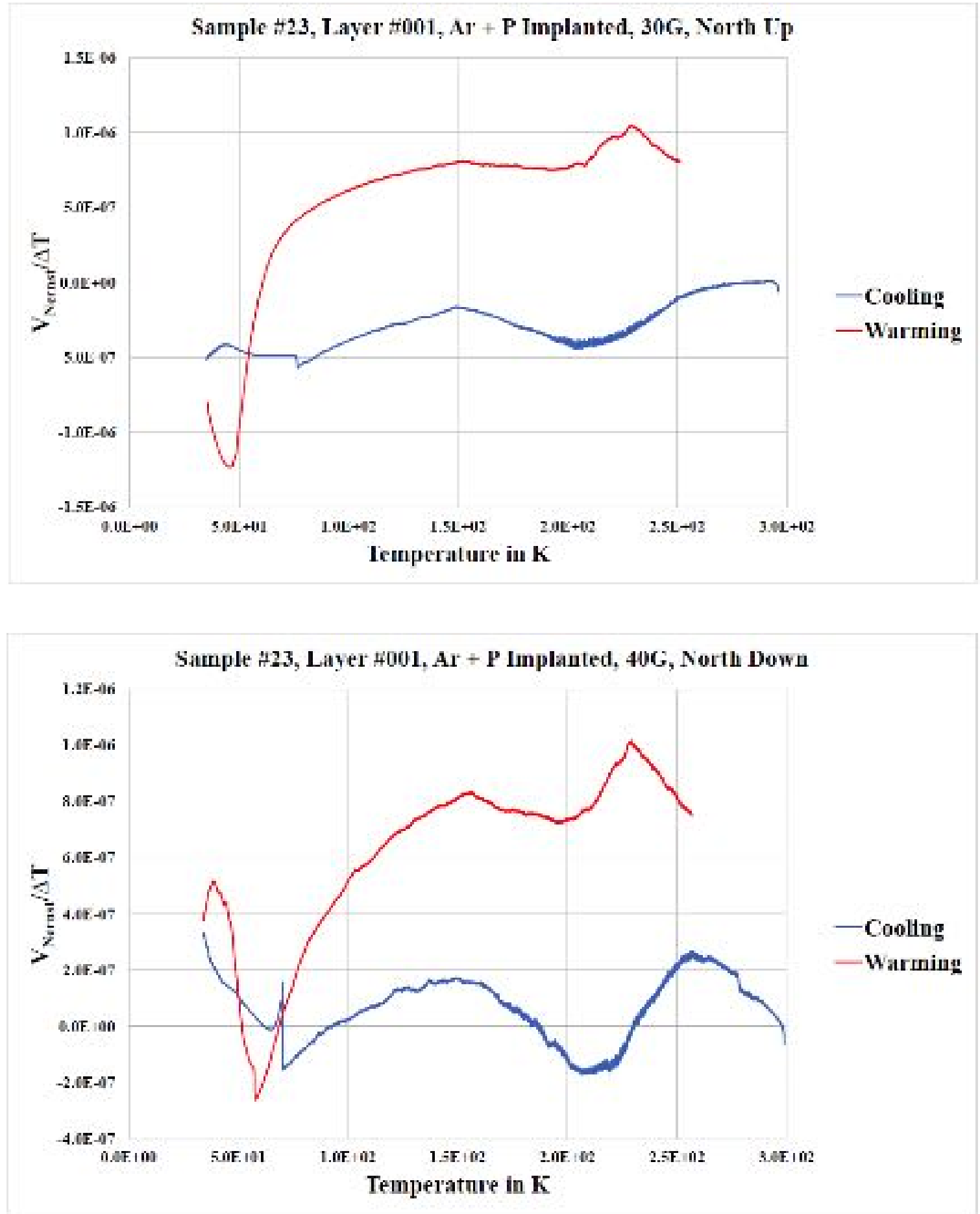

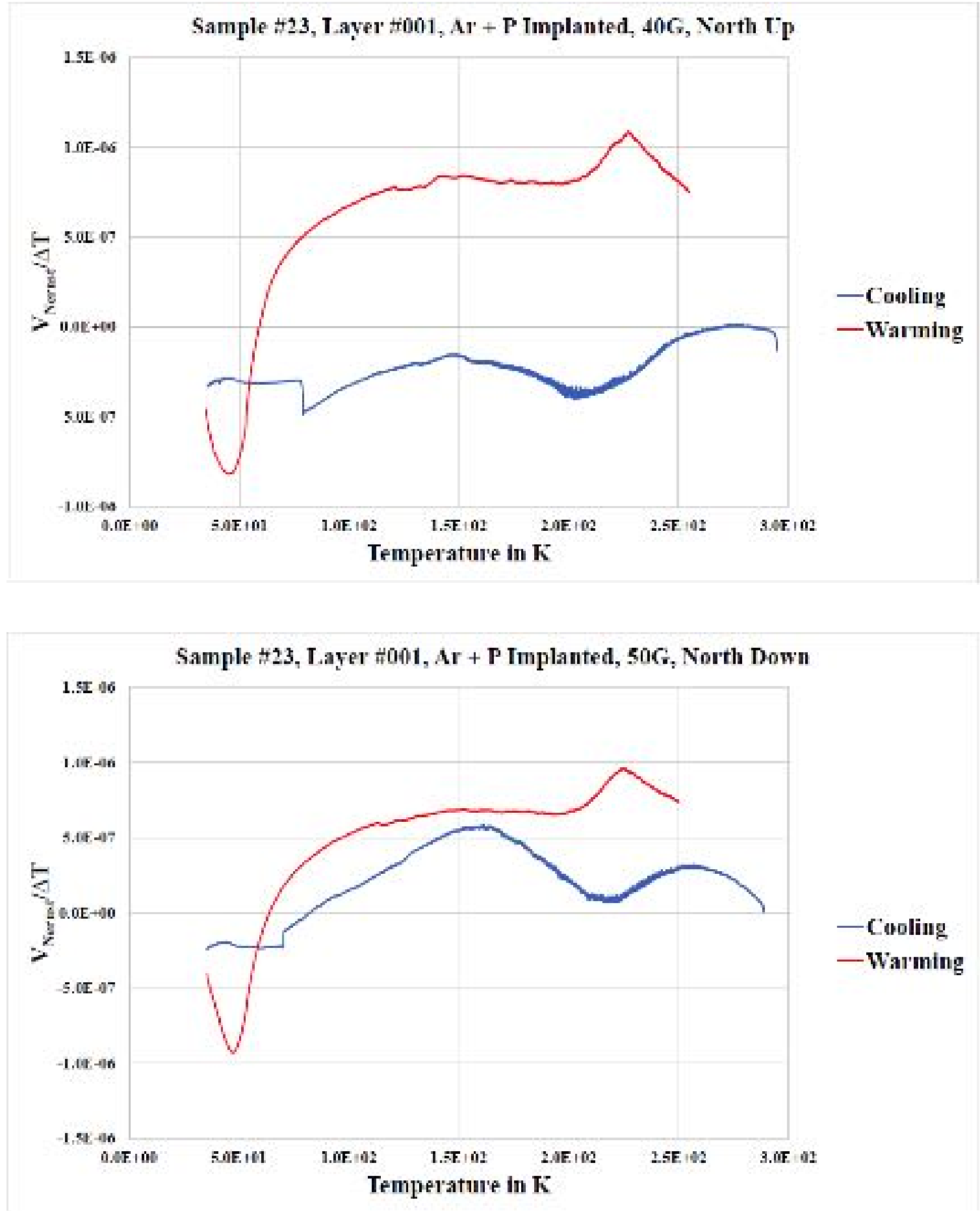

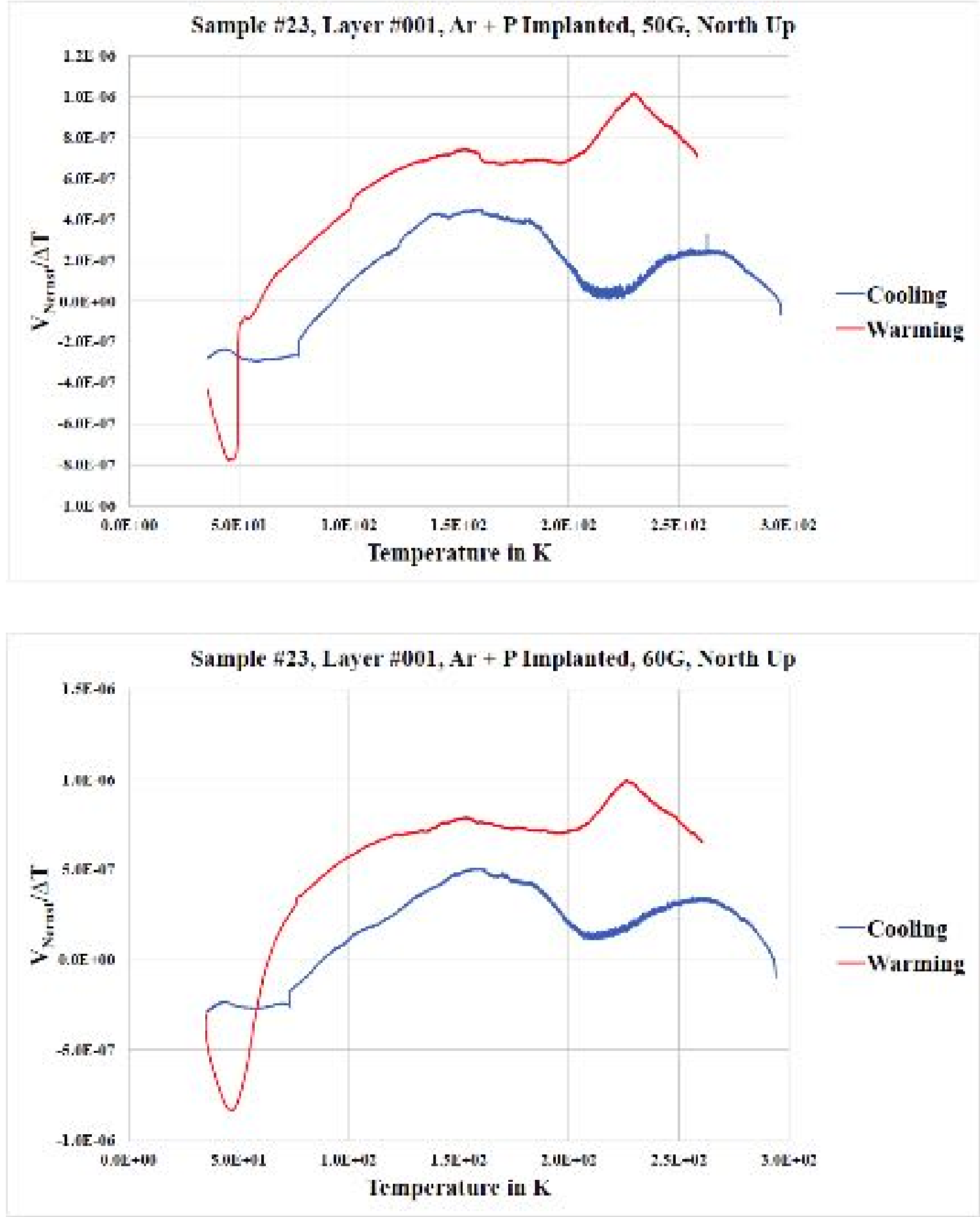

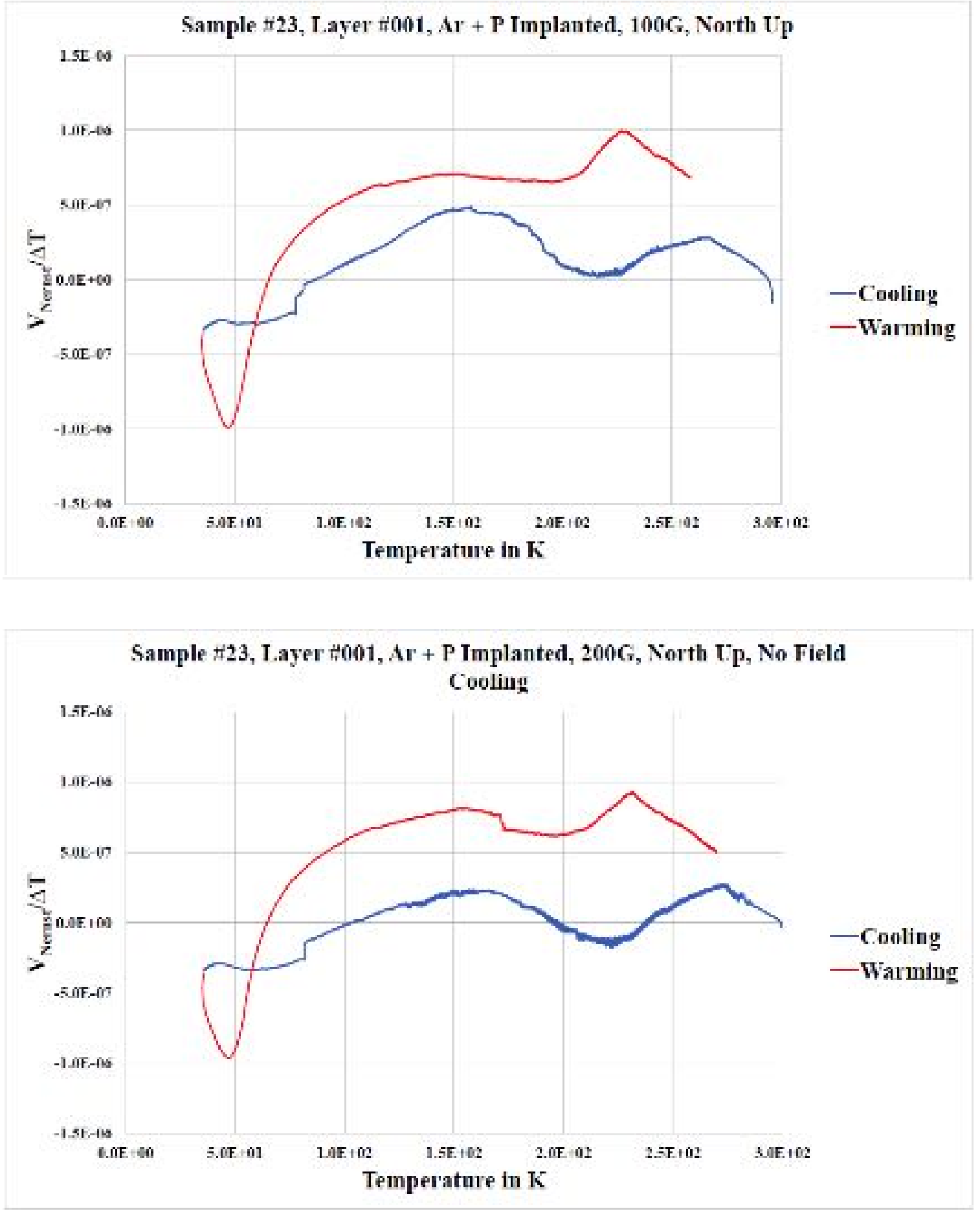

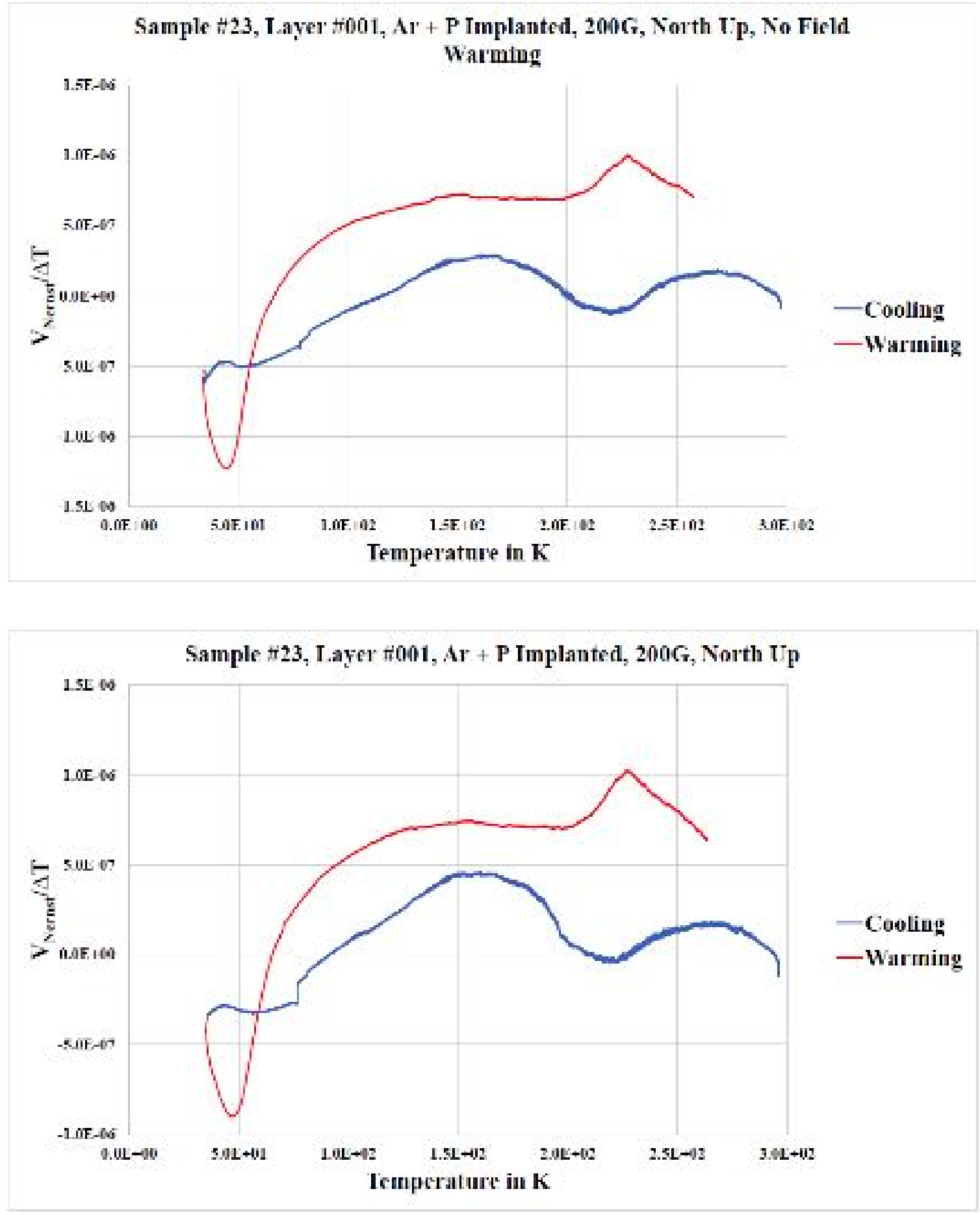

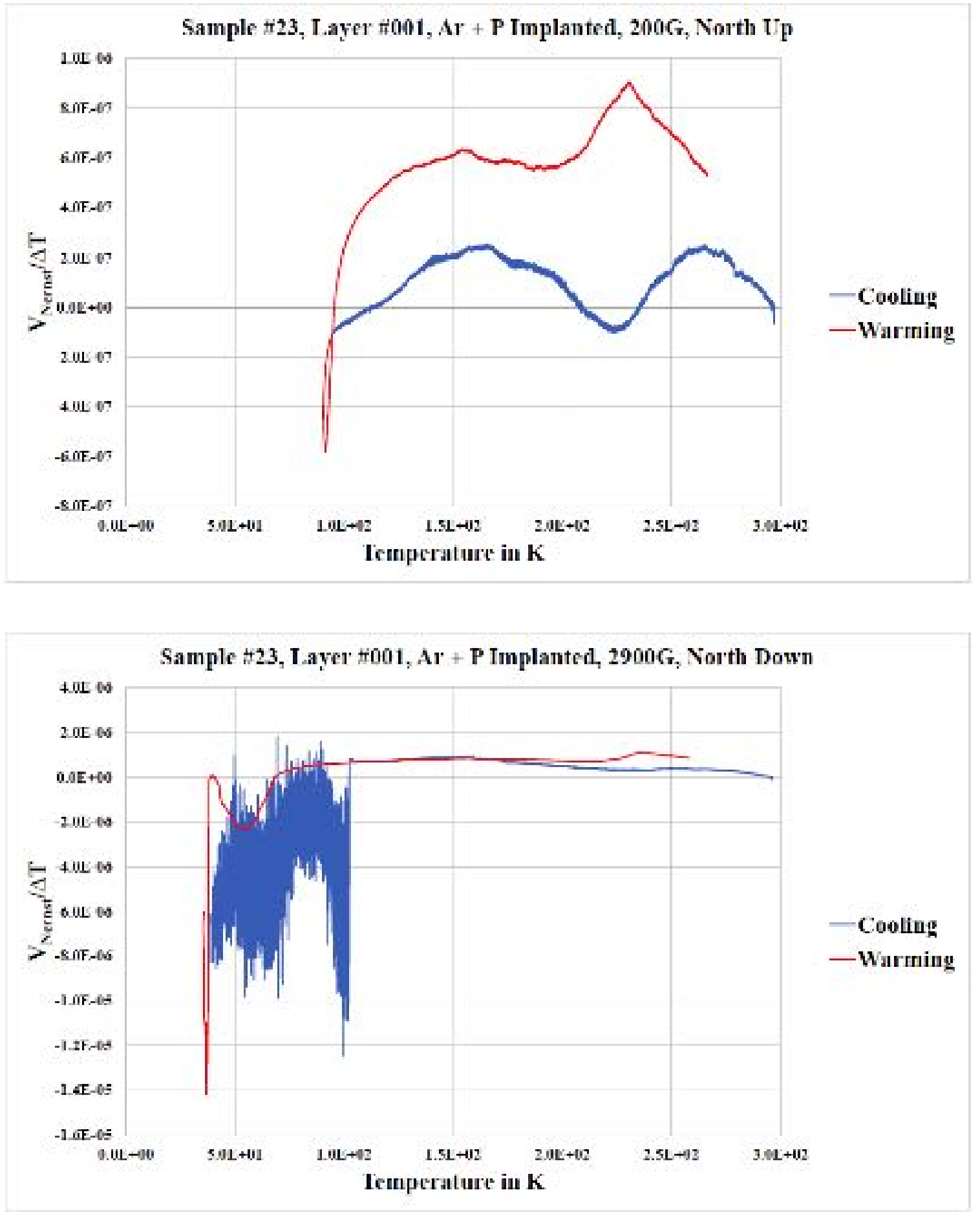


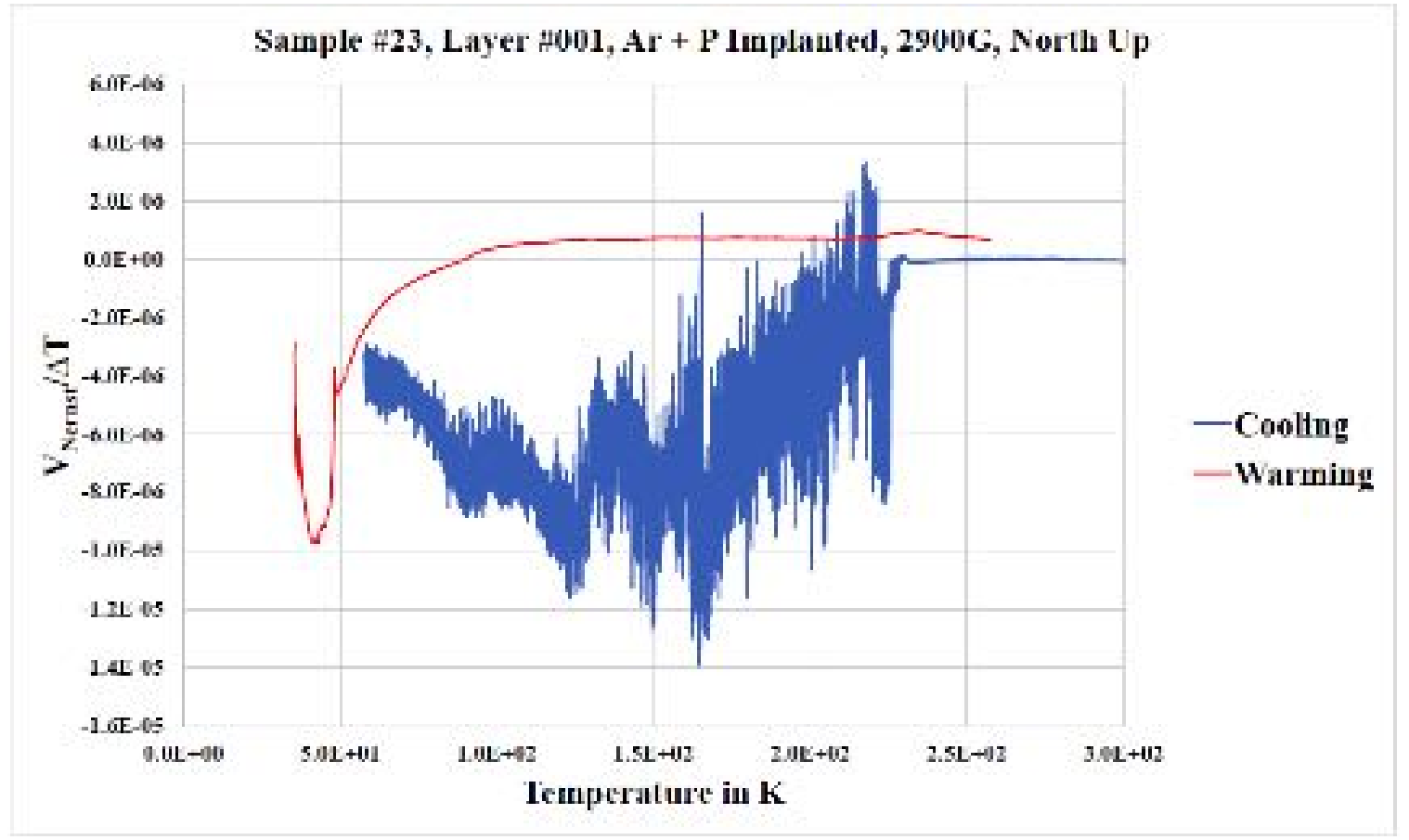


II. Sample \# 151, layer \# 008
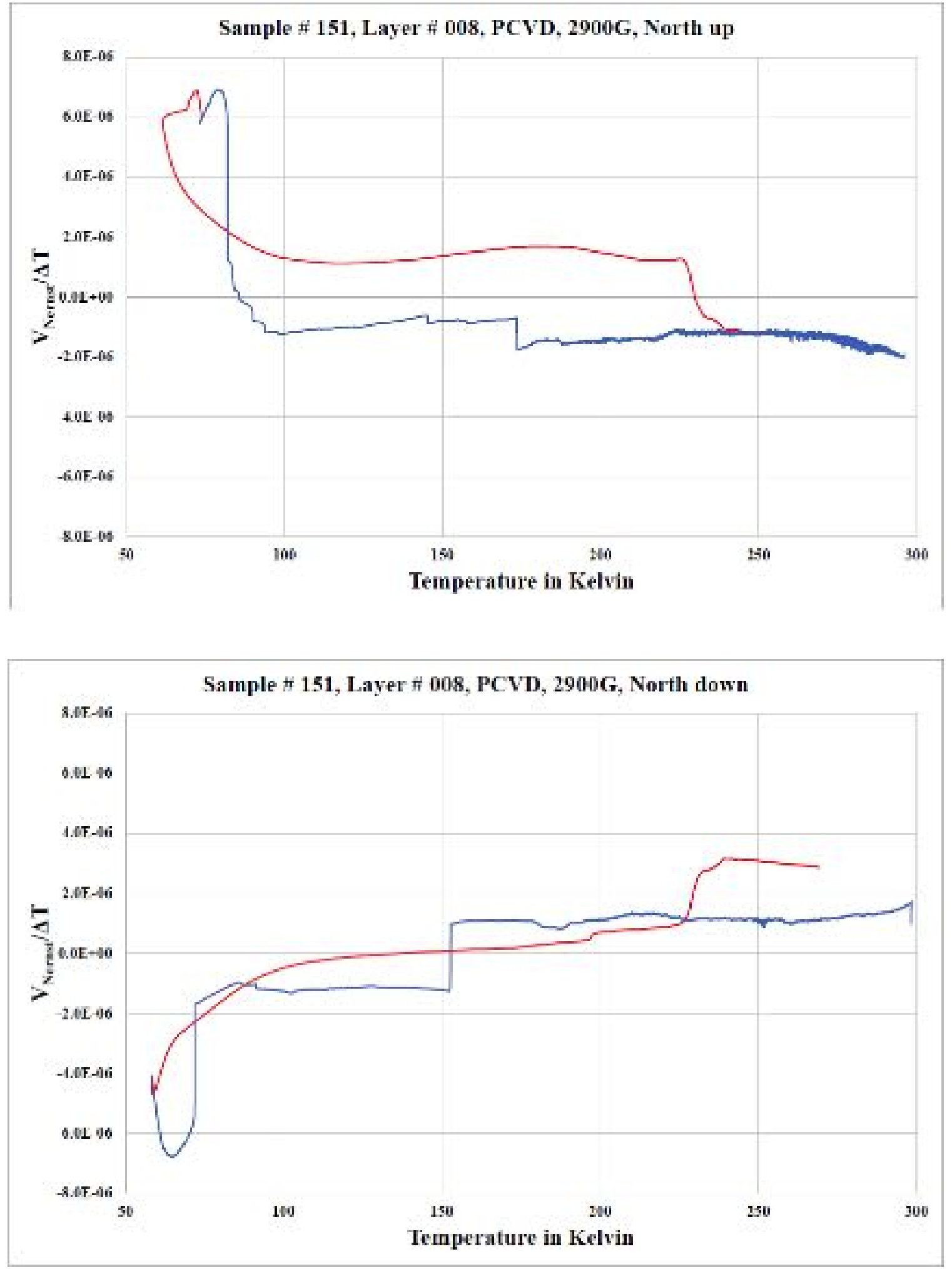

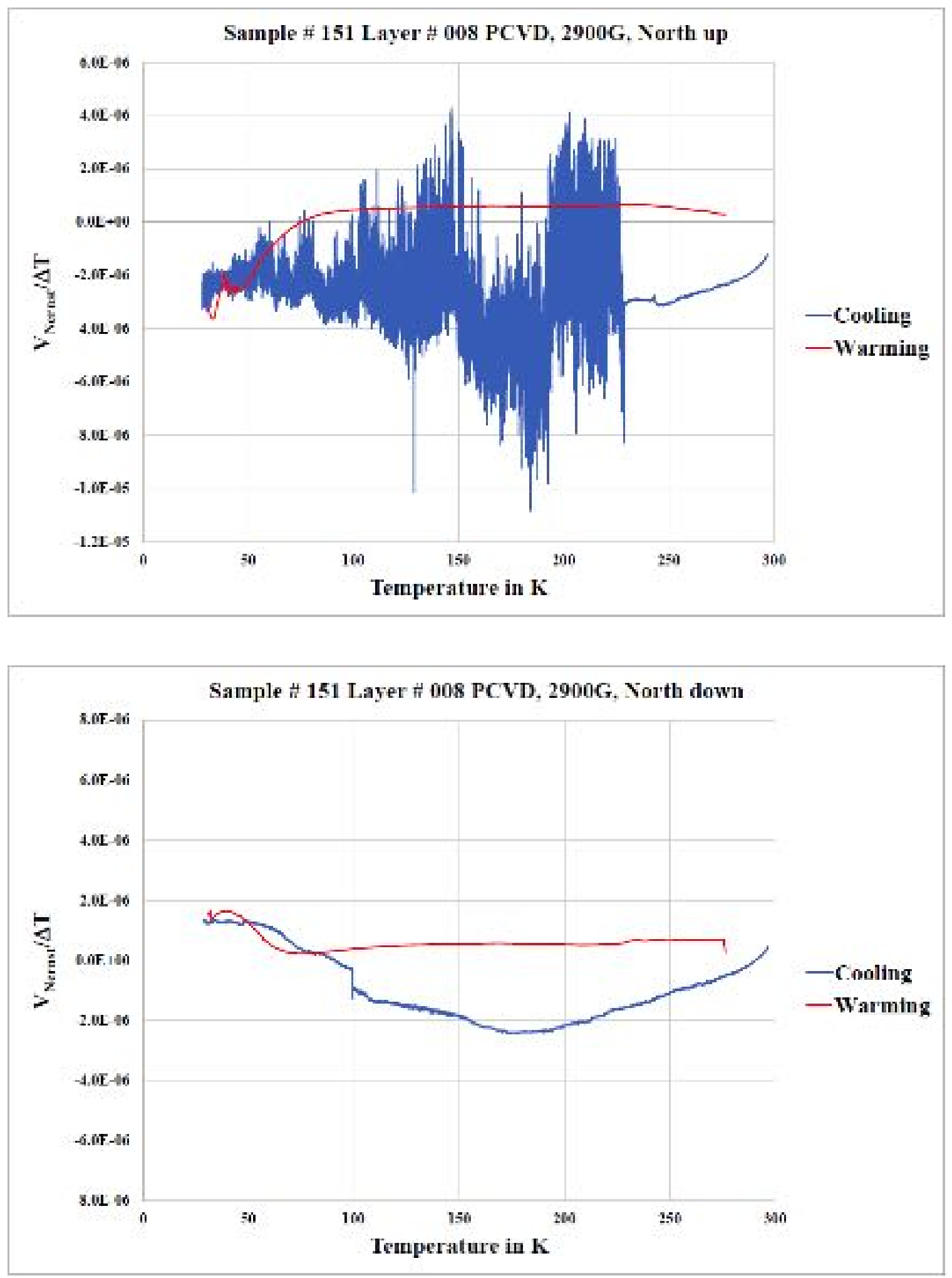

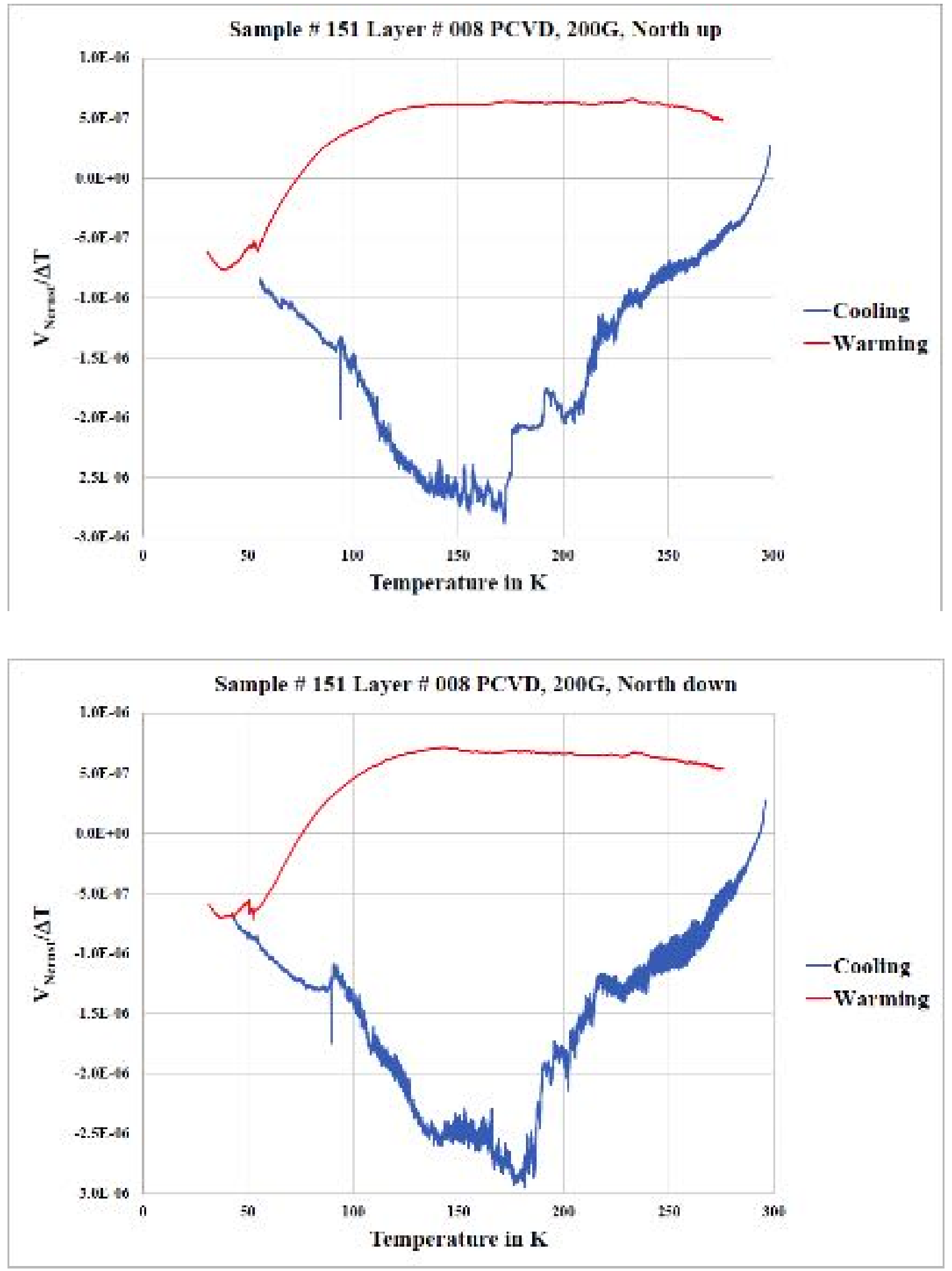

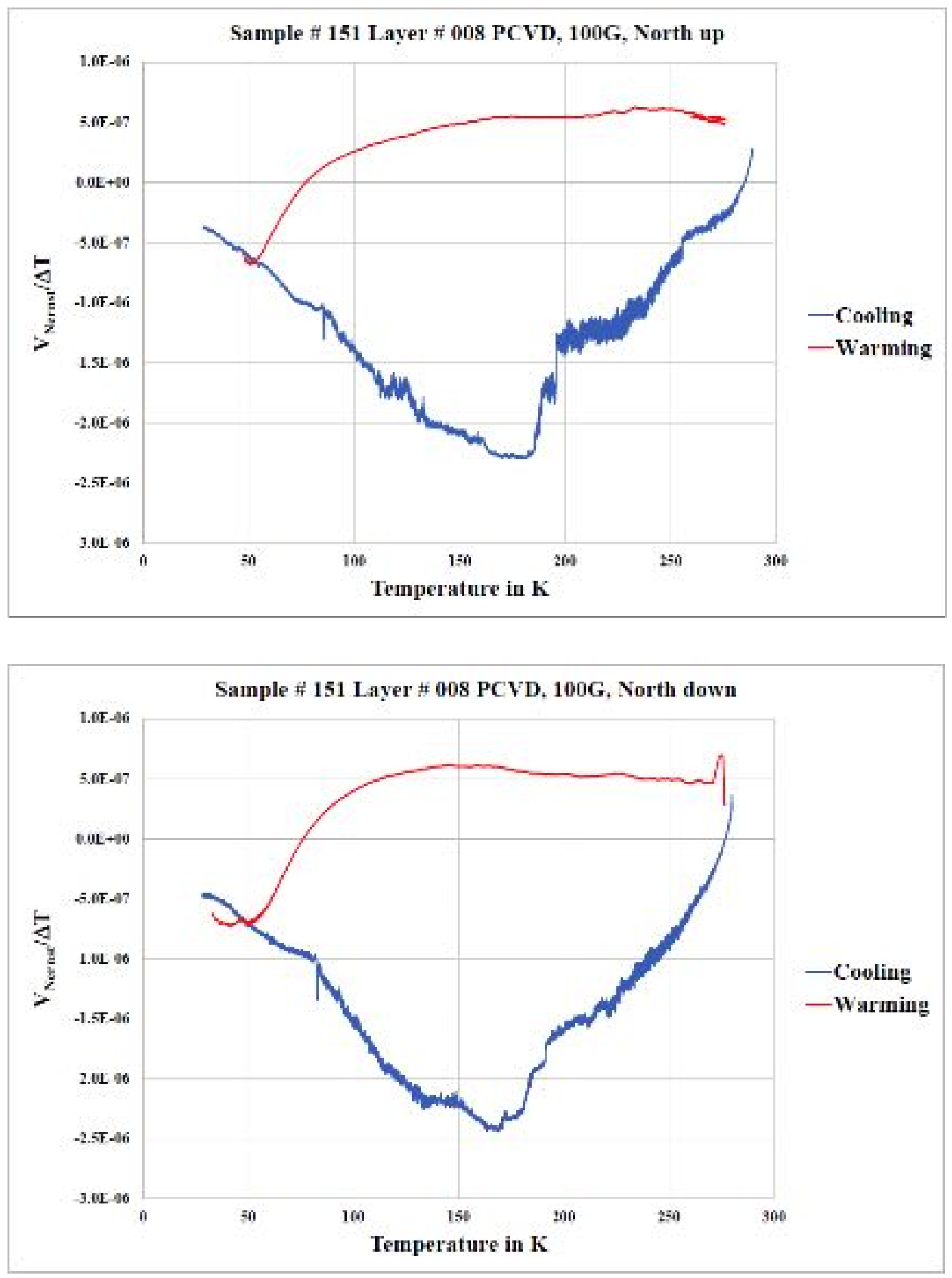

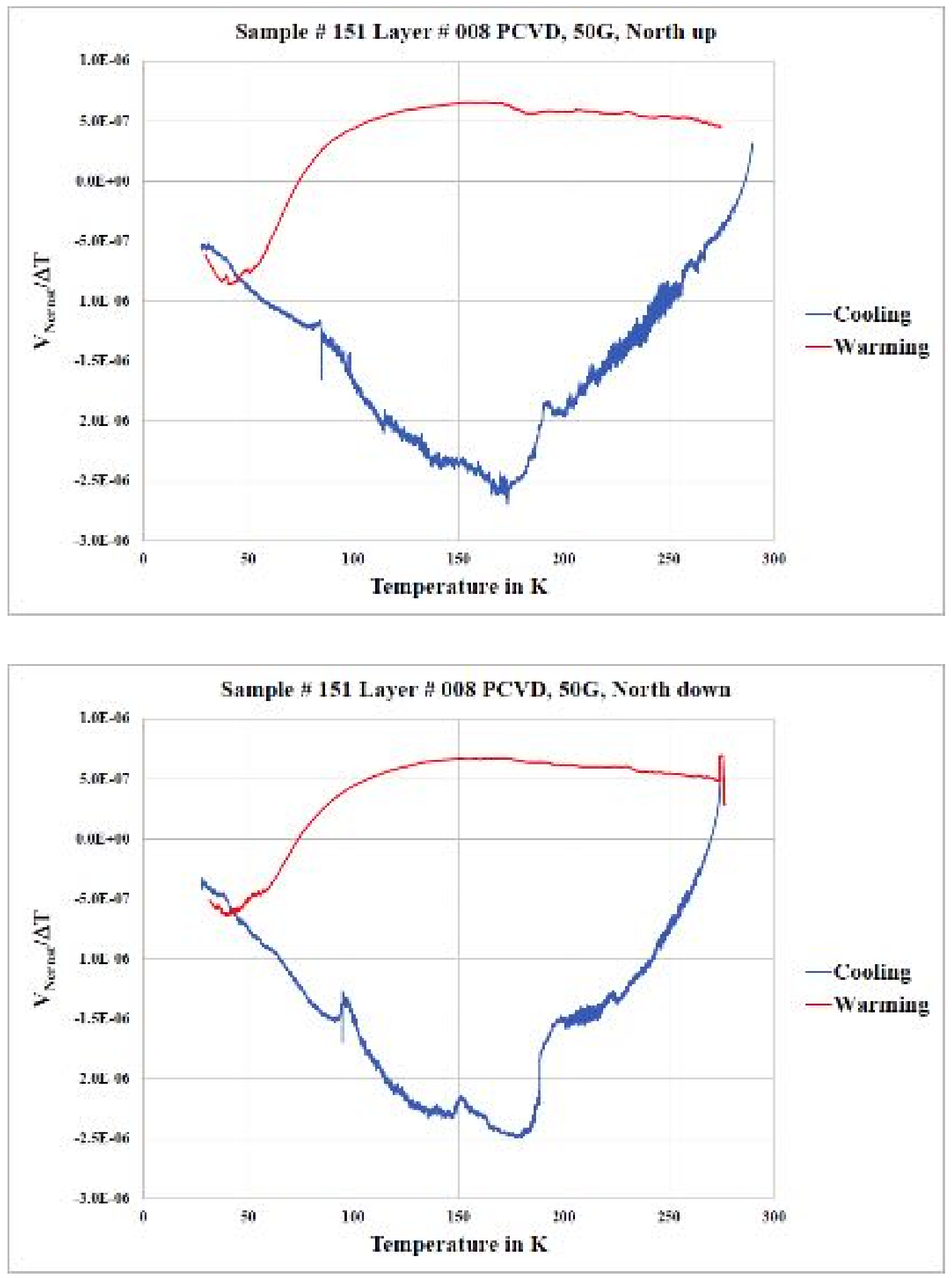

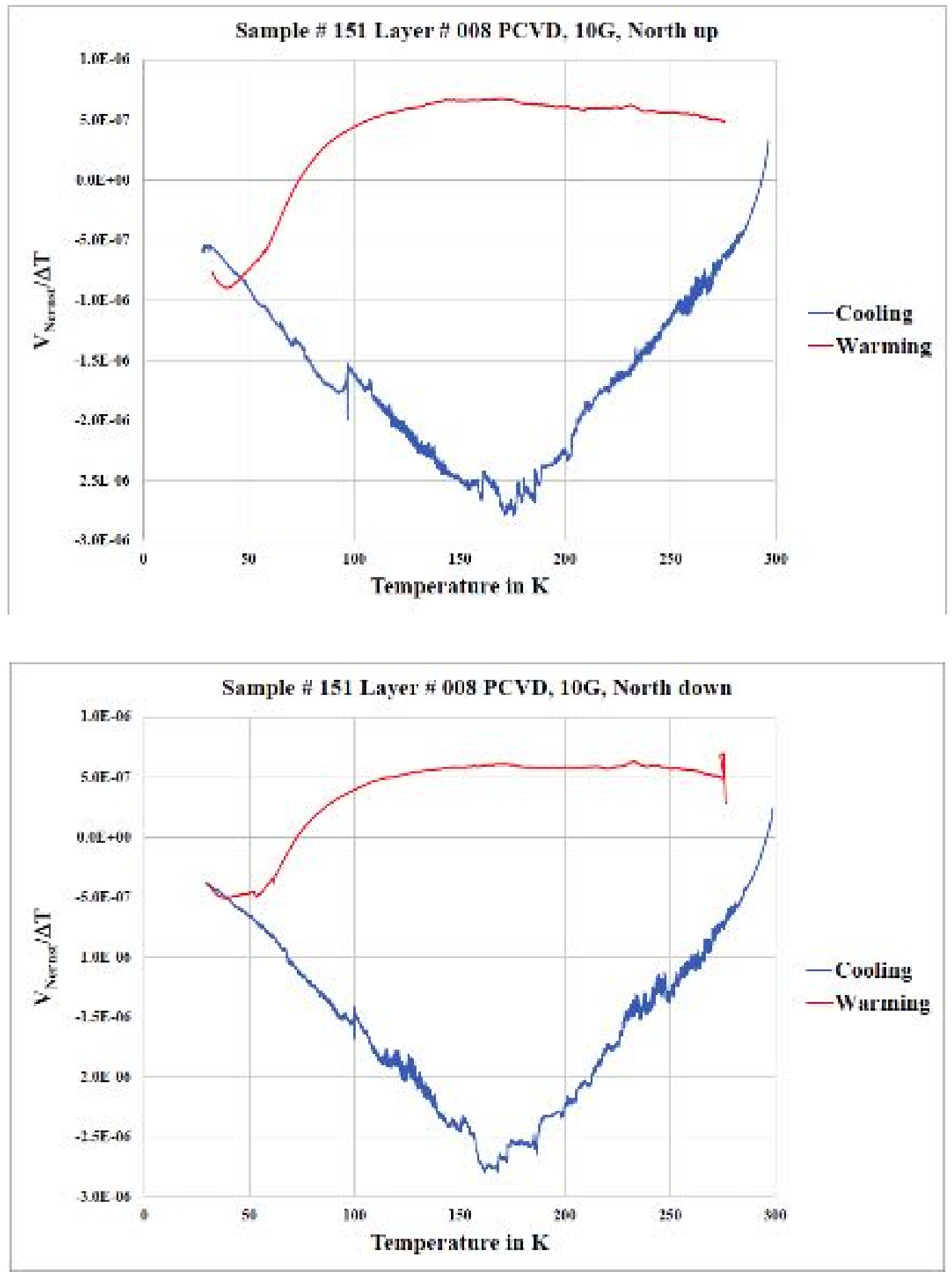


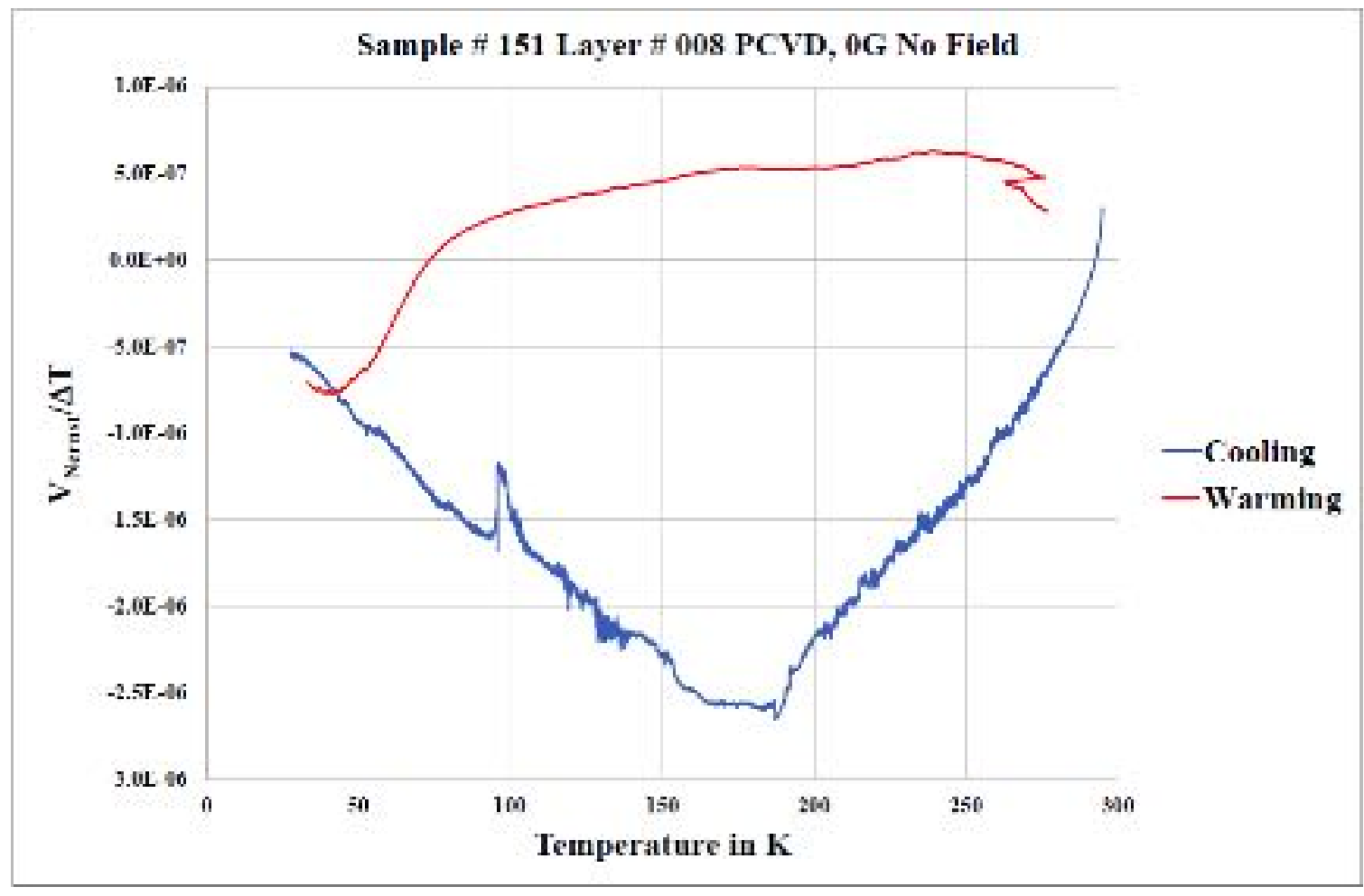


III. Sample \# 163, layer \# 009
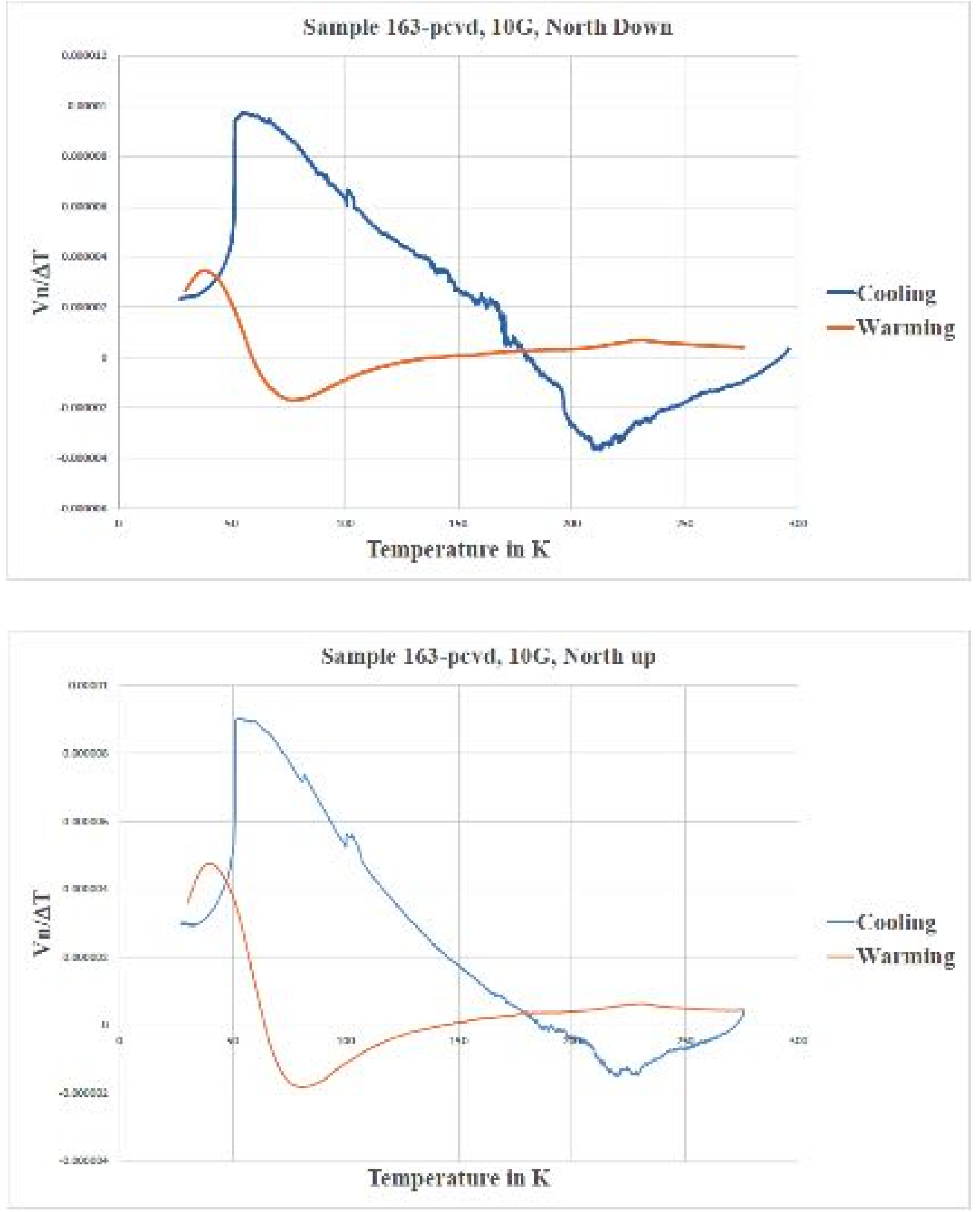

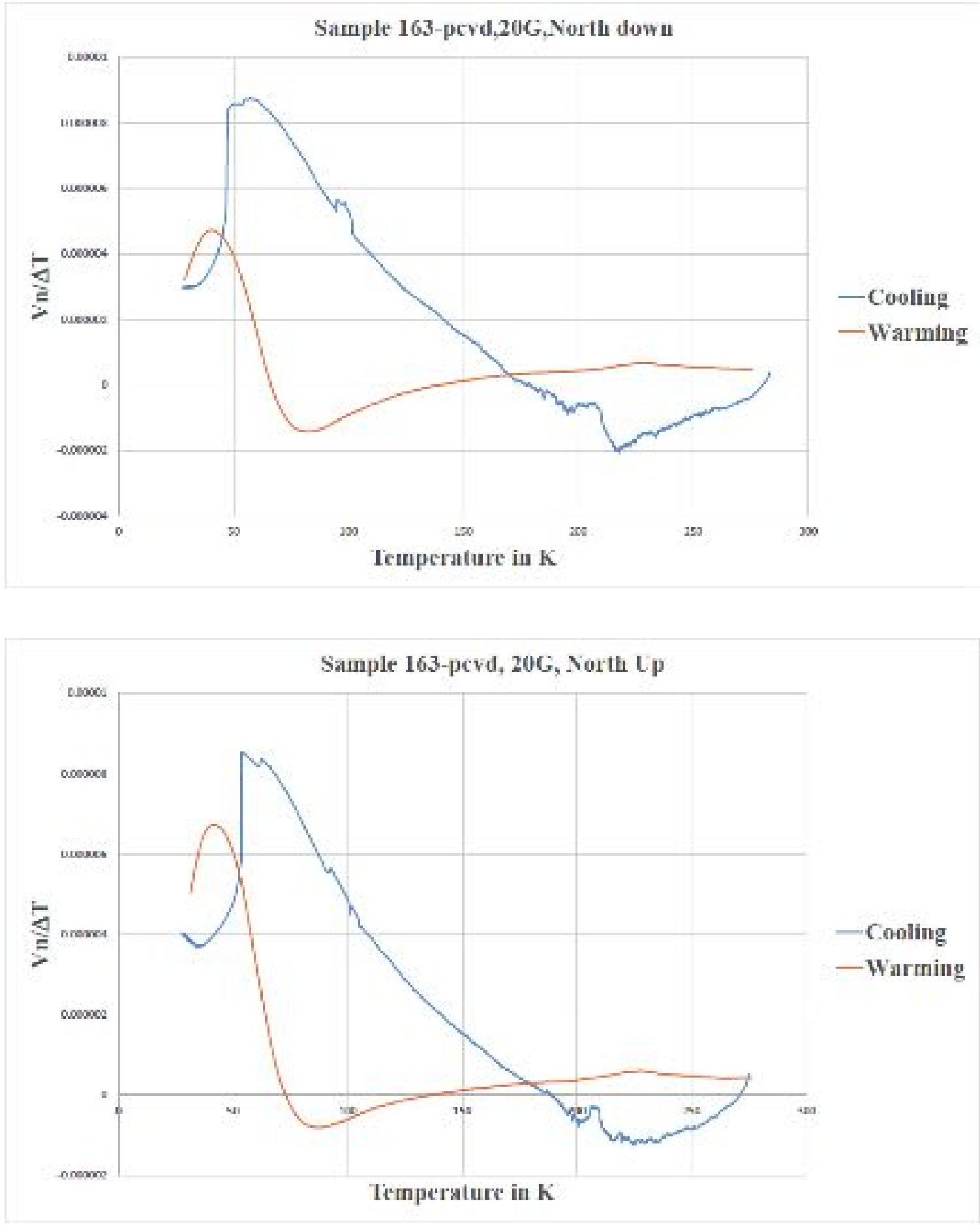

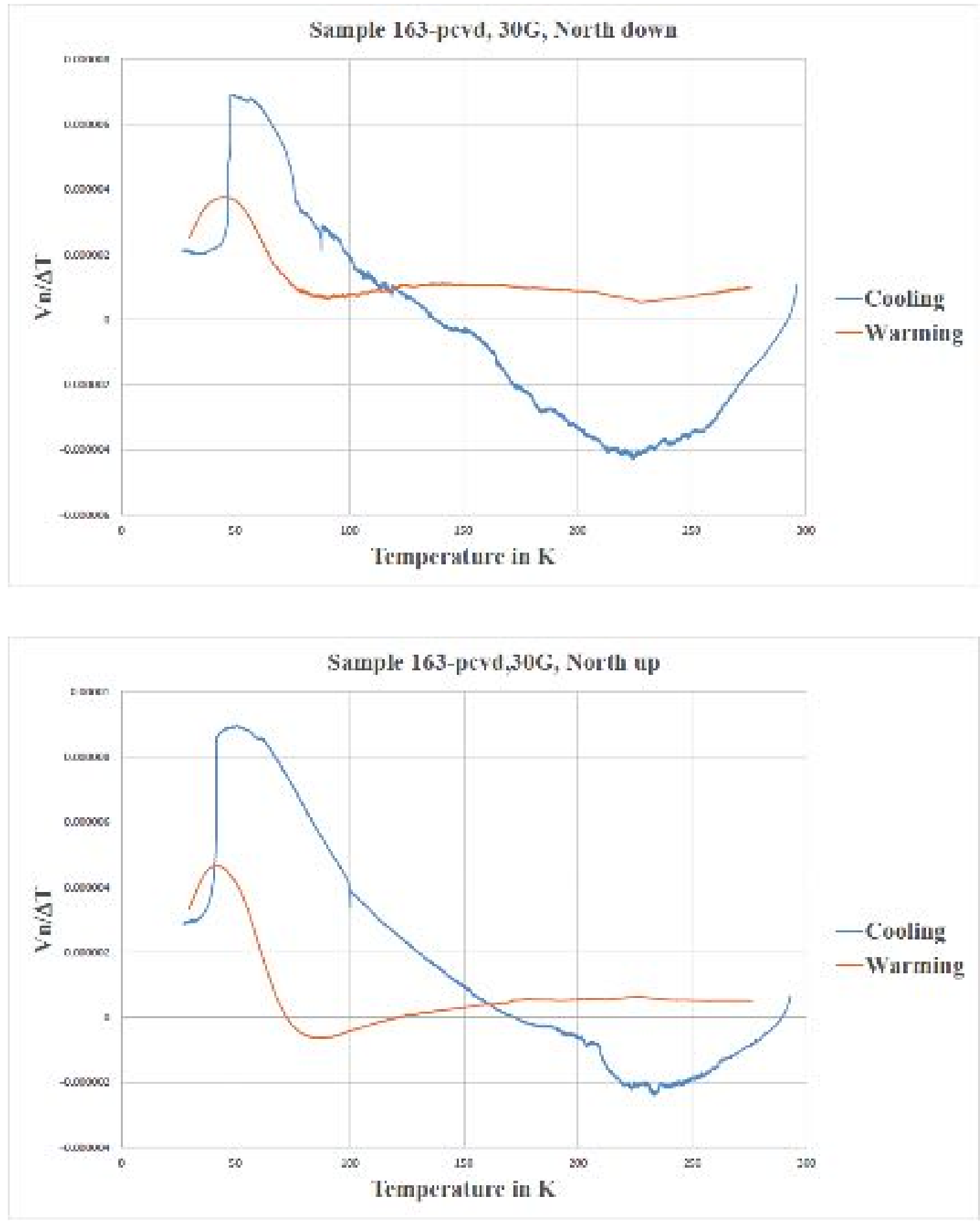

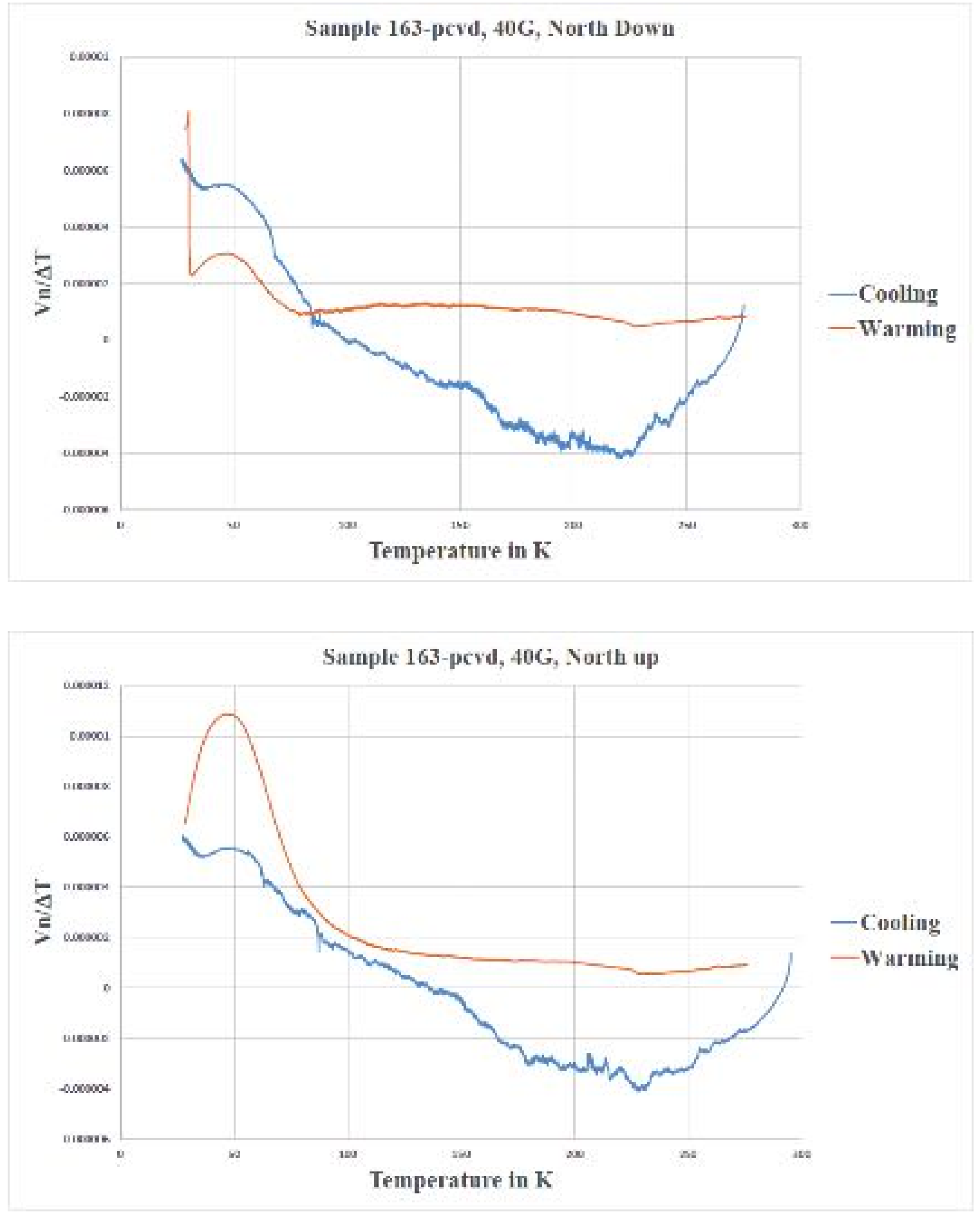
IV. Sample \# 170, layer \# 010
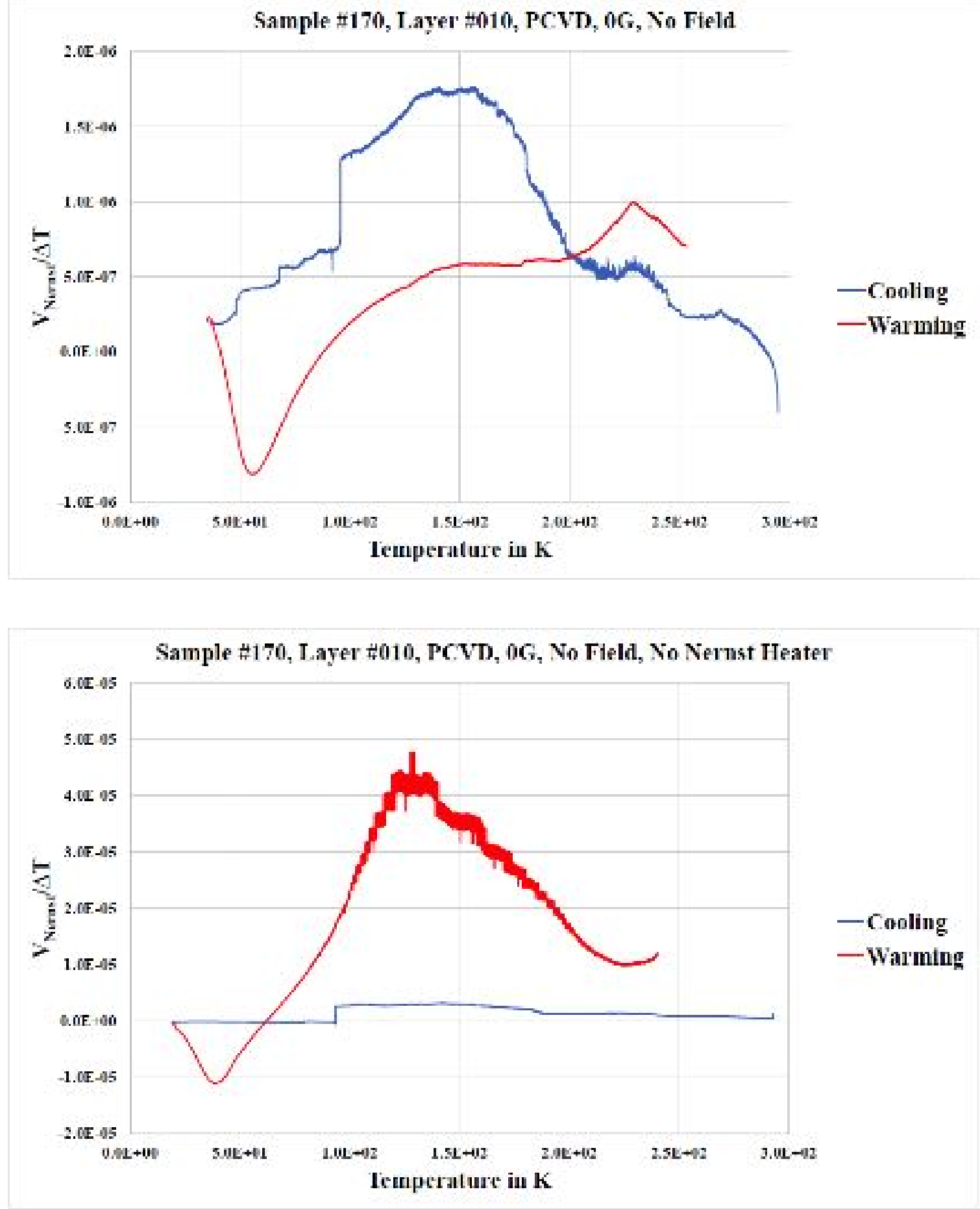

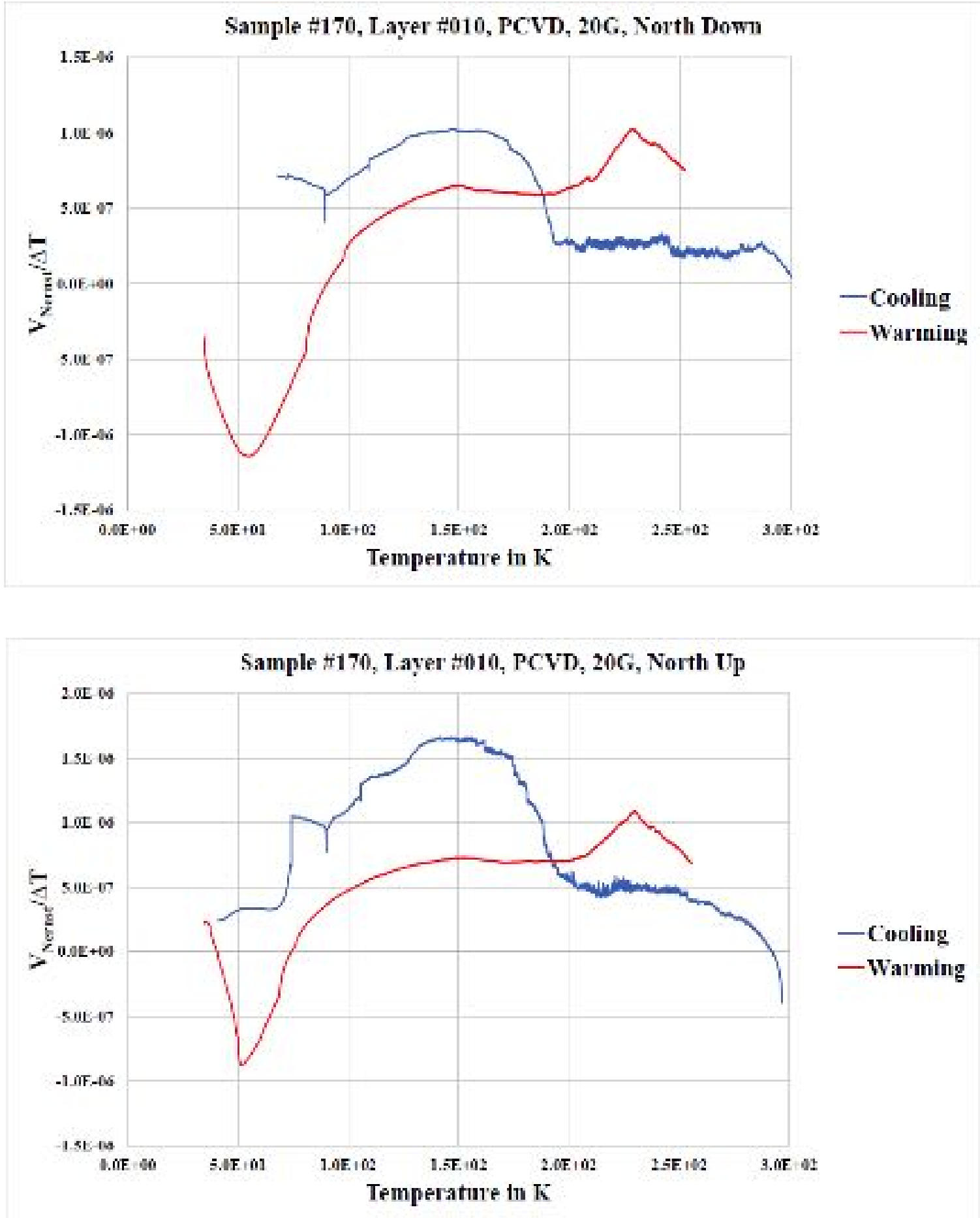

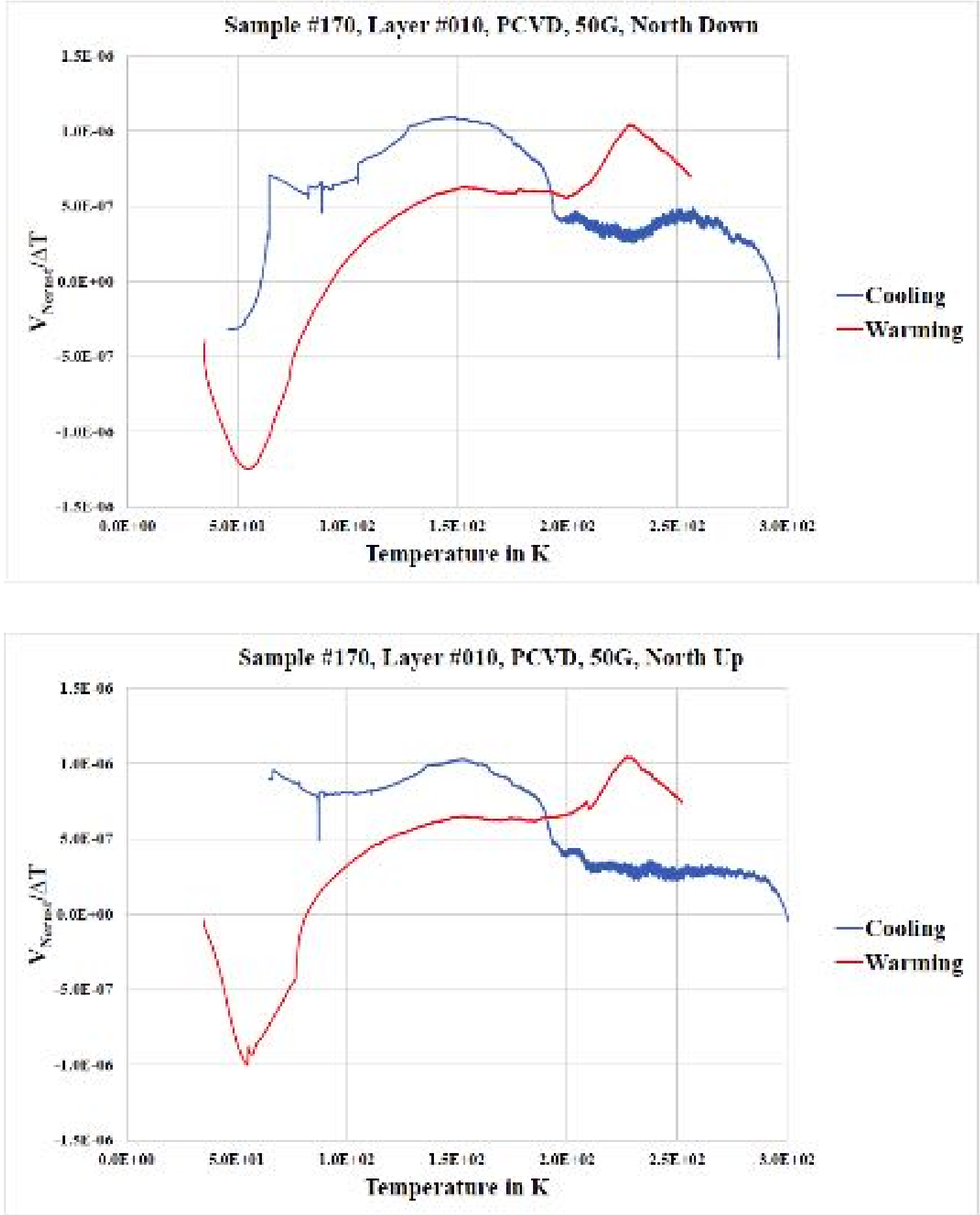

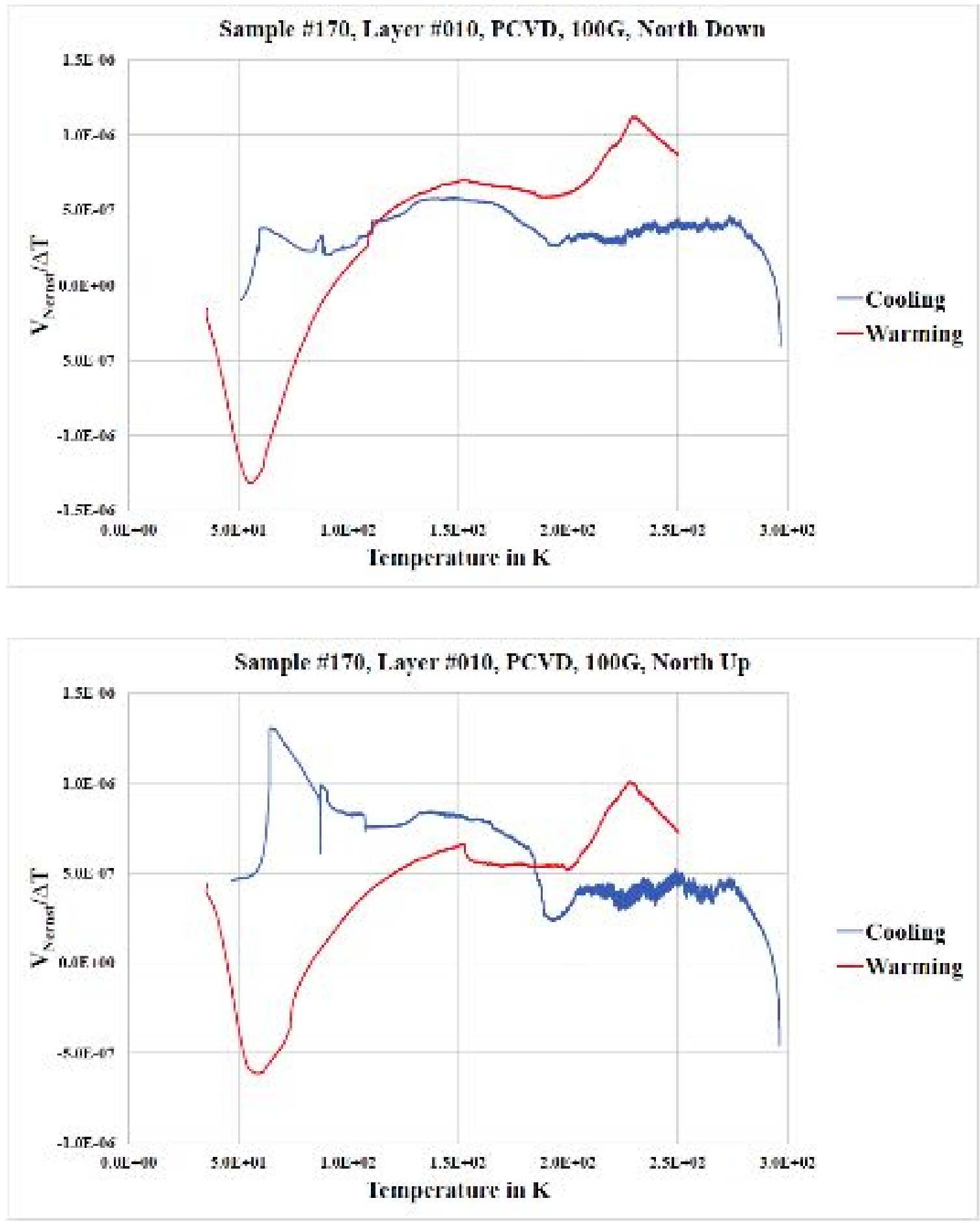

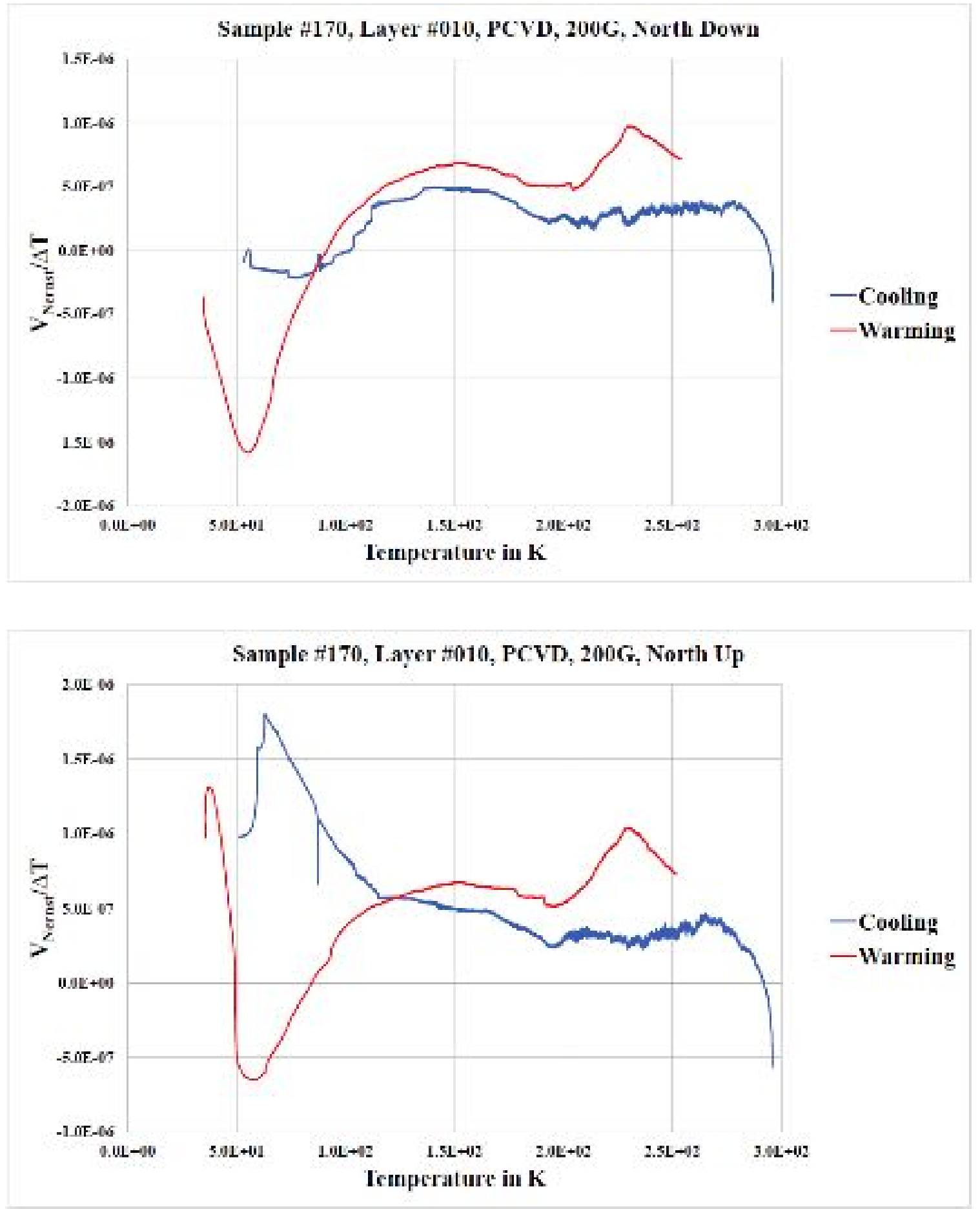


\section{V. $\quad$ Sample \# 213, layer \# 001}

Sample213-(Ar+P)-1113G V/DTNdown - V/DTNip

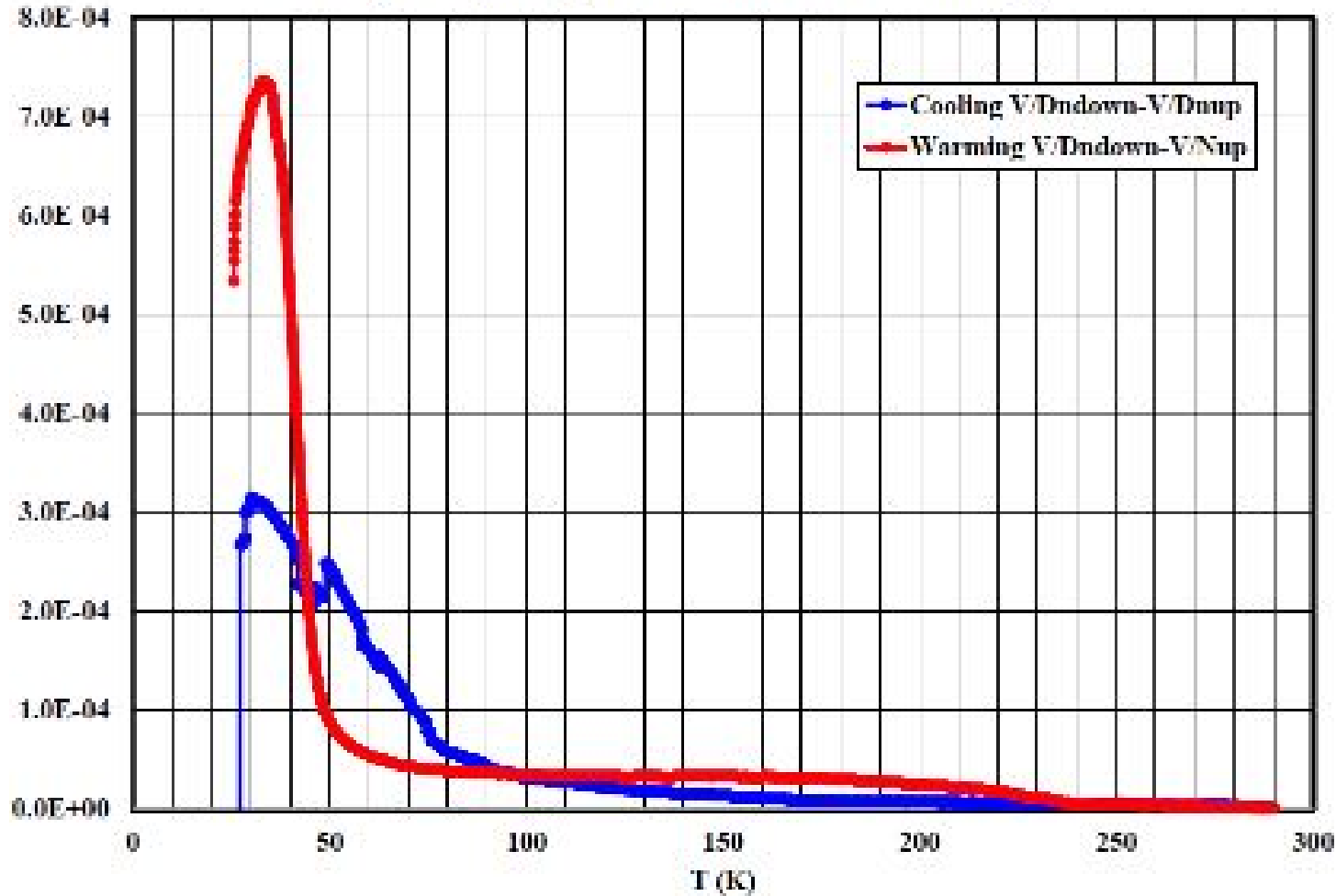

Sample213-(Ar+P)-1113G V/DTNdown - V/DTNup

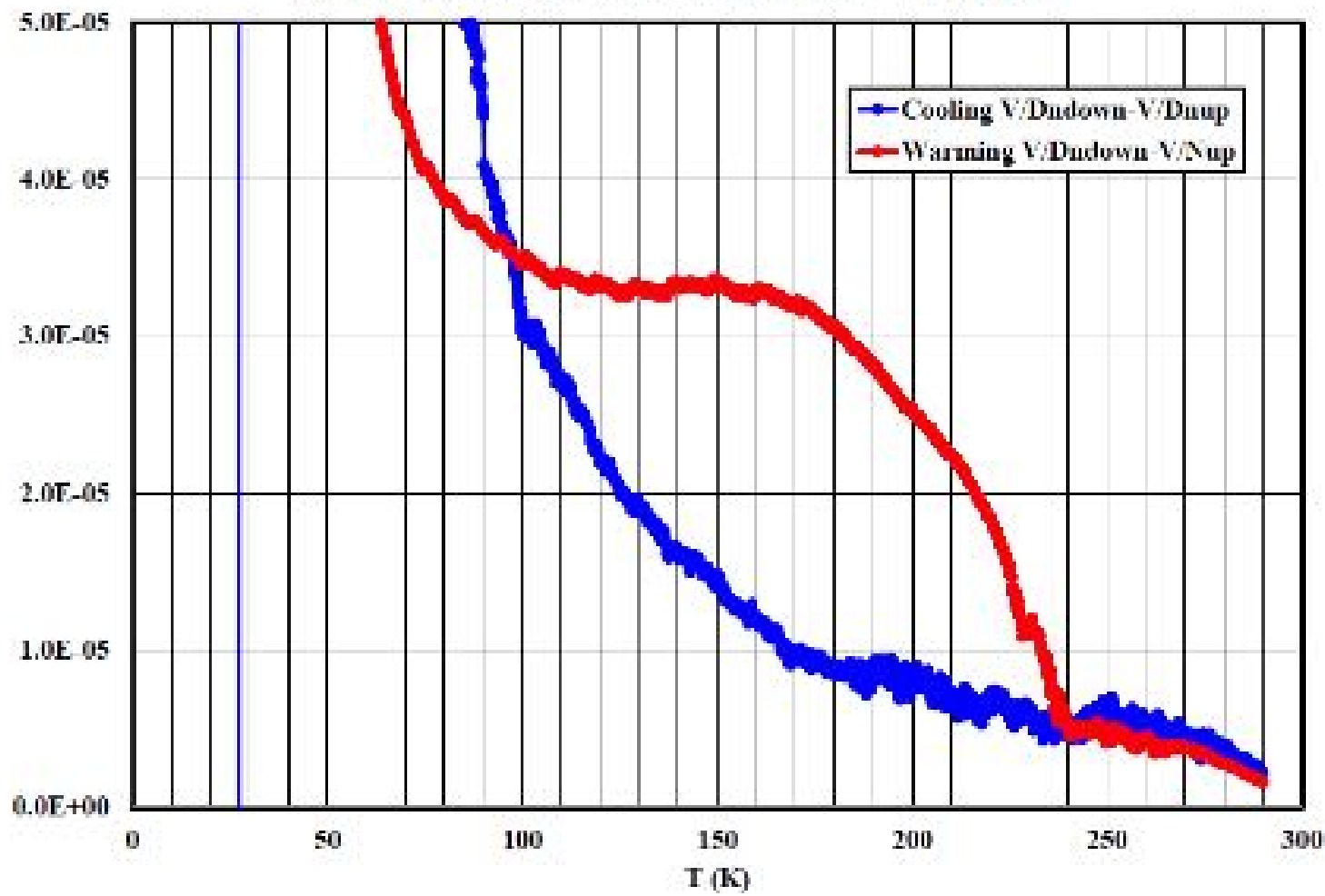


Sample213-(Ar+P)-640G V/DTNdown - V/DTNup
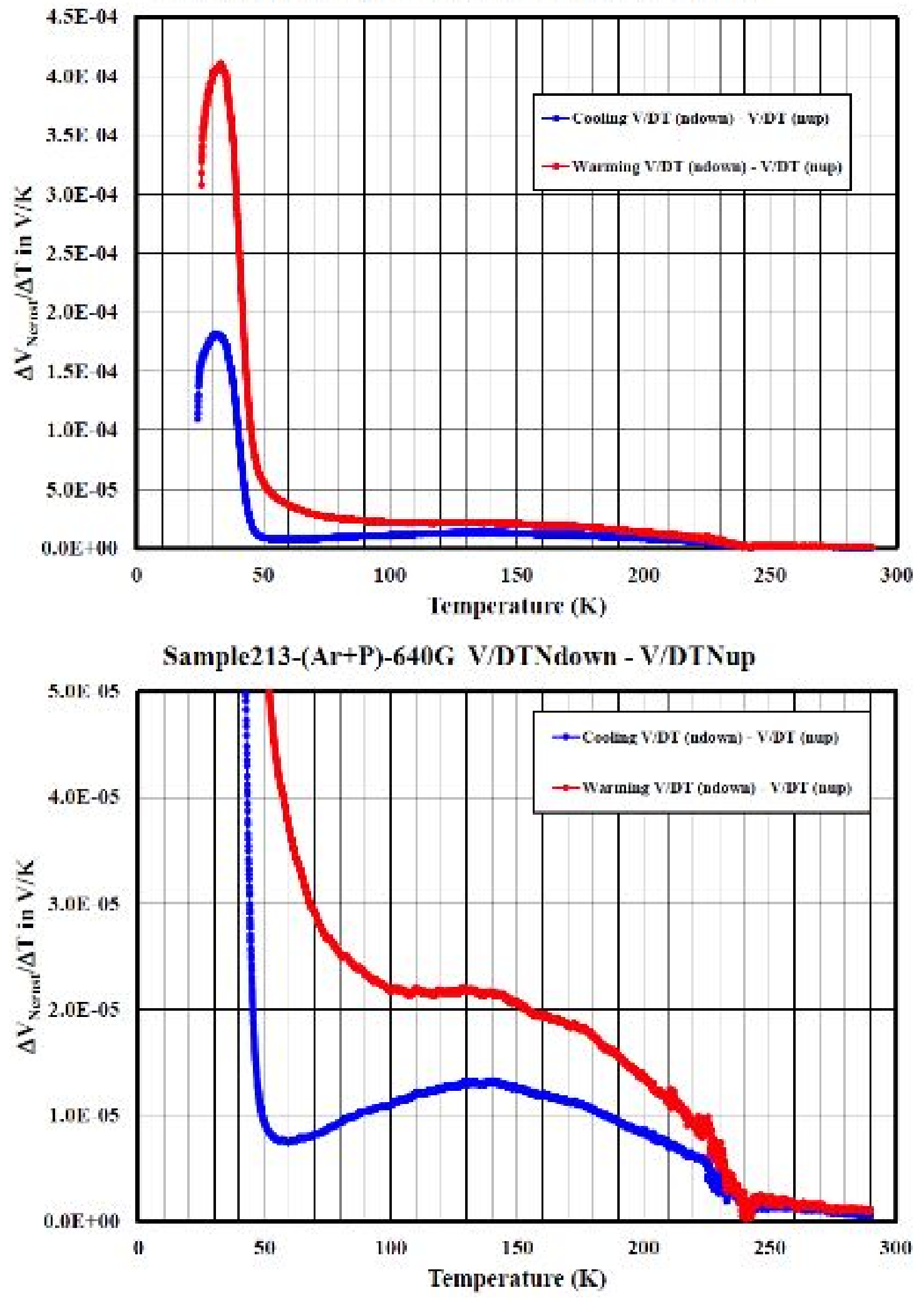
Sample213-(Ar+P)-290G

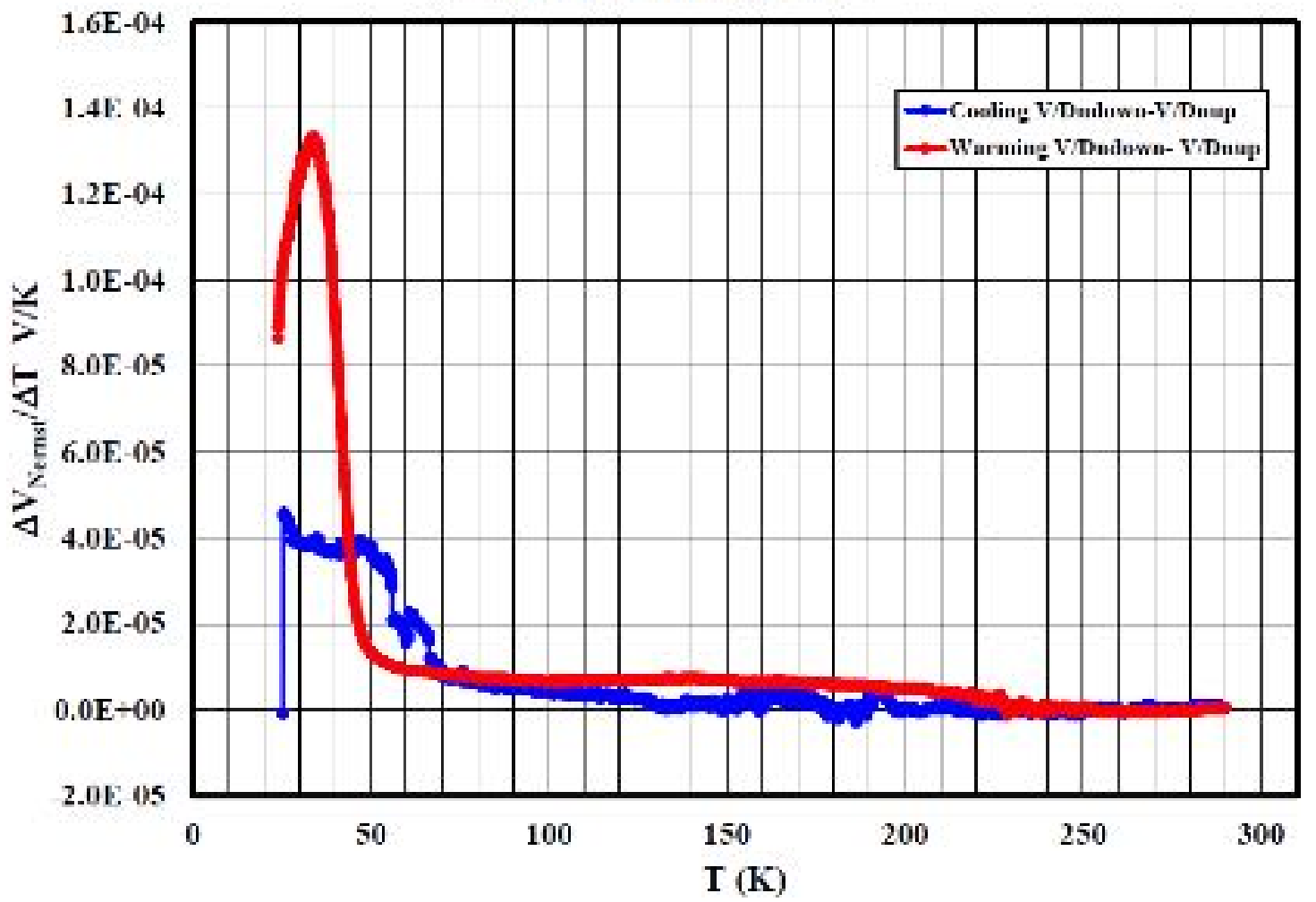

Sample213-(Ar+P)-290G

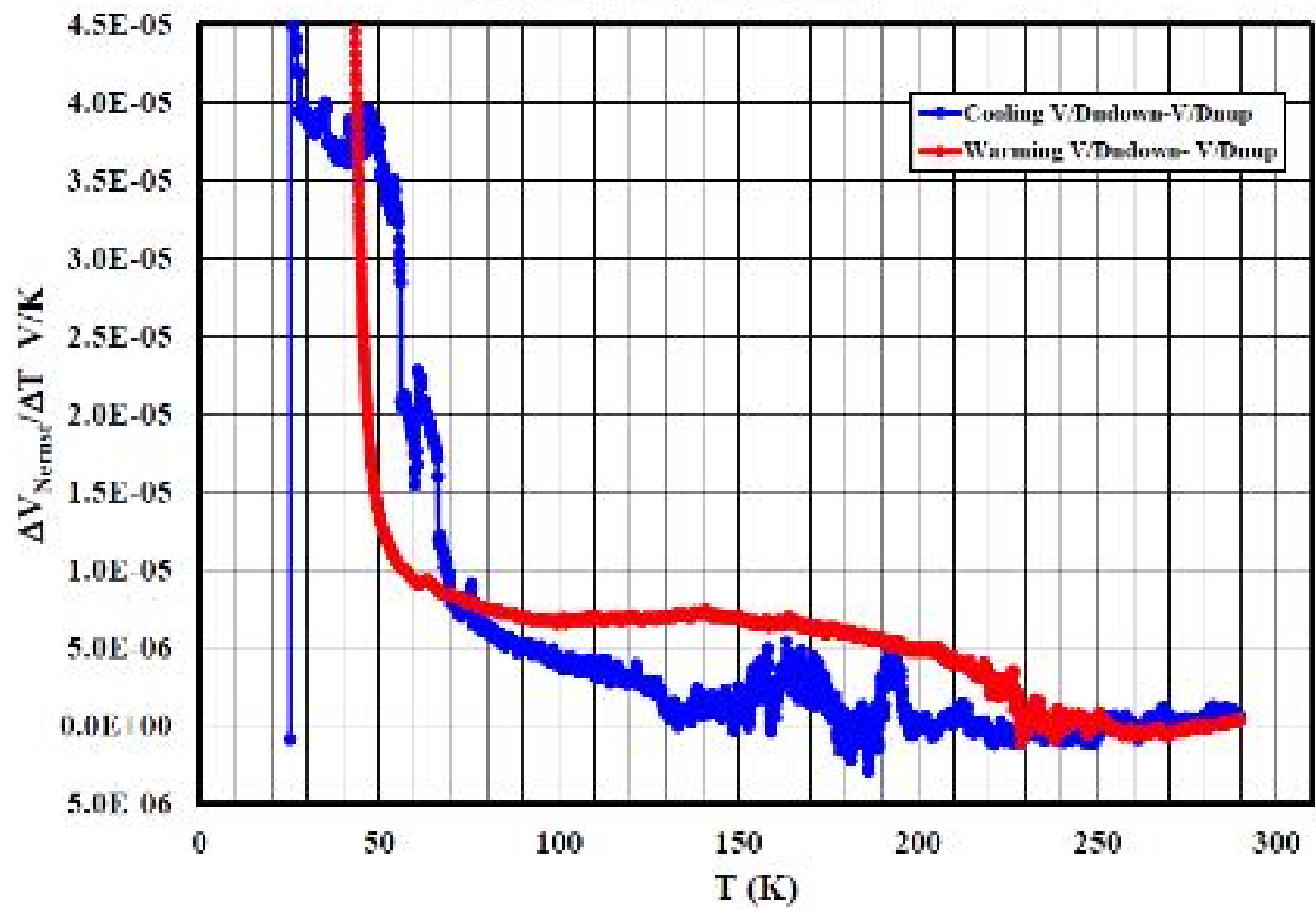




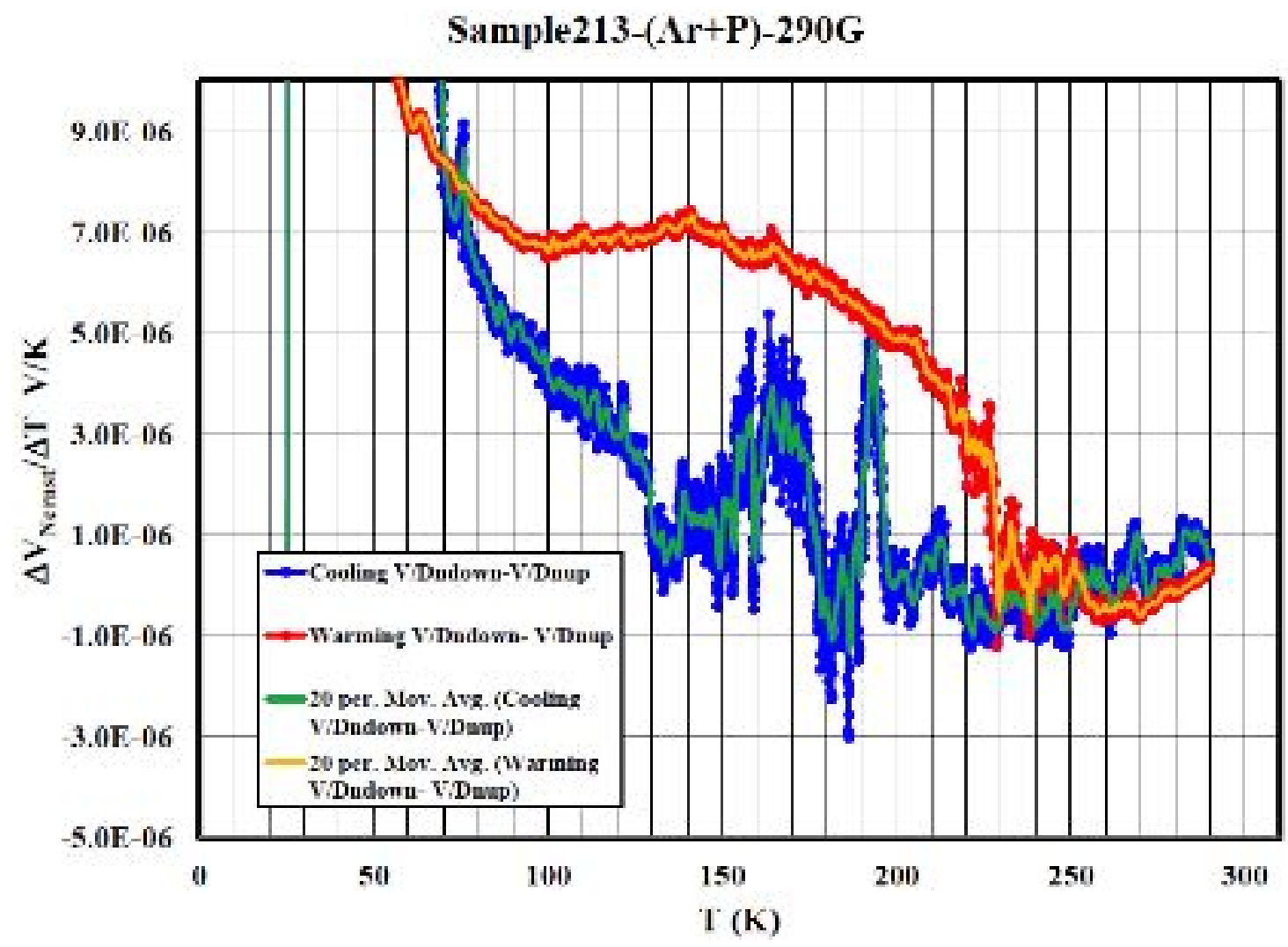

Sample 213, 120 Ganss, $V_{\text {Nerwst }}(\mathrm{NDn}) / \Delta \mathrm{T}-\mathrm{V}_{\text {Nerwat }}(\mathrm{Nup}) / \Delta \mathrm{T}$

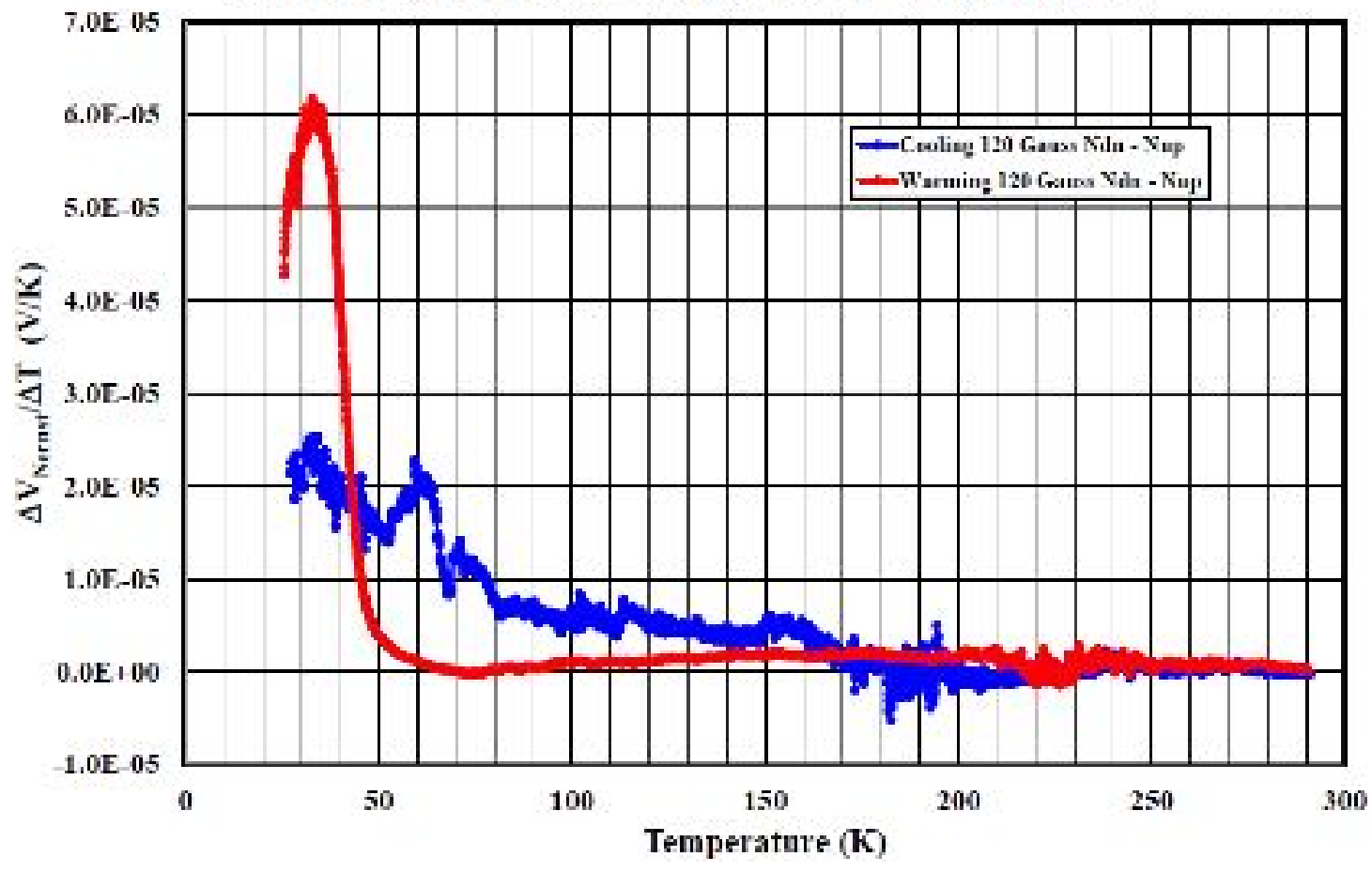




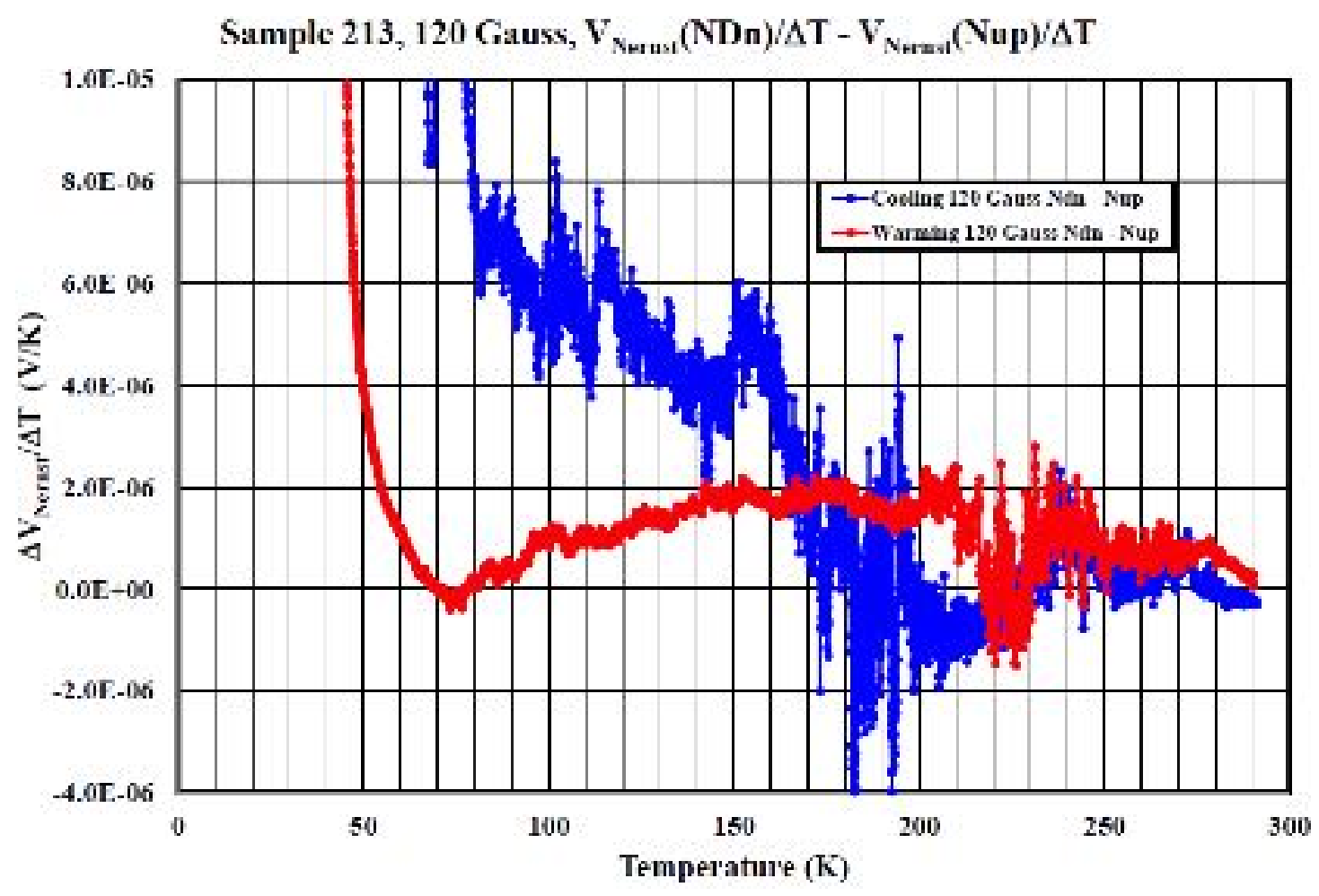

Sample 213,120 Gauss, $V_{\text {Nernst }}(\mathrm{NDn}) / \Delta T-V_{\text {Nermos }}($ Nup $) / \Delta T$

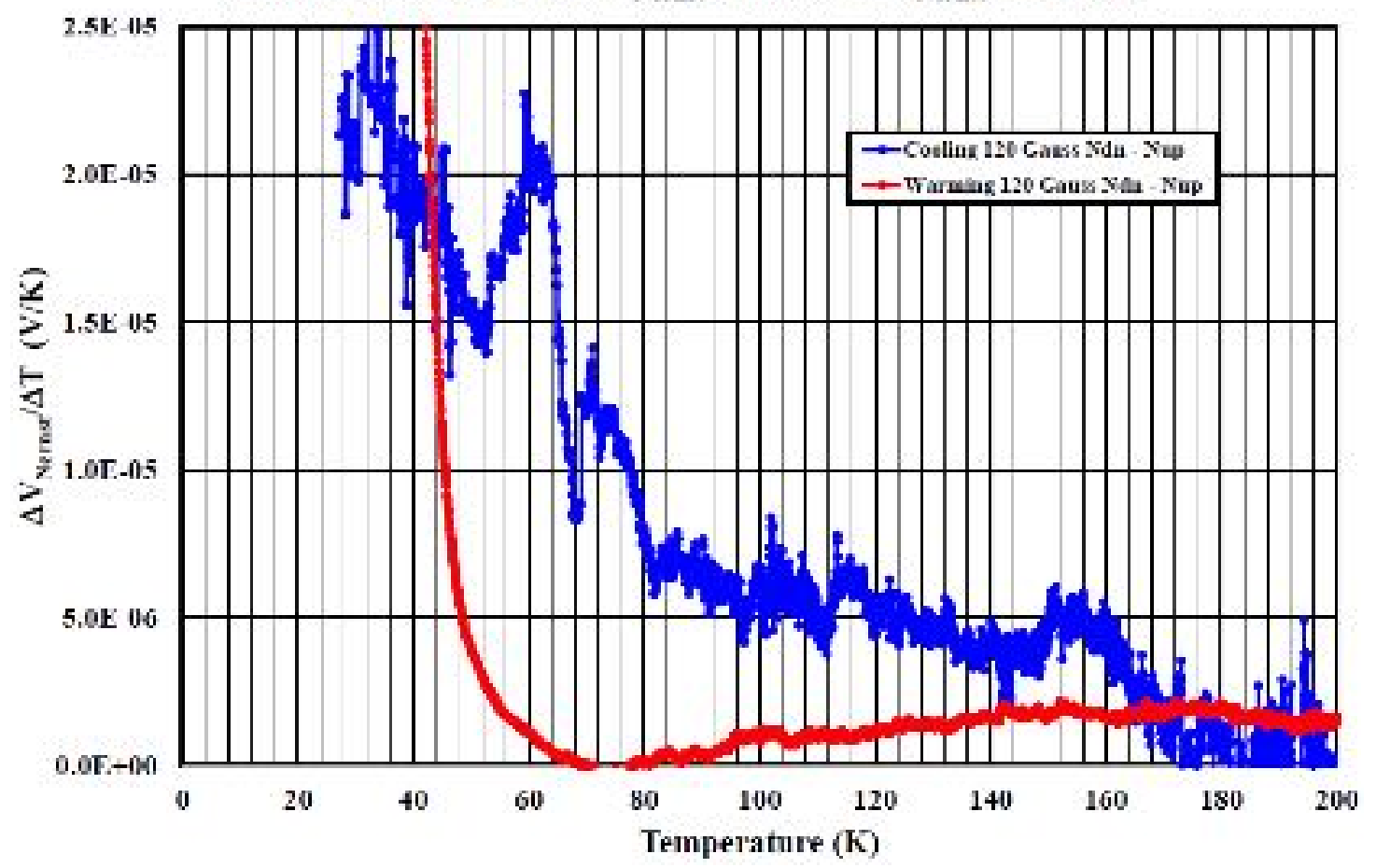


VI. Sample \# 401, layer \# 003
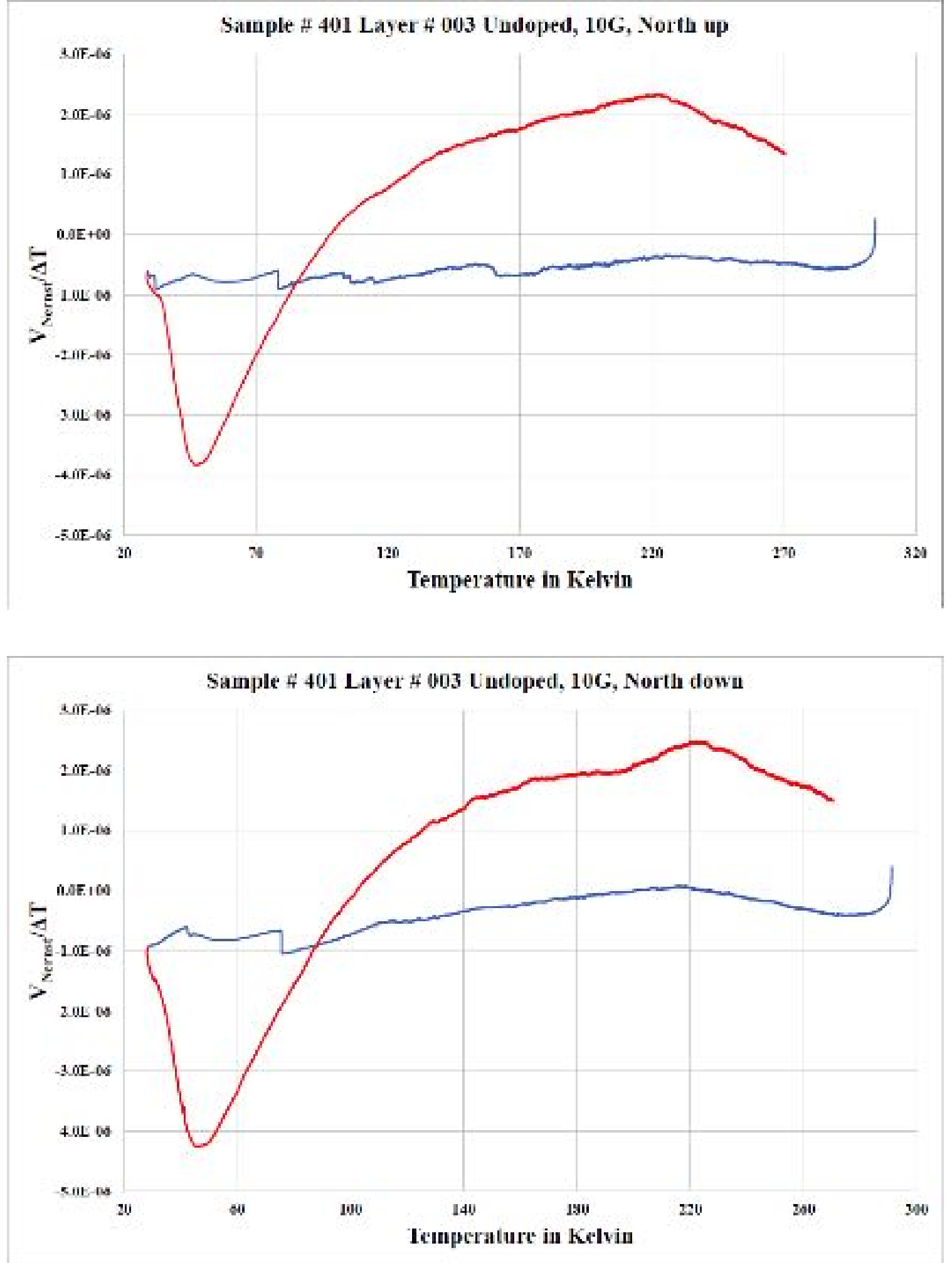

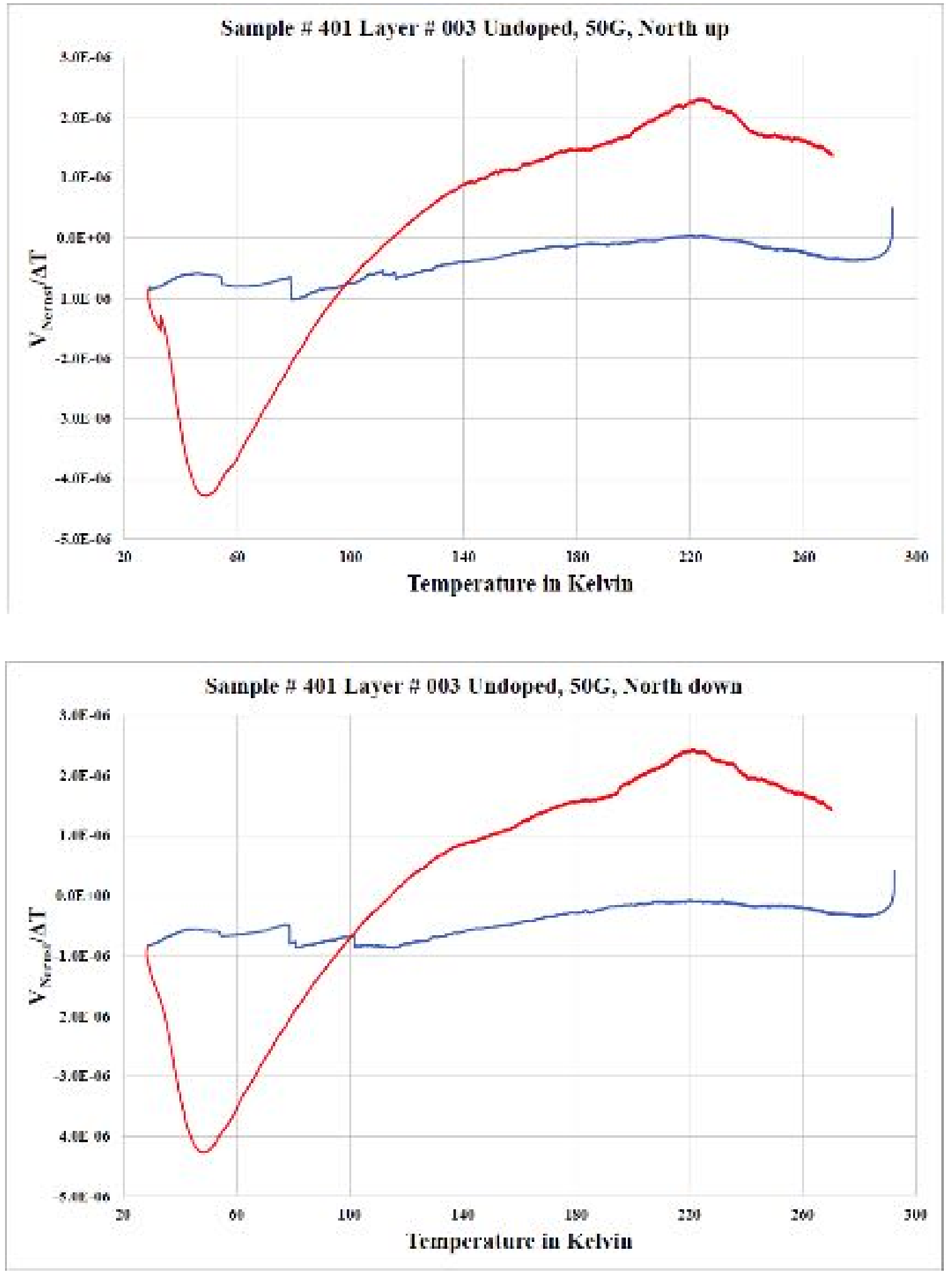

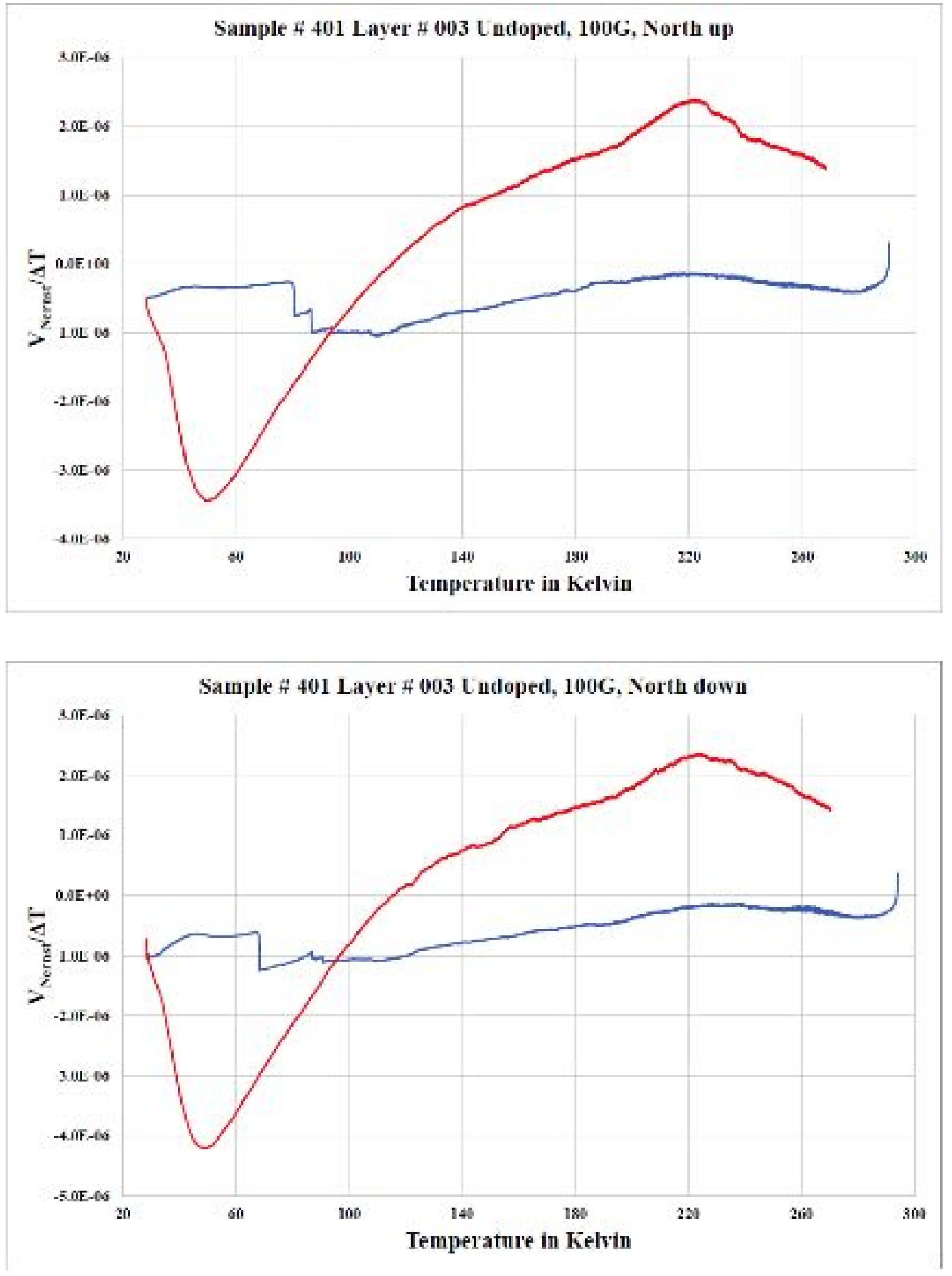

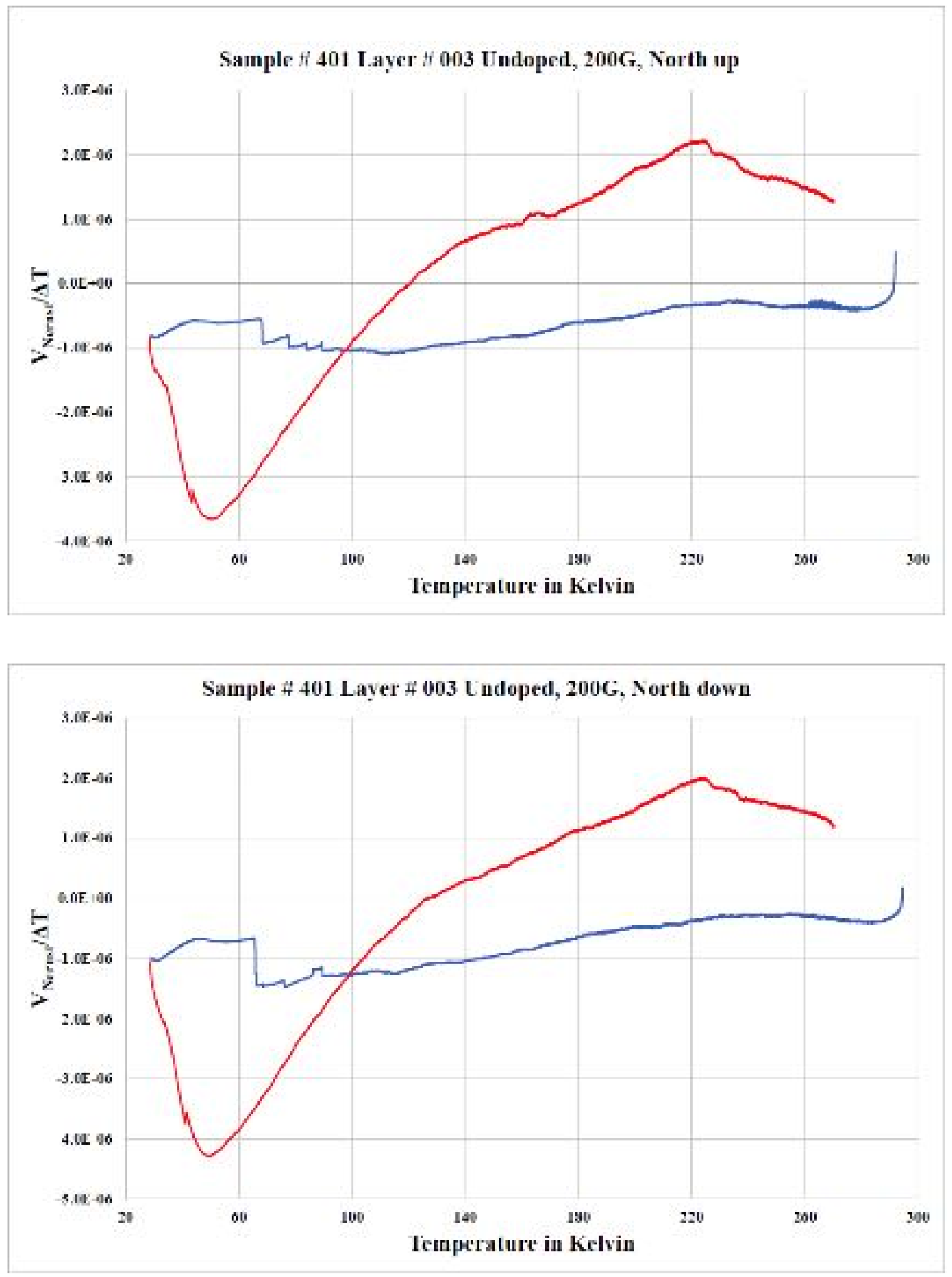

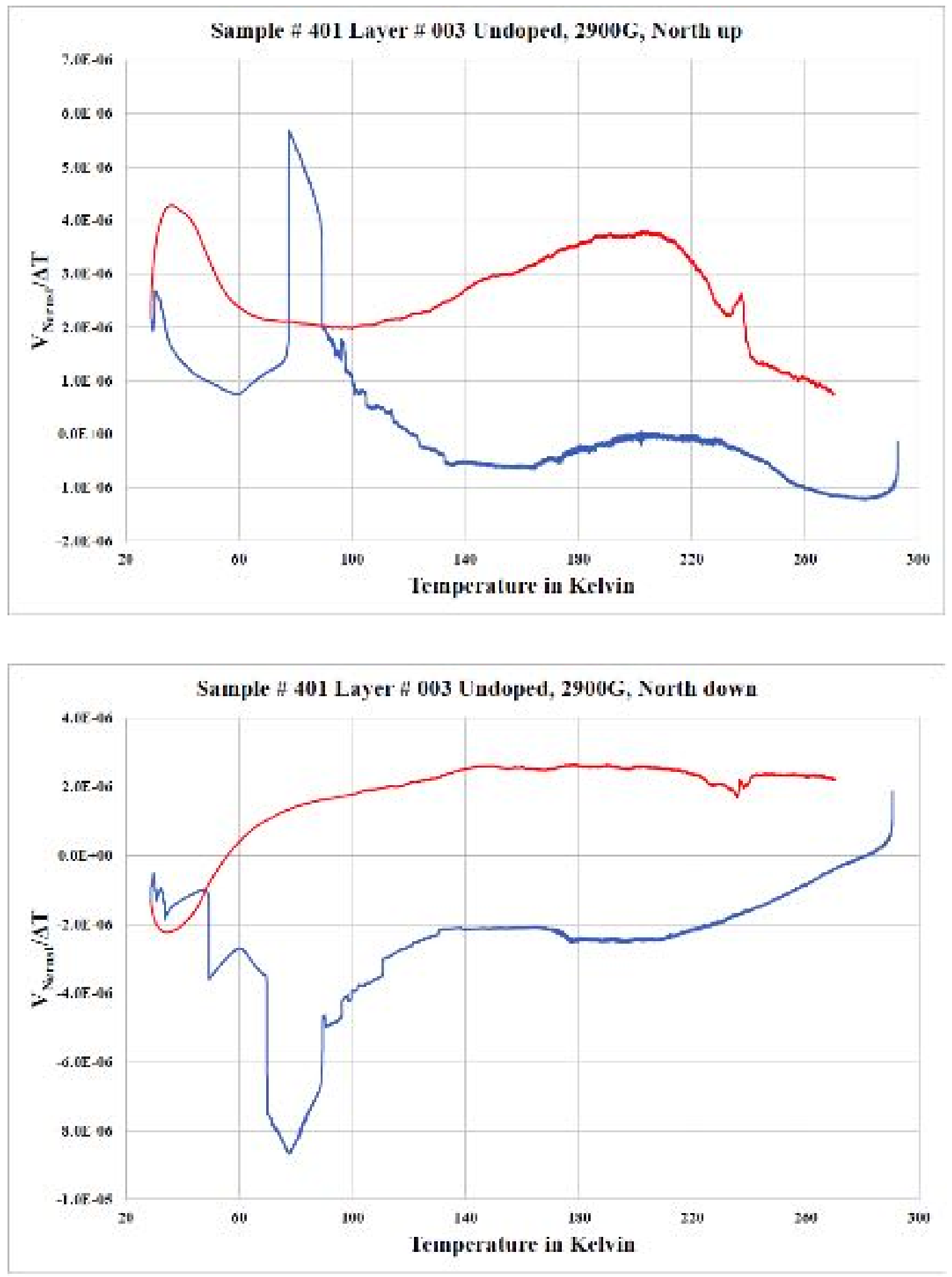
VII. Sample \# 402, layer \# 005
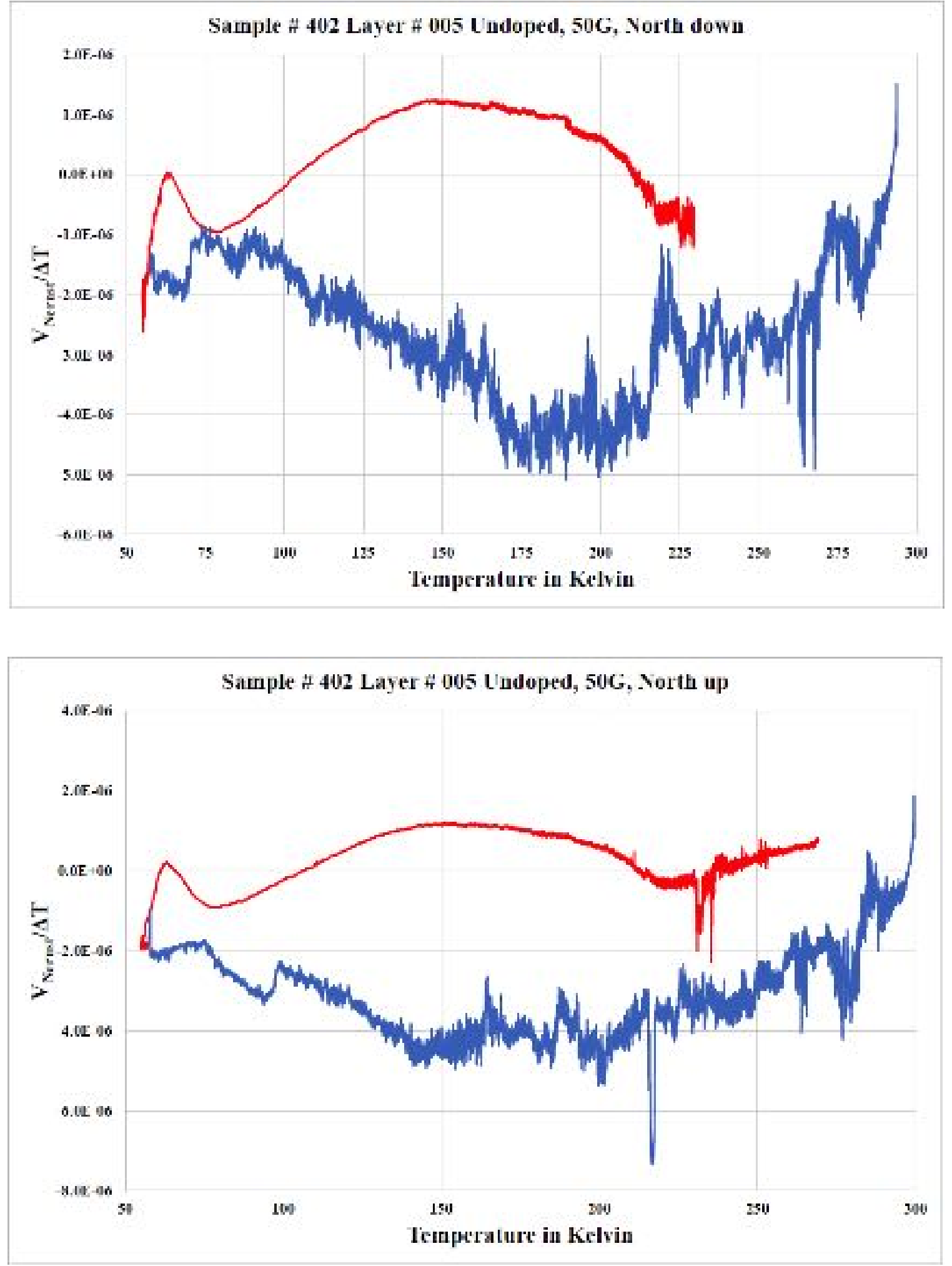

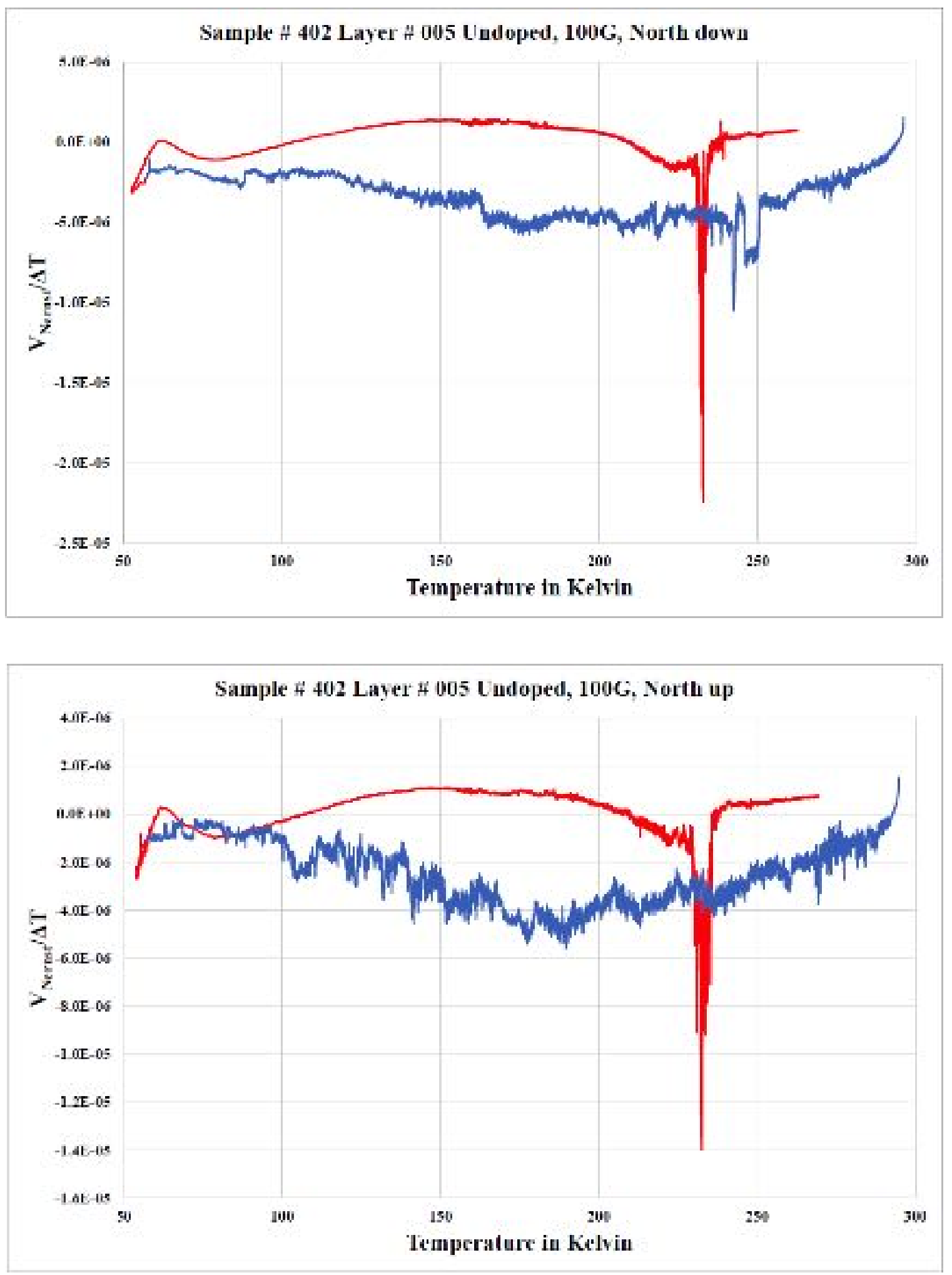

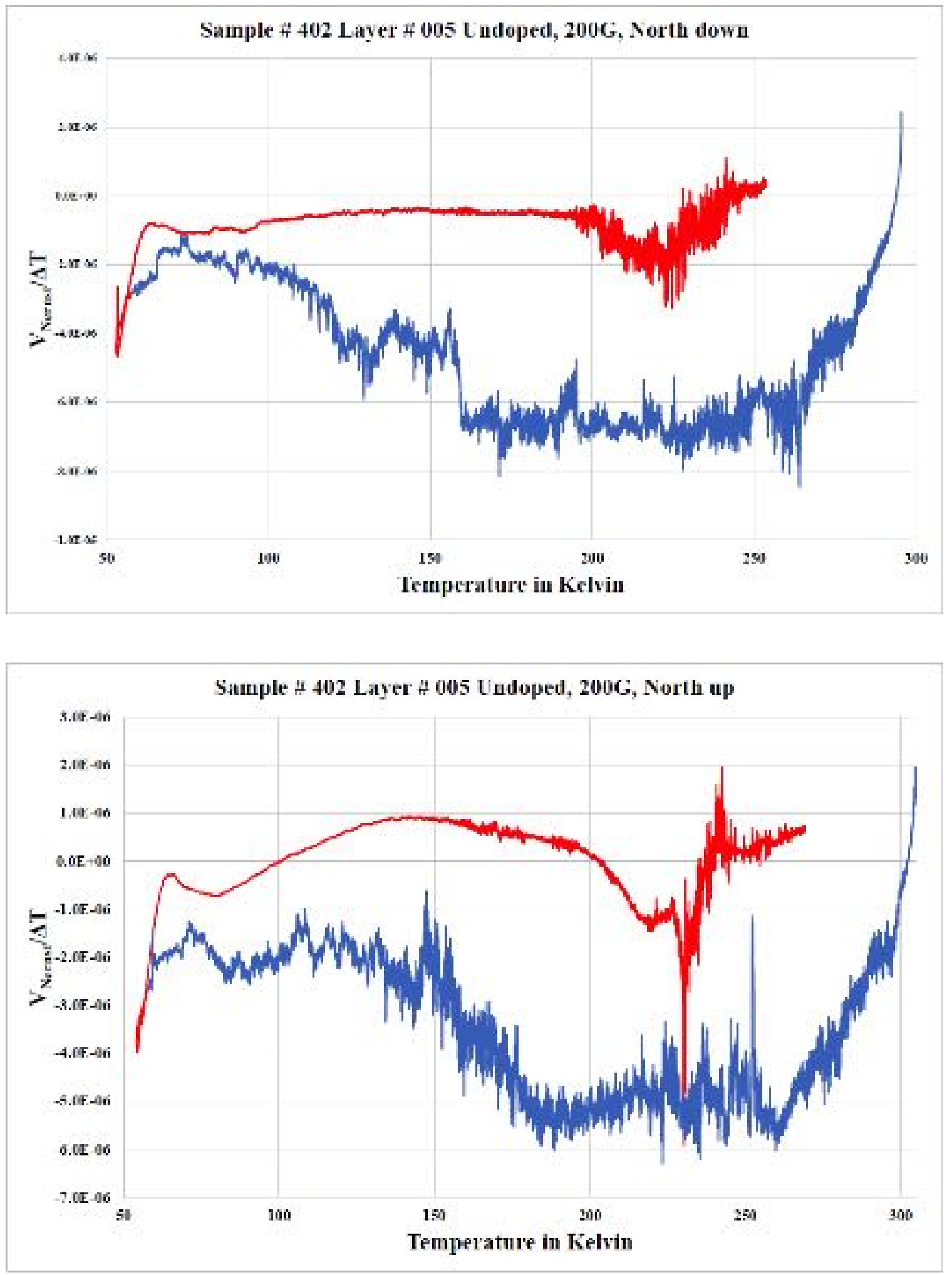

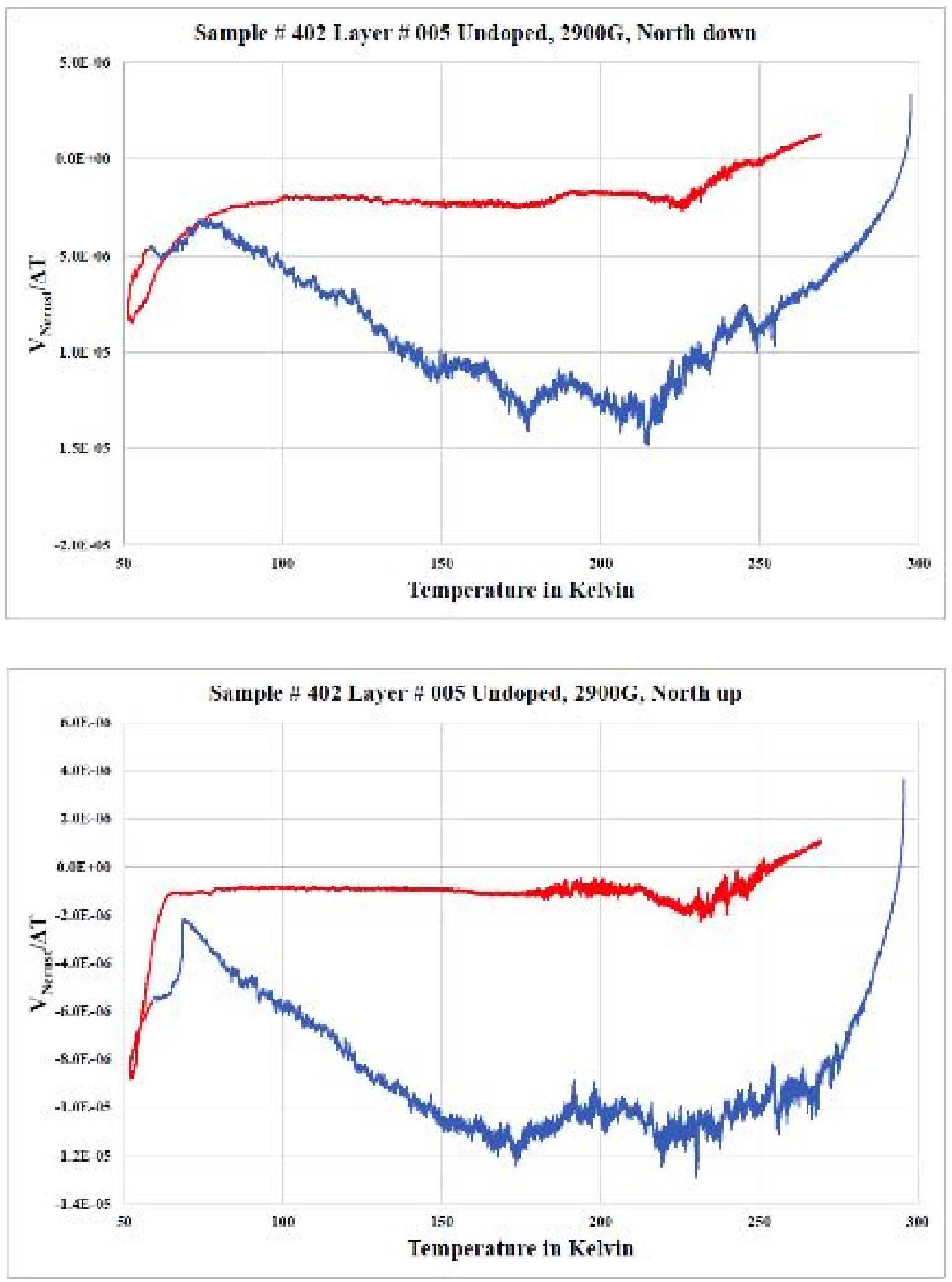


\section{Appendix 5 - Permission to use the copyrighted materials}

รามาง

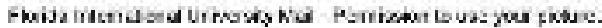

\section{Fiv'Mail}

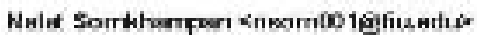

\section{Permission to use your picture.}

3 masages:

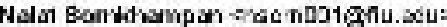

To syanga13 Jyma l.com

Fil, Fas 22, 2319 at 5:10 PW

Desr Sir

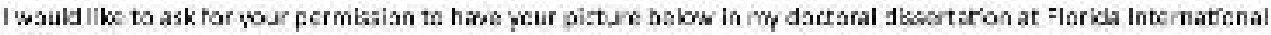
Iriversily.

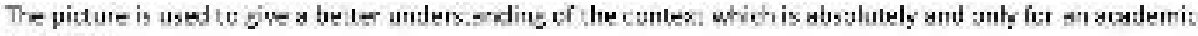
juirowe

Bะs. Reprods,

Mala. Sarvihampan

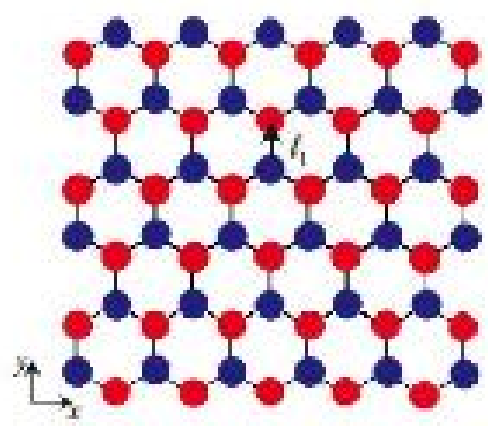

ct: 여-scyans128anal con:

Fin. Fts 22, 2j18 s! 5:18 P:

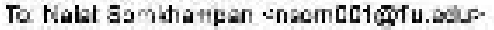

Ves, sprisom

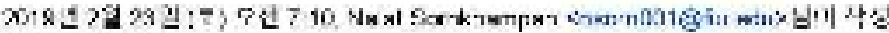

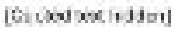

S.-7. Ek Yare

Depaitmat of Prydits, Serea Jrivadity

Secd, 136-713, south Koros

aptas 812 gogmal icom

Te + 22.2-32923100

Fiex:+\$2-2.927-3206

Nalat Bambanpan s'ciénDa1ghu cous

Fii, Fab 22,2319 at $520 \mathrm{PW}$

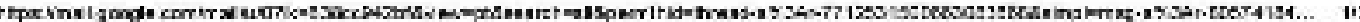


S17015

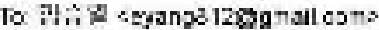

Trark gnd :ury muth

[A.rviouritien]

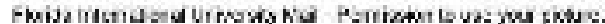

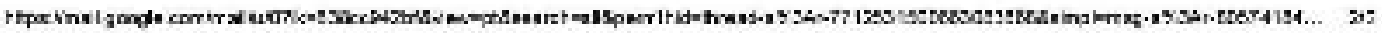




\section{FIU Maiai}

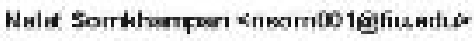

\section{Permission to use your picture.}

4 mesalgés

Nolat Bamdhampan scacendotegnu.zeus

To K.KJiz napfle.mog de

Fil, Fab 22,2010 at 5:44 PH

\section{Dear Sir.}

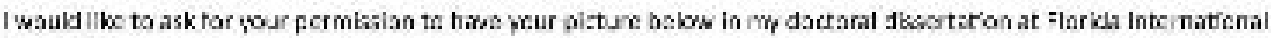

Utigensity

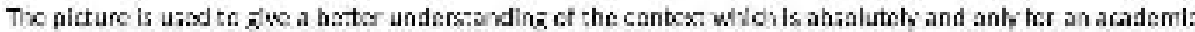
purpose

B:S- Regardi,

Mala: Sornkhampan

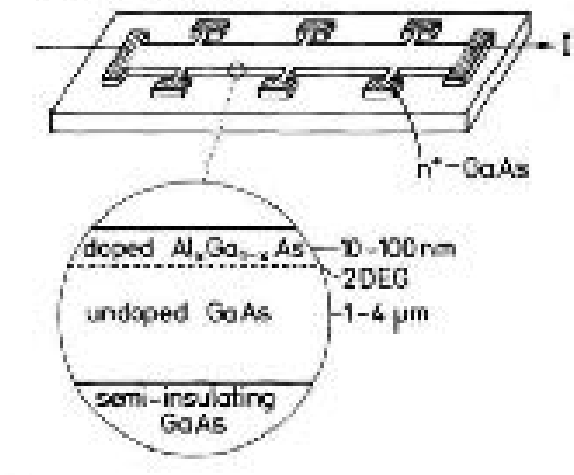

KIave won KIHzng e.K <rzinaykt mpg.Je?

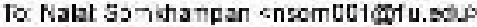

Fn, Fet 22, 2J18 st $731 \mathrm{PW}$

Dear Mr. Sonkrampaר,

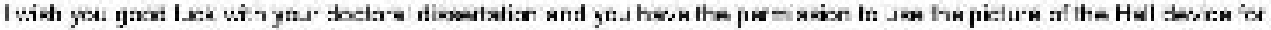

hon-eoriméreal pupéses

Wh has iegards

Klac ช. KIIIIกม

[ELskad wakisien]

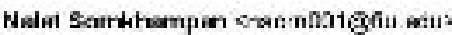

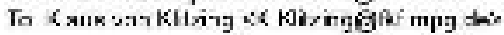

Fi, Fis 22,2019 , $743 \mathrm{PH}$

Thork yod wery much

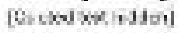

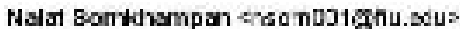

Fri, Fus 22, 2519 at 5:13 PW

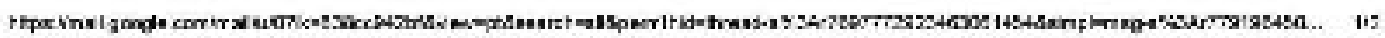


พพชา

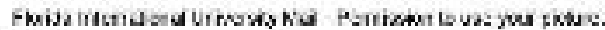

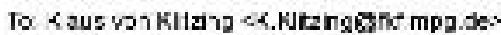

Dasar \$i

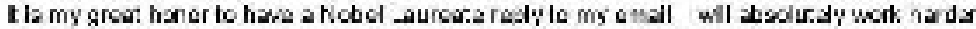

Best Ropass

Nalat Sembiamsar

[C: Leied sackisim]

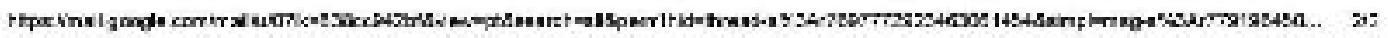




\section{FIU Mâil}

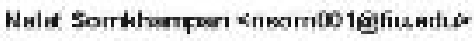

\section{Permission to use your picture.}

3 masage:

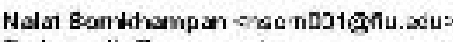

Fil, Fats 22,2310 at 5:35 PY

To ba andingeos uer cou

\section{Dear Sit,}

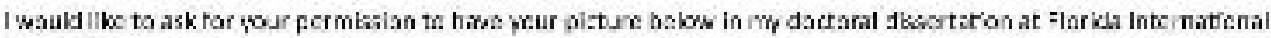

Itigensit.

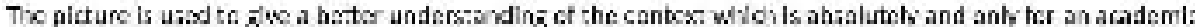
purpose

Ba:- Regardi,

Mala: Sornkhampan

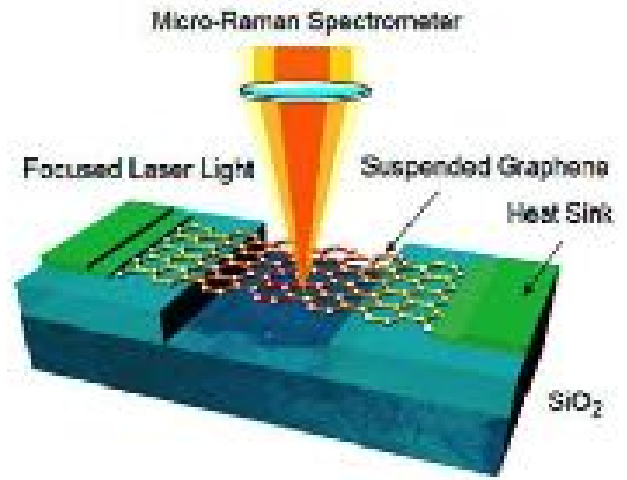

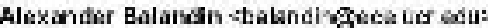

Fil, Fib 22, 2019 an 508 PM

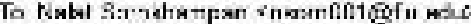

Dear Nals

Sure, pesse fes fee :ause wint the prope ki:sion:

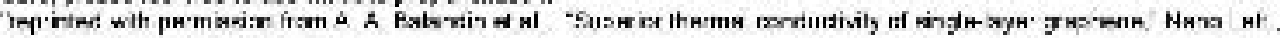

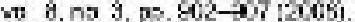

Tark gn.

$4 \sec$

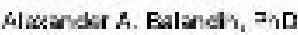

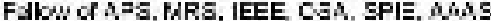

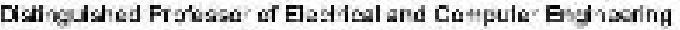

Untwerst; of Calfomia Preadarta Char Jrofeas

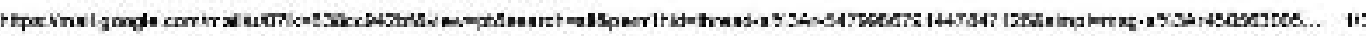




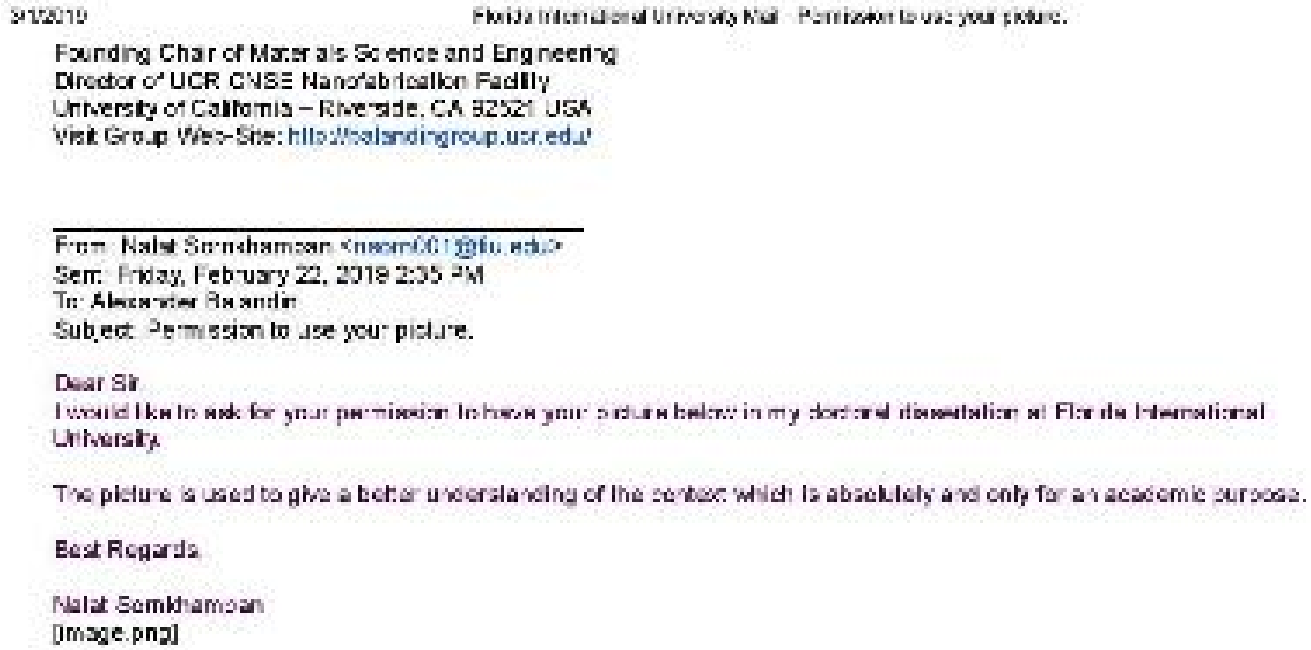




\section{FIU M̄ail}

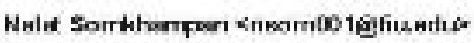

\section{Permission to use your picture.}

2 masagé:

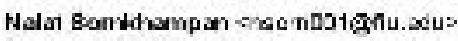

To calamDogean ed

Fil, Fabs 22,2019 at $4 ; 46 \mathrm{PH}$

\section{Desr Dr. All-km n,}

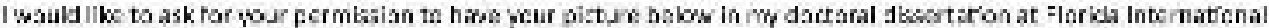

Whigersily

The picture is usad te sue a batter understonding of the cantexe whoc is absalutely and anty fer an acodemi: aunsores

Res, Regar: 1s,

Nala: 5ankhampan

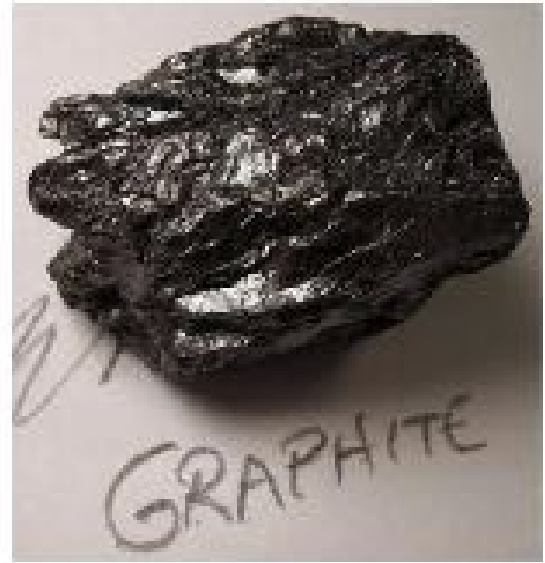

Chowdhury Al-Amin sibmin_spe27osyshoo.com:

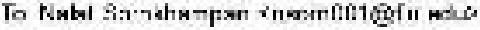

I dont mrd fusso wr.ר sppros rate calston.

Torke

Serr. Tou Yshos kasi fo- thone

[Ësikd $x x$ indfor]

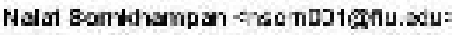

Fil, Fab 22, 2J19 at 9:46 PM

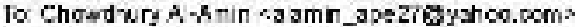

Trark ynd :nry must

[C.sud sockitim] 


\section{FIU Miail}

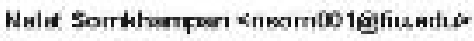

\section{Permission to use your picture.}

3 masages

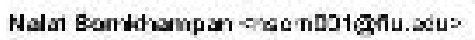

To verciragnus.eju.sg

Fil, Fabs 22,2019 at 5:19 PM

\section{Dear Sir.}

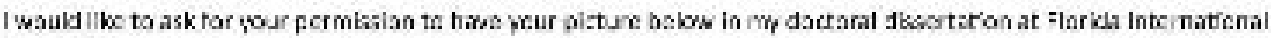

Utigensity

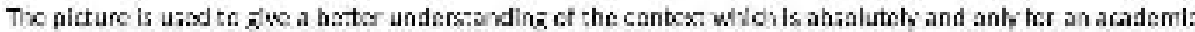
purpose

B:- Regardi,

Malar: Sornkhampan

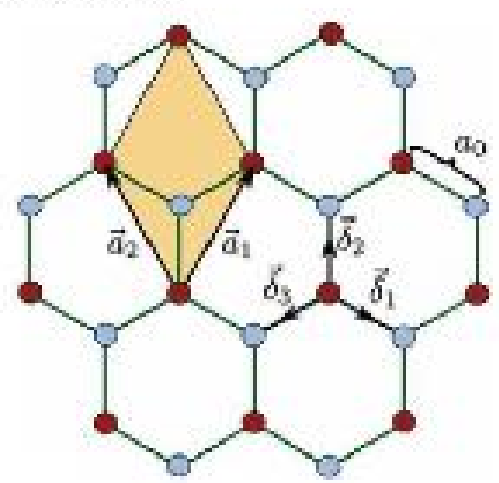

Pereira VHor Marudel asere ragnus edu.egs

$\mathrm{F} 7 \mathrm{~T}=\mathrm{sb} 22,2016 \times 1100 \mathrm{PH}$

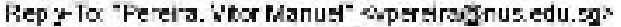

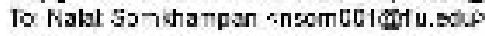

Doar Nalia

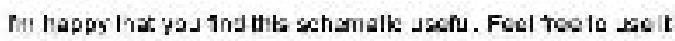

Focoards

vtor,

vtor I2. Pereira

A.sartant ב-ofeses

Departmex ot Prugies

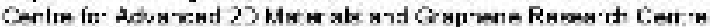

Nationsl universty of Singaps?

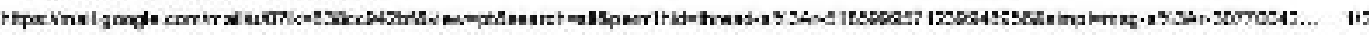


มพจา

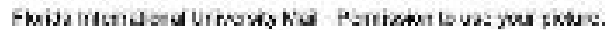

F: $35013642 \mid \mathrm{F}: 67770125$

[t.: sxd $x x / n d f=\mid$

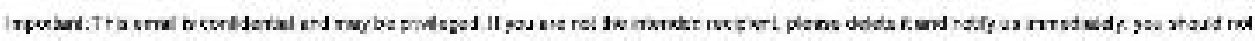

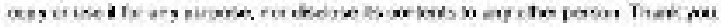

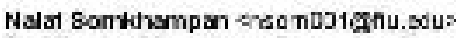

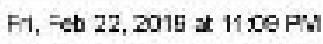

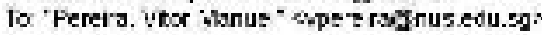

Trank ynd *ury musth

[C.eved wor hisim]

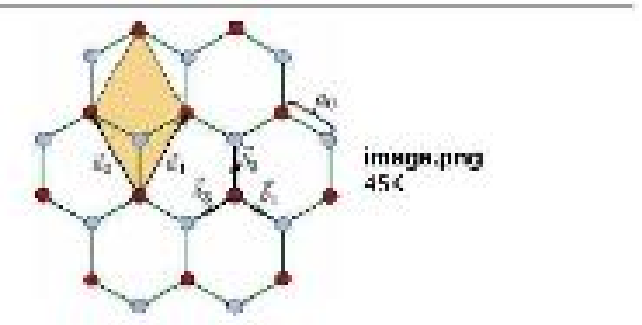

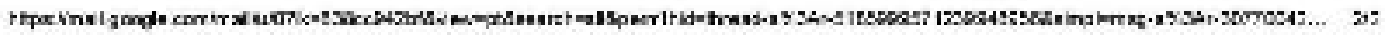




\section{FIU Maiai}

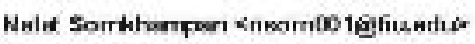

\section{Permission to use your picture.}

5 masagés

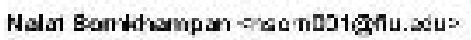

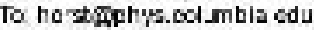

Fil, Fab 22, 2019 at 9:12 P:S

\section{Dear Sit,}

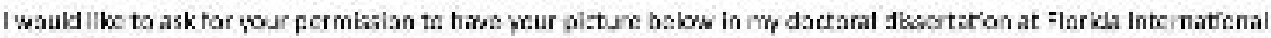

Itiversity.

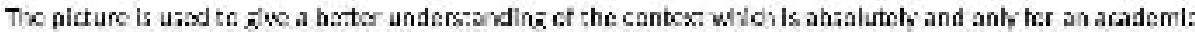
purpose

B:- Regardi,

Malar: Sornkhampan

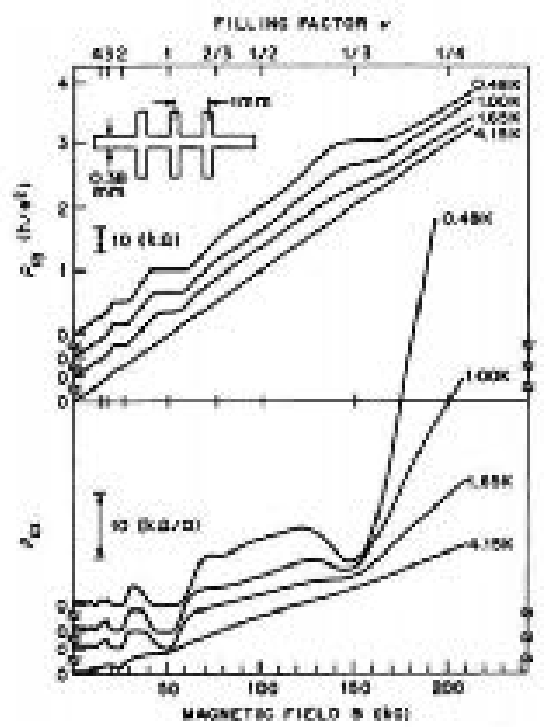

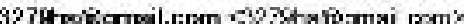

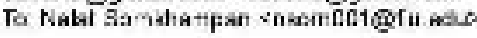

permision $\hat{x}$ 'antê

thenk you for Lsirg this graph.

beat wishes

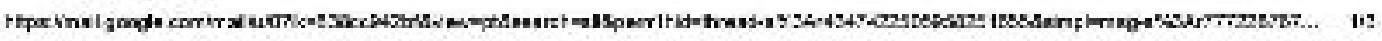




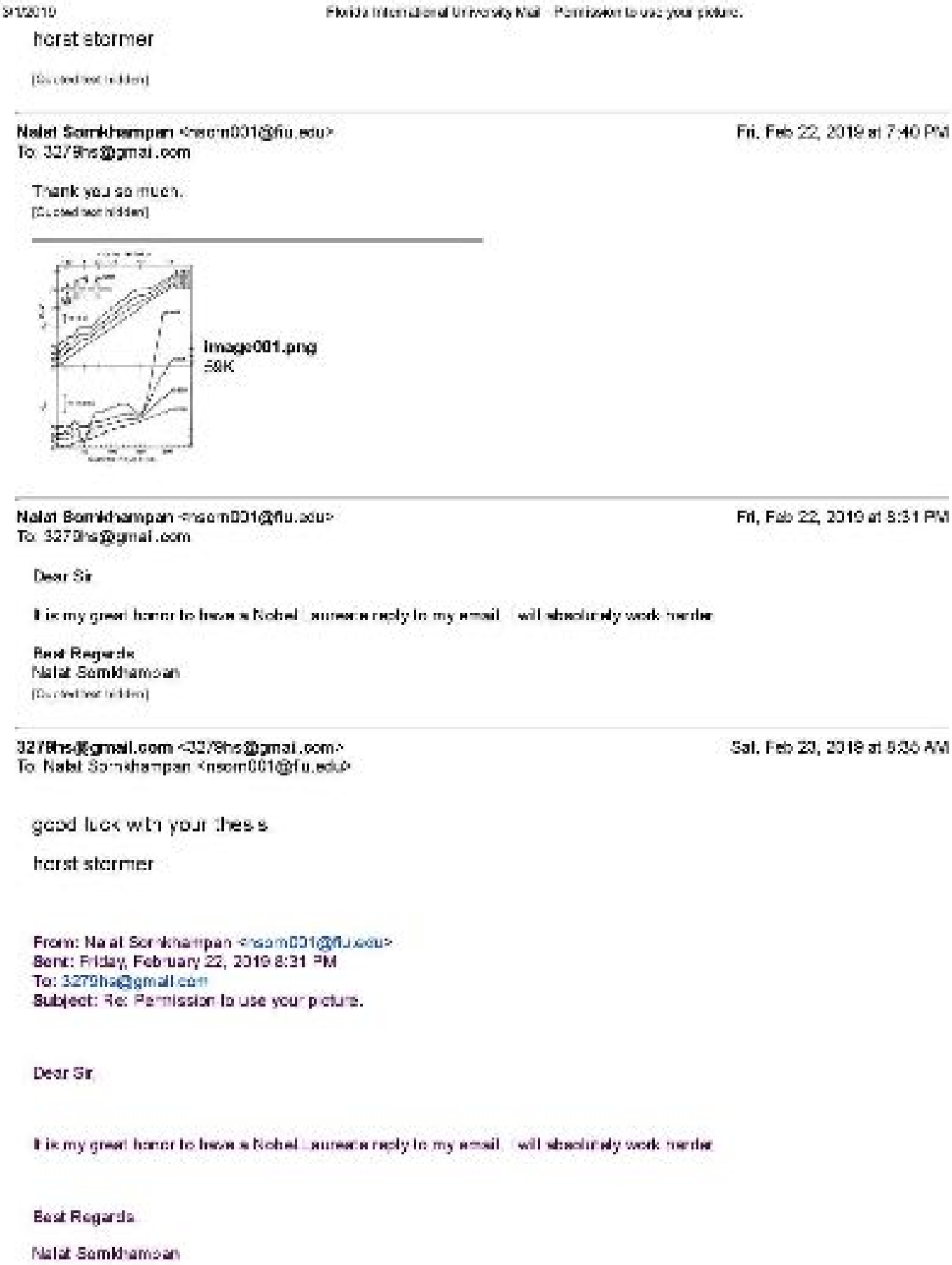

Holat Bondhampan scio nDJ10

To 327 Drapymai.com

Fil, Fab 22,2319 of $8: 31 \mathrm{PW}$

Dest \$ir

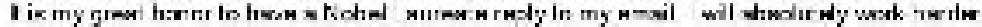

Pend Regusto

Malat Semblamsar

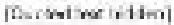

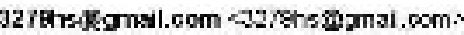

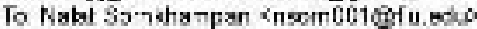

gcod lucswt? yout less

Herst stermer

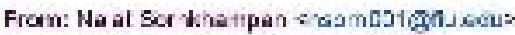

Berx: Fidsy, Fobuary z2, 2319 a:31 =k

To: $3272 h$ hagmill cut

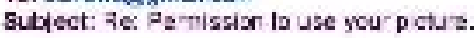

Desr $5 r$

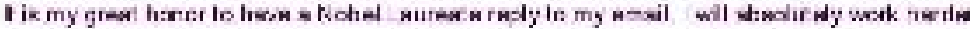

Eest Regarda

Halat Samblamsan

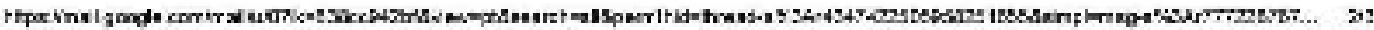




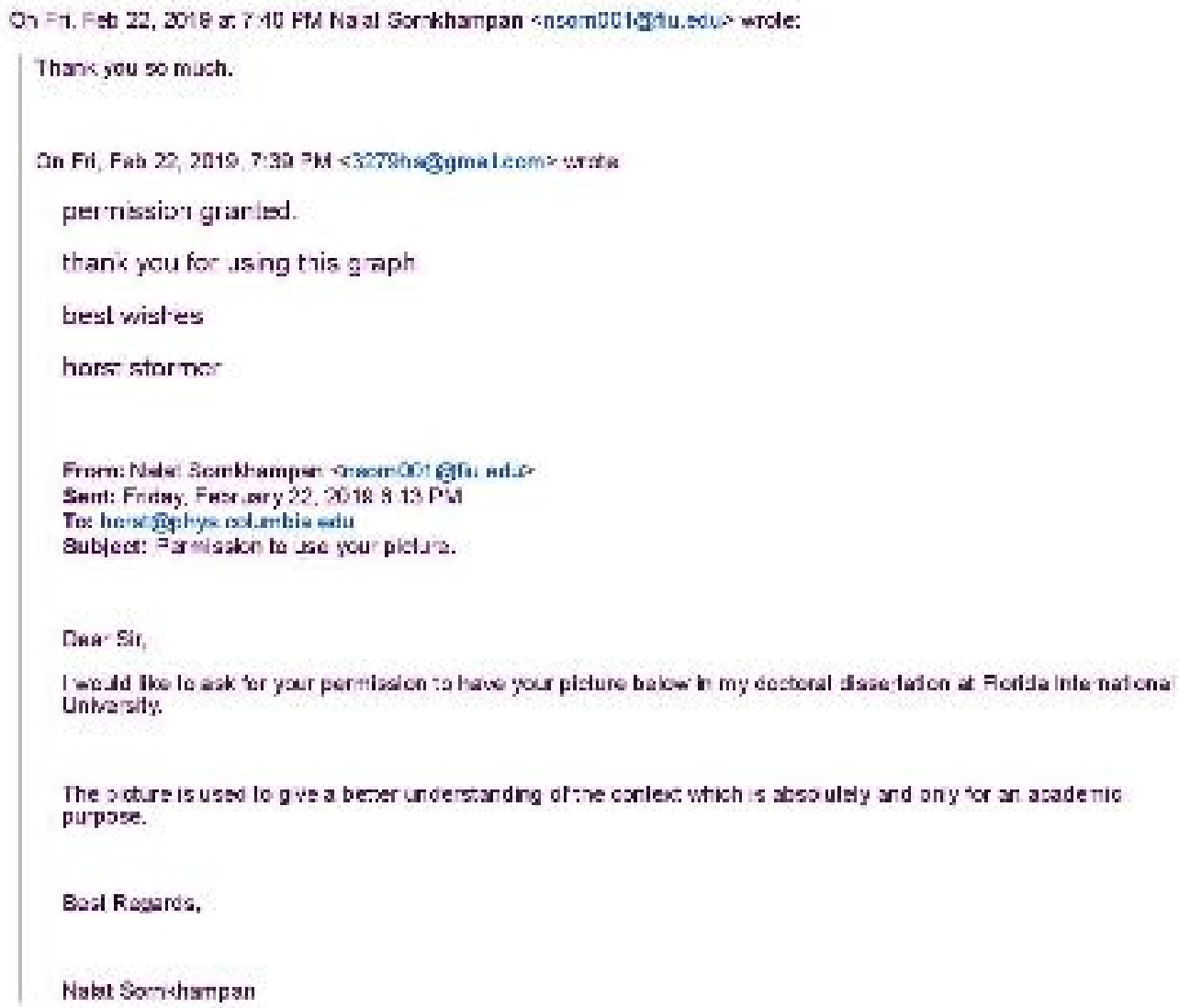




\section{FIU Maiai}

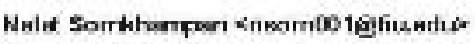

\section{Permission to use your picture.}

3 masagé:

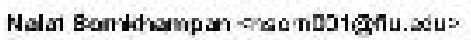

To kra.yalamanchester.sc.di

Fil, Fab 22, 2310 at $900 \mathrm{PH}$

\section{Dear Sit,}

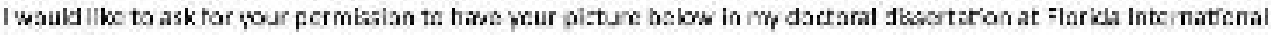

Itigensit.

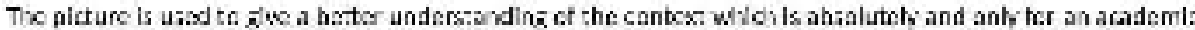
purporse

Bas: Rogardi,

Mala: Sornkhampan

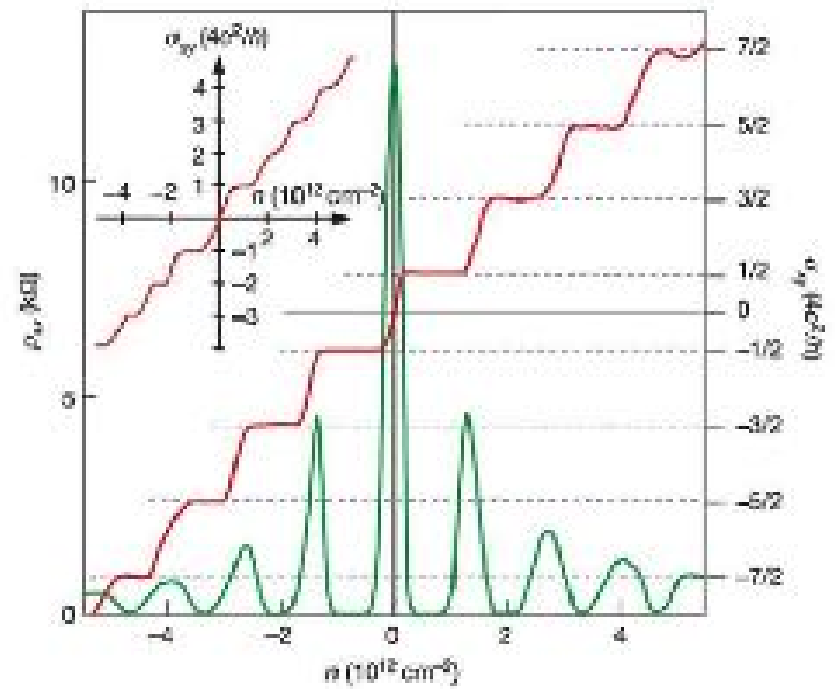

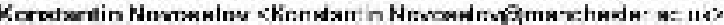

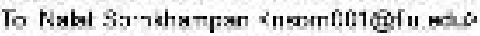

I glac yeu the permissian to use the pieture

Kosiva 


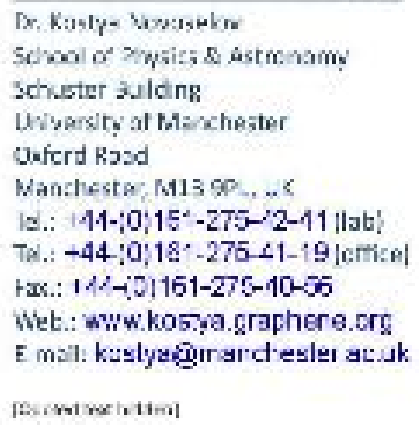




\section{FIU M̄ail}

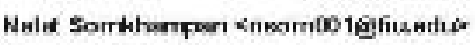

\section{Permission to use your picture.}

3 masages

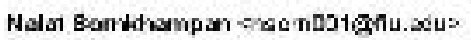

To taipptreckor.cdu

Fil, Fab 22, 2010 at $3: 20 \mathrm{PH}$

\section{Dear Sit.}

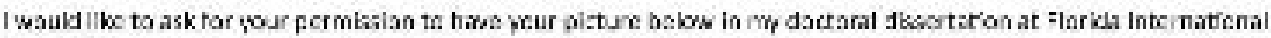

Itiversit.

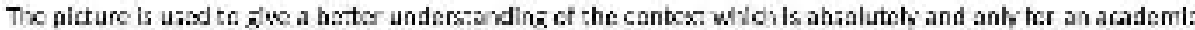
purporse

Bะ- Repards,

Mala: Somkhampan

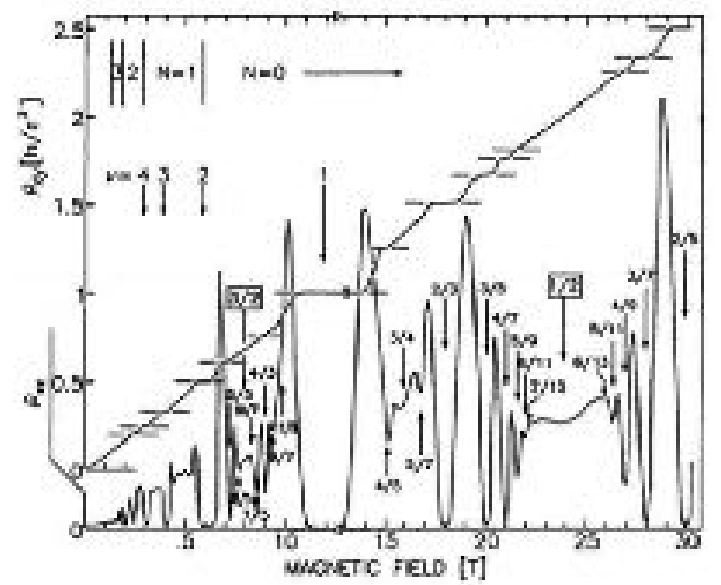

Denel C. Tsu sle las nocbon.eds:-

Eal, Fab 22, 2010 at $450 \mathrm{PH}$

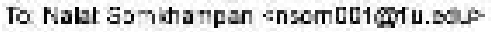

Wou hrug my po-тission. DC Tai

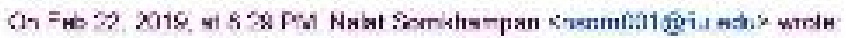

Dear sir,

I would the to ask for pour permission to have your pisture telow in mf dec.orel deser:asian s: Herids Internariansi Lniucrsity. 


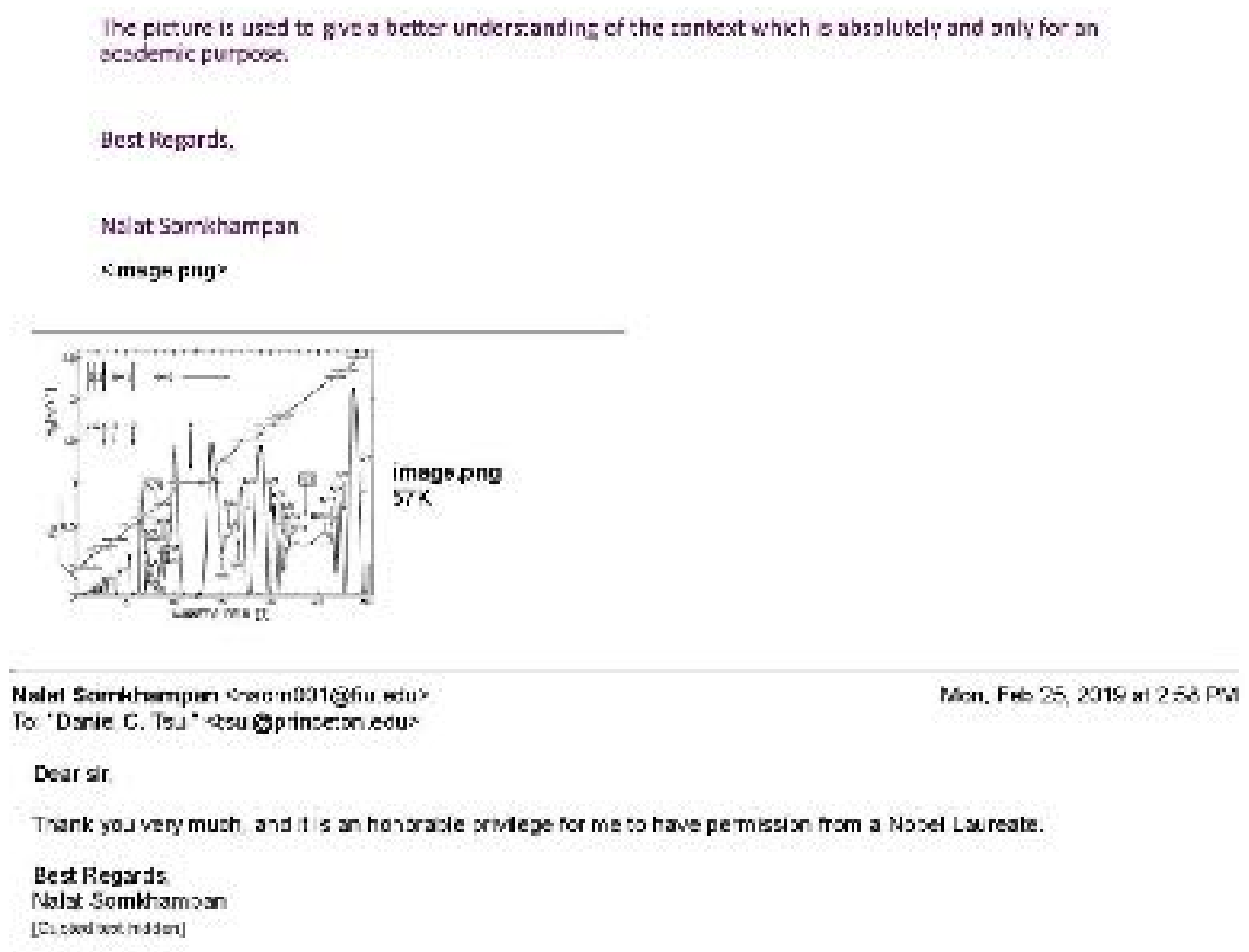




\section{FIU Maiai}

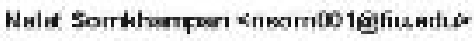

\section{Permission to use your picture.}

3 mesalgés

Nolat Bambampan scaé nDJignu.zeu:

To go cmaา

Sal, Fab 22, 2319 of $7: 43 \mathrm{~PB}$

\section{Dear Sit,}

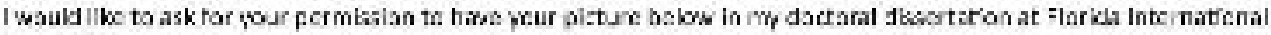

Itigensit.

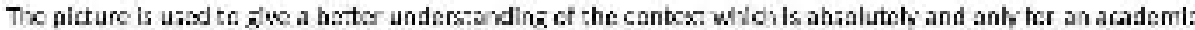
purporse

B:A- Regardi,

Malar: Sornkhampan

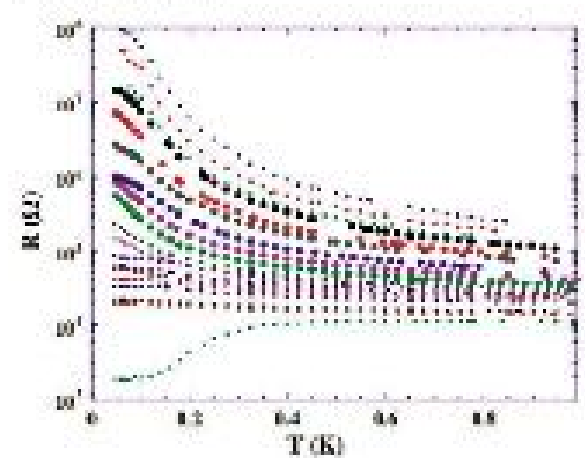

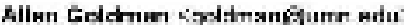

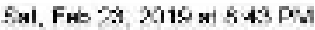

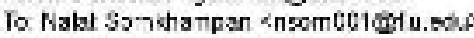

You hrue ry pamiszixn.

Alen Gokman

Serr. tort my IPad

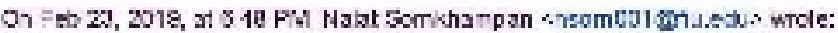

Dear Sit.

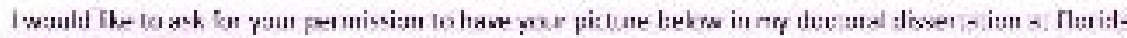
Internacianal uniwersity

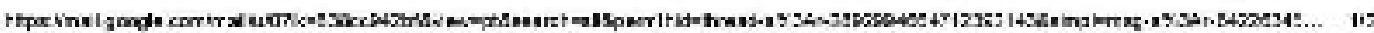


The picture is used te gre a better unders.andinz of the zantext which s absolutely and only for an scidervic punpoes.

Best Rewords,

Naist Sornkhampan

<mapepngs

Nelu Bandhampan raé nDJianu.xu:

To AlanGodmaา $<$ gowmang.mn.ets

Mon, F⿻s 25, 2319 at 2:S3 PM

Trank you wary mush

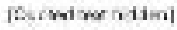




\section{FIU Maiai}

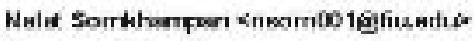

\section{Permission to use your picture.}

3 mesalgés

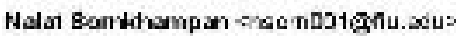

To FodNar.pasu.2d.

Eal, Fab 22, 2019 at 955 PW

\section{Dear Sit,}

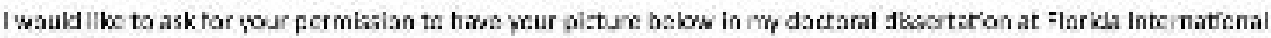

Itigensit.

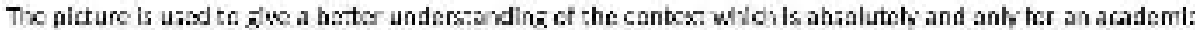
purporse

Ba:- Regardi,

Mala: Sornkhampan

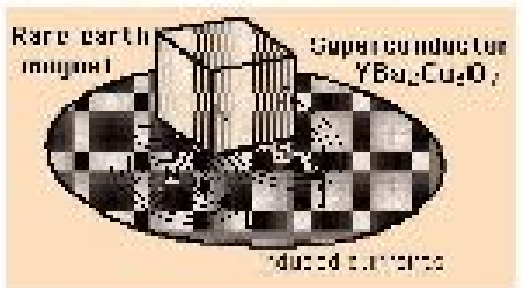

Rod Nawn entherionesall cal:

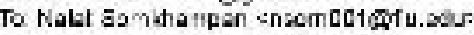

5al, Fab 79, 2019 a1 $5 \leq 8 \mathrm{PH}$

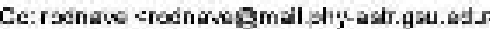

Helo, Nas!

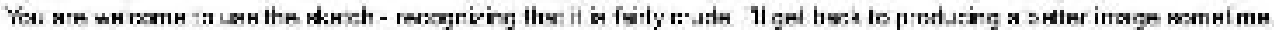

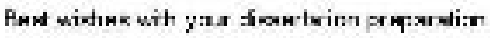

Fing;

Foce Have FodNareRas.ed.

Hysertiyci:a Prejest

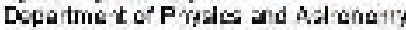

Quarsia Stale Un werst:

Alsinia, 3a 30302-5090

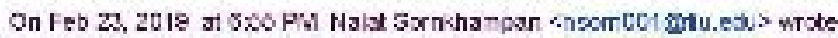

Devr Sir. 


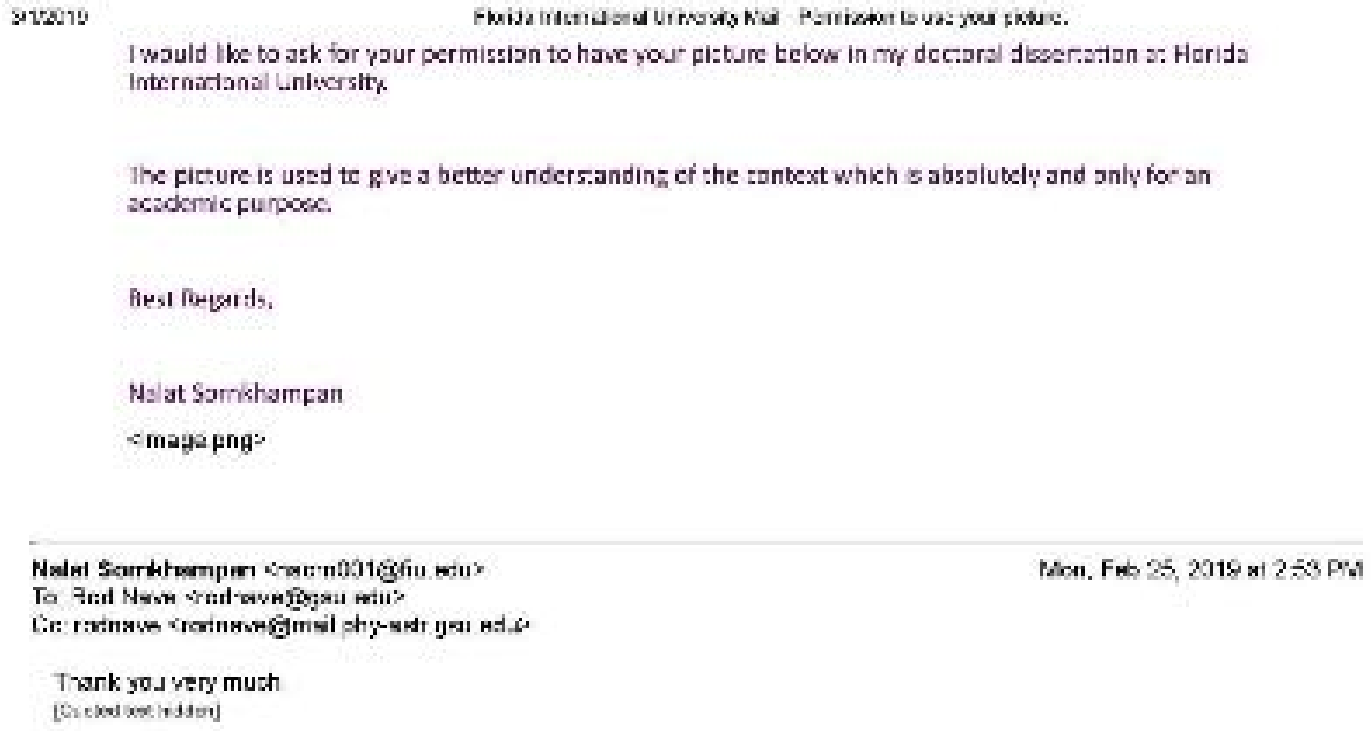




\section{FIU Maiai}

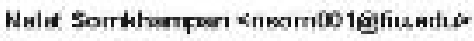

\section{Permission to use your picture.}

3 masagé:

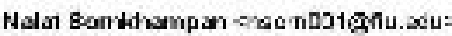

To haslandpith. 20

Sal, Fab 22, 2319 of $7: 38 \mathrm{PH}$

\section{Dear Sit,}

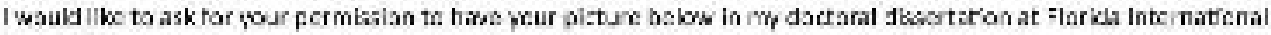
Itivensit:

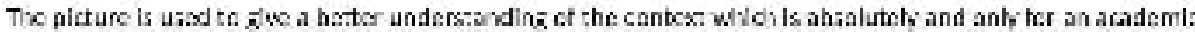
purpose

B:- Regardi,

Mala: Sornkhampan

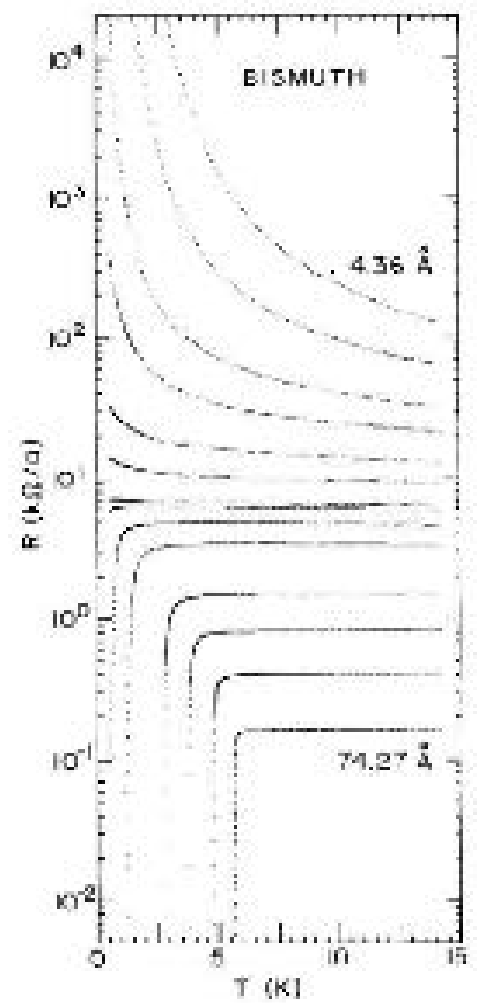

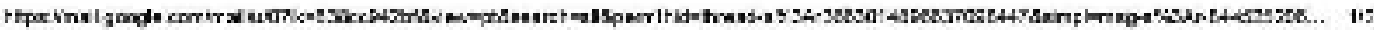




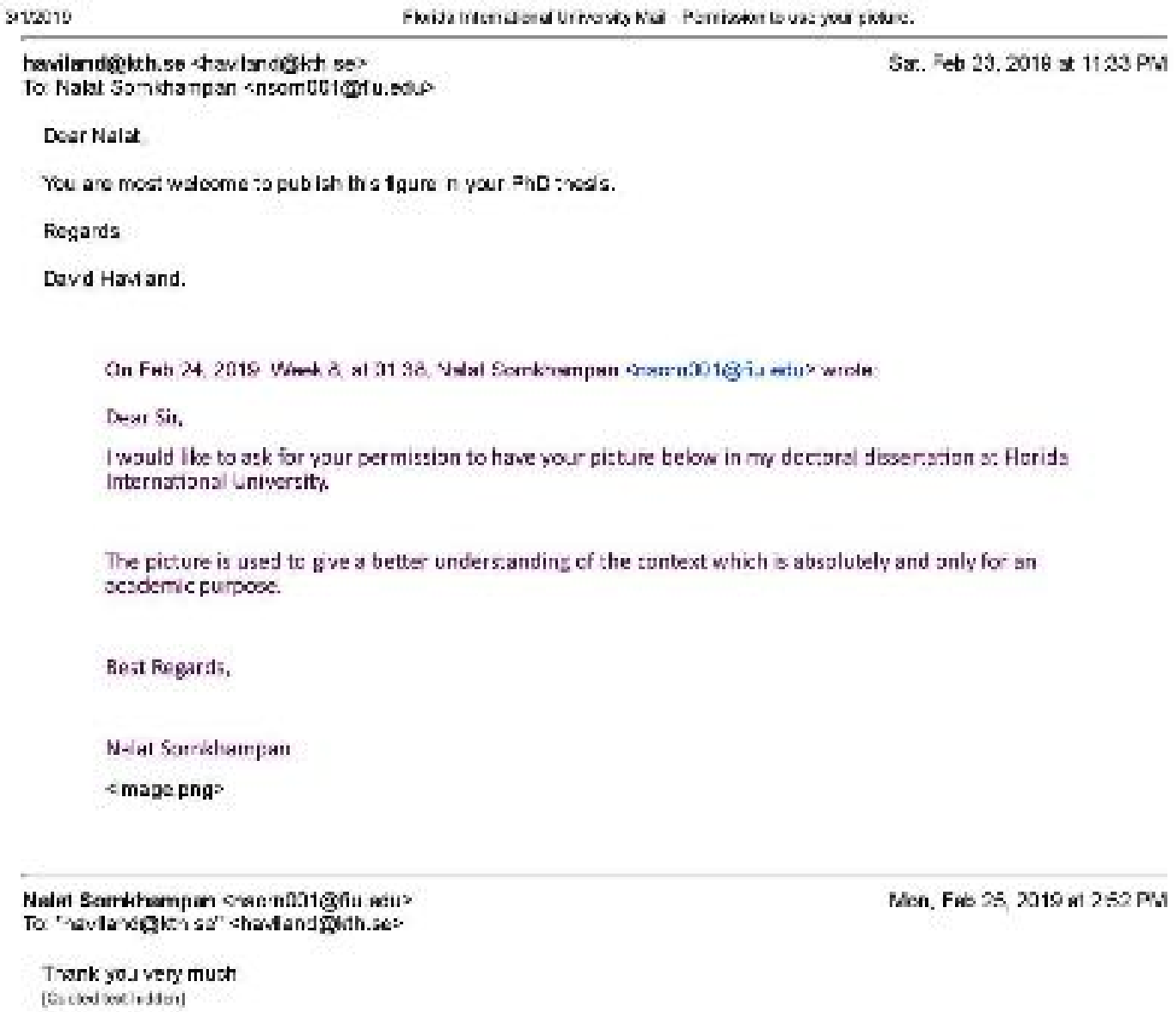




\section{FIU M̄ail}

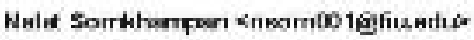

\section{Permission to use your picture.}

2 messages:

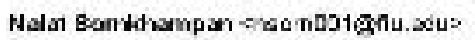

To kawakani.15groes.cdu, jarcedav.fabiangur.de

Fil, Fab 22, 2019 at 9:33 PH

\section{Dear Sir.}

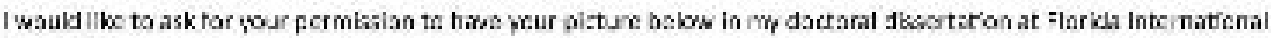

Utigensity

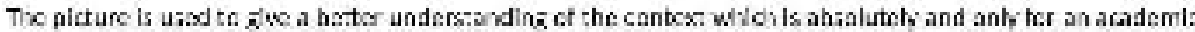
purpose

B:S- Regardi,

Mala: Sornkhampan

\begin{tabular}{|c|c|c|c|c|}
\hline \multicolumn{2}{|l|}{ Syin Chunesi } & \multirow{2}{*}{$\begin{array}{l}\text { Spin tiframe } \\
\begin{array}{l}-42 \text { ps at } 4.2 \mathrm{~K} \\
-11 \text { jo a1 } 360 \mathrm{~K}\end{array}\end{array}$} & \multirow{2}{*}{ 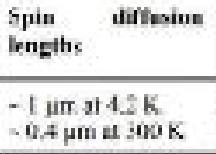 } & \multirow{2}{*}{$\begin{array}{l}\text { Spin sienals } \\
\begin{array}{l}-1 \mathrm{mR} \text { at } 42 \mathrm{~K} \\
-0 \mathrm{~S} \tan \text { a } 300 \mathrm{~K}\end{array}\end{array}$} \\
\hline Metzls & $\mathrm{Cn}$ & & & \\
\hline & Al & $\begin{array}{l}-100 \mathrm{ps} 2 \mathrm{t} 1.2 \mathrm{~K} \\
-45 \mathrm{ps} \text { at } 360 \mathrm{~K}\end{array}$ & $\begin{array}{l}-0.6 \mu \mathrm{m} \text { at } 4.2 \mathrm{~K} \\
-0.4 \mathrm{\mu m} \text { at } 300 \mathrm{~K}\end{array}$ & $\begin{array}{l}-12 \mathrm{mCa} \text { at } 42 \mathrm{~K} \\
-05 \mathrm{mQ} \text { a } 305 \mathrm{~K}\end{array}$ \\
\hline & $\mathrm{As}$ & $\begin{array}{l}-30 \text { ps at } 5 \mathrm{~K} \\
=10 \text { gs at } 300 \mathrm{~K}\end{array}$ & $\begin{array}{l}-1 \mu \mathrm{m} \text { at } 5 \mathrm{~K} \\
-0.3 \mu \mathrm{m} \text { it } 300 \mathrm{~K}\end{array}$ & $\begin{array}{l}-4 \mathrm{mll} \text { at } 5 \mathrm{~K} \\
\sim 2 \mathrm{~mL} \text { at } 36 \mathrm{~W} \mathrm{~K}\end{array}$ \\
\hline \multirow[t]{3}{*}{ Sembeond:ceser } & $\begin{array}{l}\text { Highly } \\
\text { dcped } \\
\text { Si }\end{array}$ & $\begin{array}{l}-10 \mathrm{rs} \text { al } 3 \mathrm{~K} \\
-13 \text { as at } 300 \mathrm{~K}\end{array}$ & $\begin{array}{l}-2 \mu m a 8 x \\
-0.5 \text { क } 8.370 \mathrm{~K}\end{array}$ & $\begin{array}{l}=30 \mathrm{~ms} 2 \mathrm{a} 8 \mathrm{~K} \\
=1 \mathrm{mR} \text { al } 360 \mathrm{~K}\end{array}$ \\
\hline & Casts & $\begin{array}{l}24 \text { wat } 15 \mathbb{R} \\
4 \mathrm{~ns} \times \mathrm{XK}\end{array}$ & 6 pm $x .50 \mathrm{~K}$ & $-30 m \mathrm{~m}=50 \mathrm{~K}$ \\
\hline & $\begin{array}{l}\text { Highly } \\
\text { dopod } \\
\text { Ge }\end{array}$ & $\begin{array}{l}-1 \mathrm{n} \times 21<\mathrm{K} \\
-300 \mathrm{~K} \times 100 \mathrm{~K}\end{array}$ & $-0.6 \mu \mathrm{m} n \mathrm{t} 4 \mathrm{~K}$ & $\begin{array}{l}0.1-10 x+4 x \\
0.00-110 x .200 x\end{array}$ \\
\hline \multicolumn{2}{|l|}{ Orajencas: } & 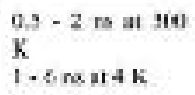 & $\begin{array}{l}3-10 \mu m a s, 400 \mathrm{~K} \\
1-100 \mu \mathrm{m} \text { fit frem } \\
\text { loal NR dvsy }\end{array}$ & 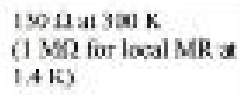 \\
\hline
\end{tabular}

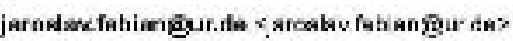

Sain, Fits 242019 al BCB AN

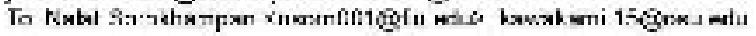

Dasar Natat

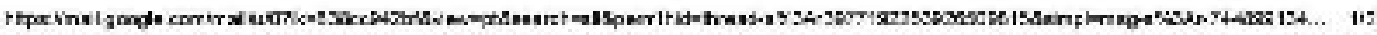


รามอาด

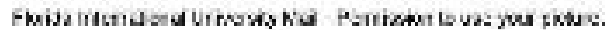

I sm tne w Ih you daרวlh s p oure.

Bext

Ja't

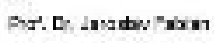

Dapaciere 7 Prgats:

Whistals of Hagerat:

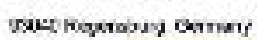

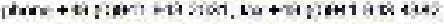

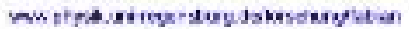

Soswots ares:

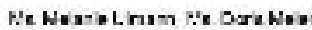

nะar ofnasto

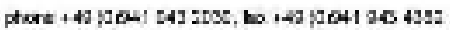

[Servaliveloind

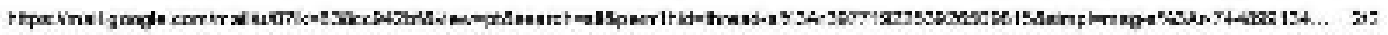




\section{FIU Maiai}

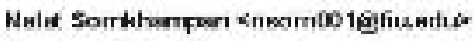

\section{Permission to use your picture.}

2 masages

Nolat Bandhampan scac nDJegnu. zeus

To cker.yasyevpephich

Fil, Fabs 22,2010 of $9: 21 \mathrm{PH}$

\section{Dear Sir.}

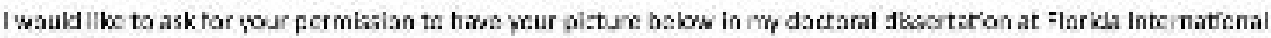

Ltivensit:

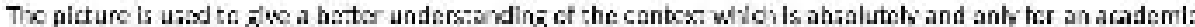
purporse

Bะล. Repards,

Mala: Sarnkhampan
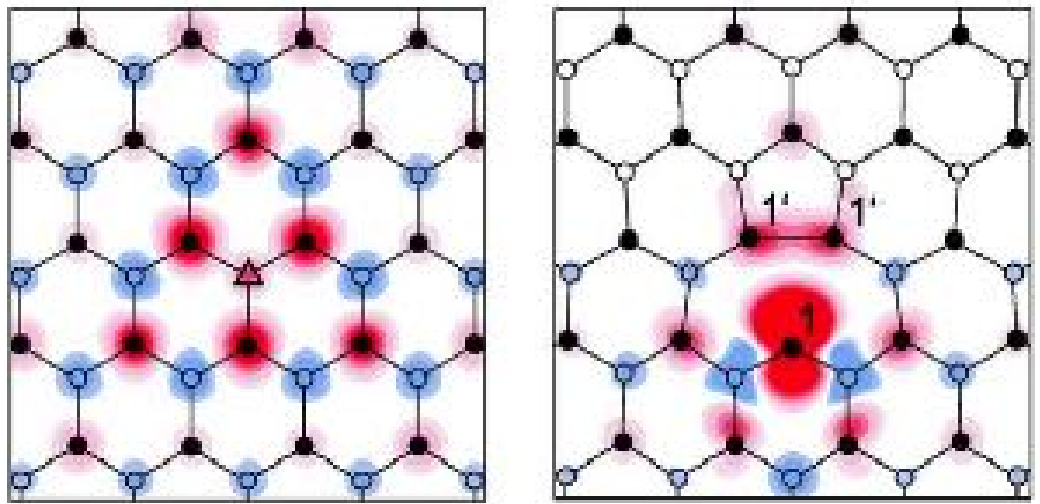

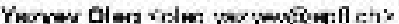

Sun, $=$ - $24,2018+11$ 16 A.

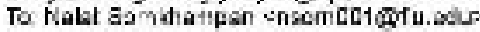

Dear Nislat.

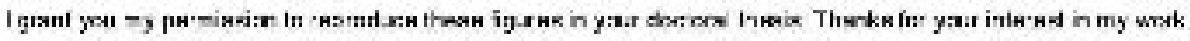

rw:arith

$\mathrm{O}=\mathrm{g}$

P.o Cin: varym

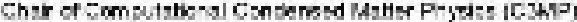

irali.tu of Pryats IIFHYS,

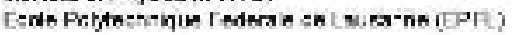

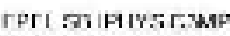

Saton 3

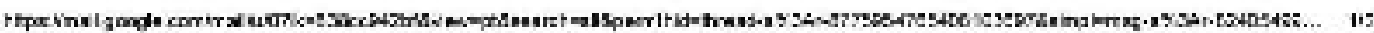


\$พชา

1015 unesene

Satzerian:

Tat: +4 21 c835405

Fas. +4121 serjetid

[nN: $\mathrm{Nm}$,

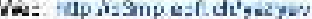

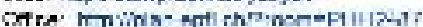

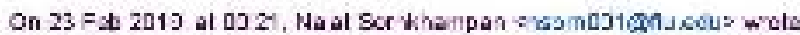

Lejr sir.

I would the to ask for pour permiszion to have your pisture below in nrf dec:oral deser:saion s: Flerids inlmun i:mal (niversilis

The citure is used te give a better unders.anding of the tuntext which is absulutely and anly for sn acsolemic Furpose.

Biest feetards.

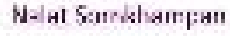

¿mage png

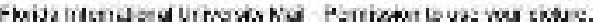

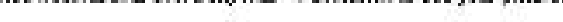

magepngr 


\section{FIU Maiai}

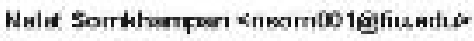

\section{Permission to use your picture.}

2 masages

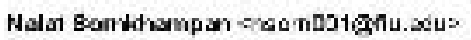

To ircegmknomasch.com

Fil, Fab 22,2010 at $5 x 6 \mathrm{PH}$

\section{Desr Miruchisgat}

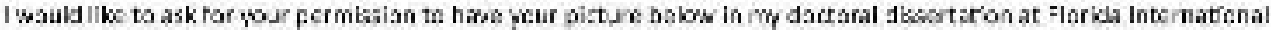
Wrighersily:

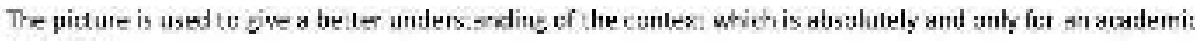
purcorote

Bะs: Rewords,

Mala: Sarrihampan

\begin{tabular}{|c|c|c|}
\hline \multirow{2}{*}{ Grade } & \multicolumn{2}{|c|}{ Mosaic Spread } \\
\cline { 2 - 3 } & Value & Accunacy \\
\hline$Z Y A$ & $0.4^{\circ}$ & $0.1^{\circ}$ \\
\hline$Z Y B$ & $0.3^{\circ}$ & $=0.2^{\circ}$ \\
\hline$Z Y H$ & $3.5^{\circ}$ & $\pm 1.5^{\circ}$ \\
\hline
\end{tabular}

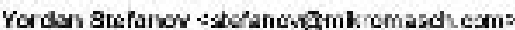

Mon, Fas 25, 2519 at $2 x 0 \mathrm{Am}$ To necrcoi pru.edu

Dasar Nala

Fral of al lha'k you b' daing o.jp podusls

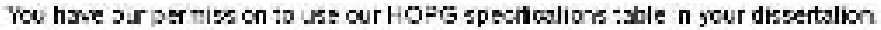

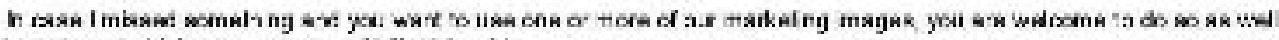

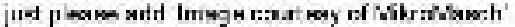

Lel me know if I san hels you whin anyting clse

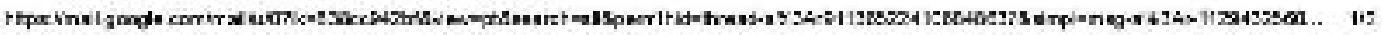


Dest regards.

Yoroan

Yordan Stefanov

CO

Kmasch

Wi brablache

rovotiox Sol juns baspris _td.

48 Jallat Curiz St., 11 ' 3 SeHE, Blaporta

Toml: 3597565 \&S ?

Fax: $+315 s 29636732$

stetanowkmikromasch.0om

WwW_smmips.com

in 0.08

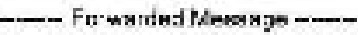

Subject: Perraissiz 1 to tye your pictu re.

Date:Fr, 22 Feb 2019 17:26:27 -0500

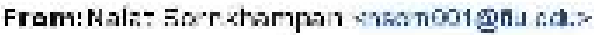

TD: rtatgrakrorissch con

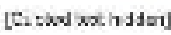

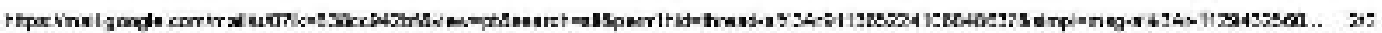




\section{FIU Maiai}

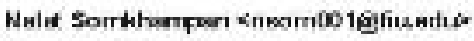

\section{Permission to use your picture.}

3 mesalgés

Nelat Bandhampan scaénajegnu.xeus:

To. P.R.Lesclagkmamisk ac.t.

Fil, Fab 22, 2019 at $324 \mathrm{PH}$

\section{Dear Sit,}

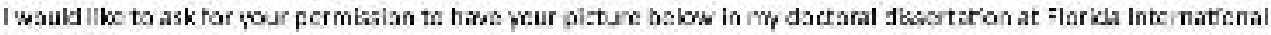
Itigensit.

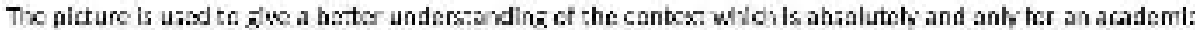
purporse

Bะ- Re⿻ard:,

Mala: Sorkhampan

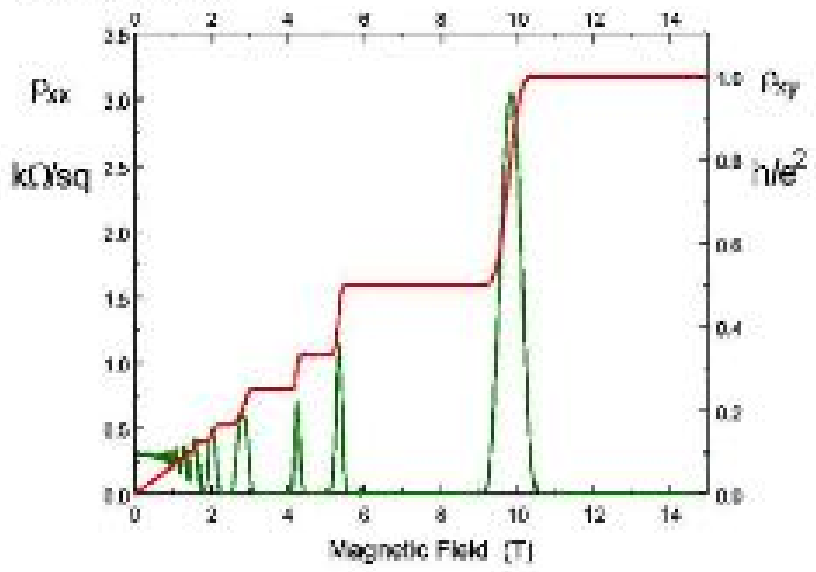

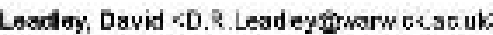

Dear Nals:

bu vre aekeme to ues the figurs in your disser:ston.

Cre: D.R Lejdis: Uniesity of Warwes (1993\%? 


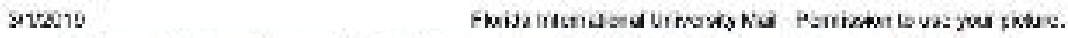

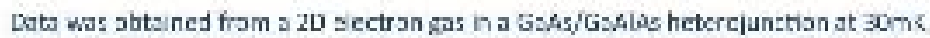

B25. regard:

Lovid

[t:ased sereir $11 \mathrm{~m}]$

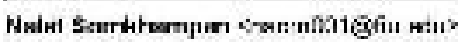

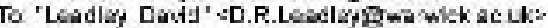

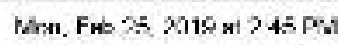

Thank yod wery mush

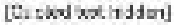




\section{Permission to use screenshots from your YouTube video.}

3 masages

Nolat Sarndhampan scac nDJegnu.zeus

Won, Fab 25, 2319 at 5:47 PH

To ran.b hucpaรuam.2s

\section{Dear Sir}

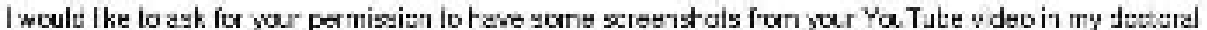

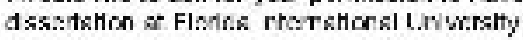

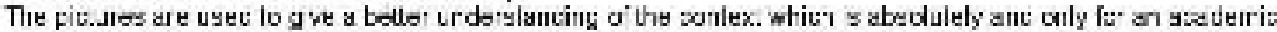
puT.ose

Pend Regje: : $\mathrm{k}$

Plalit Semblamsar

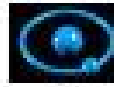

(a)

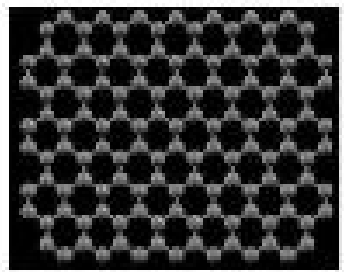

(b)

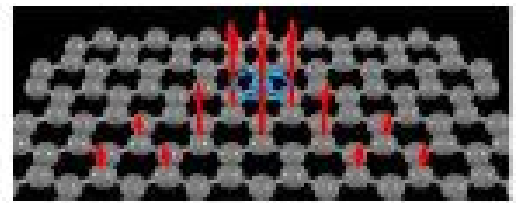

(c)

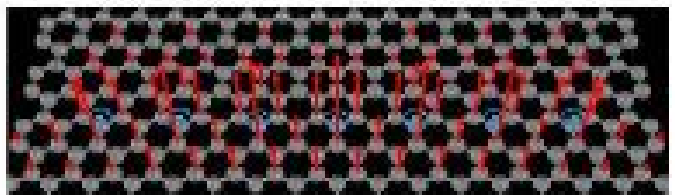

(d)

Iran Bnhuoga \%van,briwegsos.sm es

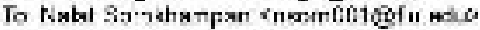

Dear Nalat

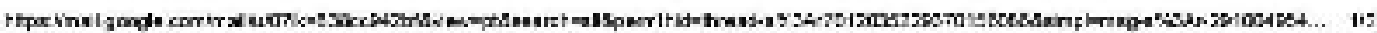


No probkm, you san use the utole vdeo st your set cowen ense.

Gose lusk whth pou doctora etasation.

ตา

[0.: : xd $x x / 1$ dfer|

Twan Arth,ets

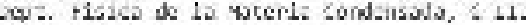

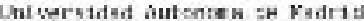

20009 rudrid, siak

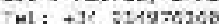

$F 2 \alpha:+32=14973: 61$

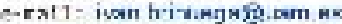

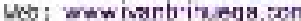

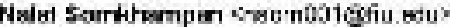

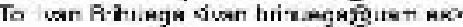

Tu*, =eb $20.2018 * 11: 15 \mathrm{~A}: 1$

Trank you very much

[E. swed $x x$ indsa] 


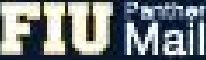

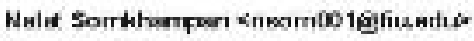

\section{Permission to use your picture}

3 masage:

Nelat Barndhampan scac nDJegnu.zeus

To havgekia ed

Tué, Fab 2E, 2010 at 500 PW

Dear Sit,

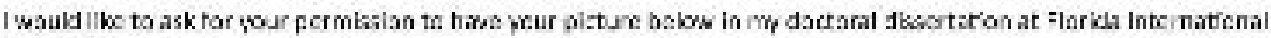

Itiversit:

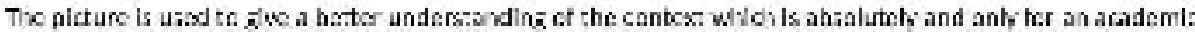
purporse

Bะ- Re⿻ardi,

Malar: Sarkihampan

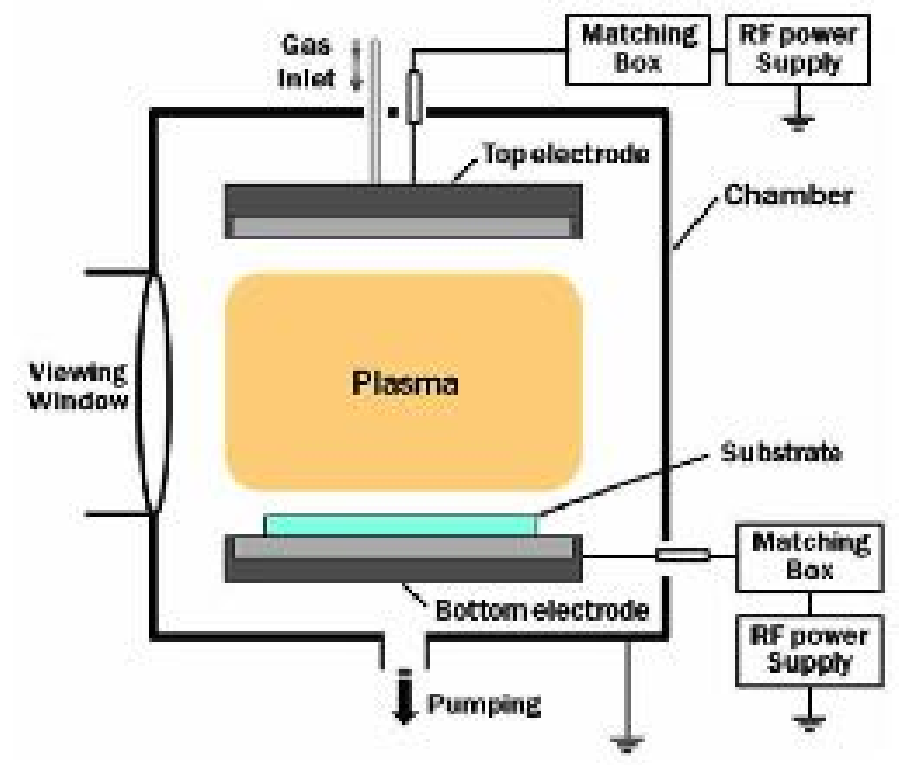

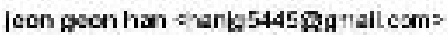

Tue, Fus 26,2019 at $3: 55 \mathrm{PW}$

To Natat 5sาishampan 4ngemCC10

Desar Naral

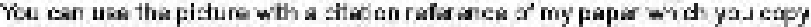

JM:T Han

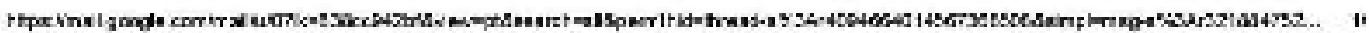


มพอาต

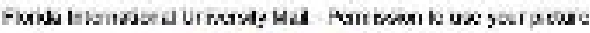

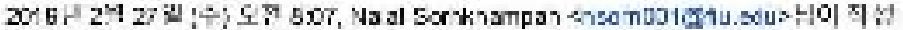

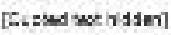

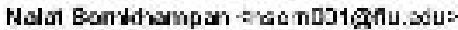

Tue, Fob 2E, 2019 at 9:13 P\$

To joon gavohan stanjy54458ytad cam?

Trark god arry muth

[Eicixd $x x$ indion]

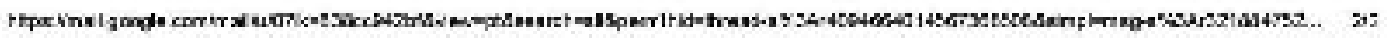




\section{FIU Maiai}

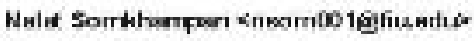

\section{Permission to use your picture}

3 masage:

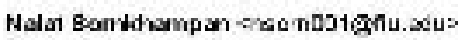

To corlactgacamaxcrialsam

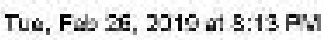

Dear Siri'tisdan,

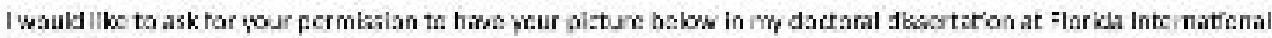

Utigensity

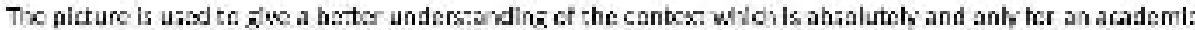
parporse

B:A- Regardi,

Mala: Sornkhampan

$$
\left.\mathrm{CH}_{4} \rightarrow \mathrm{C} \text { (Graphene }\right)+2 \mathrm{H}_{2}
$$

$\mathrm{CH}$. Nucleation Graphene

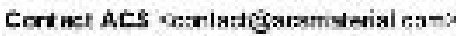

Tue, Fes 88 2013 al 1000 Pos

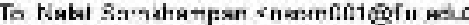

Dear Nals Sundhamsan,

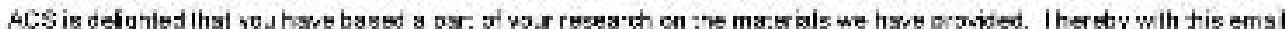

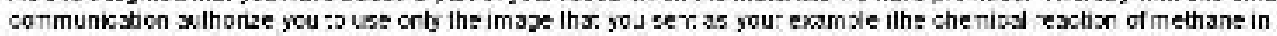

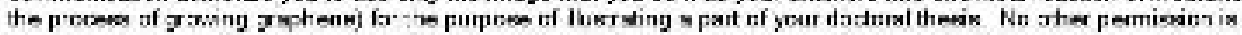

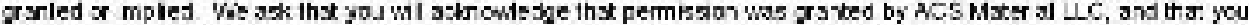
onvit lo us $x$ ind in the pisper.

Trank yn..

Firtant $P_{\text {nex }}: \mathrm{FO}$

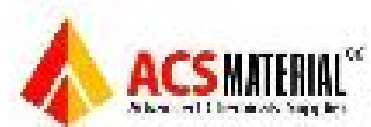

ACS B

$P+18 \times 227-3858$

$\mathrm{F}+1$ 701 518-020?

E sonis:løaranaleris .rom

whw.ses

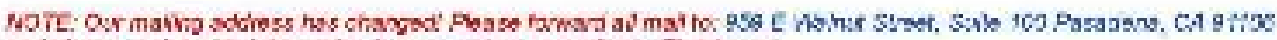

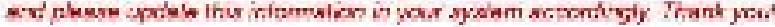

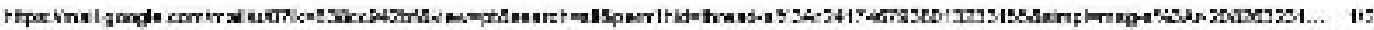


งพอาต

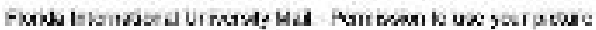

[Ci sad wah Ifer]

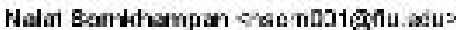

Wed, Fab 27, 2019 at 5-36 PM

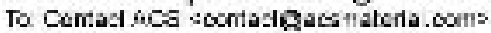

Tosk you very mush

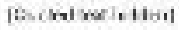

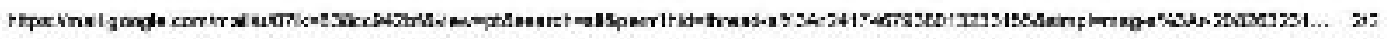




\section{Fiv'mail}

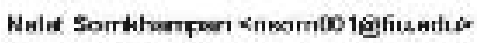

\section{Permission to use your picture}

3 masages

Nolat Bandhampan ecaénasignu.xeus:

To Injtarganudi.sulen

Tué, Fab 26, 2019 at $300 \mathrm{PSH}$

\section{Dear Sit.}

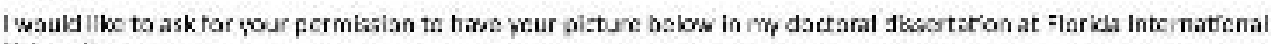
Itiversity.

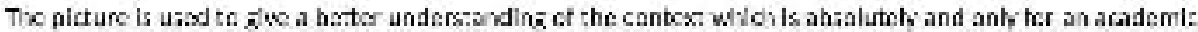
purpose

B:- Regardi,

Mala: Sornkhampan

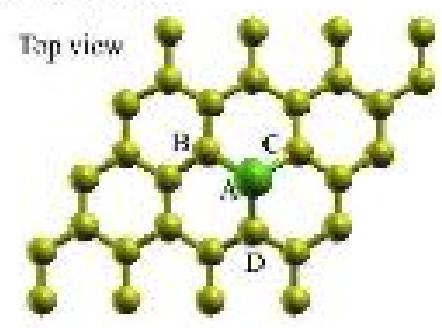

Side vicw:

P-doped graphente

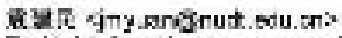

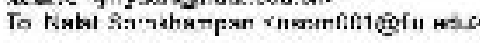

Wed. Fet 27,2018 at $157 \mathrm{~d} / \mathrm{s}$

\section{Doar Nalak}

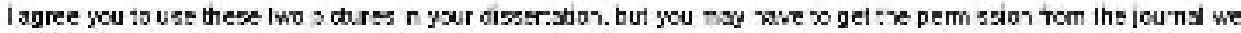
publitied :nese sibures on r..

Best wishes.

Jarmn Yusา

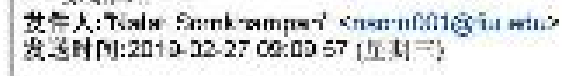

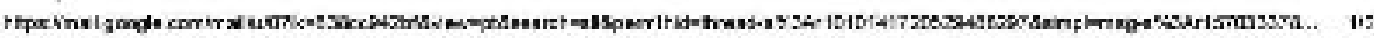




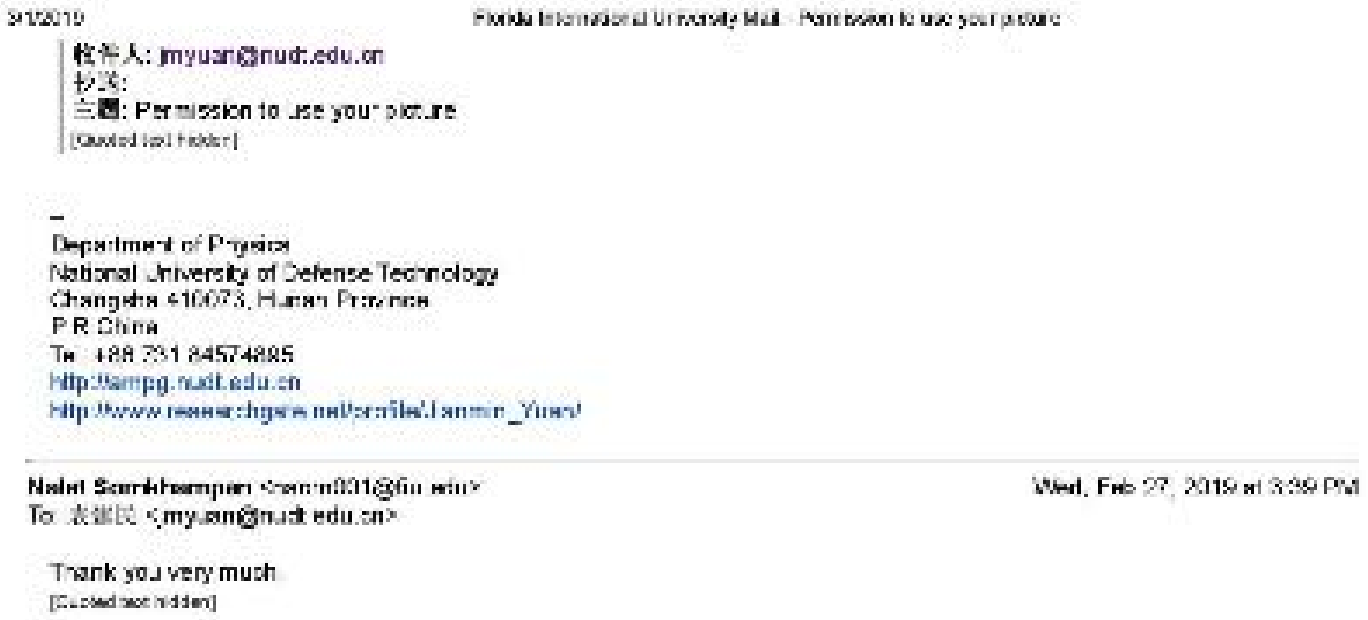




\section{FIU Miail}

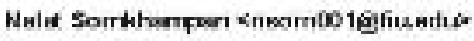

\section{Permission to use your picture}

3 masagé:

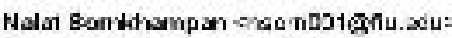

To ge hard.J Jor hamme Bplariece cam

Tué, Fab 2E, 2019 ant 7 SB PY

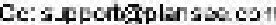

Dew Sin,

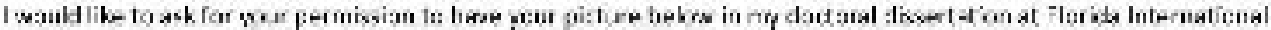

Livarsits:

The picture is used to gue a heter undersiending of the contex which is absalutely and anly for an academi: aunsoces

Bas, Regardi,

pเงla. Sarnishamfan

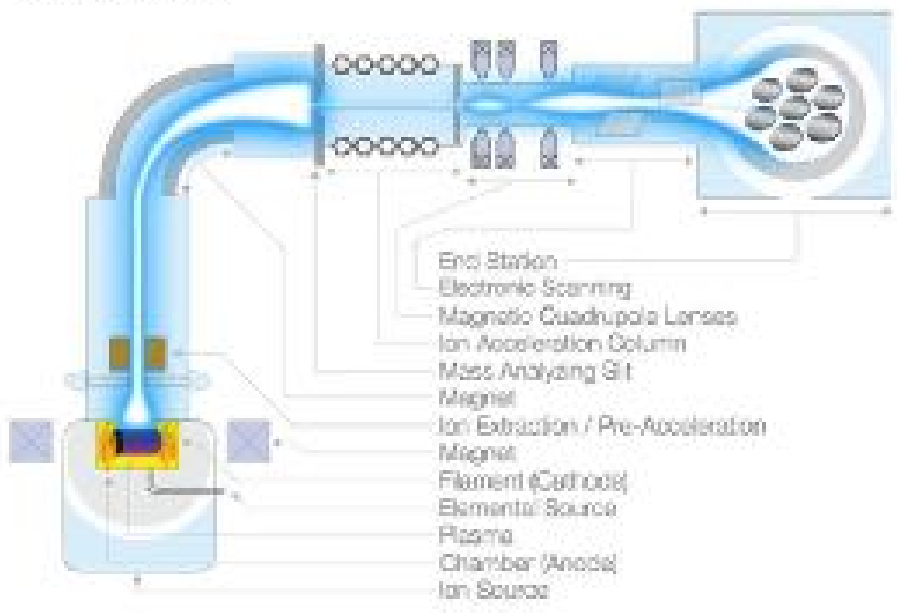

Najol Sorrkbampan kenonojolffiu.sou:

Tue. F th 26, 2J18 3! 5:15 P*1

To usaistolsees com

[C.:akd soerisim]

resend weristmi

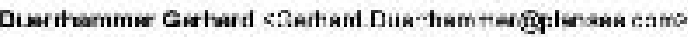

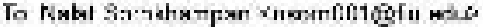

F-i. Rar 1 2019 w1 8'13 AW

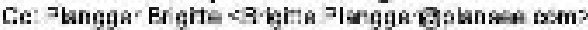

Desr Natat Sandhamsan

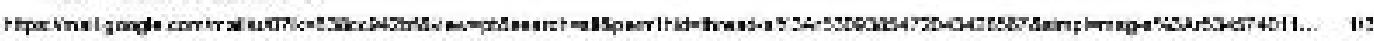




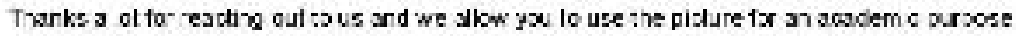

Wodd t be possisle is sา

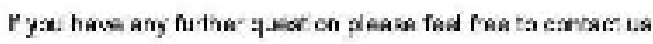

A) the best

G: hard Dirmammer

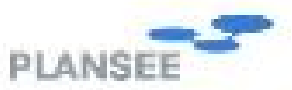

Gerhard Dürharmes

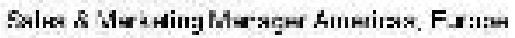

Ela ncss Unt: Scmison

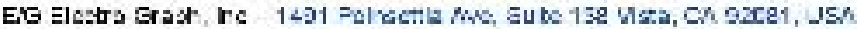

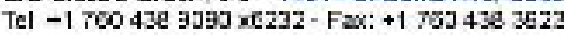

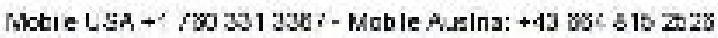

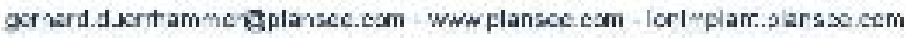

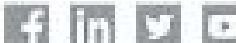

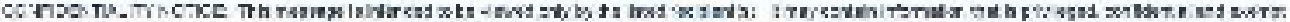

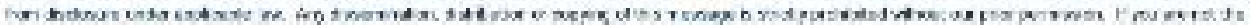

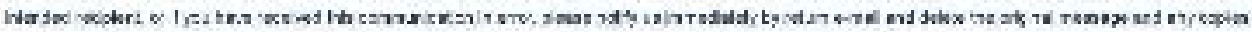

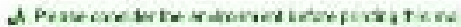

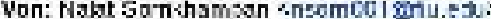

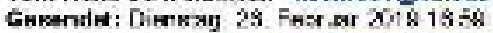

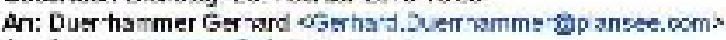

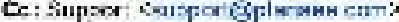

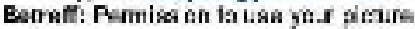

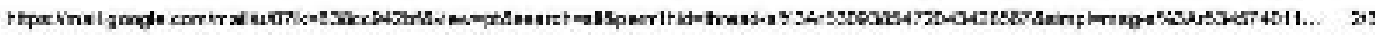




$$
- \pm x T^{-1}
$$

[C: sikd $x x / 1 d d=1]$

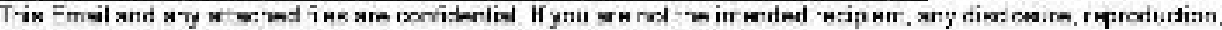

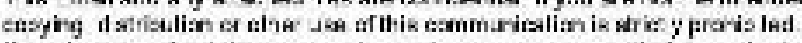

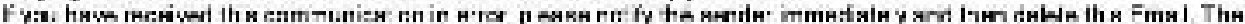

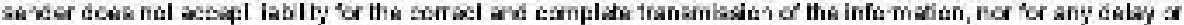

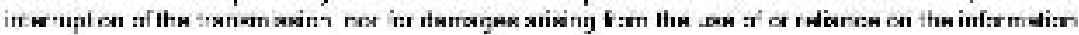

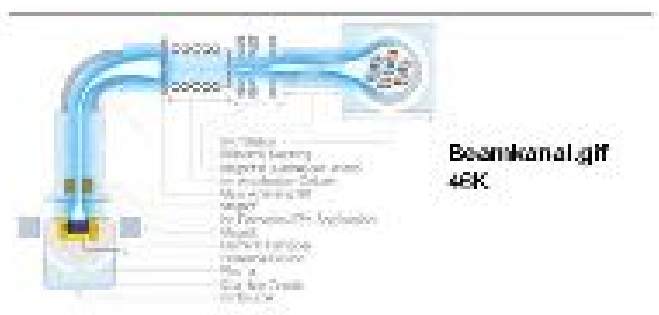




\section{FIU M̄ail}

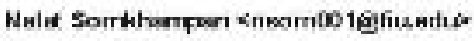

\section{Permission to use your picture.}

3 masages

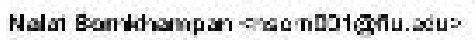

To sami.1 arasilgoabon

Tué, Fab 2E, 2019 of $7: 47$ PW

\section{Dear Sit.}

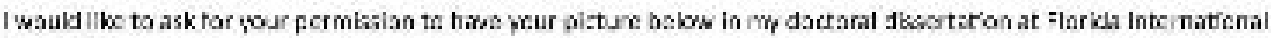

Itiversity.

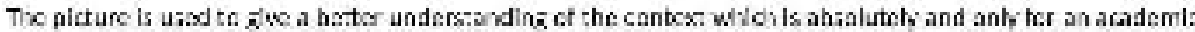
purpose

Bะ- Repards,

Mala: Sornkhampan
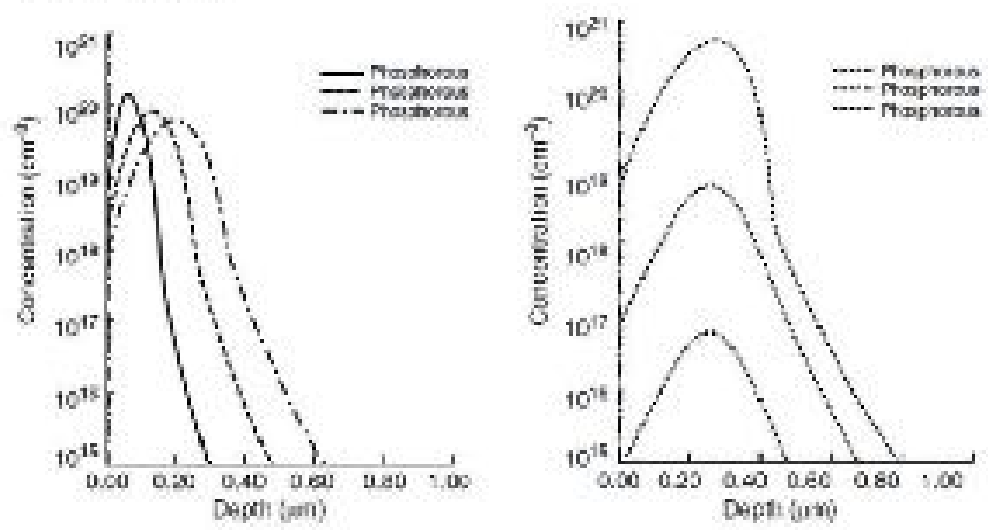

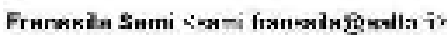

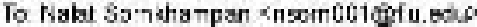

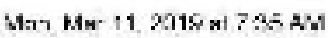

Sorty for ste response, please so ahead.

Sami Franssila

[Beneiveleliou|

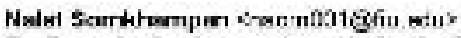

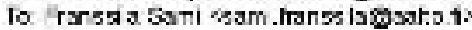

Saר $\mathrm{Ms} 11,2019$ * $200 \mathrm{Pm}$

Trark yod wery much

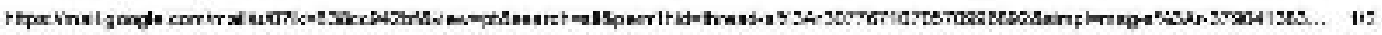


4⿻20าด

[Cisiod $x x i h d f=1]$

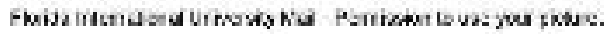

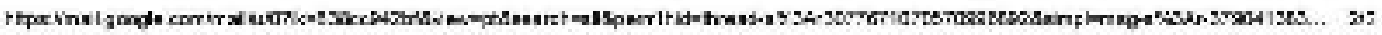


VITA

\title{
NALAT SORNKHAMPAN
}

\author{
Born Bangkok, Thailand \\ $2014-2016 \quad$ B.S., Electrical Engineering \\ Florida International University \\ Miami, FL \\ 2016 - $2017 \quad$ M.S., Electrical Engineering \\ Florida International University \\ Miami, FL \\ 2017 - $2019 \quad$ Ph.D., Electrical Engineering \\ Florida International University \\ Miami, FL \\ 2018 - present 2d Lt., Test Engineer \\ United States Air Force \\ Holloman AFB, NM
}

\section{PUBLICATION AND PRESENTATION}

[1] N. Sornkhampan, J. G. Pinzon, J. P. Zuniga, A. Woods, Y. A. Vlasov, and G. L. Larkins, "Evidence of Superconductivity and Magnetic Vortices in Phosphorous Doped Graphene at Temperatures up to Approximately $260 \mathrm{~K}$," Applied Superconductivity Conference (ASC), Seattle, Washington, 2018.

[2] A. Abbaspour, M. Sanchez, A, Sargolzaei, K. Yen, and N. Sornkhampan, "Adaptive Neural Network Based Fault Detection Design for Unmanned Quadrotor under Faults and Cyber Attacks," IEEE Xplore, 25 ${ }^{\text {th }}$ International Conference on Systems Engineering (ICSEng), 2017. 